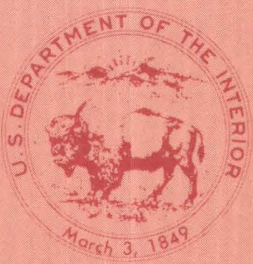

\section{United States Earthquakes, 1984}

\section{U.S. GEOLOGICAL SURVEY BULLETIN 1862}

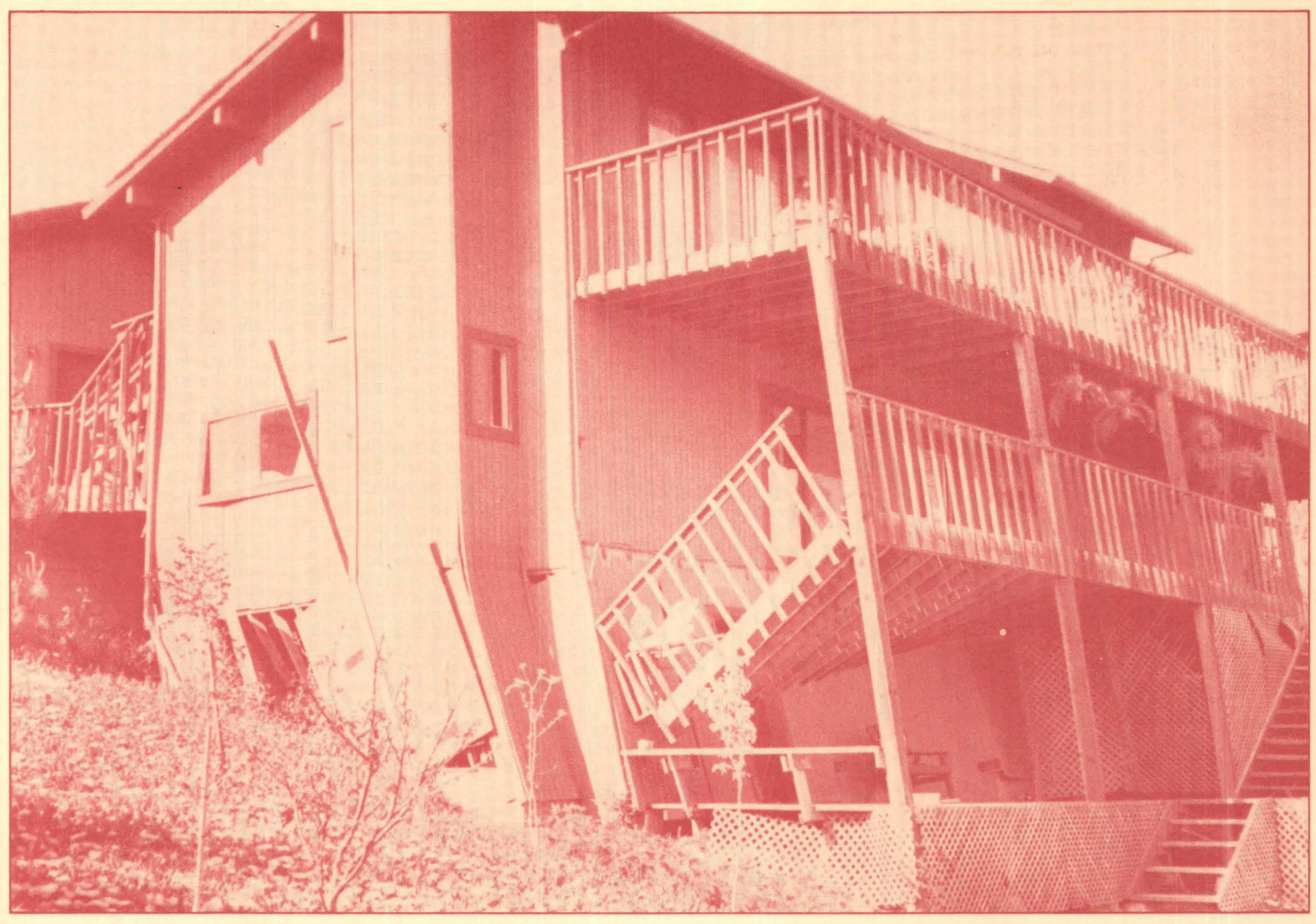




\section{AVAILABILITY OF BOOKS AND MAPS OF THE U.S. GEOLOGICAL SURVEY}

Instructions on ordering publications of the U.S. Geological Survey, along with prices of the last offerings, are given in the current-year issues of the monthly catalog "New Publications of the U.S. Geological Survey." Prices of available U.S. Geological Survey publications released prior to the current year are listed in the most recent annual "Price and Availability List." Publications that are listed in various U.S. Geological Survey catalogs (see back inside cover) but not listed in the most recent annual "Price and Availability List" are no longer available.

Prices of reports released to the open files are given in the listing "U.S. Geological Survey Open-File Reports," updated monthly, which is for sale in microfiche from the U.S. Geological Survey, Books and Open-File Reports Section, Federal Center, Box 25425, Denver, CO 80225. Reports released through the NTIS may be obtained by writing to the National Technical Information Service, U.S. Department of Commerce, Springfield, VA 22161; please include NTIS report number with inquiry.

Order U.S. Geological Survey publications by mail or over the counter from the offices given below.

\section{BY MAIL}

\section{Books}

Professional Papers, Bulletins, Water-Supply Papers, Techniques of Water-Resources Investigations, Circulars, publications of general interest (such as leaflets, pamphlets, booklets), single copies of Earthquakes \& Volcanoes, Preliminary Determination of Epicenters, and some miscellaneous reports, including some of the foregoing series that have gone out of print at the Superintendent of Documents, are obtainable by mail from

\section{U.S. Geological Survey, Books and Open-File Reports Federal Center, Box 25425 Denver, CO 80225}

Subscriptions to periodicals (Earthquakes \& Volcanoes and Preliminary Determination of Epicenters) can be obtained ONLY from the

\section{Superintendent of Documents \\ Government Printing Office \\ Washington, D.C. 20402} ments.)

(Check or money order must be payable to Superintendent of Docu-

\section{Maps}

For maps, address mail orders to

\section{U.S. Geological Survey, Map Distribution \\ Federal Center, Box 25286 \\ Denver, CO 80225}

Residents of Alaska may order maps from

\author{
Alaska Distribution Section, U.S. Geological Survey, \\ New Federal Building - Box 12 \\ 101 Twelfth Ave., Fairbanks, AK 99701
}

\section{OVER THE COUNTER}

\section{Books}

Books of the U.S. Geological Survey are available over the counter at the following Geological Survey Public Inquiries Offices, all of which are authorized agents of the Superintendent of Documents:

- WASHINGTON, D.C.--Main Interior Bldg., 2600 corridor, 18 th and C Sts., NW.

- DENVER, Colorado--Federal Bldg., Rm. 169, 1961 Stout St.

- LOS ANGELES, California--Federal Bldg., Rm. 7638, 300 N. Los Angeles St.

- MENLO PARK, California--Bldg. 3 (Stop 533), Rm. 3128, 345 Middlefield Rd.

- RESTON, Virginia--503 National Center, Rm. 1C402, 12201 Sunrise Valley Dr.

- SALT LAKE CITY, Utah--Federal Bldg., Rm. 8105, 125 South State St.

- SAN FRANCISCO, California--Customhouse, Rm. 504, 555 Battery St.

- SPOKANE, Washington--U.S. Courthouse, Rm. 678, West 920 Riverside Ave..

- ANCHORAGE, Alaska--Rm. 101, 4230 University Dr.

- ANCHORAGE, Alaska--Federal Bldg, Rm. E-146, 701 C St.

\section{Maps}

Maps may be purchased over the counter at the U.S. Geological Survey offices where books are sold (all addresses in above list) and at the following Geological Survey offices:

- ROLLA, Missouri--1400 Independence Rd.

- DENVER, Colorado--Map Distribution, Bldg. 810, Federal Center

- FAIRBANKS, Alaska--New Federal Bldg., 101 Twelfth Ave. 


\section{United States Earthquakes, 1984}

\section{BY CARL W. STOVER}

This publication summarizes data for earthquakes that occurred in the 50 states and Puerto Rico during 1984. Descriptions of individual earthquakes include hypocenters, magnitudes, intensities, and damages. The report also contains results from regional networks and data recorded by strong-motion seismographs 
DEPARTMENT OF THE INTERIOR

DONALD PAUL HODEL, Secretary

U.S. GEOLOGICAL SURVEY

Dallas L. Peck, Director

For sale by the Superintendent of Documents, U.S. Government Printing Office

Washington, D.C. 20402

Any use of trade names is for descriptive purposes only and does not imply endorsement by the U.S. Geological Survey.

\footnotetext{
Library of Congress Cataloging-in-Publication Data

Card number 75-640209
} 


\section{Contributors}

Contributors to this publication are listed below according to the information furnished or service performed:

Hypocenters and magnitudes:

John H. Minsch, U.S. Geological Survey, Denver, Colo.

Robert Y. Koyanagi, Hawaiian Volcano Observatory, U.S. Geological Survey

\section{Intensities:}

Carl W. Stover, U.S. Geological Survey, Denver, Colo.

Robert Y. Koyanagi, Hawaiian Volcano Observatory, U.S. Geological Survey

Network operations (by institution):

University of California, Berkeley

Hawaiian Volcano Observatory, U.S. Geological Survey

Kansas Geological Survey, Lawrence

Lamont-Doherty Geological Observatory, Columbia University, Palisades, N. Y.

Los Alamos National Laboratory, Los Alamos, N. Mex.

New Mexico Institute of Mining and Technology, Socorro

Oklahoma Geological Survey, Leonard

St. Louis University, Missouri

University of Nevada-Reno

University of Utah, Salt Lake City

University of Washington, Seattle

Virginia Polytechnic Institute and State University, Blacksburg

U.S. Geological Survey, Socorro, N. Mex.

Weston Observatory, Boston College, Weston, Mass.

\section{Strong-motion seismograph data:}

Ronald L. Porcella and Josephine C. Switzer, U.S. Geological Survey, Menlo Park, Calif.

Editorial assistance and manuscript preparations:

Francis W. Baldwin, U.S. Geological Survey, Denver, Colo.

Lindie R. Brewer, U.S. Geological Survey, Denver, Colo. 



\section{Introduction 1}

Discussion of tables 1

Epicenter and isoseismal maps 1

Magnitude and intensity ratings 3

Modified Mercalli intensity scale of 19315

Collaborators 8

Earthquake descriptions 10

Alabama 10

Alaska 10

Arkansas 18

California 18

California-Off the coast $\mathbf{5 1}$

Colorado 53

Connecticut 54

Delaware 54

District of Columbia 55

Georgia 55

Hawaii 57

Idaho 60

Illinois 64

Indiana 66

Kansas 66

Kentucky 66

Maine 67

Maryland 69

Missouri 69

Montana 69

Nebraska 70

Nevada 71

New Jersey 73

New Mexico 73

New York 73

North Carolina 74

Ohio 74

Oklahoma 74

Oregon 75

Pennsylvania 75

Puerto Rico 81

South Dakota 81

Tennessee 82

Texas 82

Utah 82

Vermont 83

Virginia 83

Washington 85

West Virginia 86

Wyoming 86 


\section{Network operations 135}

Eastern Aleutian seismicity, 1984: by J. J. Taber, M. A. Luckman, and S. Rosen 135

Northern and central California earthquakes, 1984: by Robert A. Uhrhammer 135

Seismicity and volcanic activity in Hawaii, 1984: by Robert Y. Koyanagi 138

Kansas-Nebraska seismicity, 1984: by Greg M. Hildebrand and Don W. Steeples 140

Central Mississippi Valley earthquakes, 1984: by W. Stauder, R. Herrmann, J. Chulick, C. Finn, P. Leu, T. Shin, K. Rinderknecht, J. Mascarenas, H. Yepes, V. John, and C. Carr 141

Nevada and eastern California earthquakes, 1984: by Ute Vetter $\mathbf{1 4 3}$

New England earthquakes, 1984: by James P. McCaffrey, S.J. 146

Seismicity of New Mexico, 1984: by Allan R. Sanford, Lawrence Jaksha, and Dan Cash 148

Seismic activity in north-central New Mexico, 1984: by Joyce J. Wolff and Daniel J. Cash 149

Socorro, New Mexico area earthquakes, 1984: by Allan R. Sanford, Shirley Wade, Kevin King, and Lawrence Jaksha 150

Oklahoma earthquakes, 1984: by James E. Lawson, Jr., and Kenneth V. Luza 151

Southeastern United States earthquakes, 1984: by G. A. Bollinger, Martin C. Chapman, and Mathew S. Sibol 153

Utah earthquakes, 1984: by Ethan D. Brown 154

Washington earthquakes, 1984: by R. S. Ludwin, S. D. Malone, and R. S. Crosson 157

Miscellaneous activities 160

Principal earthquakes of the World for $1984 \mathbf{1 6 0}$

Strong-motion seismograph data: by Ronald L. Porcella and Josephine C. Switzer 162

Introduction 162

Accelerograph data 163

\section{References cited 176}

Errata: United States earthquakes, 1983 (U.S. Geological Survey Bulletin.1698) 178

\section{Tables}

1. Summary of United States earthquakes for $1984 \mathbf{9 4}$

2. Probability of earthquake occurrence in northern and central California $\mathbf{1 3 8}$

3. $\mathrm{M}_{L} \geq 5$ earthquakes that occurred in northern and central California and adjoining areas during 1984138

4. Kansas and Nebraska earthquakes 1984140

5. Earthquakes lucated by the Sleepy Hollow, Nebraska, seismic network, 1984141

6. New England earthquakes, 1984, with coda magnitudes $\geq 2.0146$

7. Oklahoma earthquake catalog for $1984 \mathbf{1 5 3}$

8. Southeastern United States earthquakes in $1984 \mathbf{1 5 4}$

9. SEUSSN earthquake statistics $1984 \mathbf{1 5 4}$

10. Washington area hypocenters for events with magnitudes $\geq 2.7 \mathbf{1 5 8}$

11. Principal earthquakes of the world during $1984 \mathbf{1 6 0}$

12. Summary of U.S. accelerograph records recovered during $1984 \mathbf{1 6 4}$ 


\section{Figures}

1. Earthquake epicenters in the conterminous United States for 19842

2. Earthquake epicenters in Alaska for 19842

3. Earthquake epicenters in Hawaii for $1984 \mathbf{3}$

4. Epicenters in the conterminous United States for earthquakes with magnitudes $\geq 5.0$ in 19844

5. Epicenters in Alaska for earthquakes with magnitudes $\geq 5.0$ in $1984 \mathbf{4}$

6. Epicenters in Hawaii for earthquakes with magnitudes $\geq 5.0$ in 19845

7. Earthquakes in the conterminous United States that were felt or caused damage in 19846

8. Earthquakes in Alaska that were felt or caused damage in 19846

9. Earthquakes in Hawaii that were felt or caused damage in 19847

10. Isoseismal map for the southern Alaska earthquake of 14 August 1984, 010208.4 UTC 14

11. Isoseismal map for the central California earthquake of 23 January 1984, 054020.3 UTC 19

12. Isoseismal map for the central California earthquake of 27 March 1984, 033635.4 UTC 23

13. Isoseismal map for the Morgan Hill, California, earthquake of 24 April 1984, 211519.0 UTC 25

14. Photograph of damage to a home in Jackson Oaks subdivision of Morgan Hill, California 28

15. Photograph of damage to a home in Jackson Oaks subdivision of Morgan Hill, California 29

16. Photograph of damage to a chimney in San Martin, California 30

17. Isoseismal map for the Owens Valley, California, earthquake of 23 November 1984, 180825.5 UTC 48

18. Isoseismal map for the northern California earthquake of 10 September 1984, 031410.1 UTC 52

19. Isoseismal map for the Wilmington, Delaware earthquake of 19 January 1984, 230334.0 UTC 55

20. Isoseismal map for the northwestern Georgia earthquake of 9 October 1984, 115427.0 UTC 56

21. Isoseismal map for the central Idaho earthquake of 22 August 1984, 094630.2 UTC 62

22. Isoseismal map for the southwestern Indiana earthquake of 28 July 1984, 233927.4 UTC 65

23. Isoseismal map for the British Columbia-Alberta, Canada border, earthquake of 11 February 1984, 133827.0 UTC: 68

24. Isoseismal map for the southeastern Pennsylvania earthquake of 23 April 1984, 013600.1 UTC 76

25. Isoseismal map for the Cunningham, Virginia, earthquake of 17 August 1984, 180546.9 UTC 84

26. Isoseismal map for the northeastern Wyoming earthquake of 29 May 1984, 201832.6 UTC 87

27. Isoseismal map for the northeastern Wyoming earthquake of 8 September 1984, 005931.1 UTC 88

28. Isoseismal map for the eastern Wyoming earthquake of 18 October 1984, 1530 23.0 UTC 89

29. Seismicity recorded by the Shumagin Island seismic network from January 1 to December 30,1984136

30. Cross section of seismicity projected along the line A-A' in figure $29 \mathbf{1 3 7}$

31. The Shumagin seismic network, Alaska, during 1984138

32. Northern and central California seismicity during $1984 \mathbf{1 3 8}$

33. Seismograph stations for the Island of Hawaii during 1984139

34. Earthquakes in Hawaii during 1984 (0-60 kilometers depths, $M \geq 3.5) 139$

35. Southeastern Hawaii Island earthquakes $(M \geq 1.5)$ during 1984139

36. Seismograph stations in Kansas and Nebraska in January 1984140

37. Microearthquakes located by the Kansas-Nebraska seismograph network between August 1977 and December 1984141

38. Central Mississippi Valley earthquakes during 1984 within a $4^{\prime \prime}$ x $5^{\prime \prime}$ region centered at $36.5^{\prime \prime} \mathrm{N}$., $89.5^{\prime \prime}$ W. 142

39. Central Mississippi Valley earthquakes during 1984 within a $1.5^{\prime \prime}$ x $1.5^{\prime \prime}$ region centered at $36.25^{\prime \prime} \mathrm{N}$., $89.75^{\circ \prime} \mathrm{W} .142$

40. Central Mississippi Valley earthquakes during 1984 in the same region as figure 38, scaled by focal depth 143

41. Central Mississippi Valley earthquakes during 1984 in the same region as figure 39 , scaled by focal depth 143

42. The Nevada-eastern California network during 1984144

43. Seismicity of the Nevada-eastern California area during 1984144 
44. Mammoth Lakes, California, area earthquakes during 1984145

45. Earthquakes in the Nevada-eastern California area with magnitude $\geq 3.5146$

46. Northeastern United States earthquakes during $1984 \mathbf{1 4 7}$

47. Earthquakes in New Mexico during 1984148

48. Seismicity of north-central New Mexico during 1984149

49. Seismicity of the Socorro, New Mexico, area during 1984150

50. Distribution of focal depths for the 103 earthquakes plotted in figure 49150

51. Active seismograph stations in Oklahoma for 1984152

52. Distribution of Oklahoma earthquakes for 1984152

53. Southeastern United States earthquakes during $1984 \mathbf{1 5 3}$

54. Southeastern United States seismic network stations during 1984154

55. Earthquake statistics for the southeastern United States during 1984154

56. University of Utah seismograph station network during 1984155

57. Utah and adjacent areas earthquakes during 1984156

58. Earthquakes in Washington and northern Oregon during 1984158 


\section{United States Earthquakes, 1984}

\section{By Carl W. Stover, Editor}

\section{Introduction}

This publication describes all earthquakes that were reported felt in the United States and nearby territories in 1984. Its purpose is to provide a continuous history of U.S. earthquakes to be used in estimating areal seismic risk, designing earthquake-resistant structures, and answering inquiries from scientists, engineers, and the public.

The U.S. Geological Survey/National Earthquake Information Center (USGS/NEIC) collects intensity information primarily by mailing questionnaires, "Earthquake Report" forms, to postmasters and other public institutions (police departments and/or fire departments) in the earthquake area. Completed questionnaires are returned to the USGS, where they are evaluated and intensities are assigned. For damaging earthquakes, the questionnaires are supplemented by USGS field investigations. The USGS/NEIC publishes preliminary maximum intensity data for United States earthquakes in the Preliminary Determination of Epicenters, Monthly Listing (PDE) (for example, Irby and others, 1982). The latest and most complete information is published with maps, diagrams, and photographs in United States Earthquakes (now published as a USGS Bulletin), issued annually since 1928. Copies of issues prior to 1982 can be obtained from the Open-File Services Section (OFSS), Distribution Branch, U.S. Geological Survey, Box 25425, Federal Center, Denver, Colo. 80225 .

This publication is composed of four major sections: "Earthquake Descriptions," which includes a summary of macroseismic data reported for each earthquake and a chronological list of earthquakes by State (table 1); "Network Operations," which summarizes the results from local seismic networks; "Miscellaneous Activities," which contains information on principal earthquakes of the world (table 11); and "StrongMotion Seismograph Data" (table 12). The intensity and macroseismic data in "Earthquake Descriptions" are compiled from questionnaire canvasses (see previous paragraph), newspaper articles, and reports prepared by other government organizations, State institutions, local organizations, and individuals. Each de- scription includes date, hypocenter, the source of the hypocenter computation, magnitude, maximum intensity (Modified Mercalli), and/or macroseismic effects reported by localities that felt the earthquake.

\section{DISCUSSION OF TABLES}

The earthquake parameters in tables 1 and $11 \mathrm{in}$ clude date, origin time, hypocenter (epicenter and focal depth), and magnitude. Table 1 also contains the maximum observed Modified Mercalli (MM) intensity. The origin time and date are listed in Universal Coordinated Time (UTC). The epicenters were taken principally from those published in the USGS Preliminary Determination of Epicenters, Monthly Listings. These data have been updated and new data added from subsequent publications of universities or State agencies who operate seismic networks. The accuracy of the epicenters is that claimed by the institution supplying the hypocenter and is not necessarily the accuracy indicated by the number of decimals listed. The epicenters located by the USGS/NEIC have a varying degree of accuracy, usually twontenths of a degree or less. See Preliminary Determination of Epicenters, Monthly Listing, for an explanation of the accuracy of USGS hypocenters. Depths are listed to the nearest kilometer.

Magnitudes listed in the tables were furnished by cooperating institutions or determined by the USGS. The computational sources are indicated by letter codes identified in headnotes to the tables.

\section{EPICENTER AIND ISOSEISMAL MAPS}

Figures 1-3 are computer plots of all earthquake epicenters in the conterminous United States, Alaska, and Hawaii listed in table 1. Figures 4-6 show only these earthquakes that had computed magnitudes of 5.0 or larger. Each earthquake epicenter is indicated by a small circle or square. 


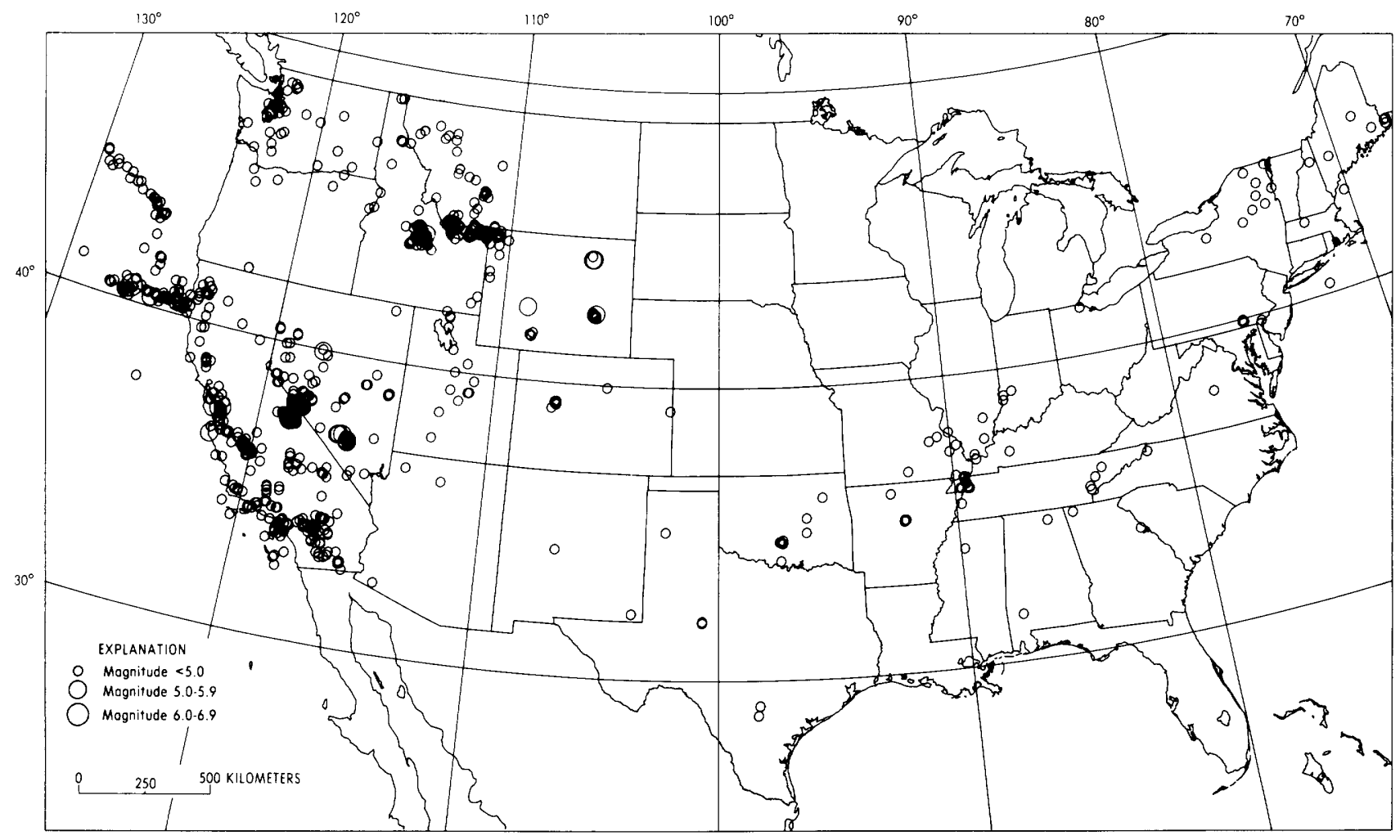

Figure 1. Earthquake epicenters in the conterminous United States for 1984 (from table 1).

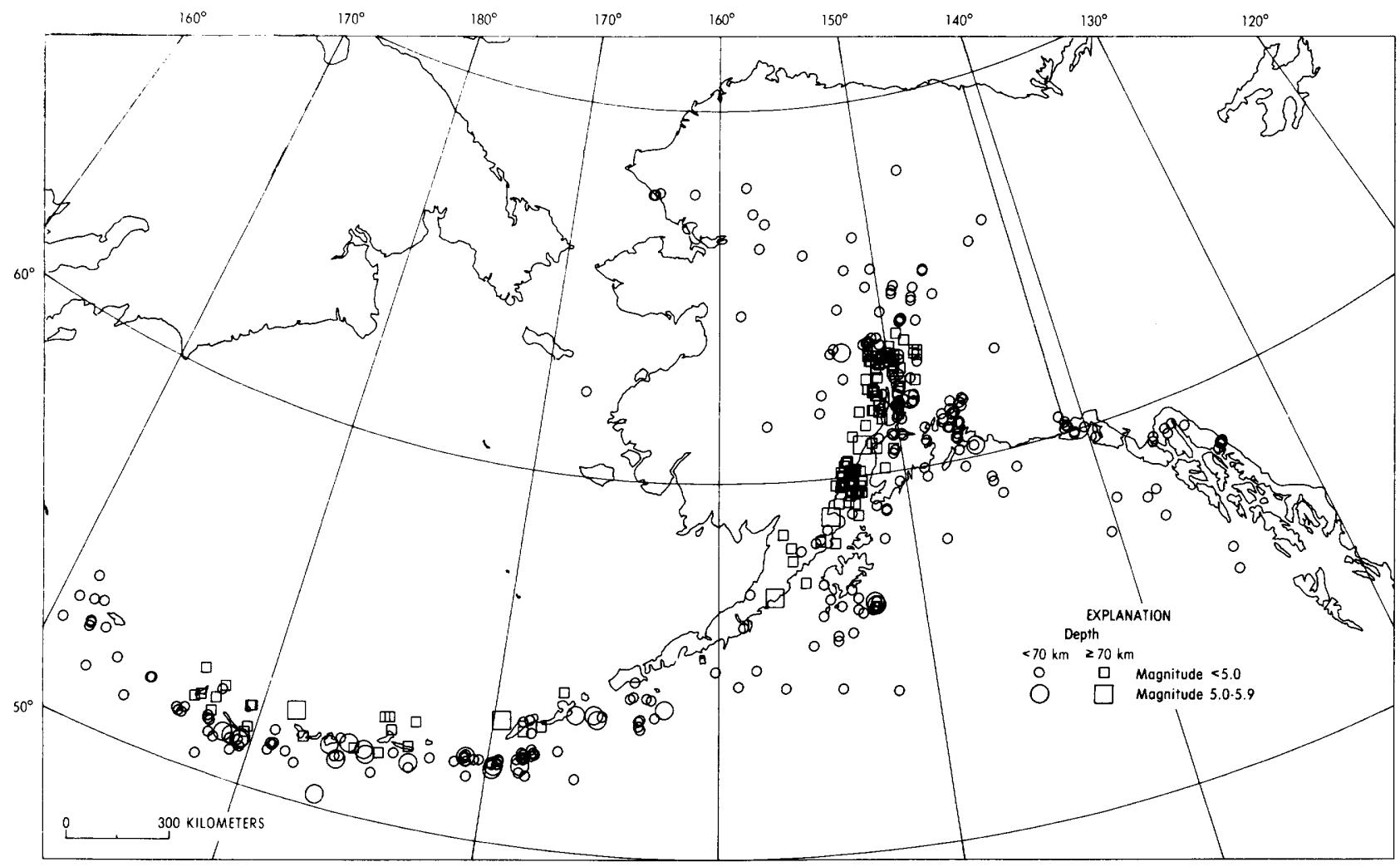

Figure 2. Earthquake epicenters in Alaska for 1984 (from table 1). 


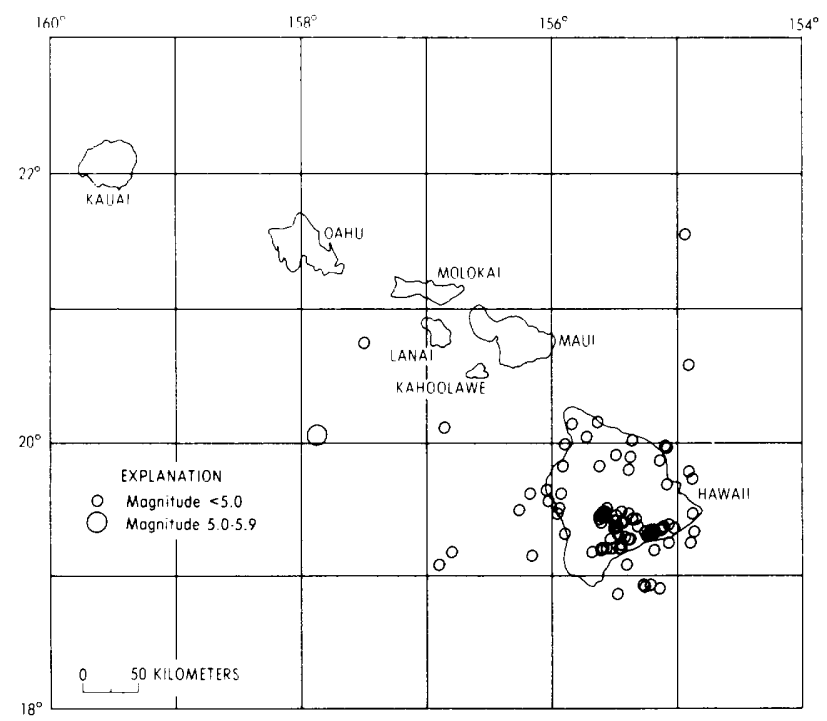

Figure 3. Earthquake epicenters in Hawaii for 1984 (from table 1).

Figures 7-9 are maps showing the maximum intensity of earthquakes in the conterminous United States, Alaska, and Hawaii. Maximum intensities are represented by Arabic numerals at the epicentral locations. Earthquakes of intensity I-IV are represented by solid circles.

The USGS/NEIC coordinates the collection of all types of earthquake information, with the special objective of correlating instrumentally determined earthquake locations with noninstrumental locations indicated by intensity data. This correlation is achieved through regional investigations of earthquakes by local organizations and the USGS. Primary data are gathered by a mail canvass of the epicentral area using questionnaire cards. A field survey is usually carried out for damaging events. When returned and analyzed, this information is used to prepare isoseismal maps which show the areal pattern of intensity associated with individual earthquakes.

The selection of earthquakes for isoseismal maps (shown in the Earthquake Descriptions) is governed largely by the size of the area affected. This means that sharp, localized shocks of intensity VI (which often occur in California) may not be represented by these maps, whereas more widely felt earthquakes of intensity V and VI (which are characteristic of the Eastern and Central States) often will be illustrated because of the larger felt areas. Arabic numerals on these computerplotted maps represent the maximum $\mathrm{MM}$ intensities at sampled localities. Isoseismal contours are a generalization of intensity data and are extrapolated in regions that have few observations. The isoseismals do not account for each intensity observation since they are drawn to show the general patterns at a level of intensity or range of intensities.

\section{MAGNITUDE AND INTENSITY RATINGS}

Magnitude, a measure of the "size" of an earthquake, is related to the energy release at the focus of an earthquake. Although the magnitude scale has neither "top" nor "bottom" values, the highest ever calculated was greater than 9.0 and the lowest was about -3.0 . On this logarithmic scale, a magnitude 6.0 shallowfocus earthquake represents elastic-wave energy about 30 times greater than that generated by a magnitude 5.0 earthquake, 900 times greater than that of a magnitude 4.0 shock, and so forth. Many factors enter into the determination of earthquake magnitude, including earthquake focal depth, frequency content of the sampled energy, and the earthquake radiation pattern. Magnitude values calculated by the USGS are based on the following five formulas:

$$
\begin{aligned}
& \text { Surface Wave Magnitude } \\
& \qquad \mathbf{M}_{s}=\log (\mathbf{A} / \mathbf{T})+1.66 \log \mathbf{D}+3.3,
\end{aligned}
$$

as adopted by the lntermational Association of Seismology and Physics of the Earth's Interior (IASPEI) Bath, (1966), p. 153, where $\mathbf{A}$ is the maximum vertical surface-wave ground amplitude, in micrometers; $\mathbf{T}$ is the period, in seconds, and $18 \leq \mathbf{T} \leq 22$; and $\mathbf{D}$ is the distance in geocentric degrees (station to epicenter), and $20^{\circ} \leq \mathbf{D} \leq 160^{\circ}$. No depth correction is made for depth less than $50 \mathrm{~km}$, and no $\mathbf{M}_{\boldsymbol{S}}$ magnitudes are computed for depths greater than $50 \mathrm{~km}$.

$$
\begin{aligned}
& \text { Body Wave Magnitude } \\
& \qquad \mathbf{m}_{b}=\log (\mathbf{A} / \mathbf{T})+\mathbf{Q}(\mathbf{D}, \mathbf{h}),
\end{aligned}
$$

as defined by Gutenberg and Richter (1956), except that $\mathbf{T}$, the period in seconds, is restricted to $0.1 \leq \mathbf{T}$ $\leq 3.0$, and $\mathbf{A}$, the ground amplitude in micrometers, is not necessarily the maximum of the $P$-wave group. $\mathbf{Q}$ is a function of distance $\mathbf{D}$ and depth $\mathbf{h}$, where $\mathbf{D} \geq 5^{\prime \prime}$.

\section{Local Magnitude

$$
\mathbf{M}_{L}=\log \mathbf{A}-\log \mathbf{A}_{0},
$$

as defined by Richter $(1958$, p. 340), where $A$ is the maximum trace amplitude in millimeters, written by a Wood-Anderson torsion seismometer, and $\log \mathbf{A}_{0}$ is a standard value as a function of distance, where the distance is $\leq 600 \mathrm{~km}$. Values of $\mathbf{M}_{L}$ are also calculated from other seismometers by conversion of recorded ground motion to the expected response of the torsion seismometer. $\mathbf{M}_{L}$ magnitudes are listed for events with depths less than 70 kilometers. 


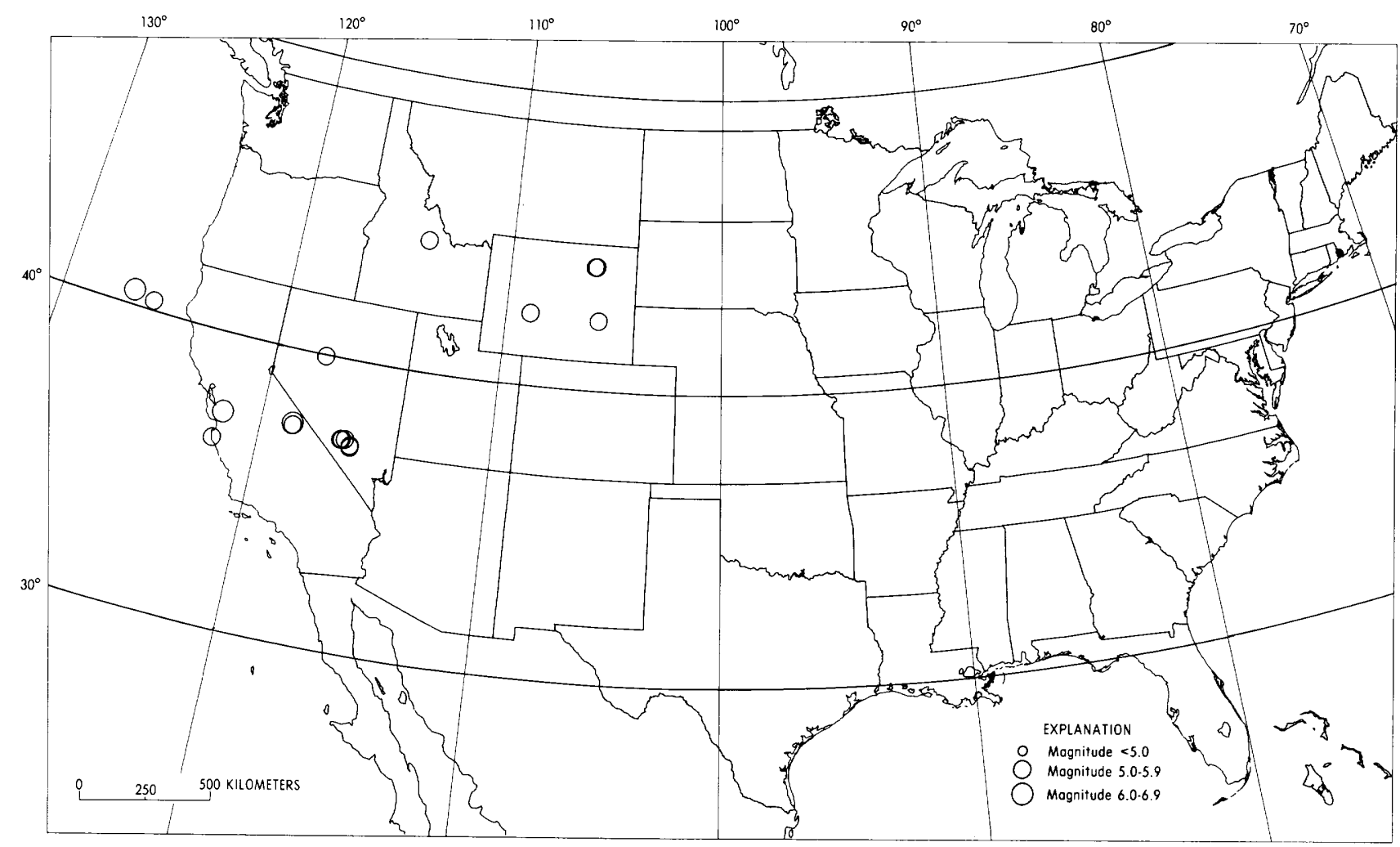

Figure 4. Epicenters in the conterminous United States for earthquakes with magnitudes $\geq 5.0$ in 1984.

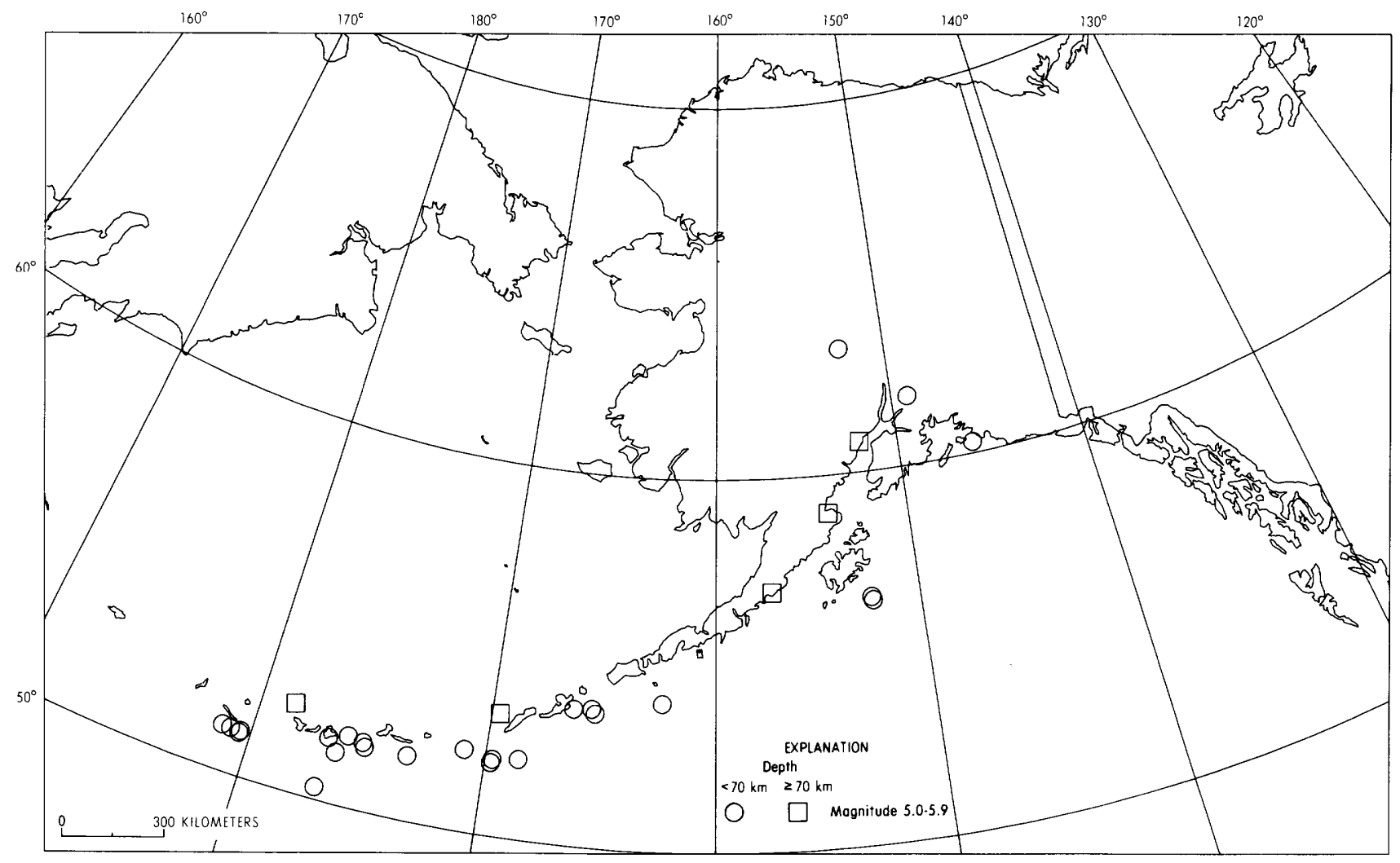

Figure 5. Epicenters in Alaska for earthquakes with magnitudes $\geq 5.0$ in 1984. 


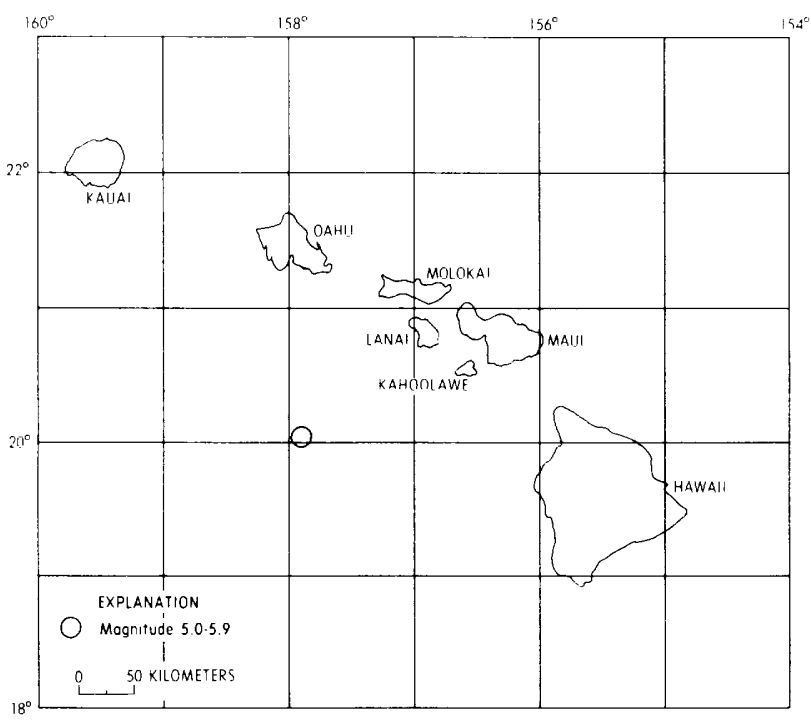

Figure 6. Epicenters in Hawaii for earthquakes with magnitudes $\geq 5.0$ in 1984 .

\section{Local Magnitude}

$$
\begin{gathered}
\mathbf{M}_{n}=3.75+0.90(\log \mathbf{D})+\log (\mathbf{A} / \mathbf{T}) \\
0.5^{\circ} \leq \mathbf{D} \leq 4^{\prime \prime}, \\
\mathbf{M}_{n}=3.30+1.66(\log \mathbf{D})+\log (\mathbf{A} / \mathbf{T}) \\
4^{\prime \prime} \leq \mathbf{D} \leq 30^{\prime \prime}
\end{gathered}
$$

as proposed by Nuttli (1973) for North America east of the Rocky Mountains, where $\mathbf{A} / \mathbf{T}$ is expressed in micrometers per second, calculated from the verticalcomponent 1 -second $L g$ waves, and $\mathbf{D}$ is the distance in geocentric degrees.

\section{Moment Magnitude}

$$
\mathbf{M}_{w}=2 / 3 \log \mathbf{M}_{,-1}-10.7
$$

as defined by Hanks and Kanamori (1979), where $\mathbf{M}_{\text {, }}$ is the seismic moment in dyne-cm.

Other types of magnitudes computed by other organizations or universities are also listed in this publication. These are defined in the following two paragraphs.

$\mathbf{M}_{D}$ designates duration or coda length magnitude. $\mathbf{M}_{D}$ is usually computed from the difference, in seconds, between $P_{n}$ - or $P_{g}$-wave arrival time and the time the final coda amplitude decreases to the background-noise amplitude. Duration magnitude scales are normally adjusted to agree with $\mathbf{M}_{L}$ or $\mathbf{M}_{n}$ estimates so that resulting magnitudes are compatible. Thus, the $\mathbf{M}_{D}$ formulas vary for different geographic regions and seismograph systems.
Some seismograph network operators determine a magnitude formula for their specific network based on a comparison of their computed magnitude values with magnitudes published from other sources; such as the VSGS. These values are usually compared with $\mathbf{m}_{h}$, $\mathbf{M}_{L}$, or $\mathbf{M}_{n}$ magnitudes. In this bulletin these types of magnitudes will be designated as $\mathbf{m}_{r}$ for body wave magnitudes $\left(\mathbf{m}_{l}\right)$ and $\mathbf{M}_{X}$ for local magnitudes $\left(\mathbf{M}_{L}\right.$ or $\mathbf{M}_{n}$ ).

Intensity, as applied to earthquakes, represents a quantity determined from the effects on people, manmade structures, and the Earth's surface (landslides, ground fissures, etc.). Intensities are assigned according to the descriptions listed in the Modified Mercalli Intensity Scale of 1931 (Wood and Neumann, 1931). There are 12 discrete steps in the MM scale (see next section). An earthquake in a populated area will have different intensities at different localities, owing to the distance from the focus of the earthquake, type of focal mechanism, local geological conditions, structural design of buildings, and the earthquake magnitude.

The text of this publication gives the intensity at locations where an earthquake was reported felt and summaries of the strongest effects. Each earthquake is further characterized by its maximum intensity, which is given in the text and in table 1 . The word "FELT" in the maximum intensity columns of table 1 indicates that only minimal or sketchy information was available. This does not imply that the earthquake was felt at a low intensity level, but indicates that the available data were not sufficient for assigning an intensity value.

Although the Modified Mercalli Intensity Scale is in many instances inadequate for present-day requirements, the scale has been the guide used by the USGS and will continue to be so until a new scale has been devised and has acceptance in the engineering and seismological communities.

\section{MODIFIED MERCALLI INTENSITY SCALE OF 1931}

Adapted from Sieberg's Mercalli-Cancani scale, modified and condensed.

I-Not felt-or, except rarely under especially favorable circumstances. Under certain conditions, at and outside the boundary of the area in which a great shock is felt: sometimes birds, animals, reported uneasy or disturbed; sometimes dizziness or nausea experienced; sometimes trees, structures, liquids, bodies of water, may sway-doors may swing very slowly. 


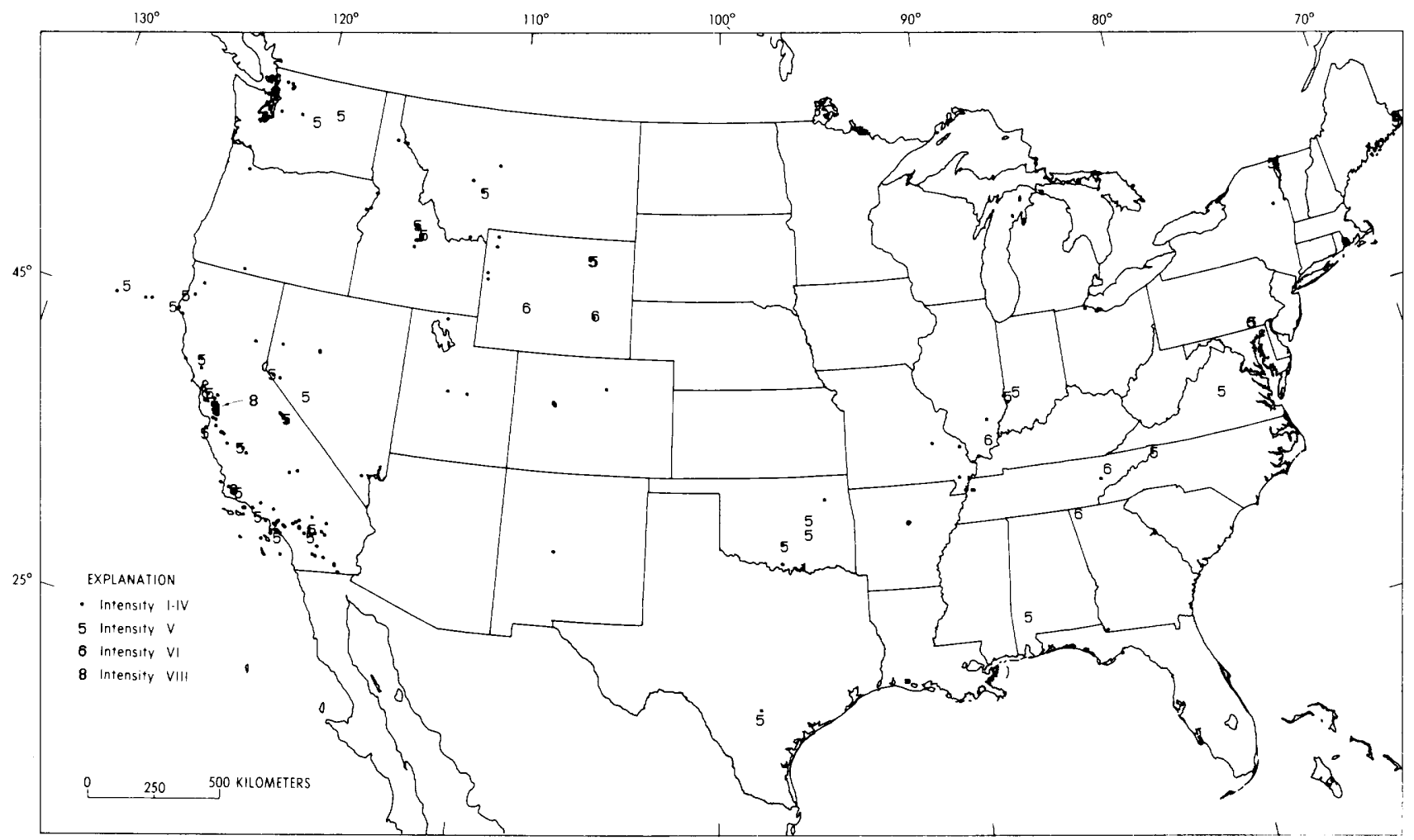

Figure 7. Earthquakes in the conterminous United States that were felt or caused damage in 1984. The intensities denote maximum observed intensity plotted at the epicenter.

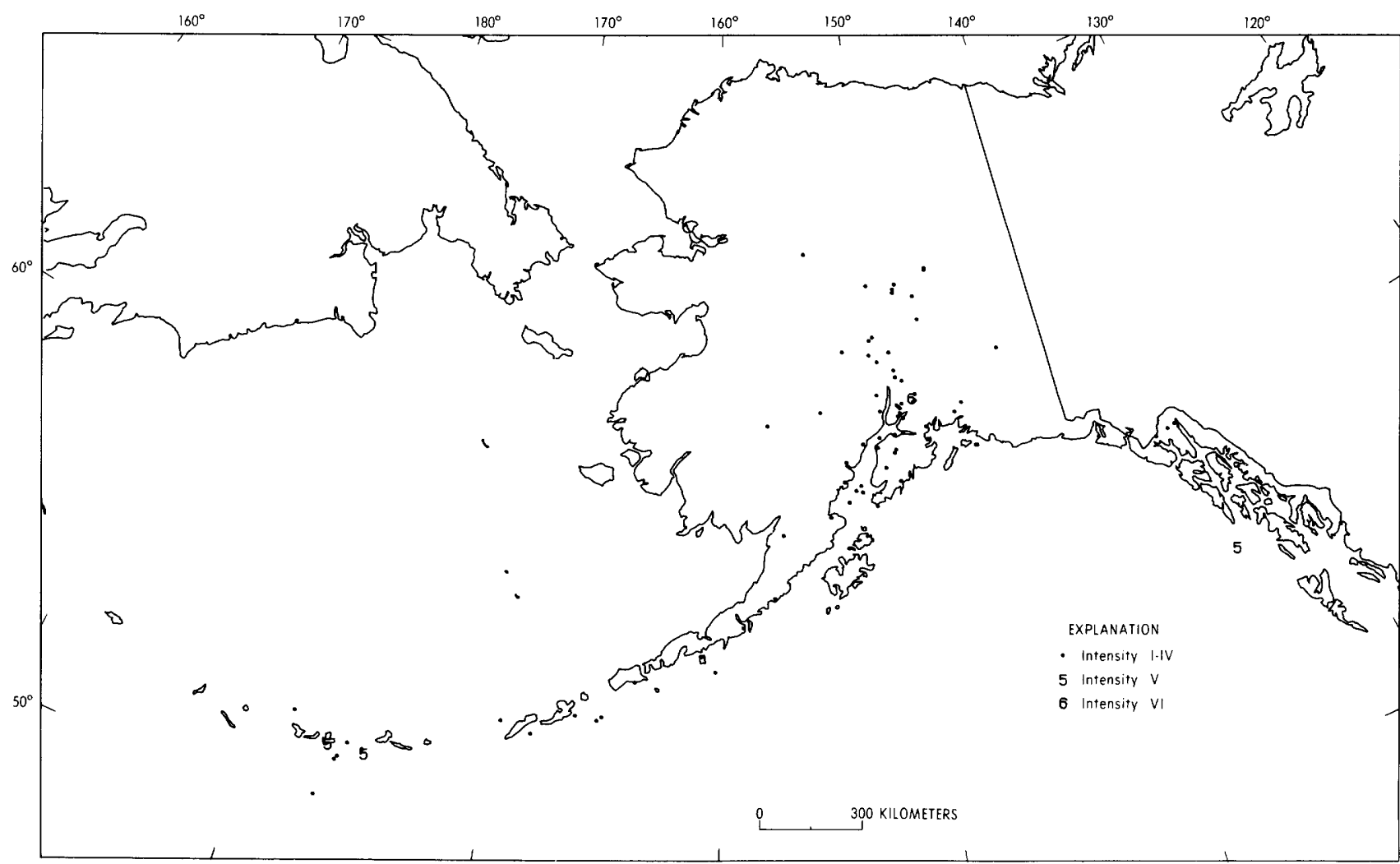

Figure 8. Earthquakes in Alaska that were felt or caused damage in 1984. The intensities denote maximum observed intensity plotted at the epicenter. 


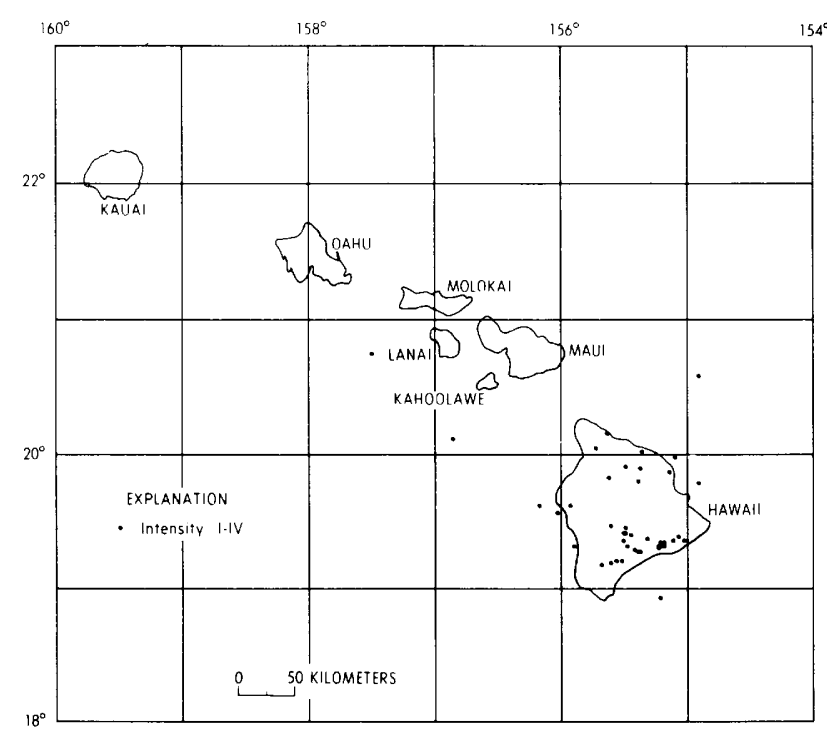

Figure 9. Earthquakes in Hawaii that were felt or caused damage in 1984. The intensities denote maximum observed intensity plotted at the epicenter.

II-Felt indoors by few, especially on upper floors, or by sensitive or nervous persons. Also, as in grade 1, but often more noticeably: sometimes hanging objects may swing, especially when delicately suspended; sometimes trees, structures, liquids, bodies of water, may sway, doors may swing very slowly; sometimes birds, animals, reported uneasy or disturbed; sometimes dizziness or nausea experienced.

III-Felt indoors by several, motion usually rapid vibration. Sometimes not recognized to be an earthquake at first. Duration estimated in some cases. Vibration like that due to passing of light or lightly loaded trucks, or heavy trucks some distance away. Hanging objects may swing slightly. Movements may be appreciable on upper levels of tall structures. Rocked standing motor cars slightly.

IV-Felt indoors by many, outdoors by few. Awakened few, especially light sleepers. Frightened no one, unless apprehensive from previous experience. Vibration like that due to passing of heavy or heavily loaded trucks. Sensation like heavy body striking building or falling of heavy objects inside. Rattling of dishes, windows, doors; glassware and crockery clink and clash. Creaking of walls, frame, especially in the upper range of this grade. Hanging objects swung, in numerous instances. Disturbed liquids in open vessels slightly. Rocked standing motor cars noticeably.

$\mathbf{V}$-Felt indoors by practically all, outdoors by many or most: outdoors direction estimated. Awakened many, or most. Frightened few-slight excitement, a few ran outdoors. Buildings trembled throughout. Broke dishes, glassware, to some extent. Cracked windows-in some cases, but not generally. Overturned vases, small or unstable objects, in many instances, with occasional fall. Hanging objects, doors, swing generally or considerably. Knocked pictures against walls, or swung them out of place. Opened, or closed, doors, shutters, abruptly. Pendulum clocks stopped, started, or ran fast or slow. Moved small objects, furnishings, the latter to slight extent. Spilled liquids in small amounts from well-filled open containers. Trees, bushes, shaken slightly.

VI-Felt by all, indoors and outdoors. Frightened many, excitement general, some alarm, many ran outdoors. Awakened all. Persons made to move unsteadily. Trees, bushes, shaken slightly to moderately. Liquid set in strong motion. Small bells rang-church, chapel, school, etc. Damage slight in poorly built buildings. Fall of plaster in small amount. Cracked plaster somewhat, especially fine cracks in chimneys in some instances. Broke dishes, glassware, in considerable quantity, also some windows. Fall of knickknacks, books, pictures. Overturned furniture in many instances. Moved furnishings of moderately heavy kind.

VII-Frightened all-general alarm, all ran outdoors. Some, or many, found it difficult to stand. Noticed by persons driving motor cars. Trees and bushes shaken moderately to strongly. Waves on ponds, lakes, and ruming water. Water turbid from mud stirred up. Incaving to some extent of sand or gravel stream banks. Rang large church bells, etc. Suspended objects made to quiver. Damage negligible in buildings of good design and construction, slight to moderate in wellbuilt ordinary buildings, considerable in poorly built or badly designed buildings, adobe houses, old walls (especially where laid up without mortar), spires, etc. Cracked chimneys to considerable extent, walls to some extent. Fall of plaster in considerable to large amount, also some stucco. Broke numerous windows, furniture to some extent. Shook down loosened brickwork and tiles. Broke weak chimneys at the roof-line (sometimes damaging roofs). Fall of cornices from towers and high buildings. Dislodged bricks and stones. Overturned heavy furniture, with damage from breaking. Damage considerable to concrete irrigation ditches.

VIII-Fright general-alarm approaches panic. Disturbed persons driving motor cars. Trees shaken strongly-branches, trunks, broken off, especially palm trees. Ejected sand and mud in small amounts. Changes: temporary, permanent; in flow of springs and wells; dry wells renewed flow; in temperature of spring and well waters. Damage slight in structures (brick) built especially to withstand earthquakes. Considerable in ordinary substantial buildings, partial collapse: 
racked, tumbled down, wooden houses in some cases; threw out panel walls in frame structures, broke off decayed piling. Fall of walls. Cracked, broke, solid stone walls seriously. Wet ground to some extent, also ground on steep slopes. Twisting, fall, of chimneys, columns, monuments, also factory stacks, towers. Moved conspicuously, overturned, very heavy furniture.

DX-Panic general. Cracked ground conspicuously. Damage considerable in (masonry) structures built especially to withstand earthquakes: Threw out of plumb some wood-frame houses built especially to withstand earthquakes; great in substantial (masonry) buildings, some collapse in large part; or wholly shifted frame buildings off foundations, racked frames; serious to reservoirs; underground pipes sometimes broken.

$\mathbf{X}$-Cracked ground, especially when loose and wet, up to widths of several inches; fissures up to a yard in width ran parallel to canal and stream banks. Landslides considerable from river banks and steep coasts. Shifted sand and mud horizontally on beaches and flat land. Changed level of water in wells. Threw water on banks of canals, lakes, rivers, etc. Damage serious to dams, dikes, embankments. Severe to well-built wooden structures and bridges, some destroyed. Developed dangerous cracks in excellent brick walls. Destroyed most masonry and frame structures, also their foundations. Bent railroad rails slightly. Tore apart, or crushed endwise, pipe lines buried in earth. Open cracks and broad wavy folds in cement pavements and asphalt road surfaces.

$\mathbf{X I}$-Disturbances in ground many and widespread varying with ground material. Broad fissures, earth slumps, and land slips in soft, wet ground. Ejected water in large amounts charged with sand and mud. Caused sea-waves ("tidal" waves) of significant magnitude. Damage severe to wood-frame structures, especially near shock centers. Great to dams, dikes, embankments often for long distances. Few, if any (masonry) structures remained standing. Destroyed large well-built bridges by the wrecking of supporting piers, or pillars. Affected yielding wooden bridges less. Bent railroad rails greatly, and thrust them endwise. Put pipe lines buried in earth completely out of service.

XII-Damage total-practically all works of construction damaged greatly or destroyed. Disturbances in ground great and varied, numerous shearing cracks. Landslides, falls of rock of significant character, slumping of river banks, etc., numerous and extensive. Wrenched loose, tore off, large rock masses. Fault slips in firm rock, with notable horizontal and vertical offset displacements. Water channels, surface and underground, disturbed and modified greatly. Dammed lakes, produced waterfalls, deflected rivers, etc. Waves seen on ground surfaces (actually seen, probably, in some cases). Distorted lines of sight.

\section{COLLABORATORS}

Active cooperation in earthquake investigations in the United States is provided by several seismological collaborators. The following served as collaborators to the USGS during 1984:

Alaska.-Staff of NOAA-Alaska Tsunami Warning Center, Palmer.

California (northern)--Bruce A. Bolt and Robert A. Uhrhammer, University of California, Berkeley.

California (southern).-Clarence R. Allen and L. K. Hutton, California Institute of Technology, Pasadena.

Canada-Staff of Seismological Service, Geological Survey of Canada, Ottawa and Staff of Pacific Geoscience Centre, Sidney, British Columbia.

Connecticut.-Robert Miller, University of Connecticut, Groton.

Delaware.-Kenneth D. Woodruff, University of Delaware, Newark.

Florida and Georgia.-Leland T. Long, Georgia Institute of Technology, Atlanta.

Hawaii.-Robert Y. Koyanagi, U.S. Geological Survey, Hawaiian Volcano Observatory, Hawaii National Park.

Idaho.-James K. Applegate, Boise State University, Boise.

Indiana.-Gary L. Parlis, Department of Geology, Indiana University, Bloomington.

Iowa.-J. P. Kopp, Loras College, Dubuque.

Kansas.-Don W. Steeples, Kansas Geological Survey, Lawrence.

Kentucky.-Ronald L. Street, University of Kentucky, Lexington.

Missouri, Illinois, Arkansas area.- Otto Nuttli and Robert B. Herrmann, Saint Louis University, Saint Louis.

Montana.-Anthony Qamar, University of Montana, Missoula.

Nevada.-Ute R. Vetter, University of Nevada, Reno.

New England.-James P. McCaffrey, S. J., Boston College, Weston, Mass.

New York.-Lynn R. Sykes, Lamont-Doherty Geological Observatory, Palisades, N.Y.

Ohio.-William R. Ott, S. J., John Carroll University, Cleveland. 
Oklahoma.-James E. Lawson, Jr., Oklahoma Geological Survey, Leonard.

Oregon.-Randy Jacobson, Oregon State University, Corvallis.

Penusylvania.-Benjamin F Howell, Jr. and Shelton Alexander, Pennsylvania State University, University Park.

South Carolina.-Pradeep Talwani, University of South Carolina, Columbia, and Joyce Bagwell, Baptist College at Charleston, Charleston.
Tennessee.-Arch C. Johnston, Tennessee Earthquake Information Center, Memphis.

Texas.-W. D. Pennington, University of Texas, Austin. Utah.-W. J. Arabasz, University of Utah, Salt Lake City.

Virginia.-G. A. Bollinger, Virginia Polytechnic Institute and State University, Blacksburg.

Washington.-Robert S. Crosson, University of Washington, Seattle.

Wyoming.-R. A. Hutchinson, National Park Service, Yellowstone National Park. 


\section{Earthquake Descriptions}

This section lists alphabetically by State (and Puerto Rico) all 1984 earthquakes that were reported felt. The origin time of each earthquake is given in Universal Coordinated Time (UTC). Time is expressed continuously from midnight to midnight, or 0 to 24 hours.

Sources of noninstrumental information (macroseismic data) in this publication include questionnaire canvasses conducted by the USGS, newspaper articles, bulletins of the Seismological Society of America, and special earthquake reports of other organizations. Instrumental data are provided by the USGS/National Earthquake Information Center, other government agencies, and universities that operate seismic networks.

Roman numerals in the earthquake descriptions refer to the Modified Mercalli Intensity Scale of 1931 (see above), which gives about equal weight to the disturbance of inanimate objects and to reactions of people. Where more than one degree of intensity is reported from a town, the town is assigned the highest intensity reported. All earthquake questionnaires or press reports that contain only minimal information, without enough detail from which to assign an intensity, are listed as "FELT."

The following codes are used to indicate sources for hypocenters, magnitudes, intensities and/or felt data: (BK) University of California, Berkeley; (BU) Montana Bureau of Mines and Geology, Butte; (DE) Delaware Geological Survey, Newark; (EN) Department of Energy, Washington, D.C.; (EP) Geophysics Division, Geological Survey of Canada, Ottawa, Ontario; (GM) U.S. Geological Survey, Menlo Park, Calif.; (GS) U.S. Geological Survey, Golden, Colo.; (HV) Hawaiian Volcano Observatory, U.S. Geological Survey, Hawaii National Park; (IU) Indiana University, Bloomington; (LA) Los Alamos National Laboratory, New Mexico; (LD) Lamont-Doherty Geological Observatory, Palisades, N.Y.; (MI) University of Michigan, Ann Arbor; (NM) New Mexico Institute of Mining and Technology, Socorro: (OS) Oregon State University, Corvallis; (PG) Pacific Geoscience Centre,
Sydney, B.C., Canada; (PM) Alaska Palmer Observatory, NOAA, Palmer, Alaska; (PS) California Institute of Technology, Pasadena; (RN) University of Nevada, Reno; (SL) St. Louis University, St. Louis, Mo.; (TC) Tennessee Earthquake Information Center, Memphis; (TU) Oklahoma Geological Survey, Leonard; (UU) University of Utah, Salt Lake City; (VP) Virginia Polytechnic Institute and State University, Blacksburg; (WA) University of Washington, Seattle; (WO) Weston Observatory, Weston, Mass. Normal depth $=33$ $\mathrm{km}$.

\section{ALABAMA}

\section{January (GS) Southwestern Alabama}

Origin time: 030420.9

Epicenter: 31.607 N., $87.808 \mathrm{~W}$.

Depth: $5 \mathrm{~km}$

Magnitude: $2.9 M_{D}(\mathrm{TC})$

Intensity V: Jackson-Dishes were knocked off tables; some pictures fell off walls (press report); a few windows cracked; a few items shook off of store shelves; hairline cracks in plaster walls; many made calls to Jackson Police Station (press report).

Intensity IV: Walker Springs.

Felt: McVay, Mexia, and Winn (press report)

\section{October (TC) Northwestern Georgia}

Origin time: 115427.0

See Georgia listing.

\section{ALASKA}

3 January (GS) Southern Alaska

Origin time: 114009.1

Epicenter: 61.738N., 149.943W.

Depth: $62 \mathrm{~km}$

Magnitude: $3.8 m_{l}(\mathrm{GS}), 3.6 M_{L}(\mathrm{PM})$

Intensity III: Anchorage (press report), Palmer (PM).

\section{January (GS) Southeastern Alaska}

Origin time: 033643.4

Epicenter: 59.254N., $136.057 \mathrm{~W}$.

Depth: $15 \mathrm{~km}$

Magnitude: $3.8 m_{l}(\mathrm{GS}), 4.3 M_{L}(\mathrm{PM})$

Felt: Haines (PM). 
12 January (GS) Southern Alaska

Origin time: 011850.2

Epicenter: 61.764N., $154.265 \mathrm{~W}$.

Depth: Normal

Magnitude: $3.3 M_{L}(\mathrm{PM})$

Intensity III: Palmer (PM).

14 January (GS) Southern Alaska

Origin time: 114424.8

Epicenter: 59.883N., 153.293W.

Depth: $142 \mathrm{~km}$

Magnitude: $4.8 m_{l},(\mathrm{GS})$

Intensity III: Homer.

23 January (GS) Fox Islands, Aleutian Islands

Origin time: 220606.5

Epicenter: 53.287N., 169.639W.

Depth: $103 \mathrm{~km}$

Magnitude: $5.3 m_{l},(\mathrm{GS})$

Intensity III: Nikolski (PM).

27 January (GS) Alaska Peninsula

Origin time: 100008.6

Epicenter: $56.169 \mathrm{~N} ., 158.807 \mathrm{~W}$.

Depth: Normal

Magnitude: $4.1 m_{l}(\mathrm{GS}), 4.1 M_{L}(\mathrm{PM})$

Intensity IV: Chignik (PM).

3 February (GS) Southern Alaska

Origin time: $2317 \quad 15.2$

Epicenter: 62.109N., 150.942W.

Depth: $72 \mathrm{~km}$

Magnitude: $4.1 m_{b}$ (GS)

Felt: Anchorage, Big Lake, and Wasilla (press report).

6 February (GS) Southern Alaska

Origin time: 071631.2

Epicenter: $61.983 \mathrm{~N} ., 148.877 \mathrm{~W}$.

Depth: $18 \mathrm{~km}$

Magnitude: $3.3 M_{L}(\mathrm{PM})$

Felt: Anchorage and Palmer areas (PM).

6 February (GS) Southern Alaska

Origin time: 074817.3

Epicenter: $62.988 \mathrm{~N} ., 150.739 \mathrm{~W}$.

Depth: $129 \mathrm{~km}$

Magnitude: $4.4 m_{l},(\mathrm{GS})$

Intensity II-III: Anchorage to Talkeetna (PM).

6 February (GS) Central Alaska

Origin time: 121535.3

Epicenter: 64.944N., 149.011W.

Depth: Normal
Magnitude: None computed.

Felt: Fairbanks (PM).

10 February (GS) Southern Alaska

Origin time: 221744.4

Epicenter: $61.515 \mathrm{~N} ., 146.329 \mathrm{~W}$.

Depth: Normal

Magnitude: $3.0 M_{L}(\mathrm{PM})$

Felt: Palmer (PM).

14 February (GS) Kenai Peninsula

Origin time: 181724.4

Epicenter: 59.721N., 150.319W.

Depth: Normal

Magnitude: $3.3 M_{L}(\mathrm{PM})$

Intensity III: Homer.

22 February (GS) Central Alaska

Origin time: 160440.8

Epicenter: $65.032 \mathrm{~N} ., 150.744 \mathrm{~W}$.

Depth: Normal

Magnitude: $4.3 M_{L}(\mathrm{PM})$

Felt: Manley Hot Springs (PM).

24 February (GS) Southern Alaska

Origin time: 064704.3

Epicenter: 60.393N., 153.106W.

Depth: $140 \mathrm{~km}$

Magnitude: $4.3 m_{l},(\mathrm{GS})$

Intensity III: Homer.

26 February (GS) Southern Alaska

Origin time: 112326.6

Epicenter: $61.376 \mathrm{~N} ., 149.751 \mathrm{~W}$.

Depth: $43 \mathrm{~km}$

Magnitude: $3.9 m_{l}(\mathrm{GS}), 3.6 M_{L}(\mathrm{PM})$

Intensity IV: Skwentna.

Intensity III: Anchorage, Eagle River (press report).

27 February (GS) Southern Alaska

Origin time: 031221.6

Epicenter: $59.747 \mathrm{~N}$., $152.450 \mathrm{~W}$.

Depth: $104 \mathrm{~km}$

Magnitude: None computed.

Felt: Homer (PM).

7 March (GS) Southern Alaska

Origin time: 023556.2

Epicenter: $61.059 \mathrm{~N}$., $148.556 \mathrm{~W}$.

Depth: Normal

Magnitude: $3.5 M_{L}(\mathrm{PM})$

Intensity II: Anchorage and Palmer (PM). 
14 March (GS) Southern Alaska

Origin time: 203252.4

Epicenter: $61.703 \mathrm{~N} ., 149.859 \mathrm{~W}$.

Depth: $67 \mathrm{~km}$

Magnitude: $4.8 m_{l}$, (GS), $4.5 M_{L}$ (PM)

Intensity IV: Eagle River, Fort Richardson, Palmer (PM), Skwentna, Sutton (PM), Wasilla (PM), Willow.

Intensity III: Anchorage, Anchorage International Airport, Chugiak, Cooper Landing, Moose Pass, and Spenard.

Felt: Talkeetna.

23 March (GS) Southern Alaska

Origin time: 083805.8

Epicenter: 58.978 N., $154.153 \mathrm{~W}$.

Depth: $119 \mathrm{~km}$

Magnitude: $5.3 m_{b}(\mathrm{GS})$

Intensity IV: Homer, Larsen Bay, Naknek, Portlock (PM).

Intensity III: Akhiok, Anchorage, Moose Pass, Port Lions, and Seldovia.

Intensity II: Anchorage International Airport, Kodiak (PM).

Felt: Anchor Point.

15 April (GS) Southern Alaska

Origin time: 165934.1

Epicenter: $60.681 \mathrm{~N} ., 148.542 \mathrm{~W}$.

Depth: $63 \mathrm{~km}$

Magnitude: $3.8 m_{l}$ (GS), 3.9 $M_{L}(\mathrm{PM})$

Felt: Anchorage (PM).

15 April (GS) Southern Alaska

Origin time: 200500.1

Epicenter: 59.610N., 152.775W.

Depth: $97 \mathrm{~km}$

Magnitude: $4.0 m_{\imath}$ (GS)

Felt: Homer.

\section{April (GS) Southern Alaska}

Origin time: 193128.7

Epicenter: 60.833N., 152.067W.

Depth: $95 \mathrm{~km}$

Magnitude: $5.1 m_{b}(\mathrm{GS})$

Intensity IV: Kasilof, Moose Pass, Ninilchik, Skwentna, Sutton, and Tyonek.

Intensity III: Anchor Point, Homer, Seward, Wasilla, and Whittier.

Intensity II: Willow.

Felt: Anchorage, Kenai, Palmer, and Valdez (PM).

19 April (GS) Southern Alaska

Origin time: 201436.3

Epicenter: 61.516N., 149.893W.
Depth: $74 \mathrm{~km}$

Magnitude: None computed.

Intensity II: Anchorage and Palmer (PM).

23 April (GS) Central Alaska

Origin time: 014236.3

Epicenter: 65.182N., 146.945W.

Depth: $15 \mathrm{~km}$

Magnitude: $4.2 M_{L}(\mathrm{PM})$

Felt: Ester and Fairbanks (PM).

23 April (GS) Central Alaska

Origin time: 023216.9

Epicenter: $65.243 \mathrm{~N} ., 146.889 \mathrm{~W}$.

Depth: $22 \mathrm{~km}$

Magnitude: $3.3 M_{L}(\mathrm{PM})$

Felt: Fairbanks (PM).

26 April (GS) Southeastern Alaska

Origin time: 181058.4

Epicenter: $61.295 \mathrm{~N}, 146.827 \mathrm{~W}$.

Depth: Normal

Magnitude: $3.4 M_{L}(\mathrm{PM})$

Intensity III: Valdez (PM).

2 May (GS) Southeastern Alaska

Origin time: 185905.7

Epicenter: 55.590N., 135.047W.

Depth: $10 \mathrm{~km}$

Magnitude: $4.9 m_{b}(\mathrm{GS}), 4.0 M_{\hookrightarrow}(\mathrm{GS}), 4.9 M_{L}(\mathrm{PM})$, $5.1 M_{L}(\mathrm{EP})$

Intensity V: Port Alexander-A few windows cracked; a few small objects overturned and fell; moving vehicles rocked slightly; windows rattled.

Intensity III: Craig, Sitka (PM).

\section{May (GS) Southern Alaska}

Origin time: 230318.9

Epicenter: $59.316 \mathrm{~N} ., 153.187 \mathrm{~W}$.

Depth: $88 \mathrm{~km}$

Magnitude: $4.8 m_{b}$ (GS)

Intensity IV: Homer, Seldovia.

Felt: Anchorage and Seward (PM).

6 May (GS) Andreanof Islands, Aleutian Islands

Origin time: 195449.1

Epicenter: $51.688 \mathrm{~N} ., 176.774 \mathrm{~W}$.

Depth: $58 \mathrm{~km}$

Magnitude: $5.6 m_{l}(\mathrm{GS}), 5.6 M_{L}(\mathrm{PM})$

Intensity V: Adak - Small objects overturned or fell; a few items shook off of store shelves; standing and moving vehicles rocked slightly; trees and bushes shook 
slightly; water splashed onto sides of ponds; felt by many.

\section{June (GS) Alaska Peninsula}

Origin time: 014421.4

Epicenter: $56.901 \mathrm{~N} ., 157.262 \mathrm{~W}$.

Depth: $94 \mathrm{~km}$

Magnitude: $5.3 m_{b}$, (GS)

Intensity IV: Chignik (PM).

11 June (GS) Southern Alaska

Origin time: 012817.0

Epicenter: $62.492 \mathrm{~N} ., 149.829 \mathrm{~W}$.

Depth: $15 \mathrm{~km}$

Magnitude: $4.0 M_{L}(\mathrm{PM})$

Intensity II: Palmer (PM).

12 June (GS) Fox Islands, Aleutian Islands

Origin time: 110915.4

Epicenter: 53.648 N., $165.218 \mathrm{~W}$.

Depth: $43 \mathrm{~km}$

Magnitude: $5.3 m_{l}$ (GS), 4.8 $M_{s}$ (GS), 5.2Ms $(\mathrm{BK})$

Intensity III: Cold Bay, Unalaska.

\section{June (GS) Southern Alaska}

Origin time: 021646.0

Epicenter: 60.960N., 150.340W.

Depth: $50 \mathrm{~km}$

Magnitude: $3.3 m_{r}$ (GS), $3.8 M_{L}$ (PM)

Intensity III: Anchorage.

14 June (GS) Southern Alaska

Origin time: 062627.8

Epicenter: 62.377N., 149.464W.

Depth: $88 \mathrm{~km}$

Magnitude: $3.6 m_{l},(\mathrm{GS})$

Intensity II: Anchorage (PM).

15 June (GS) Andreanof Islands, Aleutian Islands

Origin time: 042714.9

Epicenter: $52.335 \mathrm{~N} ., 178.550 \mathrm{~W}$.

Depth: $170 \mathrm{~km}$

Magnitude: $5.1 m_{l}(\mathrm{GS})$

Intensity II: Adak (PM).

17 June (EP) Southeastern Alaska

Origin time: 151818.0

Epicenter: 59.270N., 135.630W.

Depth: $18 \mathrm{~km}$

Magnitude: $3.1 M_{L}$ (PM)

Intensity III: Haines (PM).
23 June (EP) Southeastern Alaska

Origin time: 102204.0

Epicenter: 59.640N., 139.630W.

Depth: $18 \mathrm{~km}$

Magnitude: $3.2 M_{L}(\mathrm{EP})$

Intensity IV: Yakutat.

28 June (GS) Central Alaska

Origin time: 222306.9

Epicenter: 64.751N., 149.130W.

Depth: Normal

Magnitude: $3.6 M_{L}$ (PM)

Intensity III: Fairbanks (PM).

16 July (GS) Central Alaska

Origin time: 110942.1

Epicenter: $63.576 \mathrm{~N} ., 150.999 \mathrm{~W}$.

Depth: $15 \mathrm{~km}$

Magnitude: $3.7 m_{l},(\mathrm{GS}), 4.1 M_{L}$ (PM)

Intensity IV: Denali National Park Headquarters, Kantishna Lodge, and Wonder Lake (PM).

25 July (GS) Southern Alaska

Origin time: 045950.1

Epicenter: $62.688 \mathrm{~N} ., 149.811 \mathrm{~W}$.

Depth: $71 \mathrm{~km}$

Magnitude: $4.4 m_{b}$ (GS)

Intensity IV: Talkeetna and Willow (PM).

Intensity II: Anchorage (PM).

Felt: Palmer and Wasilla (PM).

25 July (GS) Southern Alaska

Origin time: 123645.9

Epicenter: 61.504N., 157.329W.

Depth: Normal

Magnitude: $4.1 m_{l}$ (GS), 4.8 $M_{L}$ (PM)

Intensity IV: Aniak and Akiak (PM).

Intensity II: Bethel (PM).

25 July (GS) Alaska Peninsula area

Origin time: 232853.3

Epicenter: $54.955 \mathrm{~N} ., 160.113 \mathrm{~W}$.

Depth: $53 \mathrm{~km}$

Magnitude: $5.3 m_{1},(\mathrm{GS}), 4.7 M_{L}(\mathrm{PM})$

Intensity IV: Sand Point (PM).

27 July (GS) Andreanof Islands, Aleutian Islands

Origin time: 155751.3

Epicenter: $50.317 \mathrm{~N} ., 176.868 \mathrm{~W}$.

Depth: Normal

Magnitude: $5.8 m_{1}$ (GS), 5.0M $(\mathrm{GS}), 5.7 M_{L}(\mathrm{PM})$, $5.2 M_{\hookrightarrow}(\mathrm{BK})$

Intensity IV: Adak (PM). 


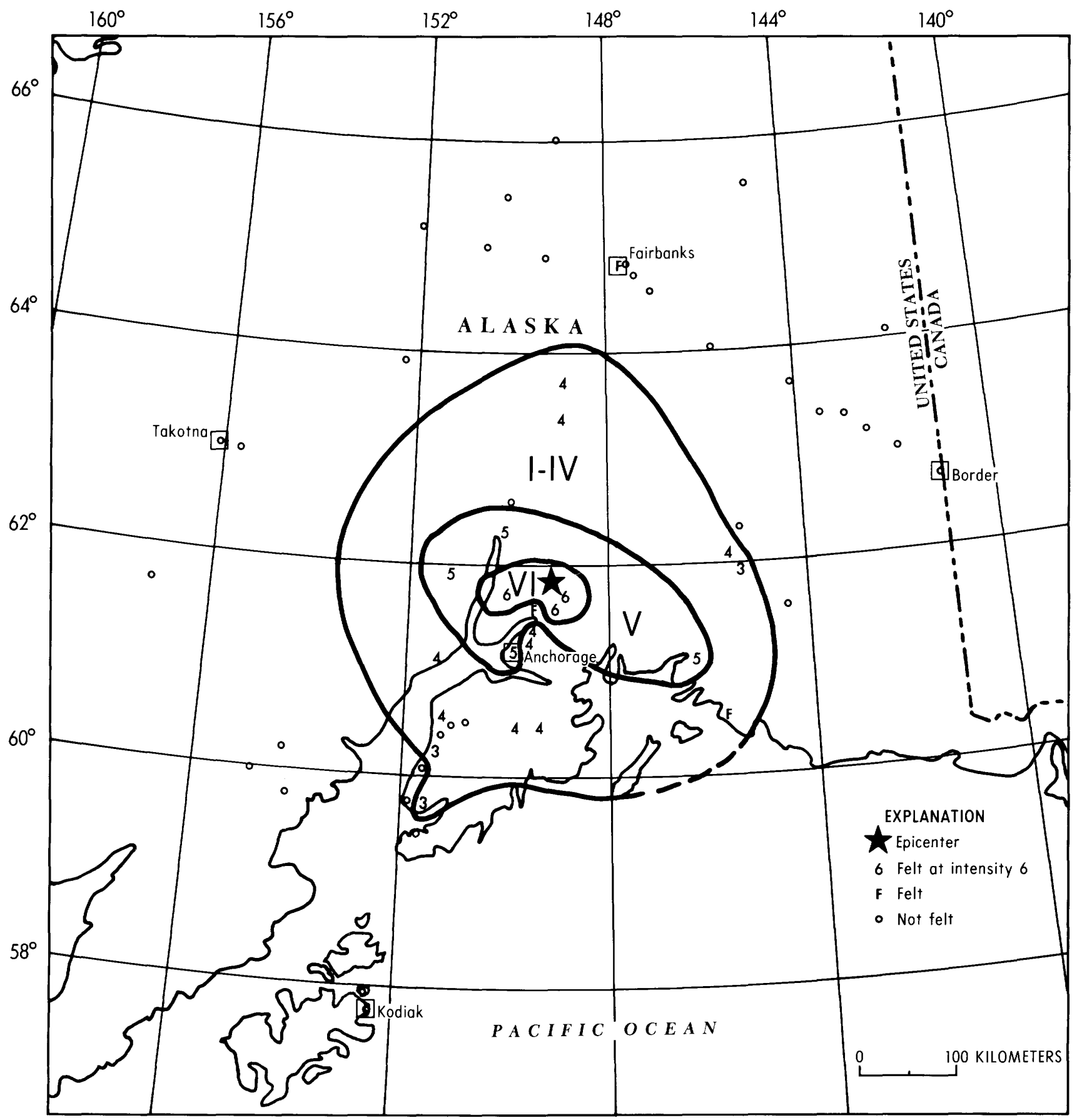

Figure 10. Isoseismal map for the southern Alaska earthquake of 14 August 1984, 010208.4 UTC. Roman numerals represent Modified Mercalli intensities between isoseismals; Arabic numerals represent intensities at specific sites. 
5 August (GS) Southern Alaska

Origin time: 141102.7

Epicenter: 61.636N., 149.823W.

Depth: $58 \mathrm{~km}$

Magnitude: $3.0 M_{L}(\mathrm{PM})$

Felt: Anchorage, Eagle River, and Palmer (PM).

7 August (GS) Southern Alaska

Origin time: 083522.7

Epicenter: 61.769N., 149.674W.

Depth: $44 \mathrm{~km}$

Magnitude: $3.4 M_{L}(\mathrm{PM})$

Intensity III: Anchorage, Eagle River, and Palmer (PM).

\section{August (GS) Southeastern Alaska}

Origin time: 073010.0

Epicenter: 59.131N., 136.771W.

Depth: $15 \mathrm{~km}$

Magnitude: $4.1 m_{1},(\mathrm{GS}), 4.2 M_{L}(\mathrm{PM})$

Felt: At several places in Glacier Bay National Park and on a tour boat in Glacier Bay.

\section{August (GS) Southern Alaska}

Origin time: 010208.4

Epicenter: 61.857N., 149.104W.

Depth: $20 \mathrm{~km}$

Magnitude: $5.7 m_{l}$ (GS), 5.2 $M_{S}(\mathrm{GS}), 5.7 M_{L}(\mathrm{PM})$, $5.4 M_{S}(\mathrm{BK})$

This earthquake was felt over an area of approximately 74,500 kilometers ${ }^{2}$ of southern Alaska (fig. 10).

\section{Intensity VI:}

Palmer - Plate glass windows shattered at Pioneer Meat Market and D and G Super; a few dishes broke; light furniture overturned; a few items shook off of store shelves; a few small objects overturned and fell; buildings shook strongly; people had difficulty standing; trees and bushes shook moderately; standing vehicles rocked slightly; small landslides occurred; felt by many.

Sutton - Some windows broke; light furniture overturned; a few dishes broke; many small items overturned and fell; many items shook off of store shelves; trees and bushes shook slightly; standing and moving vehicles shook; water splashed onto sides of lakes; buildings shook strongly; felt by all. The press reported a small dirt and rock landslide on Glenn Highway at Mile 69 near Sutton.

Willow - Hanging pictures fell; many dishes broke; many small objects overturned and fell; many items shook off of store shelves; trees and bushes shook moderately; standing vehicles rocked moderately; buildings shook strongly; people had difficulty standing; felt by many.

\section{Intensity $\mathrm{V}$ :}

Anchorage- A few dishes broke; a few small objects overturned and fell; a few items shook off of store shelves; buildings shook strongly; trees and bushes shook moderately; standing vehicles rocked slightly; felt by many.

Skwentna- A few small objects overturned and fell; a few items shook off of store shelves; trees and bushes shook strongly; buildings shook strongly; felt by many.

Talkeetna- A few items shook off of store shelves; standing vehicles rocked moderately; buildings shook strongly; felt by all.

Valdez - A few dishes broke; a few small objects overturned and fell; trees and bushes shook slightly; buildings shook slightly; people had difficulty standing; felt by many.

Intensity IV: Cantwell, Chugiak, Cooper Landing, Eagle River, Elmendorf Air Force Base, Glennallen, Kenai, McKinley Park, Moose Pass, and Tyonek.

Intensity III: Clam Gulch, Copper Center, and Homer. Felt: Cordova, Fairbanks, and Wasilla (press report).

14 August (GS) Southern Alaska

Origin time: 015437.3

Epicenter: 61.774N., 148.973W.

Depth: $24 \mathrm{~km}$

Magnitude: $4.2 M_{L}(\mathrm{PM})$

Intensity IV: Palmer and Wasilla (PM).

Felt: Anchorage to Homer (PM).

14 August (GS) Southern Alaska

Origin time: 074107.7

Epicenter: 61.784N., 148.935W.

Depth: $15 \mathrm{~km}$

Magnitude: $3.7 M_{L}(\mathrm{PM})$

Intensity III: Palmer and Wasilla (PM).

\section{August (GS) Southern Alaska}

Origin time: 043129.6

Epicenter: 60.493N., $150.478 \mathrm{~W}$.

Depth: $58 \mathrm{~km}$

Magnitude: $3.6 m_{l},(\mathrm{GS}), 4.0 M_{L}(\mathrm{PM})$

Felt: Anchorage, Kenai, Palmer, and Wasilla (PM).

\section{August (GS) Central Alaska}

Origin time: 204150.8

Epicenter: 64.554N., 147.980W.

Depth: $16 \mathrm{~km}$

Magnitude: $4.1 M_{L}(\mathrm{PM})$ 
Intensity III: Fairbanks, Fort Wainwright.

Felt: Eielson Air Force Base (PM).

26 August (GS) Southern Alaska

Origin time: 033245.7

Epicenter: 60.703N., 151.339W.

Depth: $100 \mathrm{~km}$

Magnitude: None computed.

Felt: Kenai Peninsula (PM).

30 August (GS) Queen Charlotte Islands, Canada

Origin time: 122839.7

Epicenter: 54.113N., 133.639W.

Depth: $10 \mathrm{~km}$

Magnitude: $5.5 m_{l},(\mathrm{GS}), 5.5 M_{s}(\mathrm{GS}), 5.4 M_{L}(\mathrm{PM})$

Intensity IV:

Alaska-Craig, Ketchikan.

Intensity III:

Alaska - Hydaburg, Metlakatla, Petersburg.

Felt:

British Columbia - Masset, Prince Rupert, Queen Charlotte City, and Skidegate (EP).

1 September (GS) Fox Islands, Aleutian Islands Origin time: 145816.4

Epicenter: 53.012N., 168.260W.

Depth: $61 \mathrm{~km}$

Magnitude: $4.6 m_{l},(\mathrm{GS}), 4.6 M_{L}(\mathrm{PM})$

Felt: Nikolski (PM).

\section{September (GS) Central Alaska}

Origin time: 084735.9

Epicenter: 64.847 N., $149.107 \mathrm{~W}$.

Depth: $28 \mathrm{~km}$

Magnitude: $3.8 M_{L}(\mathrm{PM})$

Intensity III: Fairbanks, Healy, and Nenana (PM).

10 September (GS) Southern Alaska

Origin time: 192231.1

Epicenter: 60.933N., 151.115W.

Depth: $5 \mathrm{~km}$

Magnitude: None computed.

Intensity III: Anchorage (PM).

20 September (GS) Southern Alaska

Origin time: 041724.4

Epicenter: $60.322 \mathrm{~N}$., $146.001 \mathrm{~W}$.

Depth: $18 \mathrm{~km}$

Magnitude: $5.5 m_{l}(\mathrm{GS}), 5.2 M_{s}(\mathrm{GS}), 5.2 M_{L}(\mathrm{PM})$,

$$
5.1 M_{s}(\mathrm{BK})
$$

Intensity IV: Anchorage, Cordova, Sutton.

Intensity III: Chitina (PM), Chugiak, Palmer (PM),
Seward (PM), Valdez (PM).

Intensity II: Eagle River.

20 September (GS) Southern Alaska

Origin time: 042804.0

Epicenter: $60.306 \mathrm{~N}$., $146.098 \mathrm{~W}$.

Depth: $26 \mathrm{~km}$

Magnitude: $5.1 m_{l}$ (GS), 4.7 $M_{S}$ (GS), 4.7 $M_{L}(\mathrm{PM})$

Intensity IV: Cordova (PM).

Intensity III: Anchorage, Chitina, Palmer, Seward, and Valdez (PM).

23 September (GS) Fox Islands, Aleutian Islands

Origin time: 170636.3

Epicenter: $53.577 \mathrm{~N}$., $165.424 \mathrm{~W}$.

Depth: Normal

Magnitude: $5.7 m_{l}(\mathrm{GS}), 5.5 M_{S}(\mathrm{GS}), 5.9 M_{L}(\mathrm{PM})$, $5.6 M_{s}(\mathrm{BK})$

Intensity IV: Unalaska.

Felt: Nikolski (PM).

24 September (GS) Southern Alaska

Origin time: 134838.9

Epicenter: 61.432N., $150.005 \mathrm{~W}$.

Depth: $46 \mathrm{~km}$

Magnitude: $3.3 M_{L}(\mathrm{PM})$

Intensity II: Anchorage (PM).

25 September (GS) Fox Islands, Aleutian Islands

Origin time: 190650.3

Epicenter: 53.663N., 166.404W.

Depth: $65 \mathrm{~km}$

Magnitude: $4.7 m_{l},(\mathrm{GS}), 5.1 M_{L}(\mathrm{PM})$

Intensity II: Unalaska (PM).

26 September (GS) Southern Alaska

Origin time: 145825.1

Epicenter: 63.168 N., $149.926 \mathrm{~W}$.

Depth: $114 \mathrm{~km}$

Magnitude: $4.3 m_{l},(\mathrm{GS})$

Intensity III: Cantwell (PM), Trappers Creek (PM).

Intensity II: Anchorage (PM).

29 September (GS) Southern Alaska

Origin time: 141915.0

Epicenter: $61.000 \mathrm{~N}:, 149.836 \mathrm{~W}$.

Depth: $62 \mathrm{~km}$

Magnitude: $4.6 m_{l}(\mathrm{GS}), 4.2 M_{L}(\mathrm{PM})$

Intensity III: Anchorage (PM).

Intensity II: Palmer, Wasilla, and Willow (PM).

3 October (GS) Eastern Alaska

Origin time: 191723.0

16 U.S. Earthquakes, 1984 
Epicenter: 62.742N., 143.714W.

Depth: $5 \mathrm{~km}$

Magnitude: $3.0 M_{L}(\mathrm{PM})$

Intensity II: Nabesna and Slana (PM).

4 October (GS) Kenai Peninsula

Origin time: 224103.3

Epicenter: 59.145N., 151.706W.

Depth: $63 \mathrm{~km}$

Magnitude: $4.6 m_{l},(\mathrm{GS}), 4.3 M_{L}$ (PM)

Intensity III: Homer (PM).

5 October (GS) Andreanof Islands, Aleutian Islands

Origin time: 154627.2

Epicenter: 51.871N., 176.016W.

Depth: $68 \mathrm{~km}$

Magnitude: $5.3 m_{1}$, (GS), 5.2 $M_{L}$ (PM)

Intensity IV: Adak (PM).

18 October (GS) Southern Alaska

Origin time: 051956.6

Epicenter: $63.176 \mathrm{~N}, 151.131 \mathrm{~W}$.

Depth: $135 \mathrm{~km}$

Magnitude: $4.7 m_{l},(\mathrm{GS})$

Intensity III: Talkeetna.

Intensity II: Palmer and Wasilla (PM).

19 October (GS) Southern Alaska

Origin time: 044444.7

Epicenter: 61.649N., 150.973W.

Depth: $77 \mathrm{~km}$

Magnitude: $4.5 m_{l}$ (GS)

Intensity II: Anchorage-Palmer area (PM).

19 October (GS) Southern Alaska

Origin time: 204306.2

Epicenter: $60.139 \mathrm{~N} ., 150.987 \mathrm{~W}$.

Depth: $97 \mathrm{~km}$

Magnitude: $3.7 m_{l}$ (GS)

Intensity II: Anchorage (PM).

25 October (GS) Andreanof Islands, Aleutian Islands

Origin time: $1237 \mathbf{1 5 . 4}$

Epicenter: $51.675 \mathrm{~N} ., 175.210 \mathrm{~W}$.

Depth: $56 \mathrm{~km}$

Magnitude: $5.3 m_{b}(\mathrm{GS}), 5.1 M_{L}(\mathrm{PM})$

Intensity V: Adak- A few items shook off of store shelves; a few small objects overturned and fell; hanging pictures swung out of place; buildings shook slightly; walls creaked; windows rattled; felt by many.
9 November (GS) Central Alaska

Origin time: 065806.9

Epicenter: 63.905N., $147.915 \mathrm{~W}$.

Depth: $23 \mathrm{~km}$

Magnitude: $3.7 m_{l}$, (GS), $4.2 M_{L}$ (PM)

Intensity IV: Ester and Fairbanks (PM).

17 November (GS) Central Alaska

Origin time: 090319.3

Epicenter: 63.330N., 152.694W.

Depth: $3 \mathrm{~km}$

Magnitude: $5.0 m_{1},(\mathrm{GS}), 5.3 M_{L}(\mathrm{PM})$

Intensity III: Lake Minchumina (PM), McGrath, Takotna.

Intensity II: Talkeetna (PM).

19 November (GS) Alaska Peninsula

Origin time: 004427.2

Epicenter: 58.567N., 156.702W.

Depth: $206 \mathrm{~km}$

Magnitude: $4.6 m_{l},(\mathrm{GS})$

Intensity II: Egegik (PM).

19 November (GS) Rat Islands, Aleutian Islands Origin time: 041042.4

Epicenter: $51.170 \mathrm{~N} ., 179.096 \mathrm{E}$.

Depth: $39 \mathrm{~km}$

Magnitude: $5.6 m_{l},(\mathrm{GS}), 5.5 M_{s}(\mathrm{GS}), 5.7 M_{L}(\mathrm{PM})$

Intensity II: Adak (PM).

19 November (GS) Andreanof Islands, Aleutian Islands

Origin time: 120637.3

Epicenter: $51.777 \mathrm{~N} ., 175.272 \mathrm{~W}$.

Depth: $58 \mathrm{~km}$

Magnitude: $5.6 m_{l}$ (GS), $5.5 M_{L}(\mathrm{PM})$

Intensity IV: Adak and Atka (PM).

23 November (GS) Fox Islands, Aleutian Islands Origin time: 103738.3

Epicenter: 54.654N., 163.849W.

Depth: Normal

Magnitude: $4.8 m_{l}$, GS

Intensity III: Cold Bay.

29 November (GS) Andreanof Islands, Aleutian Islands

Origin time: 074512.6

Epicenter: 51.466N., 176.268W.

Depth: Normal

Magnitude: $4.3 m_{l}$ (GS), 4.6 $M_{L}$ (PM)

Felt: Adak Island (PM). 
1 December (GS) Kenai Peninsula

Origin time: 182551.3

Epicenter: $60.575 \mathrm{~N} ., 150.301 \mathrm{~W}$.

Depth: $51 \mathrm{~km}$

Magnitude: $3.8 m_{l},(\mathrm{GS}), 4.1 M_{L}(\mathrm{PM})$

Intensity II: Anchorage and Soldotna (PM).

1 December (GS) Andreanof Islands, Aleutian Islands

Origin time: 184524.8

Epicenter: $51.355 \mathrm{~N} ., 176.342 \mathrm{~W}$.

Depth: Normal

Magnitude: $5.0 m_{l}$ (GS), $5.0 M_{L}$ (PM)

Intensity III: Adak Island (PM).

8 December (GS) Central Alaska

Origin time: 210747.3

Epicenter: 63.653N., 150.770W.

Depth: $24 \mathrm{~km}$

Magnitude: $4.6 m_{l}(\mathrm{GS}), 5.2 M_{S}(\mathrm{GS}), 4.6 M_{L}(\mathrm{PM})$

Felt: Fairbanks (PM).

17 December (GS) Central Alaska

Origin time: 005152.2

Epicenter: $66.067 \mathrm{~N}$., $154.489 \mathrm{~W}$.

Depth: $27 \mathrm{~km}$

Magnitude: $3.8 M_{L}(\mathrm{PM})$

Intensity II: Hughes (PM).

17 December (GS) Southern Alaska

Origin time: 183623.5

Epicenter: $59.553 \mathrm{~N} ., 152.389 \mathrm{~W}$.

Depth: $85 \mathrm{~km}$

Magnitude: None computed.

Intensity III: Homer.

\section{ARKANSAS}

12 July (TC) Central Arkansas

Origin time: 012717.6

Epicenter: $35.230 \mathrm{~N}$., $92.207 \mathrm{~W}$.

Depth: $2 \mathrm{~km}$

Magnitude: $2.6 M_{D}(\mathrm{TC}), 2.8 M_{n}(\mathrm{SL})$

Felt: Enola area (TC).

27 September (GS) Central Arkansas

Origin time: 130305.2

Epicenter: $35.202 \mathrm{~N}$, $92.187 \mathrm{~W}$.

Depth: $10 \mathrm{~km}$

Magnitude: $3.3 M_{n}(\mathrm{TU}), 3.2 M_{D}(\mathrm{TC}), 3.4 M_{n}(\mathrm{SL})$

Intensity IV: Enola.

27 September (GS) Central Arkansas

Origin time: 131604.0
Epicenter: $35.218 \mathrm{~N} ., 92.173 \mathrm{~W}$.

Depth: $10 \mathrm{~km}$

Magnitude: $2.4 M_{n}$ (TU), $2.7 M_{D}$ (TC), $3.0 M_{n}(\mathrm{SL})$

Felt: Enola area (TC).

\section{CALIFORNIA}

\section{January Central California}

Origin time: 0721

Epicenter: Not located.

Magnitude: $2.7 M_{D}(\mathrm{GM}), 2.5 M_{L}(\mathrm{PS})$

Intensity III: San Luis Obispo County (near the epicenter).

5 January (BK) Central California

Origin time: 190107.0

Epicenter: 37.377 N., $121.758 \mathrm{~W}$.

Depth: $4 \mathrm{~km}$

Magnitude: $3.3 M_{L}(\mathrm{BK}), 3.1 M_{D}(\mathrm{GM})$,

$$
M_{1}=2.7 \mathrm{E} 21(\mathrm{BK})
$$

Intensity III: San Jose, Santa Clara.

Felt: Halls Valley area (press report), Hollister (BK), parts of San Francisco (press report), Sunnyvale (press report).

9 January (PS) Imperial Valley

Origin time: 190829.7

Epicenter: $32.969 \mathrm{~N} ., 115.542 \mathrm{~W}$.

Depth: $5 \mathbf{~ k m}$

Magnitude: $2.9 M_{L}$ (PS)

Felt: Brawley (PS).

10 January (BK) Bishop area

Origin time: 151547.7

Epicenter: $37.452 \mathrm{~N}$., $118.658 \mathrm{~W}$.

Depth: $5 \mathrm{~km}$

Magnitude: $3.5 M_{L}(\mathrm{BK}), 3.7 M_{L}(\mathrm{PS})$

Felt: Mammoth Lakes (BK).

11 January (BK) Central California

Origin time: 141900.1

Epicenter: $37.367 \mathrm{~N} ., 121.758 \mathrm{~W}$.

Depth: $5 \mathrm{~km}$

Magnitude: $2.9 M_{L}(\mathrm{BK}), M_{\iota}=4.3 \mathrm{E} 20(\mathrm{BK})$

Intensity III: San Jose.

14 January (BK) Bishop area

Origin time: 004223.6

Epicenter: $37.423 \mathrm{~N}$., $118.595 \mathrm{~W}$.

Depth: $10 \mathrm{~km}$

Magnitude: $3.8 M_{L}(\mathrm{BK}), 4.0 M_{L}(\mathrm{PS}), 3.5 M_{D}(\mathrm{GM})$

Intensity IV:

California- Bishop.

Intensity III: 


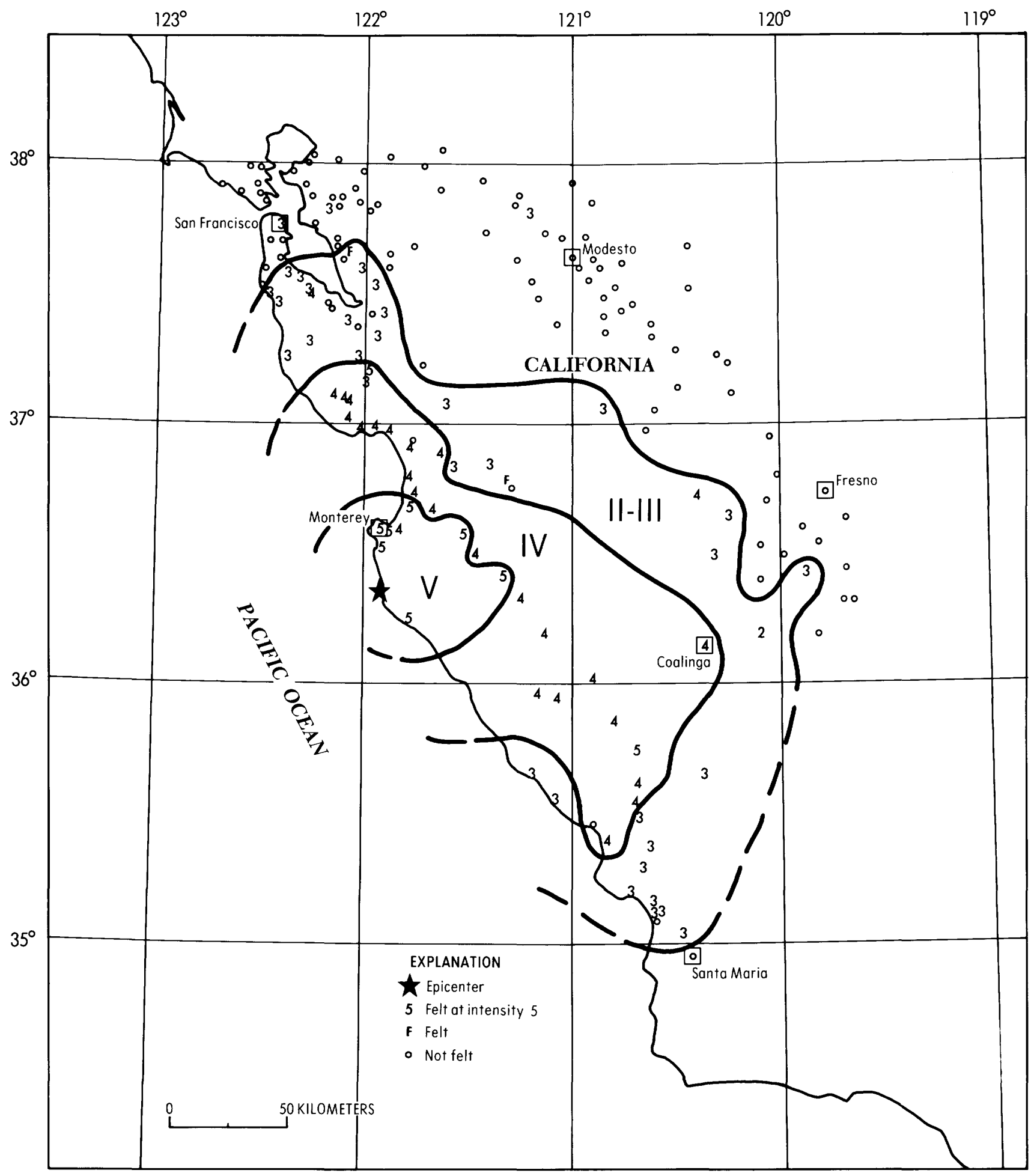

Figure 11. Isoseismal map for the central California earthquake of 23 January 1984, 054020.3 UTC. Roman numerals represent Modified Mercalli intensities between isoseismals; Arabic numerals represent intensities at specific sites. 
California - Independence, Lakeshore.

Nevada - Dyer.

\section{January (BK) Bishop area}

Origin time: 031115.0

Epicenter: $37.415 \mathrm{~N} ., 118.587 \mathrm{~W}$.

Depth: $11 \mathrm{~km}$

Magnitude: $4.0 M_{L}(\mathrm{BK}), 4.0 M_{L}(\mathrm{PS}), 3.5 M_{D}(\mathrm{GM})$

Felt: Bishop (BK).

\section{January (BK) Northern California}

Origin time: 103701.9

Epicenter: $40.268 \mathrm{~N} ., 124.357 \mathrm{~W}$.

Depth: $9 \mathrm{~km}$

Magnitude: $4.1 m_{l},(\mathrm{GS}), 4.0 M_{L}(\mathrm{BK}), 3.9 M_{D}(\mathrm{GM})$,

Felt: Petrolia (BK).

$$
M_{c}=2.2 \mathrm{E} 22(\mathrm{BK})
$$

\section{January (BK) Northern California}

Origin time: 103955.9

Epicenter: $40.290 \mathrm{~N}$., $124.383 \mathrm{~W}$.

Depth: $10 \mathrm{~km}$

Magnitude: $4.3 m_{l},(\mathrm{GS}), 3.9 M_{L}(\mathrm{BK}), 3.9 M_{D}(\mathrm{GM})$,

Felt: Petrolia (BK).

$$
M_{10}=2.1 \mathrm{E} 22(\mathrm{BK})
$$

\section{January (BK) Central California}

Origin time: 054020.3

Epicenter: $36.392 \mathrm{~N} ., 121.878 \mathrm{~W}$.

Depth: $8 \mathrm{~km}$

Magnitude: $5.1 m_{l}(\mathrm{GS}), 4.6 M_{\hookrightarrow}(\mathrm{GS}), 5.1 M_{L}(\mathrm{BK})$,

$$
M_{,}=3.5 \mathrm{E} 23(\mathrm{BK})
$$

Felt over an area of 32,400 kilometers $^{2}$ of central California (fig. 11) from the San Francisco Bay area south to San Luis Obispo. The press reported as much as 4 inches of vertical movement at the ends of the Little Sur bridge over Little Sur River about 3 kilometers north of Point Sur.

\section{Intensity V:}

Big Sur- A few windows cracked; a few items shook off of store shelves; hanging pictures fell; felt by everyone. At the River Inn glasses fell to the floor; a few bottles of liquor fell from the back bar, and everything on the walls fell (press report).

Carmel-by-the-Sea - Vibration was described as strong; people had difficulty standing or walking.

Chualar- A few small objects overturned and fell; hanging pictures swung out of place; vibration was described as strong; people had difficulty standing or walking; felt by many.

Los Gatos-Plaster walls sustained hairline cracks; hanging pictures swung out of place; buildings shook strongly; vibration was described as strong; felt by many.

Marina- A few windows cracked; trees and bushes shook slightly; standing vehicles rocked slightly; buildings shook strongly; vibration was described as strong; felt by all.

Monterey - A few small objects overturned and fell; buildings shook strongly; trees and bushes shook slightly; vibration was described as strong; felt by many. One resident described the shaking as:

"extremely sharp-like an explosion; the whole room started shaking and a stereo speaker fell, and the television fell" (press report). Some plaster fell from the ceiling of a building at the Fleet Numerical Oceanography Center.

Pacific Grove - Plaster walls sustained hairline cracks; a few windows cracked; a few dishes broke; a few items shook off of store shelves; buildings shook strongly; vibrations described as strong; felt by many.

San Miguel- Plaster walls sustained hairline cracks; trees and bushes shook slightly; standing vehicles rocked slightly; a few small objects overturned or fell; felt by many.

Soledad-A few dishes broke; a few small objects overturned and fell; felt by many.

Intensity IV: Aptos, Aromas, Ben Lomond, Boulder Creek, Bradley, Brookdale, Capitola, Castroville, Coalinga, Davenport, Felton, Gonzales, Greenfield, Jolon, King City, Lockwood, Los Banos, Mendota, Morro Bay, Moss Landing, Paso Robles, Salinas, San Ardo, San Carlos, Santa Cruz, Seaside, Soquel, Templeton, and Watsonville.

Intensity III: Arroyo Grande, Atascadero, Avila Beach, Belmont, Cambria, Cantua Creek, Canyon, El Granada, Fremont, Grover City, Half Moon Bay, Hollister, La Honda, Manteca, Millbrae, Milpitas, Mountain View, Nipomo, Pescadero, Pismo Beach, Redwood Estates, Riverdale, San Francisco, San Francisco (Bayview), San Juan Bautista, San Luis Obispo, San Martin, San Mateo, San Simeon, Santa Clara, Santa Margarita, Saratoga, Shandon, Tranquillity, and Union City.

Intensity II: Huron.

Felt: Campbell, Hayward (press report), Tres Pinos.

\section{January (BK) Central California}

Origin time: 054858.0

Epicenter: 36.365 N., 121.878W.

Depth: $10 \mathrm{~km}$ 
Magnitude: $3.6 M_{L}(\mathrm{BK}), 3.3 M_{D}(\mathrm{GM})$,

$$
M_{1,}=4.2 \mathrm{E} 21(\mathrm{BK})
$$

Felt: South of Monterey (BK).

\section{January (BK) Central California}

Origin time: 065951.3

Epicenter: $36.388 \mathrm{~N} ., 121.872 \mathrm{~W}$.

Depth: $8 \mathrm{~km}$

Magnitude: $4.5 M_{L}(\mathrm{BK}), 4.6 M_{D}(\mathrm{GM})$,

Felt: Monterey (BK).

$$
M_{,}=3.1 \mathrm{E} 22(\mathrm{BK})
$$

\section{January (PS) Southern California}

Origin time: 170316.1

Epicenter: 33.970N., 116.840W.

Depth: $15 \mathrm{~km}$

Magnitude: $2.7 M_{L}$ (PS)

Felt: Banning (PS).

\section{January (BK) Central California}

Origin time: 195821.5

Epicenter: $36.367 \mathrm{~N} ., 121.883 \mathrm{~W}$.

Depth: $9 \mathrm{~km}$

Magnitude: $3.6 M_{L}(\mathrm{BK}), 3.4 M_{L}(\mathrm{PS}), 3.1 M_{D}(\mathrm{GM})$,

Felt: South of Monterey.

$$
M_{1}=4.5 \mathrm{E} 21(\mathrm{BK})
$$

24 January (BK) Central California

Origin time: 010039.4

Epicenter: $36.372 \mathrm{~N} ., 121.880 \mathrm{~W}$.

Depth: $8 \mathrm{~km}$

Magnitude: $3.7 M_{L}(\mathrm{BK}), 3.4 M_{D}(\mathrm{GM})$,

$$
M_{1,}=1.1 \mathrm{E} 22(\mathrm{BK})
$$

Felt: Monterey (press report).

\section{January (BK) Central California}

Origin time: 044435.8

Epicenter: $36.277 \mathrm{~N}$., $120.400 \mathrm{~W}$.

Depth: $7 \mathrm{~km}$

Magnitude: $3.8 M_{L}(\mathrm{BK}), 3.6 M_{D}(\mathrm{GM}), 3.8 M_{L}(\mathrm{PS})$,

Felt: Coalinga (BK).

$$
M_{,}=6.3 \mathrm{E} 21(\mathrm{BK})
$$

\section{January (PS) Southern California}

Origin time: 200625.4

Epicenter: $33.725 \mathrm{~N}$., $118.081 \mathrm{~W}$.

Depth: $6 \mathrm{~km}$

Magnitude: $2.7 M_{L}$ (PS)

Felt: Long Beach (PS).

29 January (PS) Southern California

Origin time: 201945.9

Epicenter: $33.709 \mathrm{~N}$., 118.063W.

Depth: $6 \mathrm{~km}$
Magnitude: $2.7 M_{L}$ (PS)

Felt: Long Beach (PS).

2 February (PS) Southern California

Origin time: 214756.5

Epicenter: $33.893 \mathrm{~N} ., 116.847 \mathrm{~W}$.

Depth: $17 \mathrm{~km}$

Magnitude: $3.3 M_{L}$ (PS)

Felt: Banning (PS).

7 February (PS) Southern California

Origin time: 033330.3

Epicenter: 34.309N., 119.265W.

Depth: $0 \mathrm{~km}$

Magnitude: $3.3 M_{L}(\mathrm{PS}), 3.5 M_{D}(\mathrm{GM})$

Intensity IV: Ventura (press report).

10 February (BK) Central California

Origin time: 072325.3

Epicenter: $36.387 \mathrm{~N} ., 121.883 \mathrm{~W}$.

Depth: $12 \mathrm{~km}$

Magnitude: $4.1 M_{L}(\mathrm{BK}), 4.2 M_{D}(\mathrm{GM}), 4.1 M_{L}(\mathrm{PS})$,

$$
M_{1}=8.6 \mathrm{E} 21(\mathrm{BK})
$$

Intensity $\mathrm{V}$ :

Monterey - A few small objects overturned and fell; trees and bushes shook slightly; standing vehicles rocked slightly; buildings shook strongly; vibration was described as strong; felt by and awakened many.

Pacific Grove- Moving vehicles rocked slightly; standing vehicles rocked moderately; trees and bushes shook moderately; buildings shook strongly; felt by many.

Santa Cruz - Trees and bushes shook slightly; a few dishes broke; a few small objects overturned and fell; a few items shook off of shelves; a few windows cracked; felt by many.

Intensity IV: Big Sur, Paicines, Soquel.

Intensity III: Boulder Creek, Brookdale, King City, Marina, Soledad, Monterey Bay Academy (9 miles south of Watsonville).

Intensity II: Mount Herman.

Felt: Salinas.

11 February (BK) Central California

Origin time: 141028.8

Epicenter: $36.388 \mathrm{~N} ., 121.882 \mathrm{~W}$.

Depth: $9 \mathrm{~km}$

Magnitude: $3.4 M_{L}(\mathrm{BK}), 3.0 M_{D}(\mathrm{GM})$

Felt: Monterey area (BK).

17 February (BK) Western Nevada

Origin time: 120356.2

See Nevada listing. 
19 February (BK) Central California

Origin time: 094310.7

Epicenter: $36.277 \mathrm{~N} ., 120.323 \mathrm{~W}$.

Depth: $10 \mathrm{~km}$

Magnitude: $4.2 m_{l}(\mathrm{GS}), 4.1 M_{L}(\mathrm{BK}), 4.3 M_{D}(\mathrm{GM})$, $4.4 M_{L}(\mathrm{PS}), M_{c}=2.8 \mathrm{E} 22(\mathrm{BK})$

Intensity IV: Huron- A few windows cracked.

Felt: Coalinga area (BK).

23 February (PS) Southern California

Origin time: 173423.3

Epicenter: $33.890 \mathrm{~N} ., 116.153 \mathrm{~W}$.

Depth: $5 \mathrm{~km}$

Magnitude: $3.7 M_{L}(\mathrm{PS})$

Felt: Indio.

27 February (BK) Bishop area

Origin time: 013620.6

Epicenter: $37.375 \mathrm{~N} ., 118.598 \mathrm{~W}$.

Depth: $10 \mathrm{~km}$

Magnitude: $4.3 m_{l}(\mathrm{GS}), 4.5 M_{L}(\mathrm{BK}), 4.1 M_{L}(\mathrm{PS})$, $4.2 M_{D}(\mathrm{GM}), M_{,}=2.8 \mathrm{E} 22(\mathrm{BK})$

Intensity III: Bishop, Independence, Toms Place.

Intensity II: June Lake.

Felt: Mammoth Lakes (BK).

28 February (PS) Southern California

Origin time: 060859.3

Epicenter: $34.398 \mathrm{~N}$., $116.825 \mathrm{~W}$.

Depth: $8 \mathrm{~km}$

Magnitude: $3.4 M_{L}(\mathrm{PS})$

Felt: Lucerne Valley (PS).

29 February (PS) Southern California

Origin time: 020731.7

Epicenter: $33.136 \mathrm{~N} ., 116.071 \mathrm{~W}$.

Depth: $7 \mathrm{~km}$

Magnitude: $4.3 M_{L}$ (PS)

Intensity III: Imperial Valley area.

Felt: Painted Gorge Valley (about 5 miles west of Plaster City).

2 March (BK) Central California

Origin time: 113909.6

Epicenter: $38.805 \mathrm{~N} ., 122.795 \mathrm{~W}$.

Depth: $5 \mathrm{~km}$

Magnitude: $3.0 M_{L}(\mathrm{BK}), 3.0 M_{D}(\mathrm{GM})$,

Felt: Cobb (BK).

$$
M_{,}=7.6 \mathrm{E} 21(\mathrm{BK})
$$

5 March (BK) Mammoth Lakes area

Origin time: 114319.8

Epicenter: $37.488 \mathrm{~N}$., 118.807W.

Depth: $8 \mathrm{~km}$
Magnitude: $3.4 M_{L}(\mathrm{BK}), 3.2 M_{D}(\mathrm{GM}), 3.8 M_{L}(\mathrm{PS})$

Felt: Mammoth Lakes (BK).

6 March (PS) Southern California

Origin time: 024135.0

Epicenter: 34.180N., 117.310W.

Depth: $2 \mathrm{~km}$

Magnitude: $2.5 M_{L}$ (PS)

Intensity III: San Bernardino (press report).

7 March (BK) Mammoth Lakes area

Origin time: 083236.5

Epicenter: $37.498 \mathrm{~N}$., $118.818 \mathrm{~W}$.

Depth: $4 \mathrm{~km}$

Magnitude: $3.4 M_{L}(\mathrm{BK}), 3.3 M_{D}(\mathrm{GM}), 3.5 M_{L}(\mathrm{PS})$

Felt: Mammoth Lakes (BK).

7 March (PS) Southern California

Origin time: 202635.0

Epicenter: $33.590 \mathrm{~N}$., $118.360 \mathrm{~W}$.

Depth: $8 \mathrm{~km}$

Magnitude: $2.1 M_{L}$ (PS)

Felt: West Los Angeles (PS).

9 March (BK) Northern California

Origin time: 063533.5

Epicenter: 40.347N., 124.337W.

Depth: $5 \mathrm{~km}$

Magnitude: $3.2 M_{L}(\mathrm{BK}), 3.1 M_{D}(\mathrm{GM})$

Felt: Humboldt County (BK).

12 March (BK) Central California

Origin time: $05 \quad 1555.1$

Epicenter: $36.950 \mathrm{~N}$., $121.600 \mathrm{~W}$.

Depth: $5 \mathrm{~km}$

Magnitude: $2.9 M_{L}(\mathrm{BK}), M_{t,}=4.2 \mathrm{E} 20(\mathrm{BK})$

Intensity IV: Mesa Ranch (southeast of Gilroy-press report).

Felt: Gilroy.

18 March (BK) Central California

Origin time: 052729.9

Epicenter: $37.785 \mathrm{~N}$., $121.725 \mathrm{~W}$.

Depth: $10 \mathrm{~km}$

Magnitude: $4.1 M_{L}(\mathrm{BK}), 3.4 M_{D}(\mathrm{GM})$, $M_{c}=2.8 \mathrm{E} 22(\mathrm{BK})$

Intensity IV: Byron, Canton, Danville, Lafayette, Livermore, Oakland (Eastmont), San Jose.

Intensity III: Berkeley, Brentwood, Byron, Fair Oaks, Holt, Manteca, Moraga, Oakland, Oakley, Pleasanton, Walnut Creek.

Felt: Antioch (BK), Clayton (BK), Concord (BK), Dublin (BK), Martinez (press report), Pittsburg (BK), San Francisco (press report), San Ramon (BK). 


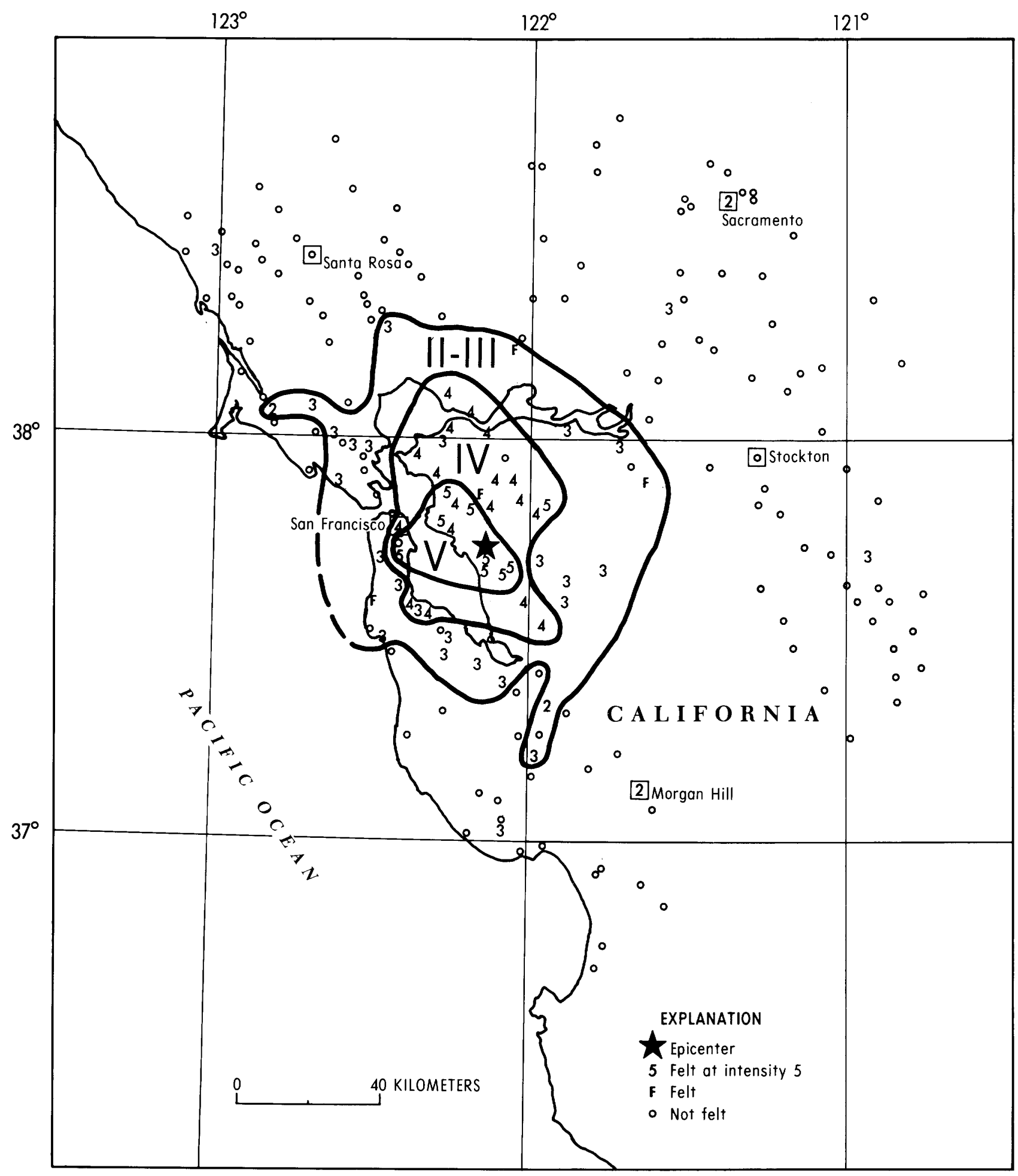

Figure 12. Isoseismal map for the central California earthquake of 27 March 1984, 033635.4 UTC. Roman numerals represent Modified Mercalli intensities between isoseismals; Arabic numerals represent intensities at specific sites. 
19 March (BK) Northern California

Origin time: 210028.5

Epicenter: $40.893 \mathrm{~N} ., 123.785 \mathrm{~W}$.

Depth: $6 \mathrm{~km}$

Magnitude: $3.4 M_{L}(\mathrm{BK}), 3.1 M_{D}(\mathrm{GM}), M_{\iota}=2.9 \mathrm{E} 21(\mathrm{BK})$

Intensity IV: Eureka- Buildings shook strongly; a few windows cracked.

Intensity III: Bayside, Kneeland.

21 March (BK) Northern California

Origin time: 111112.2

Epicenter: $39.842 \mathrm{~N} ., 120.640 \mathrm{~W}$.

Depth: $10 \mathrm{~km}$

Magnitude: $3.4 M_{L}(\mathrm{BK}), M_{1}=5.5 \mathrm{E} 21(\mathrm{BK})$

Intensity III: Portola (press report).

\section{March (PS) Southern California}

Origin time: 235203.4

Epicenter: $33.666 \mathrm{~N} ., 118.388 \mathrm{~W}$.

Depth: $2 \mathrm{~km}$

Magnitude: $2.8 M_{L}$ (PS)

Felt: Palos Verdes (PS).

26 March (BK) Central California

Origin time: 075839.9

Epicenter: $36.747 \mathrm{~N} ., 121.485 \mathrm{~W}$.

Depth: $7 \mathrm{~km}$

Magnitude: $4.0 m_{1}$ (GS), 4.0 $M_{L}(\mathrm{BK}), 4.0 M_{D}(\mathrm{GM})$, $M_{1}=1.9 \mathrm{E} 22(\mathrm{BK})$

Intensity IV: Chualar, Hollister, Pacific Grove, Salinas, San Martin, and Spreckels.

Intensity III: Moss Landing, San Juan Bautista, Santa Cruz, and Seaside.

Intensity II: Pescadero.

Felt: Redwood Estates.

\section{March (BK) Central California}

Origin time: 033635.4

Epicenter: $37.755 \mathrm{~N} ., 122.095 \mathrm{~W}$.

Depth: $7 \mathrm{~km}$

Magnitude: $4.3 m_{3},(\mathrm{GS}), 4.3 M_{L}(\mathrm{BK}), 4.7 M_{D}(\mathrm{GM})$,

$$
M_{0}=4.1 \mathrm{E} 22(\mathrm{BK})
$$

This earthquake was felt over an area of approximately 7,600 kilometers ${ }^{2}$ of the San Francisco Bay area (fig. 12).

Intensity $\mathbf{V}$ :

Berkeley - Plaster walls sustained hairline cracks; hanging pictures swung out of place; buildings shook strongly; walls creaked; windows rattled; vibration was described as strong; felt by all.

Brisbane - Motor vehicles rocked slightly; a few glassware items broke; a few items shook off of store shelves; a few small objects overturned and fell; buildings shook strongly; windows rattled; walls creaked; felt by many.

Canyon - Hanging pictures fell; buildings shook strongly; walls creaked; windows rattled; vibration was described as strong; felt by and frightened many.

Castro Valley - The interior wall of the post office sustained hairline cracks; also, ceiling tile support strips dislodged and florescent light covers became detached. Windows cracked; wine bottles fell and broke in a Safeway store on Redwood Road (press report). Walls creaked; windows rattled; vibration was described as strong; felt by and frightened many.

Diablo- A few glass items broke; a few small objects overturned and fell; hanging pictures swung out of place; buildings shook strongly; windows rattled.

Hayward - The press reported bottles fell in a liquor store. Plaster walls sustained hairline cracks; walls creaked; windows rattled; felt by many.

Oakland (Eastmont) - Plaster walls sustained cracks; water splashed onto sides of a swimming pool; a few canned goods and bottles fell from shelves; hanging pictures swung out of place; vibration was described as strong with a loud noise.

Oakland (Elmhurst) - A few small objects overturned and fell; vibration was described as strong with a loud noise; felt by many.

Oakland (Fruitvale) - A few glass items broke; a few windows cracked; a few small objects overturned; hanging pictures swung out of place; vibration was described as strong with a loud noise; felt by all and frightened many.

San Francisco (Parkside) - Plaster walls sustained hairline cracks motor vehicles rocked slightly; a few items shook off of store shelves; many small objects overturned; vibration was described as strong; felt by and frightened many.

San Leandro- The press reported Romley's Market on Lake Chabot Road closed early to clean up the bottles and other merchandise that were shaken from shelves. Buildings shook strongly; vibration was described as strong; felt by many.

San Lorenzo- A few windows cracked; a few items shook off of store shelves; a few small objects overturned and fell; buildings shook strongly; vibration was described as strong; felt by and frightened many. Intensity IV: Alameda, Alamo, Danville, El Cerrito, Fremont (bottles fell off shelves-press report) Lafayette, Martinez, Millbrae, Moraga, Oakland, Oakland (Rockridge Station), Oakland (Temescal Station), Piedmont, Port Costa, Richmond, Rodeo, San Francisco, San Mateo, Union City, Vallejo, Walnut Creek. 


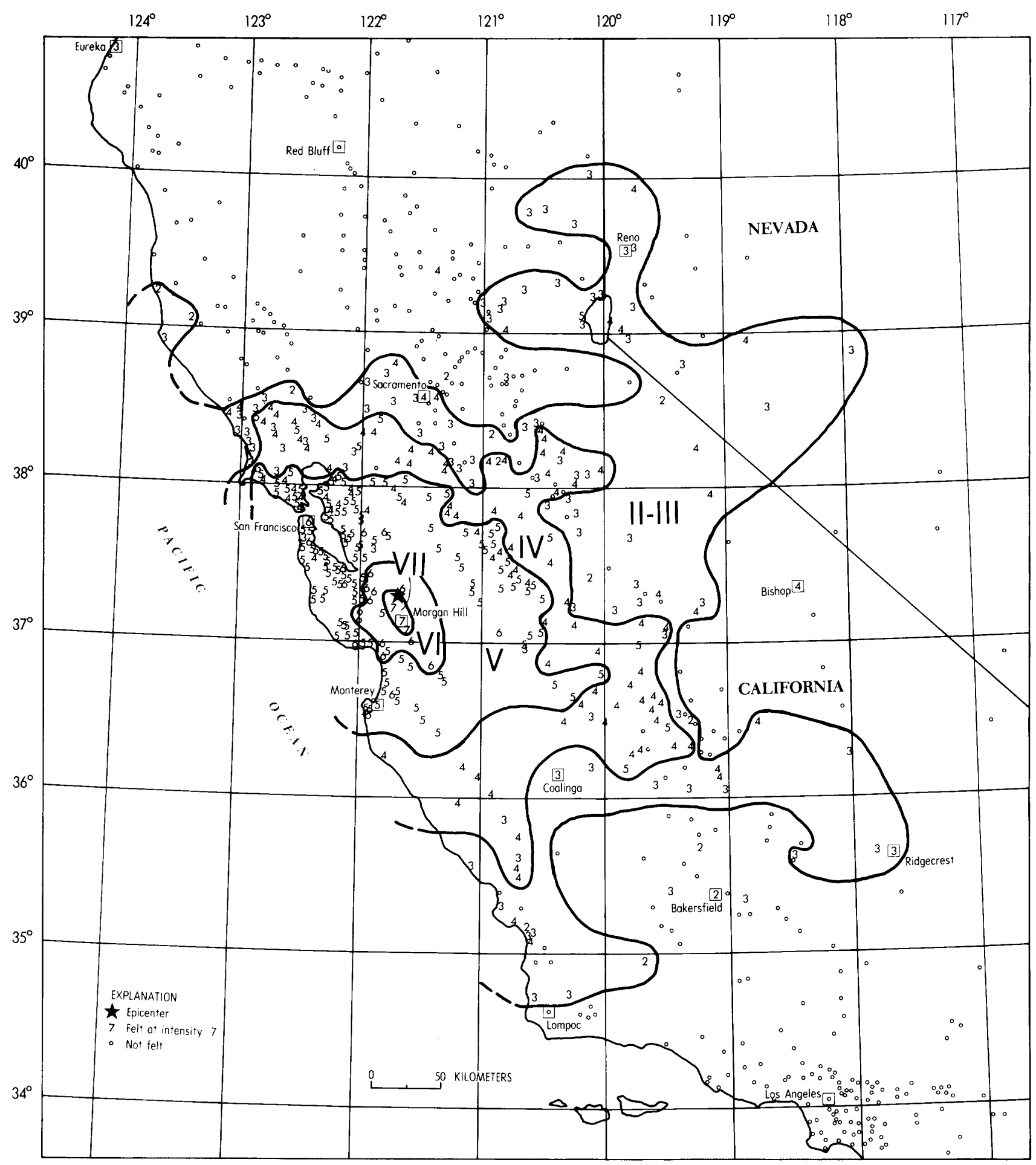

Figure 13. Isoseismal map for the Morgan Hill, California, earthquake of 24 April 1984, 211519.0 UTC. Roman numerals represent Modified Mercalli intensities between isoseismals; Arabic numerals represent intensities at specific sites. 
Intensity III: Benicia, Burlingame, Courtland, Daly City, Dublin, East Palo Alto, El Granada, Felton, Livermore (press report), Los Gatos, Monte Rio, Mountain View, Nicasio, Oakley, Pinole, Pittsburg, Pleasanton (press report), Redwood City, Riverbank, San Anselmo, San Carlos, San Francisco (Sunset), San Rafael, Sonoma, South San Francisco, Stinson Beach, Sunol, Woodacre.

Intensity II: Morgan Hill, Point Reyes Station, Sacramento, Santa Clara.

Felt: Byron, Fairfield (press report), Orinda (press report), Pacifica.

28 March (PS) Southern California

Origin time: 173620.2

Epicenter: $33.970 \mathrm{~N} ., 117.230 \mathrm{~W}$.

Depth: $13 \mathrm{~km}$

Magnitude: $2.5 M_{L}$ (PS)

Felt: Riverside (PS).

1 April (PS) Southern California

Origin time: 071702.4

Epicenter: $33.109 \mathrm{~N} ., 116.404 \mathrm{~W}$.

Depth: $11 \mathrm{~km}$

Magnitude: $3.9 M_{L}$ (PS)

Intensity IV: Julian.

Intensity III: Ocotillo.

3 April (PS) Southern California

Origin time: 062639.8

Epicenter: 33.873N., 118.241W.

Depth: 6 km

Magnitude: $2.6 M_{L}$ (PS)

Intensity IV: Gardena (press report).

10 April (GM) Mammoth Lakes area

Origin time: 071930.0

Epicenter: $37.563 \mathrm{~N} ., 118.823 \mathrm{~W}$.

Depth: $6 \mathrm{~km}$

Magnitude: $3.1 M_{D}(\mathrm{GM}), 3.6 M_{L}(\mathrm{PS})$

Felt: Mammoth Lakes (press report).

18 April (PS) Southern California

Origin time: 131902.7

Epicenter: $33.660 \mathrm{~N}$., 118.360W.

Depth: $3 \mathrm{~km}$

Magnitude: $3.1 M_{L}$ (PS)

Felt: Torrance (PS).

20 April (PS) Southern California

Origin time: 100841.3

Epicenter: 33.934N., 117.878W.

Depth: 9 km
Magnitude: $2.5 M_{L}$ (PS)

Felt: Brea (PS).

21 April (PS) Southern California

Origin time: 115843.2

Epicenter: $33.643 \mathrm{~N} ., 117.943 \mathrm{~W}$.

Depth: $6 \mathrm{~km}$

Magnitude: $3.0 M_{L}$ (PS)

Intensity III: Costa Mesa and Huntington Beach (press reports).

21 April (PS) Southern California

Origin time: 222315.1

Epicenter: $34.249 \mathrm{~N} ., 119.617 \mathrm{~W}$.

Depth: $12 \mathrm{~km}$

Magnitude: $3.4 M_{L}(\mathrm{PS}), 3.5 M_{D}(\mathrm{GM})$

Felt: Santa Barbara (press report)

21 April (PS) Southern California

Origin time: 230222.4

Epicenter: 34.249N., 119.618W.

Depth: $12 \mathrm{~km}$

Magnitude: $3.4 M_{L}(\mathrm{PS}), 3.6 M_{D}(\mathrm{GM})$

Felt: Santa Barbara (press report).

22 April (PS) Southern California

Origin time: 055328.9

Epicenter: $34.240 \mathrm{~N}$., $119.604 \mathrm{~W}$.

Depth: $14 \mathrm{~km}$

Magnitude: $3.7 M_{L}(\mathrm{PS}), 3.8 M_{D}(\mathrm{GM})$

Intensity IV: Carpinteria.

Intensity III: Isla Vista, Summerland.

Felt: Santa Barbara (PS).

23 April (PS) Imperial Valley

Origin time: 143112.9

Epicenter: $32.907 \mathrm{~N}$., $115.537 \mathrm{~W}$.

Depth: $10 \mathrm{~km}$

Magnitude: $3.0 M_{L}$ (PS)

Felt: Brawley (press report).

24 April (BK) Central California

Origin time: 045251.5

Epicenter: $36.163 \mathrm{~N} ., 120.287 \mathrm{~W}$.

Depth: $10 \mathrm{~km}$

Magnitude: $4.0 M_{L}(\mathrm{BK}), 4.1 M_{L}(\mathrm{PS})$,

$$
M_{1}=1.1 \mathrm{E} 22(\mathrm{BK})
$$

Intensity IV: Cantua Creek.

Intensity III: Huron, Riverdale.

Felt: Avenal and Coalinga (BK).

24 April (BK) Central California

Origin time: $21 \quad 1519.0$

Epicenter: 37.320 N., 121.698W. 
Depth: $8 \mathrm{~km}$

Magnitude: $5.7 m_{l}$, (GS), 6.1 $M_{c}(\mathrm{GS}), 6.2 M_{L}(\mathrm{BK})$, $6.1 M_{\hookrightarrow}(\mathrm{LD}), M_{1,}=1.1 \mathrm{E} 25(\mathrm{BK})$

This earthquake, known as the Morgan Hill earthquake, caused injuries to 27 persons (24 in Morgan Hill and three in San Martin) and estimated losses of $\$ 8.0$ million from damage to structures and their contents (Hindmarsh, 1984). Most of the damage occurred in Santa Clara County where 522 homes and 43 businesses suffered estimated losses of $\$ 5.4$ and $\$ 1.7$ million, respectively (Hindmarsh, 1984). Intensity VIII effects were confined to a very small area on two streets, Oak Ridge Lane and Oak Ridge Court, of the Jackson Oaks subdivision located east of Morgan Hill near Anderson Lake. This earthquake was felt over an area of approximately 120,000 kilometers ${ }^{2}$ (fig. 13) in California and western Nevada (Stover, 1984).

No major damage resulted from this earthquake but there was some structural damage. However, most of the dollar losses were from damage to the contents of homes and businesses. Well-engineered industrial buildings and residential structures sustained only minor damage, but many mobile homes fell off their supports causing considerable damage to the furnishings inside.

Figure 13 shows the area of intensity VII effects extending southward from the epicenter rather than centering on the epicenter as most isoseismal maps tend to do. This pattern may be explained by the conclusions of Abrahamson and Darragh (1985), that the Morgan Hill earthquake is a double event with the second event being located about $17 \mathrm{~km}$ southeast of the main shock. This location had a significant effect on the large acceleration of $1.29 \mathrm{~g}$ at Coyote Lake Dam, located about $25 \mathrm{~km}$ southeast of the main shock (Abrahamson and Darragh, 1985).

The descriptions of damage at specific locations listed under intensity VII and VIII below were taken from Bennett and Sherburne (1984), Hoose (1984), Thiel, C. C. Jr. (1985), and from press reports.

\section{Intensity VIII:}

\section{California-}

Morgan Hill (Jackson Oaks) - Five houses were condemned, two of which fell off their concrete foundations and suffered partial collapse. One of these two partially collapsed homes was located on Oak Ridge Lane, the other on Oak Ridge Court. The damage was caused by the failure of cripple walls, allowing the houses to move horizontally and fall off founda- tions (fig. 14). Other damage consisted of cracked exterior walls around garage doors and windows and a house thrown out of plumb but still on the foundation (fig. 15). Considerable damage was done to the contents of homes when china closets were thrown over and cabinet doors and drawers opened, throwing china and glassware to the floor. In one home a heavy table was overturned; chairs flung about; planters overturned and broke, and a chimney broke off falling onto another home. A woman was knocked off her feet, a dresser turned over, and a pool table overturned. One man who was at home sick in bed described the shaking to the press as follows: "The room was twisting and turning; dressers were moving back and forth. I was getting thrown around in the bed so much that all I could do was grab onto the mattress and hang on. I was totally helpless." Several buried water lines broke.

\section{Intensity VII:}

California-

Coyote-General effects reported: chimneys fell; landslides occurred; water flow was disturbed in springs or wells; hanging objects swung violently; furniture overturned; many items shook off of store shelves; a few dishes or glassware items broke; a few small objects overturned and fell; some windows broke; trees and bushes shook strongly; both standing and moving vehicles rocked moderately; buildings shook strongly; vibration was described as strong with moderate noise; felt by and frightened everyone. Specific reports of damage at three sites follow:

IBM Santa Teresa Laboratories (1 mile south on Bailey Avenue) - People could not stand on either the ground or upper floors of the four-story building. The structure was designed for seismic loading and suffered no significant structural damage. Damage consisted of fallen ceiling panels (suspended), a fallen light fixture, and rupture of buried, plastic sprinkler pipe. No plate glass windows broke.

Metcalf Substation (1 mile northwest of Coyote near U.S. Highway 101 and Metcalf Road) - A ceramic column of a circuit breaker fractured, a ceramic lightning arrester broke, four relays tripped due to vibration. All equipment, in the buildings, was anchored.

United Technologies Chemical Systems Plant (3 miles northeast of Coyote on Metcalf Road in Shingle Valley) - People had difficulty standing during the earthquake. Damage was estimated at $\$ 1.5$ million. Several steel-framed buildings had dam- 


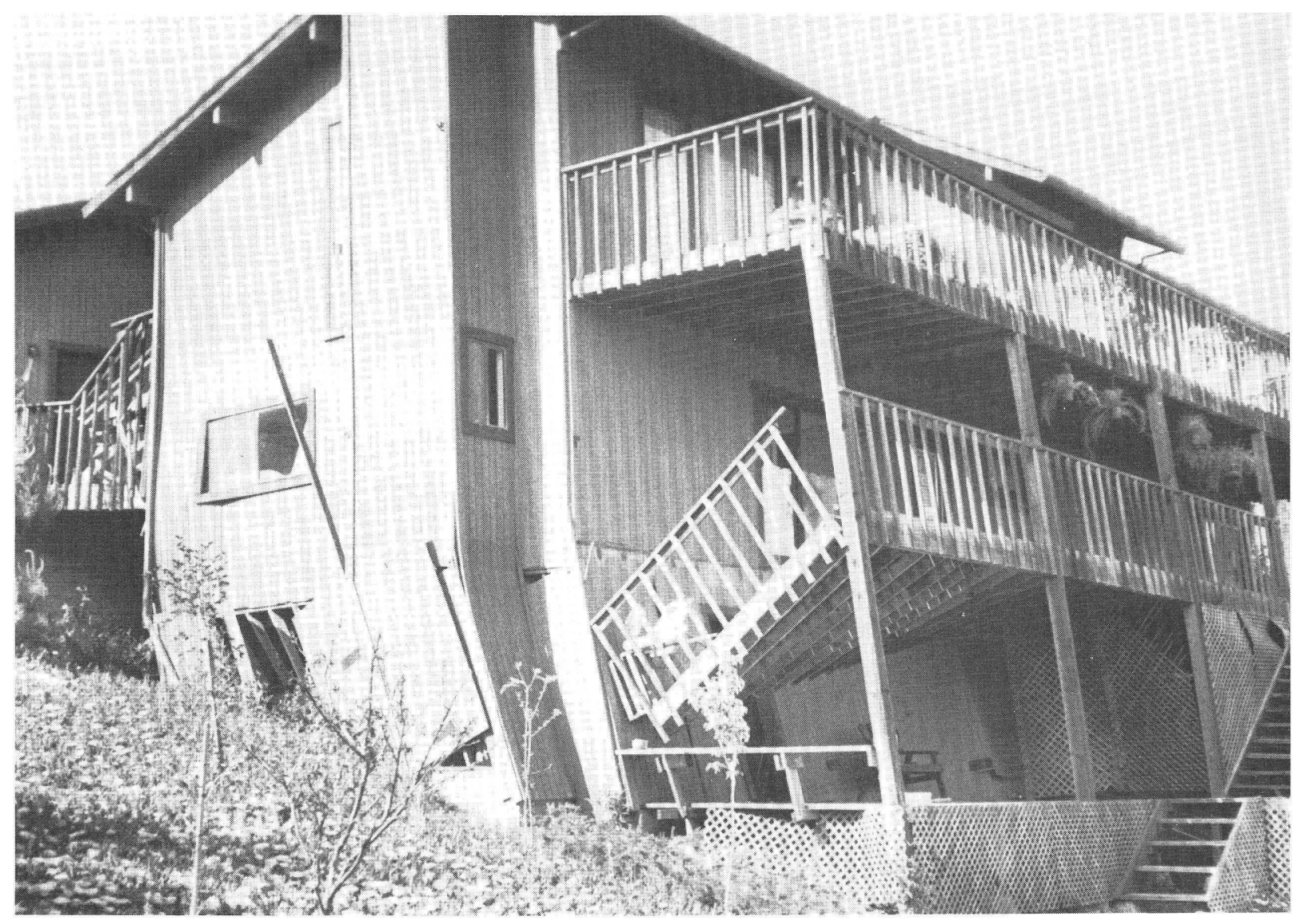

Figure 14. Damage to a home, in the Jackson Oaks subdivision of Morgan Hill,California, caused by the 24 April 1984 earthquake (photograph provided by the Morgan Hill Times). 


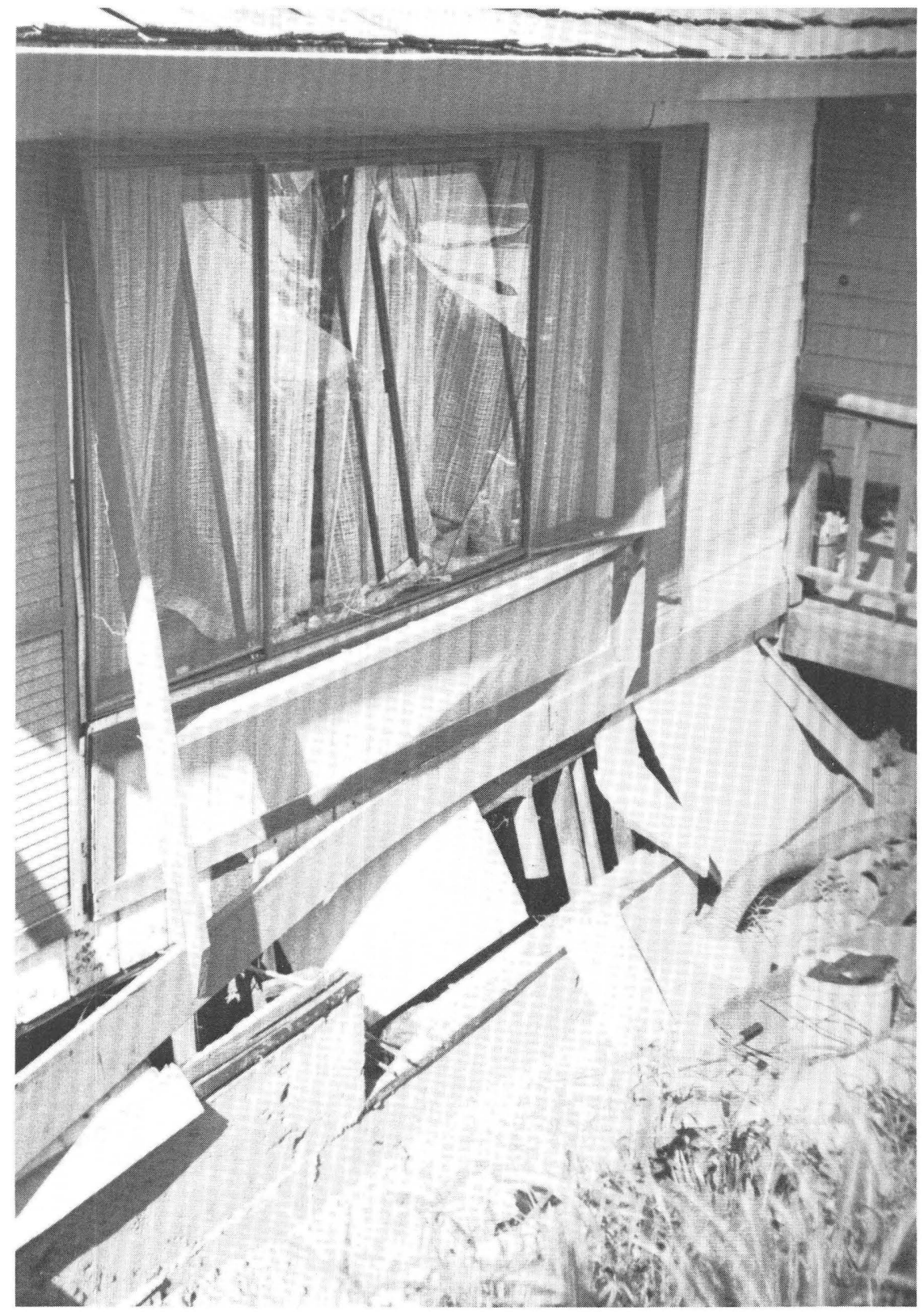

Figure 15. Damage to a home, in the Jackson Oaks subdivision of Morgan Hill, California, caused by the 24 April 1984 earthquake (photograph provided by the Morgan Hill Times). 


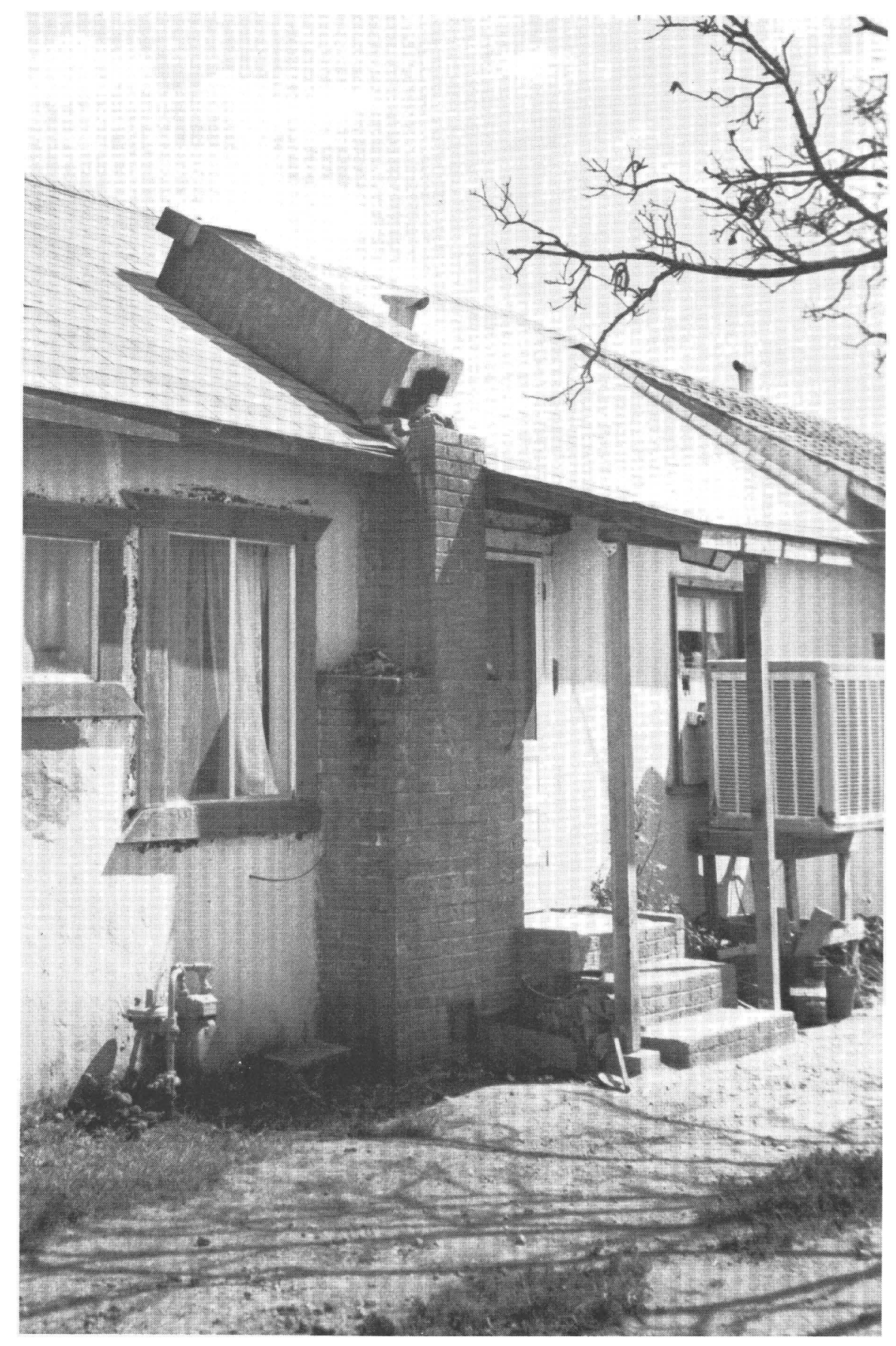

Figure 16. Damage to a chimney, at a home in San Martin, California, caused by the 24 April 1984 earthquake (photograph provided by the Morgan Hill Times). 
aged column base connections, stretched anchor bolts, buckled diagonal steel braces, and popped screws in the metal siding. Concrete buildings had cracked walls and broken weld connections, and one building had a separated wall panel from the roof. Inside the buildings many ceiling panels fell; suspended ceiling lights dislodged or fell; chain supported lights in warehouses fell; one large plate glass window fell and shattered; large amounts of glassware broke in laboratories; numerous bookcases, shelves, and filing cabinets toppled, and desk top equipment fell to the floor damaging typewriters and CRT's. Roof-mounted air conditioners slid.

Jackson Ranch (east of Anderson Lake) - A 1904 vintage ranch house shifted off its foundation and was reported to have collapsed (press report).

Morgan Hill - Many homes did not sustain structural damage but did have substantial damage to contents. Large dressers and bookcases fell over; contents of kitchen cabinets and other storage areas fell onto the floor; pictures fell off walls; furniture slid several inches in many homes, and a few windows broke. Other effects: exterior stone walls sustained cracks; plaster on interior walls cracked; many items shook off of store shelves, many glass items broke; buildings shook strongly; trees and bushes shook moderately; standing and moving vehicles rocked moderately; water splashed onto sides of swimming pools; loud earth noise heard, felt by and frightened all.

Several masonry buildings on Main Street were damaged and later condemned. Other buildings lost plate glass and had minor cracking of exterior walls and interior partitions. A chimney fell at 30 East Fifth Street, and a refrigerator overturned at Hale Lumber Company. Detailed descriptions of damage and other effects are listed below:

South Coast Realty Building at 2nd and Monterey Streets (built in 1906) - The cornice on the south side fell; brick walls cracked; and a plate glass window broke.

Pearson's Hardware and Supply at 2nd and Depot Streets- Stacked fertilizer and roll roofing outside fell to the north; paint was spilled ankle-deep on the floor inside.

Emilio Guglielmo Winery at 1480 Main Street- Approximately $400 \mathrm{ft}^{2}$ of sheetrock fell from the ceiling of a storage warehouse; three 10,250-gallon stainless steel tanks were damaged; many 8,000- gallon wooden barrels shifted on their supports; some 100-200-gallon barrels fell from racks or from stacks.

Gunther Brothers Feed and Grain Supply StoreThere was severe diagonal cracking of the east and west walls of the three-story brick tower.

Jackson Elementary School - Ceiling panels and Tbars became dislodged and fell.

Morgan Hill Elementary School- Two steam radiators fell off the walls. The ceiling and light fixtures in one room were damaged.

Britton Middle School-Cracks in concrete wall near the stage were enlarged; stucco cracked and a few acoustical ceiling tiles fell.

Three mobile home parks: Madrone Manor Mobile Estates, Windmill Mobile Estates, and Woodland Mobile Home Park sustained the most damage. One unit burned as a result of a broken gas line. Seventeen homes were shaken completely off their support systems and many partially fell. The damage consisted of bent skirts around the perimeter of the mobile homes, separation of porches from the home, damaged patio covers; broken water, gas, and sewage lines; and considerable amounts of broken household items as the interior contents were thrown about.

The bridge on East Dunne Avenue over Anderson Reservoir (about 4.5 miles east of Morgan Hill) was closed to traffic due to a rock slide and major structural damage. The damage consisted of anchor bolts sheared on one support, which knocked the pier out of plumb by about 1 foot, spalling at the base of a pier, moved girders, a cracked restrainer, and movement of the wood flooring.

The Coyote Creek bridge (on new U.S. highway 101) had some spalling on the south abutment.

Leroy Anderson Dam (about 3.5 miles northeast of Morgan Hill) - Minor seiching occurred; the crest of the dam sustained cracks.

San Martin - A chimney fell at one home (fig. 16). Other effects: contents of cabinets and refrigerators spilled out onto the floor; exterior walls cracked; sheetrock walls sustained large cracks; underground pipes broke; trees and bushes shook strongly; small landslides occurred; many items shook off of store shelves; many glassware items broke; furniture overturned; some windows broke; hanging pictures fell; 
buildings shook strongly; people had difficulty standing or walking; loud earth noise heard; felt by and frightened all. Effects at specific sites are listed below:

Coyote Dam area (about 4 miles northeast of San Martin) - Minor surficial cracks occurred on the upstream face, crest, and spillway area. A park ranger at the equipment yard located about 3 miles south of the dam on the west side of the lake could stand with difficulty. He observed vehicles bouncing and an unloaded boat trailer bouncing enough to completely leave the ground. Oxygen bottles toppled in a nearby warehouse.

San Martin Winery- (about 2.5 miles south of San Martin on Highway 101) - There were cracked pilasters in the bottling building; a wine tank wall buckled; boilers slid; small tanks slid; wine barrels fell; there was spalling of concrete supports under the tanks; a filing cabinet overturned in the office and there was extensive loss of merchandise from shelves in the tasting room.

Scarborough Lumber- The press reported that a heavy fork lift was lifted free of the ground and a large truck loaded with lumber also left the ground.

Raymond Given Elementary School- Several book shelves anchored to walls pulled loose and overturned; filling cabinets moved.

Wiltron Facility - The plywood roof diaphragm split near the southwest corner of the building; racking of the building displaced several interior walls; many ceiling panels fell; unanchored lathes and drill presses slid on the concrete floor as much as 3 inches; several light fixtures fell; liquid solder sloshed from an automatic soldering machine.

Intensity VI: The most common effects at the locations listed below: windows cracked; buildings shook strongly; many small objects overturned and fell; a few glassware items broke; a few merchandise items shook off of store shelves, sheet rock walls sustained hairline cracks; trees and bushes shook moderately; standing vehicles rocked moderately; chimneys cracked; vibration described as strong causing people difficulty in standing or walking; felt by all. Additional effects at specific locations are shown below:

Agnew (Santa Clara) - About 5\% of the buildings had some type of minor damage.

Blossom Hill (San Jose)- Underground pipes broke; a gas leak caused a fire that destroyed three stores in a shopping center; streets cracked; hanging pictures fell.

Corralitos-- A foundation cracked; light furniture overturned; moving vehicles rocked slightly.

Dublin- Some windows broke; sheetrock walls sustained large cracks; chimneys twisted; exterior cinderblock walls sustained large cracks; moving vehicles rocked moderately; water splashed onto sides of swimming pools.

Fort Ord - Light furniture overturned; underground pipes broke; water splashed on sides of swimming pools.

Gilroy-A few windows broke; people ran from stores. A crack occurred in the corner of the exterior wall of a service station at the north end of Main Street. The Old Gilroy Courhouse had cracks in the exterior walls and minor cracks in the interior walls and ceiling of the second floor. Another building along Main Street had minor cracks in exterior walls. Hanging light fixtures fell in six classrooms of South Valley Junior High School.

Hollister- Some windows broke; light furniture overturned; many dishes broke; light fixtures and ceiling tiles fell; two plate glass windows shattered.

Livermore (Chabot College, Valley Campus 3 miles northwest of Livermore)- A building terrace sustained three-inch hairline cracks; total damage was estimated at $\$ 6,000$ (press report).

Los Altos- A few light furniture items overturned; brick fences cracked; an exterior brick wall cracked.

Los Banos- Many merchandise items shook off of store shelves; moving vehicles rocked moderately; walls separated from the ceiling; water splashed onto sides of swimming pools.

Los Banos Substation (just south of State Route 152 on the west side of Interstate 5) - Waves seen on the ground were estimated between 3 and 6 inches crest to trough; $500-\mathrm{KV}$ porcelain circuit breakers were damaged by the fracturing of the porcelain, and diagonal branches on support columns failed.

Milpitas- Light furniture overturned; a foundation cracked; water splashed onto sides of swimming pools; Elmwood Rehabilitation Center was damaged.

Mount Hamilton- Hanging pictures fell; many merchandise items shook off of; underground pipes broke; moving vehicles rocked moderately; water splashed 
onto sides of swimming pools. At Lick Observatory trees shook strongly; minor damage was reported to the building housing the telescope, and a boulder rolled downhill and hit a car.

Pittsburg - Social Services building had a 14-foot-long crack in the floor; a wall shifted causing part of the ceiling to sag and the building shook violently. People in the Sun Valley Mall ran outside to the parking area (press reports).

Redwood Estates - Water splashed onto sides of swimming pools.

San Bruno-- Light furniture overturned; moving vehicles rocked slightly; water splashed onto sides of swimming pools.

San Francisco- Shattered glass fell from windows of the Western Merchandise Mart at 1355 Market Street; a cornice was knocked off a building in the 1600 block of Pacific; on pier 39 buildings on stilts shook violently. At City Hall chunks of plaster moulding fell from the second floor ceiling; one elevator was knocked off its track; several hundred people fled into the street (from press reports).

San Jose- Eastridge Shopping Mall (a three-story indoor shopping complex)- There was spalled concrete at expansion joints between balconies and bridge walkways on the upper level; three stores lost plate glass windows; suspended ceiling panels fell; a substantial portion of merchandise fell from shelves and display cases.

Mirassou Winery - Large tanks slid as much as 3 inches and sheared or pulled out anchor bolts; a section of overhead steel catwalk grating dislodged and fell; fifteen pallets of empty wine bottles overturned and the bottles broke; power was lost for several hours.

Evergreen Community College- A few suspended ceiling tiles fell; approximately 5,000 books fell from shelves in the main library; a cabinet on rollers overturned spilled several hundred cassette tapes.

Alexian Hospital- Some light cracking occurred in walls and partitions; one piece of equipment overturned.

Santa Clara County Building (North First Street and West Hedding Street) - Some walls cracked; shelving and equipment toppled; the building was evacuated after the earthquake.
San Jose City Hall- Some walls cracked.

San Felipe Ranch (8025 San Felipe Road) - A chimney on the main house (built 1914) fell; a foundation cracked; dishes fell out of cabinets; a concrete patio at the rear of one guest house was severely cracked and settled from 2-6 inches; two other guest houses had minor foundation cracks at the corners.

Santa Clara- Moving vehicles rocked slightly; water splashed onto sides of swimming pools.

Santa Cruz-- Exterior, brick-veneer walls sustained large cracks; moving vehicles rocked slightly; a plate glass window at Woolworth's in the Pacific Garden Mall fell out; part of the marble facade at Rader's Pawn Shop fell to the ground; an 18-foot support column and a few ceiling tiles cracked at DeLaveaga School Media Service Center.

Scotts Valley - Many glassware items and dishes broke; light furniture overturned; many items shook off of store shelves; underground pipes broke; moving vehicles rocked moderately.

Watsonville- Plaster and sheetrock walls sustained large cracks; reinforced concrete walls sustained cracks; many items shook off of store shelves; water splashed onto sides of swimming pools.

\section{Intensity V:}

California-

The most common effects at most of the places listed below: buildings shook strongly; a few small objects overturned or fell; vibration was described as moderate to strong; trees and bushes shook slightly to moderately; standing vehicles rocked slightly to moderately; felt by most. Additional effects at specific locations are listed below:

Alameda- Plaster walls sustained hairline cracks.

Alamo-Sheetrock wall sustained hairline cracks.

Alviso- Hanging pictures were out of place; a few merchandise items shook off of store shelves.

Antioch-A few buildings were damaged.

Aptos- Hanging pictures were out of place.

Aromas- A few merchandise items shook off of store shelves; moving vehicles rocked moderately.

Atherton- A few windows cracked; a few glassware items broke; moving vehicles rocked slightly.

Atwater- One chimney cracked; plaster walls sustained hairline cracks.

Belmont - Hanging pictures swung.

Ben Lomond-A few windows cracked; a few glassware items broke; many items shook off of store shelves. 
Berkeley - Moving vehicles rocked slightly; water splashed onto sides of swimming pools.

Bethel Island- A few glassware items broke.

Biola- A few windows cracked; a few glassware items broke; moving vehicles rocked slightly; water splashed onto sides of swimming pools.

Bolinas - Hanging pictures were out of place; a few glassware items broke; light furniture overturned; a few items shook off of store shelves; moving vehicles rocked slightly.

Boulder Creek - People had difficulty standing.

Brentwood- Items fell from grocery store shelves and broke (press report); a few windows cracked; sheetrock walls sustained hairline cracks; water splashed onto sides of swimming pools.

Brisbane - A few windows cracked; a few glassware items broke; a few items shook off of store shelves; plaster and sheetrock walls sustained hairline cracks; water splashed onto sides of swimming pools.

Brookdale - Hanging pictures were out of place; moving vehicles rocked slightly.

Burlingame- Plaster walls sustained hairline cracks; small foundation cracks.

Campbell - Hanging pictures were out of place; a few glassware items broke; a few items shook off of store shelves; plaster walls sustained hairline cracks; moving vehicles rocked moderately.

Capitola - People had difficulty standing; sheetrock walls sustained hairline cracks; moving vehicles rocked slightly to moderately.

Carmel-A few items shook off of store shelves; moving vehicles rocked moderately.

Castle Air Force Base- People had difficulty standing.

Castro Valley- Hanging pictures swung.

Castroville - Hanging pictures were out of place; moving vehicles rocked slightly.

Ceres- Sheetrock wall sustained hairline cracks; water splashed onto sides of swimming pools.

Chualar - Hanging pictures were out of place; a few items shook off of store shelves.

Concord- One picture window cracked (press report); a few items shook off of store shelves; plaster walls sustained hairline cracks.

Copperopolis- Moving vehicles rocked slightly.

Corte Madera- People had difficulty standing; a few glassware items broke.

Cressey- People had difficulty standing; moving vehicles rocked moderately; water splashed onto sides of swimming pools.

Crows Landing - Hanging pictures fell; a few items shook off of store shelves; water splashed onto sides of swimming pools.

Cupertino- A few items shook off of store shelves; water splashed onto sides of swimming pools.

Daly City - A few windows cracked; hanging pictures fell; a few items shook off of store shelves; a few glassware items broke; light furniture overturned.

Danville - People had difficulty standing; a few windows cracked; a few glass items broke; water splashed onto sides of swimming ponds.

Davenport-- Hanging pictures were out of place; a few glass items broke; a few items shook off of store shelves; plaster walls sustained hairline cracks; moving vehicles rocked slightly.

Denair - People had difficulty standing; hanging pictures were out of place; trees and bushes shook strongly; water splashed onto sides of swimming pools.

Dixon- A few windows cracked; a few items shook off of store shelves.

Dos Palos- Hanging pictures swung.

El Cerrito (press report) - People ran into the street; a telephone slid across a desk (press report).

El Granada- A few items shook off of store shelves.

El Sobrante- Moving vehicles rocked slightly.

Emeryville.

Empire- A few glassware items broke; a few items shook off of store shelves.

Fairfax.

Felton- Hanging pictures were out of place; a few items shook off of store shelves.

Forest Knolls-- Water splashed onto sides of swimming pools.

Foster City - Standing vehicles rocked strongly.

Freedom.

Fremont- A few windows cracked; sheetrock walls sustained hairline cracks; moving vehicles rocked slightly; water splashed onto sides of swimming pools.

Fremont (Irvington)- A few glassware items broke; a few items fell off of store shelves; moving vehicles rocked slightly.

Friant- Hanging pictures swung.

Glen Ellen- A few windows cracked; a few glassware items broke; a few items shook off of store shelves.

Gonzales.

Graton.

Gustine- People had difficulty standing.

Half Moon Bay-- Hanging pictures fell; moving vehicles rocked slightly.

Hayward- A few windows cracked; a few glassware 
items broke; light furniture overturned; a few items shook off of store shelves; plaster and sheetrock walls sustained hairline cracks; moving vehicles rocked slightly; water splashed onto sides of swimming pools.

Holt - Hanging pictures fell.

Inverness- Moving vehices rocked slightly.

Lafayette- Moving vehicles rocked slightly; wates splashed onto sides of swimming pools.

Lagunitas - A few glassware items broke; a few items shook off of store shelves; hanging pictures were out of place.

La Honda.

Livermore- A few items shook off of store shelves; water splashed onto sides of swimming pools.

Livingston- Plaster and sheetrock walls sustained hairline cracks; many items shook off of store shelves; a few glassware items broke; light furniture overturned; people had difficulty standing.

Loma Mar- Hanging pictures were out of place; a few items shook off of store shelves; water splashed onto sides of swimming pools.

Los Altos Hills - People had difficulty standing; there were minor gas leaks and a cracked library window at Foothills College (press report).

Los Gatos- Plaster and sheetrock walls sustained hairline cracks; a few items shook off of store shelves; many small objects overturned or fell; hanging pictures fell; standing and moving vehicles rocked slightly; felt by many.

Marina- Hanging pictures fell; a few glassware items broke; many items shook off of store shelves; plaster and sheetrock walls sustained hairline cracks.

Martinez - An entire building shook (press report).

Mendota- People had difficulty standing; grocery items fell off shelves at Mendota Food Center (press report).

Menlo Park - A few windows cracked; a few glassware items broke; sheetrock walls sustained hairline cracks; underground pipes broke; water splashed onto sides of swimming pools.

Merced- A few glassware items broke; a few items shook off of store shelves; moving vehicles rocked slightly; water splashed onto sides of swimming pools.

Millbrae- People had difficulty standing; a few items shook off of store shelves; water splashed onto sides of swimming pools.

Mill Valley- A few items shook off of store shelves; underground pipes broke.
Modesto- A few windows cracked; people had difficulty standing; plaster and sheetrock walls sustained hairline cracks.

Monterey - A few windows cracked; a few glassware items broke; a few items shook off of store shelves; plaster walls sustained hairline cracks; moving vehicles rocked moderately.

Moraga- Hanging pictures fell; a few items shook off of store shelves; plaster and sheetrock walls sustained hairline cracks; water splashed onto sides of swimming pools.

Moss Landing - People had difficulty standing in the Post Office; a few glassware items broke; light furniture overturned; a few items shook off of store shelves; moving vehicles rocked slightly; water splashed onto sides of swimming pools; chimneys twisted.

Mount Eden- Plaster walls sustained hairline cracks; moving vehicles rocked slightly.

Mountain View- One window broke; plaster walls sustained hairline cracks; moving vehicles rocked slightly; water splashed onto sides of swimming pools.

Napa- Plaster walls sustained hairline cracks; moving vehicles rocked slightly.

New Almaden - Moving vehicles rocked slightly; water splashed onto sides of swimming pools.

Newark - Hanging pictures were out of place; a few glassware items broke; a few items shook off of store shelves; plaster and sheetrock walls sustained hairline cracks; moving vehicles rocked slightly; water splashed onto sides of swimming pools.

Newman- A few items shook off of store shelves; hanging pictures were out of place; water splashed onto sides of swimming pools.

Niles.

Novato- A few windows cracked; a few items shook off of store shelves.

Oakdale- People had difficulty standing; a few items shook off of store shelves; moving vehicles rocked slightly; water splashed onto sides of swimming pools.

Oakland-A few windows cracked; a few glassware items broke; hanging pictures were out of place; plaster walls sustained hairline cracks; moving vehicles rocked slightly. Bricks fell from the upper level of Central Building; employees fled the County Courthouse (press report).

Oakland International Airport- People had difficulty 
standing; hanging pictures were out of place; plaster and sheetrock walls sustained hairline cracks; moving vehicles rocked moderately.

Pacifica-- Items shook off of grocery shelves (press report).

Pacific Grove- A few windows cracked; a few glassware items broke; plaster and sheetrock walls sustained hairline cracks; a foundation cracked; moving vehicles rocked slightly; water splashed onto sides of swimming pools.

Paicines- Moving vehicles rocked slightly.

Palo Alto- People had difficulty standing; plaster walls sustained hairline cracks; moving vehicles rocked slightly.

Patterson- A few glassware items broke; a few items shook off of store shelves; plaster and sheetrock walls sustained hairline cracks; water splashed onto sides of swimming pools.

Pescadero- Moving vehicles rocked slightly.

Pinecrest- Many items shook off of store shelves.

Pinole-A few glassware items broke; hanging pictures were out of place; a few windows cracked; plaster and sheetrock walls sustained hairline cracks; moving vehicles rocked slightly.

Pleasanton- Hanging pictures were out of place; water splashed onto sides of swimming pools; crystal glasses broke in a kitchenware shop (press report).

Point Reyes Station- Moving vehicles rocked slightly.

Port Costa - Hanging pictures were out of place.

Portola Valley.

Red Top- Moving vehicles rocked slightly; a concrete foundation cracked.

Redwood City - A few glassware items broke; a few items shook off of store shelves; hanging pictures were out of place; plaster walls sustained hairline cracks; people in one restaurant ran outside; waves were observed on an asphalt parking lot.

Richmond- Hanging pictures fell; a few windows cracked; a few glassware items broke; a few items shook off of store shelves; light furniture overturned; plaster walls sustained hairline cracks; moving vehicles rocked slightly; water splashed onto sides of swimming pools.

Ripon-- A few windows cracked; a few glassware items broke; a few items shook off of store shelves; water splashed onto sides of swimming pools.

Riverbank - People had difficulty standing; a few windows cracked; water splashed onto sides of swimming pools.

Rodeo- People had difficulty standing.
Salinas- Hanging pictures fell; a few windows cracked; a few glassware items broke; moving vehicles rocked moderately; water splashed onto sides of swimming pools.

San Carlos- A few windows cracked; a few items shook off of store shelves; moving vehicles rocked slightly.

San Francisco (Bay View).

San Francisco (Chinatown) - A few windows cracked; a few glassware items broke; plaster and sheetrock walls sustained hairline cracks.

San Francisco (Letterman)- People had difficulty standing.

San Francisco (Marina) - Hanging objects swung violently.

San Francisco (Parkside)- Felt by all.

San Francisco (Presidio)- People had difficulty standing; hanging pictures were out of place; a few glassware items broke; many items shook off of store shelves; moving vehicles rocked slightly.

San Francisco International Airport- People on the 5 th floor had difficulty standing.

San Geronimo-- Hanging pictures were out of place.

San Gregorio- Plaster and sheetrock walls sustained hairline cracks; water splashed onto sides of swimming pools.

San Jose (Cambrian Park) - A few windows cracked; a few glassware items broke; a few items shook off of store shelves; hanging pictures were out of place; plaster and sheetrock walls sustained hairline cracks; moving vehicles rocked slightly; water splashed onto sides of swimming pools.

San Jose (Saint James Park) - Hanging pictures were out of place; moving vehicles rocked moderately.

San Jose (Valley Fair) - Hanging pictures fell; a few glassware items broke; a few items shook off of store shelves; plaster and sheetrock walls sustained hairline cracks; moving vehicles rocked slightly.

San Jose (Westgate) - People had difficulty standing; a few windows cracked; a few glassware items broke; plaster or sheetrock walls sustained hairline cracks; moving vehicles rocked slightly.

San Juan Bautista-- People had difficulty standing; hanging pictures fell; a few windows cracked; a few glassware items broke; a few items shook off of store shelves; plaster and sheetrock walls sustained hairline cracks; a foundation cracked; moving vehicles rocked slightly; water splashed onto sides of swimming pools.

San Leandro- People had difficulty standing; a few 
windows cracked; a few glassware items broke; plaster and sheetrock walls sustained hairline cracks.

San Lorenzo- Hanging pictures swung.

San Mateo- Hanging pictures were out of place; moving vehicles rocked slightly; water splashed onto sides of swimming pools.

San Ramon- A few items shook off of store shelves; plaster and sheetrock walls sustainted hairline cracks; water splashed onto sides of swimming pool.

Santa Rita Park - Many items shook off of store shelves; moving vehicles rocked moderately.

Saratoga- People had difficulty standing; hanging pictures were out of place; a few glassware items broke; moving vehicles rocked slightly; water splashed onto sides of swimming pools.

Seaside.

Soledad-A few items shook off of store shelves; moving vehicles rocked slightly.

Soquel- People had difficulty standing; hanging pictures fell; a few items shook off of store shelves; moving vehicles rocked moderately.

South Dos Palos- People had difficulty standing; a few windows cracked; a few items shook off of store shelves; plaster walls sustained hairline cracks.

South San Francisco- Hanging pictures were out of place; a few glassware items broke; moving vehicles rocked slightly.

South San Leandro- Moving vehicles rocked moderately.

Spreckles- People had difficulty standing; plaster walls sustained hairline cracks; moving vehicles rocked slightly.

Stanford- Moving vehicles rocked slightly; one brace on a seventh-level bookcase broke in Old Green Library, and students got under desks (press report).

Stevinson- Hanging pictures fell; a few glassware items broke.

Stockton- A few windows cracked; a few glassware items broke; a few items shook off of store shelves; plaster and sheetrock walls sustained hairline cracks; moving vehicles rocked slightly; water splashed onto sides of swimming pools.

Stockton (Homestead) - A few windows cracked; sheetrock walls sustained hairline cracks; moving vehicles rocked slightly.

Stratford- Plaster walls sustained hairline cracks; moving vehicles rocked slightly.

Suisun City - People had difficulty standing; a few windows cracked; a few glassware items broke; a few items shook off of store shelves; plaster and sheetrock walls sustained hairline cracks; moving vehicles rocked slightly; water splashed onto sides of swimming pools.

Sunnyvale- People had difficulty standing; a few windows cracked; a few glassware items broke; a few items shook off of store shelves; plaster and sheetrock walls sustained hairline cracks; moving vehicles rocked slightly; water splashed onto sides of swimming pools.

Tiburon-- Fire engines bounced up and down for 30 seconds (press report).

Tracy- A few windows cracked.

Tranquillity - Trees and bushes shook strongly; moving vehicles rocked slightly.

Traver- A few windows cracked; a few glassware items broke.

Treasure Island.

Tres Pinos-Hanging pictures were out of place; a few glassware items broke; a few items shook off of store shelves; moving vehicles rocked slightly.

Turlock- People had difficulty standing; sheetrock walls sustained hairline cracks; water splashed onto sides of swimming pools.

Union City-A few windows cracked; a few items shook off of store shelves; hanging pictures were out of place; moving vehicles rocked moderately.

Vernalis- A few windows cracked; a few glassware items broke; a few items shook off of store shelves; water splashed onto sides of swimming pools.

Walnut Creek - Hanging pictures fell; a few windows cracked; a few glassware items broke; a few items shook off of store shelves; moving vehicles rocked slightly.

West Menlo Park - A few items shook off of store shelves; moving vehicles rocked slightly.

Westley- A few items shook off of store shelves.

West Pittsburg - A few windows cracked; a few glassware items broke; light furniture overturned; a few items shook off of store shelves; moving vehicles rocked slightly; water splashed onto sides of swimming pools.

\section{Intensity IV:}

California - Armona, Arnold, Atascadero, Auberry, Avila Beach, Ballico, Bangor, Bass Lake, Big Creek, Big Sur, Bishop, Bridgeport, Burrel, Byron, Cantua Creek, Canyon, Chowchilla, Clovis, Crockett, Del Rey, Delhi, Diablo, Douglasflat, El Nido, El Verano, Elmira, Fairfield (press report), Firebaugh, Foresthill, Fowler, French Camp, Fresno, Fulton, Goshen, Guerneville, Hickman, Hillsborough, Homewood, Hughson, Isleton, 
Jenner, Jolon, Kentfield, Kenwood, Kerman, Keyes, King City, Kingsburg, Knights Ferry, Knights Landing, La Grange, Ladera, Larkspur, Lathrop, Lee Vining, Lemoore, Lindsay, Lodi, Madera, Manteca, Mountain Ranch, Murphys, O'Neals, Oakland (Rockridge), Oakley, Oceano, Olema, Orinda (press report), Pacheco, Parlier, Pebble Beach, Piedmont, Planada, Pleasant Hill, Point Richmond, Rail Road Flat, Raisin, Rio Vista, Rohnert Park, Ross, Ryde, Sacramento, Sacramento (Parkway), Saint Helena (press report), Salida, San Anselmo, San Ardo, San Francisco (Sunset), San Joaquin, San Lucas, San Miguel, San Quentin, San Rafael, Santa Rosa, Sather Gate, Sausalito, Sebastopol, Selma (press report), Sequoia National Park, Snelling, Sonora, Stinson Beach, Strathmore, Strawberry, Temescal, Templeton, Thornton, Twain Harte, Vacaville, Vallejo, Valley Home, Valley Springs, Vineburg, Visalia, Vista Grande, Wallace, Waterford, Wilseyville, Winton, Woodacre, Woodbridge, Yountville.

Nevada - Gardnerville, Genoa, Glenbrook, Schurz. Intensity III:

California - Acampo, Ahwahnee, Alta, Altaville, Arroyo Grande, Avery, Benicia, Blairsden, Bodega, Bodega Bay, Bradley, Buttonwillow, Cambria, Carnelian Bay, Cedar Ridge, Chinese Camp, Clements, Coalinga, Coarsegold, Colfax, Colma, Corcoran, Cotati, Coulterville, Davis, Dillon Beach, Dinuba, Doyle, Dutch Flat, Edison, El Portal, Eldridge, Emigrant Gap, Eureka, Forestville, Galt, Gold Run, Groveland, Grover City, Hathaway Pines, Helm, Hood, Huron, Inyokern, Kings Beach, Lake Isabella, Lakeshore, Le Grand, Linden, Long Barn, Los Alamos, Los Osos, Loyalton, Madison, Mare Island, Marshall, Mi-Wuk Village, Monte Rio, Nicasio, Oakville, Olancha, Paso Robles, Petaluma, Pine Grove, Pioneer, Placerville, Point Arena, Porterville, Portola, Prather, Raymond, Ridgecrest, Roseland, Soda Springs, Sonoma, Soulsbyville, Steele Park, Sunol, Tahoe Vista, Tahoma, Tipton, Tomales, Valley Ford, Vandenberg Air Force Base, Victor, Villa Grande, West Sacramento, Wilton, Windsor, Winters, Yolo.

Nevada- Carson City, Gabbs, Hawthorne, Minden, Reno, Smith, Sparks.

Intensity II:

California- Albion, Burson, Catheys Valley, Ione, McFarland, New Cuyama, Philo, Pismo Beach, Roseville.

Felt:

California- Bakersfield, Calistoga, Coleville, Cutler, Occidental.

\section{April (PS) Southern California}

Origin time: 072043.5

Epicenter: $34.251 \mathrm{~N} ., 119.639 \mathrm{~W}$.

Depth: $12 \mathrm{~km}$

Magnitude: $3.5 M_{L}$ (PS)

Felt: Santa Barbara (press report).

\section{April (BK) Central California \\ Origin time: 004226.6 \\ Epicenter: $37.140 \mathrm{~N}$, $121.580 \mathrm{~W}$. \\ Depth: 5 km \\ Magnitude: $3.6 M_{L}(\mathrm{BK}), 3.1 M_{D}(\mathrm{GM})$,

$$
M_{1}=4.0 \mathrm{E} 21(\mathrm{BK})
$$

Felt: Morgan Hill (BK).

26 April (BK) Central California

Origin time: 062951.9

Epicenter: 37.138 N., $121.570 \mathrm{~W}$.

Depth: $5 \mathrm{~km}$

Magnitude: $3.7 M_{L}(\mathrm{BK}), 3.2 M_{D}(\mathrm{GM})$, $M_{,}=8.1 \mathrm{E} 21(\mathrm{BK})$

Intensity IV: Gilroy, Morgan Hill, San Martin, Saratoga.

Felt: Throughout the San Francisco Bay area (BK).

26 April (PS) Southern California

Origin time: 224416.7

Epicenter: $32.745 \mathrm{~N} ., 115.424 \mathrm{~W}$.

Depth: $13 \mathrm{~km}$

Magnitude: $3.9 M_{L}$ (PS)

Intensity IV: Calexico, Heber.

Intensity III: El Centro.

Intensity II: Westmorland.

27 April (BK) Central California

Origin time: 041023.9

Epicenter: 37.122N., 121.545W.

Depth: 8 km

Magnitude: $3.5 M_{L}(\mathrm{BK}), 3.0 M_{D}(\mathrm{GM})$, $M_{,}=3.1 \mathrm{E} 21(\mathrm{BK})$

Felt: Halls Valley area (BK). 
27 April (BK) Central California

Origin time: 164834.1

Epicenter: 37.277 N., 121.670W.

Depth: $10 \mathrm{~km}$

Magnitude: $3.4 M_{L}(\mathrm{BK}), 3.1 M_{D}(\mathrm{GM})$,

$$
M_{,}=1.1 \mathrm{E} 21(\mathrm{BK})
$$

Felt: Halls Valley area (BK).

28 April (BK) Mammoth Lakes area

Origin time: 224821.0

Epicenter: $37.622 \mathrm{~N}$., $118.897 \mathrm{~W}$.

Depth: $3 \mathrm{~km}$

Magnitude: $4.3 m_{l},(\mathrm{GS}), 4.7 M_{L}(\mathrm{BK}), 4.8 M_{L}(\mathrm{PS})$,

$$
M_{,}=5.1 \mathrm{E} 22(\mathrm{BK})
$$

Intensity IV: Mammoth Lakes.

Felt: Bishop (BK).

29 April (BK) Mammoth Lakes area

Origin time: 005033.8

Epicenter: 37.613N., 118.910W.

Depth: 5 km

Magnitude: $3.7 M_{L}(\mathrm{BK}), 3.8 M_{L}(\mathrm{PS})$

Felt: Mammoth Lakes (BK).

29 April (PS) Southern California

Origin time: 165629.8

Epicenter: $33.870 \mathrm{~N}$., 116.540W.

Depth: $11 \mathrm{~km}$

Magnitude: $2.8 M_{L}$ (PS)

Felt: Palm Springs (PS).

\section{May (BK) Central California}

Origin time: 151845.2

Epicenter: 37.288 N., 121.638 W.

Depth: $4 \mathrm{~km}$

Magnitude: $3.2 M_{L}(\mathrm{BK}), M_{c}=4.7 \mathrm{E} 20(\mathrm{BK})$

Felt: Halls Valley area (BK).

\section{May (BK) Central California}

Origin time: 130251.7

Epicenter: $37.283 \mathrm{~N}$., $121.677 \mathrm{~W}$.

Depth: $9 \mathrm{~km}$

Magnitude: $3.2 M_{L}(\mathrm{BK}), M_{c}=2.1 \mathrm{E} 20(\mathrm{BK})$

Intensity III: Morgan Hill (press report).
3 May (BK) Central California

Origin time: 130711.9

Epicenter: 37.287 N., $121.670 \mathrm{~W}$.

Depth: $10 \mathrm{~km}$

Magnitude: $4.4 M_{L}(\mathrm{BK}), M_{t}=4.1 \mathrm{E} 22(\mathrm{BK})$

Intensity V: Morgan Hill-A few windows cracked; a few glassware items broke; a few small objects overturned or fell; windows and dishes rattled; felt by many. Intensity IV: Oakland (press report), San Jose, San Leandro (press report).

Intensity III: Berkeley (press report), Hayward, Milpitas, Redwood City, Soquel.

Felt: Fremont (BK).

\section{May (BK) Central California}

Origin time: 010514.0

Epicenter: 37.735N., 122.123W.

Depth: $6 \mathrm{~km}$

Magnitude: $2.6 M_{L}(\mathrm{BK})$

Intensity III: San Ramon.

Felt: Castro Valley and San Leandro (press reports).

\section{May (BK) Central California}

Origin time: 184258.4

Epicenter: $37.292 \mathrm{~N}$., $121.685 \mathrm{~W}$.

Depth: $6 \mathrm{~km}$

Magnitude: $3.4 M_{L}(\mathrm{BK}), M_{c}=1.1 \mathrm{E} 21(\mathrm{BK})$

Felt: Morgan Hill (BK).

\section{May (BK) Central California}

Origin time: 055014.2

Epicenter: 37.105N., 121.582W.

Depth: $5 \mathrm{~km}$

Magnitude: $3.0 M_{L}(\mathrm{BK}), M_{c}=3.5 \mathrm{E} 20(\mathrm{BK})$

Felt: Morgan Hill (BK).

\section{May (BK) Northern California}

Origin time: 012540.8

Epicenter: 40.138N., 124.110W.

Depth: 5 km

Magnitude: $3.5 M_{L}(\mathrm{BK}), 3.2 M_{D}(\mathrm{GM})$,

Intensity II: Miranda.

$$
M_{,}=3.3 \mathrm{E} 21(\mathrm{BK})
$$

Felt: Myers Flat. 
7 May (PS) Southern California

Origin time: 193232.8

Epicenter: 34.661N., 119.973W.

Depth: $10 \mathrm{~km}$

Magnitude: $4.4 m_{l},(\mathrm{GS}), 4.2 M_{L}(\mathrm{PS}), 4.2 M_{L}(\mathrm{BK})$

Intensity V: Santa Ynez- Plaster walls sustained hairline cracks; trees and bushes shook strongly; windows rattled; felt by many.

Intensity IV: Buellton, Los Alamos, Los Olivos, Santa Barbara, Solvang, Summerland.

Intensity III: Casmalia, Cuyama, Goleta, Lompoc, Saticoy, Vandenberg Air Force Base.

\section{May (BK) Central California}

Origin time: 192114.0

Epicenter: 36.168 N., 120.297W.

Depth: $8 \mathrm{~km}$

Magnitude: $4.2 m_{l},(\mathrm{GS}), 4.4 M_{L}(\mathrm{BK}), 4.2 M_{L}(\mathrm{PS})$, $M_{c}=3.1 \mathrm{E} 22(\mathrm{BK})$

Intensity V: Coalinga- A few items shook off of store shelves; people had difficulty standing; some people went out into the streets; vibration was described as strong; several ceiling tiles fell to the floor of the California Highway Patrol office; buildings shook strongly; hanging pictures were out of place; felt by all.

Intensity IV: Avenal, Huron, Stratford.

Intensity II: Helm.

\section{May (PS) Southern California}

Origin time: 042614.4

Epicenter: $34.010 \mathrm{~N} ., 118.180 \mathrm{~W}$.

Depth: 13 km

Magnitude: $2.7 M_{L}$ (PS)

Felt: Montebello.

\section{May (BK) Southern California}

Origin time: 173014.2

Epicenter: 34.803 N., $120.382 \mathrm{~W}$.

Depth: $11 \mathrm{~km}$

Magnitude: $3.5 M_{L}(\mathrm{BK}), 3.6 M_{L}(\mathrm{PS})$

Intensity III: Santa Maria (press report), Vandenberg Air Force Base.

Felt: Solvang (BK).

17 May (BK) Central California

Origin time: 084303.6
Epicenter: $37.298 \mathrm{~N} ., 121.688 \mathrm{~W}$.

Depth: $6 \mathrm{~km}$

Magnitude: $3.5 M_{L}(\mathrm{BK}), 3.0 M_{D}(\mathrm{GM})$,

$$
M_{1}=3.0 \mathrm{E} 21(\mathrm{BK})
$$

Intensity IV: San Jose (Evergreen, Berryessa).

Felt: Morgan Hill (BK).

\section{May (BK) Central California}

Origin time: 124740.9

Epicenter: 36.240N., 120.853W.

Depth: $6 \mathrm{~km}$

Magnitude: $3.2 M_{L}(\mathrm{BK}), 3.2 M_{D}(\mathrm{GM})$

Felt: Avenal (BK).

\section{May (BK) Central California}

Origin time: 070821.1

Epicenter: $38.778 \mathrm{~N}$., $122.767 \mathrm{~W}$.

Depth: $5 \mathrm{~km}$

Magnitude: $2.9 M_{L}(\mathrm{BK})$

Felt: South of Clear Lake (BK).

19 May (BK) Central California

Origin time: 164950.9

Epicenter: $37.343 \mathrm{~N}$., $121.727 \mathrm{~W}$.

Depth: $6 \mathrm{~km}$

Magnitude: $3.2 M_{L}(\mathrm{BK}), M_{c}=3.9 \mathrm{E} 20(\mathrm{BK})$

Felt: Morgan Hill (BK).

24 May (GM) Central California

Origin time: 080054.0

Epicenter: $37.500 \mathrm{~N} ., 122.200 \mathrm{~W}$.

Depth: $7 \mathrm{~km}$

Magnitude: $2.6 M_{D}(\mathrm{GM})$

Intensity IV: Menlo Park and Redwood City (press report).

\section{May (BK) Central California}

Origin time: 032815.6

Epicenter: 37.265N., 121.665W.

Depth: $7 \mathrm{~km}$

Magnitude: $3.4 M_{L}(\mathrm{BK}), M_{c}=1.4 \mathrm{E} 21(\mathrm{BK})$

Felt: Morgan Hill and San Jose (BK). 
30 May (BK) Central California

Origin time: 123811.2

Epicenter: $37.298 \mathrm{~N} ., 121.687 \mathrm{~W}$.

Depth: $6 \mathrm{~km}$

Magnitude: $3.2 M_{L}(\mathrm{BK}), M_{n}=8.0 \mathrm{E} 20(\mathrm{BK})$

Felt: Halls Valley area $(\mathrm{BK})$.

\section{June (BK) Central California}

Origin time: 165620.8

Epicenter: $37.268 \mathrm{~N} ., 121.610 \mathrm{~W}$.

Depth: $6 \mathrm{~km}$

Magnitude: $4.2 M_{L}(\mathrm{BK}), 3.5 M_{D}(\mathrm{GM})$, $M_{\prime}=2.9 \mathrm{E} 22(\mathrm{BK})$

Intensity IV: Morgan Hill (press report).

Felt: San Jose (press report).

12 June (PS) Southern California

Origin time: 002752.4

Epicenter: $34.541 \mathrm{~N} ., 118.989 \mathrm{~W}$.

Depth: $12 \mathrm{~km}$

Magnitude: $4.1 M_{L}$ (PS), $4.2 M_{L}(\mathrm{BK})$

Intensity IV: Summerland, Ventura.

Intensity III: Fillmore, Oak View, Ojai (Meiners Oaks), Oxnard.

Intensity II: Gardena.

\section{June (BK) Central California}

Origin time: 035027.4

Epicenter: $37.288 \mathrm{~N}$., $121.685 \mathrm{~W}$.

Depth: $5 \mathrm{~km}$

Magnitude: $3.4 M_{L}(\mathrm{BK}), M_{c}=1.6 \mathrm{E} 21(\mathrm{BK})$

Felt: South of Clear Lake (BK).

\section{June (BK) Central California}

Origin time: 104454.0

Epicenter: $38.808 \mathrm{~N}$., $122.793 \mathrm{~W}$.

Depth: $5 \mathrm{~km}$

Magnitude: $3.0 M_{L}(\mathrm{BK}), M_{1}=2.2 \mathrm{E} 21(\mathrm{BK})$

Felt: South of Clear Lake (BK).

20 June (PS) Southern California

Origin time: 192806.3

Epicenter: 34.918 N., $120.800 \mathrm{~W}$.

Depth: $6 \mathrm{~km}$
Magnitude: $4.6 m_{l}(\mathrm{GS}), 4.2 M_{L}(\mathrm{PS}), 4.3 M_{L}(\mathrm{BK})$

Intensity IV: Arroyo Grande, Avila Beach, Grover City, Oceano, Paso Robles, Pismo Beach (a few items shaken off store shelves), San Luis Obispo (press report), Santa Margarita.

Intensity III: Cambria, Creston, Guadalupe, Harmony, Los Osos, Morro Bay, Nipomo, San Ardo, San Miguel, Shandon, Shell Beach, Vandenberg Air Force Base, Ventura.

Intensity II: Casmalia, Cayucos, Cholame.

Felt: Santa Maria (PS).

\section{June (PS) Southern California}

Origin time: 215729.9

Epicenter: $33.955 \mathrm{~N} ., 116.338 \mathrm{~W}$.

Depth: $6 \mathrm{~km}$

Magnitude: $3.5 M_{L}$ (PS)

Felt: Anza, Twentynine Palms, and Yucca Valley (PS).

25 June (BK) Central California

Origin time: 101324.1

Epicenter: $37.728 \mathrm{~N}$., $122.152 \mathrm{~W}$.

Depth: $7 \mathrm{~km}$

Magnitude: $3.1 M_{L}(\mathrm{BK}), M_{\iota}=1.7 \mathrm{E} 21(\mathrm{BK})$

Felt in parts of Alameda, Contra Costa, Marin, San Francisco, and San Mateo Counties (press report).

Intensity IV: Alameda, San Leandro.

Intensity III: Alameda Naval Air Station, Moraga.

Felt: Daly City, Hayward, Piedmont, and Richmond (press reports).

4 July (PS) Southern California

Origin time: 130050.3

Epicenter: $33.957 \mathrm{~N}$., $117.204 \mathrm{~W}$.

Depth: $13 \mathrm{~km}$

Magnitude: $3.2 M_{L}$ (PS)

Intensity IV: Perris, Sunnymead.

Felt: Riverside (PS).

6 July (PS) Central California

Origin time: 120012.7

Epicenter: $35.730 \mathrm{~N}$., $118.051 \mathrm{~W}$.

Depth: $8 \mathrm{~km}$

Magnitude: $3.9 M_{L}(\mathrm{PS}), 4.3 M_{L}(\mathrm{BK})$

Intensity III: Weldon.

Felt: Walker Pass area (PS).

6 July (PS) Central California

Origin time: 221414.0

Epicenter: 35.733N., 118.049W.

Depth: $7 \mathrm{~km}$ 
Magnitude: $3.6 M_{L}(\mathrm{PS}), 4.1 M_{L}(\mathrm{BK})$,

Felt: Onyx

$$
M_{1,}=1.7 \mathrm{E} 21(\mathrm{BK})
$$

\section{July (BK) Central California}

Origin time: 072505.2

Epicenter: $37.165 \mathrm{~N}$., $121.682 \mathrm{~W}$.

Depth: $6 \mathrm{~km}$

Magnitude: $3.1 M_{L}(\mathrm{BK}), M_{c}=1.3 \mathrm{E} 21(\mathrm{BK})$

Felt: Morgan Hill (BK).

\section{July (PS) Southern California}

Origin time: 114117.9

Epicenter: 33.704N., 118.060W.

Depth: $6 \mathrm{~km}$

Magnitude: $3.0 M_{L}(\mathrm{PS})$

Felt: Long Beach (PS).

\section{July (BK) Central California}

Origin time: 120818.5

Epicenter: $37.615 \mathrm{~N} ., 121.808 \mathrm{~W}$.

Depth: $4 \mathrm{~km}$

Magnitude: $3.3 M_{L}(\mathrm{BK}), 3.1 M_{D}(\mathrm{GM})$,

Intensity III: Agnew.

$$
M_{,}=1.4 \mathrm{E} 21(\mathrm{BK})
$$

Felt: Livermore and Pleasanton (BK).

16 July (BK) Mammoth Lakes area

Origin time: 101422.4

Epicenter: $37.625 \mathrm{~N} ., 118.960 \mathrm{~W}$.

Depth: $7 \mathrm{~km}$

Magnitude: $3.7 M_{L}(\mathrm{BK}), 4.2 M_{L}(\mathrm{PS})$

Intensity III: Mammoth Lakes.

18 July (BK) Northern California

Origin time: 194758.2

Epicenter: $41.373 \mathrm{~N}$., $123.483 \mathrm{~W}$.

Depth: $13 \mathrm{~km}$

Magnitude: $4.5 m_{l},(\mathrm{GS}), 3.6 M_{\hookrightarrow}(\mathrm{GS}), 4.0 M_{L}(\mathrm{BK})$, $4.0 M_{D}(\mathrm{GM}), M_{n}=3.1 \mathrm{E} 22(\mathrm{BK})$

Intensity III: Eureka, Forks of Salmon, Fort Jones, Gasquet, Hoopa, Orleans, Ti-Bar (30 miles south of Happy Camp).

Intensity II: Blue Lake, Callahan, Scott Bar, Yreka.

27 July (BK) Central California

Origin time: 172902.9

Epicenter: $38.775 \mathrm{~N}$., $122.770 \mathrm{~W}$.

Depth: $3 \mathrm{~km}$

Magnitude: $3.3 M_{L}(\mathrm{BK})$

Felt: Near Clear Lake (BK).

27 July (PS) Southern California

Origin time: 194207.8

Epicenter: $34.262 \mathrm{~N} ., 116.150 \mathrm{~W}$.
Depth: $6 \mathrm{~km}$

Magnitude: $3.5 M_{L}$ (PS)

Intensity III: Morongo Valley.

Felt: Twentynine Palms (PS).

29 July (BK) Central California

Origin time: 205902.9

Epicenter: $37.260 \mathrm{~N}$., $121.658 \mathrm{~W}$.

Depth: $6 \mathrm{~km}$

Magnitude: $3.2 M_{L}(\mathrm{BK}), M_{1}=3.1 \mathrm{E} 20(\mathrm{BK})$

Felt: Morgan Hill (press report).

29 July (BK) Central California

Origin time: 210518.2

Epicenter: $37.267 \mathrm{~N}$., $121.662 \mathrm{~W}$.

Depth: $7 \mathrm{~km}$

Magnitude: $3.5 M_{L}(\mathrm{BK}), M_{c}=8.7 \mathrm{E} 20(\mathrm{BK})$

Felt: Morgan Hill (press report).

31 July (BK) Central California

Origin time: 194347.9

Epicenter: $37.258 \mathrm{~N}$., $121.673 \mathrm{~W}$.

Depth: $5 \mathrm{~km}$

Magnitude: $3.2 M_{L}(\mathrm{BK}), 3.0 M_{D}(\mathrm{GM})$,

Felt: San Jose (BK).

$$
M_{c}=5.8 \mathrm{E} 20(\mathrm{BK})
$$

31 July (BK) Central California

Origin time: 205916.1

Epicenter: $37.377 \mathrm{~N} ., 121.765 \mathrm{~W}$.

Depth: $6 \mathrm{~km}$

Magnitude: $3.5 M_{L}(\mathrm{BK}), M_{,}=5.5 \mathrm{E} 20(\mathrm{BK})$

Intensity III: Fremont, San Jose, Sunol.

Felt: Foster City (BK), Morgan Hill (press report),

San Francisco (press report).

5 August (PS) Southern California

Origin time: 152855.0

Epicenter: $34.410 \mathrm{~N}$., $118.440 \mathrm{~W}$

Depth: $6 \mathrm{~km}$

Magnitude: $3.0 M_{L}$ (PS)

Felt: Newhall and Sylmar (PS).

5 August (PS) Southern California

Origin time: 153818.6

Epicenter: $34.405 \mathrm{~N}$., $118.437 \mathrm{~W}$.

Depth: $6 \mathrm{~km}$

Magnitude: $2.1 M_{L}$ (PS)

Felt: Newhall and Sylmar (PS).

6 August (BK) Central California

Origin time: 000639.8

Epicenter: 36.997 N., $121.713 \mathrm{~W}$.

Depth: 8 km 
Magnitude: $3.8 M_{L}(\mathrm{BK}), 3.2 M_{D}(\mathrm{GM})$, $M_{1}=4.0 \mathrm{E} 21(\mathrm{BK})$

Intensity IV: San Martin.

Intensity III: Mount Hermon, San Jose, Santa Cruz, Soquel, Watsonville.

Intensity II: Brookdale.

Felt: Gilroy (BK), Monterey (BK), Morgan Hill (press report), San Francisco (press report).

\section{August (PS) Southern California}

Origin time: 081436.6

Epicenter: 33.976 N., 116.713W.

Depth: $14 \mathrm{~km}$

Magnitude: $4.0 m_{l}$ (GS), $4.3 M_{L}(\mathrm{PS}), 4.5 M_{L}(\mathrm{BK})$

Intensity $\mathrm{V}$ :

Banning - A few small objects overturned and fell; a few items shook off of store shelves; standing vehicles rocked slightly; buildings shook strongly; vibration was described as strong; felt by many.

Beaumont - A few small objects overturned and fell; a few items shook off of store shelves; standing vehicles rocked slightly; buildings shook strongly; vibration was described as strong; felt by many.

Cabazon - The accelerograph at the post office was activated; felt by and awakened many.

Cathedral City - A few small objects overturned and fell; trees and bushes shook slightly; standing vehicles shook slightly; water splashed onto sides of swimming pools; hanging pictures swung.

Idyllwild - A few small objects overturned and fell; felt by many.

Morongo Valley - A few windows cracked; buildings shook strongly; awakened many.

Sun City- A few windows cracked; buildings shook strongly; vibration was described as strong.

Intensity IV: Angelus Oaks, Coachella, Colton, Hemet, Indio, Lakeview, LaVerne, Mountain Center, Palm Springs, Perris, Rancho Mirage, Riverside, San Dimas, San Jacinto, Silverado, Skyforest, Sugarloaf, Valle Vista.

Intensity III: Aguanga, Big Bear City, Calimesa, Canyon Lake, Fawnskin, Hesperia (press report), Homeland, Lemon Grove, Mecca, Murrieta, Palomar Mountain, Riverside (Arlington), Romoland, San Bernardino, Thousand Palms, West Covina.

Felt: Diamond Bar (PS), Lake Arrowhead (press report), Twin Peaks (press report).

\section{August (BK) Central California}

Origin time: 095136.1

Epicenter: 37.285 N., 121.673W.
Depth: $9 \mathrm{~km}$

Magnitude: $3.7 M_{L}(\mathrm{BK}), 3.1 M_{D}(\mathrm{GM})$, $M_{0}=6.0 \mathrm{E} 21(\mathrm{BK})$

Intensity IV: Felton (press report), Los Gatos, Morgan Hill (Jackson Oaks), Saratoga.

Intensity III: San Jose.

Intensity II: San Francisco (press report).

Felt: Morgan Hill (press report).

\section{August (BK) Central California}

Origin time: 002640.5

Epicenter: 37.333N., 121.692W.

Depth: $6 \mathrm{~km}$

Magnitude: $3.0 M_{L}(\mathrm{BK}), M_{f}=1.2 \mathrm{E} 20(\mathrm{BK})$

Felt: San Jose (BK).

16 August (BK) Central California

Origin time: 013054.6

Epicenter: $36.190 \mathrm{~N} ., 120.192 \mathrm{~W}$.

Depth: $7 \mathrm{~km}$

Magnitude: $3.2 M_{L}(\mathrm{BK}), 3.2 M_{D}(\mathrm{GM}), 3.7 M_{L}(\mathrm{PS})$

Intensity III: Avenal.

16 August (BK) Central California

Origin time: 204534.6

Epicenter: $37.263 \mathrm{~N} ., 121.663 \mathrm{~W}$.

Depth: $6 \mathrm{~km}$

Magnitude: $3.6 M_{L}(\mathrm{BK}), 3.1 M_{D}(\mathrm{GM})$,

$M_{1}=3.8 \mathrm{E} 21(\mathrm{BK})$

Intensity III: Morgan Hill (press report), New Almaden.

Felt: San Francisco, San Jose (BK), Coyote Lake area (BK).

\section{August (BK) Central California}

Origin time: 010554.3

Epicenter: $37.260 \mathrm{~N} ., 121.655 \mathrm{~W}$.

Depth: $6 \mathrm{~km}$

Magnitude: $3.6 M_{L}(\mathrm{BK}), 3.2 M_{D}(\mathrm{GM})$, $M_{1,}=3.4 \mathrm{E} 22(\mathrm{BK})$

Intensity III: Morgan Hill (press report).

7 September (BK) Central California

Origin time: 025219.5

Epicenter: 36.093N., 120.013W.

Depth: $5 \mathrm{~km}$

Magnitude: $3.5 M_{L}(\mathrm{BK}), 3.7 M_{L}(\mathrm{PS})$

Intensity IV: Avenal.

\section{September (PS) Southern California}

Origin time: 175730.3

Epicenter: $33.460 \mathrm{~N} ., 116.370 \mathrm{~W}$.

Depth: $15 \mathrm{~km}$

Magnitude: $4.1 M_{L}(\mathrm{PS}), 4.0 M_{L}(\mathrm{BK})$ 
Intensity IV: Indio, Mecca.

Intensity III: Anza, Rancho Mirage, Thousand Palms. Intensity II: Palm Springs, Thermal.

19 September (PS) Southern California

Origin time: 211118.1

Epicenter: $34.661 \mathrm{~N}$., $119.968 \mathrm{~W}$.

Depth: $1 \mathrm{~km}$

Magnitude: $3.4 M_{L}(\mathrm{PS}), 3.2 M_{D}(\mathrm{GM})$

Intensity II: Santa Barbara, Ventura.

21 September (BK) Northern California

Origin time: 030721.1

Epicenter: $40.737 \mathrm{~N} ., 124.207 \mathrm{~W}$.

Depth: $11 \mathrm{~km}$

Magnitude: $3.2 M_{L}(\mathrm{BK}), 3.3 M_{D}(\mathrm{GM})$, $M_{\text {" }}=2.1 \mathrm{E} 21(\mathrm{BK})$

Intensity $\mathrm{V}$ :

Arcata- A few items shook off of store shelves; a few small objects overturned and fell; trees and bushes shook slightly; buildings shook slightly; walls creaked; windows rattled; felt by several.

Eureka-- A few items shook off of store shelves; buildings shook strongly; trees and bushes shook slightly; hanging pictures were out of place; a wood pile collapsed; felt by many.

Intensity IV: Loleta, Samoa (a few items shaken off store shelves).

Intensity III: Bayside, Cutten, Rio Dell, Scotia, Weott.

Felt: McKinleyville (BK).

22 September (BK) Northern California

Origin time: 185203.0

Epicenter: $38.802 \mathrm{~N} ., 122.807 \mathrm{~W}$.

Depth: $1 \mathrm{~km}$

Magnitude: $4.2 m_{l},(\mathrm{GS}), 4.0 M_{L}(\mathrm{BK}), 3.3 M_{D}(\mathrm{GM})$

Intensity V:

Cobb- A few small objects overturned and fell; trees and bushes shook strongly; hanging objects swung violently in the east-west direction; hanging pictures swung; buildings shook strongly; windows rattled; felt by many.

Loch Lomond - Buildings shook strongly; a mobile home was unleveled; small landslides occurred; trees and bushes shook slightly; felt by many.

Intensity IV: Geyserville.

Intensity III: Cloverdale, Navarro.

25 September (BK) Central California

Origin time: 022957.4

Epicenter: $36.602 \mathrm{~N} ., 121.885 \mathrm{~W}$.

Depth: $5 \mathrm{~km}$
Magnitude: $2.5 M_{L}$ (BK)

Felt: Monterey Peninsula (BK).

26 September (BK) Central California

Origin time: 204606.3

Epicenter: 37.340 N., 121.733W.

Depth: $6 \mathrm{~km}$

Magnitude: $4.3 m_{l},(\mathrm{GS}), 4.4 M_{L}(\mathrm{BK}), 3.5 M_{D}(\mathrm{GM})$, $M_{.}=1.1 \mathrm{E} 23(\mathrm{BK})$

Felt throughout the San Francisco Bay area (press report).

Intensity V:

Mount Hamilton- A few items shook off of store shelves; small landslides occurred; trees and bushes shook slightly; buildings shook slightly; walls creaked; windows rattled; vibration was described as strong; vehicles rocked slightly; felt by many.

Santa Cruz- A few items shook off of store shelves; a few glassware items broke; a few small objects overturned and fell; trees and bushes shook slightly; windows rattled.

Intensity IV: Agnew, Boulder Creek, Campbell, Canyon, Coyote, Evergreen Valley College, Fremont (Irvington), Lafayette, Milpitas, Mountain View, New Almaden, Redwood Estates, San Carlos, San Jose (Westgate).

Intensity III: Alameda, Alviso, Atherton, Berkeley, Burlingame, Davenport, Fremont (Mission San Jose), La Honda, Loma Mar, Menlo Park, Morgan Hill, Mount Hermon, Oakland, Pescadero, Port Costa, Redwood City, Ross, Salinas, San Francisco, San Jose (Blossom Hill), Aptos, Stinson Beach, Woodacre.

Intensity II: Keyes, San Juan Bautista, Sunol.

Felt: Daly City (press report), Holt, Merced (BK), Modesto (press report), Monterey (press report), Newark (press report), San Anselmo, San Lorenzo, San Martin, (press report), Stockton (press report).

30 September (PS) Baja California, Mexico

Origin time: 211614.1

Epicenter: $32.499 \mathrm{~N}$., $115.267 \mathrm{~W}$.

Depth: $6 \mathrm{~km}$

Magnitude: $3.6 M_{L}$ (PS)

Felt: El Centro.

2 October (PS) Southern California

Origin time: 025916.7

Epicenter: $34.309 \mathrm{~N} ., 119.250 \mathrm{~W}$.

Depth: $2 \mathrm{~km}$

Magnitude: $3.2 M_{L}$ (PS), $3.2 M_{D}(\mathrm{GM})$

Intensity IV: Ventura. 
2 October (PS) Southern California

Origin time: 093814.5

Epicenter: $34.310 \mathrm{~N} ., 119.241 \mathrm{~W}$.

Depth: $1 \mathrm{~km}$

Magnitude: $3.3 M_{L}$ (PS), $3.3 M_{D}(\mathrm{GM})$

Intensity IV: Ventura.

3 October (PS) Southern California

Origin time: 124949.5

Epicenter: $33.984 \mathrm{~N} ., 118.665 \mathrm{~W}$.

Depth: $13 \mathrm{~km}$

Magnitude: $3.3 M_{L}$ (PS)

Felt: Santa Monica (PS).

4 October (BK) Central California

Origin time: 075312.5

Epicenter: $38.835 \mathrm{~N}$., $122.777 \mathrm{~W}$.

Depth: $5 \mathrm{~km}$

Magnitude: $2.8 M_{L}(\mathrm{BK})$

Felt: Cobb (BK).

5 October (PS) Southern California

Origin time: 073714.1

Epicenter: $33.666 \mathrm{~N}$., $116.703 \mathrm{~W}$.

Depth: $19 \mathrm{~km}$

Magnitude: $3.9 M_{L}$ (PS)

Intensity V: Hemet - A few windows cracked; a few small objects overturned or fell; buildings shook slightly; walls creaked; windows rattled; felt by many.

Intensity IV: Aguanga, Cabazon, Cathedral City, Pauma Valley, Palm Springs, Rancho Mirage.

Intensity III: Beaumont, Warner Springs.

\section{October (BK) Central California}

Origin time: 161630.0

Epicenter: $36.573 \mathrm{~N} ., 121.203 \mathrm{~W}$.

Depth: $9 \mathrm{~km}$

Magnitude: $4.0 M_{L}(\mathrm{BK}), 3.5 M_{D}(\mathrm{GM})$, $M_{1}=1.8 \mathrm{E} 22(\mathrm{BK})$

Intensity III: Hollister (press report).

10 October (PS) Southern California

Origin time: 212258.9

Epicenter: $33.138 \mathrm{~N} ., 116.501 \mathrm{~W}$.

Depth: $12 \mathrm{~km}$

Magnitude: $4.3 m_{l},(\mathrm{GS}), 4.5 M_{L}(\mathrm{PS}), 4.6 M_{L}(\mathrm{BK})$

Felt throughout the San Diego metropolitan area (press report).

Intensity IV: Borrego Springs, Boulevard, Calexico, Cathedral City, Descanso, Jacumba, Julian, Mecca, Mount Laguna, Palomar Mountain, Pine Valley, Ramona, Ranchita, Santa Ysabel, Warner Springs.
Intensity III: Aguanga, Campo, El C'ajon, El C'ajon (Bostonia), El Centro Naval Air Facility, Hemet (press report), Jamul, Lakeside, Lake Cuyamaca, Lakeview, La Mesa, Mission Viejo, North Palm Springs, Ocotillo, Pala, Potrero, Rancho Santa Fe, San Marcos, Seeley, Spring Valley.

Intensity II: Heber, 5 miles from Plaster City.

15 October (PS) Southern California

Origin time: 174404.4

Epicenter: $33.698 \mathrm{~N} ., 118.160 \mathrm{~W}$.

Depth: $2 \mathrm{~km}$

Magnitude: $3.7 M_{L}$ (PS)

Intensity IV: East Long Beach (one report of dishes falling from kitchen cabinets), Garden Grove, Laguna Niguel, Long Beach, Surfside.

Intensity III: Artesia, Norwalk.

Intensity II: Pico Rivera.

Felt: Bellflower, Cerritos, Downey, Lakewood, Lynwood, and Paramount (press reports), Torrance.

16 October (PS) Southern California

Origin time: 221850.0

Epicenter: $33.815 \mathrm{~N} ., 117.031 \mathrm{~W}$.

Depth: $14 \mathrm{~km}$

Magnitude: $3.2 M_{L}$ (PS)

Intensity III: Hemet (press report).

22 October (BK) Central California

Origin time: 040631.4

Epicenter: $36.582 \mathrm{~N} ., 121.235 \mathrm{~W}$.

Depth: $10 \mathrm{~km}$

Magnitude: $3.6 M_{L}(\mathrm{BK}), 3.3 M_{D}(\mathrm{GM}), 4.0 M_{L}(\mathrm{PS})$, $M_{,}=2.6 \mathrm{E} 21(\mathrm{BK})$

Intensity IV: Greenfield (press report), Soledad, Chualar.

Intensity III: Gonzales.

23 October (BK) Central California

Origin time: 100834.7

Epicenter: 38.705 N., $123.500 \mathrm{~W}$.

Depth: $5 \mathrm{~km}$

Magnitude: $3.1 M_{L}(\mathrm{BK}), 3.2 M_{D}(\mathrm{GM})$

Felt: Sea Ranch (BK).

\section{October (PS) Southern California}

Origin time: 103602.4

Epicenter: $34.737 \mathrm{~N}$, $120.148 \mathrm{~W}$.

Depth: $6 \mathrm{~km}$

Magnitude: $4.5 m_{l}$ (GS), $4.5 M_{L}$ (PS), $4.4 M_{L}(\mathrm{BK})$

Intensity VI:

Los Olivos - Most of the damage occurred at the Fire- 
stone Winery north of town. Several steel storage tanks were damaged slightly; one was cracked and lost 400 gallons of wine; the foundations of several large oak vats in the winery warehouse were damaged; more than 30 cases of vintage wine were destroyed; the warehouse sustained one stress crack, and the water line to the sprinkler system ruptured (from press reports). Other effects in the city: plaster and sheetrock walls sustained hairline cracks; a few windows cracked; a few dishes or glassware items broke; many small objects overturned and fell; furniture was displaced; buildings shook strongly; felt by and awakened many.

Santa Ynez- Brick fences cracked; joints between a cinderblock wall and a wood frame wall sustained large cracks; interior plaster walls sustained cracks; a few items shook off of store shelves; a few small objects overturned and fell; buildings shook strongly; walls creaked; windows rattled; felt by all and awakened many.

Intensity V:

Los Alamos- Plaster walls sustained hairline cracks; a few items shook off of store shelves; a few dishes or glassware items broke; hanging pictures fell; buildings shook strongly; walls creaked; windows rattled; felt by and awakened many.

Santa Barbara - A few windows cracked; a few light furniture pieces overturned; a few dishes or glassware items broke; a few small objects overturned and fell; buildings shook slightly; felt by and awakened many.

Solvang- A few dishes or glassware items broke; a few small objects overturned and fell; buildings shook slightly; walls creaked; windows rattled; felt by and awakened many.

Intensity IV: Buellton, Casmalia, Goleta, Lompoc, Orcutt, Summerland, Tupman, Ventura.

Intensity III: Arroyo Grande, Camarillo, Santa Maria, Vandenberg Air Force Base.

25 October (PS) Southern California

Origin time: 202933.5

Epicenter: 34.729 N., $120.158 \mathrm{~W}$.

Depth: $10 \mathrm{~km}$

Magnitude: $3.1 M_{L}(\mathrm{PS}), 3.2 M_{D}(\mathrm{GM})$

Felt: Los Olivos area (PS).

\section{October (PS) Southern California}

Origin time: 172043.5

Epicenter: $34.016 \mathrm{~N} ., 118.988 \mathrm{~W}$.

Depth: $13 \mathrm{~km}$

Magnitude: $4.3 m_{l}(\mathrm{GS}), 4.6 M_{L}(\mathrm{PS}), 4.0 M_{L}(\mathrm{BK})$
Felt in Los Angeles, Orange, Riverside, and Orange Counties.

Intensity V:

Bell-- Plaster walls sustained hairline cracks; trees and bushes shook slightly; standing vehicles rocked shightly; hanging pictures were out of place; buildings shook slightly; walls creaked; windows rattled; felt by many.

El Segundo- A few items shook off of store shelves; a few dishes or glassware items broke; a few small objects overturned and fell; buildings shook slightly; walls creaked; windows rattled; felt by many; vibration was described as strong.

Lawndale- A few small objects overturned and fell; hanging pictures were out of place; buildings shook moderately; windows rattled; vibration was described as strong; felt by and frightened many.

Long Beach - One report of a television set falling off a stand; pictures fell off a wall, and people ran out of houses (press report). Sheetrock walls sustained hairline cracks; buildings shook slightly; walls creaked; windows rattled; felt by many.

Malibu - Buildings shook strongly; hanging pictures swung; walls creaked; windows rattled; vibration described as strong; felt by many. A minor rockslide occurred on the Kanan-Dune Road about 2 miles north of the Pacific Coast Highway (press report).

Pointe Dume- About 200 cans and jars were shaken off shelves at Trancas Market; a few jars broke. A small earthslide blocked part of a local street; exterior walls cracked; and pictures were out of place (press reports).

Intensity IV: Agoura, Bakersfield, Camarillo, Canoga Park, Culver City, Glendale, Harbor City, Hawthorne, Laguna Niguel, La Habra, Los Alamitos, Los Angeles, Manhattan Beach, Marina del Rey, Oxnard, Pacific Palisades, Palos Verdes Peninsula, Paramount, Pasadena, Point Mugu Naval Air Station, Port Hueneme, Santa Paula, Sepulveda, Sierra Madre, South Gate, South Pasadena, Sunset Beach, Surfside, Thousand Oaks, Torrance, Van Nuys, Westminster, Whittier.

Intensity III: Anaheim, Atwood, Beverly Hills, Buena Park, Claremont, Culver City, Downey, Fullertin (press report), Huntington Beach, Huntington Park, Los Alamos, Los Angeles International Airport, Mission Viejo, Monterey Park, Northridge, Norwalk, Reseda, San Dimas, San Gabriel, San Pedro, Santa Monica (press report), Saugus, Seal Beach, Tarzana, Topanga, Venice, Westchester.

Intensity II: City of Industry, Compton, La Mirada, La Verne, Somis, Stanton. 
Felt: Arcadia, Costa Mesa, El Segundo, Pomona, Ventura, and West Covina (press reports).

30 October (BK) Central California

Origin time: 205006.6

Epicenter: $38.495 \mathrm{~N} ., 122.710 \mathrm{~W}$.

Depth: $4 \mathrm{~km}$

Magnitude: $2.7 M_{L}(\mathrm{BK})$

Felt: Santa Rosa (press report).

30 October (BK) Central California

Origin time: 234400.7

Epicenter: $38.500 \mathrm{~N}$., $122.705 \mathrm{~W}$.

Depth: $5 \mathrm{~km}$

Magnitude: $2.9 M_{L}(\mathrm{BK})$

Felt: Santa Rosa (press report).

31 October (BK) Central California

Origin time: 030512.0

Epicenter: $37.293 \mathrm{~N} ., 121.685 \mathrm{~W}$.

Depth: $5 \mathrm{~km}$

Magnitude: $3.2 M_{L}(\mathrm{BK}), M_{c}=1.9 \mathrm{E} 20(\mathrm{BK})$

Felt: San Jose (BK).

\section{October (BK) Central California}

Origin time: 141227.1

Epicenter: $37.305 N$ N., $121.648 \mathrm{~W}$.

Depth: $8 \mathrm{~km}$

Magnitude: $3.6 M_{L}(\mathrm{BK}), 3.0 M_{D}(\mathrm{GM})$,

$$
M_{c}=3.1 \mathrm{E} 20(\mathrm{BK})
$$

Intensity II: Santa Clara.

Felt: San Jose (BK).

1 November (BK) Central California

Origin time: 101438.1

Epicenter: 37.507 N., $122.190 \mathrm{~W}$.

Depth: $10 \mathrm{~km}$

Magnitude: $2.4 M_{L}(\mathrm{BK})$

Felt: Menlo Park (BK).

5 November (PS) Southern California

Origin time: 025927.5

Epicenter: $34.140 \mathrm{~N}$., $117.433 \mathrm{~W}$.

Depth: $4 \mathrm{~km}$

Magnitude: $3.4 M_{L}$ (PS)

Intensity IV: Colton.

Intensity III: Redlands.

Felt: Riverside (PS).

\section{November (PS) Southern California}

Origin time: 094332.6

Epicenter: $33.901 \mathrm{~N}$., $118.263 \mathrm{~W}$.

Depth: $3 \mathrm{~km}$

Magnitude: $3.2 M_{L}$ (PS)

Felt: Long Beach (PS), Redondo Beach (press report).
10 November (BK) Centra] California

Origin time: 095409.7

Epicenter: $37.235 \mathrm{~N}$., $121.647 \mathrm{~W}$.

Depth: $5 \mathrm{~km}$

Magnitude: $3.0 M_{L}(\mathrm{BK}), M_{,}=2.7 \mathrm{E} 20(\mathrm{BK})$

Felt: San Jose (BK).

19 November (PS) Central California

Origin time: 050253.3

Epicenter: $35.858 \mathrm{~N} ., 117.724 \mathrm{~W}$.

Depth: $5 \mathrm{~km}$

Magnitude: $3.1 M_{L}$ (PS)

Felt: Coso Junction area (PS).

23 November (BK) Owens Valley area

Origin time: 180825.5

Epicenter: $37.458 \mathrm{~N}$., $118.605 \mathrm{~W}$.

Depth: $12 \mathrm{~km}$

Magnitude: $5.6 m_{l}(\mathrm{GS}), 5.7 M_{s}(\mathrm{GS}), 6.1 M_{L}(\mathrm{BK})$, $6.2 M_{L}(\mathrm{PS}), M_{n}=2.6 \mathrm{E} 25(\mathrm{BK})$

This earthquake was felt over an area of approximately 114,000 kilometers $^{2}$ of California and Nevada (fig. 17).

Intensity V: The most common effects at the places listed below: windows rattled; vibration was described as moderate to strong; walls creaked; buildings shook; small objects overturned and fell; trees and bushes shook slightly to moderately; hanging pictures swung out of place; felt by most. Additional effects at specific sites are listed below:

\section{California-}

Alpaugh - Plaster walls sustained hairline cracks.

Auberry - Slumping occurred on a river bank; water splashed onto sides of swimming pools.

Bakersfield- A few windows cracked; a few dishes or glassware items broke; a few light furniture items overturned; a few items shook off of store shelves.

Benton-Shaking was strong; a parked camper bounced around (press report).

Big Creek.

Bishop- A Safeway store closed 2 hours to clean up broken bottles and spilled food items (press report); plaster and sheetrock walls sustained hairline cracks; a few dishes or glassware items broke; hanging pictures fell; standing and moving vehicles rocked moderately; a foundation cracked; a minor rockslide occurred on U.S. Highway 395 about 12 miles north of 


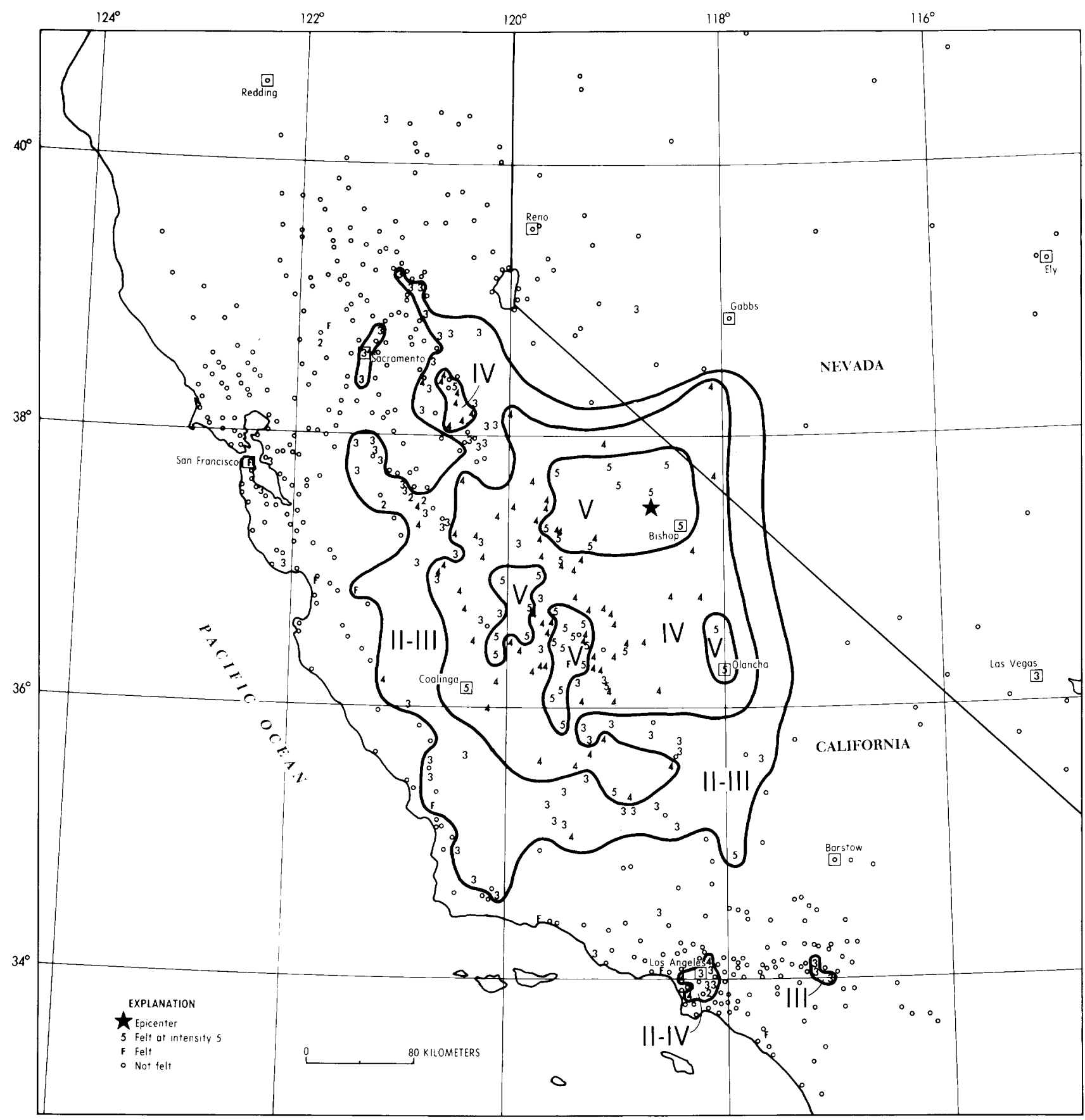

Figure 17. Isoseismal map for the Owens Valley, California, earthquake of 23 November 1984, 18 08 25.5 UTC. Roman numerals represent Modified Mercalli intensities between isoseismals; Arabic numerals represent intensities at specific sites. 
Bishop and one on U.S. Highway 6 near the InyoMono County line (press report).

Caruthers- Standing vehicles rocked slightly; water splashed onto sides of swimming pools.

Coalinga-A few items shook off of store shelves; plaster walls sustained hairline cracks; water splashed onto sides of swimming pools.

Corcoran.

Crowley Lake - A few items shook off of store shelves; standing and moving vehicles rocked moderately.

Dinuba- A few items shook off of store shelves (press report); standing and moving vehicles rocked slightly; water splashed onto sides of swimming pools.

Edwards- A few windows cracked; a few dishes or glassware items broke; a few items shook off store shelves; water splashed onto sides of swimming pools.

Five Points-A few windows cracked; standing vehicles rocked slightly.

Fresno- People had difficulty standing; plaster walls sustained hairline cracks; standing vehicles rocked moderately; moving vehicles rocked slightly; and there was a report of a cracked brick fence.

Fresno (Barton)-- Sheetrock walls sustained hairline cracks; people had difficulty standing.

Fresno (Clinter) - Standing vehicles rocked slightly.

Friant.

Helm - A few items shook off of store shelves.

June Lake- A few items shook off store shelves; standing vehicles rocked slightly.

Kingsburg.

Lindsay - A few windows cracked; a few items shook off of store shelves; standing vehicles rocked slightly.

Lone Pine- A few items shook off of store shelves; plaster and sheetrock walls sustained hairline cracks; standing vehicles rocked moderately; moving vehicles rocked slightly; water splashed onto sides of swimming pools.

Madera- People had difficulty standing; a few items shook off of store shelves; standing vehicles rocked slightly; water splashed onto sides of swimming pools.

Mammoth Lakes - A few cans and jars were shaken off store shelves; ski lifts swayed causing them to be temporarily shut down (press report).

North Fork- A few items shook off of store shelves; plaster and sheetrock walls sustained hairline cracks.

Oakhurst- A few dishes or glassware items broke.

Olancha- Plaster walls sustained hairline cracks; people had difficulty standing.
Orange Cove - A chimney cracked; water splashed onto sides of swimming pools.

Reedley-A few windows cracked.

Sanger-Standing vehicles rocked slightly.

Three Rivers.

Toms Place- People had difficulty standing; a few items shook off of store shelves; standing vehicles rocked moderately; moving vehicles rocked slightly.

Traver- A few windows cracked; a few dishes or glassware items broke; a few items shook off of store shelves; plaster and sheetrock walls sustained hairline cracks; standing and moving vehicles rocked slightly.

Visalia- A few windows cracked; a few dishes or glassware items broke; standing and moving vehicles rocked slightly; water splashed onto sides of swimming pools.

Waukena-A few items shook off of store shelves; people had difficulty standing; standing and moving vehicles rocked slightly; water splashed onto sides of swimming pools.

Wilseyville- A few items shook off of store shelves; standing vehicles rocked slightly.

Yettem-Standing vehicles rocked slightly; an interior wall separated from the ceiling.

Yosemite National Park - A few windows cracked; a few dishes or glassware items broke; a few items shook off of store shelves; plaster walls sustained hairline cracks; standing and moving vehicles rocked slightly; water splashed onto sides of lakes.

\section{Intensity IV:}

California - Ahwahnee, Angels Camp, Armona, Avenal, Avery, Badger, Bass Lake, Big Pine, Bodfish, Burrel, Calwa, Camp Nelson, Cantua Creek, Carmichael, Catheys Valley, Chowchilla, Coarsegold, Culver City, Cutler, Del Rey, Dos Palos, Dunlap, Edison, El Portal, Exeter, Farmersville, Firebaugh, Fish Camp, Fowler, Grants Grove, Hanford, Huron, Independence, Ivanhoe, Kaweah, King City, La Grange, Lakeshore, Lawndale, Lee Vining, Lemoncove, Lemoore, Lemoore Naval Air Station, Lost Hills, Maricopa, Mariposa, McFarland, Mendota, Merced, Miramonte, Mountain Ranch, O'Neals, Orosi, Pasadena (press report), Piedra, Pinecrest, Pine Grove, Planada, Porterville, Prather, Rail Road Flat, Richgrove, Riverdale, Santa Rita Park, Selma, Sequoia National Park, Shaver Lake, Squaw Valley, Stevinson, Strathmore, Sutter Creek, Tipton, Tollhouse, Turlock, Vallecito, Volcano, Wasco, Wawona, Wishon.

Nevada-Dyer, Mina. 


\section{Intensity III:}

California- Alameda, Altaville, Arnold, Atascadero, Atwater, Bell, Bryn Mawr, Buttonwillow, Caliente, Calimesa, Camino, Castaic, Castle Air Force Base, Ceres, Chester, City Of Industry, Clovis, Colfax, Delano, Delhi, Di Giorgio, Ducor, Earlimart, Elk Grove, El Nido, Fellows, French Camp, Georgetown, Grass Valley, Hathaway Pines, Highland, Holt, lowa Hill, Jackson, Kerman, Kernville, Kyburz, Lamont, Laton, Le Grand, Long Barn, Los Alamos, Los Angeles, Los Banos, Manteca (press report), McKittrick, Mi-Wuk Village, Modesto (press report), Mount Aukum, Mount Hermon, Oxnard, Paso Robles, Pico Rivera, Pollock Pines, Posey, Raymond, Ridgecrest, Rocklin, Sacramento, San Ardo, San Mateo, Santa Maria, Santa Ynez, Shafter, Shandon, Sonora, Soulsbyville, South Dos Palos, Stockton (press report), Taft, Tehachapi, Tracy, Tranquillity, Tulare, Tuolumne, Valley Springs, Wofford Heights.

Nevada - Las Vegas (press report), Schurz.

Intensity II:

California - Denair, Keyes, Paramount, Patterson, Woodland.

Felt:

California-Goshen, Hollister (press report), Knights Landing, Mission Viejo (press report), San Francisco (press report), San Luis Obispo (press report), Santa Barbara (press report), Topanga, Ventura (press report), Watsonville (press report).

\section{November (GM) Owens Valley area}

Origin time: 191234.6

Epicenter: 37.433N., 118.612W.

Depth: $14 \mathrm{~km}$

Magnitude: $4.8 m_{1}(\mathrm{GS}), 4.7 M_{S}(\mathrm{GS}), 5.5 M_{L}(\mathrm{BK})$, $5.4 M_{L}(\mathrm{PS}), M_{.,}=5.1 \mathrm{E} 23(\mathrm{BK})$

Intensity IV: Douglas Flat, Stockton (press report), Strathmore.

Intensity III: Iowa Hill.

Felt: Bishop (BK), Fresno, Mammoth Lakes (PS).

24 November (GM) Owens Valley area

Origin time: 092117.1

Epicenter: $37.473 \mathrm{~N} ., 118.577 \mathrm{~W}$.

Depth: $12 \mathrm{~km}$

Magnitude: $4.2 M_{L}(\mathrm{BK}), 4.1 M_{L}(\mathrm{PS})$, $M_{,}=6.3 \mathrm{E} 21(\mathrm{BK})$

Felt: Bishop-Mammoth Lakes area (BK).

24 November (GM) Owens Valley area

Origin time: 202532.9

Epicenter: $37.438 \mathrm{~N}$., $118.648 \mathrm{~W}$.
Depth: 9 km

Magnitude: $4.0 m_{1},(\mathrm{GS}), 4.0 M_{L}(\mathrm{BK}), 4.1 M_{L}(\mathrm{PS})$, $M_{1,}=1.1 \mathrm{E} 22(\mathrm{BK})$.

Felt: Bishop-Mammoth Lakes area (BK).

25 November (PS) Central California

Origin time: 231009.6

Epicenter: $37.451 \mathrm{~N}$., $118.620 \mathrm{~W}$.

Depth: 6 km

Magnitude: $4.3 m_{1}(\mathrm{GS}), 3.1 M_{S}(\mathrm{GS}), 4.7 M_{L}(\mathrm{PS})$,

Felt: Bishop (BK). $4.8 M_{L}(\mathrm{BK}), M_{c}=3.6 \mathrm{E} 22(\mathrm{BK})$

\section{November (BK) Owens Valley area}

Origin time: 162147.2

Epicenter: $37.448 \mathrm{~N} ., 118.653 \mathrm{~W}$.

Depth: $9 \mathrm{~km}$

Magnitude: $5.1 m_{l}(\mathrm{GS}), 4.7 M_{s}(\mathrm{GS}), 5.6 M_{L}(\mathrm{BK})$, $5.5 M_{L}(\mathrm{PS}), M_{1,}=2.4 \mathrm{E} 23(\mathrm{BK})$

One man was injured by a rock and snow slide at the mouth of the Union Carbide tungsten mine in Pine Creek Canyon about 12 miles northwest of Bishop.

\section{Intensity V:}

California-

Bishop- A few small objects fell; pictures swung; buildings shook strongly; walls creaked; windows rattled; felt by many.

\section{Intensity IV:}

California- Ahwahnee, Alpaugh, Badger, Bass Lake, Big Creek, Cantua Creek, Del Rey, Douglas Flat, Dunlap, Friant, Grant Grove, Hathaway Pines, Hornitos, Jamestown, June Lake, Kaweah, La Grange, Lone Pine, Midpines, Miramonte, Mountain Ranch, Orange Cove, Poplar, Railroad Flat, San Joaquin, Sequoia National Park, Strawberry, Visalia, Volcano, Wisseyville, Woodville.

\section{Intensity III:}

California - Avery, Big Oak Flat, Chowchilla, Cutler, Dinuba, Ducor, East Fresno, El Portal, Fish Camp, Fresno, Huron, Independence, Lemoncove, Mariposa, Piedra, Pine Grove, Raymond, Santa Rita Park, Sheep Ranch, Stevinson, Tollhouse, Vallecito, Wofford Heights.

Nevada - Schurz.

Intensity II:

California- Caruthers, North Fork.

Felt:

California - Firebaugh, Fresno (Barton), Pinecrest, Shaver Lake. 
27 November (PS) Southern California

Origin time: 223746.3

Epicenter: $34.023 \mathrm{~N} ., 117.544 \mathrm{~W}$.

Depth: $4 \mathrm{~km}$

Magnitude: $2.5 M_{L}$ (PS)

Felt: Ontario area (PS).

28 November (BK) Central California

Origin time: 115438.1

Epicenter: $37.293 \mathrm{~N} ., 121.682 \mathrm{~W}$.

Depth: $6 \mathrm{~km}$

Magnitude: $3.2 M_{L}(\mathrm{BK}), M_{n}=1.9 \mathrm{E} 20(\mathrm{BK})$

Felt: San Jose (BK).

28 November (GM) Owens Valley area

Origin time: 162326.1

Epicenter: $37.425 \mathrm{~N} ., 118.630 \mathrm{~W}$.

Depth: $8 \mathrm{~km}$

Magnitude: $4.3 m_{l}(\mathrm{GS}), 4.7 M_{L}(\mathrm{BK}), 4.7 M_{L}(\mathrm{PS})$,

Felt: Bishop.

$$
M_{o}=4.4 \mathrm{E} 22(\mathrm{BK}) \text {. }
$$

5 December (BK) Central California

Origin time: 103009.9

Epicenter: $37.728 \mathrm{~N} ., 122.128 \mathrm{~W}$.

Depth: $5 \mathrm{~km}$

Magnitude: $2.5 M_{L}(\mathrm{BK})$

Felt: Castro Valley and San Leandro (BK).

10 December (PS) Southern California

Origin time: 154448.7

Epicenter: $34.082 \mathrm{~N}$., $118.105 \mathrm{~W}$.

Depth: $5 \mathrm{~km}$

Magnitude: $2.5 M_{L}$ (PS)

Felt: El Serreno (PS).

\section{December (PS) Southern California}

Origin time: 212804.8

Epicenter: $33.890 \mathrm{~N} ., 117.821 \mathrm{~W}$.

Depth: $3 \mathrm{~km}$

Magnitude: $3.1 M_{L}$ (PS)

Felt: Santa Ana (PS).

21 December (BK) Central California

Origin time: 123904.8

Epicenter: $36.520 \mathrm{~N} ., 121.123 \mathrm{~W}$.

Depth: $5 \mathrm{~km}$

Magnitude: $3.0 M_{L}(\mathrm{BK}), 3.0 M_{D}(\mathrm{GM})$

Intensity IV: Pinnacles National Monument (press report).

24 December (GM) Central California

Origin time: 192229.4
Epicenter: $37.622 \mathrm{~N} ., 118.863 \mathrm{~W}$.

Depth: $10 \mathrm{~km}$

Magnitude: $3.8 M_{L}(\mathrm{BK}), 3.4 M_{D}(\mathrm{GM}), 4.3 M_{L}(\mathrm{PS})$

Intensity III: Mammoth Lakes.

28 December (BK) Central California

Origin time: 071942.4

Epicenter: $37.327 \mathrm{~N}$., $121.718 \mathrm{~W}$.

Depth: $6 \mathrm{~km}$

Magnitude: $3.8 M_{L}(\mathrm{BK}), 3.6 M_{D}(\mathrm{GM})$, $M_{c}=1.6 \mathrm{E} 22(\mathrm{BK})$

Intensity III: San Jose (press report).

\section{CALIFORNIA-OFF THE COAST}

27 February (PS) Southern California

Origin time: 101815.0

Epicenter: $33.471 \mathrm{~N} ., 118.061 \mathrm{~W}$.

Depth: $6 \mathrm{~km}$

Magnitude: $4.0 M_{L}(\mathrm{PS})$

Intensity V:

Huntington Beach - A few dishes or glass items broke; a few small objects overturned and fell; a few items shook off of store shelves; motor vehicles rocked slightly; windows rattled; awakened many.

Santa Ana- A few windows cracked; a few items shook off of store shelves; a few dishes or glass items broke; a few small objects overturned and fell; hanging pictures swung out of place.

Intensity IV: Garden Grove, Surfside, Westminster.

Intensity III: Avalon, Buena Park, Cypress, Midway City, Mission Viejo.

Felt: Long Beach (PS).

28 February (BK) Northern California

Origin time: 151606.7

Epicenter: 40.357N., $125.897 \mathrm{~W}$.

Depth: $5 \mathrm{~km}$

Magnitude: $4.9 m_{l}(\mathrm{GS}), 4.4 M_{s}(\mathrm{GS}), 5.2 M_{L}(\mathrm{BK})$, $4.6 M_{D}(\mathrm{GM}), M_{1}=1.5 \mathrm{E} 23(\mathrm{BK})$

Intensity IV: Honeydew, Petrolia.

Intensity III: Loleta, Piercy, Rio Dell, Whitethorn.

\section{August (BK) Northern California}

Origin time: 214553.2

Epicenter: $40.255 \mathrm{~N} ., 124.578 \mathrm{~W}$.

Depth: $5 \mathrm{~km}$

Magnitude: $4.7 m_{l},(\mathrm{GS}), 4.6 M_{S}(\mathrm{GS}), 4.7 M_{L}(\mathrm{BK})$, $M_{1,}=2.0 \mathrm{E} 23(\mathrm{BK})$

Intensity V:

Alderpoint- A few small objects overturned and fell; a few items shook off of store shelves; windows rattled. 


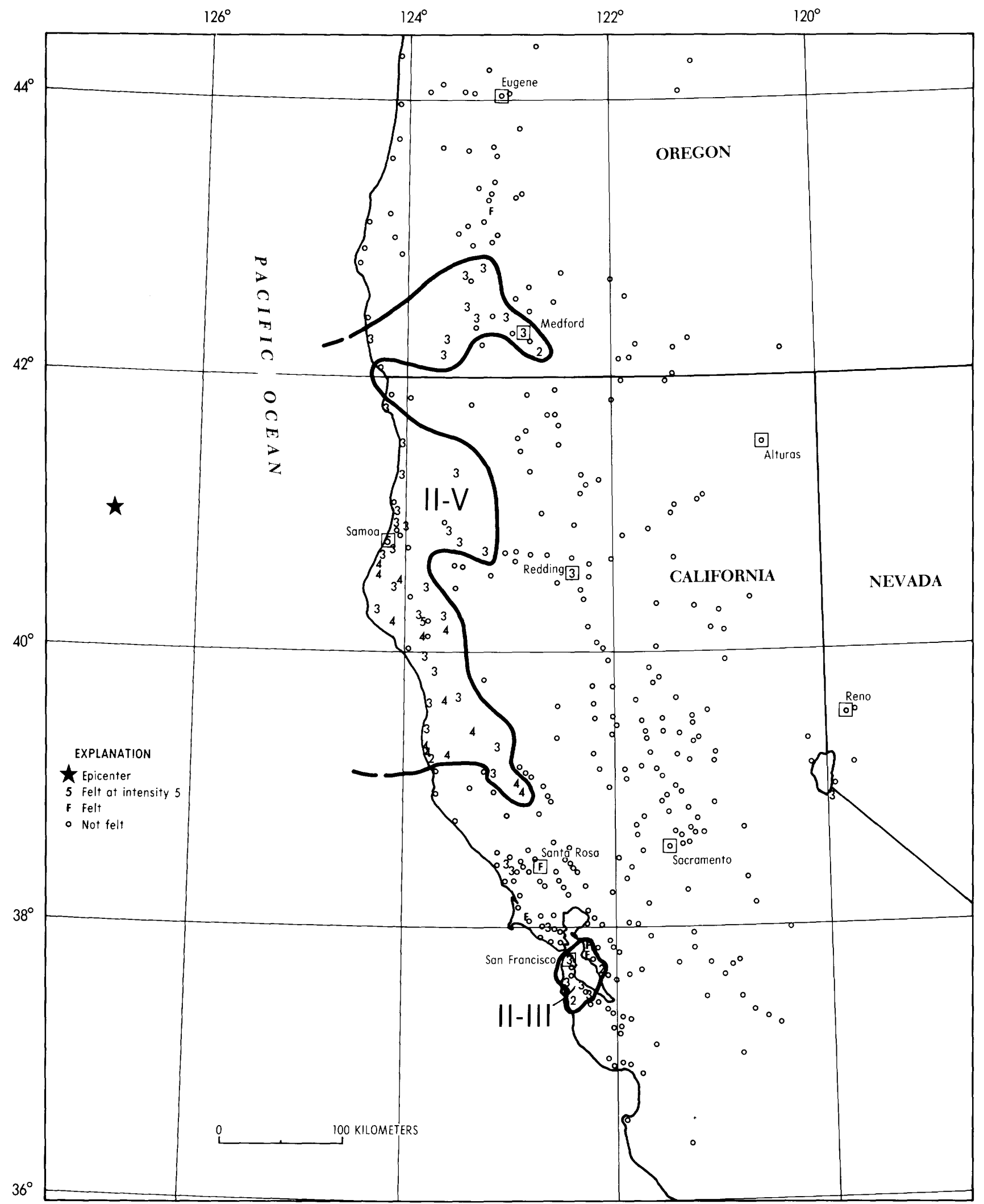

Figure 18. Isoseismal map for the northern California earthquake of 10 September 1984, 031410.1 UTC. Roman numerals represent. Modified Mercalli intensities between isoseismals; Arabic numerals represent intensities at specific sites. 
Miranda- A few items overturned and fell; trees and bushes shook slightly; buildings shook strongly.

Rio Dell- A few small objects overturned and fell; a few items shook off of store shelves; hanging pictures were out of place; buildings shook strongly.

Intensity IV: Bridgeville, Carlotta, Honeydew (a few items were shaken off store shelves), Weott, Whitethorn.

Intensity III: Blocksburg, Bridgeville, Cutten, Loleta, Myers Flat, Piercy, Trinidad.

Felt: Eureka and Ferndale (BK).

7 September (PS) Southern California

Origin time: 110313.4

Epicenter: $32.945 \mathrm{~N} ., 117.806 \mathrm{~W}$.

Depth: $6 \mathrm{~km}$

Magnitude: $4.8 m_{l},(\mathrm{GS}), 4.3 M_{L}(\mathrm{PS}), 4.3 M_{D}(\mathrm{BK})$

Intensity IV: Laguna Beach, San Clemente, San Onofre Nuclear Power Plant (press report).

10 September (GS) Northern California

Origin time: 031410.1

Epicenter: $40.503 \mathrm{~N} ., 126.831 \mathrm{~W}$.

Depth: $10 \mathrm{~km}$

Magnitude: $6.1 m_{l}(\mathrm{GS}), 6.7 M_{s}(\mathrm{GS}), 6.6 M_{L}(\mathrm{BK})$

This earthquake was felt over an area of approximately 24,000 kilometers $^{2}$ of the coastal area (fig. 18).

\section{Intensity V:}

California-

Miranda- A few small objects overturned and fell; hanging pictures swung; buildings shook slightly; walls creaked; windows rattled; felt by several.

Samoa- A few small objects overturned and fell; hanging pictures were out of place; trees and bushes shook slightly; buildings shook slightly; windows rattles; felt by several.

\section{Intensity IV:}

California - Alderpoint, Branscomb, Carlotta, Comptche, Ferndale, Finley, Honeydew, Lakeport, Little River, Loleta, Mendocino, Redway, Willits.

\section{Intensity III:}

California - Big Bar, Blocksburg, Blue Lake, Bridgeville, Burnt Ranch, Camp Meeker, Crescent City, Cutten, Eureka, Fields Landing, Fort Bragg, Iowa Hill, Klamath, Laytonville, Leggett, McKinleyville, Monte Rio, Myers Flat, Orick, Orleans, Pacifica, Petrolia, Piercy, Potter Valley, Redding, Rio Dell, Salyer, San Carlos (press report), San Francisco, San Mateo, Scotia, South Lake Tahoe, Talmage, Westhaven, Westport, Woodacre.
Oregon-Azalea, Cave Junction, Glendale, Gold Hill, Grants Pass, Medford, Merlin, Pistol River, Selma.

Intensity II:

California-- Albion, Half Moon Bay, San Lorenzo. Oregon- Ashland.

Felt:

California- Berkeley (press report), Inverness (press report), Oakland (press report), Santa Rosa (press report).

Oregon- Roseburg (press report).

\section{September (BK) Northern California}

Origin time: 183042.5

Epicenter: $40.382 \mathrm{~N} ., 125.617 \mathrm{~W}$.

Depth: $5 \mathrm{~km}$

Magnitude: $4.7 m_{1}$ (GS), $4.8 M_{L}(\mathrm{BK}), 4.1 M_{D}(\mathrm{GM})$, $M_{،}=5.6 \mathrm{E} 22(\mathrm{BK})$

Intensity IV: Fields Landing.

Intensity III: Eureka, Rio Dell.

20 September (GS) Northern California

Origin time: 190435.5

Epicenter: 40.276 N., $127.205 \mathrm{~W}$.

Depth: $10 \mathrm{~km}$

Magnitude: $4.5 m_{l},(\mathrm{GS}), 4.8 M_{L}(\mathrm{BK}), 4.2 M_{D}(\mathrm{GM})$, $M_{t}=5.7 \mathrm{E} 21(\mathrm{BK})$

Intensity III: Rio Dell.

25 November (PS) Southern California

Origin time 050850.1

Epicenter: $33.332 \mathrm{~N} ., 118.667 \mathrm{~W}$.

Depth: $14 \mathrm{~km}$

Magnitude: $3.8 M_{D}$ (PS)

Felt: Beach areas of Los Angeles (PS).

\section{COLORADO}

\section{February (GS) Denver area}

Origin time: 091819.0

Epicenter: $39.920 \mathrm{~N} ., 105.020 \mathrm{~W}$.

Depth: $5 \mathrm{~km}$

Magnitude: $2.5 M_{L}$ (GS)

Intensity III: Northglenn and Thornton (press report).

\section{April (GS) Western Colorado}

Origin time: 201657.8

Epicenter: $39.298 \mathrm{~N}$., 107.232W.

Depth: $5 \mathrm{~km}$

Magnitude: $2.2 M_{L}(\mathrm{GS})$ 
This event is the first of a series of earthquakes having magnitudes ranging from 1.1 to 3.1 which were located in the Carbondale area.

Felt: Seven miles south of Carbondale (Colorado School of Mines, Golden).

\section{April (GS) Western Colorado}

Origin time: 173056.7

Epicenter: $39.281 \mathrm{~N} ., 107.190 \mathrm{~W}$.

Depth: $5 \mathrm{~km}$

Magnitude: $3.1 M_{L}$ (GS)

Intensity IV: Carbondale.

3 May (GS) Western Colorado

Origin time: 182854.1

Epicenter: 39.304N., 107.253W.

Depth: $5 \mathrm{~km}$

Magnitude: $3.0 M_{L}$ (GS)

Felt: Carbondale.

4 May (GS) Western Colorado

Origin time: 011710.4

Epicenter: $39.334 \mathrm{~N}$., 107.274W.

Depth: $5 \mathrm{~km}$

Magnitude: $2.4 M_{L}$ (GS)

Felt: Carbondale.

4 May (GS) Western Colorado

Origin time: 021333.1

Epicenter: $39.340 \mathrm{~N}$, 107.245W.

Depth: $5 \mathrm{~km}$

Magnitude: $2.2 M_{L}$ (GS)

Felt: Carbondale.

6 May (GS) Western Colorado

Origin time: 020056.6

Epicenter: 39.335 N., 107.249W.

Depth: $5 \mathrm{~km}$

Magnitude: $2.1 M_{L}(\mathrm{GS})$

Felt: Carbondale.

6 May (GS) Western Colorado Origin time: 021249.7

Epicenter: 39.339 N., 107.248W.

Depth: $5 \mathrm{~km}$

Magnitude: $2.3 M_{L}(\mathrm{GS})$

Felt: Carbondale.

6 May (GS) Western Colorado

Origin time: 021334.4

Epicenter: $39.349 \mathrm{~N}$, 107.262W.

Depth: $5 \mathrm{~km}$

Magnitude: $2.6 M_{L}$ (GS)

Felt: Carbondale.

\section{May (GS) Western Colorado}

Origin time: 025136.9

Epicenter: 39.331N., 107.232W

Depth: $5 \mathrm{~km}$

Magnitude: $2.2 M_{L}$ (GS)

Felt: Carbondale.

\section{May Western Colorado}

Origin time: 0254

Epicenter: Not located.

Depth: None computed.

Magnitude: $2.2 M_{L}$ (GS)

Felt: Carbondale.

14 May (GS) Western Colorado

Origin time: 101417.2

Epicenter: $39.322 \mathrm{~N}$., 107.228W.

Depth: $5 \mathrm{~km}$

Magnitude: $3.2 M_{L}$ (GS)

Intensity IV: Carbondale - A few small objects fell; windows rattled; buildings shook slightly; felt by and awakened many.

Felt: Glenwood Springs (press report).

17 May (GS) Western Colorado

Origin time: 091120.2

Epicenter: $39.337 \mathrm{~N} ., 107.245 \mathrm{~W}$.

Depth: $5 \mathrm{~km}$

Magnitude: $2.4 M_{L}$ (GS)

Felt: Carbondale (press report).

25 June Western Colorado

Origin time: 1815

Epicenter: Not located.

Depth: None computed.

Magnitude: None computed.

Felt: Carbondale (telephone report).

18 October (GS) Eastern Wyoming

Origin time: 153023.0

See Wyoming listing.

\section{CONNECTICUT}

23 April (GS) Lancaster County, Pennsylvania Origin time: 013600.1

See Pennsylvania listing.

\section{DELAWARE}

19 January (GS) Wilmington area

Origin time: 230334.0

Epicenter: $39.795 \mathrm{~N} ., 75.524 \mathrm{~W}$. 


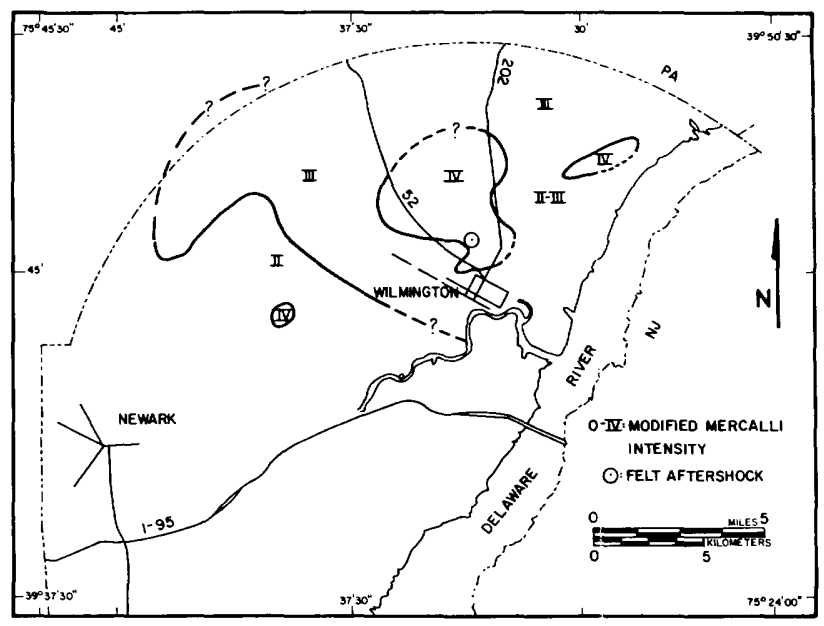

Figure 19. Isoseismal map for the Wilmington, Delaware, earthquake of 19 January 1984, 2303 34.0 UTC. Roman numerals represent Modified Mercalli intensities between isoseismals (provided by K. Woodruff, Delaware Geological Survey).

\section{DELAWA RE-Continued}

Depth: $5 \mathrm{~km}$

Magnitude: $2.4 M_{D}(\mathrm{LD}), 2.5 M_{D}(\mathrm{DE})$

This earthquake was only felt in the Wilmington suburban area (see fig. 19). Two smaller events at 0046 UTC, January 20 and 1217 UTC, February 15, magnitude $1.8 M_{D}(\mathrm{DE})$ and $1.5 M_{D}(\mathrm{DE})$ respectively, were felt in the same area.

Intensity IV: Augustine Cut-Off (press report), Baynard Boulevard and 26th street (press report), Claymont, Fairfax, Georgian Terrace (press report), Union Street, and Lancaster Avenue (press report).

Intensity III: Greenville.

Felt: Arden, Prices Corner, Wawaset Park, and Westmoreland (press reports).

\section{DISTRICT OF COLUMBIA}

23 April (GS) Lancaster County, Pennsylvania Origin time: 013600.1

See Pennsylvania listing.

\section{GEORGIA}

\section{October (TC) Northwestern Georgia}

Origin time: 115427.0

Epicenter: $34.754 \mathrm{~N} ., 85.200 \mathrm{~W}$

Depth: $12 \mathrm{~km}$

Magnitude: $4.0 M_{n}(\mathrm{GS}), 4.2 M_{n}(\mathrm{SL}), 3.8 M_{D}(\mathrm{TC})$

\section{GEORGIA-Continued}

This earthquake was felt over an area of approximately 8,500 kilometers $^{2}$ of Alabama, Georgia, and Tennessee (fig. 20).

\section{Intensity VI:}

Georgia - Seven kilometers south of Ringgold - Exterior, brick-veneer walls were cracked; a foundation cracked; plaster and sheetrock walls cracked; a tractor plow turned over; a wall clock swung; there was moderate earth noise from the east; vehicles rocked slightly; trees and bushes shook slightly; felt by and frightened all.

\section{Intensity V:}

\section{Georgia-}

Chickamauga - A few windows cracked; a few small objects overturned and fell; buildings shook slightly; walls creaked; windows rattled; felt by many.

La Fayette - A few windows cracked; a few glassware items broke; a few small objects overturned and fell; hanging pictures were out of place; buildings shook slightly; windows rattled; felt by many.

Summerville- A few windows cracked; a few glassware items broke; a few small objects overturned and fell; buildings shook slightly; windows rattled; felt by many.

Trenton- A few windows cracked; a few glassware items broke; a few small objects overturned and fell; a few items shook off of store shelves; plaster and sheetrock walls sustained hairline cracks; velicles rocked slightly; trees and bushes shook slightly; felt by many.

\section{Tennessee-}

Chattanooga- A few glassware items broke; a few small objects overturned and fell; hanging pictures were out of place; standing vehicles rocked slightly; buildings shook slightly; windows rattled; felt by many.

Intensity IV:

Alabama - Bridgeport, Fabius, Flat Rock, Higdon, Mentone, Scottsboro.

Georgia - Center Post, Cloudland, Dalton, Flintstone, Fort Oglethorpe, Graysville, Menlo, Noble, Rising Fawn, Rock Spring, Rocky Face, Rossville, Subligna, Trenton, Trion.

Tennessee - East Lake, East Ridge, Lookout Mountain, Lupton City.

Intensity III:

Alabama- Arab, Dutton, Fackler, Ider, Leesburg, Sylvania, Trenton, Union Grove.

Georgia - Crandall, Lyerly, Tunnel Hill. 


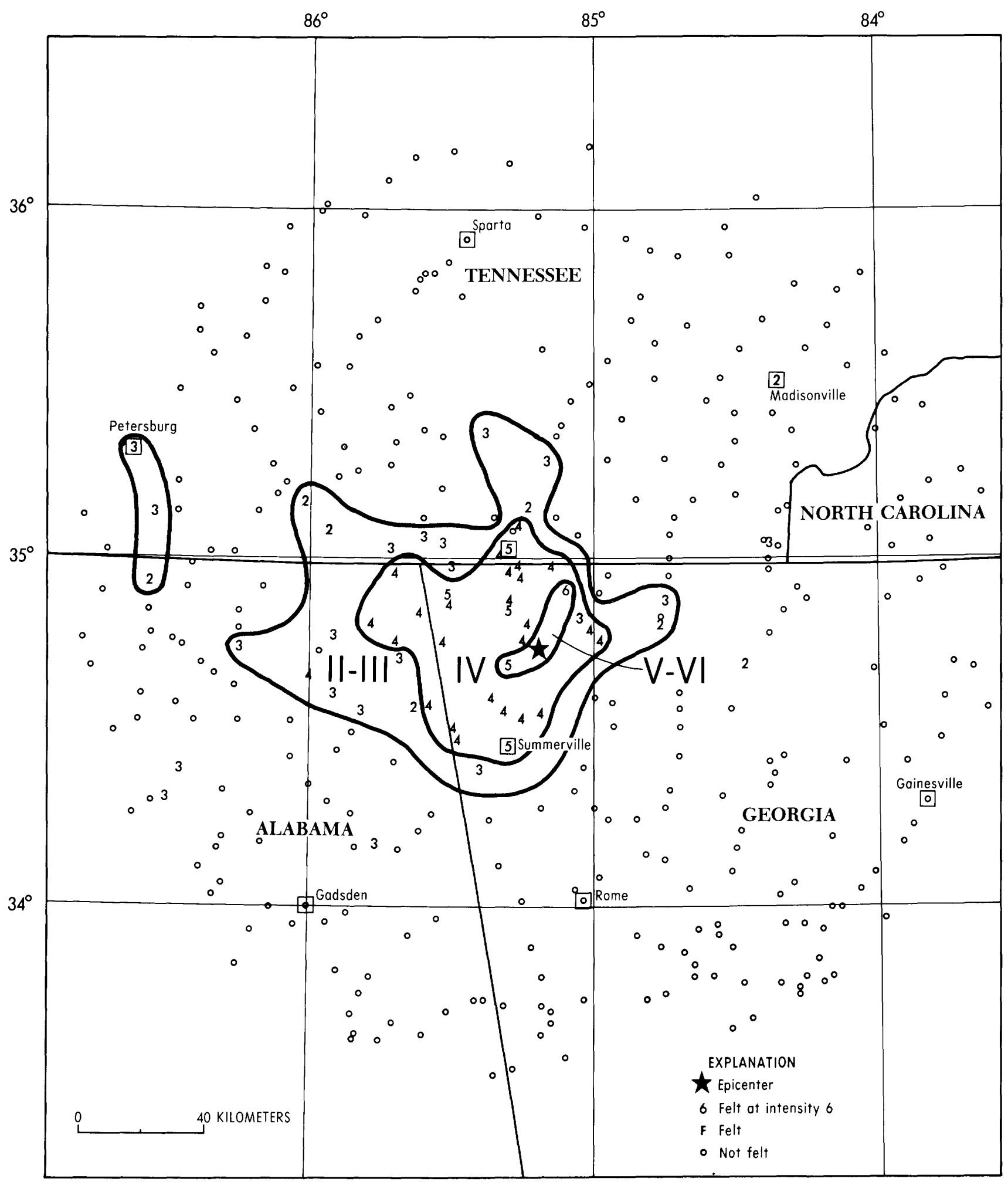

Figure 20. Isoseismal map for the northwestern Georgia earthquake of 9 October 1984, 115427.0 UTC. Roman numerals represent Modified Mercalli intensities between isoseismals; Arabic numerals represent intensities at specific sites. 
Tennessee - Ducktown, Dunlap, East Chattanooga, Fayetteville, Guild, Jasper, North Chattanooga, Petersburg, Soddy-Daisy, South Pittsburg, Whiteside.

Intensity II:

Alabama - Hammondville, Hazel Green.

Georgia-Chatsworth, Ellijay.

Tennessee- Cowan, Hixson, Madisonville, Sherwood.

\section{HAWAII}

\section{January (HV) Island of Hawaii}

Origin time: 001020.1

Epicenter: 20.588N., 154.916W.

Depth: $0 \mathrm{~km}$

Magnitude: $3.7 M_{L}(\mathrm{HV})$

Intensity IV: Hamakua.

Intensity III: Hilo, Waimea.

28 January (HV) Island of Hawaii

Origin time: 122207.8

Epicenter: 19.620N., 156.180W.

Depth: $29 \mathrm{~km}$

Magnitude: $3.7 M_{L}(\mathrm{HV})$

Intensity III: Kona.

31 January (HV) Island of Hawaii

Origin time: 062656.5

Epicenter: $19.566 \mathrm{~N} ., 156.028 \mathrm{~W}$.

Depth: $16 \mathrm{~km}$

Magnitude: $3.4 M_{L}(\mathrm{HV})$

Intensity III: Kona.

\section{February (HV) Island of Hawaii}

Origin time: 211220.9

Epicenter: $19.319 \mathrm{~N} ., 155.221 \mathrm{~W}$.

Depth: $11 \mathrm{~km}$

Magnitude: $3.5 M_{L}(\mathrm{HV})$

Intensity III: Hawaii Volcanoes National Park, Hilo, Volcano.

3 February (HV) Island of Hawaii

Origin time: 162225.1

Epicenter: 19.304N., 155.230W.

Depth: $10 \mathrm{~km}$

Magnitude: $3.5 M_{L}(\mathrm{HV})$

Intensity III: Mountain View.

4 February (HV) Island of Hawaii

Origin time: 054535.9

Epicenter: 19.192N., 155.610W.

Depth: $9 \mathrm{~km}$
Magnitude: $3.3 M_{L}(\mathrm{HV})$

Intensity III: Pahala.

15 February (HV) Island of Hawaii

Origin time: 172241.1

Epicenter: $19.399 \mathrm{~N} ., 155.455 \mathrm{~W}$.

Depth: $12 \mathrm{~km}$

Magnitude: $3.5 M_{L}(\mathrm{HV})$

Intensity IV: Pahala.

Intensity III: Hawaiian Volcano Observatory, Volcano, Volcano Golf Course.

17 February (HV) Island of Hawaii

Origin time: 124506.3

Epicenter: 19.382N., 155.074W.

Depth: $9 \mathrm{~km}$

Magnitude: $3.3 M_{L}(\mathrm{HV})$

Intensity II: Papaikou.

24 February (HV) Island of Hawaii

Origin time: 065909.6

Epicenter: $19.417 \mathrm{~N}, 155.503 \mathrm{~W}$.

Depth: $12 \mathrm{~km}$

Magnitude: $3.2 M_{L}(\mathrm{HV})$

Intensity II: Pahala.

\section{March (HV) Island of Hawaii}

Origin time: 205849.9

Epicenter: 19.322N., 155.194W.

Depth: $9 \mathrm{~km}$

Magnitude: $3.5 M_{L}(\mathrm{HV})$

Intensity III: Hilo, Mauna Loa Observatory.

Intensity II: Ainaloa, Hawaii Volcanoes National Park.

\section{March (HV) Island of Hawaii}

Origin time: 164646.0

Epicenter: $19.821 \mathrm{~N} ., 155.616 \mathrm{~W}$.

Depth: $19 \mathrm{~km}$

Magnitude: $3.1 M_{L}(\mathrm{HV})$

Intensity II: Waimea.

15 March (HV) Island of Hawaii

Origin time: 230300.8

Epicenter: 19.364N., 155.116W.

Depth: $9 \mathrm{~km}$

Magnitude: $3.4 M_{L}(\mathrm{HV})$

Intensity III: Hilo, Volcano.

$18 \mathrm{March}$ (HV) Island of Hawaii

Origin time: 052735.1

Epicenter: 19.334N., 155.200W. 
Depth: $9 \mathrm{~km}$

Magnitude: $3.1 M_{L}(\mathrm{HV})$

Intensity II: Volcano.

30 March (HV) Island of Hawaii

Origin Time: 202115.1

Epicenter: 19.970N., 155.091W

Depth: $36 \mathrm{~km}$

Magnitude: $3.8 M_{L}$ (HV)

Intensity IV: Hilo (press report).

7 April (HV) Island of Hawaii

Origin time: 044329.0

Epicenter: 19.312N., 155.229W.

Depth: $10 \mathrm{~km}$

Magnitude: $3.4 M_{L}(\mathrm{HV})$

Intensity III: Hilo, Volcano.

9 April (HV) Island of Hawaii

Origin time: 170539.2

Epicenter: $19.468 \mathrm{~N} ., 155.606 \mathrm{~W}$.

Depth: $2 \mathrm{~km}$

Magnitude: $3.7 M_{L}(\mathrm{HV})$

Intensity II: Kalapana.

9 April (HV) Island of Hawaii

Origin time: 231339.9

Epicenter: 19.310N., 155.226W.

Depth: $11 \mathrm{~km}$

Magnitude: $3.9 M_{L}(\mathrm{HV})$

Intensity IV: Hilo, Hawaii Volcanoes National Park, Volcano.

Intensity III: Kalapana, Pahoa, Pepeekeo, Pohakuloa.

22 April (HV) Island of Hawaii

Origin time: 122329.0

Epicenter: 19.305N., 155.224W.

Depth: $11 \mathrm{~km}$

Magnitude: $3.7 M_{L}(\mathrm{HV})$

Intensity III: Hilo, Volcano.

Intensity II: Glenwood, Kurtistown, Mountain View.

22 April (HV) Island of Hawaii

Origin time: 122703.6

Epicenter: 19.307N., 155.223W.

Depth: $8 \mathrm{~km}$

Magnitude: $3.8 M_{L}(\mathrm{HV})$

Intensity III: Hilo, Volcano.

Intensity II: Glenwood, Kurtistown, Mountain View.

28 April (HV) Island of Hawaii

Origin time: 094849.5

Epicenter: $19.320 \mathrm{~N} ., 155.473 \mathrm{~W}$.

Depth: $10 \mathrm{~km}$
Magnitude: $3.2 M_{L}(\mathrm{HV})$

Intensity II: Pahala.

29 April (HV) Island of Hawaii

Origin time: 064534.4

Epicenter: $19.173 \mathrm{~N} ., 155.677 \mathrm{~W}$.

Depth: $9 \mathrm{~km}$

Magnitude: $3.5 M_{L}(\mathrm{HV})$

Intensity II: Hawaiian Ocean View Estates.

1 May (HV) Island of Hawaii

Origin time: 135523.1

Epicenter: $19.211 \mathrm{~N} ., 155.569 \mathrm{~W}$.

Depth: $13 \mathrm{~km}$

Magnitude: $4.0 M_{L}(\mathrm{HV})$

Intensity III: Hawaiian Ocean View Estates, Pahala.

20 May (HV) Island of Hawaii

Origin time: 044102.7

Epicenter: $19.343 \mathrm{~N} ., 155.217 \mathrm{~W}$.

Depth: $10 \mathrm{~km}$

Magnitude: $3.3 M_{L}(\mathrm{HV})$

Intensity II: Pahala, Volcano.

8 June (HV) Island of Hawaii

Origin time: 192750.7

Epicenter: 19.201N., 155.520W.

Depth: $38 \mathrm{~km}$

Magnitude: $3.7 M_{L}(\mathrm{HV})$

Intensity IV: Pahala.

Intensity III: Ahualoa, Kohala.

12 June (HV) Island of Hawaii

Origin time: 233900.4

Epicenter: $19.624 \mathrm{~N} ., 155.930 \mathrm{~W}$.

Depth: $20 \mathrm{~km}$

Magnitude: $3.5 M_{L}(\mathrm{HV})$

Intensity II: Kona.

13 June (HV) Island of Hawaii

Origin time: 030320.1

Epicenter: $19.352 \mathrm{~N}, 155.502 \mathrm{~W}$.

Depth: $10 \mathrm{~km}$

Magnitude: $3.6 M_{L}(\mathrm{HV})$

Intensity II: Hawaiian Ocean View Estates, Red Hill.

13 June (HV) Island of Hawaii

Origin time: 115231.2

Epicenter: 19.341N., 155.213W.

Depth: $9 \mathrm{~km}$

Magnitude: $3.5 M_{L}(\mathrm{HV})$

Intensity III: Hilo.

16 June (HV) West of Lanai Island

Origin time: 182210.0 
Epicenter: 20.742N., 157.505W.

Depth: $34 \mathrm{~km}$

Magnitude: $4.0 M_{L}(\mathrm{HV})$

Intensity IV: Oahu Island-Aina Haina, Kailua, Kaneohe, Kaimuki.

Intensity III:

Maui Island- Paia, Wailuku.

Molokai Island- Kualapuu, Mauna Loa.

Oahu Island- Fort Shafter, Hawaii Kai, Honolulu (Kalama Valley).

Intensity II: Oahu Island-- Waialae-Kahala.

17 June (HV) Island of Hawaii

Origin time: 210523.5

Epicenter: 19.357N., 155.502W.

Depth: $10 \mathrm{~km}$

Magnitude: $3.1 M_{L}(\mathrm{HV})$

Intensity II: Pahala.

25 June (HV) Island of Hawaii

Origin time: 072320.0

Epicenter: $19.337 \mathrm{~N} ., 155.181 \mathrm{~W}$.

Depth: $10 \mathrm{~km}$

Magnitude: $3.5 M_{L}(\mathrm{HV})$

Intensity III: Hawaii Volcanoes National Park, Hilo, Puna, Volcano.

27 June (HV) Island of Hawaii

Origin time: 051431.3

Epicenter: $20.018 \mathrm{~N} ., 155.358 \mathrm{~W}$.

Depth: $8 \mathrm{~km}$

Magnitude: $3.3 M_{L}(\mathrm{HV})$

Intensity II: Ahualoa.

20 July (HV) Island of Hawaii

Origin time: 184747.9

Epicenter: 20.050N., 155.728W.

Depth: $7 \mathrm{~km}$

Magnitude: $3.6 M_{L}$ (HV)

Intensity III: Kohala, Kona, Waimea.

25 July (HV) Island of Hawaii

Origin time: 183238.1

Epicenter: 19.369N., 155.311W.

Depth: $31 \mathrm{~km}$

Magnitude: $3.5 M_{L}(\mathrm{HV})$

Intensity III: Pahala.

12 August (HV) Island of Hawaii

Origin time: 092930.1

Epicenter: 19.458N., 155.489W.

Depth: $10 \mathrm{~km}$

Magnitude: $3.0 M_{L}(\mathrm{HV})$
Intensity IV: Waimea.

Intensity III: Hilo.

14 August (HV) Island of Hawaii

Origin time: 201002.1

Epicenter: 19.786N., 154.913W.

Depth: $48 \mathrm{~km}$

Magnitude: $3.7 M_{L}(\mathrm{HV})$

Felt: Hilo and Kamuela (HV).

3 September (HV) Island of Hawaii

Origin time: 024314.4

Epicenter: $19.355 \mathrm{~N} ., 155.026 \mathrm{~W}$.

Depth: $7 \mathrm{~km}$

Magnitude: $3.2 M_{L}(\mathrm{HV})$

Intensity III: Kalapana.

11 September (HV) Island of Hawaii

Origin time: 095136.3

Epicenter: $19.343 \mathrm{~N} ., 155.222 \mathrm{~W}$.

Depth: $9 \mathrm{~km}$

Magnitude: $3.4 M_{L}(\mathrm{HV})$

Intensity III: Pahala.

25 September (HV) Island of Hawaii

Origin time: 072748.2

Epicenter: 19.316N., 155.903W.

Depth: $12 \mathrm{~km}$

Magnitude: $4.3 M_{L}(\mathrm{HV})$

Intensity IV: South Kona.

Intensity III: Hamakua, Keahole, North Kona, Volcano, Waimea.

27 September (HV) Island of Hawaii

Origin time: 170312.7

Epicenter: 19.323N., 155.183W.

Depth: $10 \mathrm{~km}$

Magnitude: $3.2 M_{L}(\mathrm{HV})$

Intensity III: Papaikou, Volcano

8 October (HV) Island of Hawaii

Origin Time: 100025.1

Epicenter: $20.117 \mathrm{~N} ., 156.86 \mathrm{~W}$.

Depth: $30 \mathrm{~km}$

Magnitude: $3.9 M_{L}(\mathrm{HV})$

Intensity III: Kealakekua.

9 October (HV) Island of Hawaii

Origin time: 021810.7

Epicenter: 19.915N., 155.490W.

Depth: $18 \mathrm{~km}$

Magnitude: $3.7 M_{L}(\mathrm{HV})$

Intensity IV: Ahualoa, Honokaa. 
Intensity III: Hilo, Kamuela, Kukuihaele, Pepeekeo, Waikaloa.

10 October (HV) Island of Hawaii

Origin time: 123349.7

Epicenter: $19.287 \mathrm{~N}$., $155.420 \mathrm{~W}$.

Depth: $10 \mathrm{~km}$

Magnitude: $3.0 M_{L}(\mathrm{HV})$

Intensity III: Pahala.

17 October (HV) Island of Hawaii

Origin time: 004555.8

Epicenter: 19.408 N., $155.489 \mathrm{~W}$.

Depth: $10 \mathrm{~km}$

Magnitude: $3.5 M_{L}(\mathrm{HV})$

Intensity III: Pahala.

11 November (HV) Island of Hawaii

Origin time: 153406.2

Epicenter: 18.931N., 155.222W.

Depth: $9 \mathrm{~km}$

Magnitude: $4.0 M_{L}(\mathrm{HV})$

Intensity II: Pahala.

12 November (HV) Island of Hawaii

Origin time: 050319.5

Epicenter: $19.343 \mathrm{~N} ., 155.188 \mathrm{~W}$.

Depth: $9 \mathrm{~km}$

Magnitude: $3.4 M_{L}(\mathrm{HV})$

Intensity II: Ainaloa, Hilo.

14 November (HV) Island of Hawaii

Origin time: 110556.2

Epicenter: $19.863 \mathrm{~N} ., 155.148 \mathrm{~W}$.

Depth: $16 \mathrm{~km}$

Magnitude: $3.8 M_{L}(\mathrm{HV})$

Intensity IV: Honomu.

Intensity III: Hilo, Laphoehoe, Ninoole.

Intensity II: Keaukaha.

19 November (HV) Island of Hawaii

Origin time: 003510.0

Epicenter: 20.163N., $155.636 \mathrm{~W}$.

Depth: $32 \mathrm{~km}$

Magnitude: $4.0 M_{L}(\mathrm{HV})$

Intensity III: Honokaa, Kamuela.

Intensity II: Kohala.

28 November (HV) Island of Hawaii

Origin time: 121548.5

Epicenter: $19.797 \mathrm{~N} ., 155.394 \mathrm{~W}$.

Depth: $25 \mathrm{~km}$

Magnitude: $3.1 M_{L}(\mathrm{HV})$

Intensity III: Kamuela.
5 December (HV) Island of Hawaii

Origin time: 073457.6

Epicenter: $19.896 \mathrm{~N} ., 155.377 \mathrm{~W}$.

Depth: $10 \mathrm{~km}$

Magnitude: $3.3 M_{L}(\mathrm{HV})$

Intensity III: Paauilo.

Intensity II: Hilo.

Felt: Kaumana (press report).

23 December (HV) Island of Hawaii Origin time: 161419.2

Epicenter: 19.269N., 155.381W.

Depth: $8 \mathrm{~km}$

Magnitude: $3.2 M_{L}(\mathrm{HV})$

Intensity III: Pahala.

Intensity II: Kapapala.

23 December (HV) Island of Hawaii

Origin time: 174618.5

Epicenter: $19.269 \mathrm{~N}$., $155.385 \mathrm{~W}$.

Depth: $8 \mathrm{~km}$

Magnitude: $3.2 M_{L}(\mathrm{HV})$

Intensity III: Pahala.

Intensity II: Kapapala.

26 December (HV) Island of Hawaii

Origin time: 032211.5

Epicenter: $19.980 \mathrm{~N} ., 155.099 \mathrm{~W}$.

Depth: $35 \mathrm{~km}$

Magnitude: $4.3 M_{L}(\mathrm{HV})$

Intensity III: Hilo, Honokaa.

Intensity II: Waimea.

\section{IDAHO}

19 Jamuary (GS) Western Montana

Origin time: 072542.9

See Montana listing.

24 January (GS) Central Idaho

Origin time: 210757.5

Epicenter: $44.047 \mathrm{~N} ., 114.442 \mathrm{~W}$.

Depth: $10 \mathrm{~km}$

Magnitude: $4.5 m_{l},(\mathrm{GS}), 4.6 M_{L}(\mathrm{BU})$

Intensity IV: Clayton, Stanley.

Intensity III: Darlington, Hailey, May.

Intensity II: Atlanta.

Felt: Boise and Ketchum (press report).

25 January (GS) Northern Idaho

Origin time: 193612.0

60 U.S. Earthquakes, 1984 
Epicenter: 47.500N., 116.000W.

Depth: $1 \mathrm{~km}$

Magnitude: $2.3 M_{L}$ (GS)

Rockburst in the Lucky Friday mine near Wallace, Idaho. One person killed and one injured (U.S. Bureau of Mines).

\section{January (GS) Northeastern Oregon}

Origin Time: 052915.6

See Oregon listing.

5 February (GS) Central Idaho

Origin time: 034415.8

Epicenter: $44.275 \mathrm{~N} ., 114.127 \mathrm{~W}$.

Depth: $10 \mathrm{~km}$

Magnitude: $3.9 M_{L}(\mathrm{GS}), 4.3 M_{L}(\mathrm{BU})$

Felt: Boise (press report).

11 February (PG) British Columbia-Alberta, Canada border

Origin Time: 133827.0

See Montana listing.

2 March (GS) Central Idaho

Origin time: 002945.1

Epicenter: 44.350N., 114.186W.

Depth: $10 \mathrm{~km}$

Magnitude: $4.5 m_{l}$, (GS), $4.3 M_{L}(\mathrm{GS}), 4.4 M_{D}(\mathrm{BU})$

Intensity IV: Challis.

Intensity III: Clayton, May, Stanley.

Intensity II: Cobalt.

24 March (GS) Central Idaho

Origin time: 000747.7

Epicenter: 44.740N., 114.430W.

Depth: $10 \mathrm{~km}$

Magnitude: $4.2 m_{l}$ (GS), $4.3 M_{L}(\mathrm{BU}), 3.8 M_{D}$ (UU)

Intensity V: Challis- Plasterboard walls sustained hairline cracks; trees and bushes shook slightly; a few small objects overturned and fell; hanging pictures swung out of place; felt by many.

Intensity III: Cobalt.

24 March (GS) Central Idaho

Origin time: 210316.3

Epicenter: $44.728 \mathrm{~N} ., 114.441 \mathrm{~W}$.

Depth: $10 \mathrm{~km}$

Magnitude: $3.8 M_{L}(\mathrm{GS}), 3.7 M_{L}(\mathrm{BU})$

Intensity III: Cobalt.

10 April (GS) Central Idaho

Origin time: 021018.8

Epicenter: 44.654N., 114.310W.
Depth: $10 \mathrm{~km}$

Magnitude: $3.1 M_{L}$ (GS), $3.2 M_{L}(\mathrm{BU})$

Seven earthquakes were reported to have been felt in Clayton; two were located and are listed here. The five not located occurred at 03:11, 05:23, 16:23, 16:26, and 21:30 UTC.

Felt: Clayton.

\section{April (GS) Central Idaho}

Origin time: 143054.9

Epicenter: $44.270 \mathrm{~N} ., 114.365 \mathrm{~W}$.

Depth: $10 \mathrm{~km}$

Magnitude: $3.2 M_{L}(\mathrm{BU})$

Felt: Clayton.

\section{April (GS) Central Idaho}

Origin time: 155155.9

Epicenter: $44.774 \mathrm{~N} ., 114.469 \mathrm{~W}$.

Depth: $10 \mathrm{~km}$

Magnitude: $3.7 m_{l}$ (GS), $4.1 M_{L}$ (GS), $4.0 M_{L}$ (BU)

Intensity IV: Clayton, North Fork.

12 April (GS) Central Idaho

Origin time: 090802.0

Epicenter: $44.297 \mathrm{~N}$., $114.280 \mathrm{~W}$.

Depth: $10 \mathrm{~km}$

Magnitude: $3.0 M_{L}(\mathrm{BU})$

Felt: Clayton.

\section{May Central Idaho}

Origin time: 1411

Epicenter: Not located.

Depth: None computed.

Magnitude: None computed.

Intensity III: Clayton.

22 August (GS) Central Idaho

Origin time: 094630.2

Epicenter: $44.467 \mathrm{~N}$., $114.008 \mathrm{~W}$.

Depth: $10 \mathrm{~km}$

Magnitude: $5.0 m_{l}$ (GS), 5.1 $M_{s}(\mathrm{GS}), 5.8 M_{L}$ (UU)

At least 15 aftershocks were felt at the Ingram Ranch near Challis following this event. It was felt over an area of 173,000 kilometers $^{2}$ of Idaho, Montana, and Oregon (fig. 21).

Intensity V: The most common effects at the places listed below: a few small objects overturned and fell; hanging pictures were out of place; buildings shook slightly to moderately; walls creaked; windows rattled; shaking was described as moderate to strong; felt by and awakened many. Additional effects at specific locations are listed below: 


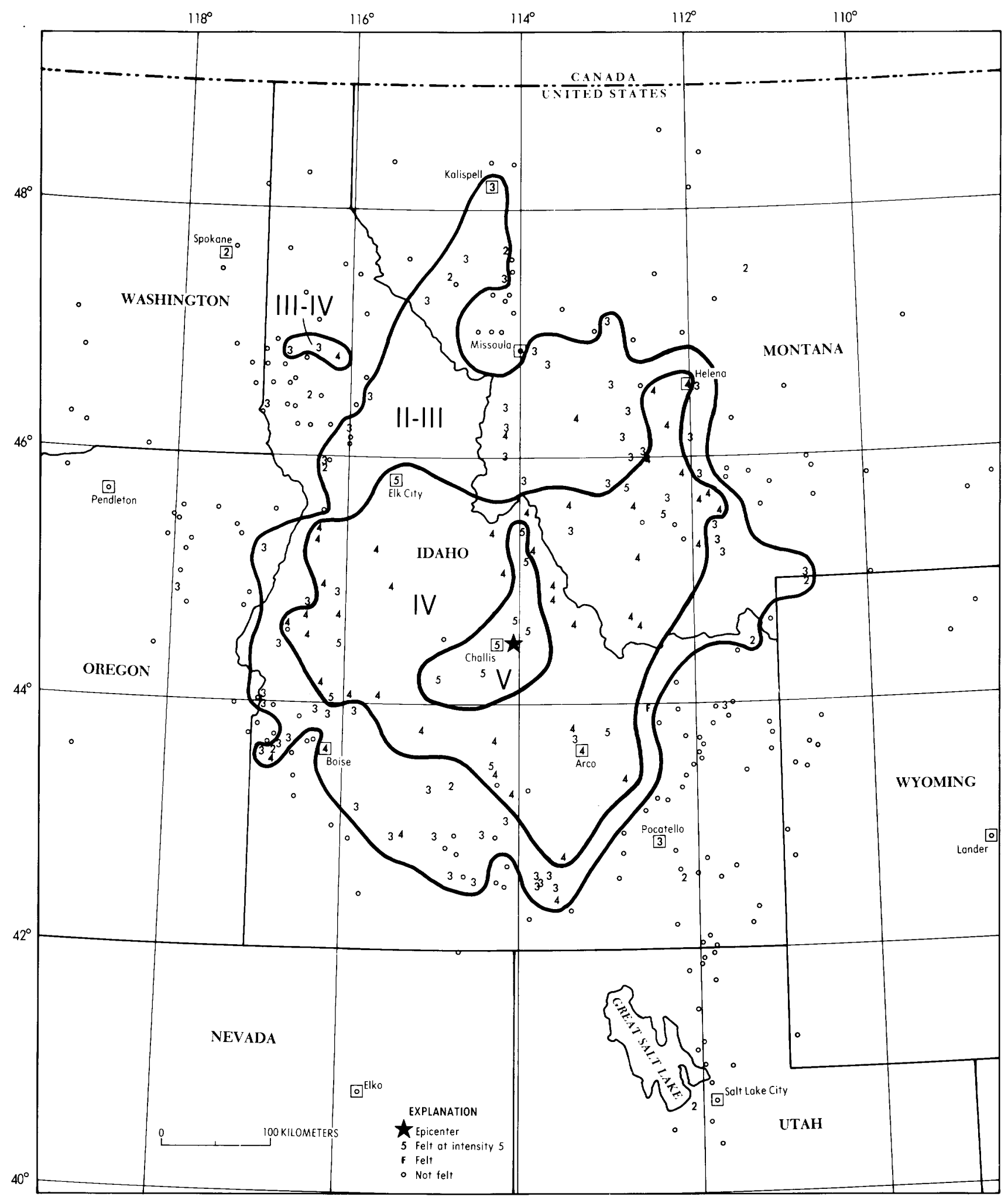

Figure 21. Isoseismal map for the central Idaho earthquake of 22 August 1984, 094630.2 UTC. Roman numerals represent Modified Mercalli intensities between isoseismals; Arabic numerals represent intensities at specific sites. 
Idaho-

Banks-- A few glassware items broke.

Cascade-- It was felt by and awakened several.

Challis - Local rockslides occurred (press report).

Clayton-- Buildings shook strongly.

Elk City.

Ellis- A few items shook off of store shelves; buildings shook strongly.

Hailey - A few windows cracked; a few glassware items broke; a few items shook off of store shelves; motor vehicles rocked slightly.

Howe.

May-- Buildings shook strongly; plaster walls sustained hairline cracks; hanging objects swung violently; felt by all.

North Fork - Buildings shook strongly; a few items shook off of store shelves.

Salmon- A few windows cracked; a few glassware items broke; a few items shook off of store shelves.

Stanley - Buildings shook strongly; a few items shook off of store shelves; motor vehicles rocked moderately; felt by all.

Montana-

Divide.

Twin Bridges-- A few glassware items broke.

Intensity IV:

Idaho- Albion, Arco, Atlanta, Atomic City, Bellevue, Boise, Carmen, Cobalt, Council, Darlington, Donnelly, Elk River, Garden Valley, Gibbonsville, Glenns Ferry, Indian Valley, Leadore, Lemhi, Lowman, Marsing, Minidoka, New Meadows, Ola, Picabo, Pollock, Riggins, Shoup, Sun Valley, Tendoy, Warm Lake, Warren, Yellow Pine.

Montana- Basin, Butte, Dell, Dillon, Elliston, Grantsdale, Harrison, Helena, Lima, Melrose, Norris, Philipsburg, Pony, Virginia City, Whitehall, Wisdom.

Intensity III:

Idaho- Bliss, Bovill, Buhl, Burley, Declo, Fruitland, Fruitvale, Greenleaf, Hammett, Heyburn, Hill City, Homedale, Horseshoe Bend, Idaho City, Kamiah, Lewiston, McCall, Middleton, Midvale, Moore, Paul, Payette, Pierce, Placerville, Pocatello, Princeton, Richfield, Rupert, Saint Anthony, Shoshone, Sweet, Twin Falls, Weiser, West Mountain, White Bird.

Montana - Anaconda, Bonner, Boulder, Cameron, Cardwell, Charlo, Clinton, Darby, Deer Lodge, East Helena, Ennis, Gardiner, Goldcreek, Hamilton, Hot Springs, Jackson, Kalispell, McAllister, Milltown, Ramsay, Saint Regis, Silver Star, Sula, Victor, Walkerville, Warmsprings, Wise River.

Oregon- Haines, Jordan Valley.
Intensity II:

Idaho - Arimo, Fairfield, Grangeville, Huston, Lenore, Macks Inn.

Montana - Great Falls, Plains, Polson.

Utah- Magna.

Washington-Spokane.

Wyoming-- Mammoth Hot Spings.

Felt:

Idaho-- Monteview.

30 August (GS) Central Idaho

Origin time: 184227.6

Epicenter: $44.452 \mathrm{~N}$., $114.126 \mathrm{~W}$.

Depth: $10 \mathrm{~km}$

Magnitude: $3.5 M_{L}$ (GS), $4.2 M_{L}(\mathrm{BU})$

Felt: Challis (press report).

30 August (GS) Central Idaho

Origin time: 210620.7

Epicenter: $44.455 \mathrm{~N}$, $114.154 \mathrm{~W}$.

Depth: $10 \mathrm{~km}$

Magnitude: $3.9 M_{L}(\mathrm{GS}), 4.4 M_{L}(\mathrm{BU})$

Felt: Challis and several small mountain towns (press report).

4 September (GS) Central Idaho

Origin time: 055041.3

Epicenter: 44.449N., 114.107W.

Depth: $10 \mathrm{~km}$

Magnitude: $3.7 M_{L}(\mathrm{GS}), 4.3 M_{L}(\mathrm{BU})$

Intensity IV: Challis.

5 September (GS) Central Idaho

Origin time: 173858.4

Epicenter: $44.421 \mathrm{~N}$., $114.183 \mathrm{~W}$.

Depth: $10 \mathrm{~km}$

Magnitude: $3.7 M_{L}(\mathrm{GS}), 4.3 M_{L}(\mathrm{BU})$

Felt: Challis (press report).

8 September (GS) Central Idaho

Origin time: 061640.1

Epicenter: $44.439 \mathrm{~N}$., $114.154 \mathrm{~W}$.

Depth: $10 \mathrm{~km}$

Magnitude: $5.0 m_{l}$ (GS), $4.4 M_{S}(\mathrm{GS}), 4.3 M_{D}(\mathrm{UU})$

Intensity IV:

Idaho- Challis (press report), Clayton, Ellis, Hailey, May, Warm Lake.

Intensity III:--

Idaho- Atlanta, Atomic City, Boise, Fruitland, Homedale, Horseshoe Bend, Lemni, Meridian, Midvale, Moore, North Fork, Pollock, Shoup, Tendoy, Yellow Pine.

Montana- Divide, Jackson, Sula. 
Intensity II:

Idaho- Cascade, Star.

Montana- Darby, Hamilton.

Oregon-- Haines.

8 September (GS) Central Idaho

Origin time: 085550.0

Epicenter: 44.444N., 114.152W.

Depth: $10 \mathrm{~km}$

Magnitude: $3.9 M_{L}$ (GS), $4.4 M_{L}(\mathrm{BU})$

Intensity III: Clayton.

Felt: Challis (press report).

8 September (GS) Central Idaho

Origin time: 113613.7

Epicenter: $44.420 \mathrm{~N}$, $114.150 \mathrm{~W}$.

Depth: $10 \mathrm{~km}$

Magnitude: $4.0 M_{L}(\mathrm{GS}), 4.2 M_{L}(\mathrm{BU})$

Felt: Challis (press report).

8 September (GS) Central Idaho

Origin time: 135637.7

Epicenter: $44.416 \mathrm{~N} ., 114.147 \mathrm{~W}$.

Depth: $10 \mathrm{~km}$

Magnitude: $4.4 m_{l},(\mathrm{GS}), 4.6 \mathrm{M}_{D}(\mathrm{BU})$

Intensity III: Clayton.

Felt: Challis (press report).

13 September (GS) Central Idaho

Origin time: 111248.9

Epicenter: 44.434N., 114.150W.

Depth: $10 \mathrm{~km}$

Magnitude: $3.9 M_{L}$ (GS), $4.4 M_{L}(\mathrm{BU})$

Intensity IV: Challis.

15 September (GS) Northern Idaho

Origin time: 025502.6

Epicenter: 47.460N., 115.987W.

Depth: $1 \mathrm{~km}$

Magnitude: $2.8 M_{L}$ (GS)

Rockburst in a mine near Wallace, Idaho. One person was killed and one injured.

18 September (GS) Central Idaho

Origin time: 150958.0

Epicenter: 44.408N., 114.124W.

Depth: $10 \mathrm{~km}$

Magnitude: $4.0 M_{L}$ (GS), $3.3 M_{D}$ (UU)

Felt: Challis (press report).

19 September (GS) Western Idaho

Origin time: 013206.9

Epicenter: 45.079N., $116.766 \mathrm{~W}$.

Depth: $5 \mathrm{~km}$

Magnitude: $3.4 M_{D}(\mathrm{GS})$

64 U.S. Earthquakes, 1984
Intensity III:

Oregon-- Halfway (OS).

Felt:

Idaho- Boise (OS).

Oregon-Richland (Zollweg and Jacobson, 1986).

30 October (GS) Central Idaho

Origin time: 230530.6

Epicenter: 44.430N., 114.112W.

Depth: $10 \mathrm{~km}$

Magnitude: $3.9 M_{L}(\mathrm{GS}), 4.2 M_{D}(\mathrm{BU})$

Intensity IV: Clayton.

Felt: Challis (telephone report).

\section{ILLINOIS}

13 February (SL) Western Kentucky

Origin time: 224245.3

See Kentucky listing.

14 February (SL) Western Kentucky

Origin time: 225610.4

See kentucky listing.

17 April (SL) Southern Illinois

Origin time: 044444.9

Epicenter: $38.410 \mathrm{~N} ., 88.480 \mathrm{~W}$.

Depth: $14 \mathrm{~km}$

Magnitude: $3.2 M_{n}(\mathrm{SL})$

Intensity IV: Bluford, Dix, Ellery (a few small objects overturned and fell), Fairfield (a few small objects overturned and fell), Griffin, Keenes, Macedonia, Mill Shoals (hairline cracks in plaster walls).

Intensity III: Barnhill, Bluford, Deff, Mount Erie, Sailor Springs.

29 June (SL) Southern Illinois

Origin time: 075829.3

Epicenter: $37.700 \mathrm{~N}$., $88.470 \mathrm{~W}$.

Depth: $2 \mathrm{~km}$

Magnitude: $4.1 M_{n}(\mathrm{GS}), 3.8 M_{n}(\mathrm{SL})$

Intensity VI:

Illinois-

Harrisburg - One house had structural damage (SL).

Intensity V:

Illinois-

Raleigh - A few small objects overturned or fell; windows, doors, and dishes rattled; felt by and awakened many.

Intensity IV:

Illinois- Equality, Herod, Karbers Ridge, Muddy, Ozark, Rosiclare, Shawneetown. 


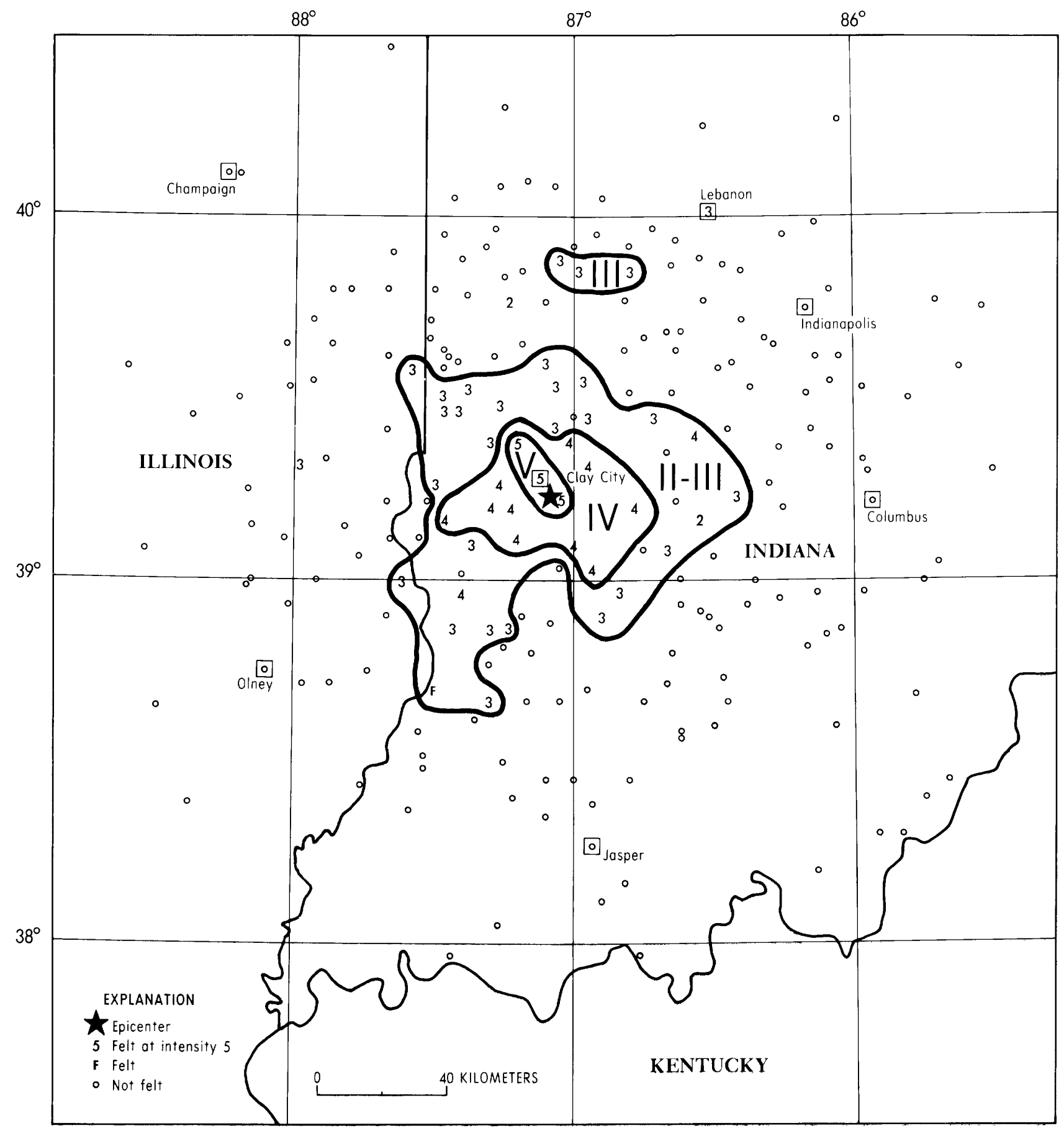

Figure 22. Isoseismal map for the southwestern Indiana earthquake of 28 July 1984, 233927.4 UTC. Roman numerals represent Modified Mercalli intensities between isoseismals; Arabic numerals represent intensities at specific sites. 
Kentucky - Tolu.

Intensity III:

Illinois- Breese, Carrier Mills, Cave in Rock, Emma, Galatia, McLeansboro, Stonefort, Tamaroa.

Kentucky - Marion, Uniontown.

Intensity II:

Missouri-Sikeston.

Felt: Illinois - Ridgway.

28 July (SL) Southwestern Indiana

Origin time: 233927.4

See Indiana listing.

30 July (SL) Southeastern Missouri

Origin time: 073346.5

See Missouri listing.

INDIANA

\section{June (SL) Southwestern Indiana}

Origin time: 182648.2

Epicenter: $38.918 \mathrm{~N} ., 87.464 \mathrm{~W}$.

Depth: $3 \mathrm{~km}$

Magnitude: $3.4 M_{n}(\mathrm{SL})$

This event may have been a mine explosion.

Intensity IV: Carlisle, Paxton (IU), Pleasantville (II).

\section{July (SL) Southwestern Indiana}

Origin time: 233927.4

Epicenter: $39.220 \mathrm{~N}$., 87.071W.

Depth: $10 \mathrm{~km}$

Magnitude: $4.0 M_{n}(\mathrm{SL})$

This earthquake was felt over an area of 6,800 kilometers $^{2}$ of the Illinois-Indiana border region (fig. 22).

Intensity V:

Indiana--

Clay City - Hanging pictures fell; a few small objects overturned and fell; plaster walls sustained hairline cracks; trees and bushes shook slightly; windows rattled; felt by many.

Coal City - A few windows cracked; a few dishes broke; a few small objects overturned and fell; interior walls sustained hairline cracks; felt by many.

Cory - Plaster walls sustained hairline cracks; a foundation cracked; a few glassware items broke; a few small objects overturned and fell; felt by many.
Intensity IV:

Indiana- Bloomfield, Bowling Green, Carlisle, Coalmont, Freedom, Hymera, Jasonville, Lewis, Linton, Midland, Paragon, Patricksburg, Shelburn, Worthington.

Intensity III:

Illinois - Casey, Palestine, Vermilion.

Indiana - Carbon, Centerpoint, Crane, Freelandville, Harmony, Koleen, Lebanon, North Terre Haute, Oaktown, Poland, Prairie Creek, Quincy, Reelsville, Riley, Roachdale, Russellville, Saint Mary-of-the-Woods, Seelyville, Stanford, Sullivan, Terre Haute, Unionville, Waveland, West Terre Haute, Westphalia, Wheatland.

Intensity II:

Indiana - Bloomington, Rockville.

Felt:

Indiana-Vincennes (IU).

\section{August (SL) Southwestern Indiana}

Origin time: 065059.5

Epicenter: $39.110 \mathrm{~N}$., $87.450 \mathrm{~W}$.

Depth: $10 \mathrm{~km}$

Magnitude: $3.1 M_{n}(\mathrm{SL})$

Intensity V: Clay City - Plaster walls sustained hairline cracks; hanging pictures fell; a few small objects overturned and fell; buildings shook slightly; walls creaked; windows rattled; felt by and awakened several. Intensity III: Bowling Green, Crane, Fairbanks, Lewis.

Felt: Terre Haute (SL).

\section{KANSAS}

\section{October (GS) Eastern Wyoming}

Origin time: 153023.0

See Wyoming listing.

\section{KENTUCKY}

\section{February (SL) Western Kentucky}

Origin time: 224245.3

Epicenter: $37.210 \mathrm{~N}$., 89.020W.

Depth: $5 \mathrm{~km}$

Magnitude: $3.2 M_{n}(\mathrm{SL}), 3.3 M_{n}(\mathrm{VP})$

Intensity IV:

Kentucky - Bandana. 
Intensity III:

Illinois - Belknap, Cairo, Grand Chain, Mounds, Tamms, Ullin.

Kentucky - Kevil, La Center.

Intensity II:

Illinois- Brokport, Perks.

Kentucky - Paducah.

14 February (SL) Western Kentucky

Origin time: 225610.4

Epicenter: $37.210 \mathrm{~N} ., 89.000 \mathrm{~W}$

Depth: $2 \mathrm{~km}$

Magnitude: $3.6 M_{n}(\mathrm{SL}) .3 .8 M_{n}(\mathrm{VP})$

Intensity IV:

Illinois-- Belknap, C'airo, Cypress, Grand Chain, Karnak, Olmsted, Perks, Simpson.

Kentucky - Bandana, Kevil.

Intensity III:

Illinois - Brownfield, Carbondale, Colp, Dongola,

Ewing, Mill Creek, Mounds, Joppa, Temple Hill,

Tamms, Ullin.

Kentucky - La Center.

Missouri- Delta, Marston, Patton.

Intensity II:-

Illinois- Brookport, Grantsburg.

Kentucky - Kirksey.

Missouri-Cape Girardeau, Chaffee.

Felt:

Kentucky - Paducah.

29 June (SL) Southern Illinois

Origin time: 075829.3

See Illinois listing.

\section{MAINE}

\section{January (EP) Southeastern Maine}

Origin time: 053433.0

Epicenter: 45.000N., 67.200W.

Depth: $8 \mathrm{~km}$

Magnitude: $2.6 M_{n}(\mathrm{EP})$

Felt: Calais (press report), Eastport.

14 January (WO) Southeastern Maine

Origin time: 090834.5

Epicenter: $45.050 \mathrm{~N} ., 67.180 \mathrm{~W}$.

Depth: $8 \mathrm{~km}$

Magnitude: $3.4 M_{L}(\mathrm{WO})$

Intensity $\mathbf{V}$ :

Maine--

Calais - A few windows cracked; a few dishes broke; a few small objects fell; a few merchandise items fell from store shelves; felt by and awakened many; vibration was described as strong.

Pembroke- A foundation cracked; plaster walls sustained hairline cracks; buildings shook strongly; felt by and awakened many; vibration was described as strong.

Intensity IV:

Maine

Cooper, Dennysville, Eastport, Lubec, Machiasport, Robbinston, Whitneyville, Woodland.

New Brunswick - St. Stephens (press report).

Intensity III:

Maine-East Machias, Machias, Perry, Whiting.

Felt:

New Brunswick - Black's Harbour and McAdam (press reports).

\section{January (WO) Southeastern Maine}

Origin time: 090933.1

Epicenter: 45.050N, 67.180W.

Depth: $8 \mathrm{~km}$

Magnitude: $3.6 M_{n}$ (WO)

This earthquake was felt at the same intensity as the one at 090834.5 above. Because it followed the earlier earthquake by about 1 minute, the felt effects could not be separated; most people thought they had felt only one earthquake.

\section{January (WO) Southeastern Maine}

Origin time: 052608.8

Epicenter: 44.893N., 67.301W.

Depth: $12 \mathrm{~km}$

Magnitude: $3.8 M_{n}(\mathrm{WO}), 3.6 M_{D}$ (WO)

Intensity V:

Maine--

Alexander- A wall cracked along a seam in a prefabricated home (press report).

Dennysville- A few windows cracked; standing and moving vehicles were rocked slightly; walls creaked; dishes rattled; felt by and awakened many.

Edmunds Township (near Dennysville) - A chimney crack and a basement wall crack were widened.

Jonesboro- A few dishes and glassware items broke; a few small objects overturned and fell; felt by many.

Machias- One chimney cracked; a few glassware items broke; a few small objects fell; felt by and awakened many.

Whitneyville - Plaster walls sustained hairline cracks; a few small objects overturned and fell; hanging pictures were out of place; felt by and awakened many. 


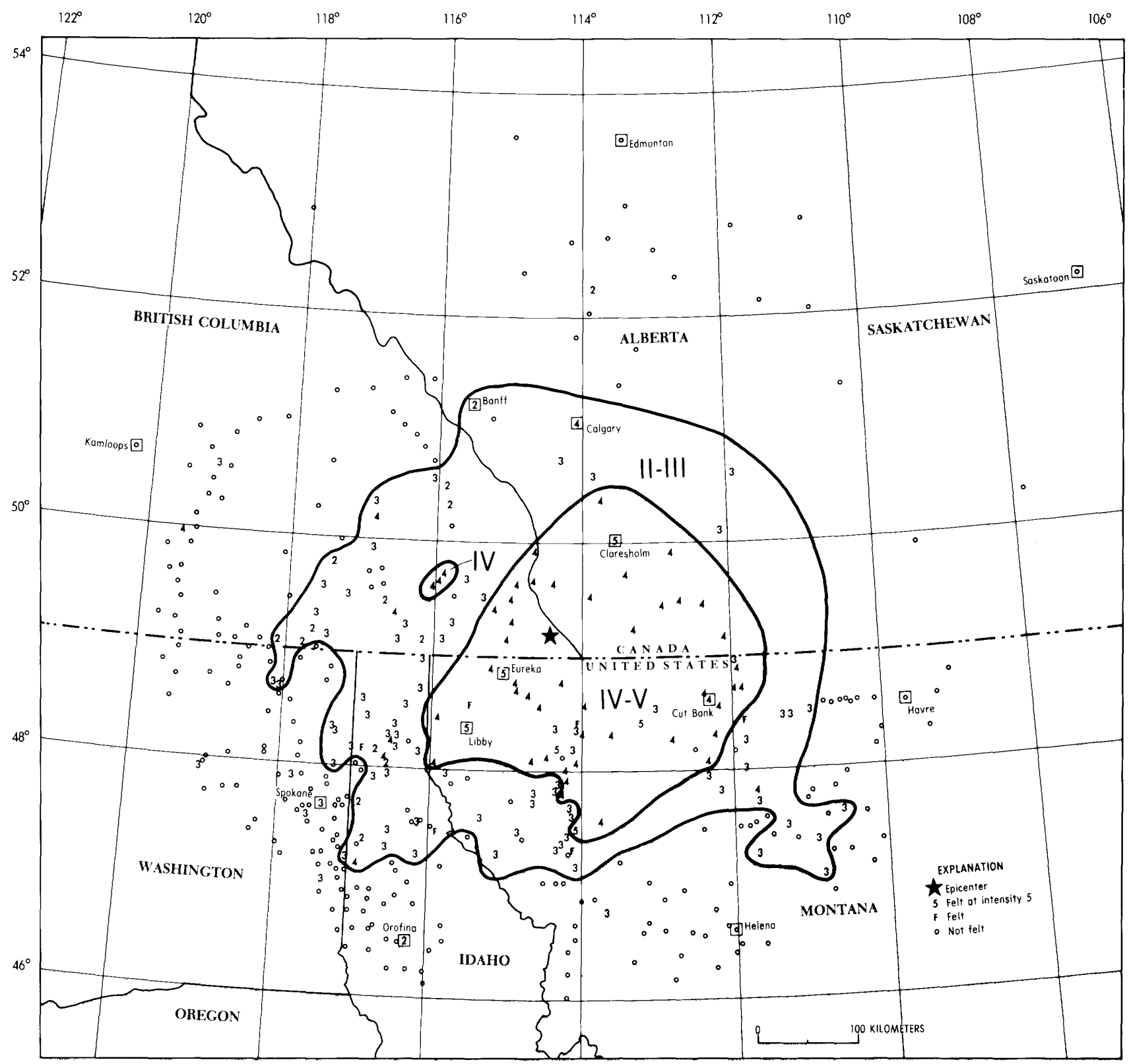

Figure 23. Isoseismal map for the British Columbia-Alberta, Canada border, earthquake of 11 February 1984, 1338 27.0 UTC. Roman numerals represent Modified Mercalli intensities between isoseismals; Arabic numerals represent intensities at specific sites. 
Intensity IV:

Maine- Bucks Harbor, Columbia Falls, Cutler (one chimney reported cracked), East Machias, Eastport, Lubec, Machiasport, Northfield, Princeton, Whiting.

Intensity III:

Maine--Addison, Beals, Cherryfield, Grand Lake Stream, Robbinston.

Felt:

Maine-Calais (press report), Perry.

New Brunswick-- Fredericton (press report).

\section{MARYLAND}

19 April (GS) Lancaster County, Pennsylvania Origin time: 045458.3

See Penusylvania listing.

23 April (GS) Lancaster County, Pennsylvania Origin time: (11 3000.1

See Pennsylvania listing.

\section{MISSOURI}

12 January (SL) Southestern Missouri

Origin time: 024815.7

Epicenter: $37.590 \mathrm{~N} ., 89.750 \mathrm{~W}$.

Depth: $2 \mathrm{~km}$

Magnitude: $3.0 M_{n}(\mathrm{SL})$

Intensity III: South of Perryville (SL).

28 January (SL) New Madrid area

Origin time: 212922.1

Epicenter: $36.610 \mathrm{~N} ., 89.920 \mathrm{~W}$.

Depth: $1 \mathrm{~km}$

Magnitude: $3.2 M_{n}(\mathrm{SL})$

Intensity IV: Gideon and Malden (SL).

14 February (SL) Western Kentucky

Origin time: 225610.4

See Kentucky listing.

29 June (SL) Southern Illinois

Origin time: 075829.3

See Illinois listing.

30 July (SL) Southeastern Missouri

Origin time: 073346.5

Epicenter: 37.828 N., $90.921 \mathrm{~W}$.

Depth: $7 \mathrm{~km}$

Magnitude: $3.0 M_{n}(\mathrm{SL})$

Felt:

Illinois Carbondale (SL).

Missouri-- Bonne Terre, Farmington, and Flat River (SL).
3 December (SL) New Madrid area

Origin time: 115544.5

Epicenter: $36.150 \mathrm{~N} ., 89.700 \mathrm{~W}$.

Depth: $12 \mathrm{~km}$

Magnitude: $3.0 M_{n}(\mathrm{SL})$

Intensity IV:

Missouri-- Cooter.

Intensity III:

Missouri-- Gobler.

Tennessee - Miston.

Felt:

Missouri-Caruthersville (SL).

\section{MONTANA}

16 January (GS) Central Montana

Origin time: 195025.2

Epicenter: $47.149 \mathrm{~N} ., 110.845 \mathrm{~W}$.

Depth: $5 \mathrm{~km}$

Magnitude: $3.3 M_{D}(\mathrm{BU})$

Intensity IV: Monarch.

Intensity III: Belt, Buffalo, Raynesford

Intensity II: Black Eagle.

19 January (GS) Western Montana

Origin time: 072542.9

Epicenter: 47.435N., 115.503W.

Depth: $1 \mathrm{~km}$

Magnitude: $2.5 M_{L}(\mathrm{GS}), 2.6 M_{D}(\mathrm{BU})$

This event may be a rockburst.

Felt:

Idaho- Mullan, Wallace, and the Lucky Friday Mine (telephone report).

11 February (PG) British Columbia-Alberta, Canada border

Origin time: 133827.0

Epicenter: $49.210 \mathrm{~N} ., 114.340 \mathrm{~W}$.

Depth: $18 \mathrm{~km}$

Magnitude: $4.5 m_{l},(\mathrm{GS})$

Felt over an area of approximately 200,000 kilometers ${ }^{2}$ of the United States and Canada. The intensity values in Canada shown on figure 23 were taken from Canadian Earthquakes, National Summary, AprilJune 1984, Seismological Service of Canada, Ottawa.

Intensity V:

Montana-

East Glacier Park - A few dishes broke; a few small objects overturned and fell; hanging pictures swung out of place; felt by many.

Earthquake Descriptions 69 
Eureka--Standing vehicles rocked slightly; a few small objects overturned and fell; walls creaked; felt by all.

Kalispell- A few small objects overturned and fell; walls creaked; awakened several; felt by many.

Libby - Trees and bushes shook slightly; a few dishes broke; a few small objects overturned and fell; hanging pictures swung out of place; felt by many.

Ronan-Plaster walls sustained hairline cracks; a few items shook off of store shelves; a few dishes broke; a few small objects overturned and fell; felt by many.

Intensity IV:

Idaho-- Cocolalla, Dover, Tensed.

Montana- Big Arm, Bigfork, Condon, Cut Bank, Dutton, Essex, Ethridge, Fortine, Heart Butte, Heron, Hungry Horse, Kevin, Kila, Lakeside, Marion, Oilmont, Olney, Pendroy, Polebridge, Rexford, Rollins, Santa Rita, Stryker, Sunburst, Trego, Troy, Valier, West Glacier.

\section{Intensity III:}

Idaho-Athol, Bayview, Calder, Clark Fork, Colburn, Coolin, Eastport, Medimont, Moyie Springs, Naples, Nordman, Ponderay, Sagle, Saint Maries, Sandpoint, Smelterville.

Montana-- Arlee, Belt, Brady, Browning, Bynum, Cascade, Charlo, Chester, Choteau, Clinton, Columbia Falls, Creston, Dixon, Elmo, Galata, Great Falls, Ledger, Lonepine, Lothair, Malmstrom Air Force Base, Moiese, Monarch, Niarada, Noxon, Pablo, Plains, Polson, Power, Proctor, Saint Regis, Shonkin, Sweetgrass, Thompson Falls, Whitefish.

Washington- Boyds, Cusick, Electric City, Elk, Ford, Four Lakes, Marcus, Newport, Spokane (Parkwater), Steptoe, Tekoa, Usk.

Intensity II:

Idaho- Careywood, Laclede, Orofino, Post Falls, Worley.

Washington-Spokane, Spokane (Hayes Park).

Felt:

Idaho- Kootenai, Priest River (press report), Wallace (press report).

Montana- Coram, Saint Ignatius (press report), Shelby (press report).

28 February (GS) Western Montana

Origin time: 003133.5

Epicenter: 46.184N., 111.497W.

Depth: $5 \mathrm{~km}$

Magnitude: $3.2 M_{L}(\mathrm{BU})$

Felt: Toston.

28 February (GS) Western Montana Origin time: 042511.4
Epicenter: 46.179 N., $111.460 \mathrm{~W}$

Depth: $5 \mathrm{~km}$

Magnitude: $3.8 M_{L}(\mathrm{BU}), 3.2 M_{D}(\mathrm{UU})$

Intensity V: Toston- A few dishes and glass items broke; a few small objects overturned and fell; buildings shook strongly; windows and dishes and rattled; felt by many.

Intensity IV: Big Sky, Radersburg.

Intensity III: McAllister, Three Forks, Willow Creek.

24 March (BU) Western Montana

Origin time: 210631.8

Epicenter: 46.547N., $112.095 \mathrm{~W}$

Depth: $11 \mathrm{~km}$

Magnitude: $3.1 M_{L}(\mathrm{BU})$

Intensity III: Helena.

7 May (GS) Southwestern Montana

Origin time: 205716.9

Epicenter: $44.662 \mathrm{~N} ., 111.851 \mathrm{~W}$.

Depth: $5 \mathrm{~km}$

Magnitude: $4.0 M_{L}$ (GS), $4.1 M_{L}(\mathrm{BU})$

Intensity III: Lima.

29 May (GS) Northeastern Wyoming Origin time: 201832.6

See Wyoming listing.

22 August (GS) Central Idaho

Origin time: 094630.2

See Idaho listing.

8 September (GS) Northeastern Wyoming Origin time: 005931.1

See Wyoming listing.

8 September (GS) Central Idaho

Origin time: 061640.1

See Idaho listing.

18 October (GS) Eastern Wyoming

Origin time: 153023.0

See Wyoming listing.

\section{NEBRASKA}

18 October (GS) Eastern Wyoming

Origin time: 153023.0

See Wyoming listing. 
14 January (BK) Bishop area

Origin time: 004223.6

See California listing.

31 January (EN) Southern Nevada

Origin time: 153000.084

Epicenter: $37.113 \mathrm{~N} ., 116.122 \mathrm{~W}$.

Depth: $0 \mathrm{~km}$

Magnitude: $4.1 m_{l}$ (GS), $4.4 M_{L}(\mathrm{BK})$

Nevada Test Site explosion "AGRINI" at $37^{\prime \prime} 08^{\circ}$ $47.18^{\prime \prime}$ N., $116^{\prime \prime} 05^{\prime} 02.62$ "W., surface elevation 1331 meters, depth of burial 564 meters.

15 February (EN) Southern Nevada

Origin time: 170000.105

Epicenter: $37.221 \mathrm{~N} ., 116.181 \mathrm{~W}$.

Depth: $0 \mathrm{~km}$

Magnitude: $5.0 m_{l},(\mathrm{GS}), 4.8 M_{L}(\mathrm{BK})$

Nevada Test Site explosion "MIDAS MYTH" at $37^{\prime \prime} 13^{\prime} 16.98^{\prime \prime} \mathrm{N}$., $116^{\prime \prime} 10^{\prime} 51.95^{\prime \prime} \mathrm{W}$., surface elevation 2076 meters, working elevation 1715 meters, tunnel shot.

16 February (GS) Western Nevada

Origin time: 111457.5

Epicenter: 39.933N., 117.759W.

Depth: $8 \mathrm{~km}$

Magnitude: $4.8 m_{l}(\mathrm{GS}), 5.2 M_{L}(\mathrm{BK})$

Intensity IV: Austin, Lovelock.

Intensity III: Fernley, Winnemucca.

Felt: Fallon (press report).

17 February (BK) Western Nevada

Origin time: 120356.2

Epicenter: $38.880 \mathrm{~N} ., 119.637 \mathrm{~W}$.

Depth: $25 \mathrm{~km}$

Magnitude: $4.1 m_{l},(\mathrm{GS}), 4.3 M_{L}(\mathrm{BK}), 4.5 M_{D}(\mathrm{GM})$,

$$
M_{1}=3.5 \mathrm{E} 22(\mathrm{BK})
$$

Intensity V:

California-

Topaz - A few small objects fell; wall creaked; windows rattled; vibration was described as strong; felt by and awakened many.

Nevada--

Carson City- A few small objects overturned and fell; hanging pictures swung out of place; windows rattled; awakened few.

Minden - A few small objects overturned; a few items shook off of store shelves; hanging pictures swung out of place; windows rattled; vibration was described as moderate; awakened many.
Wellington - A few small objects overturned and fell; hanging pictures swung out of place; windows rattled; vibration was described as moderate; awakened few.

Intensity IV:

California - Kyburz, South Lake Tahoe, Wilseyville. Nevada- Gardnerville (press report), Smith Valley.

Intensity III:

California - Avery (White Pines Subdivision), Camino, Kirkwood, Pioneer.

Intensity II:

Nevada-- Genda, Silver City.

Felt:

California - Coleville.

Nevada- Yerington (RN).

17 February (BK) Western Nevada

Origin time: 121646.6

Epicenter: $38.902 \mathrm{~N} ., 119.608 \mathrm{~W}$.

Depth: $25 \mathrm{~km}$

Magnitude: $3.6 M_{L}(\mathrm{BK}), 3.5 M_{D}(\mathrm{GM})$

Felt: Lake Tahoe area (BK).

19 February (GS) Central Nevada

Origin time: 102241.2

Epicenter: $39.976 \mathrm{~N} ., 117.825 \mathrm{~W}$.

Depth: $10 \mathrm{~km}$

Magnitude: $4.0 M_{L}(\mathrm{GS})$

Intensity IV: Fallon.

1 March (EN) Southern Nevada

Origin time: 174500.094

Epicenter: $37.066 \mathrm{~N} ., 116.046 \mathrm{~W}$.

Depth: $0 \mathrm{~km}$

Magnitude: $5.9 m_{l},(\mathrm{GS}), 4.4 M_{s}(\mathrm{GS}) 5.5 M_{L}(\mathrm{BK})$

Nevada Test Site explosion "TORUGAS" at $37^{\prime \prime} 03$ ' $56.76^{\prime \prime} \mathrm{N}$., $116^{\prime \prime} 02^{\prime} 46,66^{\prime \prime} \mathrm{W}$., surface elevation 1243 meters, depth of burial 369 meters.

\section{March (RN) Central Nevada}

Origin time: 124652.4

Epicenter: $38.819 \mathrm{~N} ., 118.007 \mathrm{~W}$.

Depth: $13 \mathrm{~km}$

Magnitude: $2.6 M_{L}(\mathrm{RN})$

Felt: Gabbs (RN).

\section{March (EN) Southern Nevada}

Origin time: 143000.084

Epicenter: $39.146 \mathrm{~N}, 116.084 \mathrm{~W}$.

Depth: $0 \mathrm{~km}$

Magnitude: $4.1 m_{b}(\mathrm{GS}), 4.2 M_{L}(\mathrm{BK})$ 
Nevada Test Site explosion "AGRINI" at $37^{\prime \prime} 08$ ' 47.18 " N., $116^{\prime \prime} 05^{\prime} 02.62^{\prime \prime} \mathrm{W}$., surface elevation 1331 meters, depth of burial 320 meters.

\section{April (BK) Ceniral California}

Origin time: 211519.0

See California listing.

\section{May (EN) Southern Nevada}

Origin time: 190500.093

Epicenter: $37.106 \mathrm{~N}$., $116.022 \mathrm{~W}$.

Depth: $0 \mathrm{~km}$

Magnitude: $5.3 m_{b}$ (GS), 4.2Ms (GS), $5.3 M_{L}(\mathrm{BK})$

Nevada Test Site explosion "MUNDO" at 37"06" $22.40^{\prime \prime}$ N., $116^{\prime \prime} 01^{\prime 2} 20.58^{\prime \prime} \mathrm{W}$., surface elevation 1319 meters, depth of burial 567 meters.

\section{May (GS) Southern Nevada}

Origin time: 200648.0

Epicenter: $37.085 \mathrm{~N}$., $115.966 \mathrm{~W}$.

Depth: $0 \mathrm{~km}$

Magnitude: $4.2 m_{l}$ (GS)

Collapse from explosion "MUNDO" listed above.

\section{May (EN) Southern Nevada}

Origin time: 130400.102

Epicenter: $37.103 \mathrm{~N}$., $116.048 \mathrm{~W}$.

Depth: $0 \mathrm{~km}$

Magnitude: $5.8 m_{l},(\mathrm{GS}), 4.1 M_{s}(\mathrm{GS}), 5.6 M_{L}(\mathrm{BK})$

Nevada Test Site explosion "CAPROCK" at 37"06" $11.28^{\prime \prime} \mathrm{N}$., 116"02'52.97"W., surface elevation 1264 meters, depth of burial 600 meters.

20 June (EN) Southern Nevada

Origin time: 151500.089

Epicenter: 37.000N., 116.043W.

Depth: $0 \mathrm{~km}$

Magnitude: $4.6 m_{t}$ (GS), $4.5 M_{L}(\mathrm{BK})$

Nevada Test Site explosion "DUORO" at $37^{\prime} 00$ " 01.36 " N., 116"02'35.01"W., surface elevation 1194 meters, depth of burial 381 meters.

\section{June (GS) Southern Nevada}

Origin time: 161000.4

Epicenter: $37.002 \mathrm{~N} ., 116.031 \mathrm{~W}$.

Magnitude: $4.2 m_{l}$ (GS), $4.1 M_{L}(\mathrm{BK})$

Collapse from explosion "DUORO" listed above.

\section{July (EN) Southern Nevada}

Origin time: 153000.084

Epicenter: $37.268 \mathrm{~N} ., 116.411 \mathrm{~W}$.
Depth: $0 \mathrm{~km}$

Magnitude: $5.3 m_{l},(\mathrm{GS}), 5.2 M_{L}(\mathrm{BK})$

Nevada Test Site explosion "KAPPELI" at 37"16' $03.96^{\prime \prime}$ N., $116^{\prime \prime} 24^{\prime} 38.31$ "W., surface elevation 2010 meters, depth of burial 640 meters.

2 August (EN) Southern Nevada

Origin time: 150000.094

Epicenter: $37.017 \mathrm{~N} ., 116.008 \mathrm{~W}$.

Depth: $0 \mathrm{~km}$

Magnitude: $4.7 m_{l},(\mathrm{GS}), 4.4 M_{L}(\mathrm{BK})$

Nevada Test Site explosion "CORREO" at $37^{\prime \prime} 01$ ' 01.50 " N., $116^{\prime \prime} 00$ '27.52" W., surface elevation 1209 meters, depth of burial 335 meters.

19 August (GS) Western Nevada

Origin time: 022234.2

Epicenter: $38.356 \mathrm{~N} ., 118.062 \mathrm{~W}$.

Depth: $5 \mathrm{~km}$

Magnitude: $3.8 m_{l},(\mathrm{GS}), 4.1 M_{L}(\mathrm{BK}), 4.3 M_{L}(\mathrm{PS})$

Intensity V: Luning- A few small objects overturned and fell; hanging pictures were out of place; walls creaked; windows rattled; felt by several.

Intensity IV: Mina.

30 August (EN) Southern Nevada

Origin time: 144500.102

Epicenter: 37.090 N., $115.998 \mathrm{~W}$.

Depth: $0 \mathrm{~km}$

Magnitude: $4.5 m_{l},(\mathrm{GS}), 4.3 M_{L}(\mathrm{BK})$

Nevada Test Site explosion "DOLCETTO" at $37^{\prime \prime} 05$ ' 23.26"N., $115^{\prime \prime} 599^{\prime} 52.73$ "W., surface elevation 1318 meters, depth of burial 366 meters.

13 September (EN) Southern Nevada

Origin time: 140000.0

Epicenter: 37.087 N., $116.071 \mathrm{~W}$.

Depth: $0 \mathrm{~km}$

Magnitude: $5.0 m_{l}(\mathrm{GS}), 5.0 M_{L}(\mathrm{BK})$

Nevada Test Site explosion "BRETON" at 37"05" $12.07^{\prime \prime}$ N., $116^{\prime \prime} 04^{\prime} 16.23^{\prime \prime} \mathrm{W}$., surface elevation 1265 meters, depth of burial 483 meters.

13 September (GS) Southern Nevada

Origin time: 210136.7

Epicenter: $37.073 \mathrm{~N} ., 116.097 \mathrm{~W}$.

Depth: $0 \mathrm{~km}$

Magnitude: $4.3 m_{l},(\mathrm{GS})$

Collapse from explosion "BRETON" listed above.

21 October (BK) Western Nevada

Origin time: 145133.8 
Epicenter: $38.810 \mathrm{~N} ., 119.297 \mathrm{~W}$.

Depth: $16 \mathrm{~km}$

Magnitude: $3.9 M_{L}(\mathrm{BK}), 3.8 M_{D}(\mathrm{GM})$

Intensity IV: Smith, Wellington, Yerington.

21 October (BK) Western Nevada

Origin time: 145511.8

Epicenter: $38.830 \mathrm{~N} ., 119.317 \mathrm{~W}$.

Depth: $6 \mathrm{~km}$

Magnitude: $3.5 M_{L}(\mathrm{BK}), 3.6 M_{D}(\mathrm{GM})$

Felt: Yerington (press report).

22 October (GS) Western Nevada

Origin time: 112740.7

Epicenter: 39.967 N., 119.479W.

Depth: $5 \mathrm{~km}$

Magnitude: $3.9 M_{L}(\mathrm{BK}), 3.3 M_{D}(\mathrm{GM})$

Intensity IV: Sutcliffe (press report).

Intensity III: Nixon.

10 November (EN) Southern Nevada

Origin time: 164000.089

Epicenter: $37.000 \mathrm{~N} ., 116.017 \mathrm{~W}$.

Depth: $0 \mathrm{~km}$

Magnitude: $4.5 m_{l}$ (GS), $4.4 M_{L}$ (BK)

Nevada Test Site explosion "VILLITA" at $37 " 40$ ' 00.36 "N., $116^{\prime \prime} 01$ '02.55"W., surface elevation 1205 meters, depth of burial 373 meters.

23 November (BK) Owens Valley area

Origin time: 180825.5

See California listing.

26 November (BK) Owens Valley area

Origin time: 162147.2

See California listing.

4 December (GS) Southern Nevada

Origin time: 205306.3

Epicenter: $36.091 \mathrm{~N} ., 115.069 \mathrm{~W}$.

Depth: $5 \mathrm{~km}$

Magnitude: $2.9 M_{L}$ (GS)

Intensity III: Henderson (press report), East Las Vegas, Las Vegas (eastern part of the city).

9 December (EN) Southern Nevada

Origin time: 194000.089

Epicenter: $37.270 \mathrm{~N} ., 116.498 \mathrm{~W}$.

Depth: $0 \mathrm{~km}$

Magnitude: $5.5 m_{1}$ (GS), $5.4 M_{L}(\mathrm{BK})$

Nevada Test Site explosion "EGMONT" at $37^{\prime \prime} 16$ ' $12.28 "$ N., $116^{\prime \prime} 29^{\prime} 51.28$ "W., surface elevation 1867 me- ters, depth of burial 551 meters.

15 December (EN) Southern Nevada

Origin time: 144500.0

Epicenter: $37.281 \mathrm{~N} ., 116.305 \mathrm{~W}$.

Depth: $0 \mathrm{~km}$

Magnitude: $5.4 m_{1},(\mathrm{GS}), 5.4 M_{L}(\mathrm{BK})$

Nevada Test Site explosion "TIERRA" at 37 " 16 " 52.05 " N., 116"18'19.48"W., surface elevation 2145 meters, depth of burial 640 meters.

\section{NEW JERSEY}

23 April (GS) Lancaster County, Pennsylvania Origin time: 013600.1

See Pennsylvania.

NEW MEXICO

26 August (GS) Central New Mexico

Origin time: 021954.0

Epicenter: $34.310 \mathrm{~N}$., $106.800 \mathrm{~W}$.

Depth: $5 \mathrm{~km}$

Magnitude: $2.9 M_{L}$ (GS), $2.6 M_{D}$ (LA)

Felt: La Joya and Contreras (NM).

\section{NEW YORK}

23 April (GS) Lancaster County, Pennsylvania Origin time: 013600.1

See Pennsylvania listing.

20 August (LD) Northeastern New York

Origin time: 105817.0

Epicenter: $44.875 \mathrm{~N}$., $73.480 \mathrm{~W}$.

Depth: $11 \mathrm{~km}$

Magnitude: $3.2 M_{D}(\mathrm{LD})$

Intensity $\mathrm{V}$ :

New York -

Chazy - A few glassware items broke; a few small objects overturned and fell; a few items shook off of store shelves; windows rattled; trees and bushes shook slightly; felt by many.

\section{Vermont-}

Isle La Motte- Plaster and sheetrock walls sustained hairline cracks; cracked chimney; trees and bushes shook slightly; standing and moving vehicles rocked slightly; buildings shook slightly; windows rattled; felt by and awakened several. 


\section{Intensity IV:}

New York - Altona, Beekmantown, Dannemora, Mooers, Rouses Point, West Chazy.

Intensity III:

New York - Champlain, Mooers Forks, Plattsburgh, Schuyler Falls.

Intensity II:

New York - Ellenburg Center, Redford.

Vermont-Grand Isle.

23 October (LD) Eastern New York

Origin time: 062621.5

Epicenter: 43.592N., 73.937W.

Depth: $1 \mathrm{~km}$

Magnitude: $3.2 M_{n}(\mathrm{GS}), 3.4 M_{n}(\mathrm{LD})$

Most of the questionnaires containing reports of earth noise termed it moderate to loud.

Intensity IV: Athol, Bakers Mills, Bolton Landing, Brant Lake, Chestertown (Loon Lake), Cleverdale, Corinth, Glens Falls, Johnsburg, Lake Luzerne, Minerva, Newcomb, North Creek, North River, Northville, Pottersville, Riparius, Sabael, Schroon Lake, Speculator, Stony Creek, Warrensburg, Weverton.

Intensity III: Adirondack, Chestertown, Diamond Point, Dresden, Hadley, Hague, Indian Lake, Kattskill Bay, Lake Pleasant, Minerva, Northumberland, Northville, Porter Corners, Saratoga Springs, Silver Bay, South Schroon, Wells, Wilton.

Heard: Crown Point, Lake George, Queensbury, Sabbath Day Point.

\section{NORTH CAROLINA}

\section{October (TC) Western North Carolina}

Origin time: 185841.8

Epicenter: $36.364 \mathrm{~N} ., 81.677 \mathrm{~W}$.

Depth: $11 \mathrm{~km}$

Magnitude: $3.1 M_{n}(\mathrm{VP}), 3.2 M_{D}(\mathrm{TC})$

Felt in Ashe, Avery, and Watauga Counties in North Carolina, and in Johnson County, Tennessee.

Intensity V:

North Carolina-

Boone- A few small objects overturned and fell; hanging pictures were out of place; buildings shook slightly; windows rattled; felt by many.

Creston - Plaster walls sustained hairline cracks; a few items shook off of store shelves; a few dishes or glassware items broke; a few small objects overturned and fell; hanging pictures were out of place; build- ings shook slightly; windows rattled; trees and bushes shook slightly; standing and moving vehicles rocked slightly; vibration was described as strong; felt by many.

Vilas-A few small objects overturned and fell; trees and bushes shook slightly; buildings shook slightly; walls creaked; windows rattled; vibration was described as strong; felt by many.

Zionville- A few small objects overturned and fell; trees and bushes shook slightly; pictures swung; buildings shook slightly; walls creaked; windows rattled; felt by many.

Tennessee--

Trade- A few windows cracked; a few items shook off of store shelves; a few dishes or glassware items broke; a few small objects overturned and fell; buildings shook slightly; walls creaked; windows rattled; felt by all.

Intensity IV:

North Carolina- Blowing Rock, Fleetwood, Sugar Grove, Triplett, Todd, Warrensville.

Intensity III:

North Carolina - Deep Gap, Valle Crucis, West Jefferson.

Intensity II:

North Carolina - Lansing.

\section{OHIO}

14 January (GS) Northern Ohio

Origin time: 201431.2

Epicenter: $41.645 \mathrm{~N}, 83.427 \mathrm{~W}$.

Depth: $5 \mathrm{~km}$

Magnitude: $2.5 M_{D}(\mathrm{MI}), 3.0 M_{n}(\mathrm{EP})$

Intensity IV: Harbor View, Oregon.

Intensity III: Walbridge.

Felt: Eastern part of Toledo.

OKLAHOMA

6 January (TU) Northeastern Oklahoma

Origin time: 171449.8

Epicenter: $36.161 \mathrm{~N} ., 95.582 \mathrm{~W}$.

Depth: $5 \mathrm{~km}$

Magnitude: $2.5 M_{D}(\mathrm{TU})$

Intensity IV: Five kilometers west of Inola (TU).

Felt: Inola.

24 January (TU) Central Oklahoma

Origin time: 153409.6

Epicenter: $35.033 \mathrm{~N}$, $96.366 \mathrm{~W}$.

Depth: $5 \mathrm{~km}$

74 U.S. Earthquakes, 1984 
Magnitude: $2.8 M_{n}(\mathrm{TU})$

Intensity V: Holdenville (TU).

3 February (TU) Central Oklahoma

Origin time: 043828.0

Epicenter: 34.665 N., $97.356 \mathrm{~W}$.

Depth: $5 \mathrm{~km}$

Magnitude: $3.2 M_{n}$ (TU), $3.2 M_{n}$ (GS), 3.1 $M_{D}$ ((TC)

Intensity V: Thirteen kilometers north of Elmore City (TU).

Intensity IV: Elmore City (TU).

Intensity III: Pauls Valley.

Intensity II: Maysville (TU).

10 February (TU) Central Oklahoma

Origin time: 183913.6

Epicenter: 34.049 N., $97.415 \mathrm{~W}$.

Depth: $5 \mathrm{~km}$

Magnitude: $2.2 M_{D}(\mathrm{TU})$

Intensity IV: One kilometer north of seismograph station WLO, Love County (TU).

\section{March (TU) Central Oklahoma}

Origin time: 114202.4

Epicenter: $35.514 \mathrm{~N} ., 96.301 \mathrm{~W}$.

Depth: $5 \mathrm{~km}$

Magnitude: $2.6 M_{n}(\mathrm{TU})$

Intensity V: Castle and Okemah (TU).

20 November (TU) Southern Oklahoma

Origin time: 105732.0

Epicenter: 34.707 N., 97.410W.

Depth: $5 \mathrm{~km}$

Magnitude: $3.1 M_{n}(\mathrm{TU})$

Intensity IV: Antioch (TU).

Intensity III: Elmore City (TU).

Felt: Paoli (TU).

\section{OREGON}

31 January (GS) Northeastern Oregon

Origin time: 052915.6

Epicenter: 45.579N., $116.571 \mathrm{~W}$.

Depth: $5 \mathrm{~km}$

Magnitude: $3.5 M_{L}(\mathrm{GS}), 4.1 M_{L}(\mathrm{BU})$

Intensity IV:

Idaho- Lucile, Pollock.

Oregon- Imnaha.

Intensity III:

Idaho- Riggins, White Bird.

Washington-Clarkston.

30 July (GM) Southern Oregon

Origin time: 213904.5
Epicenter: $42.150 \mathrm{~N} ., 121.877 \mathrm{~W}$.

Depth: $8 \mathrm{~km}$

Magnitude: $3.5 M_{D}(\mathrm{GM}), 2.9 M_{L}(\mathrm{BK})$

Intensity IV: Klamath Falls (OS).

10 August (GS) Northeastern Oregon

Origin time: 072638.3

Epicenter: 44.986N., 116.952W.

Depth: $5 \mathrm{~km}$

Magnitude: $3.5 M_{L}(\mathrm{GS}), 4.0 M_{L}(\mathrm{BU})$

Intensity IV: Halfway.

Felt: Richland.

22 August (GS) Central Idaho

Origin time: 094630.2

See Idaho listing.

8 September (GS) Central Idaho

Origin time: 061640.1

See Idaho listing.

10 September (GS) Northern California

Origin time: 031410.1

See California-off-the-coast listing.

19 September (GS) Western Idaho

Origin time: 013206.9

See Idaho listing.

11 December (WA) Northwestern Oregon

Origin time: 063449.9

Epicenter: 45.469N., 122.829W.

Depth: $22 \mathrm{~km}$

Magnitude: $2.5 M_{D}$ (WA)

Felt: Portland (West Slope).

\section{PENNSYLVANIA}

19 April (GS) Lancaster County, Pennsylvania

Origin time: 045458.3

Epicenter: $39.923 \mathrm{~N} ., 76.316 \mathrm{~W}$.

Depth: $5 \mathrm{~km}$

Magnitude: $3.0 M_{n}(\mathrm{VP}), 2.9 M_{D}(\mathrm{LD})$

Intensity $\mathrm{V}$ :

Airville- A few items shook off of store shelves; a few dishes or glassware items broke; a few small objects overturned and fell; buildings shook slightly; windows and dishes rattled; felt by and awakened many.

East Petersburg- A few windows cracked; a few dishes or glassware items broke; a few small objects overturned and fell; awakened several. 


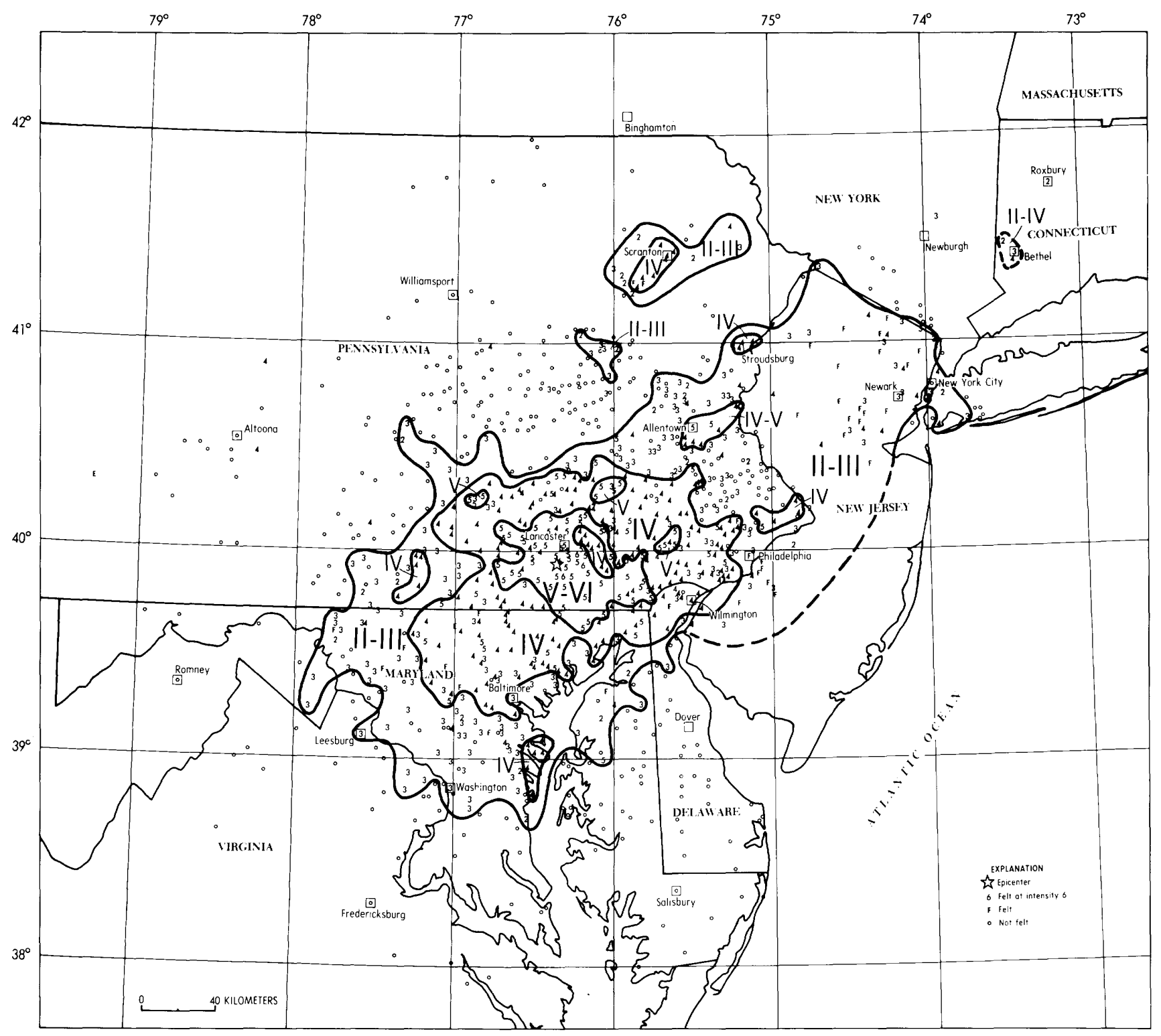

Figure 24. Isoseismal map for the southeastern Pennsylvania earthquake of 23 April 1984, 013600.1 UTC. Roman numerals represent Modified Mercalli intensities between isoseismals; Arabic numerals represent intensities at specific sites. 
Holtwood- Motor vehicles rocked slightly; a few windows cracked; a few dishes or glassware items broke; a few items shook off of store shelves; a few small objects overturned and fell; buildings shook strongly; felt by and awakened many.

New Providence- A few windows cracked; hanging pictures swung; buildings shook slightly; windows and dishes rattled; felt by many and awakened few.

Red Lion - A few items shook off of store shelves; a few dishes or glassware items broke; a few small objects overturned and fell; hanging pictures swung; awakened several.

Reinholds- Plaster walls sustained hairline cracks; motor vehicles rocked; a few items shook off of store shelves; a few windows cracked; a few small objects overturned and fell; hanging pictures swung out of place; windows and dishes rattled.

\section{Intensity IV:}

Pennsylvania - Bausman (a few small objects fell), Conestoga, Dallastown, East Prospect, Fawn Grove, Lancaster, Mount Gretna, Mount Joy (a few items were shaken off store shelves), Mountville, Neffsville, Peach Bottom, Quentin, Refton, Rohrerstown, Ronks, Stewartstown, Strasburg, Washington Boro, Wrightsville.

Intensity III:

Maryland-- Freeland, Pylesville, Whiteford, Whitehall.

Pennsylvania - Bart, Colebrook, Cornwall, Delta, Drumore, Elm, Intercourse, Leola, Lititz, Maytown, Millersville, New Park, Parkesburg, Penryn, Quarryville, West Willow, Windsor.

Intensity II:

Pennsylvania-- Gap, Railroad, Seven Valleys.

\section{April (GS) Southeastern Pennsylvania}

Origin time: 013600.1

Epicenter: $39.921 \mathrm{~N} ., 76.355 \mathrm{~W}$.

Depth: $5 \mathrm{~km}$

Magnitude: $4.2 m_{1}$ (GS), $4.1 M_{n}(\mathrm{GS}), 4.4 M_{n}(\mathrm{TU})$,

$$
\text { 4.1 } M_{D}(\mathrm{LD})
$$

This earthquake was reported felt in eight States and the District of Columbia. Most of the felt area was in the States of Pennsylvania, Maryland, and New Jersey with Connecticut, Delaware, New York, Virginia, and West Virginia reporting it felt in a few localities. This event generated a high level of sound that was emphasized in many of the reports (and described as being similiar to an explosion or a loud clap of thunder (Scharnberger and Howell, 1985). It was felt over approximately 49,000 kilometers ${ }^{2}$ (fig. 24). The af- tershocks were located during the period of April 24 through April 28, 1984 (Armbruster and Seeber, 1987). Some of the data used to assign intensities in the epicentral region were furnished by $C$. K. Scharnberger, Millersville University, Pennsylvania.

\section{Intensity VI:}

Pennsylvania -

Conestoga - Plaster fell from the ceiling; concrete walls of a basement and cistern cracked; garage shifted $1 / 2$ inch off its foundation; crystals fell from a chandelier; a sidewalk cracked; a few glassware items broke; a few items shook off of store shelves; a few windows cracked; felt by and frightened many.

Lampeter- A foundation cracked; brick fences cracked; plaster walls sustained hairline cracks; a few items shook off of store shelves; a few dishes or glassware items broke; a few small objects overturned and fell; hanging pictures swung out of place.

Mount Nebo-- Plaster wall cracked.

New Providence-- A foundation cracked; interior walls sustained hairline cracks; moving vehicles rocked moderately; trees and bushes shook slightly; a few items shook off of store shelves; a basement floor cracked; a few dishes or glassware items broke; a few small objects overturned and fell; hanging pictures fell; felt by all and frightened many.

Intensity V: The most common effects at the places listed below: a few small objects overturned and fell; a few dishes broke; buildings shook slightly; windows rattled; vibration was described as moderate to strong; felt by many to all; frightened many; noise was described as moderate to loud. Additional effects are noted after each place listed below:

\section{Maryland--}

Benson- Dry wall sustained hairline cracks; hanging pictures swung out of place.

Boring - Buildings shook strongly.

Conowingo- Hanging pictures fell.

Darlington - Hanging pictures swung out of place; windows cracked.

Elk Mills- Hanging pictures were out of place; buildings shook strongly.

Elkton - Windows cracked.

Glenwood-Windows cracked.

Joppa- Windows cracked; buildings shook strongly.

Rising Sun-- Windows cracked.

Union Bridge - Standing vehicles rocked slightly.

Upperco.

Whiteford- Plaster walls sustained hairline cracks. 


\section{Pennsylvania-}

Airville- A foundation cracked; plaster walls sustained hairline cracks; moving vehicles rocked slightly; a few items shook off of store shelves; windows cracked.

Akron-Windows cracked.

Allentown-Windows cracked; a few items shook off of store shelves; trees and bushes shook slightly.

Bainbridge - A few items shook off of store shelves; hanging pictures were out of place.

Bausman-Shaking described as strong.

Bernville- Windows cracked.

Bethlehem-- Plaster walls sustained hairline cracks; hanging pictures were out of place.

Blue Ball.

Bridgeport- People ran out of their homes (press report).

Brogue- A few items shook off of store shelves.

Brownstown-Windows cracked.

Camp Hill- Felt while driving a car.

Chatham - Hanging pictures were out of place.

Chester Springs- Windows cracked; buildings shook strongly; hanging pictures were out of place.

Coatesville-Windows cracked.

Cochranville- People had difficulty standing.

Codorus-Windows cracked.

Columbia- Windows cracked; a few items shook off of store shelves; light furniture overturned.

Cornwall.

Craley- A few items shook off of store shelves.

Dallastown-Windows cracked; hanging pictures were out of place; trees and bushes shook moderately.

Delta- Plaster walls sustained hairline cracks.

Downingtown- A few items shook off of store shelves; trees and bushes shook slightly; vehicles rocked slightly; buildings shook strongly.

Drexel Hill.

East Greenville- Hanging pictures fell.

East Prospect- Standing vehicles rocked slightly; trees and bushes shook moderately; buildings shook strongly.

Ephrata- Standing and moving vehicles rocked slightly.

Exton.

Gap- A few items shook off of store shelves; buildings shook strongly.

Glenmoore-- Buildings shook strongly; hanging pictures were out of place.

Hamburg - A few items shook off of store shelves.
Harrisburg- There was cracked plaster in an apartment building at 2644 Green Street (press report).

Holtwood-Windows cracked; buildings shook strongly; a few items shook off of store shelves; vehicles rocked slightly.

Honey Brook - Plaster walls sustained hairline cracks.

Huntingdon Valley- A few items shook off of store shelves.

Kinzers- Plaster walls sustained hairline cracks; hanging pictures were out of place.

Kirkwood-Windows cracked.

Lancaster.

Landenberg - Windows cracked.

Landisville- Hanging pictures were out of place; standing vehicles rocked slightly; trees and bushes shook slightly.

Lebanon- Hanging pictures fell.

Lititz - Plaster walls sustained hairline cracks; windows cracked; vehicles rocked slightly; trees and bushes shook slightly.

Littlestown - A few items shook off of store shelves; hanging pictures were out of place.

Loganville- Plaster walls sustained hairline cracks; windows cracked; trees and bushes shook slightly.

Lyndon-Shook dishes off a rack (press report).

Manheim-A few items shook off of store shelves.

Marietta.

Marticville- Hanging pictures fell; a rafter was cracked; a few items shook off of store shelves.

Maytown.

Millersville.

Mohnton.

Morgantown- Vehicles rocked slightly; trees and bushes shook slightly.

Mount Joy-Trees and bushes shook moderately; a few items shook off of store shelves; hanging pictures were out of place; buildings shook strongly.

Mount Wolf- Windows cracked; trees and bushes shook slightly; vehicles rocked slightly.

Mountville.

Myerstown - Sidewalks cracked.

Neffsville-- Windows cracked; hanging pictures were out of place.

New Cumberland- A few items shook off of store shelves; trees and bushes shook slightly; vehicles rocked slightly.

New Holland- Windows cracked; plaster walls sustained hairline cracks; trees and bushes shook slightly; standing vehicles rocked slightly. 
New London- Plaster walls sustained hairline cracks; hanging pictures were out of place; trees and bushes shook slightly.

Newtown Square - Windows cracked; plaster walls sustained hairline cracks; a foundation cracked; trees and bushes shook slightly; a few items shook off of store shelves; hanging pictures were out of place; cracks formed in wet ground.

Oxford-Windows cracked; a few items shook off of store shelves.

Parkesburg- Plaster walls sustained hairline cracks; buildings shook strongly; trees and bushes shook slightly; standing vehicles rocked slightly; cracks formed in wet ground.

Peach Bottom-Windows cracked.

Pequea- Hanging pictures fell; a loose screen door fell from hinges.

Quarryville- Plaster walls sustained hairline cracks.

Rawlinsville - Cabinet and closed doors popped open; pendulum clocks stopped.

Red Lion-Windows cracked; a few items shook off of store shelves.

Refton- Hanging pictures were out of place.

Reinholds - A few items shook off of store shelves; plaster walls sustained hairline cracks; vehicles rocked slightly; trees and bushes shook slightly.

Smoketown - A few items shook off of store shelves; basement wall cracked (press report).

Strasburg- Plaster-board walls sustained hairline cracks; hanging pictures were out of place.

Wernersville- Windows cracked; plaster walls sustained hairline cracks; trees and bushes shook slightly; light furniture overturned.

West Grove - Trees and bushes shook moderately; vehicles rocked slightly to moderately.

West Lawn- Windows cracked; a few items shook off of store shelves.

West Willow- A few items shook off store shelves; hanging pictures were out of place; cabinet and closet doors opened.

Willow Street.

York- A few items shook off of store shelves; hanging pictures fell.

York Haven- Plaster walls sustained hairline cracks.

Intensity IV:

Connecticut--- Dunbury (press report).

Delaware- Bear, Centerville (press report), Claymont (press report), Hockessin, Montchanin, Newark, Wilmington, Winterthur, Yorklyn.
Maryland- Aberdeen Proving Ground, Arnold (press report), Ashton, Bel Air, Bentley Springs, Brooklyn Park (press report), Butler, Cape Saint Claire (press report), Childs, Colora, Cooksville, Detour, Edgewater (press report), Edgewood, Ellicott City, Fallston, Finksburg, Forest Hill, Fork, Freeland, Garrison, Gibson Island (press report), Glen Arm (press report), Glenelg, Hampstead, Havre De Grace, Ilchester, Jarrettsville, Kingsville (press report), Linthicum Heights, Long Green, Maryland Line, Millers, Mount Airy, New Windsor, North East, Odenton, Parkton, Perry Point, Perryville, Pylesville, Randallstown, Reisterstown, Severna Park, Shady Side, Smithsburg, Sparks Glencoe, Street, Taneytown, Towson (press report), Unionville (press report), Westminster (press report), White Hall, Woodstock.

New Jersey - Clifton (press report), Frankfort (press report), Gloucestar City (press report), Jersey City (press report), Oakland (press report), Passaic (press report), Penns Grove (press report), Pennsville (press report), South Plainfield (press report), Washington (press report), West Milford (press report), Woodbridge (press report).

New York - Manhattan Beach (press report), Washington Heights (press report).

Pennsylvania - Annville (press report), Aspers (press report), Aston, Atglen, Aboca, Avon, Bangor, Bart, Bendersville, Biglerville (press report), Bird In Hand, Birdsboro (press report), Blue Bell, Boiling Springs, Bowmansville, Boyertown (press report), Broomall, Bryn Mawr, Campbelltown, Chadds Ford, Chester (press report), Christiana, Clarks Summit, Colebrook, Conshohocken, Darby, Denver, Dillsburg, Dover, Drumore, East Petersburg, East Stroudsburg (press report), East Texas, East York, Easton, Edgemont, Elizabethtown, Elm, Emigsville, Emmaus, Exeter (press report), Fairfield, Fairless Hills (press report), Fawn Grove, Feasterville (press report), Flourtown, Flowing Spring (press report), Folcroft, Franklintown, Geigertown, Gettysburg (press report), Glen Rock, Glenolden, Goodville, Gordonville, Gradyville, Green Lane, Hanover (press report), Hatboro, Hellam, Hellertown, Hersey, Honesdale, Hopeland, Hummelstown (press report), Intercourse, Kelton, Kemblesville, Kennett Square, Kleinfeltersville, Laurys Station, Lawn, Lenhartsville, Lenni, Leola, Lewisberry, Lewisville, Limekiln, Limeport, Lincoln University, Linglestown, Lionville, Lower Providence (press report), Lyndell, Macungie, Malvern, Manchester, Martindale, Mechanicsburg, Mendenhall, Middletown, Modena, Morton, 
Narvon, New Freedom, New Park, Newbury (press report), Newmanstown, Ninepoints, Norristown, North York (press report), Nottingham, Olyphant, Palmyra, Paradise, Parker Ford, Perkiomenville (press report), Philipsburg (press report), Phoenixville, Pine Forge, Plains (press report), Plymouth Neeting, Pocopson, Porters Sideling, Potts Grove (press report), Pottstown (press report), Prospect Park, Pughtown (press report), Quentin, Railroad, Reading, Reamstown, Rexmont, Rheems, Richland, Richlandtown, Ridley Park, Robesonia, Ronks, Rose Valley (press report), Royersford (press report), Sadsburyville, Saint Peters, Salisbury Heights (press report), Schaefferstown, Scranton (press report), Seven Valleys, Shawnee On Delaware (press report), Shrewsbury, Silver Spring, Soudersburg, Spring City, Spring Grove, Stevens, Stewartstown, Stroudsburg (press report), Sumneytown, Talmage, Terre Hill, Thorndale, Toughkenamon, Trevose (press report), Valley Forge, Villanove, Wagontown, Washington Boro, Wellsville, West Chester, Westtown, Windsor, Witmer, Woodlyn, Wrightsville, Wyomissing, Yardley (press report), York New Salem.

\section{Intensity III:}

Connecticut - Bethel (press report).

District of Columbia- Washington.

Delaware-- Greenville (press report), New Castle, Odessa.

Maryland- Aberdeen, Abingdon, Adelphi, Baltimore, Beltsville, Braddock Heights, Bradshaw, Brookeville, Buckeystown, Burtonsville, Camp Springs, Cascade, Cavetown, Charlestown, Chase, Churchville, Clarksburg, Clinton, Crownsville, Davidsonville, Dayton, Emmitsburg, Friendship International Airport, Funkstown, Gambrills, Garrett Park, Glen Burnie, Glyndon, Hagerstown, Harmans, Highland, Hydes, Jefferson, Keymar, Libertytown, Lisbon, LuthervilleTimonium, Manchester, Maugansville, Middletown, Millington, Monrovia, Owings Mills, Pasadena, Perry Hall, Pikesville, Poolesville, Rock Hall, Rohrersville, Savage, Severn, Silver Spring, Simpsonville, Spencerville, Stevenson, Walkersville, West Friendship, West River.

New Jersey - East Newark (press report), Edison (press report), Lindenwold (press report), Mahwah (press report), Middlesex (press report), New Providence (press report), Newark (press report), Newton (press report), Pompton Lakes (press report), Stillwater (press report), Totowa (press report), Woodbury (press report), Woodstown.
New York - Brooklyn, New York, Pearl River (press report), Port Jervis, Tappan, Wappingers Falls, Woodmere.

Pennsylvania-- Abbottstown, Aquashicola, Arendtsville, Avondale, Bally, Bath, Bechtelsville, Birchrunville, Blandon, Bowers, Breinigsville, Bristol, Brodmall, Bryn Athyn, Chalfont, Chambersburg, Chester Heights, Clifton Heights, Collegeville, Conyngham, Coopersburg, Devault, Devon, Douglassville, Duncannon, Durham, East Berlin, East Earl, Elizabethville, Felton, Folsom, Gardners, Glenville, Grantham, Hawley, High Spire, Holmes, Immaculata, Leesport, Lehighton, Lemoyne, Lyon Station, Mary D, Marysville, Maxatawny, McSherrystown, Media, Milford Square, Minisink Hills, Mount Aetna, Mount Carmel, Mount Holly Springs, Nanticoke (press report), Narberth, Nazareth, Neffs, New Bloomfield, New Kingstown, New Oxford, Newport (press report), Oakland Mills, Old Zionsville, Oley, Orefield, Oreland, Palmerton, Penryn, Perkasie, Quakake, Rehrersburg, Riegelsville, Rouzerville, Salunga, Schnecksville, Scotland, Sellersville, Shoemakersville, Sinking Spring, Slatington, South Mountain (press report), Springtown, Steelton, Stockertown, Swarthmore, Sybertsville, Tatamy, Temple, Thomasville, Thornton, Topton, Treichlers, Walnutport, Wayne, Weston, Windgap, Womelsdorf, Worcester, Wyncote, York Springs, Zionsville.

Virginia- Fairfax, Falls Church, Leesburg, Vienna.

West Virginia- Charles Town, Kearneysville.

Intensity II:

Maryland-Chestertown, Clarksville, Earleville, Hyattsville, North Beach, Riva, Saint James.

New Jersey - Willingboro (press report).

New York - Flushing.

Pennsylvania - Barto, Cherryville, Dallas (press report), Danielsville, Lake Winola (press report), Lansdale, Mexico, Milnesville, Morrisville, Mount Cobb (press report), Nescopeck, New Tripoli, Orrtanna, Palm, Pipersville, Quincy, Trucksville (press report), Wilkes-Barre (press report).

Connecticut - New Fairfield (press report), Roxbury (press report).

Felt:

Delaware-- Marshallton.

Maryland - Annapolis Junction, Frederick (press report), Halfway (press report), Lineboro (press report), Mount Pleasant (press report), Still Pond, Sykesville (press report), Winfield (press report), Woodsboro (press report).

New Jersey - Andover (press report), Berkeley Heights (press report), Bernardsville (press report), 
Clementon (press report), Collingswood (press report), East Brunswick (press report), Fanwood (press report), Garfield (press report), Haddan (press report), High Bridge (press report), Holland (press report), Jefferson (press report), Lebanon (press report), Oaklyn (press report), Old Bridge (press report), Paramus (press report), Pequannock (press report), Pine Hill (press report), Piscataway (press report), Rahway (press report), Randolph (press report), Ringwood (press report), Roselle (press report), Scotch Plains (press report), Somerdale (press report), South Bound Brook (press report), South Plainfield (press report), Summit (press report), Trenton (press report), Wanaque (press report), Warren (press report).

New York- Westhampton (press report).

Pennsylvania-Ambler, Conewago Heights (press report), Dauphin (press report), Derry (press report), Haverford (press report), Inkerman (press report), Philadelphia (press report), Southampton (press report), Swoyersville (press report), Upper Darby (press report).

\section{May Lancaster County, Pennsylvania}

Origin time: 1015

Epicenter: Not located.

Depth: None computed.

Magnitude: None computed.

Intensity IV: Mount Nebo, Pequea, Truce, and Willow Street (press report).

\section{May Lancaster County, Pennsylvania}

Origin time: 0110

Epicenter: Not located

Depth: None computed.

Magnitude: None computed.

Intensity IV: Conestoga.

\section{September Southeastern, Pennsylvania}

Origin time: 1543

Epicenter: Not located.

Depth: None computed.

Magnitude: 1.7 Unknown (Millersville University)

Intensity IV: Lancaster, 780 Eden Road, and Lancaster Bible College, 901 Eden Road (press reports).

Intensity III: Manheim (press report).

\section{PUERTO RICO}

19 March (GS) Puerto Rico Trench

Origin time: 121446.9

Epicenter: $19.451 \mathrm{~N} ., 64.921 \mathrm{~W}$.

Depth: $14 \mathrm{~km}$

Magnitude: $5.0 m_{l},(\mathrm{GS}), 4.2 M_{s}(\mathrm{GS})$
Intensity IV: Isla Verde International

Airport.

Intensity III: Caguas, Morovis.

Intensity II: Guayama, Gurabo.

30 March (GS) Eastern Puerto Rico

Origin time: 145607.9

Epicenter $18.346 \mathrm{~N} ., 65.689 \mathrm{~W}$.

Depth: Normal

Magnitude: None completed.

Felt: Arecibo, San Juan.

5 April (GS) Puerto Rico Trench

Origin time: 015315.7

Epicenter: 19.397N., 64.888W.

Depth: Normal

Magnitude: $5.0 m_{l}$ (GS)

Intensity IV: Comerio, San Juan.

Intensity III: Aguas Buenas, Cayey, Guayama, Guaraquao.

Intensity II: Santurce.

6 April Puerto Rico

Origin time: 0928

Epicenter: Not located

Depth: None computed.

Magnitude: None computed.

Intensity III: Aibonito and Cayey (San Juan Observatory).

2 October (GS) Southern Puerto Rico

Origin time: 151324.0

Epicenter: $17.844 \mathrm{~N}$., $66.644 \mathrm{~W}$.

Depth: $18 \mathrm{~km}$

Magnitude: $4.8 m_{b},(\mathrm{GS})$

Intensity IV: Ponce.

Felt: Yauco (press report).

10 October (GS) Eastern Puerto Rico

Origin time: 222820.8

Epicenter: $18.217 \mathrm{~N} ., 65.745 \mathrm{~W}$.

Depth: Normal.

Magnitude: None computed.

Felt: Cidra (telephone report).

\section{SOUTH DAKOTA}

8 September (GS) Northeastern Wyoming

Origin time: 005931.1

See Wyoming listing.

18 October (GS) Eastern Wyoming

Origin time: 153023.0

See Wyoming listing. 
14 February (TC) Eastern Tennessee

Origin time: 205430.9

Epicenter: $36.125 \mathrm{~N} ., 83.737 \mathrm{~W}$.

Depth: $10 \mathrm{~km}$

Magnitude: $3.5 M_{D}(\mathrm{TC}), 3.6 M_{n}(\mathrm{VP})$

Intensity VI: Blaine - Some windows broke; a few buildings were reported damaged; buildings shook strongly; windows rattled; vibration was described as strong; felt by and frightened many.

Intensity V: New Market-A few windows cracked; a few small objects overturned; walls creaked; windows and dishes rattled; vibration was described as strong.

Intensity IV: Kodak, Mascot, Strawberry Plains.

Intensity III: Knoxville, Luttrell, Maynardville, Powder Springs.

Felt: Fountain City.

\section{March (TC) Eastern Tennessee}

Origin time: 232611.4

Epicenter: $35.832 \mathrm{~N}$., $84.050 \mathrm{~W}$.

Depth: $7 \mathrm{~km}$

Magnitude: $3.0 M_{L}$ (GS), $3.0 M_{D}$ (TC)

Intensity IV: Fountain City, Knoxville, Louisville, Rockford.

Intensity III: Alcoa, Knoxville (Burlington), Maryville.

Intensity II: Vonore.

26 June (SL) Western Tennessee

Origin time: 151519.9

Epicenter: $36.102 \mathrm{~N}$., 89.391W.

Depth: $12 \mathrm{~km}$

Magnitude: $3.2 M_{n}(\mathrm{SL})$

Intensity III: Dyersburg, Lenox, and Nauvoo (TC).

6 September (SL) Northwestern Tennessee

Origin time: 160608.1

Epicenter: $36.100 \mathrm{~N}$., $89.350 \mathrm{~W}$.

Depth: $9 \mathrm{~km}$

Magnitude: $2.9 M_{n}(\mathrm{SL})$

Felt: Brazil (SL) and Millsfield (TC).

9 October (TC) Northwestern Georgia

Origin Time: 115427.0

See Georgia listing.

22 October (TC) Western North Carolina

Origin Time: 185841.8

See North Carolina listing.

3 December (SL) New Madrid area

Origin time: 115544.5

See Missouri listing.
3 March (GS) Southern Texas

Origin time: 010326.5

Epicenter: 28.852N., 98.461W.

Depth: $5 \mathrm{~km}$

Magnitude: $3.8 M_{n}(\mathrm{GS}), 3.9 M_{n}(\mathrm{TU})$

Intensity V: Jourdanton- The press reported a concrete driveway at a home dropped 2 inches due to earth settlement; people ran out of their homes. Other effects: plaster walls sustained hairline cracks; a few items shook off of store shelves; vibration was described as strong; objects overturned or fell; buildings shook strongly; hanging pictures swung out of place; felt by many. Broken windows and cracked brick walls were also reported.

Intensity IV: Christine, Pleasanton, Poteet.

Intensity III: Leming.

3 March Southern Texas

Origin time: 0158

Epicenter: Not located.

Depth: None computed.

Magnitude: None computed.

Felt: Pleasanton.

25 March Southern Texas

Origin time 0445

Epicenter: Not located.

Depth: None computed.

Magnitude: None computed.

Intensity III: Pleasanton, Poteet.

8 August (GS) Central Texas

Origin time: 013127.3

Epicenter: 29.133N., 98.362W.

Depth: $5 \mathrm{~km}$

Magnitude: $3.0 M_{n}(\mathrm{GS}), 3.1 M_{n}(\mathrm{TU})$

Intensity IV: Pleasanton (press report).

\section{UTAH}

21 March (UU) Central Utah

Origin time: 111930.4

Epicenter: 39.344N., 111.109W.

Depth: $0 \mathrm{~km}$

Magnitude: $3.5 M_{L}$ (GS), $2.9 M_{L}$ (UU)

Felt: Epicentral area (UU).

16 August (UU) Central Utah

Origin time: 141921.7

Epicenter: $39.392 \mathrm{~N}$., 111.936W.

Depth: $6 \mathrm{~km}$

Magnitude: $3.7 M_{L}(\mathrm{UU})$

Intensity IV: Ephraim, Fayette, Gunnison, Levan, Manti. 
Intensity III: Chester, Leamington, Moroni, Oak City, Scipio, Wales.

\section{August (GS) Central Idaho}

Origin time: 094630.2

See Idaho listing.

\section{October (UU) Northwestern Utah}

Origin time: 232356.5

Epicenter: $41.805 \mathrm{~N} ., 112.402 \mathrm{~W}$.

Depth: $4 \mathrm{~km}$

Magnitude: $3.4 M_{L}$ (UU)

Intensity IV: Howell.

Intensity III: Garland, Portage.

18 October (GS) Eastern Wyoming

Origin time: 153023.0

See Wyoming listing.

\section{VERMONT}

\section{August (LD) Northeastern New York}

Origin time: 105817.0

See New York listing.

\section{VIRGINIA}

23 April (GS) Lancaster County, Pennsylvania Origin time: 013600.1

See Pennsylvania listing.

\section{August (VP) Central Virginia}

Origin time: 180546.9

Epicenter: $37.868 \mathrm{~N} ., 78.324 \mathrm{~W}$.

Depth: $8 \mathrm{~km}$

Magnitude: $4.2 M_{n}(\mathrm{VP}), 3.9 M_{n}(\mathrm{GS}), 4.0 M_{D}(\mathrm{VP})$

This earthquake, named the Cunningham, Virginia, earthquake, was felt over a 22,000-kilometer ${ }^{2}$ area of central Virginia (see fig. 25). It had no detectable foreshocks or aftershocks (Davison and others, 1984). Some of the intensities listed below were based on a newspaper questionnaire canvass by G. A. Bollinger, Virginia Polytechnic and State University, Blacksburg.

Intensity V:

Charlottesville- A few glassware items broke; a few windows cracked; a few small objects overturned and fell; a few items shook off of store shelves; hanging pictures were out of place; buildings shook strongly; windows and dishes rattled; rumbling sounds were heard; felt by many.

Columbia- A few small objects fell; dishes and windows rattled; felt by all.

Cunningham ( 3 miles west) - A few bricks cracked at the end of a house; a crack in a garage floor lengthened; a resident had difficulty standing; loud booming noise was heard; a house shook strongly; dishes and mirror rattled. (6 miles east)- $\mathbf{A}$ farm had two windows broken in a barn and two doors stuck in the house which were difficult to open. $(0.5$ miles west)- A smoke detecter fell off a wall; pictures were crooked; dishes rattled in kitchen cabinets; it was described as a loud explosion.

Gordonsville- A heavy iron bed rocked and moved away from the wall; small objects shifted and a few overturned; windows and dishes rattled.

Keene- A few small objects overturned and fell; hanging pictures swung felt by many.

Palmyra-- Windows broke; a few small objects fell; hanging pictures were out of place; water splashed onto sides of ponds; walls creaked; windows and dishes rattled; felt by many.

Piney River- A few small objects overturned and fell; hanging pictures were out of place; windows and dishes rattled; felt by many.

Schuyler- A few glassware items broke; a few windows broke; a few small objects overturned and fell; hanging pictures were out of place; buildings shook strongly; felt by many.

Scottsville (5 miles east) - Vibration was strong; everything rattled; a crack in an old (1837) brick foundation widened. (4 miles south on State Highway 723) - A flower pot fell off a window sill; a loud rumbling noise was heard; a couch moved about 3 inches across the floor.

Intensity IV: Alberene, Amherst, Andersonville, Arvonia, Batesville (2.7 miles southwest on State Highway 151), Bon Air, Boyd Tavern, Bremo Bluff, Brightwood, Buckingham, Cartersville, Colonial Heights, Covesville, Crozet, Cumberland ( 3 miles southwest on State Highway 629), Dillyn, Doswell, Dyke, near Ellisville, Flat Rock ( 2 miles east on U.S. Highway 60 and 5 miles southwest on U.S. Highway 60), Fork Union, Fredericksburg, Goochland, Hadensville, Ivy, Kents Store, Keswick, Lexington (4 miles northeast), Lignum (press report), Locust Grove (Lake Of The Woods), Louisa, Lovingston, Lynchburg, Midlothian, Monroe, New Canton, North Garden, Orange, Powhatan (0.8 miles south), Rice, Richmond (north and west side), Shelby (on State Highway 621, 2 miles west of U.S. 


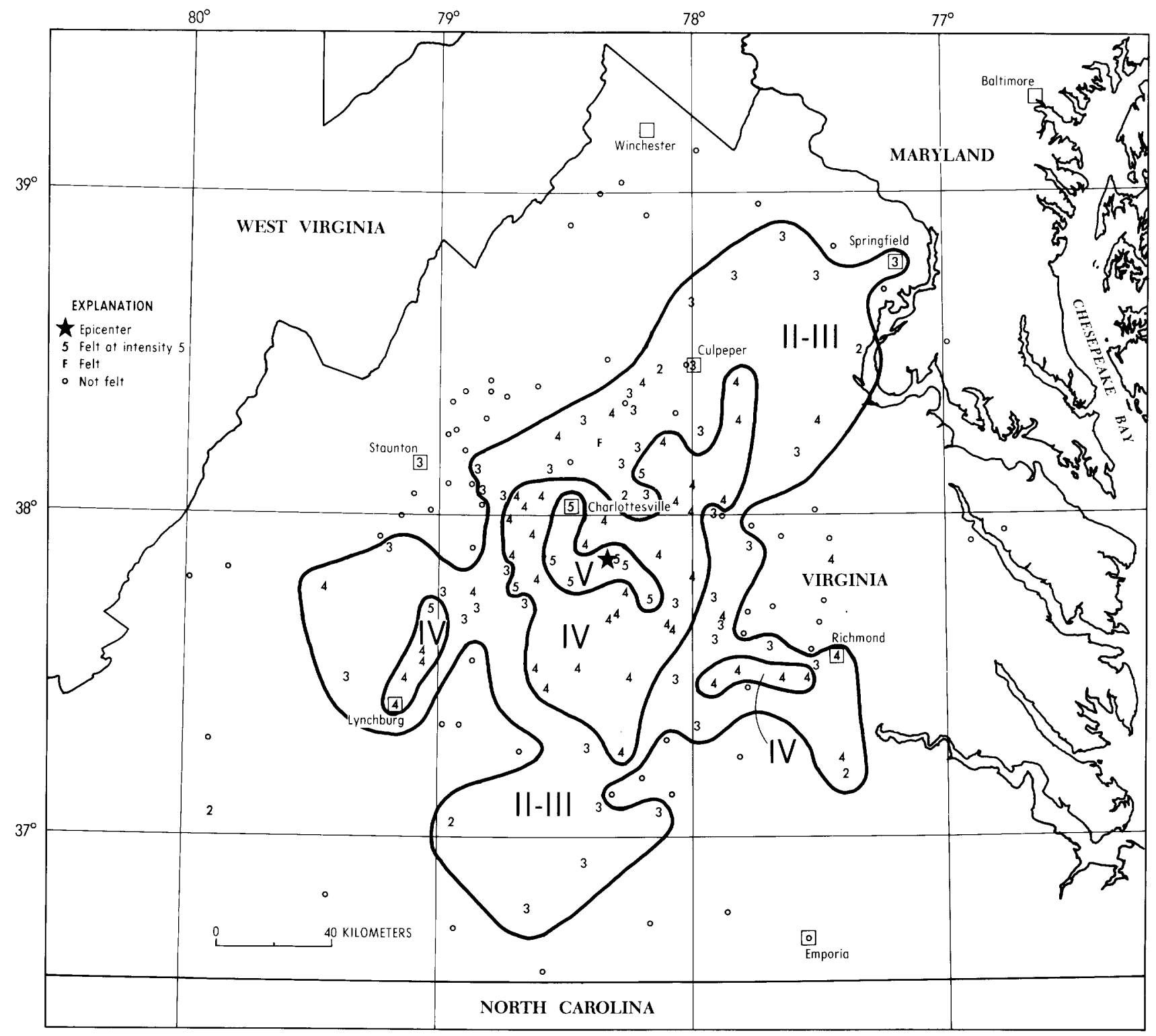

Figure 25. Isoseismal map for the Cunningham, Virginia, earthquake of 17 August 1984, 180546.9 UTC. Roman numerals represent Modified Mercalli intensities between isoseismals; Arabic numerals represent intensities at specific sites. 
Highway 29), Sweet Briar, Tamworth, Trevilians (press report), Troy, Wares Crossroads (junction of U.S. Highway 522 and State Highway 208), Woodridge (crossroads of State Highways 619 and 620 ).

Intensity III: Amelia, Amissville (2 miles south), Aroda (north of State Highway 230 on State Highway 607), Arrington, Ballsville (1 mile east), Barboursville, Big Island, Boswells Tavern (0.25 miles south), Buckner (2.5 miles south on State Highway 609), Cartersville (on State Highway 647), Chase City, Crewe, Crimora, Culpeper (5 miles east on State Highway 669), Faber, Farmville, Fife, Fort Mitchell, Free Union, Gravel Hill (at intersection of State Highways 617 and 668), Greenwood, Haymarket (at intersection of State Highway 234 and U.S. Highway 15), Howardsville, Jefferson, Kents Store (north on the shore of Lake Anna), Madison, Maidens, Manakin Sabot, Manassas, Meherrin, Mineral, Partlow, Roseland, Sandy Hook, Shipman, Somerset, Spotsylvania, Springfield (press report), Stanardsville, Staunton, Unionville, Vesuvius, Warrenton, Waynesboro.

Intensity II: Brookneal, Cobham, Culpeper, Petersburg, Quantico, Reva (3 miles southwest), Wirtz.

Felt: Ruckersville.

\section{WASHINGTON}

31 January (GS) Northeastern Oregon

Origin time: 052915.6

See Oregon listing.

11 February (PG) British Columbia-Alberta, Canada border

Origin time: 133827.0

See Montana listing.

16 March (WA) Northwestern Washington

Origin time: 023530.8

Epicenter: $48.482 \mathrm{~N}$., $121.685 \mathrm{~W}$.

Depth: $5 \mathrm{~km}$

Magnitude: $2.6 M_{D}$ (WA)

Felt: Concrete and Rockport (press report).

16 March (WA) Northwestern Washington

Origin time: 171637.2

Epicenter: $48.454 \mathrm{~N}$., $121.705 \mathrm{~W}$.

Depth: $0 \mathrm{~km}$

Magnitude: $3.4 M_{D}$ (WA), $3.0 M_{L}(\mathrm{GS})$

Felt: Concrete and Rockport (press report).

11 April (WA) Central Washington

Origin time: 030742.1

Epicenter: 47.535 N., $120.186 \mathrm{~W}$.
Depth: $8 \mathrm{~km}$

Magnitude: $3.6 M_{L}$ (GS), $4.3 M_{D}$ (WA)

Intensity $\mathrm{V}$ :

Chelan- A few items fell off of store shelves; a few glassware items or dishes broke; a few small objects overturned and fell; buildings shook slightly; dishes rattled; felt by many.

Entiat - Plaster walls sustained hairline cracks; a few windows cracked; hanging pictures swung out of place; a few items fell off of store shelves; felt by many.

Orondo- Plaster walls sustained hairline cracks; trees and bushes shook slightly; walls creaked; vibration was described as strong; felt by many.

Intensity IV: Ardenvoir, Coulee Dam, George, Grand Coulee, Malaga, Palisades, Royal City, Waterville, Wenatchee, Wilson Creek.

Intensity III: Beverly, Creston, Cunningham, Electric City, Elmer City, Ephrata, Keller, Marlin, Pateros, Quincy, Rock Island, Vantage.

Intensity II: Monitor, Stratford.

Felt: Leavenworth (WA), Yakima (press report).

27 April (WA) Western Washington

Origin time: 230658.1

Epicenter: 47.609N., 121.964W.

Depth: $10 \mathrm{~km}$

Magnitude: $2.9 M_{D}$ (WA)

Intensity IV: Near Fall City (WA).

\section{June (WA) Western Washington}

Origin time: 125721.0

Epicenter: $47.415 \mathrm{~N} ., 122.702 \mathrm{~W}$.

Depth: $21 \mathrm{~km}$

Magnitude: $3.5 M_{L}$ (GS), $3.6 M_{D}$ (WA), $3.1 M_{L}$ (EP)

Intensity IV: Port Orchard.

4 June (WA) Southwestern Washington

Origin time: 044601.7

Epicenter: $46.214 \mathrm{~N}$., $123.062 \mathrm{~W}$.

Depth: $53 \mathrm{~km}$

Magnitude: $3.7 M_{D}$ (WA)

Intensity IV: Kelso and Longview (press report).

Felt: Centralia and Lexington (press report)

10 July (WA) Northwestern Washington

Origin time: 124233.4

Epicenter: $48.612 \mathrm{~N} ., 122.057 \mathrm{~W}$.

Depth: $3 \mathrm{~km}$

Magnitude: $2.4 M_{L}(\mathrm{GS}), 2.9 M_{D}(\mathrm{WA})$

Intensity III: Lyman and Mount Vernon. 
22 August (GS) Central Idaho

Origin time: 094630.2

See Idaho listing.

24 August (WA) Central Washington

Origin time: 044307.2

Epicenter: $47.650 \mathrm{~N} ., 120.955 \mathrm{~W}$.

Depth: $1 \mathrm{~km}$

Magnitude: $2.8 M_{L}(\mathrm{GS}), 3.0 M_{D}$ (WA)

Felt: Fish Lake area.

10 October (WA) Central Washington

Origin time: 032418.2

Epicenter: 47.904N., 119.079W.

Depth: $15 \mathrm{~km}$

Magnitude: $2.5 M_{L}(\mathrm{GS}), 3.0 M_{D}(\mathrm{WA})$

Intensity V:

Electric City - A few glassware items broke; a few small objects fell; hanging pictures were out of place; buildings shook strongly; windows rattled; loud noise was heard; felt by many.

Elmer City - A few small objects overturned and fell; buildings shook slightly; windows rattled; felt by many.

Grand Coulee- A few small objects overturned and a few fell; hanging pictures were out of place; buildings shook slightly; walls creaked; windows rattled; felt by many.

Intensity IV: Coulee Dam.

Intensity III: Hartline.

2 December (WA) Northwesterm Washington

Origin time: 161724.6

Epicenter: $48.592 \mathrm{~N}$., $121.806 \mathrm{~W}$.

Depth: $0 \mathrm{~km}$

Magnitude: $3.3 M_{L}(\mathrm{GS}), 3.1 M_{D}(\mathrm{WA})$

Intensity III: Birdsview (6 miles west of Concrete), Cape Horn Development (4 miles west of Concrete), Concrete, Hamilton.

3 December (WA) Northwestern Washington

Origin time: 190321.9

Epicenter: 48.588N., $121.808 \mathrm{~W}$.

Depth: $0 \mathrm{~km}$

Magnitude: $2.9 M_{L}(\mathrm{GS}), 3.2 M_{D}(\mathrm{WA})$

Intensity III: Birdsview ( 6 miles west of Concrete), Cape Horn Development (4 miles west of Concrete).

\section{WEST VIRGINIA}

23 April (GS) Lancaster County, Pennsylvania

Origin time: 013600.1

See Pennsylvania listing.
5 January (GS) Western Wyoming

Origin time: 201024.3

Epicenter: $43.316 \mathrm{~N}$., $110.757 \mathrm{~W}$.

Depth: $5 \mathrm{~km}$

Magnitude: $3.0 M_{L}$ (GS), $3.3 M_{L}(\mathrm{UU})$

Felt: Jackson (telephone report).

24 January (GS) Yellowstone National Park

Origin time: 071247.1

Epicenter: 44.453N., 110.519W.

Depth: $5 \mathrm{~km}$

Magnitude: $3.3 M_{L}(\mathrm{BU})$

Intensity IV: Grant Village- Awakened many; rattled household items; pictures were swung out of place.

24 March (GS) Northwestern Wyoming

Origin time: 052151.8

Epicenter: $43.329 \mathrm{~N} ., 110.782 \mathrm{~W}$

Depth: $5 \mathrm{~km}$

Magnitude: $2.8 M_{L}(\mathrm{GS})$

Intensity II: Teton Village.

Felt: Jackson (telephone report)

29 May (GS) Northeastern Wyoming

Origin time: 201832.6

Epicenter: $44.232 \mathrm{~N}$., $105.965 \mathrm{~W}$.

Depth: $18 \mathrm{~km}$

Magnitude: $5.0 m_{l},(\mathrm{GS})$

This earthquake was felt over an area of about 56,000 kilometers $^{2}$ of Wyoming (Stover, 1985) (see fig. 26).

Intensity $\mathrm{V}$ :

Wyoming-

Casper- A few windows cracked; hanging pictures were out of place and a few fell; a few items shook off of store shelves; a few glassware items broke; a few small objects overturned or fell; trees and bushes shook slightly; standing and moving vehicles rocked slightly; felt by many.

Gillette- A few windows cracked; a few glassware items broke; a few small objects overturned or fell; a few items shook off of store shelves; trees and bushes shook slightly; moving and standing vehicles rocked slightly; felt by many.

Intensity IV:

Wyoming - Arvada, Kaycee, Keeline, Linch, Midwest, Mills, Moorcroft, Shawnee.

Intensity III:

Montana- Biddle, Birney, Broadus, Busby, Hardin, Olive, Otter.

Wyoming - Alcova, Buffalo, Clearmont, Cowley, Edgerton, Hamilton Dome, Hulett, Lusk, Newcastle, 


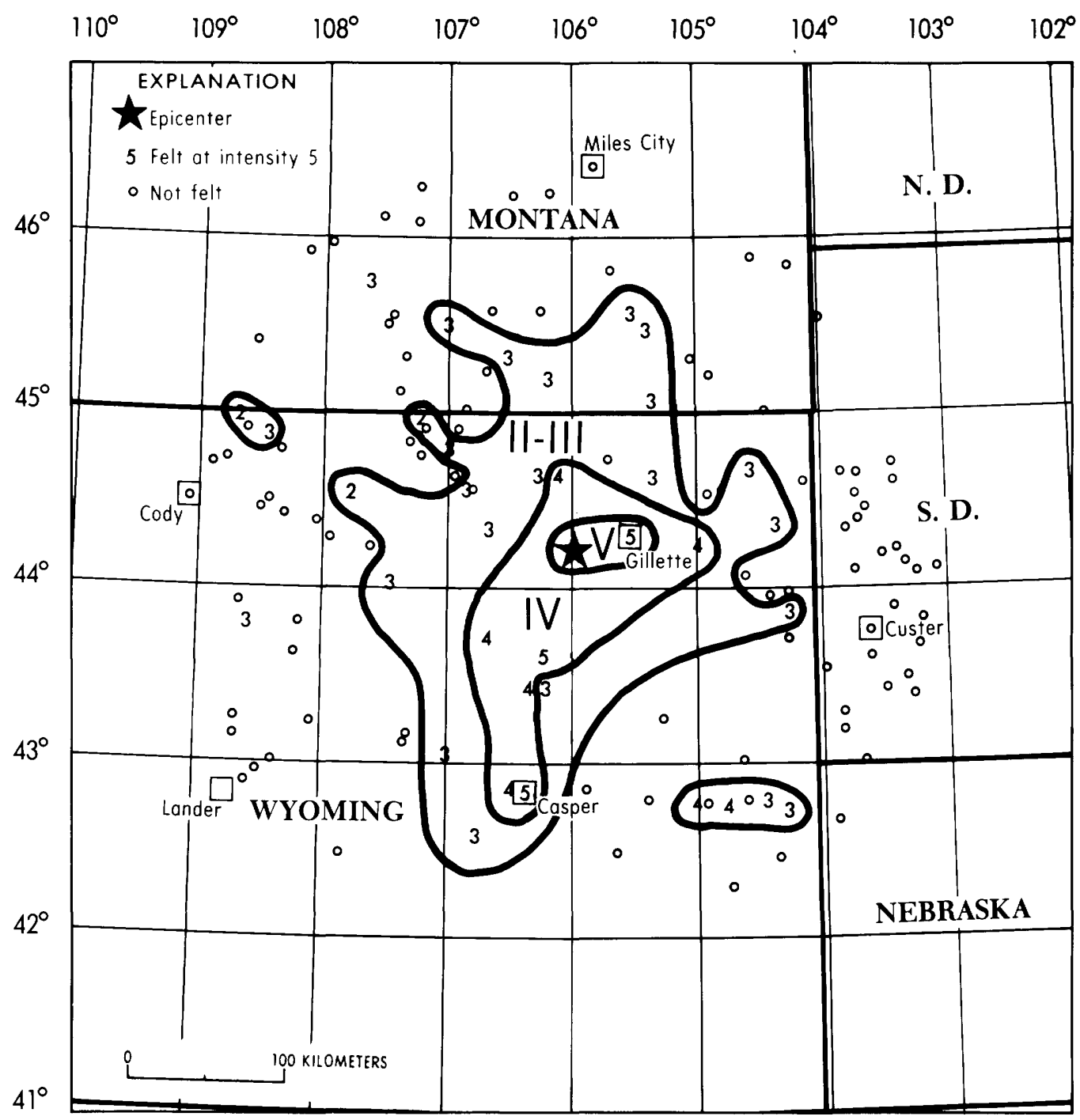

Figure 26. Isoseismal map for the northeastern Wyoming earthquake of 29 May 1984, 201832.6 UTC. Roman numerals represent Modified Mercalli intensities between isoseismals; Arabic numerals represent intensities at specific sites.

\section{WYOMING-Continued}

Node, Powder River, Sheridan, Story, Sundance (press report), Ten Sleep, Weston.

Intensity II:

Wyoming - Frannie, Parkman, Shell.

\section{July Yellowstone National Park}

Origin time: 0710

Epicenter: Not located.

Depth: None computed.

Magnitude: None computed.

Intensity IV: Grant Village and West Thumb (R. A. Hutchinson, Park Geologist, National Park Service).

\author{
WYOMING-Continued \\ 30 July Yellowstone National Park \\ Origin time: $0712 / 0713 / 0718$ \\ Epicenter: Not located. \\ Depth: None computed. \\ Magnitude: None computed. \\ Intensity II: Grant Village and West Thumb (R. A. \\ Hutchinson, Park Geologist, National Park Service).

\section{August Yellowstone National Park \\ Origin time: 0037} \\ Epicenter: Not located. \\ Depth: None computed.
}




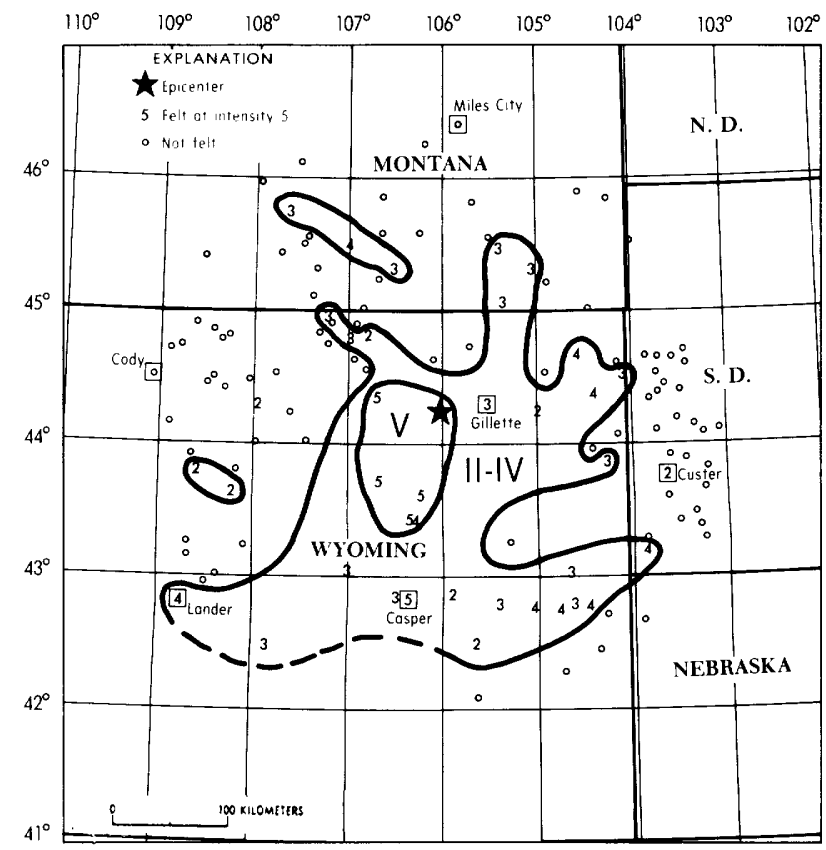

Figure 27. Isoseismal map for the northeastern Wyooming earthquake of 8 September 1984, 005931.1 UTC. Roman numerals represent Modified Mercalli intensities between isoseismals; Arabic numerals represent intensities at specific sites.

\section{WYOMING - Continued}

Magnitude: None computed.

Intensity III: Old Faithful (R. A. Hutchinson, Park Geologist National Park Service).

\section{August (GS) Central Idaho}

Origin time: 094630.2

See Idaho listing.

\section{September (GS) Northeastern Wyoming}

Origin time: 005931.1

Epicenter: 44.240N., 106.019W.

Depth: $20 \mathrm{~km}$

Magnitude: $5.1 m_{b}(\mathrm{GS}), 5.6 M_{L}(\mathrm{BU})$

This earthquake was felt over an area of approximately 68,000 kilometers ${ }^{2}$ of Montana, Wyoming, and South Dakota (Stover, 1985) (see fig. 27).

\section{Intensity V:}

Wyoming-

Buffalo- A few windows cracked; a few small objects overturned and fell; buildings shook slightly; windows rattled; felt by several.

Casper- A few windows cracked; a few items shook off of store shelves; a few small objects overturned and fell; hanging pictures were out of place; trees and bushes shook slightly; motor vehicles rocked slightly;

\section{WYOMING-Continued}

buildings shook slightly; walls creaked; windows rattled; felt by many.

Kaycee-- Plaster and sheetrock walls sustained hairline cracks; trees and bushes shook slightly; hanging pictures swung; buildings shook slightly; walls creaked; windows rattled; felt by many.

Linch-A few small objects overturned; trees and bushes shook slightly; motor vehicles rocked slightly; buildings shook slightly; walls creaked; windows rattled; felt by all.

Midwest - Plaster and sheetrock walls sustained hairline cracks; trees and bushes shook slightly; a few dishes cracked; hanging pictures swung; buildings shook slightly; walls creaked; windows rattled; felt by many.

Intensity IV:

Montana- Busby.

South Dakota - Provo.

Wyoming - Edgerton, Hulett, Keeline, Lusk, Shawnee (a report of small object fallen), Sundance.

Intensity III:

Montana- Belle Creek, Biddle, Birney, Boyes, Hardin.

Wyoming - Beulah, Douglas, Gillette, Jeffrey City, Lance Creek, Lander (press report), Manville, Mills, Newcastle, Parkman, Powder River, Sheridan, Shirley Basin.

Intensity II:

Montana- Broadus.

South Dakota- Custer.

Wyoming- Basin (press rep-ort), Glendo, Glenrock, Hamilton Dome, Moorcroft, Thermopolis, Wyarno.

Felt:

Montana-Great Falls (press report).

1 October Yellowstone National Park

Origin time: 0450

Epicenter: Not located.

Depth: None computed.

Magnitude: None computed.

Seven earthquakes on this date were felt in the Canyon area with a MM intensity IV. The times of the other six events were $0547,1011,1117,1502,1748$, and 1915 UTC (R. A. Hutchinson, Park Geologist National Park Service).

6 October (GS) Yellowstone National Park

Origin time: 203013.5

Epicenter: $44.743 \mathrm{~N}$., $110.528 \mathrm{~W}$.

Depth: $5 \mathrm{~km}$

Magnitude: $3.2 M_{L}(\mathrm{GS}), 3.7 M_{L}(\mathrm{BU})$

Eight earthquakes were felt in the Canyon area on October 6-7 between the hrs. of 2000 UTC and 1405 


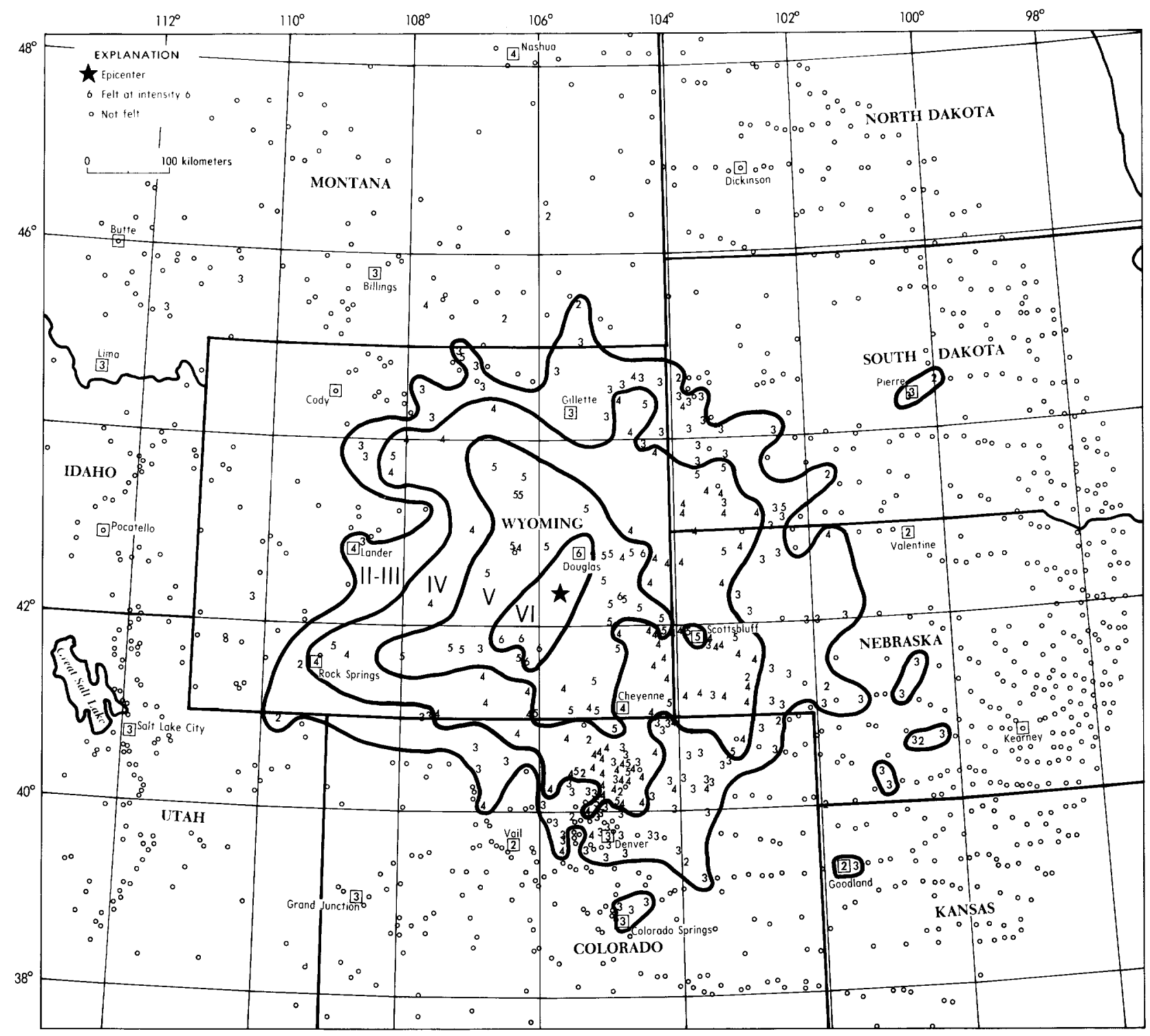

Figure 28. Isoseismal map for the eastern Wyoming earthquake of 18 October 1984, 153023.0 UTC. Roman numerals represent Modified Mercalli intensities between isoseismals; Arabic numerals represent intensities at specific sites. 
UTC. Seven of these events were felt at intensity II or III but were too small to locate (R. A. Hutchinson, Park Geologist, National Park Service).

Intensity IV: Canyon.

\author{
18 October (GS) Eastern Wyoming \\ Origin time: 153023.0 \\ Epicenter: 42.375N., 105.720W. \\ Depth: Normal \\ Magnitude: $5.4 m_{l},(\mathrm{GS}), 5.1 M_{s}(\mathrm{GS}), 5.5 M_{L}(\mathrm{GS})$, \\ $5.3 M_{n}(\mathrm{TU})$
}

This earthquake was felt over an area of about 287,000 kilometers $^{2}$ covering parts of Colorado, Kansas, Montana, Nebraska, South Dakota, Utah, and Wyoming (Stover, 1985) (fig. 28). Even though this event was felt over a large area it caused only minor damage characterized by cracked brick and cinderblock walls, cracked chimneys, and broken underground pipes.

An unusual report of damage caused by this earthquake was from Golden, Colorado, about 300 kilometers south of the epicenter. Damage occurred in two five-story buildings which were part of a 10 building complex of the Golden Ridge Condominiums located near the juncture of U.S. Highway 6 and Colorado State Highway 93 . The damage consisted of some foundation failure, numerous wall cracks and a gas leak in one of the buildings. All of the damage may not be attributed to the earthquake; some of it may be due to construction methods or soil conditions and may have existed prior to the earthquake.

Intensity VI: The most common effects at the places listed below: plaster and sheetrock on interior walls cracked; a few dishes and glassware broke; a few items shook off of store shelves; standing and moving vehicles rocked; small objects overturned and fell; a few windows cracked; buildings shook strongly; felt by many. Additional effects are listed by locality below:

\section{Wyoming-}

Casper- Chimneys cracked; underground pipes broke; exterior brick and cinderblock walls cracked; interior walls separated from ceiling or floor, light furniture overturned.

Douglas - Exterior brick and brick veneer walls sustained large cracks; foundations cracked; chimneys cracked; concrete bridges had slight damage; a few windows broke; hanging pictures fell; a brick wall cracked at Douglas City Hall.
Guernsey - Exterior cinderblock walls sustained large cracks; a foundation cracked; chimneys cracked; hanging pictures fell.

Hanna- Exterior brick walls sustained large cracks; a foundation cracked.

Lusk - Chimneys cracked; light furnitue overturned; exterior brick and cinderblock walls sustained hairline cracks.

McFadden- A foundation cracked.

Medicine Bow- Exterior brick and concrete walls sustained large cracks; tombstones were displaced; hanging pictures fell; light furniture overturned. Walls cracked at Medicine Bow High School (press report).

Rock River- Exterior brick walls sustained large cracks; flow was disturbed in well water; a foundation cracked; chimneys cracked; hanging pictures fell; hanging objects swung violently.

Shirley Basin - Exterior cinderblock walls sustained hairline cracks; a foundation cracked; underground pipes broke. Walls cracked at the elementary school (press report).

Shirley Basin (Heward Ranch about 10 miles northeast) - There were cracks in the upstairs ceiling and one big long crack formed in the basement ceiling. The house was shaken violently (press report).

Intensity V: The most common effects at the places listed below: a few small objects overturned and fell; pictures swung or were moved out of place; buildings shook slightly; walls creaked; windows and dishes rattled; felt by many. Additional effects at specific localities are listed below:

\section{Colorado-}

Carr- Standing vehicles rocked slightly.

Eaton- A few dishes or glassware items broke; a few items shook off of store shelves; plaster walls sustained hairline cracks; trees and bushes shook slightly.

Frederick - Plaster walls sustained hairline cracks; a few windows cracked; a few dishes or glassware items broke.

Glen Haven-- A few items shook off of store shelves; a few dishes or glassware items broke; standing and moving vehicles rocked slightly; trees and bushes shook slightly.

Greeley - A few windows cracked; plaster and sheetrock walls sustained hairline cracks; a foundation cracked.

Niwot- A few items shook off of store shelves; a few dishes or glassware items broke.

Sterling - A few dishes or glassware items broke; standing and moving vehicles rocked slightly; trees and bushes shook slightly (press report). 


\section{Nebraska-}

Angora.

Mitchell-A few items shook off of store shelves; standing vehicles rocked slightly; trees and bushes shook slightly.

Scottsbluff-A few windows cracked; a few items shook off of store shelves; a few dishes or glassware items broke; hanging pictures fell; standing and moving vehicles rocked slightly.

Whitney.

South Dakota--

Fairburn-Standing vehicles rocked slightly.

Porcupine-- A few dishes or glassware items broke; plaster walls sustained hairline cracks.

Pringle-- A few dishes or glassware items broke; standing vehicles rocked slightly; trees and bushes shook slightly.

\section{Wyoming -}

Alcova- A few items shook off of store shelves; trees and bushes shook moderately; standing and moving vehicles rocked slightly.

Bill-A few windows cracked.

Chugwater-A few dishes or glassware items broke; a few items shook off of store shelves; trees and bushes shook moderately.

Dwyer- Buildings shook strongly; trees and bushes shook moderately; standing vehicles rocked moderately.

Edgerton- A few items were shaken off store shelves; a foundation cracked; standing vehicles rocked moderately.

Elk Mountain - Plaster and sheetrock walls sustained hairline cracks; standing vehicles rocked moderately.

Fort Laramie - Plaster walls sustained hairline cracks; a few windows cracked.

Glendo- A few items shook off of store shelves; buildings shook strongly; trees and bushes shook slightly.

Glenrock-Buildings shook strongly.

Granite Canon.

Hartville- A few windows cracked; buildings shook strongly; trees and bushes shook slightly.

Horse Creek- A few windows cracked; a few dishes or glassware items broke; trees and bushes shook slightly; standing and moving vehicles shook slightly.

Huntley - Trees and bushes shook slighlty; standing vehicles shook slightly.

Jelm-Plaster and sheetrock walls sustained hairline cracks; a few items shook off of store shelves; a few dishes or glassware items broke; trees and bushes shook slightly.
Kaycee- Sheetrock walls sustained hairline cracks; a few items shook off of store shelves; a few dishes or glassware items broke; buildings shook strongly; trees and bushes shook slightly.

Kirby.

Linch-Sheetrock walls sustained hairline cracks; trees and bushes shook slightly; standing vehicles rocked slightly.

Lost Springs- Buildings shook strongly.

Manville-A few windows cracked; a few items shook off of store shelves; a few dishes or glassware items broke; sheetrock walls sustained hairline cracks; buildings shook strongly.

Midwest-- A few items shook off of store shelves; hanging pictures fell; buildings shook strongly; trees and bushes shook moderately; standing and moving vehicles rocked moderately.

Mills- Plaster walls sustained hairline cracks; a few dishes or glassware items broke; trees and bushes shook slightly.

Pine Bluffs - Trees and bushes shook slightly; standing and moving vehicles rocked slightly.

Ranchester.

Rawlins- A few items shook off of store shelves; trees and bushes shook slightly.

Shawnee- Plaster walls sustained hairline cracks; buildings shook strongly; trees and bushes shook slightly; standing vehicles rocked moderately; moving vehicles rocked slightly.

Sinclair- Plaster and sheetrock walls sustained hairline cracks; a few windows cracked; a few dishes or glassware items broke; trees and bushes shook slightly.

Sundance- A few items shook off of store shelves.

Superior- - Plaster walls sustained hairline cracks; trees and bushes shook slightly.

Tie Siding- A few dishes or glassware items broke; plaster walls sustained hairline cracks.

Torrington- Plaster and sheetrock walls sustained hairline cracks; a few items shook off of store shelves; a few dishes or glassware items broke; moving vehicles rocked slightly.

Wamsutter- Plaster walls sustained hairline cracks; a few windows cracked.

Wheatland- Plaster and sheetrock walls sustained hairline cracks; a few items shook off of store shelves; a few dishes or glassware items broke; trees and bushes shook slightly; standing and moving vehicles rocked slightly. 
Intensity IV:

Colorado- Ault, Bellvue, Berthoud, Boulder, Briggsdale, Brush, Cowdrey, Dacono, Estes Park, Evans, Firestone, Fort Collins, Fort Lupton, Golden, Grand Lake, Iliff, Johnstown, Keenesburg, Kersey, La Salle, Laporte, Longmont, Loveland, Lucerne, Masonville, Mead, Red Feather Lakes, Severance, Toponas, Wellington, Wheat Ridge, Wiggins, Windsor.

Montana- Nashua, Saint Xavier.

Nebraska- Bayard, Bridgeport, Broadwater, Bushnell, Chadron, Gering, Gurley, Harrison, Henry, Kimball, Lyman, Marsland, McGrew, Merriman, Minatare, Morrill, Potter, Sidney.

South Dakota - Ardmore, Edgemont, Hot Springs, Igloo (press report), Lead, Oelrichs, Oglala (press report), Oral, Provo.

Wyoming - Albin, Bairoil, Buffalo, Buford, Carlile, Carpenter, Centennial, Cheyenne, Encampment, Evansville, Foxpark, Francis E. Warren Air Force Base, Hawk Springs, Hulett, Jay Em, Keeline, Lagrange, Lance Creek, Lander, Laramie, Meriden, Newcastle, Node, Point Of Rocks, Powder River, Rock Springs, Saratoga, Savery, Slater, Ten Sleep, Thermopolis, Upton, Van Tassell, Veteran, Worland, Yoder.

Intensity III:

Colorado- Allenspark, Arvada, Association Camp, Atwood, Aurora, Bennett, Black Forest, Brighton, Clark, Coalmont, Colorado Springs, Crook, Deer Trail, Denver, Empire, Englewood, Erie, Fort Morgan, Galeton, Georgetown, Granby, Grand Junction, Grover, Haxtun, Hereford, Highlands, Hillrose, Hoyt, Hygiene, Lafayette, Limon, Littleton, Loretto, Louisville, Louviers, Lyons, Merino, Milliken, New Raymer, Nunn, Orchard, Parker, Peetz, Peyton, Pierce, Roggen, Snyder, Steamboat Springs, Stoneham, Strasburg, Thornton, Timnath, U.S. Air Force Academy, Weldona, Westminster, Winter Park, Woodrow.

Kansas - Edson.

Montana- Biddle, Billings, Ennis, Lima, Livingston. Nebraska- Alliance, Ashby, Bingham, Brule, Chappell, Dix, Farnam, Hay Springs, Hemingford, Lisco, Lodgepole, Maywood, Meadow Grove, Melbeta, North Platte, Oshkosh, Palisade, Paxton, Rushville, Stapleton, Trenton, Whitman.

South Dakota - Custer, Deadwood, Fort Pierre, Hermosa, Hill City, Manderson, Nemo, Pierre, Pine Ridge, Rapid City, Rochford, Smithwick, Spearfish, Sturgis, Wasta, Wounded Knee.

Utah-Salt Lake City.

Wyoming - Aladdin, Alva, Baggs, Devils Tower, Dixon, Four Corners, Gillette, Grass Creek, Hamilton
Dome, Hudson, Hyattville, LaBarge, Moorcroft, Osage, Oshoto, Parkman, Recluse, Shell, Sheridan, Story, Walcott.

Intensity II:

Colorado-- Agate, Drake, Livermore, Nederland, Platteville, Sedgwick, Vail.

Kansas - Coolidge, Goodland.

Montana- Birney, Broadus, Miles City.

Nebraska - Curtis, Dalton, Haigler, Lemoyne, Valentine.

South Dakota - Blunt, Wanblee.

Utah-Manila.

Wyoming-Green River.

Felt:

South Dakota - Belle Fourche, Deerfield (press report).

\section{October (GS) Eastern Wyoming}

Origin time: 155737.3

Epicenter: 42.365 N., $105.805 \mathrm{~W}$.

Depth: Normal

Magnitude: $4.5 m_{b}(\mathrm{GS}), 4.2 M_{L}(\mathrm{GS})$

Intensity IV: Heward Ranch about 10 miles northeast of Shirley Basin (press report).

\section{October (GS) Northeastern Wyoming}

Origin time: 190800.1

Epicenter: 44.351N., 105.995W.

Depth: $20 \mathrm{~km}$

Magnitude: $2.5 M_{L}$ (GS)

Felt: Gillette (telephone report).

3 November (GS) Western Wyoming

Origin time: 042306.0

Epicenter: 43.551N., $110.813 \mathrm{~W}$.

Depth: $5 \mathrm{~km}$

Magnitude: $2.6 M_{L}(\mathrm{GS}), 3.1 M_{L}(\mathrm{BU})$

Intensity III: Moose.

\section{November (GS) Western Wyoming}

Origin time: 093008.5

Epicenter: 42.494N., 108.854W.

Depth: $5 \mathrm{~km}$

Magnitude: $5.0 m_{l},(\mathrm{GS}), 4.1 M_{S}(\mathrm{GS}), 5.1 M_{L}(\mathrm{UU})$, $4.9 M_{L}(\mathrm{BU})$

\section{Intensity VI:}

Lander- Police Department reported 50 buildings had cracked walls and some cracked windows and foundations (press report). There was some glass breakage at the hospital; a few items shook off of store shelves; a few small objects overturned and fell; hanging pictures were out of place; buildings shook moderately; 
walls creaked loudly; windows rattled; vibration was described as strong; felt by and awakened many.

\section{Intensity V:}

Fort Washakie - Small objects moved; hanging pictures were out of place; buildings shook strongly; felt by and awakened many.

South Pass City- A few items shook off of store shelves; light furniture overturned; a few dishes or glassware items broke; a few windows cracked; a few small objects overturned and fell; hanging pictures were out of place; buildings shook moderately; walls creaked; windows rattled loudly; vibration was described as strong; people had difficulty standing or walking; felt by and awakened all.

Superior- Plaster walls sustained hairline cracks; a few small objects fell; buildings shook slightly; hanging pictures swung; walls creaked; windows rattled loudly; felt by and awakened many.

Intensity IV: Farson, Hudson, Jefferson City, Riverton, Saratoga, Thermopolis.

Intensity III: Etna, LaBarge, Point of Rocks.

Felt: Arapahoe. 
The following codes are used to indicate the source of hypocenters and/or magnitudes: (BK) University of California, Berkeley; (BU) Montana Bureau of Mines and Geology, Butte; (EN) Department of Energy, Washington, D.C.; (EP) Geophysics Division, Geologic Survey of Canada, Ottawa; (GM) U.S. Geological Survey, Menlo Park, Calif.; (GS) U.S. Geological Survey, Golden, Colo.; (GT) Georgia Institute of Technology, Atlanta; (HV) Hawaiian Volcano Observatory, U.S. Geological Survey, Hawaii National Park; (LD) Lamont-Doherty Geological Observatory, Palisades, N.Y.; (MI) University of Michigan, Ann Arbor; (PM) Palmer Observatory, NOAA, Palmer Alaska;
(PS) California Institute of Technology, Pasadena; (RN) University of Nevada, Reno; (SL) St. Louis University, St. Louis, Mo.; (TC) Tennessee Earthquake Information Center, Memphis; (TU) Oklahoma Geological Survey, Leonard; (UU) University of Utah, Salt Lake City; (VP) Virginia Polytechnic Institute and State University, Blacksburg; (WA) University of Washington, Seattle; (WO) Weston Observatory, Weston, Mass. Normal depth $(\mathrm{km})=33 \mathrm{~N}$. AST $=$ Alaska Standard Time, CST = Central Standard Time, EST = Eastern Standard Time, HST = Hawaii Standard Time, MST $=$ Mountain Standard Time, PST $=\mathrm{Pa}$ cific Standard Time, YST $=$ Yukon Standard Time. Leaders (...) indicate information is not available ]

\begin{tabular}{|c|c|c|c|c|c|c|c|c|c|c|c|c|c|}
\hline \multirow{2}{*}{ Date } & \multicolumn{2}{|c|}{$\begin{array}{l}\text { Origin time } \\
\text { (UTC) }\end{array}$} & \multirow{2}{*}{$\begin{array}{c}\text { Letitude } \\
\text { (') }\end{array}$} & \multirow{2}{*}{$\begin{array}{c}\text { Longitude } \\
\left({ }^{\circ}\right)\end{array}$} & \multirow{2}{*}{$\begin{array}{l}\text { Depth } \\
(\mathbf{k m})\end{array}$} & \multirow{2}{*}{$\begin{array}{l}\text { Hypo- } \\
\text { center } \\
\text { source }\end{array}$} & \multicolumn{3}{|c|}{ Magnitude } & \multirow{2}{*}{$\begin{array}{l}\text { Maximum } \\
\text { intensity }\end{array}$} & \multicolumn{3}{|c|}{ Local time } \\
\hline & hr $\mathrm{min}$ & sec & & & & & $\mathbf{m b}$ & MS & $\begin{array}{l}\text { ML. Mn } \\
M D, M w\end{array}$ & & Date & Hour & $\begin{array}{l}\text { Time } \\
\text { zome }\end{array}$ \\
\hline
\end{tabular}

ALABAMA

\begin{tabular}{|c|c|c|c|c|c|c|c|c|c|c|c|c|c|c|c|}
\hline $\begin{array}{l}\text { JAN. } \\
\text { AUG. }\end{array}$ & $\begin{array}{l}6 \\
9\end{array}$ & $\begin{array}{l}03 \\
02\end{array}$ & $\begin{array}{l}04 \\
42\end{array}$ & $\begin{array}{l}20.9 \\
35.8\end{array}$ & $\begin{array}{l}31.607 \mathrm{~N} . \\
34.629 \mathrm{~N} .\end{array}$ & $\begin{array}{l}87.808 w \\
86.295 w .\end{array}$ & $\begin{array}{l}5 \\
8\end{array}$ & $\begin{array}{l}\text { GS } \\
\text { TC }\end{array}$ & $\begin{array}{l}\cdots \\
\cdots\end{array}$ & $\begin{array}{l}\ldots \\
\ldots\end{array}$ & $\begin{array}{l}\text { 2. } 9 \mathrm{MD}(\mathrm{TC}) \\
\text { 3. } 2 \mathrm{MD}(\mathrm{TC})\end{array}$ & $\begin{array}{c}V \\
\ldots\end{array}$ & $\begin{array}{l}\text { JAN. } \\
\text { AUG. }\end{array}$ & $\begin{array}{l}5 \\
8\end{array}$ & $\begin{array}{lll}21: 04 & \text { CST } \\
20: 42 & \text { CST }\end{array}$ \\
\hline
\end{tabular}

\begin{tabular}{|c|c|c|c|c|c|c|c|c|c|c|c|c|c|c|c|c|}
\hline \multicolumn{17}{|c|}{ ALASKA } \\
\hline $\begin{array}{l}\text { JAN. } \\
\text { JAN. } \\
\text { JAN. } \\
\text { JAN. } \\
\text { JAN. }\end{array}$ & $\begin{array}{l}2 \\
3 \\
3 \\
4 \\
4\end{array}$ & $\begin{array}{l}16 \\
11 \\
18 \\
17 \\
20\end{array}$ & $\begin{array}{l}10 \\
40 \\
52 \\
03 \\
04\end{array}$ & $\begin{array}{l}18.1 \\
09.1 \\
46.8 \\
28.4 \\
35.0\end{array}$ & $\begin{array}{l}56.354 \mathrm{~N} . \\
61.738 \mathrm{~N} . \\
63.015 \mathrm{~N} . \\
51.138 \mathrm{~N} . \\
65.473 \mathrm{~N} .\end{array}$ & $\begin{array}{l}152.981 W . \\
149.943 W . \\
149.775 W . \\
174.287 E . \\
150.310 W .\end{array}$ & $\begin{array}{l}33 \mathrm{~N} \\
62 \\
115 \\
33 \mathrm{~N} \\
33 \mathrm{~N}\end{array}$ & $\begin{array}{l}\text { GS } \\
\text { GS } \\
\text { GS } \\
\text { GS } \\
\text { GS }\end{array}$ & $\begin{array}{l}5.0 \\
3.8 \\
\ldots \\
4.9 \\
\ldots\end{array}$ & $\begin{array}{l}\cdots \\
\cdots \\
4.9 \\
\ldots\end{array}$ & $\begin{array}{c}\text { 4. } 5 \mathrm{ML}(P M) \\
3.6 \mathrm{ML}(P M) \\
\ldots \\
\text { 4. } \mathrm{BML}(\mathrm{PM})\end{array}$ & $\begin{array}{l}\dot{I I} \\
\ldots \\
\ldots \\
\ldots\end{array}$ & $\begin{array}{l}\text { JAN. } \\
\text { JAN. } \\
\text { JAN. } \\
\text { JAN. } \\
\text { JAN. }\end{array}$ & $\begin{array}{l}2 \\
3 \\
3 \\
4 \\
4\end{array}$ & $\begin{array}{l}07: 10 \\
02: 40 \\
09: 52 \\
07: 03 \\
11: 04\end{array}$ & $\begin{array}{l}\text { YST } \\
\text { YST } \\
\text { YST } \\
\text { AST } \\
\text { YST }\end{array}$ \\
\hline $\begin{array}{l}\text { JAN. } \\
\text { JAN. } \\
\text { JAN. } \\
\text { JAN. } \\
\text { JAN. }\end{array}$ & $\begin{array}{r}5 \\
6 \\
7 \\
8 \\
10\end{array}$ & $\begin{array}{l}21 \\
21 \\
11 \\
03 \\
22\end{array}$ & $\begin{array}{l}41 \\
36 \\
59 \\
36 \\
04\end{array}$ & $\begin{array}{l}48.0 \\
26.7 \\
35.7 \\
43.4 \\
33.4\end{array}$ & $\begin{array}{l}51.296 \mathrm{~N} . \\
63.985 \mathrm{~N} . \\
51.907 \mathrm{~N} . \\
59.254 \mathrm{~N} . \\
51.469 \mathrm{~N} .\end{array}$ & $\begin{array}{l}179.194 W . \\
148.943 W . \\
176.398 W . \\
136.857 W . \\
172.304 E .\end{array}$ & $\begin{array}{l}53 \\
33 N \\
57 \\
15 \\
33 N\end{array}$ & $\begin{array}{l}\text { GS } \\
\text { GS } \\
\text { GS } \\
\text { GS } \\
\text { GS }\end{array}$ & $\begin{array}{l}5.2 \\
\cdots .4 \\
3.8 \\
4.6\end{array}$ & $\begin{array}{l}4.8 \\
\ldots \\
\ldots \\
\ldots \\
\ldots\end{array}$ & $\begin{array}{c}\text { 4. } 9 M L(P M) \\
\text { 2. } 9 M L(P M) \\
\text { 3. } 7 M L(P M) \\
\text { 4. 3ML(PM) } \\
\ldots\end{array}$ & $\begin{array}{c}\cdots \\
\cdots \\
\text { FË } \\
\cdots\end{array}$ & $\begin{array}{l}\text { JAN. } \\
\text { JAN. } \\
\text { JAN. } \\
\text { JAN. } \\
\text { JAN. }\end{array}$ & $\begin{array}{r}5 \\
6 \\
7 \\
7 \\
10\end{array}$ & $\begin{array}{l}11: 41 \\
12: 36 \\
01: 59 \\
18: 36 \\
12: 04\end{array}$ & $\begin{array}{l}\text { AST } \\
\text { YST } \\
\text { AST } \\
\text { YST } \\
\text { AST }\end{array}$ \\
\hline $\begin{array}{l}\text { JAN. } \\
\text { JAN. } \\
\text { JAN. } \\
\text { JAN. } \\
\text { JAN. }\end{array}$ & $\begin{array}{l}11 \\
12 \\
13 \\
13 \\
14\end{array}$ & $\begin{array}{l}20 \\
01 \\
09 \\
19 \\
06\end{array}$ & $\begin{array}{l}49 \\
18 \\
22 \\
33 \\
53\end{array}$ & $\begin{array}{l}30.9 \\
50.2 \\
12.0 \\
50.9 \\
05.4\end{array}$ & $\begin{array}{l}61.843 \mathrm{~N} \\
61.764 \mathrm{~N} \\
59.950 \mathrm{~N} \\
60.198 \mathrm{~N} \\
51.859 \mathrm{~N}\end{array}$ & $\begin{array}{l}149.696 w . \\
154.265 W . \\
140.690 W . \\
152.658 W . \\
168.331 W .\end{array}$ & $\begin{array}{l}47 \\
33 \mathrm{~N} \\
18 \\
116 \\
33 \mathrm{~N}\end{array}$ & $\begin{array}{l}\text { GS } \\
\text { GS } \\
\text { EP } \\
\text { GS } \\
\text { GS }\end{array}$ & $\begin{array}{l}\ldots \\
\ldots \\
\dot{3} . \dot{9} \\
4.8\end{array}$ & $\begin{array}{l}\cdots \\
\cdots \\
\cdots \\
\cdots \\
\cdots\end{array}$ & $\begin{array}{c}\text { 3. } 2 M L(P M) \\
\text { 3. 3ML(PM) } \\
\text { 3. } 3 M L(E P) \\
\text { 4. } 6 \mathrm{ML} \text { (PM) }\end{array}$ & $\begin{array}{l}\dot{I} \text { II } \\
\cdots \\
\cdots \\
\cdots\end{array}$ & $\begin{array}{l}\text { JAN. } \\
\text { JAN. } \\
\text { JAN. } \\
\text { JAN. } \\
\text { JAN. }\end{array}$ & $\begin{array}{l}11 \\
11 \\
13 \\
13 \\
13\end{array}$ & $\begin{array}{l}11: 49 \\
16: 18 \\
00: 22 \\
10: 33 \\
21: 53\end{array}$ & $\begin{array}{l}\text { YST } \\
\text { YST } \\
\text { YST } \\
\text { YST } \\
\text { AST }\end{array}$ \\
\hline $\begin{array}{l}\text { JAN. } \\
\text { JAN. } \\
\text { JAN. } \\
\text { JAN. } \\
\text { JAN. }\end{array}$ & $\begin{array}{l}14 \\
14 \\
14 \\
15 \\
15\end{array}$ & $\begin{array}{l}11 \\
11 \\
22 \\
07 \\
07\end{array}$ & $\begin{array}{l}04 \\
44 \\
04 \\
10 \\
30\end{array}$ & $\begin{array}{l}21.4 \\
24.8 \\
47.0 \\
52.7 \\
05.1\end{array}$ & $\begin{array}{l}52.635 \mathrm{~N} . \\
59.883 \mathrm{~N} . \\
56.484 \mathrm{~N} . \\
56.621 \mathrm{~N} . \\
56.487 \mathrm{~N} .\end{array}$ & $\begin{array}{l}177.124 E . \\
153.293 W . \\
152.272 W . \\
152.406 W . \\
152.393 W .\end{array}$ & $\begin{array}{r}85 \\
142 \\
33 N \\
33 N \\
33 N\end{array}$ & $\begin{array}{l}\text { GS } \\
\text { GS } \\
\text { GS } \\
\text { GS } \\
\text { GS }\end{array}$ & $\begin{array}{l}4.3 \\
4.8 \\
4.5 \\
5.1 \\
4.8\end{array}$ & $\begin{array}{l}\cdots \\
\cdots \\
5.1 \\
4.9\end{array}$ & $\begin{array}{c}\ldots \\
\text { 4. } 6 \mathrm{ML}(\mathrm{PM}) \\
\text { 5. } 3 \mathrm{ML}(\mathrm{PM}) \\
\text { 4. } 2 \mathrm{ML}(\mathrm{PM})\end{array}$ & $\begin{array}{l}\because \ddot{I} \\
\ldots \\
\cdots \\
\cdots\end{array}$ & $\begin{array}{l}\text { JAN. } \\
\text { JAN. } \\
\text { JAN. } \\
\text { JAN. } \\
\text { JAN. }\end{array}$ & $\begin{array}{l}14 \\
14 \\
14 \\
14 \\
14\end{array}$ & $\begin{array}{l}01: 04 \\
02: 44 \\
13: 04 \\
22: 10 \\
22: 30\end{array}$ & $\begin{array}{l}\text { AST } \\
\text { YST } \\
\text { YST } \\
\text { YST } \\
\text { YST }\end{array}$ \\
\hline $\begin{array}{l}\text { JAN. } \\
\text { JAN. } \\
\text { JAN. } \\
\text { JAN. } \\
\text { JAN. }\end{array}$ & $\begin{array}{l}15 \\
15 \\
15 \\
17 \\
17\end{array}$ & $\begin{array}{l}12 \\
12 \\
19 \\
05 \\
10\end{array}$ & $\begin{array}{l}02 \\
28 \\
14 \\
49 \\
25\end{array}$ & $\begin{array}{l}44.3 \\
39.5 \\
47.1 \\
14.0 \\
38.0\end{array}$ & $\begin{array}{l}56.491 \mathrm{~N} . \\
56.503 \mathrm{~N} . \\
63.167 \mathrm{~N} . \\
59.753 \mathrm{~N} . \\
52.199 \mathrm{~N} .\end{array}$ & $\begin{array}{l}152.345 w . \\
152.319 w . \\
148.369 w . \\
153.472 W . \\
169.530 w .\end{array}$ & $\begin{array}{c}33 N \\
33 N \\
111 \\
135 \\
33 N\end{array}$ & $\begin{array}{l}\text { GS } \\
\text { GS } \\
\text { GS } \\
\text { GS } \\
\text { GS }\end{array}$ & $\begin{array}{l}4.4 \\
5.3 \\
\ldots \\
4.7\end{array}$ & $\begin{array}{l}\cdots \\
4.7 \\
\cdots \\
\cdots \\
\cdots\end{array}$ & $\begin{array}{c}\text { 3. } 7 M L(P M) \\
\text { 5. OML(PM) } \\
\ldots \\
\ldots \\
\ldots\end{array}$ & $\begin{array}{l}\cdots \\
\cdots \\
\cdots \\
\cdots \\
\cdots\end{array}$ & $\begin{array}{l}\text { JAN. } \\
\text { JAN. } \\
\text { JAN. } \\
\text { JAN. } \\
\text { JAN. }\end{array}$ & $\begin{array}{l}15 \\
15 \\
15 \\
16 \\
17\end{array}$ & $\begin{array}{l}03: 02 \\
03: 28 \\
10: 14 \\
20: 49 \\
00: 25\end{array}$ & $\begin{array}{l}\text { YST } \\
\text { YST } \\
\text { YST } \\
\text { YST } \\
\text { AST }\end{array}$ \\
\hline
\end{tabular}




\begin{tabular}{|c|c|c|c|c|c|c|c|c|c|c|c|c|c|}
\hline \multirow{2}{*}{ Date } & \multicolumn{2}{|c|}{$\begin{array}{l}\text { Origin time } \\
\text { (UTC) }\end{array}$} & \multirow{2}{*}{$\begin{array}{c}\text { Latitude } \\
\left({ }^{\circ}\right)\end{array}$} & \multirow{2}{*}{$\begin{array}{l}\text { Longitude } \\
\left({ }^{\circ}\right)\end{array}$} & \multirow{2}{*}{$\begin{array}{c}\text { Depth } \\
(\mathbf{k m})\end{array}$} & \multirow{2}{*}{$\begin{array}{l}\text { Hypo- } \\
\text { center } \\
\text { source }\end{array}$} & \multicolumn{3}{|c|}{ Magnitude } & \multirow{2}{*}{$\begin{array}{l}\text { Maximum } \\
\text { intensity }\end{array}$} & \multicolumn{3}{|c|}{ Lucal time } \\
\hline & hr $\min$ & $\sec$ & & & & & $\mathbf{m b}$ & MS & $\begin{array}{l}\text { ML, Mn } \\
\text { MD, Mw }\end{array}$ & & Date & Hour & $\begin{array}{l}\text { Time } \\
\text { zome }\end{array}$ \\
\hline
\end{tabular}

\section{ALASKA-Continued}

\begin{tabular}{|c|c|c|c|c|c|c|c|c|c|c|c|c|c|c|c|c|}
\hline $\begin{array}{l}\text { JAN. } \\
\text { JAN. } \\
\text { JAN. } \\
\text { JAN. } \\
\text { JAN. }\end{array}$ & $\begin{array}{l}18 \\
20 \\
23 \\
25 \\
27\end{array}$ & $\begin{array}{l}01 \\
18 \\
22 \\
21 \\
10\end{array}$ & $\begin{array}{l}18 \\
23 \\
06 \\
19 \\
00\end{array}$ & $\begin{array}{l}12.2 \\
28.2 \\
06.5 \\
55.1 \\
08.6\end{array}$ & $\begin{array}{l}60.930 \mathrm{~N} \\
60.027 \mathrm{~N} . \\
53.287 \mathrm{~N} \\
61.383 \mathrm{~N} \\
56.169 \mathrm{~N}\end{array}$ & $\begin{array}{l}147.186 W . \\
153.352 W . \\
169.639 w . \\
146.977 w . \\
158.807 W .\end{array}$ & $\begin{array}{l}33 \mathrm{~N} \\
139 \\
103 \\
33 \mathrm{~N} \\
33 \mathrm{~N}\end{array}$ & $\begin{array}{l}\text { GS } \\
\text { GS } \\
\text { GS } \\
\text { GS } \\
\text { GS }\end{array}$ & $\begin{array}{l}4.1 \\
5.3 \\
\ldots .1\end{array}$ & $\begin{array}{l}\cdots \\
\cdots \\
\cdots \\
\ldots\end{array}$ & $\begin{array}{c}\text { 3. } 5 M L(P M) \\
\ldots \\
\ldots \\
\text { 3. } 2 \mathrm{ML}(P M) \\
4.1 M L(P M)\end{array}$ & $\begin{array}{l}\text { I I I } \\
\text { IV }\end{array}$ & $\begin{array}{l}\text { JAN. } \\
\text { JAN. } \\
\text { JAN. } \\
\text { JAN. } \\
\text { JAN. }\end{array}$ & $\begin{array}{l}17 \\
20 \\
23 \\
25 \\
27\end{array}$ & $\begin{array}{l}16: 18 \\
09: 23 \\
12: 06 \\
12: 19 \\
01: 00\end{array}$ & $\begin{array}{l}\text { YST } \\
\text { YST } \\
\text { AST } \\
\text { YST } \\
\text { YST }\end{array}$ \\
\hline $\begin{array}{l}\text { JAN. } \\
\text { JAN. } \\
\text { JAN. } \\
\text { JAN. } \\
\text { JAN. }\end{array}$ & $\begin{array}{l}27 \\
28 \\
28 \\
30 \\
30\end{array}$ & $\begin{array}{l}10 \\
12 \\
19 \\
04 \\
05\end{array}$ & $\begin{array}{l}51 \\
47 \\
48 \\
04 \\
03\end{array}$ & $\begin{array}{l}22.2 \\
41.2 \\
52.4 \\
41.3 \\
56.5\end{array}$ & $\begin{array}{l}67.986 \mathrm{~N} \\
55.783 \mathrm{~N} \\
67.727 \mathrm{~N} . \\
67.737 \mathrm{~N} \\
67.789 \mathrm{~N} .\end{array}$ & $\begin{array}{l}147.370 w . \\
154.297 W . \\
164.614 W . \\
164.589 w . \\
164.215 W .\end{array}$ & $\begin{array}{l}10 \\
33 N \\
33 N \\
33 N \\
33 N\end{array}$ & $\begin{array}{l}\text { GS } \\
\text { GS } \\
\text { GS } \\
\text { GS } \\
\text { GS }\end{array}$ & $\begin{array}{l}4.6 \\
4.7 \\
\ldots \\
\ldots \\
\ldots\end{array}$ & $\begin{array}{l}\ldots \\
\cdots \\
\cdots \\
\cdots\end{array}$ & $\begin{array}{l}\text { 4. } 0 M L(P M) \\
\text { 3. } 4 M L(P M) \\
\text { 3. } 2 M L(P M) \\
\text { 3. } 2 M L(P M)^{2}\end{array}$ & $\begin{array}{l}\cdots \\
\cdots \\
\cdots \\
\ldots\end{array}$ & $\begin{array}{l}\text { JAN. } \\
\text { JAN. } \\
\text { JAN. } \\
\text { JAN. } \\
\text { JAN. }\end{array}$ & $\begin{array}{l}27 \\
28 \\
28 \\
29 \\
29\end{array}$ & $\begin{array}{l}01: 51 \\
03: 47 \\
10: 48 \\
19: 04 \\
20: 03\end{array}$ & $\begin{array}{l}\text { YST } \\
\text { YST } \\
\text { YST } \\
\text { YST } \\
\text { YST }\end{array}$ \\
\hline $\begin{array}{l}\text { JAN. } \\
\text { JAN. } \\
\text { JAN. } \\
\text { FEB. } \\
\text { FEB. }\end{array}$ & $\begin{array}{r}30 \\
30 \\
3 \\
3\end{array}$ & $\begin{array}{l}05 \\
18 \\
18 \\
07 \\
23\end{array}$ & $\begin{array}{l}24 \\
01 \\
39 \\
13 \\
17\end{array}$ & $\begin{array}{l}06.4 \\
03.9 \\
08.3 \\
57.0 \\
15.2\end{array}$ & $\begin{array}{l}13 N \\
84 N \\
72 N \\
30 N \\
09 N\end{array}$ & $\begin{array}{l}22 W . \\
21 W . \\
89 w . \\
00 W . \\
42 W .\end{array}$ & $\begin{array}{l}33 N \\
33 N \\
33 N \\
18 \\
72\end{array}$ & $\begin{array}{l}\text { GS } \\
\text { GS } \\
\text { GS } \\
\text { EP } \\
\text { GS }\end{array}$ & $\begin{array}{l}\ldots .8 \\
\ldots \\
4.1\end{array}$ & $\begin{array}{l}\ldots \\
\ldots \\
\cdots \\
\cdots\end{array}$ & $\begin{array}{c}\text { 3. 3ML(PM) } \\
\text { 4. OML (PM) } \\
\text { 3. OML (PM) } \\
\text { 3. 3ML(EP) } \\
\text {... }\end{array}$ & $\begin{array}{c}\ldots \\
\cdots \\
\cdots \\
\text { FELT }\end{array}$ & $\begin{array}{l}\text { JA } \\
\text { JA } \\
\text { JA } \\
\text { FE } \\
\text { FE }\end{array}$ & $\begin{array}{r}29 \\
30 \\
30 \\
2 \\
3\end{array}$ & $\begin{array}{l}20: 24 \\
09: 01 \\
09: 39 \\
22: 13 \\
14: 17\end{array}$ & YST \\
\hline $\begin{array}{l}\text { FEB. } \\
\text { FEB. } \\
\text { FEB. } \\
\text { FEB. } \\
\text { FEB. }\end{array}$ & $\begin{array}{r}6 \\
6 \\
6 \\
8 \\
10\end{array}$ & $\begin{array}{l}07 \\
07 \\
12 \\
01 \\
22\end{array}$ & $\begin{array}{l}16 \\
48 \\
15 \\
19 \\
17\end{array}$ & $\begin{array}{l}31.2 \\
17.3 \\
35.3 \\
17.3 \\
44.4\end{array}$ & $\begin{array}{l}61.983 \mathrm{~N} \\
62.988 \mathrm{~N} \\
64.944 \mathrm{~N} \\
62.092 \mathrm{~N} \\
61.515 \mathrm{~N}\end{array}$ & & $\begin{array}{l}18 \\
129 \\
33 \mathrm{~N} \\
81 \\
33 \mathrm{~N}\end{array}$ & $\begin{array}{l}G S \\
G S \\
G S \\
G S \\
G S\end{array}$ & $\begin{array}{l}\ldots \\
4.4 \\
\ldots \\
\ldots\end{array}$ & $\begin{array}{l}\cdots \\
\cdots \\
\cdots \\
\cdots\end{array}$ & $\begin{array}{c}\text { 3. 3ML(PM) } \\
\ldots \\
\ldots \\
\ldots \\
\text { 3. } 0 \mathrm{ML}(\mathrm{PM})\end{array}$ & $\begin{array}{l}\text { FELT } \\
\text { III } \\
\text { FELT } \\
\text { FËLT }\end{array}$ & $\begin{array}{l}\text { FEB. } \\
\text { FEB. } \\
\text { FEB. } \\
\text { FEB. }\end{array}$ & $\begin{array}{r}5 \\
5 \\
6 \\
7 \\
10\end{array}$ & $\begin{array}{l}22: 16 \\
22: 48 \\
03: 15 \\
16: 19 \\
13: 17\end{array}$ & ST \\
\hline $\begin{array}{l}E B . \\
E B . \\
E B . \\
E B . \\
E B .\end{array}$ & $\begin{array}{l}12 \\
12 \\
12 \\
13 \\
14\end{array}$ & $\begin{array}{l}03 \\
04 \\
09 \\
18 \\
05\end{array}$ & $\begin{array}{l}28 \\
48 \\
01 \\
05 \\
43\end{array}$ & $\begin{array}{l}57.7 \\
00.9 \\
46.8 \\
52.1 \\
39.8\end{array}$ & $\begin{array}{l}66.324 \mathrm{~N} \\
60.269 \mathrm{~N} \\
59.232 \mathrm{~N} \\
55.686 \mathrm{~N} . \\
64.488 \mathrm{~N}\end{array}$ & $\begin{array}{l}157.322 W \\
140.990 W \\
145.441 W \\
154.332 W \\
158.690 W .\end{array}$ & $\begin{array}{l}33 N \\
15 \\
33 N \\
33 N \\
33 N\end{array}$ & $\begin{array}{l}\text { GS } \\
\text { GS } \\
\text { GS } \\
\text { GS } \\
\text { GS }\end{array}$ & $\begin{array}{l}3.7 \\
\ldots \\
4.9 \\
\ldots\end{array}$ & $\begin{array}{l}\ldots \\
\ldots \\
4.8 \\
\ldots\end{array}$ & $\begin{array}{c}\text { 4.OML(PM) } \\
\text { 3. OML(PM) } \\
\ldots \\
\ldots\end{array}$ & $\cdots$ & & $\begin{array}{l}11 \\
11 \\
12 \\
13 \\
13\end{array}$ & $\begin{array}{l}18: 28 \\
19: 48 \\
00: 01 \\
09: 05 \\
20: 43\end{array}$ & YST \\
\hline $\begin{array}{l}\text { FEB. } \\
\text { FEB. } \\
\text { FEB. } \\
\text { FEB. } \\
\text { FEB. }\end{array}$ & $\begin{array}{l}14 \\
15 \\
15 \\
16 \\
16\end{array}$ & $\begin{array}{l}18 \\
13 \\
14 \\
02 \\
14\end{array}$ & $\begin{array}{l}17 \\
52 \\
01 \\
02 \\
17\end{array}$ & $\begin{array}{l}24.4 \\
55.8 \\
35.0 \\
27.2 \\
37.6\end{array}$ & $\begin{array}{l}59.721 \mathrm{~N} \\
52.263 \mathrm{~N} \\
62.285 \mathrm{~N} \\
52.367 \mathrm{~N} \\
56.432 \mathrm{~N}\end{array}$ & $\begin{array}{l}150.319 W . \\
178.139 E . \\
167.725 W \\
170.522 E . \\
153.244 W .\end{array}$ & $\begin{array}{l}33 N \\
33 N \\
33 N \\
33 N \\
33 N\end{array}$ & $\begin{array}{l}\text { GS } \\
\text { GS } \\
\text { GS } \\
\text { GS } \\
\text { GS }\end{array}$ & $\begin{array}{l}3.8 \\
4.1 \\
4.8 \\
4.6\end{array}$ & $\begin{array}{l}\ldots \\
\ldots \\
\ldots \\
\ldots\end{array}$ & $\begin{array}{c}\text { 3. } 3 \mathrm{ML}(\mathrm{PM}) \\
\text { 4. } 1 \mathrm{ML}(\mathrm{PM}) \\
\ldots \\
\ldots \\
\ldots\end{array}$ & $\begin{array}{l}\text { II I } \\
\ldots \\
\ldots \\
\ldots \\
\cdots\end{array}$ & $\begin{array}{l}\text { FEB. } \\
\text { FEB. } \\
\text { FEB. } \\
\text { FEB. }\end{array}$ & $\begin{array}{l}14 \\
15 \\
15 \\
15 \\
16\end{array}$ & $\begin{array}{l}09: 17 \\
03: 52 \\
05: 01 \\
16: 02 \\
05: 17\end{array}$ & $\begin{array}{l}\text { ASI } \\
\text { YST }\end{array}$ \\
\hline $\begin{array}{l}\text { FEB. } \\
\text { FEB. } \\
\text { FEB. } \\
\text { FEB. } \\
\text { FEB. }\end{array}$ & $\begin{array}{l}17 \\
21 \\
22 \\
22 \\
23\end{array}$ & $\begin{array}{l}16 \\
16 \\
01 \\
16 \\
10\end{array}$ & $\begin{array}{l}47 \\
31 \\
08 \\
04 \\
06\end{array}$ & $\begin{array}{l}53.9 \\
49.0 \\
24.0 \\
40.8 \\
47.9\end{array}$ & $\begin{array}{l}3.154 \mathrm{~N} \\
2.944 \mathrm{~N} \\
9.120 \mathrm{~N} \\
5.032 \mathrm{~N} \\
2.608 \mathrm{~N}\end{array}$ & $\begin{array}{l}.534 W . \\
620 W \\
.060 W \\
.744 W \\
.881 W\end{array}$ & $\begin{array}{l}137 \\
111 \\
18 \\
33 \mathrm{~N} \\
33 \mathrm{~N}\end{array}$ & $\begin{array}{l}E \\
G \\
G\end{array}$ & $\begin{array}{l}\ldots \\
\cdots \\
\cdots\end{array}$ & $\begin{array}{l}\cdots \\
\cdots \\
\cdots \\
\ldots\end{array}$ & $\begin{array}{c}\ldots \\
\text { 3. } 1 \mathrm{ML}(\mathrm{EP}) \\
\text { 4. 3ML(PM) } \\
\ldots\end{array}$ & $\begin{array}{c}\text { FELT } \\
\ldots\end{array}$ & & $\begin{array}{l}17 \\
21 \\
21 \\
22 \\
23\end{array}$ & $\begin{array}{l}07: 47 \\
07: 31 \\
16: 08 \\
07: 04 \\
01: 06\end{array}$ & YST \\
\hline $\begin{array}{l}\text { FEB. } \\
\text { FEB. } \\
\text { FEB. } \\
\text { FEB. } \\
\text { FEB. }\end{array}$ & $\begin{array}{l}24 \\
25 \\
26 \\
26\end{array}$ & $\begin{array}{l}06 \\
11 \\
07 \\
11 \\
15\end{array}$ & $\begin{array}{l}47 \\
12 \\
55 \\
23 \\
39\end{array}$ & $\begin{array}{l}04.3 \\
06.8 \\
20.6 \\
26.6 \\
06.9\end{array}$ & $\begin{array}{l}\text { N. } \\
\text { N. } \\
\text { N. } \\
\text { N. } \\
\text { N. }\end{array}$ & & $\begin{array}{l}140 \\
48 \\
33 \mathrm{~N} \\
43 \\
56\end{array}$ & $G$ & $\begin{array}{l}4.3 \\
4.8 \\
3.9 \\
3.9 \\
4.4\end{array}$ & $\begin{array}{l}\cdots \\
\ldots \\
\ldots \\
\ldots \\
\cdots\end{array}$ & $\begin{array}{c}\text { 4. } 7 M L(P M) \\
\text { 3.6ML (PM) } \\
\text { 3. } 6 M L(P M) \\
\ldots\end{array}$ & $\begin{array}{l}\text { I I I } \\
\ldots \\
\cdots \\
\text { IV } \\
\cdots\end{array}$ & & $\begin{array}{l}23 \\
24 \\
24 \\
26 \\
26\end{array}$ & $\begin{array}{l}21: 47 \\
01: 12 \\
22: 55 \\
02: 23 \\
06: 39\end{array}$ & $\begin{array}{l}\text { YST } \\
\text { YST }\end{array}$ \\
\hline $\begin{array}{l}\text { FEB. } \\
\text { FEB. } \\
\text { FEB. } \\
\text { MAR. } \\
\text { MAR. }\end{array}$ & $\begin{array}{r}26 \\
27 \\
27 \\
1 \\
2\end{array}$ & $\begin{array}{l}21 \\
03 \\
10 \\
02 \\
13\end{array}$ & $\begin{array}{l}54 \\
12 \\
27 \\
09 \\
01\end{array}$ & $\begin{array}{l}45.4 \\
21.6 \\
10.2 \\
49.6 \\
51.9\end{array}$ & $\begin{array}{l}60.908 \mathrm{~N} \\
59.747 \mathrm{~N} \\
56.972 \mathrm{~N} \\
57.201 \mathrm{~N} \\
62.552 \mathrm{~N}\end{array}$ & $\begin{array}{l}800 w . \\
450 w . \\
461 w . \\
786 w . \\
773 w .\end{array}$ & $\begin{array}{l}33 N \\
104 \\
33 N \\
33 N \\
95\end{array}$ & $\begin{array}{l}G \\
G \\
G \\
G\end{array}$ & $\begin{array}{l}\cdots \\
4.6 \\
4.1 \\
\cdots\end{array}$ & $\begin{array}{l}\ldots \\
\ldots \\
\cdots \\
\cdots\end{array}$ & $\begin{array}{c}\text { 3. } 5 M L(P M) \\
\ldots \ldots \\
\text { 4. } 5 \mathrm{ML}(\mathrm{PM}) \\
\text { 4.0ML(PM) } \\
\ldots\end{array}$ & $\begin{array}{c}\text { FELT } \\
\ldots\end{array}$ & $\begin{array}{l}\text { FE } \\
\text { FE } \\
F E \\
\text { FE } \\
\text { MA }\end{array}$ & $\begin{array}{r}26 \\
26 \\
27 \\
29 \\
2\end{array}$ & $\begin{array}{l}12: 54 \\
18: 12 \\
01: 27 \\
17: 09 \\
04: 01\end{array}$ & $\begin{array}{l}\text { YST } \\
\text { YST } \\
\text { YST }\end{array}$ \\
\hline $\begin{array}{l}\text { MAR. } \\
\text { MAR. } \\
\text { MAR. } \\
\text { MAR. } \\
\text { MAR. }\end{array}$ & $\begin{array}{l}2 \\
2 \\
3 \\
4 \\
7\end{array}$ & $\begin{array}{l}13 \\
14 \\
01 \\
08 \\
02\end{array}$ & $\begin{array}{l}20 \\
57 \\
28 \\
04 \\
35\end{array}$ & $\begin{array}{l}18.6 \\
30.4 \\
10.3 \\
52.5 \\
56.2\end{array}$ & $\begin{array}{l}61.426 \mathrm{~N} \\
62.357 \mathrm{~N} \\
64.024 \mathrm{~N} \\
55.009 \mathrm{~N} \\
61.059 \mathrm{~N}\end{array}$ & $\begin{array}{l}146.281 W . \\
148.636 W . \\
148.984 W . \\
158.210 W . \\
148.556 W .\end{array}$ & $\begin{array}{l}33 N \\
82 \\
33 N \\
33 N \\
33 N\end{array}$ & $\begin{array}{l}\text { GS } \\
\text { GS } \\
\text { GS } \\
\text { GS } \\
\text { GS }\end{array}$ & 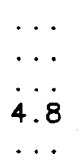 & $\begin{array}{l}\cdots \\
\ldots \\
\ldots\end{array}$ & $\begin{array}{l}\text { 3. } 9 M L(P M) \\
\ldots \ldots \\
\text { 3. } 3 M L(P M) \\
\text { 4. } 1 M L(P M) \\
\text { 3. } 5 M L(P M)\end{array}$ & II & $\begin{array}{l}\text { MAR. } \\
\text { MAR. } \\
\text { MAR. } \\
\text { MAR. }\end{array}$ & $\begin{array}{l}2 \\
2 \\
2 \\
3 \\
6\end{array}$ & $\begin{array}{l}04: 20 \\
05: 57 \\
16: 28 \\
23: 04 \\
17: 35\end{array}$ & $\begin{array}{l}\text { YST } \\
\text { YST } \\
\text { YST } \\
\text { YST } \\
\text { YST }\end{array}$ \\
\hline
\end{tabular}


Table 1. Summary of U.S. earthquakes for 1984-Continued

\begin{tabular}{|c|c|c|c|c|c|c|c|c|c|c|c|c|c|}
\hline \multirow{2}{*}{ Date } & \multicolumn{2}{|c|}{$\begin{array}{c}\text { Origin time } \\
\text { (UTC) }\end{array}$} & \multirow{2}{*}{$\begin{array}{c}\text { Latitude } \\
\left.\text { ( }{ }^{\circ}\right)\end{array}$} & \multirow{2}{*}{$\begin{array}{c}\text { Longitude } \\
\left({ }^{\circ}\right)\end{array}$} & \multirow{2}{*}{$\begin{array}{c}\text { Depth } \\
(\mathbf{k m})\end{array}$} & \multirow{2}{*}{$\begin{array}{l}\text { Hypo- } \\
\text { center } \\
\text { source }\end{array}$} & \multicolumn{3}{|c|}{ Magnitude } & \multirow{2}{*}{$\begin{array}{l}\text { Maximum } \\
\text { intensity }\end{array}$} & \multicolumn{3}{|c|}{ local time } \\
\hline & hr $\min$ & sec & & & & & $\mathbf{m b}$ & MS & $\begin{array}{l}\text { ML, Mn } \\
\text { MD, Mw }\end{array}$ & & Date & Hour & $\begin{array}{l}\text { Time } \\
\text { rone }\end{array}$ \\
\hline
\end{tabular}

ALASKA-Continued

\begin{tabular}{|c|c|c|c|c|c|c|c|c|c|c|c|c|c|c|c|c|}
\hline $\begin{array}{l}\text { MAR. } \\
\text { MAR. } \\
\text { MAR. } \\
\text { MAR. } \\
\text { MAR. }\end{array}$ & $\begin{array}{r}7 \\
8 \\
8 \\
9 \\
10\end{array}$ & $\begin{array}{l}07 \\
00 \\
13 \\
17 \\
00\end{array}$ & $\begin{array}{l}16 \\
00 \\
04 \\
00 \\
56\end{array}$ & $\begin{array}{l}13.6 \\
50.3 \\
05.4 \\
54.4 \\
25.2\end{array}$ & $\begin{array}{l}52.007 \mathrm{~N} \\
51.986 \mathrm{~N} \\
54.205 \mathrm{~N} \\
52.804 \mathrm{~N} \\
61.870 \mathrm{~N}\end{array}$ & $\begin{array}{l}177.959 E . \\
171.430 W . \\
167.015 W . \\
173.332 W . \\
149.470 W .\end{array}$ & $\begin{array}{c}137 \\
33 \mathrm{~N} \\
138 \\
250 \\
54\end{array}$ & $\begin{array}{l}\text { GS } \\
\text { GS } \\
\text { GS } \\
\text { GS } \\
\text { GS }\end{array}$ & $\begin{array}{l}4.4 \\
4.6 \\
4.7 \\
4.4 \\
\ldots\end{array}$ & $\begin{array}{l}\cdots \\
\cdots \\
\cdots \\
\ldots\end{array}$ & $\begin{array}{c}\cdots \\
\cdots \\
\cdots \\
\text { 3. } \ddot{O M L}(P M)\end{array}$ & $\begin{array}{l}\cdots \\
\cdots \\
\cdots \\
\cdots \\
\cdots\end{array}$ & $\begin{array}{l}\text { MAR. } \\
\text { MAR. } \\
\text { MAR. } \\
\text { MAR. } \\
\text { MAR. }\end{array}$ & $\begin{array}{l}6 \\
7 \\
8 \\
9 \\
9\end{array}$ & $\begin{array}{l}21: 16 \\
14: 00 \\
04: 04 \\
07: 00 \\
15: 56\end{array}$ & $\begin{array}{l}\text { AST } \\
\text { AST } \\
\text { AST } \\
\text { AST } \\
\text { YST }\end{array}$ \\
\hline $\begin{array}{l}\text { MAR. } \\
\text { MAR. } \\
\text { MAR. } \\
\text { MAR. } \\
\text { MAR. }\end{array}$ & $\begin{array}{l}11 \\
11 \\
12 \\
13 \\
14\end{array}$ & $\begin{array}{l}02 \\
23 \\
21 \\
09 \\
20\end{array}$ & $\begin{array}{l}53 \\
10 \\
16 \\
33 \\
32\end{array}$ & $\begin{array}{l}13.4 \\
31.4 \\
14.0 \\
09.0 \\
52.4\end{array}$ & $\begin{array}{l}60.130 \mathrm{~N} . \\
59.601 \mathrm{~N} . \\
54.202 \mathrm{~N} \\
54.563 \mathrm{~N} \\
61.703 \mathrm{~N} .\end{array}$ & $\begin{array}{l}140.936 w . \\
152.925 w . \\
164.071 w . \\
159.114 w . \\
149.859 w .\end{array}$ & $\begin{array}{c}15 \\
109 \\
59 \\
33 \mathrm{~N} \\
67\end{array}$ & $\begin{array}{l}\text { GS } \\
\text { GS } \\
\text { GS } \\
\text { GS } \\
\text { GS }\end{array}$ & $\begin{array}{l}\ldots \\
\ldots .1 \\
4.8 \\
4.8\end{array}$ & $\begin{array}{l}\ldots \\
\cdots \\
\cdots \\
\cdots\end{array}$ & $\begin{array}{c}\text { 3. } 4 M L(P M) \\
\ldots \ldots \\
\text { 4. } 9 \mathrm{ML}(P M) \\
\text { 4. } 1 \mathrm{ML}(P M) \\
\text { 4.5ML(PM) }\end{array}$ & $\begin{array}{l}\cdots \\
\cdots \\
\cdots \\
\text { IV }\end{array}$ & $\begin{array}{l}\text { MAR. } \\
\text { MAR. } \\
\text { MAR. } \\
\text { MAR. } \\
\text { MAR. }\end{array}$ & $\begin{array}{l}10 \\
11 \\
12 \\
13 \\
14\end{array}$ & $\begin{array}{l}17: 53 \\
14: 10 \\
12: 16 \\
00: 33 \\
11: 32\end{array}$ & $\begin{array}{l}\text { YST } \\
\text { YST } \\
\text { YST } \\
\text { YST } \\
\text { YST }\end{array}$ \\
\hline $\begin{array}{l}\text { MAR. } \\
\text { MAR. } \\
\text { MAR. } \\
\text { MAR. } \\
\text { MAR. }\end{array}$ & $\begin{array}{l}20 \\
20 \\
21 \\
22 \\
23\end{array}$ & $\begin{array}{l}01 \\
05 \\
23 \\
19 \\
08\end{array}$ & $\begin{array}{l}01 \\
14 \\
34 \\
18 \\
38\end{array}$ & $\begin{array}{l}17.2 \\
35.6 \\
00.2 \\
41.4 \\
05.8\end{array}$ & $\begin{array}{l}012 \mathrm{~N} . \\
286 \mathrm{~N} \\
522 \mathrm{~N} \\
047 \mathrm{~N} . \\
978 \mathrm{~N}\end{array}$ & $\begin{array}{l}882 W . \\
170 W . \\
659 E . \\
043 W . \\
153 W .\end{array}$ & $\begin{array}{l}33 N \\
143 \\
75 \\
162 \\
119\end{array}$ & $\begin{array}{l}\text { GS } \\
\text { GS } \\
\text { GS } \\
\text { GS } \\
\text { GS }\end{array}$ & $\begin{array}{l}\cdots \\
4.8 \\
\dot{4} . \dot{3}\end{array}$ & $\begin{array}{l}\ldots \\
\cdots \\
\cdots \\
\cdots \\
\cdots\end{array}$ & $\begin{array}{c}\text { 3. 3ML(PM) } \\
\ldots \\
\ldots \\
\ldots \\
\ldots\end{array}$ & $\begin{array}{l}\cdots \\
\cdots \\
\cdots \\
\text { IV }\end{array}$ & $\begin{array}{l}\text { MAR. } \\
\text { MAR. } \\
\text { MAR. } \\
\text { MAR. } \\
\text { MAR. }\end{array}$ & $\begin{array}{l}19 \\
19 \\
21 \\
22 \\
22\end{array}$ & $\begin{array}{l}16: 01 \\
20: 14 \\
13: 34 \\
10: 18 \\
23: 38\end{array}$ & $\begin{array}{l}\text { YST } \\
\text { YST } \\
\text { AST } \\
\text { YST } \\
\text { YST }\end{array}$ \\
\hline $\begin{array}{l}\text { MAR. } \\
\text { MAR. } \\
\text { MAR. } \\
\text { MAR. } \\
\text { MAR. }\end{array}$ & $\begin{array}{l}23 \\
24 \\
24 \\
24 \\
24\end{array}$ & $\begin{array}{l}13 \\
06 \\
16 \\
21 \\
22\end{array}$ & $\begin{array}{l}54 \\
08 \\
43 \\
04 \\
43\end{array}$ & $\begin{array}{l}00.5 \\
34.0 \\
34.1 \\
41.1 \\
37.6\end{array}$ & $\begin{array}{l}62.975 \mathrm{~N} \\
59.283 \mathrm{~N} \\
61.805 \mathrm{~N} \\
52.332 \mathrm{~N} \\
52.371 \mathrm{~N}\end{array}$ & & $\begin{array}{l}33 N \\
79 \\
66 \\
29 \\
33 N\end{array}$ & $\begin{array}{l}\text { GS } \\
\text { GS } \\
\text { GS }\end{array}$ & $\begin{array}{l}\cdots \\
\cdots \\
5.7 \\
5.4 \\
5.1\end{array}$ & $\begin{array}{l}\ldots \\
\ldots \\
5.0 \\
\ldots\end{array}$ & $\begin{array}{l}\text { 3. } 4 M L(P M) \\
\ldots \\
\text { 4. } 8 M L(P M) \\
\text { 4. } 9 M L(P M) \\
\text { 4. } 3 M L(P M)\end{array}$ & $\begin{array}{l}\cdots \\
\cdots \\
\cdots \\
\cdots\end{array}$ & $\begin{array}{l}\text { MAR. } \\
\text { MAR. } \\
\text { MAR. } \\
\text { MAR. } \\
\text { MAR. }\end{array}$ & $\begin{array}{l}23 \\
23 \\
24 \\
24 \\
24\end{array}$ & $\begin{array}{l}04: 54 \\
21: 08 \\
07: 43 \\
11: 04 \\
13: 43\end{array}$ & $\begin{array}{l}\text { YST } \\
\text { YST } \\
\text { YST } \\
\text { AST } \\
\text { AST }\end{array}$ \\
\hline $\begin{array}{l}\text { MAR. } \\
\text { MAR. } \\
\text { MAR. } \\
\text { MAR. } \\
\text { MAR. }\end{array}$ & $\begin{array}{l}24 \\
27 \\
28 \\
29 \\
29\end{array}$ & $\begin{array}{l}22 \\
18 \\
07 \\
12 \\
18\end{array}$ & $\begin{array}{l}49 \\
02 \\
46 \\
05 \\
52\end{array}$ & $\begin{array}{l}58.2 \\
04.5 \\
08.8 \\
14.2 \\
01.0\end{array}$ & $\begin{array}{l}52.320 \mathrm{~N} \\
52.011 \mathrm{~N} \\
50.964 \mathrm{~N} \\
61.689 \mathrm{~N} \\
59.728 \mathrm{~N}\end{array}$ & $\begin{array}{l}168.523 W . \\
169.708 W . \\
178.379 E \\
151.281 W . \\
148.792 W .\end{array}$ & $\begin{array}{l}33 N \\
35 \\
33 N \\
99 \\
33 N\end{array}$ & $\begin{array}{l}\text { GS } \\
\text { GS } \\
\text { GS } \\
\text { GS } \\
\text { GS }\end{array}$ & $\begin{array}{l}4.9 \\
5.0 \\
4.3 \\
\ldots . \\
\ldots\end{array}$ & $\begin{array}{l}5.3 \\
\ldots \\
\ldots \\
\ldots\end{array}$ & $\begin{array}{c}\ldots \\
\cdots \\
\cdots \\
\text { 3. } 4 \mathrm{ML}(\mathrm{PM})\end{array}$ & $\begin{array}{l}\cdots \\
\cdots \\
\cdots \\
\cdots \\
\cdots\end{array}$ & $\begin{array}{l}\text { MAR. } \\
\text { MAR. } \\
\text { MAR. } \\
\text { MAR. } \\
\text { MAR. }\end{array}$ & $\begin{array}{l}24 \\
27 \\
27 \\
29 \\
29\end{array}$ & $\begin{array}{l}12: 49 \\
08: 02 \\
21: 46 \\
03: 05 \\
09: 52\end{array}$ & $\begin{array}{l}\text { AST } \\
\text { AST } \\
\text { AST } \\
\text { YST } \\
\text { YST }\end{array}$ \\
\hline $\begin{array}{l}\text { MAR. } \\
\text { MAR. } \\
\text { MAR. } \\
\text { MAR. } \\
\text { APR. }\end{array}$ & $\begin{array}{r}29 \\
30 \\
31 \\
31 \\
4\end{array}$ & $\begin{array}{l}23 \\
22 \\
04 \\
12 \\
12\end{array}$ & $\begin{array}{l}03 \\
05 \\
58 \\
35 \\
02\end{array}$ & $\begin{array}{l}27.1 \\
39.6 \\
04.7 \\
17.9 \\
32.9\end{array}$ & $\begin{array}{l}51.049 \mathrm{~N} \\
51.374 \mathrm{~N} \\
51.334 \mathrm{~N} \\
51.478 \mathrm{~N} \\
51.429 \mathrm{~N}\end{array}$ & $\begin{array}{l}178.164 \mathrm{E} \\
177.949 \mathrm{E} \\
178.026 \mathrm{E} \\
176.896 \mathrm{E} \\
176.486 \mathrm{~W}\end{array}$ & $\begin{array}{l}33 N \\
53 \\
54 \\
33 N \\
64\end{array}$ & $\begin{array}{l}\text { GS } \\
\text { GS } \\
\text { GS } \\
\text { GS } \\
\text { GS }\end{array}$ & $\begin{array}{l}4.9 \\
4.9 \\
4.8 \\
4.5 \\
4.6\end{array}$ & $\begin{array}{l}4.3 \\
\cdots \\
\cdots \\
\cdots\end{array}$ & $\begin{array}{c}\ldots \\
\text { 4. } 3 \mathrm{ML}(\mathrm{PM}) \\
\text { 4. } 3 \mathrm{ML}(\mathrm{PM}) \\
\ldots\end{array}$ & $\begin{array}{l}\cdots \\
\cdots \\
\cdots \\
\cdots \\
\cdots\end{array}$ & $\begin{array}{l}\text { MAR. } \\
\text { MAR. } \\
\text { MAR. } \\
\text { MAR. } \\
\text { APR. }\end{array}$ & $\begin{array}{r}29 \\
30 \\
30 \\
31 \\
4\end{array}$ & $\begin{array}{l}13: 03 \\
12: 05 \\
18: 58 \\
02: 35 \\
02: 02\end{array}$ & $\begin{array}{l}\text { AST } \\
\text { AST } \\
\text { AST } \\
\text { AST } \\
\text { AST }\end{array}$ \\
\hline $\begin{array}{l}\text { APR. } \\
\text { APR. } \\
\text { APR. } \\
\text { APR. } \\
\text { APR. }\end{array}$ & $\begin{array}{l}4 \\
6 \\
7 \\
8 \\
8\end{array}$ & $\begin{array}{l}12 \\
18 \\
00 \\
00 \\
20\end{array}$ & $\begin{array}{l}13 \\
05 \\
53 \\
41 \\
24\end{array}$ & $\begin{array}{l}45.3 \\
39.2 \\
44.4 \\
24.6 \\
31.8\end{array}$ & $\begin{array}{l}61.574 \mathrm{~N} \\
62.282 \mathrm{~N} \\
59.784 \mathrm{~N} \\
51.358 \mathrm{~N} \\
59.618 \mathrm{~N}\end{array}$ & $\begin{array}{l}146.190 W . \\
151.172 W . \\
152.762 W . \\
179.596 \mathrm{E} . \\
152.653 W .\end{array}$ & $\begin{array}{r}66 \\
87 \\
100 \\
92 \\
115\end{array}$ & $\begin{array}{l}\text { GS } \\
\text { GS } \\
\text { GS }\end{array}$ & $\begin{array}{l}\cdots \\
\ldots \\
4.3 \\
\ldots\end{array}$ & $\begin{array}{l}\ldots \\
\ldots \\
\cdots \\
\cdots \\
\cdots\end{array}$ & $\begin{array}{l}9 M L(P I \\
\ldots \\
\ldots \\
\ldots \\
\ldots\end{array}$ & $\begin{array}{l}\cdots \\
\ldots \\
\ldots \\
\cdots \\
\cdots\end{array}$ & $\begin{array}{l}\text { APR. } \\
\text { APR. } \\
\text { APR. } \\
\text { APR. } \\
\text { APR. }\end{array}$ & $\begin{array}{l}4 \\
6 \\
6 \\
7 \\
8\end{array}$ & $\begin{array}{l}03: 13 \\
09: 05 \\
15: 53 \\
14: 41 \\
11: 24\end{array}$ & $\begin{array}{l}\text { YST } \\
\text { YST } \\
\text { YST } \\
\text { AST } \\
\text { YST }\end{array}$ \\
\hline $\begin{array}{l}\text { APR. } \\
\text { APR. } \\
\text { APR. } \\
\text { APR. } \\
\text { APR. }\end{array}$ & $\begin{array}{l}10 \\
10 \\
12 \\
14 \\
15\end{array}$ & $\begin{array}{l}03 \\
14 \\
14 \\
11 \\
16\end{array}$ & $\begin{array}{l}20 \\
36 \\
23 \\
14 \\
59\end{array}$ & $\begin{array}{l}49.3 \\
03.6 \\
58.7 \\
26.7 \\
34.1\end{array}$ & $\begin{array}{l}2.789 \mathrm{~N} \\
2.280 \mathrm{~N} \\
0.471 \mathrm{~N} \\
8.994 \mathrm{~N} \\
0.681 \mathrm{~N}\end{array}$ & $\begin{array}{l}150.793 w . \\
151.375 W . \\
141.229 W . \\
151.232 W . \\
148.542 W\end{array}$ & $\begin{array}{r}99 \\
100 \\
15 \\
56 \\
63\end{array}$ & $\begin{array}{l}\text { GS } \\
\text { GS } \\
\text { GS }\end{array}$ & $\begin{array}{l}3.0 \\
3.9 \\
3.8\end{array}$ & $\begin{array}{l}\cdots \\
\cdots \\
\cdots \\
\cdots\end{array}$ & $\begin{array}{c}\ldots \\
\ldots \\
\text { 4. } 2 M L(P M) \\
\text { 3.9ML (PM) } \\
\text { 3. } 9 M L(P M)\end{array}$ & $\begin{array}{c}\cdots \\
\cdots \\
\cdots \\
\text { FELT }\end{array}$ & $\begin{array}{l}\text { APR. } \\
\text { APR. } \\
\text { APR. } \\
\text { APR. } \\
\text { APR. }\end{array}$ & $\begin{array}{r}9 \\
10 \\
12 \\
14 \\
15\end{array}$ & $\begin{array}{l}18: 20 \\
05: 36 \\
05: 23 \\
02: 14 \\
07: 59\end{array}$ & $\begin{array}{l}\text { YST } \\
\text { YST } \\
\text { YST } \\
\text { YST } \\
\text { YST }\end{array}$ \\
\hline $\begin{array}{l}\text { APR. } \\
\text { APR. } \\
\text { APR. } \\
\text { APR. } \\
\text { APR. }\end{array}$ & $\begin{array}{l}15 \\
17 \\
18 \\
18 \\
18\end{array}$ & $\begin{array}{l}20 \\
00 \\
06 \\
10 \\
13\end{array}$ & $\begin{array}{l}05 \\
28 \\
55 \\
57 \\
33\end{array}$ & $\begin{array}{l}00.1 \\
28.0 \\
46.5 \\
11.6 \\
12.5\end{array}$ & $\begin{array}{l}59.610 \mathrm{~N} \\
50.976 \mathrm{~N} \\
52.046 \mathrm{~N} \\
59.781 \mathrm{~N} \\
62.243 \mathrm{~N}\end{array}$ & $\begin{array}{l}152.775 w . \\
178.035 w . \\
169.753 w . \\
146.750 w . \\
154.131 W .\end{array}$ & $\begin{array}{l}97 \\
33 N \\
33 N \\
33 N \\
33 N\end{array}$ & $\begin{array}{l}\text { GS } \\
\text { GS } \\
\text { GS }\end{array}$ & $\begin{array}{l}4.0 \\
4.7 \\
5.0 \\
3.9 \\
\ldots\end{array}$ & $\begin{array}{l}\cdots \\
\cdots \\
\cdots \\
\cdots \\
\cdots\end{array}$ & $\begin{array}{c}\ldots \\
4.8 \mathrm{ML}(\mathrm{PM}) \\
3.6 \mathrm{ML}(\mathrm{PM}) \\
3.5 \mathrm{ML}(\mathrm{PM})\end{array}$ & $\begin{array}{c}\text { FELT } \\
\cdots \\
\cdots \\
\cdots \\
\cdots\end{array}$ & २. & $\begin{array}{l}15 \\
16 \\
17 \\
18 \\
18\end{array}$ & $\begin{array}{l}11: 05 \\
14: 28 \\
20: 55 \\
01: 57 \\
04: 33\end{array}$ & $\begin{array}{l}\text { YST } \\
\text { AST } \\
\text { AST } \\
\text { YST } \\
\text { YST }\end{array}$ \\
\hline $\begin{array}{l}\text { APR. } \\
\text { APR. } \\
\text { APR. }\end{array}$ & $\begin{array}{l}18 \\
18 \\
18 \\
18 \\
19\end{array}$ & $\begin{array}{l}18 \\
19 \\
20 \\
20 \\
01\end{array}$ & $\begin{array}{l}46 \\
31 \\
20 \\
44 \\
18\end{array}$ & $\begin{array}{l}29.1 \\
28.7 \\
22.4 \\
34.0 \\
17.3\end{array}$ & $\begin{array}{l}60.833 \mathrm{~N} \\
63.045 \mathrm{~N} \\
57.030 \mathrm{~N} \\
61.193 \mathrm{~N}\end{array}$ & $\begin{array}{l}152.509 \mathrm{~W} . \\
152.067 \mathrm{~W} . \\
151.023 \mathrm{~W} . \\
137.580 \mathrm{~W} . \\
147.409 \mathrm{~W} .\end{array}$ & $\begin{array}{l}33 \mathrm{~N} \\
95 \\
131 \\
18 \\
10\end{array}$ & $\begin{array}{l}\text { GS } \\
\text { GS } \\
\text { EP } \\
\text { GS }\end{array}$ & $\begin{array}{l}4.2 \\
5.1 \\
4.4 \\
\ldots . \\
\ldots .\end{array}$ & $\begin{array}{l}\cdots \\
\cdots \\
\cdots \\
\cdots \\
\cdots\end{array}$ & $\begin{array}{c}\cdots \\
\cdots \\
\text { 3. } 1 \mathrm{ML}(\mathrm{EP}) \\
\text { 3. } O \mathrm{ML}(\mathrm{PM})\end{array}$ & $\begin{array}{l}\cdots \\
\text { IV } \\
\ldots \\
\ldots\end{array}$ & $A$ & $\begin{array}{l}18 \\
18 \\
18 \\
18 \\
18\end{array}$ & $\begin{array}{l}09: 46 \\
10: 31 \\
11: 20 \\
11: 44 \\
16: 18\end{array}$ & $\begin{array}{l}\text { YST } \\
\text { YST } \\
\text { YST } \\
\text { YST } \\
\text { YST }\end{array}$ \\
\hline
\end{tabular}


Table 1. Summary of U.S. earthquakes for 1984--Continued

\begin{tabular}{|c|c|c|c|c|c|c|c|c|c|c|c|c|c|}
\hline \multirow{2}{*}{ Date } & \multicolumn{2}{|c|}{$\begin{array}{l}\text { Origin time } \\
\text { (UTC) }\end{array}$} & \multirow{2}{*}{$\begin{array}{c}\text { Latitude } \\
\left({ }^{\circ}\right)\end{array}$} & \multirow{2}{*}{$\begin{array}{c}\text { Longitude } \\
\text { ( })\end{array}$} & \multirow{2}{*}{$\begin{array}{c}\text { Depth } \\
(\mathbf{k m})\end{array}$} & \multirow{2}{*}{$\begin{array}{l}\text { Hypo- } \\
\text { center } \\
\text { source }\end{array}$} & \multicolumn{3}{|c|}{ Magnitude } & \multirow{2}{*}{$\begin{array}{l}\text { Maximum } \\
\text { intensity }\end{array}$} & \multicolumn{3}{|c|}{ Local time } \\
\hline & hr $\min$ & $\sec$ & & & & & mb & MS & $\begin{array}{l}\text { ML, Mn } \\
\text { MD, Mw }\end{array}$ & & Date & Hour & $\begin{array}{l}\text { Time } \\
\text { zome }\end{array}$ \\
\hline
\end{tabular}

ALASKA-Continued

\begin{tabular}{|c|c|c|c|c|c|c|c|c|c|c|c|c|c|c|c|c|}
\hline $\begin{array}{l}\text { APR. } \\
\text { APR. } \\
\text { APR. } \\
\text { APR. } \\
\text { APR. }\end{array}$ & $\begin{array}{l}19 \\
19 \\
20 \\
23 \\
23\end{array}$ & $\begin{array}{l}18 \\
20 \\
04 \\
01 \\
02\end{array}$ & $\begin{array}{l}39 \\
14 \\
24 \\
42 \\
32\end{array}$ & $\begin{array}{l}46.7 \\
36.3 \\
47.8 \\
36.3 \\
16.9\end{array}$ & $\begin{array}{l}66.410 \mathrm{~N} . \\
61.516 \mathrm{~N} . \\
61.723 \mathrm{~N} . \\
65.182 \mathrm{~N} . \\
65.243 \mathrm{~N} .\end{array}$ & $\begin{array}{l}151.120 W . \\
149.893 W . \\
152.089 W \\
146.945 W \\
146.889 w\end{array}$ & $\begin{array}{l}33 N \\
74 \\
119 \\
15 \\
22\end{array}$ & $\begin{array}{l}\text { GS } \\
\text { GS } \\
\text { GS } \\
\text { GS } \\
\text { GS }\end{array}$ & $\begin{array}{l}\cdots \\
\ldots \\
4.5 \\
\ldots\end{array}$ & $\begin{array}{l}\cdots \\
\cdots \\
\cdots \\
\cdots \\
\cdots\end{array}$ & $\begin{array}{c}\text { 4. } 7 M L(P M) \\
\ldots \\
\ldots \\
\text { 4. } 2 M L(P M) \\
\text { 3. } 3 M L(P M)\end{array}$ & $\begin{array}{r}\text { II } \\
\text { FELTT }\end{array}$ & $\begin{array}{l}\text { APR. } \\
\text { APR. } \\
\text { APR. } \\
\text { APR. } \\
\text { APR. }\end{array}$ & $\begin{array}{l}19 \\
19 \\
19 \\
22 \\
22\end{array}$ & $\begin{array}{l}09: 39 \\
11: 14 \\
19: 24 \\
16: 42 \\
17: 32\end{array}$ & $\begin{array}{l}\text { YST } \\
\text { YST } \\
\text { YST } \\
\text { YST } \\
\text { YST }\end{array}$ \\
\hline $\begin{array}{l}\text { APR. } \\
\text { APR. } \\
\text { APR. } \\
\text { APR. } \\
\text { APR. }\end{array}$ & $\begin{array}{l}23 \\
24 \\
25 \\
26 \\
27\end{array}$ & $\begin{array}{l}18 \\
14 \\
07 \\
18 \\
12\end{array}$ & $\begin{array}{l}21 \\
56 \\
08 \\
10 \\
29\end{array}$ & $\begin{array}{l}01.0 \\
21.9 \\
38.3 \\
58.4 \\
13.6\end{array}$ & $\begin{array}{l}57.770 \mathrm{~N} . \\
64.522 \mathrm{~N} . \\
58.329 \mathrm{~N} . \\
61.295 \mathrm{~N} . \\
52.633 \mathrm{~N} .\end{array}$ & $\begin{array}{l}137.610 w . \\
146.681 w . \\
154.775 w . \\
146.827 w . \\
167.014 W .\end{array}$ & $\begin{array}{c}18 \\
4 \\
106 \\
33 N \\
33 N\end{array}$ & $\begin{array}{l}\text { EP } \\
\text { GS } \\
\text { GS } \\
\text { GS } \\
\text { GS }\end{array}$ & $\begin{array}{l}\cdots \\
\ddot{3} .8 \\
\ddot{4} . \dot{9}\end{array}$ & $\begin{array}{l}\cdots \\
\cdots \\
\cdots \\
\cdots\end{array}$ & $\begin{array}{c}\text { 3. } 4 M L(E P) \\
\text { 3. } 0 M L(P M) \\
\ldots \\
\text { 3. } 4 M L(P M) \\
4.6 M L(P M)\end{array}$ & $\begin{array}{l}\cdots \\
\cdots \\
\text { III } \\
\cdots\end{array}$ & $\begin{array}{l}\text { APR. } \\
\text { APR. } \\
\text { APR. } \\
\text { APR. } \\
\text { APR. }\end{array}$ & $\begin{array}{l}23 \\
24 \\
24 \\
26 \\
27\end{array}$ & $\begin{array}{l}09: 21 \\
05: 56 \\
22: 08 \\
09: 10 \\
03: 29\end{array}$ & $T$ \\
\hline $\begin{array}{l}\text { APR. } \\
\text { APR. } \\
\text { APR. } \\
\text { MAY } \\
\text { MAY }\end{array}$ & $\begin{array}{r}28 \\
29 \\
30 \\
2 \\
2\end{array}$ & $\begin{array}{l}10 \\
10 \\
16 \\
00 \\
09\end{array}$ & $\begin{array}{l}05 \\
57 \\
14 \\
22 \\
11\end{array}$ & $\begin{array}{l}51.8 \\
55.6 \\
14.2 \\
52.1 \\
45.5\end{array}$ & $\begin{array}{l}51.943 \mathrm{~N} . \\
63.173 \mathrm{~N} . \\
59.578 \mathrm{~N} . \\
61.252 \mathrm{~N} . \\
57.262 \mathrm{~N} .\end{array}$ & $\begin{array}{l}169.792 W . \\
150.855 W . \\
152.581 W . \\
146.905 W . \\
155.678 W .\end{array}$ & $\begin{array}{l}33 N \\
137 \\
88 \\
33 N \\
114\end{array}$ & $\begin{array}{l}\text { GS } \\
\text { GS } \\
\text { GS } \\
\text { GS } \\
\text { GS }\end{array}$ & $\begin{array}{l}5.4 \\
\ldots \\
\ldots \\
4.3\end{array}$ & $\begin{array}{l}4.7 \\
\ldots \\
\cdots \\
\cdots \\
\cdots\end{array}$ & $\begin{array}{c}\text { 5. } 1 \mathrm{ML}(\mathrm{PM}) \\
\ldots \\
\ldots \\
\text { 3. } 5 \mathrm{ML}(\mathrm{PM})\end{array}$ & $\ldots$ & $\begin{array}{l}\text { APR. } \\
\text { APR. } \\
\text { APR. } \\
\text { MAY } \\
\text { MAY }\end{array}$ & $\begin{array}{r}28 \\
29 \\
30 \\
1 \\
2\end{array}$ & $\begin{array}{l}00: 05 \\
01: 57 \\
07: 14 \\
15: 22 \\
00: 11\end{array}$ & . \\
\hline $\begin{array}{l}\text { MAY } \\
\text { MAY } \\
\text { MAY } \\
\text { MAY } \\
\text { MAY }\end{array}$ & $\begin{array}{l}2 \\
4 \\
4 \\
5 \\
6\end{array}$ & $\begin{array}{l}18 \\
23 \\
23 \\
00 \\
03\end{array}$ & $\begin{array}{l}59 \\
02 \\
03 \\
30 \\
52\end{array}$ & $\begin{array}{l}05.7 \\
39.9 \\
18.9 \\
42.8 \\
25.9\end{array}$ & $\begin{array}{l}55.590 \mathrm{~N} . \\
60.718 \mathrm{~N} . \\
59.316 \mathrm{~N} . \\
52.591 \mathrm{~N} . \\
61.656 \mathrm{~N} .\end{array}$ & $\begin{array}{l}135.047 W \\
146.809 W \\
153.187 W \\
168.156 W \\
146.186 W .\end{array}$ & $\begin{array}{l}10 \\
33 N \\
88 \\
33 N \\
33 N\end{array}$ & $\begin{array}{l}\text { GS } \\
\text { GS } \\
\text { GS } \\
\text { GS } \\
\text { GS }\end{array}$ & $\begin{array}{l}4.9 \\
4.8 \\
4.4 \\
\ldots\end{array}$ & $\begin{array}{l}4.0 \\
\cdots \\
\cdots \\
\cdots\end{array}$ & $\begin{array}{c}4.9 M L(P M) \\
\ldots \\
\ldots \\
\text { 3. } 0 M L(P M)\end{array}$ & $\begin{array}{c}\mathrm{V} \\
\mathrm{IV}\end{array}$ & $\begin{array}{l}\text { MAY } \\
\text { MAY } \\
\text { MAY } \\
\text { MAY } \\
\text { MAY }\end{array}$ & $\begin{array}{l}2 \\
4 \\
4 \\
4 \\
5\end{array}$ & $\begin{array}{l}09: 59 \\
14: 02 \\
14: 03 \\
15: 30 \\
18: 52\end{array}$ & $\begin{array}{l}T \\
T \\
T \\
T \\
T \\
T\end{array}$ \\
\hline $\begin{array}{l}\text { MAY } \\
\text { MAY } \\
\text { MAY } \\
\text { MAY } \\
\text { MAY }\end{array}$ & $\begin{array}{r}6 \\
15 \\
17 \\
17 \\
19\end{array}$ & $\begin{array}{l}19 \\
23 \\
20 \\
20 \\
03\end{array}$ & $\begin{array}{l}54 \\
37 \\
02 \\
07 \\
44\end{array}$ & $\begin{array}{l}49.1 \\
44.0 \\
58.8 \\
51.2 \\
53.5\end{array}$ & $\begin{array}{l}51.688 \mathrm{~N} . \\
59.910 \mathrm{~N} . \\
51.374 \mathrm{~N} . \\
51.324 \mathrm{~N} . \\
60.134 \mathrm{~N} .\end{array}$ & $\begin{array}{l}176.774 W . \\
140.690 W \\
176.540 E \\
176.645 E \\
141.078 W\end{array}$ & $\begin{array}{l}58 \\
18 \\
33 N \\
33 N \\
15\end{array}$ & $\begin{array}{l}\text { GS } \\
\text { EP } \\
\text { GS } \\
\text { GS } \\
\text { GS }\end{array}$ & $\begin{array}{l}5.6 \\
3.8 \\
4.9 \\
4.2\end{array}$ & $\begin{array}{l}\cdots \\
\cdots \\
\cdots \\
\cdots\end{array}$ & $\begin{array}{c}\text { 5. } 6 \mathrm{ML}(\mathrm{PM}) \\
\text { 3. } 8 \mathrm{ML}(\mathrm{EP}) \\
\ldots \\
\ldots \\
\text { 4. } 2 \mathrm{ML}(\mathrm{PM})\end{array}$ & $\begin{array}{l}\quad v \\
\cdots \\
\cdots\end{array}$ & $\begin{array}{l}\text { MAY } \\
\text { MAY } \\
\text { MAY } \\
\text { MAY } \\
\text { MAY }\end{array}$ & $\begin{array}{r}6 \\
15 \\
17 \\
17 \\
18\end{array}$ & $\begin{array}{l}09: 54 \\
14: 37 \\
10: 02 \\
10: 07 \\
18: 44\end{array}$ & $\begin{array}{l}T \\
T \\
T \\
T \\
T \\
T\end{array}$ \\
\hline $\begin{array}{l}\text { MAY } \\
\text { MAY } \\
\text { MAY } \\
\text { MAY } \\
\text { MAY }\end{array}$ & $\begin{array}{l}21 \\
21 \\
22 \\
23 \\
25\end{array}$ & $\begin{array}{l}18 \\
19 \\
08 \\
18 \\
09\end{array}$ & $\begin{array}{l}47 \\
23 \\
14 \\
36 \\
30\end{array}$ & $\begin{array}{l}03.1 \\
39.8 \\
53.5 \\
00.5 \\
09.8\end{array}$ & $\begin{array}{l}58.220 \mathrm{~N} \\
62.583 \mathrm{~N} \\
63.100 \mathrm{~N} \\
55.587 \mathrm{~N} \\
63.343 \mathrm{~N} .\end{array}$ & $\begin{array}{l}156.298 W . \\
151.416 W . \\
150.361 W . \\
155.465 W . \\
151.109 W .\end{array}$ & $\begin{array}{l}161 \\
100 \\
143 \\
33 N \\
33 N\end{array}$ & $\begin{array}{l}\text { GS } \\
\text { GS } \\
\text { GS } \\
\text { GS } \\
\text { GS }\end{array}$ & $\begin{array}{l}4.6 \\
\ldots .6 \\
3.5 \\
\ldots\end{array}$ & $\begin{array}{l}\cdots \\
\cdots \\
\cdots \\
\cdots \\
\cdots\end{array}$ & $\begin{array}{c}\cdots \\
\cdots \\
\cdots \\
\text { 4. } 4 \mathrm{ML}(\mathrm{PM}) \\
\text { 3. } \theta \mathrm{ML}(\mathrm{PM})\end{array}$ & $\ldots$ & $\begin{array}{l}\text { MAY } \\
\text { MAY } \\
\text { MAY } \\
\text { MAY } \\
\text { MAY }\end{array}$ & $\begin{array}{l}21 \\
21 \\
21 \\
23 \\
25\end{array}$ & $\begin{array}{l}09: 47 \\
10: 23 \\
23: 14 \\
09: 36 \\
00: 30\end{array}$ & $Y S$ \\
\hline $\begin{array}{l}\text { MAY } \\
\text { MAY } \\
\text { MAY } \\
\text { MAY } \\
\text { MAY }\end{array}$ & $\begin{array}{l}25 \\
26 \\
28 \\
28 \\
28\end{array}$ & $\begin{array}{l}14 \\
04 \\
00 \\
05 \\
10\end{array}$ & $\begin{array}{l}52 \\
14 \\
05 \\
17 \\
56\end{array}$ & $\begin{array}{l}15.2 \\
25.9 \\
41.0 \\
54.7 \\
33.2\end{array}$ & $\begin{array}{l}59.508 \mathrm{~N} . \\
53.752 \mathrm{~N} . \\
58.340 \mathrm{~N} . \\
59.297 \mathrm{~N} . \\
67.957 \mathrm{~N} .\end{array}$ & $\begin{array}{l}153.014 W . \\
171.186 \mathrm{E} . \\
133.550 W . \\
153.685 W . \\
158.085 W .\end{array}$ & $\begin{array}{l}89 \\
33 N \\
18 \\
101 \\
33 N\end{array}$ & $\begin{array}{l}\text { GS } \\
\text { GS } \\
\text { EP } \\
\text { GS } \\
\text { GS }\end{array}$ & $\begin{array}{l}3.4 \\
4.0 \\
\cdots \\
4.8 \\
4.1\end{array}$ & $\begin{array}{l}\cdots \\
\cdots \\
\cdots \\
\cdots \\
\cdots\end{array}$ & $\begin{array}{c}\ldots \\
\text { 3. } 4 \mathrm{ML}(\mathrm{EP}) \\
\text { 3. } \ddot{\mathrm{ML}} \dot{\mathrm{M}}(\mathrm{PM})\end{array}$ & $\ldots$ & $\begin{array}{l}\text { MAY } \\
\text { MAY } \\
\text { MAY } \\
\text { MAY } \\
\text { MAY }\end{array}$ & $\begin{array}{l}25 \\
25 \\
27 \\
27 \\
28\end{array}$ & $\begin{array}{l}05: 52 \\
18: 14 \\
15: 05 \\
20: 17 \\
01: 56\end{array}$ & $\begin{array}{l}\text { AST } \\
\text { YST } \\
\text { YST } \\
\text { YST }\end{array}$ \\
\hline $\begin{array}{l}\text { MAY } \\
\text { MAY } \\
\text { MAY } \\
\text { MAY } \\
\text { MAY }\end{array}$ & $\begin{array}{l}28 \\
29 \\
29 \\
29 \\
30\end{array}$ & $\begin{array}{l}14 \\
04 \\
08 \\
15 \\
12\end{array}$ & $\begin{array}{l}38 \\
14 \\
12 \\
19 \\
41\end{array}$ & $\begin{array}{l}34.2 \\
06.1 \\
37.7 \\
26.5 \\
21.3\end{array}$ & $\begin{array}{l}63.127 \mathrm{~N} . \\
60.572 \mathrm{~N} . \\
58.380 \mathrm{~N} . \\
51.950 \mathrm{~N} . \\
51.176 \mathrm{~N} .\end{array}$ & $\begin{array}{l}150.696 W . \\
146.848 W . \\
154.760 W . \\
166.213 W . \\
178.720 E .\end{array}$ & $\begin{array}{l}138 \\
33 N \\
33 N \\
33 N \\
48\end{array}$ & $\begin{array}{l}\text { GS } \\
\text { GS } \\
\text { GS } \\
\text { GS } \\
\text { GS }\end{array}$ & $\begin{array}{l}3.4 \\
4.3 \\
4.5 \\
4.9\end{array}$ & $\begin{array}{l}\cdots \\
\cdots \\
\cdots \\
\cdots\end{array}$ & $\begin{array}{l}\text { 3. } O M L(P M) \\
\text { 4. 3ML(PM) } \\
\text { 4.3ML(PM) } \\
\text { 4.8ML(PM) }\end{array}$ & $\begin{array}{l}\cdots \\
\cdots \\
\cdots \\
\cdots\end{array}$ & $\begin{array}{l}\text { MAY } \\
\text { MAY } \\
\text { MAY } \\
\text { MAY } \\
\text { MAY }\end{array}$ & $\begin{array}{l}28 \\
28 \\
28 \\
29 \\
30\end{array}$ & $\begin{array}{l}05: 38 \\
19: 14 \\
23: 12 \\
06: 19 \\
02: 41\end{array}$ & $\begin{array}{l}\text { YST } \\
\text { YST } \\
\text { YST } \\
\text { AST } \\
\text { AST }\end{array}$ \\
\hline $\begin{array}{l}\text { MAY } \\
\text { MAY } \\
\text { MAY } \\
\text { JUNE } \\
\text { JUNE }\end{array}$ & $\begin{array}{r}30 \\
31 \\
31 \\
2 \\
3\end{array}$ & $\begin{array}{l}12 \\
06 \\
21 \\
08 \\
13\end{array}$ & $\begin{array}{l}58 \\
07 \\
43 \\
49 \\
56\end{array}$ & $\begin{array}{l}02.7 \\
07.0 \\
59.3 \\
15.9 \\
06.3\end{array}$ & $\begin{array}{l}51.174 \mathrm{~N} . \\
58.320 \mathrm{~N} . \\
53.034 \mathrm{~N} . \\
60.249 \mathrm{~N} . \\
61.468 \mathrm{~N} .\end{array}$ & $\begin{array}{l}178.709 E . \\
133.610 W . \\
170.852 E . \\
146.956 W . \\
150.839 W .\end{array}$ & $\begin{array}{l}40 \\
18 \\
33 N \\
33 N \\
89\end{array}$ & $\begin{array}{l}\text { GS } \\
\text { EP } \\
\text { GS } \\
\text { GS } \\
\text { GS }\end{array}$ & $\begin{array}{l}5.0 \\
5.0 \\
3.2\end{array}$ & $\begin{array}{l}\ldots \\
\ldots \\
4.4 \\
\ldots \\
\ldots\end{array}$ & $\begin{array}{l}\text { 5. } 1 \mathrm{ML}(\mathrm{PM}) \\
\text { 3. } 3 \mathrm{ML}(\mathrm{EP}) \\
\ldots \ldots \\
\text { 3. } 2 \mathrm{ML}(\mathrm{PM})\end{array}$ & $\begin{array}{l}\cdots \\
\cdots \\
\cdots\end{array}$ & $\begin{array}{l}\text { MAY } \\
\text { MAY } \\
\text { MAY } \\
\text { JUNE } \\
\text { JUNE }\end{array}$ & $\begin{array}{r}30 \\
30 \\
31 \\
1 \\
3\end{array}$ & $\begin{array}{l}02: 58 \\
21: 07 \\
11: 43 \\
23: 49 \\
04: 56\end{array}$ & $\begin{array}{l}\text { AST } \\
\text { YST } \\
\text { AST } \\
\text { YST } \\
\text { YST }\end{array}$ \\
\hline $\begin{array}{l}\text { JUNE } \\
\text { JUNE } \\
\text { JUNE } \\
\text { JUNE } \\
\text { JUNE }\end{array}$ & $\begin{array}{l}4 \\
4 \\
4 \\
4 \\
5\end{array}$ & $\begin{array}{l}05 \\
05 \\
09 \\
17 \\
01\end{array}$ & $\begin{array}{l}43 \\
53 \\
38 \\
19 \\
44\end{array}$ & $\begin{array}{l}42.0 \\
57.2 \\
41.8 \\
59.2 \\
21.4\end{array}$ & $\begin{array}{l}63.047 \mathrm{~N} \\
61.057 \mathrm{~N} \\
64.530 \mathrm{~N} \\
52.112 \mathrm{~N} \\
56.901 \mathrm{~N}\end{array}$ & $\begin{array}{l}150.522 W . \\
152.613 W . \\
152.661 W . \\
170.999 W . \\
157.262 W .\end{array}$ & $\begin{array}{l}119 \\
150 \\
33 \mathrm{~N} \\
33 \mathrm{~N} \\
94\end{array}$ & $\begin{array}{l}\text { GS } \\
\text { GS } \\
\text { GS } \\
\text { GS } \\
\text { GS }\end{array}$ & $\begin{array}{l}\cdots \\
\cdots \\
5.2 \\
5.3\end{array}$ & $\begin{array}{l}\cdots \\
\cdots \\
\cdots \\
\ldots \\
\ldots\end{array}$ & $\begin{array}{c}3 . \dot{7} \dot{M L}(P M) \\
\ldots \\
\ldots\end{array}$ & IV & $\begin{array}{l}\text { JUNE } \\
\text { JUNE } \\
\text { JUNE } \\
\text { JUNE } \\
\text { JUNE }\end{array}$ & $\begin{array}{l}3 \\
3 \\
4 \\
4 \\
4\end{array}$ & $\begin{array}{l}20: 43 \\
20: 53 \\
00: 38 \\
07: 19 \\
16: 44\end{array}$ & $\begin{array}{l}\text { YST } \\
\text { YST } \\
\text { AST } \\
\text { YST }\end{array}$ \\
\hline
\end{tabular}


Table 1. Summary of U.S. earthquakes for 1984-Continued

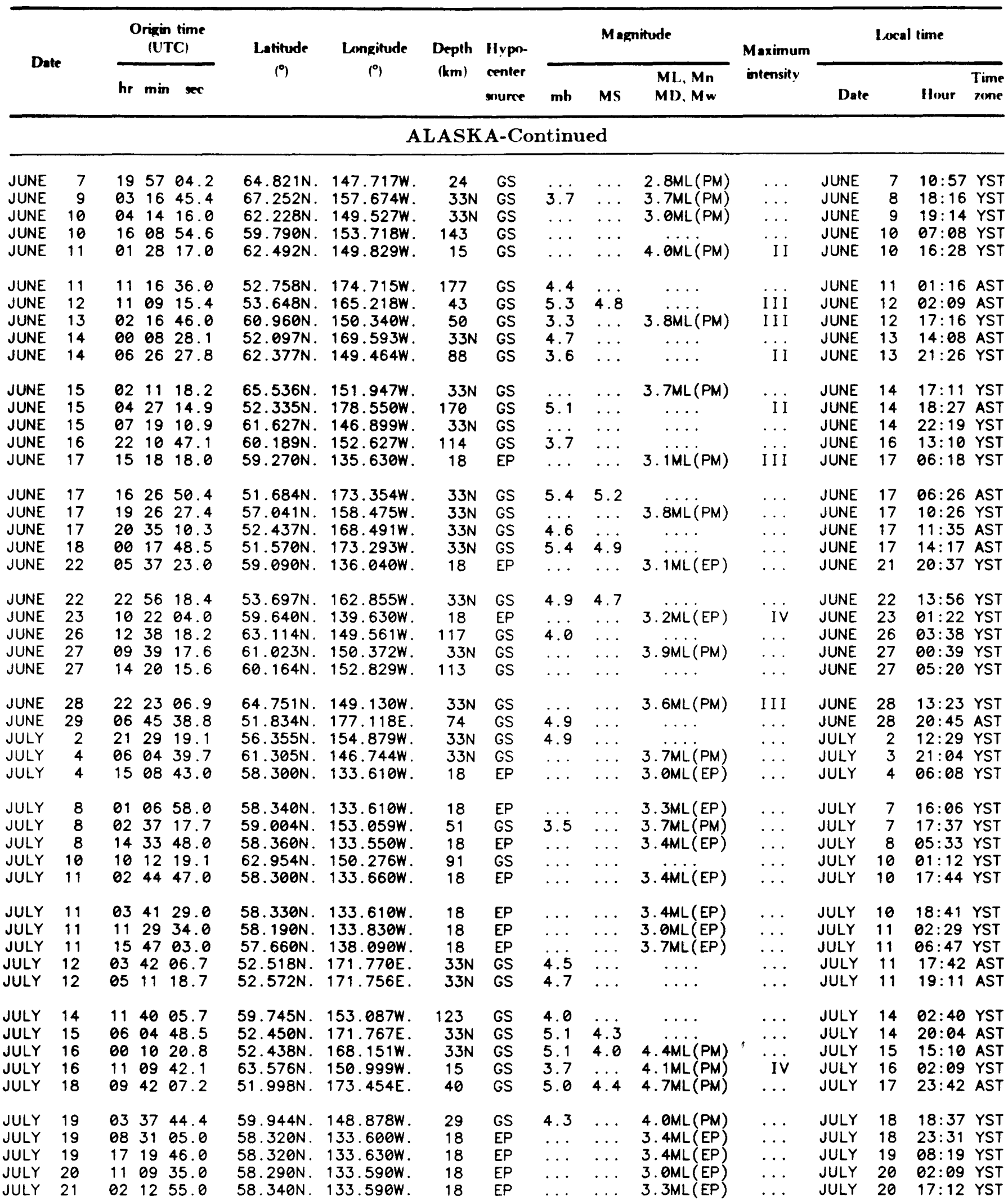


Table 1. Summary of U.S. earthquakes for 1984-Continued

\begin{tabular}{|c|c|c|c|c|c|c|c|c|c|c|c|c|c|}
\hline \multirow{2}{*}{ Date } & \multicolumn{2}{|c|}{$\begin{array}{l}\text { Origin time } \\
\text { (UTC) }\end{array}$} & \multirow{2}{*}{$\begin{array}{c}\text { Latitude } \\
\left({ }^{\circ}\right)\end{array}$} & \multirow{2}{*}{$\begin{array}{l}\text { Longitude } \\
\text { (') }\end{array}$} & \multirow{2}{*}{$\begin{array}{l}\text { Depth } \\
\text { (km) }\end{array}$} & \multirow{2}{*}{$\begin{array}{l}\text { Hypo- } \\
\text { center } \\
\text { source }\end{array}$} & \multicolumn{3}{|c|}{ Magnitude } & \multirow{2}{*}{$\begin{array}{l}\text { Maximum } \\
\text { intensity }\end{array}$} & \multicolumn{3}{|c|}{ Local time } \\
\hline & hr $\min$ & $\sec$ & & & & & $\mathbf{m b}$ & MS & $\begin{array}{l}\text { ML. Mn } \\
\text { MD. Mw }\end{array}$ & & Date & Hour & $\begin{array}{l}\text { Time } \\
\text { zone }\end{array}$ \\
\hline
\end{tabular}

ALASKA-Continured

\begin{tabular}{|c|c|c|c|c|c|c|c|c|c|c|c|c|c|c|c|c|}
\hline $\begin{array}{l}\text { JULY } \\
\text { JULY } \\
\text { JULY } \\
\text { JULY } \\
\text { JULY }\end{array}$ & $\begin{array}{l}21 \\
24 \\
24 \\
25 \\
25\end{array}$ & $\begin{array}{l}07 \\
00 \\
15 \\
04 \\
12\end{array}$ & $\begin{array}{l}11 \\
48 \\
50 \\
59 \\
36\end{array}$ & $\begin{array}{l}39.0 \\
40.3 \\
01.7 \\
50.1 \\
45.9\end{array}$ & $\begin{array}{l}58.340 \mathrm{~N} . \\
62.790 \mathrm{~N} . \\
56.761 \mathrm{~N} . \\
62.688 \mathrm{~N} . \\
61.504 \mathrm{~N} .\end{array}$ & $\begin{array}{l}133.560 W . \\
149.681 W . \\
153.156 w . \\
149.811 W . \\
157.329 W .\end{array}$ & $\begin{array}{c}18 \\
101 \\
33 \mathrm{~N} \\
71 \\
33 \mathrm{~N}\end{array}$ & $\begin{array}{l}\text { EP } \\
\text { GS } \\
\text { GS } \\
\text { GS } \\
\text { GS }\end{array}$ & $\begin{array}{l}\cdots \\
4.3 \\
4.4 \\
4.1\end{array}$ & $\begin{array}{l}\cdots \\
\cdots \\
\cdots \\
\cdots\end{array}$ & $\begin{array}{c}3.5 \mathrm{ML}(\mathrm{EP}) \\
4.5 \mathrm{ML}(P M) \\
\ldots \ldots \\
4.8 \mathrm{ML}(\mathrm{PM})\end{array}$ & $\begin{array}{l}\cdots \\
\cdots \\
\text { IV } \\
\text { IV }\end{array}$ & $\begin{array}{l}\text { JULY } \\
\text { JULY } \\
\text { JULY } \\
\text { JULY } \\
\text { JULY }\end{array}$ & $\begin{array}{l}20 \\
23 \\
24 \\
24 \\
25\end{array}$ & $\begin{array}{l}22: 11 \\
15: 48 \\
06: 50 \\
19: 59 \\
03: 36\end{array}$ & $\begin{array}{l}\text { YST } \\
\text { YST } \\
\text { YST } \\
\text { YST } \\
\text { YST }\end{array}$ \\
\hline $\begin{array}{l}\text { JULY } \\
\text { JULY } \\
\text { JULY } \\
\text { JULY } \\
\text { JULY }\end{array}$ & $\begin{array}{l}25 \\
27 \\
27 \\
27 \\
29\end{array}$ & $\begin{array}{l}23 \\
07 \\
15 \\
23 \\
10\end{array}$ & $\begin{array}{l}28 \\
39 \\
57 \\
22 \\
37\end{array}$ & $\begin{array}{l}53.3 \\
50.3 \\
51.3 \\
50.2 \\
11.6\end{array}$ & $\begin{array}{l}54.955 \mathrm{~N} . \\
60.058 \mathrm{~N} . \\
50.317 \mathrm{~N} . \\
51.764 \mathrm{~N} . \\
60.993 \mathrm{~N} .\end{array}$ & $\begin{array}{l}160.113 W . \\
152.950 W . \\
176.868 w . \\
175.713 W . \\
146.712 W .\end{array}$ & $\begin{array}{c}53 \\
106 \\
33 \mathrm{~N} \\
190 \\
33 \mathrm{~N}\end{array}$ & $\begin{array}{l}\text { GS } \\
\text { GS } \\
\text { GS } \\
\text { GS } \\
\text { GS }\end{array}$ & $\begin{array}{l}5.3 \\
\ldots .8 \\
5.8 \\
\ldots\end{array}$ & $\begin{array}{l}\ldots \\
5.0 \\
\ldots \\
\ldots\end{array}$ & $\begin{array}{c}\text { 4. } 7 \mathrm{ML}(P M) \\
\ldots \ldots \\
5.7 \mathrm{ML}(P M) \\
\ldots \ldots \dot{0 M L}(P M)\end{array}$ & $\begin{array}{l}\text { IV } \\
\ldots \\
\text { IV } \\
\ldots \\
\ldots\end{array}$ & $\begin{array}{l}\text { JULY } \\
\text { JULY } \\
\text { JULY } \\
\text { JULY } \\
\text { JULY }\end{array}$ & $\begin{array}{l}25 \\
26 \\
27 \\
27 \\
29\end{array}$ & $\begin{array}{l}14: 28 \\
22: 39 \\
05: 57 \\
13: 22 \\
01: 37\end{array}$ & $\begin{array}{l}\text { YST } \\
\text { YST } \\
\text { AST } \\
\text { AST } \\
\text { YST }\end{array}$ \\
\hline $\begin{array}{l}\text { JULY } \\
\text { JULY } \\
\text { JULY } \\
\text { JULY } \\
\text { JULY }\end{array}$ & $\begin{array}{l}29 \\
30 \\
30 \\
30 \\
30\end{array}$ & $\begin{array}{l}22 \\
09 \\
13 \\
16 \\
21\end{array}$ & $\begin{array}{l}04 \\
07 \\
38 \\
32 \\
14\end{array}$ & $\begin{array}{l}57.7 \\
47.8 \\
29.7 \\
15.4 \\
28.2\end{array}$ & $\begin{array}{l}52.521 \mathrm{~N} . \\
59.872 \mathrm{~N} \\
60.846 \mathrm{~N} \\
57.852 \mathrm{~N} \\
52.082 \mathrm{~N} .\end{array}$ & $\begin{array}{l}168.065 W \\
152.343 W \\
151.533 W \\
156.309 W \\
179.496 \mathrm{E} .\end{array}$ & $\begin{array}{l}33 N \\
93 \\
94 \\
152 \\
143\end{array}$ & $\begin{array}{l}\text { GS } \\
\text { GS } \\
\text { GS } \\
\text { GS } \\
\text { GS }\end{array}$ & $\begin{array}{l}4.7 \\
4.2 \\
\ldots \\
\therefore .8\end{array}$ & $\begin{array}{l}\ldots \\
\ldots \\
\cdots \\
\cdots \\
\cdots\end{array}$ & $\begin{array}{l}\cdots \\
\cdots \\
\cdots \\
\cdots \\
\cdots\end{array}$ & $\begin{array}{l}\cdots \\
\cdots \\
\cdots \\
\cdots\end{array}$ & $\begin{array}{l}\text { JULY } \\
\text { JULY } \\
\text { JULY } \\
\text { JULY } \\
\text { JULY }\end{array}$ & $\begin{array}{l}29 \\
30 \\
30 \\
30 \\
30\end{array}$ & $\begin{array}{l}13: 04 \\
00: 07 \\
04: 38 \\
07: 32 \\
11: 14\end{array}$ & $\begin{array}{l}\text { AST } \\
\text { YST } \\
\text { YST } \\
\text { YST } \\
\text { AST }\end{array}$ \\
\hline $\begin{array}{l}\text { ULY } \\
\text { UG. } \\
\text { UG. } \\
\text { UG. } \\
\text { UG. }\end{array}$ & $\begin{array}{r}30 \\
1 \\
2 \\
3 \\
5\end{array}$ & $\begin{array}{l}22 \\
17 \\
04 \\
15 \\
14\end{array}$ & $\begin{array}{l}03 \\
30 \\
50 \\
38 \\
11\end{array}$ & $\begin{array}{l}24.5 \\
54.7 \\
32.6 \\
15.5 \\
02.7\end{array}$ & $\begin{array}{l}53.681 \mathrm{~N} . \\
51.931 \mathrm{~N} . \\
60.632 \mathrm{~N} . \\
58.891 \mathrm{~N} . \\
61.636 \mathrm{~N} .\end{array}$ & $\begin{array}{l}165.581 w . \\
172.474 w . \\
146.935 w . \\
145.063 w . \\
149.823 w .\end{array}$ & $\begin{array}{l}33 N \\
33 N \\
33 N \\
33 N \\
58\end{array}$ & $\begin{array}{l}\text { GS } \\
\text { GS } \\
\text { GS } \\
\text { GS } \\
\text { GS }\end{array}$ & $\begin{array}{l}4.9 \\
4.7 \\
\cdots \\
\cdots \\
\cdots\end{array}$ & $\begin{array}{l}5.0 \\
\ldots \\
\cdots \\
\cdots \\
\cdots\end{array}$ & $\begin{array}{c}\ldots \\
3.7 M L(P M) \\
3.7 M L(P M) \\
3.6 M L(P M)\end{array}$ & $\begin{array}{c}\cdots \\
\cdots \\
\cdots \\
\cdots \\
F \dot{E} \dot{T}\end{array}$ & $\begin{array}{l}\text { JULY } \\
\text { AUG. } \\
\text { AUG. } \\
\text { AUG. } \\
\text { AUG. }\end{array}$ & $\begin{array}{r}30 \\
1 \\
1 \\
3 \\
5\end{array}$ & $\begin{array}{l}13: 03 \\
07: 30 \\
19: 50 \\
06: 38 \\
05: 11\end{array}$ & $\begin{array}{l}\text { AST } \\
\text { AST } \\
\text { YST } \\
\text { YST } \\
\text { YST }\end{array}$ \\
\hline $\begin{array}{l}\text { UUG. } \\
\text { UGG. } \\
\text { UUG. } \\
\text { IUG. } \\
\text { UUG. }\end{array}$ & $\begin{array}{l}5 \\
6 \\
6 \\
7 \\
7\end{array}$ & $\begin{array}{l}17 \\
07 \\
20 \\
06 \\
08\end{array}$ & $\begin{array}{l}34 \\
09 \\
24 \\
37 \\
35\end{array}$ & $\begin{array}{l}30.0 \\
26.7 \\
52.8 \\
22.3 \\
22.7\end{array}$ & $\begin{array}{l}62.721 \mathrm{~N} . \\
63.529 \mathrm{~N} . \\
51.269 \mathrm{~N} \\
51.306 \mathrm{~N} \\
61.769 \mathrm{~N} .\end{array}$ & $\begin{array}{l}149.346 W . \\
150.911 W . \\
179.124 W . \\
179.091 W . \\
149.674 W .\end{array}$ & $\begin{array}{l}93 \\
33 N \\
57 \\
56 \\
44\end{array}$ & $\begin{array}{l}\text { GS } \\
\text { GS } \\
\text { GS } \\
\text { GS } \\
\text { GS }\end{array}$ & $\begin{array}{l}\ldots \\
4.6 \\
5.0 \\
\ldots\end{array}$ & $\begin{array}{l}\cdots \\
\cdots \\
\cdots \\
\cdots\end{array}$ & $\begin{array}{c}\ldots \\
\ldots \\
\text { 4. } 9 \mathrm{ML}(P M) \\
\text { 3. } 4 \mathrm{ML}(\mathrm{PM})\end{array}$ & $\begin{array}{l}\ldots \\
\cdots \\
\cdots \\
\text { III }\end{array}$ & $\begin{array}{l}\text { AUG. } \\
A \cup G . \\
A \cup G . \\
A \cup G . \\
\text { AUG. }\end{array}$ & $\begin{array}{l}5 \\
5 \\
6 \\
6 \\
6\end{array}$ & $\begin{array}{l}08: 34 \\
22: 09 \\
10: 24 \\
20: 37 \\
23: 35\end{array}$ & $\begin{array}{l}\text { YST } \\
\text { YST } \\
\text { AST } \\
\text { AST } \\
\text { YST }\end{array}$ \\
\hline $\begin{array}{l}\text { UUG. } \\
\text { AUG. } \\
\text { UUG. } \\
\text { UUG. }\end{array}$ & $\begin{array}{r}7 \\
8 \\
9 \\
11 \\
11\end{array}$ & $\begin{array}{l}14 \\
13 \\
14 \\
07 \\
23\end{array}$ & $\begin{array}{l}15 \\
37 \\
43 \\
30 \\
31\end{array}$ & $\begin{array}{l}28.8 \\
16.7 \\
42.4 \\
10.0 \\
42.5\end{array}$ & $\begin{array}{l}58.248 \mathrm{~N} \\
61.354 \mathrm{~N} \\
62.027 \mathrm{~N} \\
59.131 \mathrm{~N} \\
63.079 \mathrm{~N}\end{array}$ & $\begin{array}{l}154.012 W . \\
151.736 W . \\
149.726 W . \\
136.771 W . \\
150.368 W .\end{array}$ & $\begin{array}{r}110 \\
100 \\
59 \\
15 \\
130\end{array}$ & $\begin{array}{l}\text { GS } \\
\text { GS } \\
\text { GS } \\
\text { GS } \\
\text { GS }\end{array}$ & $\begin{array}{l}\ldots \\
\ldots \\
4.1 \\
\ldots\end{array}$ & $\begin{array}{l}\cdots \\
\cdots \\
\cdots \\
\cdots\end{array}$ & $\begin{array}{l}\text { 3. } 2 M L(P M) \\
\text { 4. } 2 M L(P M)\end{array}$ & $\begin{array}{c}\cdots \\
\cdots \\
\text { FELT } \\
\ldots\end{array}$ & & $\begin{array}{r}7 \\
8 \\
9 \\
10 \\
11\end{array}$ & $\begin{array}{l}05: 15 \\
04: 37 \\
05: 43 \\
22: 30 \\
14: 31\end{array}$ & $\begin{array}{l}\text { YST } \\
\text { YST } \\
\text { YST } \\
\text { YST } \\
\text { YST }\end{array}$ \\
\hline $\begin{array}{l}\text { AUG. } \\
\text { AUG. } \\
\text { AUG. } \\
\text { AUG. } \\
\text { AUG. }\end{array}$ & $\begin{array}{l}12 \\
13 \\
14 \\
14 \\
14\end{array}$ & $\begin{array}{l}21 \\
07 \\
01 \\
01 \\
07\end{array}$ & $\begin{array}{l}18 \\
10 \\
02 \\
54 \\
41\end{array}$ & $\begin{array}{l}50.1 \\
58.0 \\
08.4 \\
37.3 \\
07.7\end{array}$ & $\begin{array}{l}62.415 \mathrm{~N} \\
63.670 \mathrm{~N} \\
61.857 \mathrm{~N} \\
61.774 \mathrm{~N} \\
61.784 \mathrm{~N} .\end{array}$ & $\begin{array}{l}148.880 W . \\
149.311 W . \\
149.104 W . \\
148.973 W . \\
148.935 W .\end{array}$ & $\begin{array}{l}33 \mathrm{~N} \\
124 \\
20 \\
24 \\
15\end{array}$ & $\begin{array}{l}\text { GS } \\
\text { GS } \\
\text { GS } \\
\text { GS } \\
\text { GS }\end{array}$ & $\begin{array}{l}\ldots \\
5.7 \\
\ldots \\
\ldots\end{array}$ & $\begin{array}{l}\ldots \\
\ldots \\
5.2 \\
\ldots \\
\ldots\end{array}$ & $\begin{array}{c}\ldots \\
\text { 5. } 7 \mathrm{ML}(\mathrm{PM}) \\
\text { 4. } 2 \mathrm{ML}(\mathrm{PM}) \\
\text { 3. } 7 \mathrm{ML}(\mathrm{PM})\end{array}$ & $\begin{array}{l}\cdots \\
\ddot{V I} \\
\text { IV } \\
\text { III }\end{array}$ & & $\begin{array}{l}12 \\
12 \\
13 \\
13 \\
13\end{array}$ & $\begin{array}{l}12: 18 \\
22: 10 \\
16: 02 \\
16: 54 \\
22: 41\end{array}$ & $\begin{array}{l}\text { YST } \\
\text { YST } \\
\text { YST } \\
\text { YST } \\
\text { YST }\end{array}$ \\
\hline $\begin{array}{l}\text { AUG. } \\
A \cup G . \\
A \cup G . \\
A \cup G . \\
A \cup G .\end{array}$ & $\begin{array}{l}14 \\
16 \\
17 \\
17 \\
19\end{array}$ & $\begin{array}{l}12 \\
18 \\
10 \\
20 \\
00\end{array}$ & $\begin{array}{l}34 \\
26 \\
01 \\
26 \\
41\end{array}$ & $\begin{array}{l}36.3 \\
51.3 \\
13.6 \\
59.6 \\
42.7\end{array}$ & $\begin{array}{l}66.158 \mathrm{~N} . \\
54.164 \mathrm{~N} . \\
54.486 \mathrm{~N} . \\
53.121 \mathrm{~N} . \\
51.714 \mathrm{~N} .\end{array}$ & $\begin{array}{l}142.445 W \\
151.752 W \\
156.929 W \\
171.492 E \\
177.988 W\end{array}$ & $\begin{array}{l}33 N \\
33 N \\
33 N \\
33 N \\
116\end{array}$ & $\begin{array}{l}\text { GS } \\
\text { GS } \\
\text { GS } \\
\text { GS } \\
\text { GS }\end{array}$ & $\begin{array}{l}\ldots \\
4.6 \\
4.6 \\
4.4\end{array}$ & $\begin{array}{l}\ldots \\
\ldots \\
4.1 \\
\ldots \\
\ldots\end{array}$ & $\begin{array}{c}\text { 3. } 9 \mathrm{ML}(\mathrm{PM}) \\
\text { 4. } \mathrm{MLL}(\mathrm{PM}) \\
4.5 \mathrm{ML}(\mathrm{PM}) \\
\ldots \\
\ldots\end{array}$ & $\begin{array}{l}\cdots \\
\cdots \\
\cdots \\
\cdots\end{array}$ & $\begin{array}{l}A \cup G . \\
A \cup G . \\
A \cup G . \\
A \cup G . \\
A \cup G .\end{array}$ & $\begin{array}{l}14 \\
16 \\
17 \\
17 \\
18\end{array}$ & $\begin{array}{l}03: 34 \\
09: 26 \\
01: 01 \\
10: 26 \\
14: 41\end{array}$ & $\begin{array}{l}\text { YST } \\
\text { YST } \\
\text { YST } \\
\text { AST } \\
\text { AST }\end{array}$ \\
\hline $\begin{array}{l}\text { AUG. } \\
A \cup G . \\
\text { AUG. } \\
\text { AUG. } \\
\text { AUG. }\end{array}$ & $\begin{array}{l}19 \\
20 \\
21 \\
22 \\
23\end{array}$ & $\begin{array}{l}04 \\
14 \\
18 \\
14 \\
09\end{array}$ & $\begin{array}{l}31 \\
19 \\
18 \\
35 \\
44\end{array}$ & $\begin{array}{l}29.6 \\
59.7 \\
03.2 \\
27.2 \\
28.7\end{array}$ & $\begin{array}{l}60.493 \mathrm{~N} . \\
64.297 \mathrm{~N} . \\
55.845 \mathrm{~N} . \\
56.798 \mathrm{~N} . \\
60.188 \mathrm{~N} .\end{array}$ & $\begin{array}{l}150.478 w . \\
150.099 w . \\
153.571 w . \\
154.506 w . \\
140.999 w .\end{array}$ & $\begin{array}{l}58 \\
18 \\
33 \mathrm{~N} \\
33 \mathrm{~N} \\
15\end{array}$ & $\begin{array}{l}\text { GS } \\
\text { GS } \\
\text { GS } \\
\text { GS } \\
\text { GS }\end{array}$ & $\begin{array}{l}3.6 \\
\ldots \\
\ldots \\
\ldots \\
\ldots\end{array}$ & $\begin{array}{l}\ldots \\
\cdots \\
\cdots \\
\cdots\end{array}$ & $\begin{array}{l}\text { 4. } 0 M L(P M) \\
3.2 M L(P M) \\
4.3 M L(P M) \\
4.2 M L(P M) \\
3.2 M L(P M)\end{array}$ & $\begin{array}{c}\text { FELT } \\
\cdots \\
\cdots \\
\cdots \\
\cdots\end{array}$ & $\begin{array}{l}\text { AUG. } \\
\text { AUG. } \\
\text { AUG. } \\
\text { AUG. } \\
\text { AUG. }\end{array}$ & $\begin{array}{l}18 \\
20 \\
21 \\
22 \\
23\end{array}$ & $\begin{array}{l}19: 31 \\
05: 19 \\
09: 18 \\
05: 35 \\
00: 44\end{array}$ & $\begin{array}{l}\text { YST } \\
\text { YST } \\
\text { YST } \\
\text { YST } \\
\text { YST }\end{array}$ \\
\hline $\begin{array}{l}\text { AUG. } \\
\text { AUG. } \\
\text { AUG. } \\
\text { AUG. } \\
\text { AUG. }\end{array}$ & $\begin{array}{l}23 \\
24 \\
26 \\
27 \\
27\end{array}$ & $\begin{array}{l}20 \\
18 \\
03 \\
06 \\
20\end{array}$ & $\begin{array}{l}41 \\
28 \\
32 \\
38 \\
41\end{array}$ & $\begin{array}{l}50.8 \\
32.4 \\
45.7 \\
13.9 \\
13.5\end{array}$ & $\begin{array}{l}64.554 \mathrm{~N} \\
59.492 \mathrm{~N} \\
60.703 \mathrm{~N} \\
59.008 \mathrm{~N} \\
59.832 \mathrm{~N}\end{array}$ & $\begin{array}{l}147.980 W . \\
144.104 W . \\
151.339 W . \\
151.201 W . \\
153.413 W .\end{array}$ & $\begin{array}{c}16 \\
33 \mathrm{~N} \\
100 \\
33 \mathrm{~N} \\
127\end{array}$ & $\begin{array}{l}\text { GS } \\
\text { GS } \\
\text { GS } \\
\text { GS } \\
\text { GS }\end{array}$ & $\begin{array}{l}3.9 \\
\ldots \\
4.5\end{array}$ & $\begin{array}{l}\cdots \\
\cdots \\
\cdots\end{array}$ & $\begin{array}{c}\text { 4. } 1 M L(P M) \\
\text { 4. OML (PM) } \\
\text { 4. } \ldots \mathrm{OML}(P M) \\
\ldots\end{array}$ & $\begin{array}{c}\text { III } \\
\text { FELT } \\
\ldots \\
\ldots\end{array}$ & $\begin{array}{l}\text { AUG. } \\
\text { AUG. } \\
\text { AUG. } \\
\text { AUG. } \\
\text { AUG. }\end{array}$ & $\begin{array}{l}23 \\
24 \\
25 \\
26 \\
27\end{array}$ & $\begin{array}{l}11: 41 \\
09: 28 \\
18: 32 \\
21: 38 \\
11: 41\end{array}$ & $\begin{array}{l}\text { YST } \\
\text { YST } \\
\text { YST } \\
\text { YST } \\
\text { YST }\end{array}$ \\
\hline
\end{tabular}


Table 1. Summary of U.S. earthquakes for 1984-Continued

\begin{tabular}{|c|c|c|c|c|c|c|c|c|c|c|c|c|c|}
\hline \multirow{2}{*}{ Date } & \multicolumn{2}{|c|}{$\begin{array}{l}\text { Origin time } \\
\text { (UTC) }\end{array}$} & \multirow{2}{*}{$\begin{array}{c}\text { Latitude } \\
\left({ }^{\circ}\right)\end{array}$} & \multirow{2}{*}{$\begin{array}{c}\text { Longitude } \\
\text { (०) }\end{array}$} & \multirow{2}{*}{$\begin{array}{c}\text { Depth } \\
(\mathbf{k m})\end{array}$} & \multirow{2}{*}{$\begin{array}{l}\text { Hypo- } \\
\text { center } \\
\text { source }\end{array}$} & \multicolumn{3}{|c|}{ Magnitude } & \multirow{2}{*}{$\begin{array}{l}\text { Maximum } \\
\text { intensity }\end{array}$} & \multicolumn{3}{|c|}{ Local time } \\
\hline & hr $\min$ & $\sec$ & & & & & $\mathbf{m b}$ & MS & $\begin{array}{l}\text { ML, } M n \\
M D, M w\end{array}$ & & Date & Ilour & $\begin{array}{l}\text { Time } \\
\text { zone }\end{array}$ \\
\hline
\end{tabular}

ALASKA-Continued

\begin{tabular}{|c|c|c|c|c|c|c|c|c|c|c|c|c|c|c|c|c|}
\hline $\begin{array}{l}\text { AUG. } \\
\text { AUG. } \\
\text { SEPT. } \\
\text { SEPT. } \\
\text { SEPT. }\end{array}$ & $\begin{array}{r}29 \\
31 \\
1 \\
1 \\
1\end{array}$ & $\begin{array}{l}07 \\
22 \\
14 \\
15 \\
22\end{array}$ & $\begin{array}{l}20 \\
03 \\
58 \\
30 \\
55\end{array}$ & $\begin{array}{l}45.6 \\
31.0 \\
16.4 \\
18.1 \\
51.7\end{array}$ & $\begin{array}{l}60.430 \mathrm{~N} . \\
58.320 \mathrm{~N} . \\
53.012 \mathrm{~N} . \\
52.127 \mathrm{~N} . \\
53.488 \mathrm{~N} .\end{array}$ & $\begin{array}{l}153.009 w . \\
133.610 w . \\
168.260 w . \\
170.570 w . \\
163.574 w .\end{array}$ & $\begin{array}{l}150 \\
18 \\
61 \\
33 N \\
33 N\end{array}$ & $\begin{array}{l}\text { GS } \\
\text { EP } \\
\text { GS } \\
\text { GS } \\
\text { GS }\end{array}$ & $\begin{array}{l}\cdots \\
4.6 \\
4.8 \\
4.9\end{array}$ & $\begin{array}{l}\cdots \\
\cdots \\
\cdots \\
\cdots \\
\cdots\end{array}$ & $\begin{array}{c}\text { 3. } 4 \mathrm{ML}(\mathrm{EP}) \\
4.6 \mathrm{ML}(\mathrm{PM}) \\
\ldots \ldots \\
4.5 \mathrm{ML}(\mathrm{PM})\end{array}$ & $\begin{array}{c}\cdots \\
\text { FELT } \\
\cdots \\
\cdots\end{array}$ & $\begin{array}{l}\text { AUG. } \\
\text { AUG. } \\
\text { SEPT. } \\
\text { SEPT. } \\
\text { SEPT. }\end{array}$ & $\begin{array}{r}28 \\
31 \\
1 \\
1 \\
1\end{array}$ & $\begin{array}{l}22: 20 \\
13: 03 \\
05: 58 \\
05: 30 \\
13: 55\end{array}$ & $\begin{array}{l}\text { YST } \\
\text { YST } \\
\text { AST } \\
\text { AST } \\
\text { YST }\end{array}$ \\
\hline $\begin{array}{l}\text { SEPT } \\
\text { SEPT. } \\
\text { SEPT. } \\
\text { SEPT. } \\
\text { SEPT. }\end{array}$ & $\begin{array}{l}1 \\
2 \\
3 \\
4 \\
5\end{array}$ & $\begin{array}{l}23 \\
06 \\
10 \\
08 \\
18\end{array}$ & $\begin{array}{l}33 \\
56 \\
04 \\
47 \\
24\end{array}$ & $\begin{array}{l}13.0 \\
12.0 \\
03.2 \\
35.9 \\
02.8\end{array}$ & $\begin{array}{l}58.320 \mathrm{~N} . \\
58.310 \mathrm{~N} . \\
58.003 \mathrm{~N} \\
64.847 \mathrm{~N} \\
59.393 \mathrm{~N}\end{array}$ & $\begin{array}{l}133.620 w . \\
133.590 w . \\
148.383 w . \\
149.107 w . \\
145.461 w .\end{array}$ & $\begin{array}{l}18 \\
18 \\
33 \mathrm{~N} \\
28 \\
33 \mathrm{~N}\end{array}$ & $\begin{array}{l}\text { EP } \\
\text { EP } \\
\text { GS } \\
\text { GS } \\
\text { GS }\end{array}$ & $\begin{array}{l}\ldots \\
\ldots \\
\ldots \\
\ddot{4} .6\end{array}$ & $\begin{array}{l}\ldots \\
\cdots \\
\cdots \\
\cdots \\
\cdots\end{array}$ & $\begin{array}{l}\text { 3. } 4 M L(E P) \\
\text { 3. } 5 M L(E P) \\
\text { 3. } 4 M L(P M) \\
\text { 3. } 8 M L(P M) \\
\text { 4. } 2 M L(P M)\end{array}$ & $\begin{array}{l}\ldots \\
\cdots \\
\text { II I } \\
\ldots\end{array}$ & $\begin{array}{l}\text { SEPT. } \\
\text { SEPT. } \\
\text { SEPT. } \\
\text { SEPT. } \\
\text { SEPT. }\end{array}$ & $\begin{array}{l}1 \\
1 \\
3 \\
3 \\
5\end{array}$ & $\begin{array}{l}14: 33 \\
21: 56 \\
01: 04 \\
23: 47 \\
09: 24\end{array}$ & $\begin{array}{l}\text { ST } \\
\text { ST } \\
\text { STT } \\
\text { STT }\end{array}$ \\
\hline $\begin{array}{l}\text { SEPT. } \\
\text { SEPT. } \\
\text { SEPT. } \\
\text { SEPT. } \\
\text { SEPT. }\end{array}$ & $\begin{array}{r}7 \\
10 \\
10 \\
15 \\
15\end{array}$ & $\begin{array}{l}07 \\
09 \\
19 \\
08 \\
12\end{array}$ & $\begin{array}{l}19 \\
57 \\
22 \\
50 \\
51\end{array}$ & $\begin{array}{l}19.8 \\
47.9 \\
31.1 \\
44.6 \\
58.3\end{array}$ & $\begin{array}{l}63.060 \mathrm{~N} \\
52.577 \mathrm{~N} \\
60.933 \mathrm{~N} \\
67.783 \mathrm{~N} \\
61.722 \mathrm{~N}\end{array}$ & $\begin{array}{l}148.396 W . \\
172.482 E . \\
151.115 W . \\
161.771 W . \\
151.182 W .\end{array}$ & $\begin{array}{l}111 \\
33 \mathrm{~N} \\
5 \\
33 \mathrm{~N} \\
85\end{array}$ & $\begin{array}{l}\text { GS } \\
\text { GS } \\
\text { GS } \\
\text { GS } \\
\text { GS }\end{array}$ & $\begin{array}{l}\ldots \\
4.9 \\
\ldots \\
\ldots \\
\ldots\end{array}$ & $\begin{array}{l}\ldots \\
\ldots \\
\cdots \\
\cdots \\
\cdots\end{array}$ & $\begin{array}{l}\ldots \\
\ldots \\
\cdots \\
\cdots \\
\cdots\end{array}$ & $\begin{array}{l}\ldots \\
\text { III } \\
\ldots\end{array}$ & $\begin{array}{l}\text { SEPT. } \\
\text { SEPT. } \\
\text { SEPT. } \\
\text { SEPT. } \\
\text { SEPT. }\end{array}$ & $\begin{array}{r}6 \\
9 \\
10 \\
14 \\
15\end{array}$ & $\begin{array}{l}22: 19 \\
23: 57 \\
10: 22 \\
23: 50 \\
03: 51\end{array}$ & $\begin{array}{l}S T \\
\text { ST } \\
\text { ST }\end{array}$ \\
\hline $\begin{array}{l}\text { SEPT. } \\
\text { SEPT. } \\
\text { SEPT. } \\
\text { SEPT. } \\
\text { SEPT. }\end{array}$ & $\begin{array}{l}20 \\
20 \\
22 \\
23 \\
24\end{array}$ & $\begin{array}{l}04 \\
04 \\
18 \\
17 \\
13\end{array}$ & $\begin{array}{l}17 \\
28 \\
02 \\
06 \\
48\end{array}$ & $\begin{array}{l}24.4 \\
04.0 \\
23.4 \\
36.3 \\
38.9\end{array}$ & $\begin{array}{l}60.322 \mathrm{~N} . \\
60.306 \mathrm{~N} . \\
60.143 \mathrm{~N} . \\
53.577 \mathrm{~N} . \\
61.432 \mathrm{~N} .\end{array}$ & $\begin{array}{l}146.001 W . \\
146.098 W . \\
152.342 W . \\
165.424 W . \\
150.005 W .\end{array}$ & $\begin{array}{c}18 \\
26 \\
112 \\
33 \mathrm{~N} \\
46\end{array}$ & $\begin{array}{l}\text { GS } \\
\text { GS } \\
\text { GS } \\
\text { GS } \\
\text { GS }\end{array}$ & $\begin{array}{l}5.5 \\
5.1 \\
\ldots .7 \\
\ldots .\end{array}$ & $\begin{array}{l}5.2 \\
4.7 \\
\ldots .5 \\
5 .\end{array}$ & $\begin{array}{c}\text { 5. } 2 M L(P M) \\
\text { 4. } 7 M L(P M) \\
\ldots \ldots \\
\text { 5. } 9 M L(P M) \\
\text { 3. } 3 M L(P M)\end{array}$ & $\begin{array}{l}\text { IV } \\
\text { IV } \\
\ldots \\
\text { IV } \\
\text { II }\end{array}$ & $\begin{array}{l}\text { SEPT. } \\
\text { SEPT. } \\
\text { SEPT. } \\
\text { SEPT. } \\
\text { SEPT. }\end{array}$ & $\begin{array}{l}19 \\
19 \\
22 \\
23 \\
24\end{array}$ & $\begin{array}{l}19: 17 \\
19: 28 \\
09: 02 \\
08: 06 \\
04: 48\end{array}$ & $\begin{array}{l}\text { YST } \\
\text { YST } \\
\text { YST } \\
\text { AST } \\
\text { YST }\end{array}$ \\
\hline $\begin{array}{l}\text { SEPT. } \\
\text { SEPT. } \\
\text { SEPT. } \\
\text { SEPT. } \\
\text { SEPT. }\end{array}$ & $\begin{array}{l}25 \\
26 \\
26 \\
26 \\
27\end{array}$ & $\begin{array}{l}19 \\
09 \\
14 \\
20 \\
09\end{array}$ & $\begin{array}{l}06 \\
53 \\
58 \\
30 \\
62\end{array}$ & $\begin{array}{l}50.3 \\
39.5 \\
25.1 \\
46.4 \\
52.8\end{array}$ & $\begin{array}{l}53.663 \mathrm{~N} . \\
53.380 \mathrm{~N} . \\
63.168 \mathrm{~N} . \\
51.180 \mathrm{~N} . \\
59.836 \mathrm{~N} .\end{array}$ & $\begin{array}{l}166.404 W . \\
163.526 W \\
149.926 W \\
178.510 W \\
153.048 W\end{array}$ & $\begin{array}{c}65 \\
33 \mathrm{~N} \\
114 \\
33 \mathrm{~N} \\
128\end{array}$ & $\begin{array}{l}\text { GS } \\
\text { GS } \\
\text { GS } \\
\text { GS } \\
\text { GS }\end{array}$ & $\begin{array}{l}4.7 \\
4.7 \\
4.3 \\
4.9 \\
\ldots .\end{array}$ & $\begin{array}{l}\ldots \\
\cdots \\
\cdots \\
\cdots \\
\cdots\end{array}$ & $\begin{array}{c}\text { 5. } 1 \mathrm{ML}(\mathrm{PM}) \\
\ldots \\
\ldots \\
\ldots \\
\ldots\end{array}$ & $\begin{array}{l}\text { I I } \\
\text { I I I } \\
\ldots \\
\ldots\end{array}$ & $\begin{array}{l}\text { SEPT. } \\
\text { SEPT. } \\
\text { SEPT. } \\
\text { SEPT. } \\
\text { SEPT. }\end{array}$ & $\begin{array}{l}25 \\
26 \\
26 \\
26 \\
27\end{array}$ & $\begin{array}{l}10: 06 \\
00: 53 \\
05: 58 \\
10: 30 \\
00: 02\end{array}$ & $\begin{array}{l}\text { AST } \\
\text { YST } \\
\text { YST } \\
\text { AST } \\
\text { YST }\end{array}$ \\
\hline $\begin{array}{l}\text { SEPT } \\
\text { SEPT. } \\
\text { SEPT. } \\
\text { SEPT. } \\
\text { SEPT. }\end{array}$ & $\begin{array}{l}27 \\
28 \\
29 \\
29 \\
29\end{array}$ & $\begin{array}{l}11 \\
10 \\
68 \\
13 \\
14\end{array}$ & $\begin{array}{l}20 \\
24 \\
02 \\
14 \\
19\end{array}$ & $\begin{array}{l}07.1 \\
04.7 \\
22.3 \\
58.1 \\
15.0\end{array}$ & $\begin{array}{l}63.456 \mathrm{~N} . \\
62.182 \mathrm{~N} . \\
61.802 \mathrm{~N} . \\
63.513 \mathrm{~N} . \\
61.000 \mathrm{~N}\end{array}$ & $\begin{array}{l}153.191 \mathrm{w} . \\
151.176 \mathrm{~W} . \\
150.930 \mathrm{w} . \\
151.110 \mathrm{w} . \\
149.836 \mathrm{~W} .\end{array}$ & $\begin{array}{c}33 \mathrm{~N} \\
123 \\
94 \\
33 \mathrm{~N} \\
62\end{array}$ & $\begin{array}{l}\text { GS } \\
\text { GS } \\
\text { GS } \\
\text { GS } \\
\text { GS }\end{array}$ & $\begin{array}{l}\ldots \\
\ldots \\
\ldots \\
4.6\end{array}$ & $\begin{array}{l}\cdots \\
\cdots \\
\cdots \\
\cdots \\
\cdots\end{array}$ & $\begin{array}{c}\text { 3. } 4 M L(P M) \\
\ldots \\
\ldots \\
\text { 2. } 7 M L(P M) \\
\text { 4. } 2 M L(P M)\end{array}$ & $\begin{array}{l}\ldots \\
\cdots \\
\cdots \\
\text { III }\end{array}$ & $\begin{array}{l}\text { SEPT. } \\
\text { SEPT. } \\
\text { SEPT. } \\
\text { SEPT. } \\
\text { SEPT. }\end{array}$ & $\begin{array}{l}27 \\
28 \\
28 \\
29 \\
29\end{array}$ & $\begin{array}{l}02: 20 \\
01: 24 \\
23: 02 \\
04: 14 \\
05: 19\end{array}$ & $\begin{array}{l}\text { YST } \\
\text { YST } \\
\text { YST } \\
\text { YST } \\
\text { YST }\end{array}$ \\
\hline $\begin{array}{l}\text { OCT } \\
\text { OCT } \\
\text { OCT } \\
\text { OCT } \\
\text { OCT. }\end{array}$ & $\begin{array}{l}1 \\
2 \\
2 \\
3 \\
3\end{array}$ & $\begin{array}{l}08 \\
12 \\
17 \\
04 \\
19\end{array}$ & $\begin{array}{l}40 \\
05 \\
43 \\
15 \\
17\end{array}$ & $\begin{array}{l}25.1 \\
09.4 \\
10.9 \\
02.5 \\
23.0\end{array}$ & $\begin{array}{l}51.609 \mathrm{~N} . \\
52.142 \mathrm{~N} . \\
52.095 \mathrm{~N} . \\
54.212 \mathrm{~N} . \\
62.742 \mathrm{~N} .\end{array}$ & $\begin{array}{l}177.951 \mathrm{E} . \\
173.469 \mathrm{~W} . \\
179.590 \mathrm{E} . \\
163.321 \mathrm{~W} . \\
143.714 \mathrm{~W} .\end{array}$ & $\begin{array}{c}149 \\
91 \\
175 \\
33 \mathrm{~N} \\
5\end{array}$ & $\begin{array}{l}\text { GS } \\
\text { GS } \\
\text { GS } \\
\text { GS } \\
\text { GS }\end{array}$ & $\begin{array}{l}4.4 \\
4.5 \\
4.6 \\
4.6 \\
\ldots .\end{array}$ & $\begin{array}{l}\ldots \\
\cdots \\
\cdots \\
\cdots \\
\cdots\end{array}$ & $\begin{array}{c}\ldots \\
\ldots \\
\ldots \\
3.0 \mathrm{ML}(\mathrm{PM})\end{array}$ & $\begin{array}{l}\cdots \\
\cdots \\
\text { I I }\end{array}$ & $\begin{array}{l}\text { SEPT. } \\
\text { OCT. } \\
\text { OCT. } \\
\text { OCT. } \\
\text { OCT. }\end{array}$ & $\begin{array}{r}30 \\
2 \\
2 \\
2 \\
3\end{array}$ & $\begin{array}{l}22: 40 \\
02: 05 \\
07: 43 \\
19: 15 \\
10: 17\end{array}$ & T \\
\hline $\begin{array}{l}\text { OCT. } \\
\text { OCT. } \\
\text { OCT. } \\
\text { OCT. } \\
\text { OCT. }\end{array}$ & $\begin{array}{l}3 \\
4 \\
4 \\
5 \\
6\end{array}$ & $\begin{array}{l}23 \\
06 \\
22 \\
15 \\
02\end{array}$ & $\begin{array}{l}42 \\
41 \\
41 \\
46 \\
09\end{array}$ & $\begin{array}{l}30.5 \\
57.0 \\
03.3 \\
27.2 \\
58.4\end{array}$ & $\begin{array}{l}63.344 \mathrm{~N} . \\
57.910 \mathrm{~N} . \\
59.145 \mathrm{~N} . \\
51.871 \mathrm{~N} . \\
63.947 \mathrm{~N} .\end{array}$ & $\begin{array}{l}153.382 W . \\
139.570 W . \\
151.706 \mathrm{~W} . \\
176.016 \mathrm{~W} . \\
148.967 \mathrm{~W} .\end{array}$ & $\begin{array}{l}33 N \\
18 \\
63 \\
68 \\
5\end{array}$ & $\begin{array}{l}\text { GS } \\
\text { EP } \\
\text { GS } \\
\text { GS } \\
\text { GS }\end{array}$ & $\begin{array}{l}\ldots \\
4.6 \\
5.3 \\
\ldots\end{array}$ & $\begin{array}{l}\ldots \\
\cdots \\
\cdots \\
\cdots \\
\cdots\end{array}$ & $\begin{array}{l}\text { 3. } 2 M L(P M) \\
\text { 3.5ML (EP) } \\
\text { 4. } 3 M L(P M) \\
\text { 5. } 2 M L(P M) \\
\text { 3.1ML(PM) }\end{array}$ & $\begin{array}{l}\ldots \\
\text { I I I } \\
\text { IV } \\
\ldots\end{array}$ & $\begin{array}{l}\text { OCT. } \\
\text { OCT. } \\
\text { OCT. } \\
\text { OCT. } \\
\text { OCT. }\end{array}$ & $\begin{array}{l}3 \\
3 \\
4 \\
5 \\
5\end{array}$ & $\begin{array}{l}14: 42 \\
21: 41 \\
13: 41 \\
05: 46 \\
17: 09\end{array}$ & $\begin{array}{l}\text { AS } \\
\text { YS }\end{array}$ \\
\hline $\begin{array}{l}\text { OCT. } \\
\text { OCT. } \\
\text { OCT. } \\
\text { OCT. } \\
\text { OCT. }\end{array}$ & $\begin{array}{l}7 \\
7 \\
7 \\
8 \\
8\end{array}$ & $\begin{array}{l}16 \\
17 \\
23 \\
00 \\
21\end{array}$ & $\begin{array}{l}35 \\
24 \\
42 \\
49 \\
10\end{array}$ & $\begin{array}{l}23.9 \\
54.9 \\
46.0 \\
37.9 \\
09.8\end{array}$ & $\begin{array}{l}63.432 \mathrm{~N} . \\
63.507 \mathrm{~N} . \\
59.990 \mathrm{~N} . \\
63.232 \mathrm{~N} . \\
61.716 \mathrm{~N} .\end{array}$ & $\begin{array}{l}150.398 W . \\
151.321 W . \\
140.120 W . \\
151.128 W . \\
150.834 W .\end{array}$ & $\begin{array}{l}5 \\
5 \\
18 \\
33 \mathrm{~N} \\
56\end{array}$ & $\begin{array}{l}\text { GS } \\
\text { GS } \\
\text { EP } \\
\text { GS } \\
\text { GS }\end{array}$ & $\begin{array}{l}\cdots \\
\cdots \\
\cdots\end{array}$ & $\begin{array}{l}\ldots \\
\cdots \\
\cdots \\
\cdots \\
\cdots\end{array}$ & $\begin{array}{l}\text { 3. } 3 M L(P M) \\
\text { 3. } 7 M L(P M) \\
\text { 3.1ML (EP) } \\
\text { 3. } 2 M L(P M) \\
3.5 M L(P M)\end{array}$ & $\begin{array}{l}\ldots \\
\ldots \\
\ldots \\
\cdots \\
\cdots\end{array}$ & $\begin{array}{l}\text { OCT. } \\
\text { OCT. } \\
\text { OCT. } \\
\text { OCT. } \\
\text { OCT. }\end{array}$ & $\begin{array}{l}7 \\
7 \\
7 \\
7 \\
8\end{array}$ & $\begin{array}{l}07: 35 \\
08: 24 \\
14: 42 \\
15: 49 \\
12: 10\end{array}$ & $\mathrm{~T}$ \\
\hline $\begin{array}{l}\text { OCT. } \\
\text { OCT. } \\
\text { OCT. } \\
\text { OCT. } \\
\text { OCT. }\end{array}$ & $\begin{array}{l}10 \\
11 \\
13 \\
13 \\
14\end{array}$ & $\begin{array}{l}21 \\
19 \\
18 \\
19 \\
04\end{array}$ & $\begin{array}{l}38 \\
30 \\
42 \\
01 \\
49\end{array}$ & $\begin{array}{l}09.7 \\
32.4 \\
58.8 \\
28.3 \\
38.3\end{array}$ & $\begin{array}{l}51.086 \mathrm{~N} . \\
53.292 \mathrm{~N} . \\
53.588 \mathrm{~N} . \\
53.638 \mathrm{~N} . \\
62.249 \mathrm{~N} .\end{array}$ & $\begin{array}{l}179.275 w . \\
168.721 w . \\
163.596 w . \\
163.518 w . \\
149.577 w .\end{array}$ & $\begin{array}{l}33 N \\
33 N \\
33 N \\
33 N \\
76\end{array}$ & $\begin{array}{l}\text { GS } \\
\text { GS } \\
\text { GS } \\
\text { GS } \\
\text { GS }\end{array}$ & $\begin{array}{l}4.8 \\
4.2 \\
5.0 \\
4.8 \\
3.8\end{array}$ & $\begin{array}{l}\cdots \\
\cdots \\
\cdots \\
\cdots \\
\cdots\end{array}$ & $\begin{array}{c}\ldots \\
4 . \dot{5 M L}(P M) \\
\ldots \\
\ldots\end{array}$ & $\begin{array}{l}\cdots \\
\cdots \\
\cdots \\
\cdots\end{array}$ & $\begin{array}{l}\text { OCT. } \\
\text { OCT. } \\
\text { OCT. } \\
\text { OCT. } \\
\text { OCT. }\end{array}$ & $\begin{array}{c}10 \\
11 \\
13 \\
13 \\
13\end{array}$ & $\begin{array}{c}11: 38 \\
09: 30 \\
09: 42 \\
10: 01 \\
19: 49\end{array}$ & $\begin{array}{r}\text { YST } \\
\text { YST }\end{array}$ \\
\hline
\end{tabular}


Table 1. Summary of U.S. earthquakes for 1984-Continued

\begin{tabular}{|c|c|c|c|c|c|c|c|c|c|c|c|c|c|}
\hline \multirow{2}{*}{ Date } & \multicolumn{2}{|c|}{$\begin{array}{l}\text { Origin time } \\
\text { (UTC) }\end{array}$} & \multirow{2}{*}{$\begin{array}{c}\text { Latitude } \\
\left(^{\circ}\right)\end{array}$} & \multirow{2}{*}{$\begin{array}{c}\text { Longitude } \\
\left({ }^{\circ}\right)\end{array}$} & \multirow{2}{*}{$\begin{array}{c}\text { Depth } \\
(\mathbf{k m})\end{array}$} & \multirow{2}{*}{$\begin{array}{l}\text { IIypo- } \\
\text { center } \\
\text { surece }\end{array}$} & \multicolumn{3}{|c|}{ Magnitude } & \multirow{2}{*}{$\begin{array}{l}\text { Maximum } \\
\text { intensity }\end{array}$} & \multicolumn{3}{|c|}{ Local time } \\
\hline & hr $\min$ & sec & & & & & $\mathbf{m b}$ & MS & $\begin{array}{l}\text { ML. Mn } \\
\text { MD. Mw }\end{array}$ & & Date & Hour & $\begin{array}{l}\text { Time } \\
\text { none }\end{array}$ \\
\hline
\end{tabular}

ALASKA-Continued

\begin{tabular}{|c|c|c|c|c|c|c|c|c|c|c|c|c|c|c|c|c|}
\hline $\begin{array}{l}\text { OCT. } \\
\text { OCT. } \\
\text { OCT. } \\
\text { OCT. } \\
\text { OCT. }\end{array}$ & $\begin{array}{l}14 \\
14 \\
15 \\
15 \\
15\end{array}$ & $\begin{array}{l}10 \\
14 \\
03 \\
04 \\
05\end{array}$ & $\begin{array}{l}19 \\
33 \\
25 \\
32 \\
44\end{array}$ & $\begin{array}{l}48.9 \\
47.6 \\
52.2 \\
56.2 \\
34.4\end{array}$ & $\begin{array}{l}60.928 \mathrm{~N} \\
53.389 \mathrm{~N} \\
52.455 \mathrm{~N} \\
51.953 \mathrm{~N} \\
52.279 \mathrm{~N}\end{array}$ & $\begin{array}{l}147.298 W . \\
168.435 w . \\
168.595 w . \\
168.643 w . \\
168.732 W .\end{array}$ & $\begin{array}{l}40 \\
33 N \\
33 N \\
33 N \\
33 N\end{array}$ & $\begin{array}{l}\text { GS } \\
\text { GS } \\
\text { GS } \\
\text { GS } \\
\text { GS }\end{array}$ & $\begin{array}{l}3.2 \\
4.6 \\
4.6 \\
4.7 \\
5.0\end{array}$ & $\begin{array}{l}\ldots \\
\ldots \\
\ddot{4} .4 \\
4.4\end{array}$ & $\begin{array}{c}\text { 3. } 4 M L(P M) \\
\ldots \\
\ldots \\
\ldots \\
\ldots\end{array}$ & $\begin{array}{l}\cdots \\
\cdots \\
\cdots \\
\cdots\end{array}$ & $\begin{array}{l}\text { OCT. } \\
\text { OCT. } \\
\text { OCT. } \\
\text { OCT. } \\
\text { OCT. }\end{array}$ & $\begin{array}{l}14 \\
14 \\
14 \\
14 \\
14\end{array}$ & $\begin{array}{l}01: 19 \\
05: 33 \\
17: 25 \\
18: 32 \\
19: 44\end{array}$ & $\begin{array}{l}\text { YST } \\
\text { AST } \\
\text { AST } \\
\text { AST } \\
\text { AST }\end{array}$ \\
\hline $\begin{array}{l}\text { OCT. } \\
\text { OCT. } \\
\text { OCT. } \\
\text { OCT. } \\
\text { OCT. }\end{array}$ & $\begin{array}{l}16 \\
17 \\
17 \\
18 \\
18\end{array}$ & $\begin{array}{l}06 \\
13 \\
17 \\
05 \\
23\end{array}$ & $\begin{array}{l}58 \\
53 \\
57 \\
19 \\
47\end{array}$ & $\begin{array}{l}38.2 \\
47.2 \\
27.0 \\
56.6 \\
26.5\end{array}$ & $\begin{array}{l}52.105 \mathrm{~N} \\
52.483 \mathrm{~N} \\
51.653 \mathrm{~N} \\
63.176 \mathrm{~N} \\
62.981 \mathrm{~N}\end{array}$ & $\begin{array}{l}169.883 W . \\
168.027 W . \\
179.195 W . \\
151.131 W . \\
148.216 W .\end{array}$ & $\begin{array}{c}33 N \\
33 N \\
33 N \\
135 \\
82\end{array}$ & $\begin{array}{l}\text { GS } \\
\text { GS } \\
\text { GS } \\
\text { GS } \\
\text { GS }\end{array}$ & $\begin{array}{l}4.4 \\
4.9 \\
4.7 \\
4.7 \\
3.6\end{array}$ & $\begin{array}{l}\cdots \\
\cdots \\
\cdots \\
\cdots \\
\cdots\end{array}$ & $\begin{array}{c}\text { 4. 6ML(PM) } \\
\text { 4. } 1 \mathrm{ML}(\mathrm{PM}) \\
\ldots \\
\ldots\end{array}$ & $\begin{array}{l}\cdots \\
\cdots \\
\text { III } \\
\cdots\end{array}$ & $\begin{array}{l}\text { OCT. } \\
\text { OCT. } \\
\text { OCT. } \\
\text { OCT. } \\
\text { OCT. }\end{array}$ & $\begin{array}{l}15 \\
17 \\
17 \\
17 \\
18\end{array}$ & $\begin{array}{l}20: 58 \\
04: 53 \\
07: 57 \\
20: 19 \\
14: 47\end{array}$ & $\begin{array}{l}\text { AST } \\
\text { AST } \\
\text { AST } \\
\text { YST } \\
\text { YST }\end{array}$ \\
\hline $\begin{array}{l}\text { OCT } . \\
\text { OCT. } \\
\text { OCT. } \\
\text { OCT. } \\
\text { OCT. }\end{array}$ & $\begin{array}{l}19 \\
19 \\
20 \\
21 \\
23\end{array}$ & $\begin{array}{l}04 \\
20 \\
15 \\
14 \\
16\end{array}$ & $\begin{array}{l}44 \\
43 \\
41 \\
12 \\
39\end{array}$ & $\begin{array}{l}44.7 \\
06.2 \\
45.1 \\
19.1 \\
53.9\end{array}$ & $\begin{array}{l}61.649 \mathrm{~N} \\
60.139 \mathrm{~N} \\
52.140 \mathrm{~N} \\
64.453 \mathrm{~N} \\
54.984 \mathrm{~N}\end{array}$ & $\begin{array}{l}150.973 W . \\
150.987 W . \\
168.566 W . \\
147.990 W . \\
135.167 W .\end{array}$ & $\begin{array}{l}77 \\
97 \\
33 N \\
15 \\
10\end{array}$ & $\begin{array}{l}\text { GS } \\
G S \\
G S \\
G S \\
G S\end{array}$ & $\begin{array}{l}4.5 \\
3.7 \\
5.0 \\
3.9\end{array}$ & $\begin{array}{l}\cdots \\
\cdots \\
\cdots \\
\cdots \\
\cdots\end{array}$ & $\begin{array}{c}\cdots \\
\ldots \\
\text { 3. } \ddot{9 M L}(P M)\end{array}$ & $\begin{array}{l}\text { II } \\
\text { I I } \\
\cdots \\
\cdots \\
\cdots\end{array}$ & $\begin{array}{l}\text { OCT. } \\
\text { OCT. } \\
\text { OCT. } \\
\text { OCT. } \\
\text { OCT. }\end{array}$ & $\begin{array}{l}18 \\
19 \\
20 \\
21 \\
23\end{array}$ & $\begin{array}{l}19: 44 \\
11: 43 \\
05: 41 \\
05: 12 \\
07: 39\end{array}$ & $\begin{array}{l}\text { YST } \\
\text { YST } \\
\text { AST } \\
\text { YST } \\
\text { YST }\end{array}$ \\
\hline $\begin{array}{l}\text { OCT. } \\
\text { OCT. } \\
\text { OCT. } \\
\text { OCT. } \\
\text { OCT. }\end{array}$ & $\begin{array}{l}25 \\
29 \\
29 \\
31 \\
31\end{array}$ & $\begin{array}{l}12 \\
01 \\
23 \\
01 \\
04\end{array}$ & $\begin{array}{l}37 \\
39 \\
04 \\
17 \\
40\end{array}$ & $\begin{array}{l}15.4 \\
11.4 \\
43.6 \\
37.5 \\
05.0\end{array}$ & $\begin{array}{l}51.675 \mathrm{~N} . \\
65.711 \mathrm{~N} . \\
51.125 \mathrm{~N} \\
52.796 \mathrm{~N} \\
51.173 \mathrm{~N}\end{array}$ & $\begin{array}{l}175.210 W . \\
143.617 W \\
179.484 E . \\
174.507 W . \\
179.559 E .\end{array}$ & $\begin{array}{l}56 \\
33 \mathrm{~N} \\
39 \\
189 \\
57\end{array}$ & $\begin{array}{l}\text { GS } \\
\text { GS } \\
\text { GS } \\
\text { GS } \\
\text { GS }\end{array}$ & $\begin{array}{l}5.3 \\
\cdots .2 \\
5.2 \\
4.5 \\
5.1\end{array}$ & $\begin{array}{l}\ldots \\
\ldots \\
5.2 \\
\ldots \\
\ldots\end{array}$ & $\begin{array}{c}\text { 5. } 1 \mathrm{ML}(P M) \\
\text { 3. } \mathrm{MML}(\mathrm{PM}) \\
5.3 \mathrm{ML}(\mathrm{PM}) \\
\ldots \ldots \\
5.4 \mathrm{ML}(\mathrm{PM})\end{array}$ & $\begin{array}{l}\quad v \\
\ldots \\
\cdots \\
\cdots \\
\cdots\end{array}$ & $\begin{array}{l}\text { OCT. } \\
\text { OCT. } \\
\text { OCT. } \\
\text { OCT. } \\
\text { OCT. }\end{array}$ & $\begin{array}{l}25 \\
28 \\
29 \\
30 \\
30\end{array}$ & $\begin{array}{l}02: 37 \\
16: 39 \\
13: 04 \\
15: 17 \\
18: 40\end{array}$ & $\begin{array}{l}\text { AST } \\
\text { YST } \\
\text { AST } \\
\text { AST } \\
\text { AST }\end{array}$ \\
\hline $\begin{array}{l}\text { NOV. } \\
\text { NOV. } \\
\text { NOV. } \\
\text { NOV. } \\
\text { NOV. }\end{array}$ & $\begin{array}{l}1 \\
2 \\
3 \\
8 \\
8\end{array}$ & $\begin{array}{l}08 \\
17 \\
13 \\
09 \\
13\end{array}$ & $\begin{array}{l}18 \\
45 \\
18 \\
37 \\
02\end{array}$ & $\begin{array}{l}00.7 \\
43.9 \\
52.3 \\
31.9 \\
00.1\end{array}$ & $\begin{array}{l}60.634 \mathrm{~N} \\
58.945 \mathrm{~N} \\
54.406 \mathrm{~N} \\
52.151 \mathrm{~N} \\
52.181 \mathrm{~N}\end{array}$ & $\begin{array}{l}150.445 W . \\
152.712 W . \\
154.296 W . \\
171.036 W . \\
170.999 W .\end{array}$ & $\begin{array}{l}73 \\
86 \\
33 N \\
33 N \\
33 N\end{array}$ & $\begin{array}{l}\text { GS } \\
\text { GS } \\
\text { GS } \\
\text { GS } \\
\text { GS }\end{array}$ & $\begin{array}{l}4.0 \\
4.6 \\
4.8 \\
5.4\end{array}$ & $\begin{array}{l}\cdots \\
\cdots \\
4.5 \\
5.3\end{array}$ & $\begin{array}{c}\cdots \\
\text { 4. } 3 \mathrm{ML}(\mathrm{PM}) \\
4.8 \mathrm{ML}(\mathrm{PM}) \\
5.5 \mathrm{ML}(\mathrm{PM})\end{array}$ & $\begin{array}{l}\cdots \\
\cdots \\
\cdots \\
\cdots\end{array}$ & $\begin{array}{l}\text { OCT. } \\
\text { NOV. } \\
\text { NOV. } \\
\text { NOV. } \\
\text { NOV. }\end{array}$ & $\begin{array}{r}31 \\
2 \\
3 \\
7 \\
8\end{array}$ & $\begin{array}{l}23: 18 \\
08: 45 \\
04: 18 \\
23: 37 \\
03: 02\end{array}$ & $\begin{array}{l}\text { YST } \\
\text { YST } \\
\text { YST } \\
\text { AST } \\
\text { AST }\end{array}$ \\
\hline $\begin{array}{l}\text { NOV. } \\
\text { NOV. } \\
\text { NOV. } \\
\text { NOV. } \\
\text { NOV. }\end{array}$ & $\begin{array}{r}8 \\
8 \\
9 \\
9 \\
11\end{array}$ & $\begin{array}{l}14 \\
17 \\
06 \\
19 \\
05\end{array}$ & $\begin{array}{l}53 \\
48 \\
58 \\
51 \\
45\end{array}$ & $\begin{array}{l}55.3 \\
03.6 \\
06.9 \\
39.2 \\
42.3\end{array}$ & $\begin{array}{l}52.187 \mathrm{~N} \\
52.024 \mathrm{~N} \\
63.905 \mathrm{~N} \\
62.973 \mathrm{~N} \\
61.291 \mathrm{~N}\end{array}$ & $\begin{array}{l}170.958 W . \\
170.947 w . \\
147.915 W . \\
149.968 W . \\
147.472 W .\end{array}$ & $\begin{array}{c}33 N \\
33 N \\
23 \\
105 \\
33 N\end{array}$ & $\begin{array}{l}\text { GS } \\
\text { GS } \\
\text { GS } \\
\text { GS } \\
\text { GS }\end{array}$ & $\begin{array}{l}4.6 \\
4.7 \\
3.7 \\
\ldots . \\
\ldots\end{array}$ & $\begin{array}{l}\cdots \\
\cdots \\
\cdots \\
\cdots \\
\cdots\end{array}$ & $\begin{array}{c}\text { 4. } 4 M L(P M) \\
\text { 4. } 2 M L(P M) \\
\ldots \ldots \\
\text { 4. } 2 M L(P M)\end{array}$ & $\begin{array}{l}\cdots \\
\text { IV } \\
\ldots \\
\ldots\end{array}$ & $\begin{array}{l}\text { NOV. } \\
\text { NOV. } \\
\text { NOV. } \\
\text { NOV. } \\
\text { NOV. }\end{array}$ & $\begin{array}{r}8 \\
8 \\
8 \\
9 \\
10\end{array}$ & $\begin{array}{l}04: 53 \\
07: 48 \\
21: 58 \\
10: 51 \\
20: 45\end{array}$ & $\begin{array}{l}\text { AST } \\
\text { AST } \\
\text { YST } \\
\text { YST } \\
\text { YST }\end{array}$ \\
\hline $\begin{array}{l}\text { NOV. } \\
\text { NOV. } \\
\text { NOV. } \\
\text { NOV. } \\
\text { NOV. }\end{array}$ & $\begin{array}{l}12 \\
13 \\
13 \\
13 \\
14\end{array}$ & $\begin{array}{l}08 \\
00 \\
13 \\
23 \\
19\end{array}$ & $\begin{array}{l}16 \\
37 \\
08 \\
29 \\
40\end{array}$ & $\begin{array}{l}32.0 \\
49.9 \\
24.8 \\
44.4 \\
09.4\end{array}$ & $\begin{array}{l}57.090 \mathrm{~N} \\
62.092 \mathrm{~N} \\
51.884 \mathrm{~N} \\
61.801 \mathrm{~N} \\
53.059 \mathrm{~N}\end{array}$ & $\begin{array}{l}140.350 W . \\
150.641 W . \\
174.028 W . \\
149.717 W . \\
168.653 W .\end{array}$ & $\begin{array}{l}18 \\
32 \\
33 N \\
30 \\
82\end{array}$ & $\begin{array}{l}\text { EP } \\
G S \\
\text { GS } \\
\text { GS } \\
\text { GS }\end{array}$ & $\begin{array}{l}\cdots \\
4.6 \\
\cdots \\
4.2\end{array}$ & $\begin{array}{l}\cdots \\
\cdots \\
\cdots \\
\cdots\end{array}$ & $\begin{array}{c}\text { 3. } 0 M L(E P) \\
\text { 3. } 0 M L(P M) \\
4.9 M L(P M) \\
\text { 3. } 8 M L(P M) \\
\ldots .\end{array}$ & $\begin{array}{l}\cdots \\
\cdots \\
\cdots \\
\cdots\end{array}$ & $\begin{array}{l}\text { NOV. } \\
\text { NOV. } \\
\text { NOV. } \\
\text { NOV. } \\
\text { NOV. }\end{array}$ & $\begin{array}{l}11 \\
12 \\
13 \\
13 \\
14\end{array}$ & $\begin{array}{l}23: 16 \\
15: 37 \\
03: 08 \\
14: 29 \\
09: 40\end{array}$ & $\begin{array}{l}\text { YST } \\
\text { YST } \\
\text { AST } \\
\text { YST } \\
\text { AST }\end{array}$ \\
\hline $\begin{array}{l}\text { NOV. } \\
\text { NOV. } \\
\text { NOV. } \\
\text { NOV. } \\
\text { NOV. }\end{array}$ & $\begin{array}{l}17 \\
18 \\
18 \\
19 \\
19\end{array}$ & $\begin{array}{l}09 \\
04 \\
08 \\
00 \\
04\end{array}$ & $\begin{array}{l}03 \\
36 \\
29 \\
44 \\
10\end{array}$ & $\begin{array}{l}19.3 \\
30.6 \\
20.0 \\
27.2 \\
42.4\end{array}$ & $\begin{array}{l}63.330 \mathrm{~N} \\
63.119 \mathrm{~N} \\
56.601 \mathrm{~N} \\
58.567 \mathrm{~N} \\
51.170 \mathrm{~N}\end{array}$ & $\begin{array}{l}152.694 W . \\
148.204 W . \\
152.319 W \\
156.702 W . \\
179.096 E .\end{array}$ & $\begin{array}{c}3 \\
94 \\
33 N \\
206 \\
39\end{array}$ & $\begin{array}{l}\text { GS } \\
\text { GS } \\
\text { GS } \\
\text { GS } \\
\text { GS }\end{array}$ & $\begin{array}{l}5.0 \\
4.8 \\
4.6 \\
5.6\end{array}$ & $\begin{array}{l}\ldots \\
\cdots \\
\ldots \\
5.5\end{array}$ & $\begin{array}{c}\text { 5. } 3 M L(P M) \\
\ldots \\
4.2 M L(P M) \\
\text { 5. } 7 M L(P M)\end{array}$ & $\begin{array}{l}\text { I I I } \\
\cdots \\
\cdots \\
\text { I I } \\
\text { I I }\end{array}$ & $\begin{array}{l}\text { NOV. } \\
\text { NOV. } \\
\text { NOV. } \\
\text { NOV. } \\
\text { NOV. }\end{array}$ & $\begin{array}{l}17 \\
17 \\
17 \\
18 \\
18\end{array}$ & $\begin{array}{l}00: 03 \\
19: 36 \\
23: 29 \\
15: 44 \\
18: 10\end{array}$ & $\begin{array}{l}\text { YST } \\
\text { YST } \\
\text { YST } \\
\text { YST } \\
\text { AST }\end{array}$ \\
\hline $\begin{array}{l}\text { NOV. } \\
\text { NOV. } \\
\text { NOV. } \\
\text { NOV. } \\
\text { NOV. }\end{array}$ & $\begin{array}{l}19 \\
22 \\
22 \\
22 \\
22\end{array}$ & $\begin{array}{l}12 \\
04 \\
04 \\
04 \\
05\end{array}$ & $\begin{array}{l}06 \\
15 \\
21 \\
38 \\
58\end{array}$ & $\begin{array}{l}37.3 \\
14.5 \\
16.5 \\
21.6 \\
01.0\end{array}$ & $\begin{array}{l}51.777 \mathrm{~N} . \\
51.128 \mathrm{~N} \\
51.066 \mathrm{~N} \\
50.777 \mathrm{~N} \\
50.954 \mathrm{~N} .\end{array}$ & $\begin{array}{l}175.272 W . \\
179.416 E . \\
179.093 E \\
179.240 E \\
179.596 E .\end{array}$ & $\begin{array}{l}58 \\
33 N \\
33 N \\
33 N \\
33 N\end{array}$ & $\begin{array}{l}\text { GS } \\
\text { GS } \\
\text { GS } \\
\text { GS } \\
\text { GS }\end{array}$ & $\begin{array}{l}5.6 \\
4.8 \\
4.5 \\
4.6 \\
4.5\end{array}$ & $\begin{array}{l}\cdots \\
\cdots \\
\cdots \\
\cdots \\
\cdots\end{array}$ & $\begin{array}{c}5.5 \mathrm{ML}(\mathrm{PM}) \\
4.8 \mathrm{ML}(\mathrm{PM}) \\
\ldots \\
\ldots \\
\ldots\end{array}$ & $\begin{array}{l}\text { IV } \\
\cdots \\
\cdots \\
\cdots \\
\cdots\end{array}$ & $\begin{array}{l}\text { NOV. } \\
\text { NOV. } \\
\text { NOV. } \\
\text { NOV. } \\
\text { NOV. }\end{array}$ & $\begin{array}{l}19 \\
21 \\
21 \\
21 \\
21\end{array}$ & $\begin{array}{l}02: 06 \\
18: 15 \\
18: 21 \\
18: 38 \\
19: 58\end{array}$ & $\begin{array}{l}\text { AST } \\
\text { AST } \\
\text { AST } \\
\text { AST } \\
\text { AST }\end{array}$ \\
\hline $\begin{array}{l}\text { NOV. } \\
\text { NOV. } \\
\text { NOV. } \\
\text { NOV. } \\
\text { NOV. }\end{array}$ & $\begin{array}{l}22 \\
23 \\
23 \\
24 \\
25\end{array}$ & $\begin{array}{l}13 \\
10 \\
16 \\
10 \\
08\end{array}$ & $\begin{array}{l}10 \\
37 \\
16 \\
42 \\
49\end{array}$ & $\begin{array}{l}58.1 \\
38.3 \\
24.5 \\
24.1 \\
55.1\end{array}$ & $\begin{array}{l}62.965 \mathrm{~N} \\
54.654 \mathrm{~N} \\
52.149 \mathrm{~N} \\
60.011 \mathrm{~N} \\
58.291 \mathrm{~N}\end{array}$ & $\begin{array}{l}149.602 W \\
163.849 W \\
170.384 W \\
153.359 W \\
155.072 W .\end{array}$ & $\begin{array}{c}107 \\
33 \mathrm{~N} \\
33 \mathrm{~N} \\
161 \\
33 \mathrm{~N}\end{array}$ & $\begin{array}{l}\text { GS } \\
\text { GS } \\
\text { GS } \\
\text { GS } \\
\text { GS }\end{array}$ & $\begin{array}{l}4.8 \\
4.6 \\
4.1 \\
4.2\end{array}$ & $\begin{array}{l}\cdots \\
\cdots \\
\cdots \\
\cdots\end{array}$ & $\begin{array}{c}\cdots \\
\cdots \\
\cdots \\
4.5 \mathrm{ML}(P M)\end{array}$ & $\begin{array}{l}\text { III } \\
\ldots \\
\ldots \\
\ldots\end{array}$ & $\begin{array}{l}\text { NOV. } \\
\text { NOV. } \\
\text { NOV. } \\
\text { NOV. } \\
\text { NOV. }\end{array}$ & $\begin{array}{l}22 \\
23 \\
23 \\
24 \\
24\end{array}$ & $\begin{array}{l}04: 10 \\
01: 37 \\
06: 16 \\
01: 42 \\
23: 49\end{array}$ & $\begin{array}{l}\text { YST } \\
\text { YST } \\
\text { AST } \\
\text { YST } \\
\text { YST }\end{array}$ \\
\hline
\end{tabular}


Table 1. Summary of U.S. earthquakes for 1984-Continued

\begin{tabular}{|c|c|c|c|c|c|c|c|c|c|c|c|c|c|}
\hline \multirow{2}{*}{ Date } & \multicolumn{2}{|c|}{$\begin{array}{l}\text { Origin time } \\
\text { (UTC) }\end{array}$} & \multirow{2}{*}{$\begin{array}{l}\text { Latitude } \\
\text { (०) }\end{array}$} & \multirow{2}{*}{$\begin{array}{l}\text { Longitude } \\
\text { (i) }\end{array}$} & \multirow{2}{*}{$\begin{array}{l}\text { Depth } \\
(\mathbf{k m})\end{array}$} & \multirow{2}{*}{$\begin{array}{l}\text { Hypo- } \\
\text { center } \\
\text { source }\end{array}$} & \multicolumn{3}{|c|}{ Magnitude } & \multirow{2}{*}{$\begin{array}{l}\text { Meximum } \\
\text { intensity }\end{array}$} & \multicolumn{3}{|c|}{ Local time } \\
\hline & hr $\min$ & $\sec$ & & & & & $\mathbf{m b}$ & MS & $\begin{array}{l}\text { ML. Mn } \\
\text { MD. Mw }\end{array}$ & & Date & Hour & $\begin{array}{l}\text { Time } \\
\text { Tone }\end{array}$ \\
\hline
\end{tabular}

ALASKA-Continued

\begin{tabular}{|c|c|c|c|c|c|c|c|c|c|c|c|c|c|c|c|c|}
\hline $\begin{array}{l}\text { NOV. } \\
\text { NOV. } \\
\text { NOV. } \\
\text { NOV. } \\
\text { NOV. }\end{array}$ & $\begin{array}{l}25 \\
26 \\
26 \\
26 \\
27\end{array}$ & $\begin{array}{l}09 \\
01 \\
02 \\
06 \\
02\end{array}$ & $\begin{array}{l}58 \\
42 \\
31 \\
13 \\
08\end{array}$ & $\begin{array}{l}57.1 \\
13.4 \\
39.9 \\
08.9 \\
00.1\end{array}$ & $\begin{array}{l}53.190 \mathrm{~N} \\
62.902 \mathrm{~N} \\
62.910 \mathrm{~N} \\
59.614 \mathrm{~N} \\
54.241 \mathrm{~N}\end{array}$ & $\begin{array}{l}171.869 E . \\
150.536 W . \\
150.574 W . \\
152.888 W . \\
163.857 W .\end{array}$ & $\begin{array}{l}33 \mathrm{~N} \\
126 \\
100 \\
119 \\
33 \mathrm{~N}\end{array}$ & $\begin{array}{l}\text { GS } \\
\text { GS } \\
\text { GS } \\
\text { GS } \\
\text { GS }\end{array}$ & $\begin{array}{l}4.1 \\
\ldots \\
\cdots \\
4.0 \\
4.8\end{array}$ & $\begin{array}{l}\cdots \\
\cdots \\
\cdots \\
\cdots\end{array}$ & $\begin{array}{c}\cdots \\
\cdots \\
\cdots \\
\cdots \\
4.2 \mathrm{ML}(P M)\end{array}$ & $\begin{array}{l}\cdots \\
\cdots \\
\cdots \\
\cdots\end{array}$ & $\begin{array}{l}\text { NOV. } \\
\text { NOV. } \\
\text { NOV. } \\
\text { NOV. } \\
\text { NOV. }\end{array}$ & $\begin{array}{l}24 \\
25 \\
25 \\
25 \\
26\end{array}$ & $\begin{array}{l}23: 58 \\
16: 42 \\
17: 31 \\
21: 13 \\
17: 08\end{array}$ & $\begin{array}{l}\text { AST } \\
\text { YST } \\
\text { YST } \\
\text { YST } \\
\text { YST }\end{array}$ \\
\hline $\begin{array}{l}\text { NOV. } \\
\text { NOV. } \\
\text { NOV. } \\
\text { NOV. } \\
\text { NOV. }\end{array}$ & $\begin{array}{l}29 \\
29 \\
29 \\
29 \\
30\end{array}$ & $\begin{array}{l}06 \\
07 \\
15 \\
22 \\
01\end{array}$ & $\begin{array}{l}09 \\
45 \\
32 \\
35 \\
19\end{array}$ & $\begin{array}{l}08.5 \\
12.6 \\
03.5 \\
36.6 \\
38.5\end{array}$ & $\begin{array}{l}51.233 \mathrm{~N} . \\
51.466 \mathrm{~N} . \\
51.884 \mathrm{~N} . \\
51.850 \mathrm{~N} . \\
64.022 \mathrm{~N} .\end{array}$ & $\begin{array}{l}174.832 W . \\
176.268 W . \\
175.114 \mathrm{E} . \\
175.076 \mathrm{E} . \\
148.802 \mathrm{~W} .\end{array}$ & $\begin{array}{l}33 N \\
33 N \\
47 \\
47 \\
33 N\end{array}$ & $\begin{array}{l}\text { GS } \\
\text { GS } \\
\text { GS } \\
\text { GS } \\
\text { GS }\end{array}$ & $\begin{array}{l}4.6 \\
4.3 \\
4.8 \\
4.5 \\
\cdots\end{array}$ & $\begin{array}{l}\ldots \\
4.2 \\
\ldots \\
\ldots\end{array}$ & $\begin{array}{c}\text { 4. } 6 \mathrm{ML}(P M) \\
4.6 \mathrm{ML}(\mathrm{PM}) \\
\ldots \\
\ldots \\
\text { 3. } 6 \mathrm{ML}(\mathrm{PM})\end{array}$ & $\begin{array}{c}\text { FELT } \\
\ldots \\
\ldots \\
\ldots\end{array}$ & $\begin{array}{l}\text { NOV. } \\
\text { NOV. } \\
\text { NOV. } \\
\text { NOV. } \\
\text { NOV. }\end{array}$ & $\begin{array}{l}28 \\
28 \\
29 \\
29 \\
29\end{array}$ & $\begin{array}{l}20: 09 \\
21: 45 \\
05: 32 \\
12: 35 \\
16: 19\end{array}$ & $\begin{array}{l}\text { AST } \\
\text { AST } \\
\text { AST } \\
\text { AST } \\
\text { YST }\end{array}$ \\
\hline $\begin{array}{l}\text { DEC. } \\
\text { DEC. } \\
\text { DEC. } \\
\text { DEC. } \\
\text { DEC. }\end{array}$ & $\begin{array}{l}1 \\
1 \\
1 \\
2 \\
3\end{array}$ & $\begin{array}{l}17 \\
18 \\
18 \\
09 \\
05\end{array}$ & $\begin{array}{l}17 \\
25 \\
45 \\
43 \\
53\end{array}$ & $\begin{array}{l}30.8 \\
51.3 \\
24.8 \\
02.7 \\
54.3\end{array}$ & $\begin{array}{l}52.352 \mathrm{~N} . \\
60.575 \mathrm{~N} . \\
51.355 \mathrm{~N} . \\
59.782 \mathrm{~N} . \\
52.175 \mathrm{~N} .\end{array}$ & $\begin{array}{l}178.195 E . \\
150.301 W . \\
176.342 W . \\
153.364 W . \\
169.531 W .\end{array}$ & $\begin{array}{c}160 \\
51 \\
33 \mathrm{~N} \\
149 \\
33 \mathrm{~N}\end{array}$ & $\begin{array}{l}\text { GS } \\
\text { GS } \\
\text { GS } \\
\text { GS } \\
\text { GS }\end{array}$ & $\begin{array}{l}4.5 \\
3.8 \\
5.0 \\
\ldots .8\end{array}$ & $\begin{array}{l}\cdots \\
\cdots \\
\cdots \\
\cdots \\
\cdots\end{array}$ & $\begin{array}{c}\text { 4. 1ML(PM) } \\
\text { 5. OML(PM) } \\
\ldots \\
\ldots\end{array}$ & $\begin{array}{l}\text { I I } \\
\text { I I I } \\
\cdots \\
\cdots\end{array}$ & $\begin{array}{l}\text { DEC. } \\
\text { DEC. } \\
\text { DEC. } \\
\text { DEC. } \\
\text { DEC. }\end{array}$ & $\begin{array}{l}1 \\
1 \\
1 \\
2 \\
2\end{array}$ & $\begin{array}{l}07: 17 \\
09: 25 \\
08: 45 \\
00: 43 \\
19: 53\end{array}$ & $\begin{array}{l}\text { AST } \\
\text { YST } \\
\text { AST } \\
\text { YST } \\
\text { AST }\end{array}$ \\
\hline $\begin{array}{l}\text { DEC. } \\
\text { DEC. } \\
\text { DEC. } \\
\text { DEC. } \\
\text { DEC. }\end{array}$ & $\begin{array}{l}3 \\
3 \\
4 \\
5 \\
5\end{array}$ & $\begin{array}{l}12 \\
22 \\
19 \\
07 \\
13\end{array}$ & $\begin{array}{l}22 \\
51 \\
42 \\
52 \\
58\end{array}$ & $\begin{array}{l}45.1 \\
29.5 \\
26.5 \\
39.5 \\
55.6\end{array}$ & $\begin{array}{l}51.943 \mathrm{~N} \\
63.474 \mathrm{~N} \\
60.104 \mathrm{~N} \\
62.645 \mathrm{~N} \\
61.839 \mathrm{~N}\end{array}$ & $\begin{array}{l}177.330 \mathrm{E} \\
148.889 \mathrm{~W} \\
152.752 \mathrm{~W} \\
152.760 \mathrm{~W} \\
149.711 \mathrm{~W} .\end{array}$ & $\begin{array}{r}90 \\
114 \\
133 \\
5 \\
39\end{array}$ & $\begin{array}{l}\text { GS } \\
\text { GS } \\
\text { GS } \\
\text { GS } \\
\text { GS }\end{array}$ & $\begin{array}{l}4.9 \\
\ldots \\
\ldots \\
\ldots \\
\ldots\end{array}$ & $\begin{array}{l}\cdots \\
\cdots \\
\cdots \\
\cdots\end{array}$ & $\begin{array}{c}\cdots \\
\cdots \\
2.9 M \\
3.9 M L(P M) \\
3 M L(P M)\end{array}$ & $\begin{array}{l}\ldots \\
\ldots \\
\cdots \\
\cdots\end{array}$ & $\begin{array}{l}\text { DEC. } \\
\text { DEC. } \\
\text { DEC. } \\
\text { DEC. } \\
\text { DEC. }\end{array}$ & $\begin{array}{l}3 \\
3 \\
4 \\
4 \\
5\end{array}$ & $\begin{array}{l}02: 22 \\
13: 51 \\
10: 42 \\
22: 52 \\
04: 58\end{array}$ & $\begin{array}{l}\text { AST } \\
\text { YST } \\
\text { YST } \\
\text { YST } \\
\text { YST }\end{array}$ \\
\hline $\begin{array}{l}\text { DEC. } \\
\text { DEC. } \\
\text { DEC. } \\
\text { DEC. } \\
\text { DEC. }\end{array}$ & $\begin{array}{r}7 \\
8 \\
9 \\
10 \\
12\end{array}$ & $\begin{array}{l}16 \\
21 \\
04 \\
18 \\
04\end{array}$ & $\begin{array}{l}27 \\
07 \\
23 \\
50 \\
50\end{array}$ & $\begin{array}{l}55.3 \\
47.3 \\
18.2 \\
21.3 \\
54.4\end{array}$ & $\begin{array}{l}51.668 \mathrm{~N} \\
63.653 \mathrm{~N} . \\
63.638 \mathrm{~N} \\
62.573 \mathrm{~N} \\
61.659 \mathrm{~N}\end{array}$ & $\begin{array}{l}170.817 w . \\
150.770 w . \\
150.771 w . \\
149.505 w . \\
150.965 w .\end{array}$ & $\begin{array}{l}33 N \\
24 \\
28 \\
24 \\
76\end{array}$ & $\begin{array}{l}\text { GS } \\
\text { GS } \\
\text { GS } \\
\text { GS } \\
\text { GS }\end{array}$ & $\begin{array}{l}4.9 \\
4.6 \\
3.7 \\
3.8\end{array}$ & $\begin{array}{l}4.3 \\
5.2 \\
\cdots \\
\cdots \\
\cdots\end{array}$ & $\begin{array}{l}\text { 4. } 2 M L(P M) \\
\text { 4.6ML (PM) } \\
\text { 4. } 3 M L(P M) \\
\text { 2. } 9 M L(P M)\end{array}$ & $\begin{array}{c}\text { FELT } \\
\ldots \\
\cdots \\
\cdots\end{array}$ & $\begin{array}{l}\text { DEC. } \\
\text { DEC. } \\
\text { DEC. } \\
\text { DEC. } \\
\text { DEC. }\end{array}$ & $\begin{array}{r}7 \\
8 \\
8 \\
10 \\
11\end{array}$ & $\begin{array}{l}06: 27 \\
12: 07 \\
19: 23 \\
09: 50 \\
19: 50\end{array}$ & $\begin{array}{l}\text { AST } \\
\text { YST } \\
\text { YST } \\
\text { YST } \\
\text { YST }\end{array}$ \\
\hline $\begin{array}{l}\text { DEC. } \\
\text { DEC. } \\
\text { DEC. } \\
\text { DEC. } \\
\text { DEC. }\end{array}$ & $\begin{array}{l}12 \\
16 \\
17 \\
19 \\
19\end{array}$ & $\begin{array}{l}15 \\
09 \\
18 \\
00 \\
05\end{array}$ & $\begin{array}{l}50 \\
15 \\
36 \\
51 \\
19\end{array}$ & $\begin{array}{l}32.7 \\
44.7 \\
23.5 \\
52.2 \\
45.9\end{array}$ & $\begin{array}{l}66.973 \mathrm{~N} \\
51.327 \mathrm{~N} \\
59.553 \mathrm{~N} \\
66.067 \mathrm{~N} \\
58.123 \mathrm{~N}\end{array}$ & $\begin{array}{l}156.956 W \\
176.837 \mathrm{E} . \\
152.389 W \\
154.489 W \\
155.821 W\end{array}$ & $\begin{array}{l}33 N \\
33 N \\
85 \\
27 \\
33 N\end{array}$ & $\begin{array}{l}\text { GS } \\
\text { GS } \\
\text { GS } \\
\text { GS } \\
\text { GS }\end{array}$ & $\begin{array}{l}4.3 \\
\ldots \\
\ldots \\
\ldots\end{array}$ & $\begin{array}{l}\cdots \\
\cdots \\
\cdots \\
\cdots \\
\cdots\end{array}$ & $\begin{array}{c}\text { 3. 3ML (PM) } \\
\text { 4. } 7 \mathrm{ML}(\mathrm{PM}) \\
\ldots \ldots \\
\text { 3. } \mathrm{BML}(\mathrm{PM}) \\
\ldots\end{array}$ & $\begin{array}{l}\cdots \\
\text { III } \\
\text { II } \\
\cdots\end{array}$ & $\begin{array}{l}\text { DEC. } \\
\text { DEC. } \\
\text { DEC. } \\
\text { DEC. } \\
\text { DEC. }\end{array}$ & $\begin{array}{l}12 \\
15 \\
17 \\
18 \\
18\end{array}$ & $\begin{array}{l}06: 50 \\
23: 15 \\
09: 36 \\
15: 51 \\
20: 19\end{array}$ & $\begin{array}{l}\text { YST } \\
\text { AST } \\
\text { YST } \\
\text { YST } \\
\text { YST }\end{array}$ \\
\hline $\begin{array}{l}\text { DEC. } \\
\text { DEC. } \\
\text { DEC. } \\
\text { DEC. }\end{array}$ & $\begin{array}{l}20 \\
20 \\
21 \\
21 \\
22\end{array}$ & $\begin{array}{l}03 \\
22 \\
07 \\
09 \\
10\end{array}$ & $\begin{array}{l}48 \\
26 \\
51 \\
46 \\
27\end{array}$ & $\begin{array}{l}40.8 \\
12.1 \\
19.2 \\
51.3 \\
38.8\end{array}$ & $\begin{array}{l}56.564 \mathrm{~N} \\
63.348 \mathrm{~N} \\
63.605 \mathrm{~N} \\
52.474 \mathrm{~N} \\
60.980 \mathrm{~N}\end{array}$ & $\begin{array}{l}154.041 W . \\
149.842 W \\
150.495 W \\
174.353 w \\
146.614 W\end{array}$ & $\begin{array}{c}36 \\
122 \\
33 \mathrm{~N} \\
133 \\
9\end{array}$ & $\begin{array}{l}\text { GS } \\
G S \\
G S \\
G S \\
G S\end{array}$ & $\begin{array}{l}4.7 \\
\ldots \\
\ldots .3 \\
\ldots\end{array}$ & $\begin{array}{l}\cdots \\
\cdots \\
\cdots \\
\cdots\end{array}$ & $\begin{array}{c}\text { 4. } 5 \mathrm{ML}(P M) \\
\text { 3. } 3 \mathrm{ML}(P M) \\
\text { 3. } \mathrm{OML}(P M)\end{array}$ & $\begin{array}{l}\cdots \\
\cdots \\
\cdots\end{array}$ & $\begin{array}{l}\text { DEC. } \\
\text { DEC. } \\
\text { DEC. } \\
\text { DEC. } \\
\text { DEC. }\end{array}$ & $\begin{array}{l}19 \\
20 \\
20 \\
20 \\
22\end{array}$ & $\begin{array}{l}18: 48 \\
13: 26 \\
22: 51 \\
23: 46 \\
01: 27\end{array}$ & $\begin{array}{l}\text { YST } \\
\text { YST } \\
\text { YST } \\
\text { AST } \\
\text { YST }\end{array}$ \\
\hline $\begin{array}{l}\text { DEC. } \\
\text { DEC. } \\
\text { OEC. } \\
\text { DEC. } \\
\text { OEC. }\end{array}$ & $\begin{array}{l}23 \\
24 \\
27 \\
27 \\
27\end{array}$ & $\begin{array}{l}22 \\
13 \\
09 \\
20 \\
23\end{array}$ & $\begin{array}{l}01 \\
29 \\
55 \\
50 \\
11\end{array}$ & $\begin{array}{l}58.6 \\
56.4 \\
30.2 \\
56.6 \\
32.4\end{array}$ & $\begin{array}{l}62.205 \mathrm{~N} \\
58.627 \mathrm{~N} \\
53.925 \mathrm{~N} \\
53.218 \mathrm{~N} \\
51.787 \mathrm{~N}\end{array}$ & $\begin{array}{l}151.012 W . \\
154.393 W . \\
162.417 W . \\
167.897 W . \\
174.639 W .\end{array}$ & $\begin{array}{c}98 \\
33 \mathrm{~N} \\
33 \mathrm{~N} \\
175 \\
97\end{array}$ & $\begin{array}{l}\text { GS } \\
G S \\
G S \\
G S \\
G S\end{array}$ & $\begin{array}{l}\cdots \\
\cdots \\
5.0 \\
4.2 \\
4.3\end{array}$ & $\begin{array}{l}\cdots \\
\cdots \\
\cdots \\
\cdots\end{array}$ & $\begin{array}{c}\text { 3. } 4 \mathrm{ML}(\mathrm{PM}) \\
\ldots \\
\ldots \\
\ldots\end{array}$ & $\begin{array}{l}\cdots \\
\cdots \\
\cdots \\
\cdots\end{array}$ & $\begin{array}{l}\text { DEC. } \\
\text { DEC. } \\
\text { DEC. } \\
\text { DEC. } \\
\text { DEC. }\end{array}$ & $\begin{array}{l}23 \\
24 \\
27 \\
27 \\
27\end{array}$ & $\begin{array}{l}13: 01 \\
04: 29 \\
00: 55 \\
11: 50 \\
13: 11\end{array}$ & $\begin{array}{l}\text { YST } \\
\text { YST } \\
\text { YST } \\
\text { AST } \\
\text { AST }\end{array}$ \\
\hline EC. & $\begin{array}{l}28 \\
29\end{array}$ & $\begin{array}{l}04 \\
03\end{array}$ & $\begin{array}{l}16 \\
02\end{array}$ & $\begin{array}{l}17.0 \\
12.6\end{array}$ & $\begin{array}{l}50.392 \mathrm{~N} \\
60.117 \mathrm{~N}\end{array}$ & $\begin{array}{l}177.926 \mathrm{E} . \\
153.388 \mathrm{~W} .\end{array}$ & $\begin{array}{l}33 N \\
166\end{array}$ & $\begin{array}{l}\text { GS } \\
\text { GS }\end{array}$ & $\begin{array}{l}4.8 \\
4.0\end{array}$ & $\cdots$ & $\ldots$ & $\cdots$ & DEC. & $\begin{array}{l}27 \\
28\end{array}$ & $\begin{array}{l}18: 16 \\
18: 02\end{array}$ & $\begin{array}{l}\text { AST } \\
\text { YST }\end{array}$ \\
\hline
\end{tabular}

\section{ARIZONA}

APR. $14 \quad 09 \quad 15 \quad 06.8 \quad 36.503 \mathrm{~N}, 113.383 \mathrm{~W}$. JULY $7 \quad 18 \quad 1459.0 \quad 32.460 \mathrm{~N} .114 .010 \mathrm{~W}$. JULY $18 \quad 14 \quad 2931.8 \quad 36.216 \mathrm{~N} .111 .844 \mathrm{~W}$.

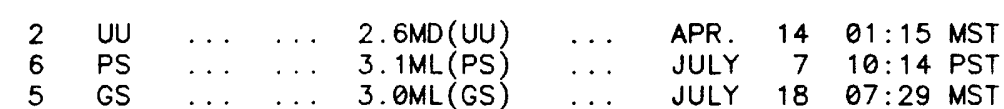


Table 1. Summary of U.S. earthquakes for 1984-Continued

\begin{tabular}{|c|c|c|c|c|c|c|c|c|c|c|c|c|c|}
\hline \multirow{2}{*}{ Date } & \multicolumn{2}{|c|}{$\begin{array}{l}\text { Origin time } \\
\text { (UTC) }\end{array}$} & \multirow{2}{*}{$\begin{array}{c}\text { Latitude } \\
\left({ }^{\circ}\right)\end{array}$} & \multirow{2}{*}{$\begin{array}{c}\text { Longitude } \\
\text { (') }\end{array}$} & \multirow{2}{*}{$\begin{array}{c}\text { Depth } \\
\text { (km) }\end{array}$} & \multirow{2}{*}{$\begin{array}{l}\text { Hypo- } \\
\text { center } \\
\text { source }\end{array}$} & \multicolumn{3}{|c|}{ Magnitude } & \multirow{2}{*}{$\begin{array}{l}\text { Maximum } \\
\text { intensity }\end{array}$} & \multicolumn{3}{|c|}{ Lexal time } \\
\hline & hr $\min$ & $\sec$ & & & & & $\mathbf{m b}$ & MS & $\begin{array}{l}\text { ML, Mn } \\
\text { MD. Mw }\end{array}$ & & Date & Hour & $\begin{array}{l}\text { Time } \\
\text { nome }\end{array}$ \\
\hline
\end{tabular}

ARKANSAS

JUNE $17 \quad 00 \quad 41 \quad 39.1 \quad 36.126 \mathrm{~N} .92 .731 W$. $\begin{array}{lllll}\text { JULY } & 12 & 01 & 27 & 17.6\end{array}$ $\begin{array}{lllll}\text { SEPT. } 27 & 13 & 03 & 05.2\end{array}$ SEPT. $27 \quad 13 \quad 16 \quad 04.0$ $35.230 \mathrm{~N} .92 .207 \mathrm{~W}$ $35.262 \mathrm{~N} .92 .187 \mathrm{~W}$. $35.218 \mathrm{~N}, 92.173 W$.

$\begin{array}{rr}5 & \text { GS } \\ 2 & \text { TC } \\ 10 & \text { GS } \\ 10 & \text { GS }\end{array}$

$\ldots \quad \ldots \quad 2.8 \mathrm{Mn}(\mathrm{TU})$

2. $6 \mathrm{MD}(\mathrm{TC})$

3. $3 \mathrm{Mn}$ (TU)

2. $4 \mathrm{Mn}(\mathrm{TU})$
JUNE $16 \quad 18: 41 \quad$ CST JULY $11 \quad 19: 27$ CST SEPT. $27 \quad 07: 03$ CST SEPT. $27 \quad 07: 16$ CST

\section{CALIFORNIA}

\begin{tabular}{|c|c|c|c|c|c|c|c|c|c|c|c|c|c|c|c|c|}
\hline $\begin{array}{l}\text { JAN. } \\
\text { JAN. } \\
\text { JAN. } \\
\text { JAN. } \\
\text { JAN. }\end{array}$ & $\begin{array}{l}1 \\
2 \\
2 \\
2 \\
3\end{array}$ & $\begin{array}{l}18 \\
00 \\
05 \\
09 \\
07\end{array}$ & $\begin{array}{l}27 \\
51 \\
00 \\
29 \\
40\end{array}$ & $\begin{array}{l}55.2 \\
32.5 \\
12.2 \\
43.1 \\
34.7\end{array}$ & $\begin{array}{l}36.364 \mathrm{~N} \\
35.962 \mathrm{~N} . \\
35.951 \mathrm{~N} \\
38.043 \mathrm{~N} . \\
36.116 \mathrm{~N} .\end{array}$ & $\begin{array}{l}120.395 w . \\
118.332 W . \\
118.329 w . \\
118.202 W . \\
120.177 w .\end{array}$ & $\begin{array}{r}19 \\
4 \\
3 \\
6 \\
6\end{array}$ & $\begin{array}{l}\text { PS } \\
\text { PS } \\
\text { PS } \\
\text { PS } \\
\text { PS }\end{array}$ & $\begin{array}{l}\cdots \\
\cdots \\
\cdots \\
\cdots\end{array}$ & $\begin{array}{l}\ldots \\
\ldots \\
\ldots \\
\ldots \\
\ldots\end{array}$ & $\begin{array}{l}\text { 3. 3ML(PS) } \\
3.4 M L(P S) \\
3.2 M L(P S) \\
3.3 M L(P S) \\
3.0 M L(P S)\end{array}$ & $\begin{array}{l}\cdots \\
\cdots \\
\cdots \\
\cdots\end{array}$ & $\begin{array}{l}\text { JAN. } \\
\text { JAN. } \\
\text { JAN. } \\
\text { JAN. } \\
\text { JAN. }\end{array}$ & $\begin{array}{l}1 \\
1 \\
1 \\
2 \\
2\end{array}$ & $\begin{array}{l}10: 27 \\
16: 51 \\
21: 00 \\
01: 29 \\
23: 40\end{array}$ & $\begin{array}{l}\text { PST } \\
\text { PST } \\
\text { PST } \\
\text { PST } \\
\text { PST }\end{array}$ \\
\hline $\begin{array}{l}\text { JAN. } \\
\text { JAN. } \\
\text { JAN. } \\
\text { JAN. } \\
\text { JAN. }\end{array}$ & $\begin{array}{l}5 \\
6 \\
8 \\
9 \\
9\end{array}$ & $\begin{array}{l}19 \\
05 \\
11 \\
17 \\
19\end{array}$ & $\begin{array}{l}01 \\
00 \\
24 \\
17 \\
08\end{array}$ & $\begin{array}{l}07.0 \\
12.8 \\
14.2 \\
35.6 \\
29.7\end{array}$ & $\begin{array}{l}7.377 \mathrm{~N} \\
5.957 \mathrm{~N} \\
6.776 \mathrm{~N} \\
8.013 \mathrm{~N} \\
2.969 \mathrm{~N}\end{array}$ & $\begin{array}{l}121.758 w . \\
118.337 w . \\
119.924 w . \\
121.966 w . \\
115.542 W\end{array}$ & $\begin{array}{r}4 \\
6 \\
6 \\
41 \\
5\end{array}$ & $\begin{array}{l}\text { BK } \\
\text { PS } \\
\text { PS } \\
\text { GM } \\
\text { PS }\end{array}$ & $\begin{array}{l}\ldots \\
\ldots \\
\cdots \\
\ldots\end{array}$ & $\begin{array}{l}\ldots \\
\ldots \\
\ldots \\
\ldots\end{array}$ & $\begin{array}{l}\text { 3. 3ML(BK) } \\
\text { 3. } 2 M L(P S) \\
\text { 3. } 0 M L(P S) \\
\text { 3.5MD(GM) } \\
\text { 2.9ML(PS) }\end{array}$ & $\begin{array}{c}\text { III } \\
\cdots \\
\cdots \\
\text { FELT }\end{array}$ & $\begin{array}{l}\text { JAN. } \\
\text { JAN. } \\
\text { JAN. } \\
\text { JAN. } \\
\text { JAN. }\end{array}$ & $\begin{array}{l}5 \\
5 \\
8 \\
9 \\
9\end{array}$ & $\begin{array}{l}11: 01 \\
21: 00 \\
03: 24 \\
09: 17 \\
11: 08\end{array}$ & $\begin{array}{l}\text { PST } \\
\text { PST } \\
\text { PST } \\
\text { PST } \\
\text { PST }\end{array}$ \\
\hline $\begin{array}{l}\text { JAN. } \\
\text { JAN. } \\
\text { JAN. } \\
\text { JAN. } \\
\text { JAN. }\end{array}$ & $\begin{array}{l}10 \\
11 \\
12 \\
12 \\
12\end{array}$ & $\begin{array}{l}15 \\
14 \\
04 \\
15 \\
22\end{array}$ & $\begin{array}{l}15 \\
19 \\
22 \\
47 \\
45\end{array}$ & $\begin{array}{l}47.7 \\
00.1 \\
39.3 \\
16.3 \\
48.8\end{array}$ & $\begin{array}{l}37.452 \mathrm{~N} \\
37.367 \mathrm{~N} . \\
36.300 \mathrm{~N} \\
35.917 \mathrm{~N} \\
36.397 \mathrm{~N} .\end{array}$ & $\begin{array}{l}118.658 w . \\
121.758 w . \\
120.407 w . \\
118.328 w . \\
120.415 w .\end{array}$ & $\begin{array}{r}5 \\
5 \\
15 \\
1 \\
16\end{array}$ & $\begin{array}{l}\text { BK } \\
\text { PS } \\
\text { PS } \\
\text { PS }\end{array}$ & $\begin{array}{l}\ldots \\
\ldots \\
\ldots \\
\ldots\end{array}$ & $\begin{array}{l}\ldots \\
\cdots \\
\cdots \\
\cdots\end{array}$ & $\begin{array}{l}\text { 3. } 5 M L(B K) \\
2.9 M L(B K) \\
3.2 M L(P S) \\
3.1 M L(P S) \\
3.1 M L(P S)\end{array}$ & $\begin{array}{c}\text { FELT } \\
\text { I I I } \\
\cdots \\
\cdots \\
\cdots\end{array}$ & $\begin{array}{l}\text { JAN. } \\
\text { JAN. } \\
\text { JAN. } \\
\text { JAN. } \\
\text { JAN. }\end{array}$ & $\begin{array}{l}10 \\
11 \\
11 \\
12 \\
12\end{array}$ & $\begin{array}{l}07: 15 \\
06: 18 \\
20: 22 \\
07: 47 \\
14: 45\end{array}$ & I \\
\hline $\begin{array}{l}\text { JAN. } \\
\text { JAN. } \\
\text { JAN. } \\
\text { JAN. } \\
\text { JAN. }\end{array}$ & $\begin{array}{l}13 \\
14 \\
14 \\
14 \\
14\end{array}$ & $\begin{array}{l}09 \\
00 \\
03 \\
03 \\
10\end{array}$ & $\begin{array}{l}41 \\
42 \\
11 \\
13 \\
37\end{array}$ & $\begin{array}{l}41.3 \\
23.6 \\
15.0 \\
53.5 \\
01.9\end{array}$ & $\begin{array}{l}4.048 \mathrm{~N} \\
7.423 \mathrm{~N} \\
7.415 \mathrm{~N} \\
7.349 \mathrm{~N} \\
0.268 \mathrm{~N}\end{array}$ & $\begin{array}{l}117.254 w . \\
118.595 w . \\
118.587 w . \\
118.593 w . \\
124.357 w .\end{array}$ & $\begin{array}{r}15 \\
10 \\
11 \\
6 \\
9\end{array}$ & $\begin{array}{l}\text { BK } \\
\text { BK } \\
\text { PS } \\
\text { BK }\end{array}$ & $\begin{array}{l}\ldots \\
\ldots \\
\cdots \\
4.1\end{array}$ & $\begin{array}{l}\cdots \\
\cdots \\
\cdots \\
\cdots \\
\cdots\end{array}$ & $\begin{array}{l}\text { 3. } 2 M L(P S) \\
\text { 3.8ML(BK) } \\
\text { 4.0ML(BK) } \\
\text { 3.1ML(PS) } \\
\text { 4. } 1 M L(B K)\end{array}$ & $\begin{array}{l}\ddot{\text { IV }} \\
\text { FELT } \\
\text { FELT }\end{array}$ & & $\begin{array}{l}13 \\
13 \\
13 \\
13 \\
14\end{array}$ & $\begin{array}{l}01: 41 \\
16: 42 \\
19: 11 \\
19: 13 \\
02: 37\end{array}$ & $\begin{array}{l}\text { PST } \\
\text { PST } \\
\text { PST }\end{array}$ \\
\hline $\begin{array}{l}\text { JAN. } \\
\text { JAN. } \\
\text { JAN. } \\
\text { JAN. } \\
\text { JAN. }\end{array}$ & $\begin{array}{l}14 \\
15 \\
15 \\
16 \\
16\end{array}$ & $\begin{array}{l}10 \\
15 \\
17 \\
02 \\
04\end{array}$ & $\begin{array}{l}39 \\
51 \\
54 \\
48 \\
06\end{array}$ & $\begin{array}{l}55.9 \\
32.8 \\
20.2 \\
06.7 \\
12.4\end{array}$ & $\begin{array}{l}40.290 \mathrm{~N} \\
36.073 \mathrm{~N} \\
34.332 \mathrm{~N} \\
36.082 \mathrm{~N} \\
35.928 \mathrm{~N}\end{array}$ & $\begin{array}{l}124.383 W . \\
120.222 W . \\
116.460 W . \\
120.235 W . \\
118.302 W .\end{array}$ & $\begin{array}{r}10 \\
11 \\
2 \\
11 \\
0\end{array}$ & $\begin{array}{l}\text { BK } \\
\text { GM } \\
\text { PS } \\
\text { GM }\end{array}$ & $\begin{array}{l}4.3 \\
\ldots \\
\ldots \\
\ldots \\
\ldots\end{array}$ & $\begin{array}{l}\cdots \\
\cdots \\
\cdots \\
\cdots\end{array}$ & $\begin{array}{l}\text { 3. } 9 M L(B K) \\
\text { 3. } 1 \mathrm{MD}(\mathrm{GM}) \\
\text { 3.1ML(PS) } \\
\text { 3. } 0 \mathrm{MD}(\mathrm{GM}) \\
\text { 3. } 4 \mathrm{ML}(\mathrm{PS})\end{array}$ & $\begin{array}{c}\text { FELT } \\
\ldots \\
\ldots \\
\ldots \\
\ldots\end{array}$ & $\begin{array}{l}\text { JAN. } \\
\text { JAN. } \\
\text { JAN. } \\
\text { JAN. } \\
\text { JAN. }\end{array}$ & $\begin{array}{l}14 \\
15 \\
15 \\
15 \\
15\end{array}$ & $\begin{array}{l}02: 39 \\
07: 51 \\
09: 54 \\
18: 48 \\
20: 06\end{array}$ & $\begin{array}{l}\text { PST } \\
\text { PST } \\
\text { PST }\end{array}$ \\
\hline $\begin{array}{l}\text { JAN. } \\
\text { JAN. } \\
\text { JAN. } \\
\text { JAN. } \\
\text { JAN. }\end{array}$ & $\begin{array}{l}17 \\
18 \\
18 \\
20 \\
20\end{array}$ & $\begin{array}{l}21 \\
16 \\
17 \\
08 \\
12\end{array}$ & $\begin{array}{l}28 \\
13 \\
25 \\
38 \\
07\end{array}$ & $\begin{array}{l}44.4 \\
08.9 \\
24.4 \\
49.2 \\
50.4\end{array}$ & $\begin{array}{l}35.937 \mathrm{~N} . \\
35.953 \mathrm{~N} . \\
35.959 \mathrm{~N} . \\
36.199 \mathrm{~N} . \\
35.958 \mathrm{~N} .\end{array}$ & $\begin{array}{l}118.324 w . \\
117.969 w . \\
117.966 w . \\
118.301 w . \\
117.967 w .\end{array}$ & $\begin{array}{l}2 \\
7 \\
8 \\
6 \\
9\end{array}$ & $\begin{array}{l}\text { PS } \\
\text { PS } \\
\text { PS } \\
\text { PS } \\
\text { PS }\end{array}$ & $\begin{array}{l}\ldots \\
\cdots \\
\cdots \\
\cdots \\
\cdots\end{array}$ & $\begin{array}{l}\cdots \\
\cdots \\
\cdots \\
\cdots\end{array}$ & $\begin{array}{l}\text { 3. } 0 M L(P S) \\
3.1 M L(P S) \\
3.2 M L(P S) \\
3.1 M L(P S) \\
3.3 M L(P S)\end{array}$ & $\begin{array}{l}\ldots \\
\cdots \\
\cdots \\
\cdots\end{array}$ & $\begin{array}{l}\text { JAN. } \\
\text { JAN. } \\
\text { JAN. } \\
\text { JAN. } \\
\text { JAN. }\end{array}$ & $\begin{array}{l}17 \\
18 \\
18 \\
20 \\
20\end{array}$ & $\begin{array}{l}13: 28 \\
08: 13 \\
09: 25 \\
00: 38 \\
04: 07\end{array}$ & $\begin{array}{l}P S \\
P S \\
P S\end{array}$ \\
\hline $\begin{array}{l}\text { JAN. } \\
\text { JAN. } \\
\text { JAN. } \\
\text { JAN. } \\
\text { JAN. }\end{array}$ & $\begin{array}{l}23 \\
23 \\
23 \\
23 \\
23\end{array}$ & $\begin{array}{l}05 \\
05 \\
05 \\
06 \\
06\end{array}$ & $\begin{array}{l}40 \\
44 \\
48 \\
05 \\
59\end{array}$ & $\begin{array}{l}20.3 \\
38.1 \\
58.0 \\
10.5 \\
51.3\end{array}$ & $\begin{array}{l}36.392 \mathrm{~N} . \\
36.395 \mathrm{~N} . \\
36.365 \mathrm{~N} . \\
36.373 \mathrm{~N} . \\
36.388 \mathrm{~N} .\end{array}$ & $\begin{array}{l}121.878 w . \\
121.862 W \\
121.878 w . \\
121.885 w \\
121.872 w\end{array}$ & $\begin{array}{r}8 \\
2 \\
10 \\
8 \\
8\end{array}$ & $\begin{array}{l}\text { BK } \\
\text { BK } \\
\text { BK } \\
\text { BK } \\
\text { BK }\end{array}$ & $\begin{array}{l}5.1 \\
\ldots \\
\ldots \\
\ldots \\
\ldots\end{array}$ & $\begin{array}{l}4.6 \\
\ldots \\
\ldots \\
\ldots \\
\ldots\end{array}$ & $\begin{array}{l}\text { 5. } 1 \mathrm{ML}(\mathrm{BK}) \\
\text { 3. } 1 \mathrm{ML}(\mathrm{BK}) \\
\text { 3. } \mathrm{MLL}(\mathrm{BK}) \\
\text { 3. } 6 \mathrm{ML}(\mathrm{BK}) \\
\text { 4.5ML(BK) }\end{array}$ & $\begin{array}{r}V \\
\text { FËLT } \\
\text { FËLT }\end{array}$ & $\begin{array}{l}\text { JAN. } \\
\text { JAN. } \\
\text { JAN. } \\
\text { JAN. } \\
\text { JAN. }\end{array}$ & $\begin{array}{l}22 \\
22 \\
22 \\
22 \\
22\end{array}$ & $\begin{array}{l}21: 40 \\
21: 44 \\
21: 48 \\
22: 05 \\
22: 59\end{array}$ & $\begin{array}{l}\text { PST } \\
\text { PST } \\
\text { PST } \\
\text { PST } \\
\text { PST }\end{array}$ \\
\hline $\begin{array}{l}\text { JAN. } \\
\text { JAN. } \\
\text { JAN. } \\
\text { JAN. } \\
\text { JAN. }\end{array}$ & $\begin{array}{l}23 \\
23 \\
23 \\
24 \\
25\end{array}$ & $\begin{array}{l}10 \\
17 \\
19 \\
01 \\
05\end{array}$ & $\begin{array}{l}53 \\
03 \\
58 \\
00 \\
26\end{array}$ & $\begin{array}{l}53.8 \\
16.1 \\
21.5 \\
39.4 \\
27.7\end{array}$ & $\begin{array}{l}36.377 \mathrm{~N} . \\
33.970 \mathrm{~N} . \\
36.367 \mathrm{~N} . \\
36.372 \mathrm{~N} . \\
33.890 \mathrm{~N} .\end{array}$ & $\begin{array}{l}121.870 w . \\
116.840 w . \\
121.883 w . \\
121.880 w . \\
116.144 w .\end{array}$ & $\begin{array}{r}8 \\
15 \\
9 \\
8 \\
2\end{array}$ & $\begin{array}{l}\text { BK } \\
\text { PS } \\
\text { BK } \\
\text { BK } \\
\text { PS }\end{array}$ & $\begin{array}{l}\ldots \\
\cdots \\
\cdots \\
\cdots\end{array}$ & $\begin{array}{l}\ldots \\
\ldots \\
\cdots \\
\cdots \\
\cdots\end{array}$ & $\begin{array}{l}\text { 3. } 0 \mathrm{ML}(\mathrm{BK}) \\
2.7 \mathrm{ML}(\mathrm{PS}) \\
\text { 3.6ML(BK) } \\
\text { 3. } 7 \mathrm{ML}(\mathrm{BK}) \\
\text { 3. } 0 \mathrm{ML}(\mathrm{PS})\end{array}$ & $\begin{array}{c}\text { FELT } \\
\text { FELT } \\
\text { FELT } \\
\ldots\end{array}$ & $\begin{array}{l}\text { JAN. } \\
\text { JAN. } \\
\text { JAN. } \\
\text { JAN. } \\
\text { JAN. }\end{array}$ & $\begin{array}{l}23 \\
23 \\
23 \\
23 \\
24\end{array}$ & $\begin{array}{l}02: 53 \\
09: 03 \\
11: 58 \\
17: 00 \\
21: 26\end{array}$ & $\begin{array}{l}\text { PST } \\
\text { PST } \\
\text { PST } \\
\text { PST } \\
\text { PST }\end{array}$ \\
\hline $\begin{array}{l}\text { JAN. } \\
\text { JAN. } \\
\text { JAN. } \\
\text { JAN. } \\
\text { JAN. }\end{array}$ & $\begin{array}{l}26 \\
27 \\
27 \\
27 \\
27\end{array}$ & $\begin{array}{l}06 \\
04 \\
09 \\
15 \\
15\end{array}$ & $\begin{array}{l}07 \\
44 \\
06 \\
41 \\
41\end{array}$ & $\begin{array}{l}16.9 \\
35.8 \\
10.4 \\
54.7 \\
54.8\end{array}$ & $\begin{array}{l}36.140 \mathrm{~N} . \\
36.277 \mathrm{~N} . \\
33.058 \mathrm{~N} . \\
35.943 \mathrm{~N} . \\
35.937 \mathrm{~N} .\end{array}$ & $\begin{array}{l}120.143 w . \\
120.400 w . \\
116.196 w . \\
120.086 w . \\
120.158 w .\end{array}$ & $\begin{array}{r}4 \\
7 \\
6 \\
15 \\
12\end{array}$ & $\begin{array}{l}\text { BK } \\
\text { BK } \\
\text { PS } \\
\text { PS } \\
\text { GM }\end{array}$ & $\begin{array}{l}\cdots \\
\cdots \\
\cdots \\
\ldots\end{array}$ & $\begin{array}{l}\ldots \\
\ldots \\
\cdots \\
\ldots\end{array}$ & $\begin{array}{l}\text { 3. } 2 \mathrm{ML}(\mathrm{BK}) \\
3.8 \mathrm{ML}(\mathrm{BK}) \\
3.0 \mathrm{ML}(\mathrm{PS}) \\
3.4 \mathrm{ML}(\mathrm{PS}) \\
3.3 \mathrm{MD}(\mathrm{GM})\end{array}$ & $\begin{array}{c}\text { FELT } \\
\ldots \\
\ldots \\
\ldots\end{array}$ & $\begin{array}{l}\text { JAN. } \\
\text { JAN. } \\
\text { JAN. } \\
\text { JAN. } \\
\text { JAN. }\end{array}$ & $\begin{array}{l}25 \\
26 \\
27 \\
27 \\
27\end{array}$ & $\begin{array}{l}22: 07 \\
20: 44 \\
01: 06 \\
07: 41 \\
07: 41\end{array}$ & $\begin{array}{l}\text { PST } \\
\text { PST } \\
\text { PST } \\
\text { PST } \\
P S T\end{array}$ \\
\hline
\end{tabular}


Table 1. Summary of U.S. earthquakes for 1984-Continued

\begin{tabular}{|c|c|c|c|c|c|c|c|c|c|c|c|c|c|}
\hline \multirow{2}{*}{ Date } & \multicolumn{2}{|c|}{$\begin{array}{l}\text { Origin time } \\
\text { (UTC) }\end{array}$} & \multirow{2}{*}{$\begin{array}{c}\text { Latitude } \\
\left({ }^{\circ}\right)\end{array}$} & \multirow{2}{*}{$\begin{array}{c}\text { Longitude } \\
\text { (1) }\end{array}$} & \multirow{2}{*}{$\begin{array}{c}\text { Depth } \\
(\mathbf{k m})\end{array}$} & \multirow{2}{*}{$\begin{array}{l}\text { Hypo- } \\
\text { center } \\
\text { source }\end{array}$} & \multicolumn{3}{|c|}{ Magnitude } & \multirow{2}{*}{$\begin{array}{l}\text { Maximum } \\
\text { intensity }\end{array}$} & \multicolumn{3}{|c|}{ Lecal time } \\
\hline & hr $\min$ & $\sec$ & & & & & $\mathbf{m b}$ & MS & $\begin{array}{l}\text { ML, Mn } \\
\text { MD, Mw }\end{array}$ & & Date & Hour & $\begin{array}{l}\text { Time } \\
\text { zone }\end{array}$ \\
\hline
\end{tabular}

CALIFORNIA-Continued

\begin{tabular}{|c|c|c|c|c|c|c|c|c|c|c|c|c|c|c|c|c|}
\hline $\begin{array}{l}\text { JAN. } \\
\text { JAN. } \\
\text { JAN. } \\
\text { JAN. } \\
\text { FEB. }\end{array}$ & $\begin{array}{r}28 \\
29 \\
29 \\
30 \\
1\end{array}$ & $\begin{array}{l}10 \\
20 \\
20 \\
01 \\
06\end{array}$ & $\begin{array}{l}11 \\
06 \\
19 \\
33 \\
39\end{array}$ & $\begin{array}{l}55.2 \\
25.4 \\
45.9 \\
31.2 \\
33.3\end{array}$ & $\begin{array}{l}36.245 \mathrm{~N} \\
33.725 \mathrm{~N} \\
33.709 \mathrm{~N} \\
41.217 \mathrm{~N} \\
33.536 \mathrm{~N}\end{array}$ & $\begin{array}{l}120.235 W \\
118.081 W \\
118.063 W \\
123.347 W \\
116.800 W\end{array}$ & $\begin{array}{l}7 \\
6 \\
6 \\
5 \\
0\end{array}$ & $\begin{array}{l}\text { PS } \\
\text { PS } \\
\text { PS } \\
\text { BK } \\
\text { PS }\end{array}$ & $\begin{array}{l}\cdots \\
\ldots \\
\cdots \\
\cdots\end{array}$ & $\begin{array}{l}\cdots \\
\ldots \\
\cdots \\
\cdots \\
\cdots\end{array}$ & $\begin{array}{l}\text { 3. } 3 M L(P S) \\
2.7 M L(P S) \\
2.7 M L(P S) \\
\text { 3. } 0 M L(B K) \\
\text { 3.1ML(PS) }\end{array}$ & $\begin{array}{c}\text { FELT } \\
\text { FELT } \\
\ldots \\
\ldots\end{array}$ & $\begin{array}{l}\text { JAN. } \\
\text { JAN. } \\
\text { JAN. } \\
\text { JAN. } \\
\text { JAN. }\end{array}$ & $\begin{array}{l}28 \\
29 \\
29 \\
29 \\
31\end{array}$ & $\begin{array}{l}02: 11 \\
12: 06 \\
12: 19 \\
17: 33 \\
22: 39\end{array}$ & $\begin{array}{l}\text { PST } \\
\text { PST } \\
\text { PST } \\
\text { PST } \\
\text { PST }\end{array}$ \\
\hline $\begin{array}{l}\text { FEB. } \\
\text { FEB. } \\
\text { FEB. } \\
\text { FEB. } \\
\text { FEB. }\end{array}$ & $\begin{array}{l}2 \\
4 \\
4 \\
5 \\
5\end{array}$ & $\begin{array}{l}21 \\
02 \\
22 \\
10 \\
20\end{array}$ & $\begin{array}{l}47 \\
29 \\
47 \\
58 \\
39\end{array}$ & $\begin{array}{l}56.5 \\
12.1 \\
39.1 \\
45.9 \\
45.5\end{array}$ & $\begin{array}{l}33.893 \mathrm{~N} . \\
37.404 \mathrm{~N} \\
35.953 \mathrm{~N} \\
37.653 \mathrm{~N} . \\
34.536 \mathrm{~N} .\end{array}$ & $\begin{array}{l}116.847 W . \\
118.557 W . \\
117.968 W . \\
119.057 W . \\
118.905 W .\end{array}$ & $\begin{array}{r}17 \\
6 \\
6 \\
10 \\
16\end{array}$ & $\begin{array}{l}\text { PS } \\
\text { PS } \\
\text { PS } \\
\text { BK } \\
\text { PS }\end{array}$ & $\begin{array}{l}\ldots \\
\ldots \\
\ldots \\
\ldots \\
\cdots\end{array}$ & $\begin{array}{l}\ldots \\
\ldots \\
\ldots \\
\cdots \\
\cdots\end{array}$ & $\begin{array}{l}\text { 3. } 3 M L(P S) \\
3.1 M L(P S) \\
3.5 M L(P S) \\
3.8 M L(B K) \\
3.2 M L(P S)\end{array}$ & $\begin{array}{c}\text { FELT } \\
\ldots \\
\ldots \\
\cdots \\
\ldots\end{array}$ & $\begin{array}{l}\text { FEB. } \\
\text { FEB. } \\
\text { FEB. } \\
\text { FEB. } \\
\text { FEB. }\end{array}$ & $\begin{array}{l}2 \\
3 \\
4 \\
5 \\
5\end{array}$ & $\begin{array}{l}13: 47 \\
18: 29 \\
14: 47 \\
02: 58 \\
12: 39\end{array}$ & $\begin{array}{l}\text { PST } \\
\text { PST } \\
\text { PST } \\
\text { PST } \\
\text { PST }\end{array}$ \\
\hline $\begin{array}{l}\text { FEB. } \\
\text { FEB. } \\
\text { FEB. } \\
\text { FEB. } \\
\text { FEB. }\end{array}$ & $\begin{array}{r}7 \\
10 \\
10 \\
11 \\
11\end{array}$ & $\begin{array}{l}03 \\
06 \\
07 \\
14 \\
14\end{array}$ & $\begin{array}{l}33 \\
33 \\
23 \\
10 \\
25\end{array}$ & $\begin{array}{l}30.3 \\
14.7 \\
25.3 \\
28.8 \\
14.5\end{array}$ & $\begin{array}{l}34.309 \mathrm{~N} . \\
36.110 \mathrm{~N} . \\
36.387 \mathrm{~N} . \\
36.388 \mathrm{~N} \\
34.536 \mathrm{~N}\end{array}$ & $\begin{array}{l}119.265 W \\
120.186 W \\
121.883 W \\
121.882 W \\
118.914 W\end{array}$ & $\begin{array}{r}0 \\
16 \\
12 \\
9 \\
18\end{array}$ & $\begin{array}{l}\text { PS } \\
\text { PS } \\
\text { BK } \\
\text { BK } \\
\text { PS }\end{array}$ & $\begin{array}{l}\ldots \\
\ldots \\
\ldots \\
\ldots \\
\ldots\end{array}$ & $\begin{array}{l}\ldots \\
\ldots \\
\ldots \\
\cdots \\
\cdots\end{array}$ & $\begin{array}{l}\text { 3. 3ML(PS) } \\
\text { 3. 3ML(PS) } \\
\text { 4. } 1 \mathrm{ML}(\mathrm{BK}) \\
\text { 3. } 4 \mathrm{ML}(\mathrm{BK}) \\
\text { 3. } 0 \mathrm{ML}(\mathrm{PS})\end{array}$ & $\begin{array}{c}\text { IV } \\
\cdots \\
\text { FELT } \\
\cdots\end{array}$ & $\begin{array}{l}\text { FEB. } \\
\text { FEB. } \\
\text { FEB. } \\
\text { FEB. } \\
\text { FEB. }\end{array}$ & $\begin{array}{r}6 \\
9 \\
9 \\
11 \\
11\end{array}$ & $\begin{array}{l}19: 33 \\
22: 33 \\
23: 23 \\
06: 10 \\
06: 25\end{array}$ & $\begin{array}{l}\text { PST } \\
\text { PST } \\
\text { PST } \\
\text { PST } \\
\text { PST }\end{array}$ \\
\hline $\begin{array}{l}\text { FEB. } \\
\text { FEB. } \\
\text { FEB. } \\
\text { FEB. } \\
\text { FEB. }\end{array}$ & $\begin{array}{l}11 \\
11 \\
14 \\
14 \\
17\end{array}$ & $\begin{array}{l}19 \\
19 \\
03 \\
16 \\
01\end{array}$ & $\begin{array}{l}04 \\
30 \\
24 \\
18 \\
23\end{array}$ & $\begin{array}{l}30.3 \\
01.1 \\
00.1 \\
37.2 \\
02.6\end{array}$ & $\begin{array}{l}40.383 \mathrm{~N} . \\
35.932 \mathrm{~N} . \\
35.834 \mathrm{~N} . \\
35.941 \mathrm{~N} . \\
36.179 \mathrm{~N} .\end{array}$ & $\begin{array}{l}124.357 W . \\
115.813 W . \\
118.309 W . \\
118.322 W . \\
120.202 W .\end{array}$ & $\begin{array}{l}5 \\
6 \\
3 \\
0 \\
8\end{array}$ & $\begin{array}{l}\text { BK } \\
\text { PS } \\
\text { GM } \\
\text { PS } \\
\text { PS }\end{array}$ & $\begin{array}{l}\ldots \\
\ldots \\
\ldots \\
\ldots \\
\ldots\end{array}$ & $\begin{array}{l}\cdots \\
\cdots \\
\cdots \\
\cdots\end{array}$ & $\begin{array}{l}\text { 3. } 1 \mathrm{ML}(\mathrm{BK}) \\
\text { 3. } 2 \mathrm{ML}(\mathrm{PS}) \\
\text { 3. } 1 \mathrm{MD}(\mathrm{GM}) \\
\text { 3. } 1 \mathrm{ML}(\mathrm{PS}) \\
\text { 3.5ML(PS) }\end{array}$ & $\begin{array}{l}\ldots \\
\ldots \\
\ldots \\
\ldots\end{array}$ & $\begin{array}{l}\text { FEB. } \\
\text { FEB. } \\
\text { FEB. } \\
\text { FEB. } \\
\text { FEB. }\end{array}$ & $\begin{array}{l}11 \\
11 \\
13 \\
14 \\
16\end{array}$ & $\begin{array}{l}11: 04 \\
11: 30 \\
19: 24 \\
08: 18 \\
17: 23\end{array}$ & $\begin{array}{l}\text { PST } \\
\text { PST } \\
\text { PST } \\
\text { PST } \\
\text { PST }\end{array}$ \\
\hline $\begin{array}{l}\text { FEB. } \\
\text { FEB. } \\
\text { FEB. } \\
\text { FEB. } \\
\text { FEB. }\end{array}$ & $\begin{array}{l}19 \\
19 \\
19 \\
23 \\
27\end{array}$ & $\begin{array}{l}09 \\
09 \\
09 \\
17 \\
01\end{array}$ & $\begin{array}{l}37 \\
42 \\
43 \\
34 \\
36\end{array}$ & $\begin{array}{l}46.8 \\
53.1 \\
10.7 \\
23.3 \\
20.6\end{array}$ & $\begin{array}{l}36.289 \mathrm{~N} . \\
36.233 \mathrm{~N} . \\
36.277 \mathrm{~N} . \\
33.890 \mathrm{~N} . \\
37.375 \mathrm{~N}\end{array}$ & $\begin{array}{l}120.311 W . \\
120.372 W . \\
120.323 w . \\
116.153 w . \\
118.598 w .\end{array}$ & $\begin{array}{r}8 \\
51 \\
10 \\
5 \\
10\end{array}$ & $\begin{array}{l}\text { PS } \\
\text { GM } \\
\text { BK } \\
\text { PS } \\
\text { BK }\end{array}$ & $\begin{array}{l}\ldots \\
\ldots \\
4.2 \\
4.3\end{array}$ & $\begin{array}{l}\cdots \\
\cdots \\
\cdots \\
\cdots \\
\cdots\end{array}$ & $\begin{array}{l}\text { 3. } 2 M L(P S) \\
\text { 3. } 6 \mathrm{MD}(\mathrm{GM}) \\
\text { 4. } 1 \mathrm{ML}(\mathrm{BK}) \\
\text { 3. } 7 \mathrm{ML}(\mathrm{PS}) \\
\text { 4. } 5 \mathrm{ML}(\mathrm{BK})\end{array}$ & $\begin{array}{r}\cdots \\
\text { FELTT } \\
\text { FELT } \\
\text { I I I }\end{array}$ & $\begin{array}{l}\text { FEB. } \\
\text { FEB. } \\
\text { FEB. } \\
\text { FEB. } \\
\text { FEB. }\end{array}$ & $\begin{array}{l}19 \\
19 \\
19 \\
23 \\
26\end{array}$ & $\begin{array}{l}01: 37 \\
01: 42 \\
01: 43 \\
09: 34 \\
17: 36\end{array}$ & $\begin{array}{l}\text { PST } \\
\text { PST } \\
\text { PST } \\
\text { PST } \\
\text { PST }\end{array}$ \\
\hline $\begin{array}{l}\text { FEB. } \\
\text { FEB. } \\
\text { MAR. } \\
\text { MAR. } \\
\text { MAR. }\end{array}$ & $\begin{array}{r}28 \\
29 \\
1 \\
2 \\
4\end{array}$ & $\begin{array}{l}06 \\
02 \\
03 \\
11 \\
06\end{array}$ & $\begin{array}{l}08 \\
07 \\
19 \\
39 \\
12\end{array}$ & $\begin{array}{l}59.3 \\
31.7 \\
38.5 \\
09.6 \\
05.5\end{array}$ & $\begin{array}{l}34.398 \mathrm{~N} \\
33.136 \mathrm{~N} \\
35.869 \mathrm{~N} \\
38.805 \mathrm{~N} \\
37.475 \mathrm{~N}\end{array}$ & $\begin{array}{l}116.825 w . \\
116.071 w . \\
116.841 w . \\
122.795 w . \\
118.845 W .\end{array}$ & $\begin{array}{l}8 \\
7 \\
6 \\
5 \\
6\end{array}$ & $\begin{array}{l}\text { PS } \\
\text { PS } \\
\text { PS } \\
\text { BK } \\
\text { PS }\end{array}$ & $\begin{array}{l}\ldots \\
\ldots \\
\ldots \\
\ldots \\
\cdots\end{array}$ & $\begin{array}{l}\cdots \\
\cdots \\
\cdots \\
\cdots \\
\cdots\end{array}$ & $\begin{array}{l}\text { 3. } 4 M L(P S) \\
\text { 4. 3ML(PS) } \\
\text { 3. } 0 M L(P S) \\
\text { 3. } 0 M L(B K) \\
\text { 3. } 0 M L(P S)\end{array}$ & $\begin{array}{c}\text { FELT } \\
\text { III } \\
\text { FELT } \\
\ldots\end{array}$ & $\begin{array}{l}\text { FEB. } \\
\text { FEB. } \\
\text { FEB. } \\
\text { MAR. } \\
\text { MAR. }\end{array}$ & $\begin{array}{r}27 \\
28 \\
29 \\
2 \\
3\end{array}$ & $\begin{array}{l}22: 08 \\
18: 07 \\
19: 19 \\
03: 39 \\
22: 12\end{array}$ & $\begin{array}{l}\text { PST } \\
\text { PST } \\
\text { PST } \\
\text { PST } \\
\text { PST }\end{array}$ \\
\hline $\begin{array}{l}\text { MAR. } \\
\text { MAR. } \\
\text { MAR. } \\
\text { MAR. } \\
\text { MAR. }\end{array}$ & $\begin{array}{l}4 \\
5 \\
5 \\
6 \\
6\end{array}$ & $\begin{array}{l}11 \\
10 \\
11 \\
02 \\
10\end{array}$ & $\begin{array}{l}18 \\
50 \\
43 \\
41 \\
41\end{array}$ & $\begin{array}{l}29.7 \\
53.4 \\
19.8 \\
35.0 \\
43.5\end{array}$ & $\begin{array}{l}36.269 \mathrm{~N} \\
37.577 \mathrm{~N} \\
37.488 \mathrm{~N} \\
34.180 \mathrm{~N} \\
35.822 \mathrm{~N}\end{array}$ & $\begin{array}{l}120.414 W . \\
118.492 W . \\
118.807 W . \\
117.310 W . \\
118.302 W .\end{array}$ & $\begin{array}{r}12 \\
5 \\
8 \\
2 \\
14\end{array}$ & $\begin{array}{l}\text { PS } \\
\text { BK } \\
\text { BK } \\
\text { PS } \\
\text { GM }\end{array}$ & $\begin{array}{l}\ldots \\
\ldots \\
\ldots \\
\ldots \\
\cdots\end{array}$ & $\begin{array}{l}\cdots \\
\cdots \\
\cdots \\
\cdots \\
\cdots\end{array}$ & $\begin{array}{l}\text { 3. } 4 \mathrm{ML}(\mathrm{PS}) \\
\text { 3. } 1 \mathrm{ML}(\mathrm{BK}) \\
\text { 3. } 4 \mathrm{ML}(\mathrm{BK}) \\
\text { 2. } \mathrm{ML}(\mathrm{PS}) \\
\text { 3. } \mathrm{MD}(\mathrm{GM})\end{array}$ & $\begin{array}{c}\text { FE } \dot{E} \dot{T} \\
\text { I I I } \\
\ldots\end{array}$ & $\begin{array}{l}\text { MAR. } \\
\text { MAR. } \\
\text { MAR. } \\
\text { MAR. } \\
\text { MAR. }\end{array}$ & $\begin{array}{l}4 \\
5 \\
5 \\
5 \\
6\end{array}$ & $\begin{array}{l}03: 18 \\
02: 50 \\
03: 43 \\
18: 41 \\
02: 41\end{array}$ & $\begin{array}{l}\text { PST } \\
\text { PST } \\
\text { PST } \\
\text { PST } \\
\text { PST }\end{array}$ \\
\hline $\begin{array}{l}\text { IAR. } \\
\text { IAR. } \\
\text { IAR. } \\
\text { IAR. } \\
\text { IAR. }\end{array}$ & $\begin{array}{r}7 \\
7 \\
7 \\
9 \\
12\end{array}$ & $\begin{array}{l}03 \\
08 \\
20 \\
06 \\
04\end{array}$ & $\begin{array}{l}32 \\
32 \\
26 \\
35 \\
00\end{array}$ & $\begin{array}{l}36.7 \\
36.5 \\
35.0 \\
33.5 \\
32.7\end{array}$ & $\begin{array}{l}34.569 \mathrm{~N} . \\
37.498 \mathrm{~N} \\
33.590 \mathrm{~N} \\
40.347 \mathrm{~N} \\
36.182 \mathrm{~N}\end{array}$ & $\begin{array}{l}115.917 W . \\
118.818 W . \\
118.360 W . \\
124.337 W . \\
120.161 W .\end{array}$ & $\begin{array}{r}6 \\
4 \\
8 \\
5 \\
12\end{array}$ & $\begin{array}{l}\text { PS } \\
\text { BK } \\
\text { PS } \\
\text { BK } \\
\text { PS }\end{array}$ & $\begin{array}{l}\ldots \\
\ldots \\
\ldots \\
\ldots \\
\ldots\end{array}$ & $\begin{array}{l}\ldots \\
\cdots \\
\cdots \\
\cdots \\
\cdots\end{array}$ & $\begin{array}{l}\text { 3. } 8 M L(P S) \\
\text { 3. } 4 M L(B K) \\
2.1 M L(P S) \\
\text { 3. } 2 M L(B K) \\
\text { 3. } 2 M L(P S)\end{array}$ & $\begin{array}{c}\text { FELT } \\
\text { FELT } \\
\text { FELT } \\
\ldots\end{array}$ & $\begin{array}{l}\text { MAR. } \\
\text { MAR. } \\
\text { MAR. } \\
\text { MAR. } \\
\text { MAR. }\end{array}$ & $\begin{array}{r}6 \\
7 \\
7 \\
8 \\
11\end{array}$ & $\begin{array}{l}19: 32 \\
00: 32 \\
12: 26 \\
22: 35 \\
20: 00\end{array}$ & $\begin{array}{l}\text { PST } \\
\text { PST } \\
\text { PST } \\
\text { PST } \\
\text { PST }\end{array}$ \\
\hline $\begin{array}{l}\text { MAR. } \\
\text { MAR. } \\
\text { MAR. } \\
\text { MAR. } \\
\text { MAR. }\end{array}$ & $\begin{array}{l}12 \\
12 \\
13 \\
13 \\
13\end{array}$ & $\begin{array}{l}05 \\
10 \\
04 \\
04 \\
04\end{array}$ & $\begin{array}{l}15 \\
17 \\
49 \\
53 \\
55\end{array}$ & $\begin{array}{l}55.1 \\
51.0 \\
04.6 \\
22.5 \\
24.1\end{array}$ & $\begin{array}{l}36.950 \mathrm{~N} \\
34.910 \mathrm{~N} \\
37.496 \mathrm{~N} \\
37.497 \mathrm{~N} \\
37.489 \mathrm{~N}\end{array}$ & $\begin{array}{l}121.600 W \\
119.020 W \\
118.866 W \\
118.846 W \\
118.841 W\end{array}$ & $\begin{array}{r}5 \\
13 \\
6 \\
6 \\
6\end{array}$ & $\begin{array}{l}\text { BK } \\
\text { PS } \\
\text { PS } \\
\text { PS } \\
\text { PS }\end{array}$ & $\begin{array}{l}\ldots \\
\ldots \\
\ldots \\
\ldots \\
\ldots\end{array}$ & $\begin{array}{l}\cdots \\
\cdots \\
\cdots \\
\cdots\end{array}$ & $\begin{array}{l}\text { 2. } 9 M L(B K) \\
\text { 3. } 5 M L(P S) \\
\text { 3. } 3 M L(P S) \\
\text { 3. } 1 M L(P S) \\
\text { 3. } 2 M L(P S)\end{array}$ & $\begin{array}{c}\text { FELT } \\
\ldots \\
\ldots\end{array}$ & $\begin{array}{l}\text { MAR. } \\
\text { MAR. } \\
\text { MAR. } \\
\text { MAR. } \\
\text { MAR. }\end{array}$ & $\begin{array}{l}11 \\
12 \\
12 \\
12 \\
12\end{array}$ & $\begin{array}{l}21: 15 \\
02: 17 \\
20: 49 \\
20: 53 \\
20: 55\end{array}$ & $\begin{array}{l}\text { PST } \\
\text { PST } \\
\text { PST } \\
\text { PST } \\
\text { PST }\end{array}$ \\
\hline $\begin{array}{l}\text { MAR. } \\
\text { MAR. } \\
\text { MAR. } \\
\text { MAR. } \\
\text { MAR. }\end{array}$ & $\begin{array}{l}15 \\
15 \\
16 \\
18 \\
19\end{array}$ & $\begin{array}{l}12 \\
14 \\
19 \\
05 \\
21\end{array}$ & $\begin{array}{l}45 \\
09 \\
53 \\
27 \\
00\end{array}$ & $\begin{array}{l}52.6 \\
50.2 \\
21.3 \\
29.9 \\
28.5\end{array}$ & $\begin{array}{l}35.836 \mathrm{~N} \\
35.716 \mathrm{~N} \\
33.703 \mathrm{~N} \\
37.785 \mathrm{~N} \\
40.893 \mathrm{~N}\end{array}$ & $\begin{array}{l}119.531 W . \\
121.426 w . \\
116.737 w . \\
121.725 W . \\
123.785 W .\end{array}$ & $\begin{array}{r}6 \\
3 \\
19 \\
10 \\
6\end{array}$ & $\begin{array}{l}\text { PS } \\
\text { PS } \\
\text { PS } \\
\text { BK } \\
\text { BK }\end{array}$ & $\begin{array}{l}\ldots \\
\ldots \\
\ldots \\
\ldots\end{array}$ & $\begin{array}{l}\cdots \\
\cdots \\
\cdots \\
\cdots \\
\cdots\end{array}$ & $\begin{array}{l}\text { 3. } 2 \mathrm{ML}(\mathrm{PS}) \\
\text { 3. } \mathrm{ML}(\mathrm{PS}) \\
\text { 3. } 0 \mathrm{ML}(\mathrm{PS}) \\
\text { 4. } 1 \mathrm{ML}(\mathrm{BK}) \\
\text { 3. } 4 \mathrm{ML}(\mathrm{BK})\end{array}$ & $\begin{array}{l}\text { IV } \\
\text { IV }\end{array}$ & $\begin{array}{l}\text { MAR. } \\
\text { MAR. } \\
\text { MAR. } \\
\text { MAR. } \\
\text { MAR. }\end{array}$ & $\begin{array}{l}15 \\
15 \\
16 \\
17 \\
19\end{array}$ & $\begin{array}{l}04: 45 \\
06: 09 \\
11: 53 \\
21: 27 \\
13: 00\end{array}$ & $\begin{array}{l}\text { PST } \\
\text { PST } \\
\text { PST } \\
\text { PST } \\
\text { PST }\end{array}$ \\
\hline
\end{tabular}


Table 1. Summary of U.S. earthquakes for 1984-Continued

\begin{tabular}{|c|c|c|c|c|c|c|c|c|c|c|c|c|}
\hline \multirow{2}{*}{ Date } & $\begin{array}{c}\text { Origin time } \\
\text { (UTC) }\end{array}$ & \multirow{2}{*}{$\begin{array}{c}\text { Latitude } \\
\left(^{\circ}\right)\end{array}$} & \multirow{2}{*}{$\begin{array}{c}\text { Longitude } \\
\text { (०) }\end{array}$} & \multirow{2}{*}{$\begin{array}{l}\text { Depth } \\
(\mathbf{k m})\end{array}$} & \multirow{2}{*}{$\begin{array}{l}\text { Hypo- } \\
\text { center } \\
\text { source }\end{array}$} & \multicolumn{3}{|c|}{ Magnitude } & \multirow{2}{*}{$\begin{array}{l}\text { Maximum } \\
\text { intensity }\end{array}$} & \multicolumn{3}{|c|}{ Ioxal time } \\
\hline & hr $\min \mathrm{sec}$ & & & & & $\mathbf{m b}$ & MS & $\begin{array}{l}\text { ML. Mn } \\
\text { MD. Mw }\end{array}$ & & Date & Hour & $\begin{array}{l}\text { Time } \\
\text { zone }\end{array}$ \\
\hline
\end{tabular}

\section{CALIFORNIA-Continued}

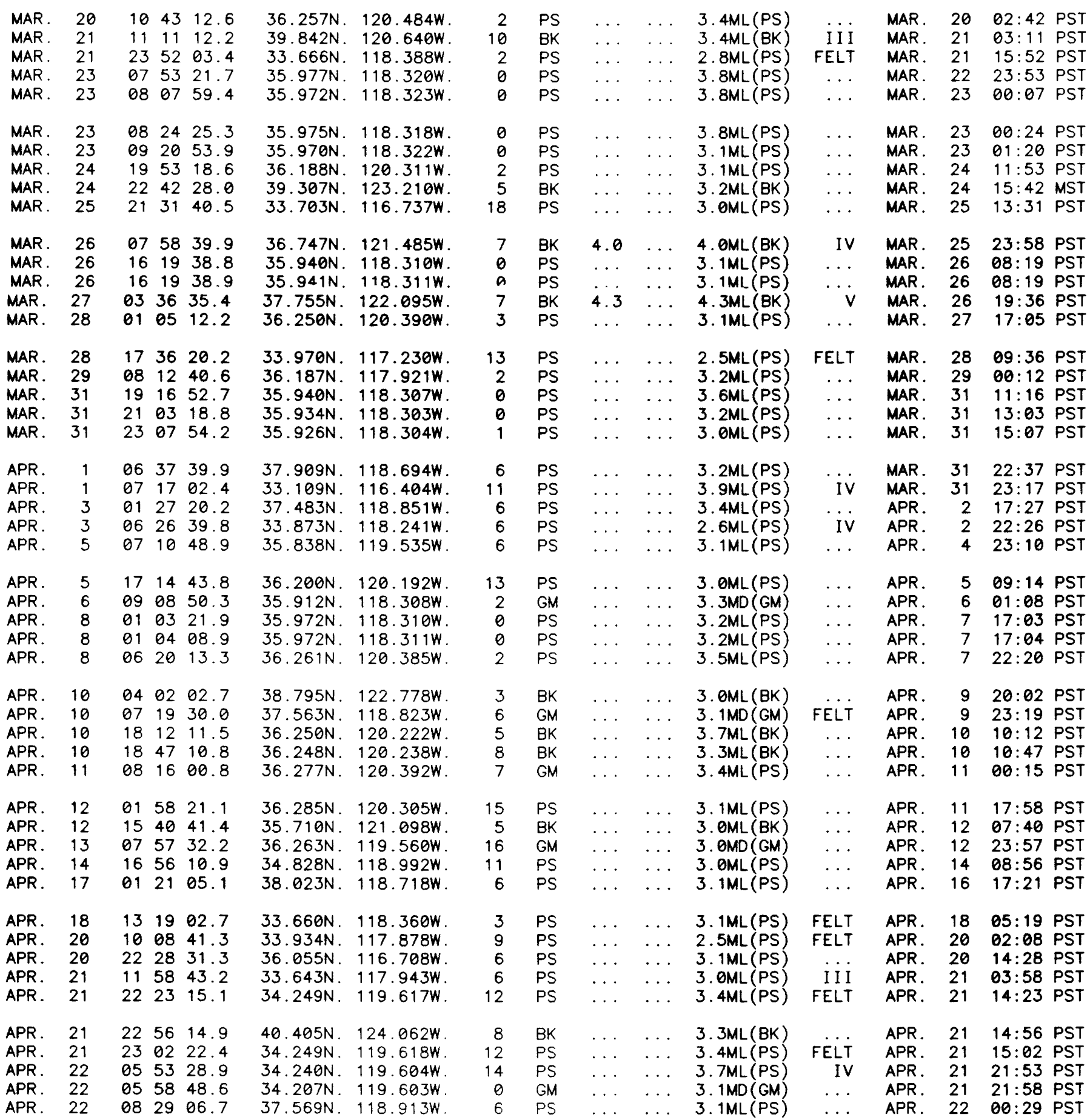


Table 1. Summary of U.S. earthquakes for 1984-Continued

\begin{tabular}{|c|c|c|c|c|c|c|c|c|c|c|c|c|c|}
\hline \multirow{2}{*}{ Date } & \multicolumn{2}{|c|}{$\begin{array}{l}\text { Origin time } \\
\text { (UTC) }\end{array}$} & \multirow{2}{*}{$\begin{array}{c}\text { Latitude } \\
\left.\text { ( }{ }^{\circ}\right)\end{array}$} & \multirow{2}{*}{$\begin{array}{c}\text { Lomgitude } \\
\left({ }^{\circ}\right)\end{array}$} & \multirow{2}{*}{$\begin{array}{c}\text { Depth } \\
\text { (km) }\end{array}$} & \multirow{2}{*}{$\begin{array}{l}\text { Iypo- } \\
\text { center } \\
\text { source }\end{array}$} & \multicolumn{3}{|c|}{ Magnitude } & \multirow{2}{*}{$\begin{array}{l}\text { Maximum } \\
\text { intensity }\end{array}$} & \multicolumn{3}{|c|}{ Local time } \\
\hline & hr $\min$ & $\sec$ & & & & & $\mathbf{m b}$ & MS & $\begin{array}{l}\text { ML, Mn } \\
M D, M w\end{array}$ & & Date & Hour & $\begin{array}{l}\text { Time } \\
\text { nome }\end{array}$ \\
\hline
\end{tabular}

CALIFORNIA-Continued

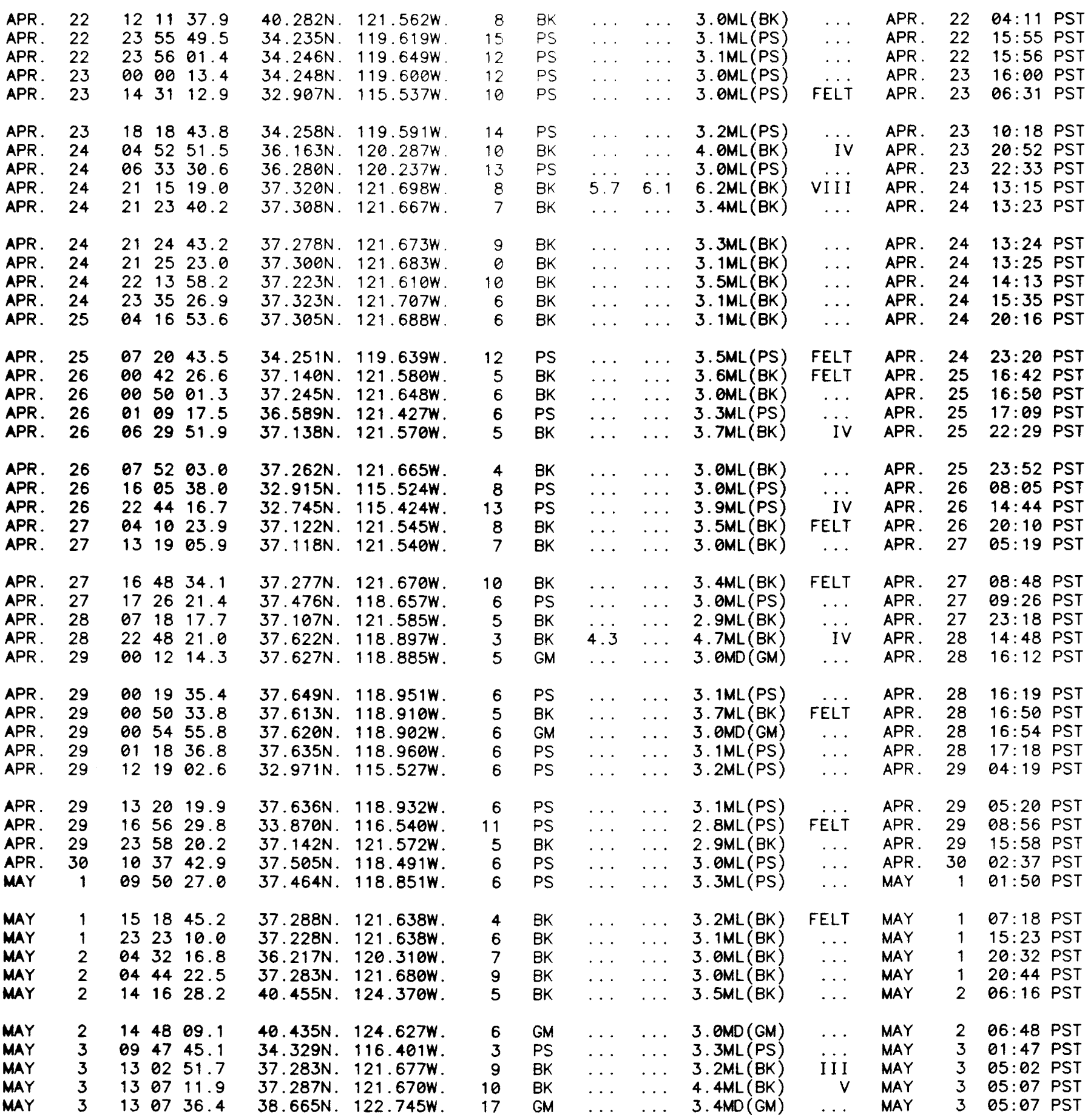


Table 1. Summary of U.S. earthquakes for 1984--Continued

\begin{tabular}{|c|c|c|c|c|c|c|c|c|c|c|c|c|}
\hline \multirow{2}{*}{ Dale } & $\begin{array}{c}\begin{array}{c}\text { Origin time } \\
\text { (UTC) }\end{array} \\
\end{array}$ & \multirow{2}{*}{$\begin{array}{c}\text { Latitude } \\
\left.\text { ( }{ }^{\circ}\right)\end{array}$} & \multirow{2}{*}{$\begin{array}{c}\text { Longitude } \\
\text { () }\end{array}$} & \multirow{2}{*}{$\begin{array}{c}\text { Depth } \\
(\mathbf{k m})\end{array}$} & \multirow{2}{*}{$\begin{array}{l}\text { Hypo- } \\
\text { center } \\
\text { source }\end{array}$} & \multicolumn{3}{|c|}{ Magnitude } & \multirow{2}{*}{$\begin{array}{l}\text { Maximum } \\
\text { intensity }\end{array}$} & \multicolumn{3}{|c|}{ Local time } \\
\hline & hr $\min \sec$ & & & & & $m b$ & MS & $\begin{array}{l}M L, M n \\
M I, M w\end{array}$ & & Date & Hour & $\begin{array}{l}\text { Time } \\
\text { Tone }\end{array}$ \\
\hline
\end{tabular}

CALIFORNIA-Continued

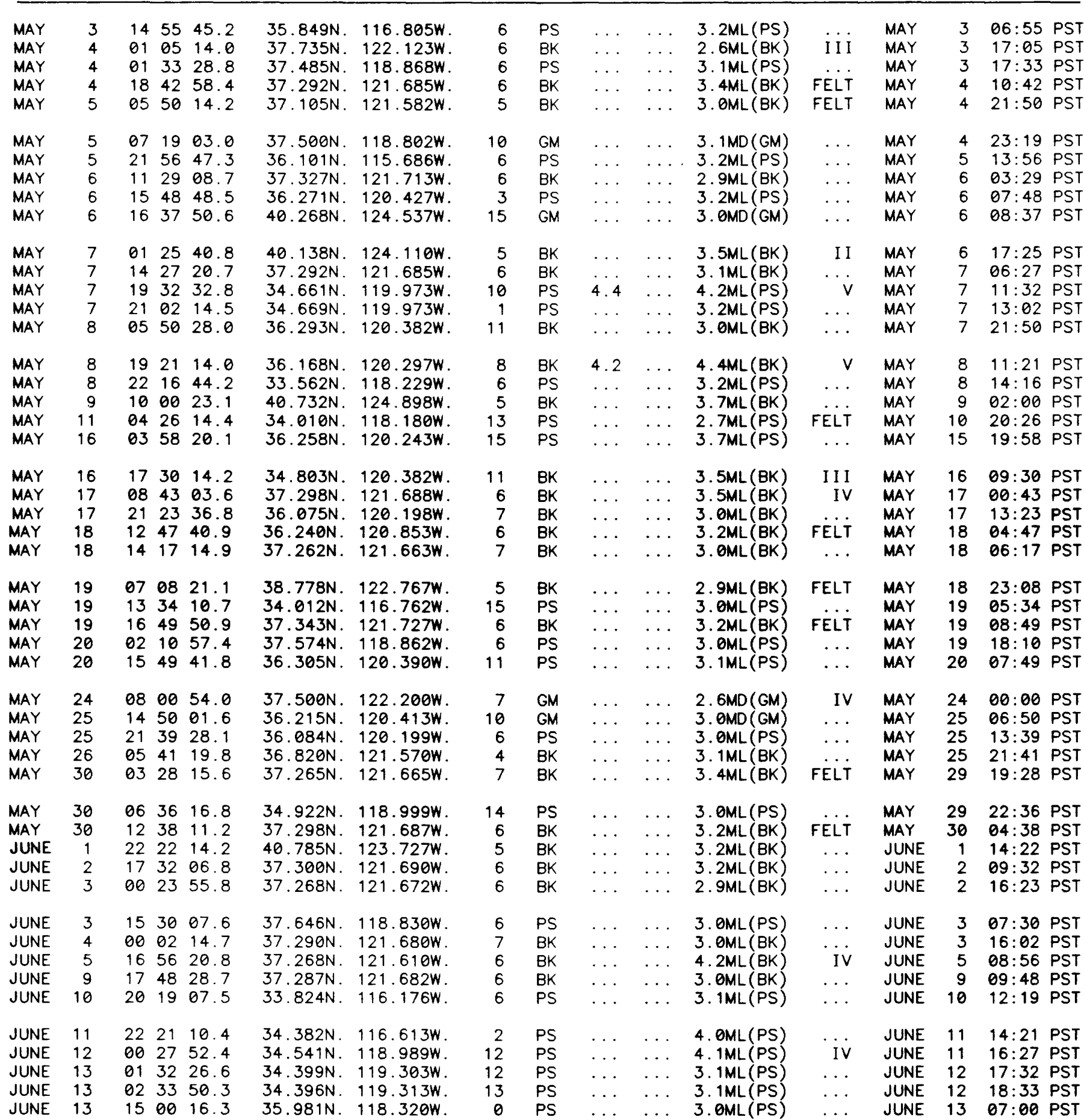


Table 1. Summary of U.S. earthquakes for 1984-Continued

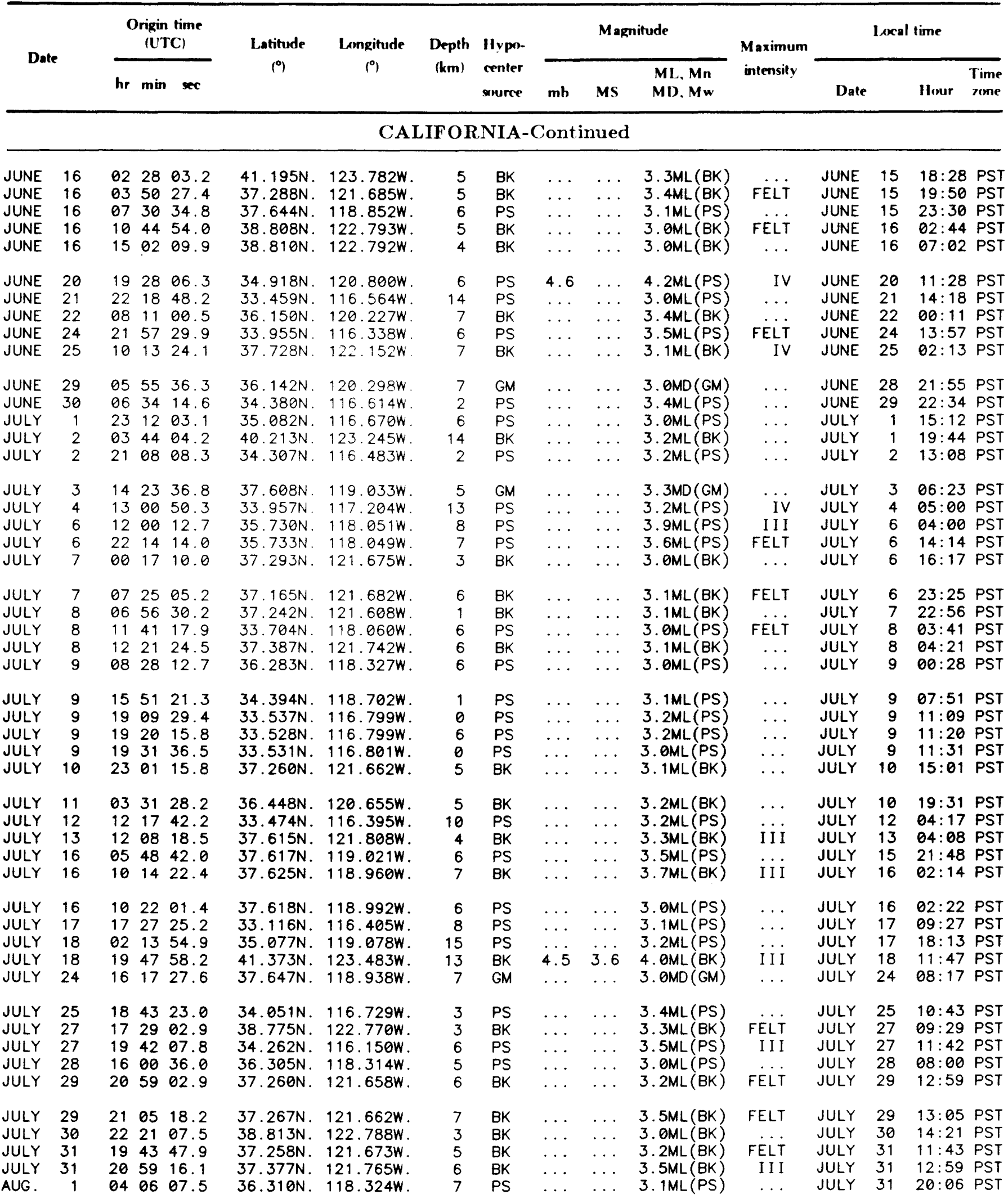


Table 1. Summary of U.S. earthquakes for 1984-Continued

\begin{tabular}{|c|c|c|c|c|c|c|c|c|c|c|c|c|c|}
\hline \multirow{2}{*}{ Date } & \multicolumn{2}{|c|}{$\begin{array}{l}\text { Origin time } \\
\text { (UTC) }\end{array}$} & \multirow{2}{*}{$\begin{array}{c}\text { Latitude } \\
\left({ }^{\circ}\right)\end{array}$} & \multirow{2}{*}{$\begin{array}{c}\text { Longitude } \\
\text { (०) }\end{array}$} & \multirow{2}{*}{$\begin{array}{c}\text { Depth } \\
(\mathbf{k m})\end{array}$} & \multirow{2}{*}{$\begin{array}{l}\text { Hypo- } \\
\text { center } \\
\text { source }\end{array}$} & \multicolumn{3}{|c|}{ Magnitude } & \multirow{2}{*}{$\begin{array}{l}\text { Maximum } \\
\text { intensity }\end{array}$} & \multicolumn{3}{|c|}{ Iocal time } \\
\hline & hr $\min$ & $\sec$ & & & & & $\mathbf{m b}$ & MS & $\begin{array}{l}\text { ML, Mn } \\
\text { MD, Mw }\end{array}$ & & Date & Hour & $\begin{array}{l}\text { Time } \\
\text { zone }\end{array}$ \\
\hline
\end{tabular}

CALIFORNIA-Continued

\begin{tabular}{|c|c|c|c|c|c|c|c|c|c|c|c|c|c|c|c|c|}
\hline $\begin{array}{l}\text { AUG. } \\
\text { AUG. } \\
\text { AUG. } \\
\text { AUG. } \\
\text { AUG. }\end{array}$ & $\begin{array}{l}2 \\
3 \\
5 \\
5 \\
5\end{array}$ & $\begin{array}{l}03 \\
17 \\
09 \\
15 \\
15\end{array}$ & $\begin{array}{l}55 \\
42 \\
38 \\
28 \\
38\end{array}$ & $\begin{array}{l}38.6 \\
52.6 \\
51.3 \\
55.0 \\
18.6\end{array}$ & $\begin{array}{l}36.463 \mathrm{~N} . \\
40.368 \mathrm{~N} . \\
34.406 \mathrm{~N} . \\
34.410 \mathrm{~N} . \\
34.405 \mathrm{~N} .\end{array}$ & $\begin{array}{l}120.300 W . \\
124.048 W . \\
118.445 W . \\
118.440 W . \\
118.437 W .\end{array}$ & $\begin{array}{l}6 \\
5 \\
6 \\
6 \\
6\end{array}$ & $\begin{array}{l}\text { PS } \\
\text { BK } \\
\text { PS } \\
\text { PS } \\
\text { PS }\end{array}$ & $\begin{array}{l}\cdots \\
\cdots \\
\cdots \\
\cdots\end{array}$ & $\begin{array}{l}\cdots \\
\cdots \\
\cdots \\
\cdots \\
\ldots\end{array}$ & $\begin{array}{l}3.5 M L(P S) \\
3.1 M L(B K) \\
3.2 M L(P S) \\
3.0 M L(P S) \\
2.1 M L(P S)\end{array}$ & $\begin{array}{l}\text { FELT } \\
\text { FELT }\end{array}$ & $\begin{array}{l}\text { AUG. } \\
A \cup G . \\
A \cup G . \\
A \cup G . \\
A \cup G .\end{array}$ & $\begin{array}{l}1 \\
3 \\
5 \\
5 \\
5\end{array}$ & $\begin{array}{l}19: 55 \\
09: 42 \\
81: 38 \\
07: 28 \\
07: 38\end{array}$ & $\begin{array}{l}\text { PST } \\
\text { PST } \\
\text { PST } \\
\text { PST } \\
\text { PST }\end{array}$ \\
\hline $\begin{array}{l}\text { AUG. } \\
\text { AUG. } \\
\text { AUG. } \\
\text { AUG. } \\
\text { AUG. }\end{array}$ & $\begin{array}{r}6 \\
6 \\
9 \\
9 \\
13\end{array}$ & $\begin{array}{l}00 \\
08 \\
00 \\
13 \\
09\end{array}$ & $\begin{array}{l}06 \\
14 \\
19 \\
18 \\
51\end{array}$ & $\begin{array}{l}39.8 \\
36.6 \\
42.7 \\
49.3 \\
36.1\end{array}$ & $\begin{array}{l}36.997 \mathrm{~N} . \\
33.976 \mathrm{~N} . \\
37.432 \mathrm{~N} \\
37.302 \mathrm{~N} . \\
37.285 \mathrm{~N} .\end{array}$ & $\begin{array}{l}121.713 W . \\
116.713 W . \\
118.677 W . \\
121.662 W . \\
121.673 W .\end{array}$ & $\begin{array}{r}8 \\
14 \\
6 \\
9 \\
9\end{array}$ & $\begin{array}{l}\text { BK } \\
\text { PS } \\
\text { PS } \\
\text { BK } \\
\text { BK }\end{array}$ & $\begin{array}{l}\cdots \\
4.0 \\
\ldots \\
\ldots\end{array}$ & $\begin{array}{l}\cdots \\
\cdots \\
\cdots \\
\cdots \\
\cdots\end{array}$ & $\begin{array}{l}\text { 3. } 8 M L(B K) \\
\text { 4. 3ML (PS) } \\
\text { 3. } 0 \mathrm{ML}(\mathrm{PS}) \\
\text { 3. } 0 \mathrm{ML}(\mathrm{BK}) \\
\text { 3. } 7 \mathrm{ML}(\mathrm{BK})\end{array}$ & $\begin{array}{l}\text { IV } \\
\text { V } \\
\ldots \\
\cdots \\
\text { IV }\end{array}$ & $\begin{array}{l}A \cup G . \\
A \cup G . \\
A \cup G . \\
A \cup G . \\
A \cup G .\end{array}$ & $\begin{array}{r}5 \\
6 \\
8 \\
9 \\
13\end{array}$ & $\begin{array}{l}16: 06 \\
00: 14 \\
16: 19 \\
05: 18 \\
01: 51\end{array}$ & $\begin{array}{l}\text { PST } \\
\text { PST } \\
\text { PST } \\
\text { PST } \\
\text { PST }\end{array}$ \\
\hline $\begin{array}{l}A \cup G . \\
A \cup G . \\
A \cup G . \\
A \cup G . \\
A \cup G .\end{array}$ & $\begin{array}{l}14 \\
14 \\
15 \\
16 \\
16\end{array}$ & $\begin{array}{l}05 \\
21 \\
00 \\
01 \\
11\end{array}$ & $\begin{array}{l}55 \\
05 \\
26 \\
30 \\
24\end{array}$ & $\begin{array}{l}33.5 \\
41.9 \\
40.5 \\
54.6 \\
44.6\end{array}$ & $\begin{array}{l}37.499 \mathrm{~N} . \\
40.948 \mathrm{~N} . \\
37.333 \mathrm{~N} . \\
36.190 \mathrm{~N} . \\
36.112 \mathrm{~N} .\end{array}$ & $\begin{array}{l}121.537 W . \\
123.332 W . \\
121.692 W . \\
120.192 W . \\
120.180 W .\end{array}$ & $\begin{array}{l}5 \\
5 \\
6 \\
7 \\
7\end{array}$ & $\begin{array}{l}\text { GS } \\
\text { BK } \\
\text { BK } \\
\text { BK } \\
\text { BK }\end{array}$ & $\begin{array}{l}\cdots \\
\cdots \\
\cdots \\
\cdots\end{array}$ & $\begin{array}{l}\ldots \\
\cdots \\
\cdots \\
\cdots \\
\cdots\end{array}$ & $\begin{array}{l}\text { 2. } 9 M L(B K) \\
\text { 3. } 2 M L(B K) \\
\text { 3. } 9 M L(B K) \\
\text { 3. } 2 M L(B K) \\
\text { 3. } 1 M L(B K)\end{array}$ & $\begin{array}{l}\ldots \\
\text { FELT } \\
\text { III } \\
\ldots\end{array}$ & $\begin{array}{l}A \cup G . \\
A \cup G . \\
A \cup G . \\
A \cup G . \\
A \cup G .\end{array}$ & $\begin{array}{l}13 \\
14 \\
14 \\
15 \\
16\end{array}$ & $\begin{array}{l}21: 55 \\
13: 05 \\
16: 26 \\
17: 30 \\
03: 24\end{array}$ & $\begin{array}{l}\text { ST } \\
\text { ST } \\
\text { ST } \\
\text { ST } \\
\text { ST }\end{array}$ \\
\hline $\begin{array}{l}A \cup G . \\
A \cup G . \\
A \cup G . \\
A \cup G . \\
A \cup G .\end{array}$ & $\begin{array}{l}16 \\
16 \\
18 \\
20 \\
21\end{array}$ & $\begin{array}{l}12 \\
20 \\
12 \\
16 \\
20\end{array}$ & $\begin{array}{l}55 \\
45 \\
17 \\
26 \\
01\end{array}$ & $\begin{array}{l}37.2 \\
34.6 \\
33.3 \\
25.5 \\
34.7\end{array}$ & $\begin{array}{l}40.962 \mathrm{~N} . \\
37.263 \mathrm{~N} . \\
37.247 \mathrm{~N} . \\
37.478 \mathrm{~N} . \\
37.230 \mathrm{~N} .\end{array}$ & $\begin{array}{l}123.448 W . \\
121.663 W . \\
121.647 W . \\
118.575 W . \\
121.587 w .\end{array}$ & $\begin{array}{r}10 \\
6 \\
6 \\
11 \\
5\end{array}$ & $\begin{array}{l}\text { BK } \\
\text { BK } \\
\text { BK } \\
\text { GM } \\
\text { GS }\end{array}$ & $\begin{array}{l}\cdots \\
\cdots \\
\cdots \\
\cdots\end{array}$ & $\begin{array}{l}\cdots \\
\cdots \\
\cdots \\
\cdots \\
\cdots\end{array}$ & $\begin{array}{l}\text { 3. } 1 \mathrm{ML}(\mathrm{BK}) \\
\text { 3. } 6 \mathrm{ML}(\mathrm{BK}) \\
2.9 \mathrm{ML}(\mathrm{BK}) \\
\text { 3. } \mathrm{ML}(\mathrm{BK}) \\
2.9 \mathrm{ML}(\mathrm{BK})\end{array}$ & $\begin{array}{c}\text { FELT } \\
\ldots \\
\ldots \\
\ldots\end{array}$ & $\begin{array}{l}A \cup G . \\
A \cup G . \\
A \cup G . \\
A \cup G . \\
A \cup G .\end{array}$ & $\begin{array}{l}16 \\
16 \\
18 \\
20 \\
21\end{array}$ & $\begin{array}{l}04: 55 \\
12: 45 \\
04: 17 \\
08: 26 \\
12: 01\end{array}$ & $\begin{array}{l}\text { PST } \\
\text { PST } \\
\text { PST } \\
\text { PST } \\
\text { PST }\end{array}$ \\
\hline $\begin{array}{l}\text { AUG. } \\
\text { AUG. } \\
\text { AUG. } \\
\text { AUG. } \\
\text { AUG. }\end{array}$ & $\begin{array}{l}23 \\
24 \\
24 \\
25 \\
30\end{array}$ & $\begin{array}{l}09 \\
01 \\
01 \\
13 \\
20\end{array}$ & $\begin{array}{l}35 \\
05 \\
06 \\
00 \\
20\end{array}$ & $\begin{array}{l}09.4 \\
54.3 \\
22.4 \\
48.8 \\
37.8\end{array}$ & $\begin{array}{l}36.141 \mathrm{~N} . \\
37.260 \mathrm{~N} . \\
38.772 \mathrm{~N} . \\
41.123 \mathrm{~N} . \\
36.277 \mathrm{~N} .\end{array}$ & $\begin{array}{l}120.118 w . \\
121.655 W . \\
122.783 w . \\
123.275 W . \\
120.273 w .\end{array}$ & $\begin{array}{r}6 \\
6 \\
6 \\
21 \\
7\end{array}$ & $\begin{array}{l}\text { PS } \\
\text { BK } \\
\text { GM } \\
\text { BK } \\
\text { PS }\end{array}$ & $\begin{array}{l}\cdots \\
\cdots \\
\cdots \\
\cdots \\
\cdots\end{array}$ & $\begin{array}{l}\cdots \\
\cdots \\
\cdots \\
\cdots \\
\cdots\end{array}$ & $\begin{array}{l}\text { 3. } 3 M L(P S) \\
\text { 3. } 6 M L(B K) \\
3.1 M D(G M) \\
\text { 3. } 8 M L(B K) \\
\text { 3. } 2 M L(P S)\end{array}$ & $\begin{array}{l}\text { I I I } \\
\ldots \\
\ldots \\
\ldots\end{array}$ & $\begin{array}{l}A \cup G . \\
A \cup G . \\
A \cup G . \\
A \cup G . \\
A \cup G .\end{array}$ & $\begin{array}{l}23 \\
23 \\
23 \\
25 \\
30\end{array}$ & $\begin{array}{l}01: 35 \\
17: 05 \\
17: 06 \\
05: 00 \\
12: 20\end{array}$ & $\begin{array}{l}\text { PST } \\
\text { PST } \\
\text { PST } \\
\text { PST } \\
\text { PST }\end{array}$ \\
\hline $\begin{array}{l}\text { AUG. } \\
\text { SEPT. } \\
\text { SEPT. } \\
\text { SEPT. } \\
\text { SEPT. }\end{array}$ & $\begin{array}{r}30 \\
4 \\
5 \\
6 \\
7\end{array}$ & $\begin{array}{l}20 \\
17 \\
05 \\
10 \\
02\end{array}$ & $\begin{array}{l}41 \\
02 \\
56 \\
04 \\
52\end{array}$ & $\begin{array}{l}22.7 \\
02.8 \\
05.4 \\
33.5 \\
19.5\end{array}$ & $\begin{array}{l}36.105 \mathrm{~N} . \\
37.260 \mathrm{~N} . \\
36.293 \mathrm{~N} . \\
33.997 \mathrm{~N} . \\
36.093 \mathrm{~N} .\end{array}$ & $\begin{array}{l}120.288 W . \\
121.658 W . \\
120.352 W . \\
117.033 w . \\
120.013 W .\end{array}$ & $\begin{array}{r}12 \\
7 \\
2 \\
6 \\
5\end{array}$ & $\begin{array}{l}\text { BK } \\
\text { BK } \\
\text { GM } \\
\text { PS } \\
\text { BK }\end{array}$ & $\begin{array}{l}\cdots \\
\cdots \\
\cdots \\
\cdots\end{array}$ & $\begin{array}{l}\cdots \\
\cdots \\
\cdots \\
\cdots\end{array}$ & $\begin{array}{l}\text { 3. } 0 M L(B K) \\
\text { 3. } 4 M L(B K) \\
\text { 3. } 0 M D(G M) \\
\text { 3. } 0 M L(P S) \\
\text { 3. } 5 M L(B K)\end{array}$ & $\begin{array}{l}\ldots \\
\cdots \\
\cdots \\
\text { IV }\end{array}$ & $\begin{array}{l}\text { AUG. } \\
\text { SEPT. } \\
\text { SEPT. } \\
\text { SEPT. } \\
\text { SEPT. }\end{array}$ & $\begin{array}{r}30 \\
4 \\
4 \\
6 \\
6\end{array}$ & $\begin{array}{l}12: 41 \\
09: 02 \\
21: 56 \\
02: 04 \\
18: 52\end{array}$ & $\begin{array}{l}\text { PST } \\
\text { PST } \\
\text { PST } \\
\text { PST } \\
\text { PST }\end{array}$ \\
\hline $\begin{array}{l}\text { SEPT. } \\
\text { SEPT. } \\
\text { SEPT. } \\
\text { SEPT. } \\
\text { SEPT. }\end{array}$ & $\begin{array}{r}7 \\
7 \\
7 \\
11 \\
13\end{array}$ & $\begin{array}{l}05 \\
14 \\
17 \\
12 \\
18\end{array}$ & $\begin{array}{l}44 \\
51 \\
57 \\
48 \\
36\end{array}$ & $\begin{array}{l}56.0 \\
51.2 \\
30.3 \\
35.5 \\
43.4\end{array}$ & $\begin{array}{l}37.413 \mathrm{~N} . \\
35.980 \mathrm{~N} . \\
33.460 \mathrm{~N} . \\
33.485 \mathrm{~N} . \\
35.735 \mathrm{~N} .\end{array}$ & $\begin{array}{l}118.376 w . \\
117.333 w . \\
116.370 w . \\
116.421 W . \\
116.634 W .\end{array}$ & $\begin{array}{r}6 \\
0 \\
15 \\
10 \\
6\end{array}$ & $\begin{array}{l}\text { PS } \\
\text { PS } \\
\text { PS } \\
\text { PS } \\
\text { PS }\end{array}$ & $\begin{array}{l}\cdots \\
\cdots \\
\cdots \\
\cdots\end{array}$ & $\begin{array}{l}\cdots \\
\cdots \\
\cdots \\
\cdots \\
\cdots\end{array}$ & $\begin{array}{l}\text { 3. } 0 M L(P S) \\
\text { 3. } 7 M L(P S) \\
\text { 4.1ML(PS) } \\
\text { 3.2ML(PS) } \\
\text { 3.2ML(PS) }\end{array}$ & $\begin{array}{l}\cdots \\
\text { IV } \\
\cdots \\
\cdots\end{array}$ & $\begin{array}{l}\text { SEPT. } \\
\text { SEPT. } \\
\text { SEPT. } \\
\text { SEPT. } \\
\text { SEPT. }\end{array}$ & $\begin{array}{r}6 \\
7 \\
7 \\
11 \\
13\end{array}$ & $\begin{array}{l}21: 44 \\
06: 51 \\
09: 57 \\
04: 48 \\
10: 36\end{array}$ & $\begin{array}{l}\text { PST } \\
\text { PST } \\
\text { PST } \\
\text { PST } \\
\text { PST }\end{array}$ \\
\hline $\begin{array}{l}\text { SEPT. } \\
\text { SEPT. } \\
\text { SEPT. } \\
\text { SEPT. } \\
\text { SEPT. }\end{array}$ & $\begin{array}{l}13 \\
19 \\
21 \\
21 \\
21\end{array}$ & $\begin{array}{l}22 \\
21 \\
03 \\
13 \\
13\end{array}$ & $\begin{array}{l}29 \\
11 \\
07 \\
50 \\
51\end{array}$ & $\begin{array}{l}01.6 \\
18.1 \\
21.1 \\
50.1 \\
10.6\end{array}$ & $\begin{array}{l}32.962 \mathrm{~N} . \\
34.661 \mathrm{~N} . \\
40.737 \mathrm{~N} \\
37.552 \mathrm{~N} . \\
37.592 \mathrm{~N} .\end{array}$ & $\begin{array}{l}117.803 w . \\
119.968 W . \\
124.207 W . \\
118.837 w . \\
118.813 W .\end{array}$ & $\begin{array}{r}6 \\
1 \\
11 \\
12 \\
15\end{array}$ & $\begin{array}{l}\text { PS } \\
\text { PS } \\
\text { BK } \\
\text { GM } \\
\text { GM }\end{array}$ & $\begin{array}{l}\ldots \\
\cdots \\
\cdots \\
\cdots \\
\cdots\end{array}$ & $\begin{array}{l}\cdots \\
\cdots \\
\cdots \\
\cdots \\
\cdots\end{array}$ & $\begin{array}{l}\text { 3. } 3 M L(P S) \\
3.4 M L(P S) \\
3.2 M L(B K) \\
3.2 M D(G M) \\
3.2 M L(P S)\end{array}$ & $\begin{array}{l}\ddot{I I} \\
V \\
\cdots \\
\cdots\end{array}$ & $\begin{array}{l}\text { SEPT. } \\
\text { SEPT. } \\
\text { SEPT. } \\
\text { SEPT. } \\
\text { SEPT. }\end{array}$ & $\begin{array}{l}13 \\
19 \\
20 \\
21 \\
21\end{array}$ & $\begin{array}{l}14: 29 \\
13: 11 \\
19: 07 \\
05: 50 \\
05: 51\end{array}$ & $\begin{array}{l}\text { PST } \\
\text { PST } \\
\text { PST } \\
\text { PST } \\
\text { PST }\end{array}$ \\
\hline $\begin{array}{l}\text { SEPT. } \\
\text { SEPT. } \\
\text { SEPT. } \\
\text { SEPT. } \\
\text { SEPT. }\end{array}$ & $\begin{array}{l}22 \\
22 \\
22 \\
25 \\
26\end{array}$ & $\begin{array}{l}18 \\
18 \\
19 \\
02 \\
20\end{array}$ & $\begin{array}{l}50 \\
52 \\
50 \\
29 \\
46\end{array}$ & $\begin{array}{l}42.3 \\
03.0 \\
56.0 \\
57.4 \\
06.3\end{array}$ & $\begin{array}{l}36.200 \mathrm{~N} . \\
38.802 \mathrm{~N} . \\
36.215 \mathrm{~N} . \\
36.602 \mathrm{~N} . \\
37.340 \mathrm{~N} .\end{array}$ & $\begin{array}{l}120.347 W . \\
122.807 W . \\
118.320 W . \\
121.885 W . \\
121.733 W .\end{array}$ & $\begin{array}{l}8 \\
1 \\
6 \\
5 \\
6\end{array}$ & $\begin{array}{l}\text { GM } \\
\text { BK } \\
\text { GM } \\
\text { BK } \\
\text { BK }\end{array}$ & $\begin{array}{l}\ddot{4.2} \\
\ldots \\
\ddot{4} .3\end{array}$ & $\begin{array}{l}\ldots \\
\cdots \\
\cdots \\
\cdots \\
\cdots\end{array}$ & $\begin{array}{l}\text { 3. } 0 M D(G M) \\
\text { 4. } 0 M L(B K) \\
\text { 3. } 0 M D(G M) \\
\text { 2. } 5 M L(B K) \\
\text { 4. } 4 M L(B K)\end{array}$ & $\begin{array}{r}\ddot{V} \\
\text { FEL } \\
v\end{array}$ & $\begin{array}{l}\text { SEPT. } \\
\text { SEPT. } \\
\text { SEPT. } \\
\text { SEPT. } \\
\text { SEPT. }\end{array}$ & $\begin{array}{l}22 \\
22 \\
22 \\
24 \\
26\end{array}$ & $\begin{array}{l}10: 50 \\
10: 52 \\
11: 50 \\
18: 29 \\
12: 46\end{array}$ & $\begin{array}{l}\text { ST } \\
\text { ST } \\
\text { ST } \\
\text { ST } \\
\text { ST }\end{array}$ \\
\hline $\begin{array}{l}\text { SEPT. } \\
\text { SEPT. } \\
\text { SEPT. } \\
\text { SEPT. } \\
\text { SEPT. }\end{array}$ & $\begin{array}{l}26 \\
26 \\
28 \\
29 \\
30\end{array}$ & $\begin{array}{l}20 \\
22 \\
15 \\
17 \\
09\end{array}$ & $\begin{array}{l}46 \\
06 \\
11 \\
14 \\
23\end{array}$ & $\begin{array}{l}30.4 \\
10.9 \\
05.9 \\
49.9 \\
16.1\end{array}$ & $\begin{array}{l}38.713 \mathrm{~N} . \\
38.118 \mathrm{~N} . \\
35.983 \mathrm{~N} . \\
37.753 \mathrm{~N} . \\
33.468 \mathrm{~N} .\end{array}$ & $\begin{array}{l}122.757 W . \\
118.720 W . \\
120.180 W . \\
118.272 W . \\
116.588 W .\end{array}$ & $\begin{array}{r}5 \\
6 \\
14 \\
12 \\
12\end{array}$ & $\begin{array}{l}\text { GM } \\
\text { PS } \\
\text { GM } \\
\text { GM } \\
\text { PS }\end{array}$ & $\begin{array}{l}\cdots \\
\cdots \\
\cdots \\
\cdots\end{array}$ & $\begin{array}{l}\cdots \\
\cdots \\
\cdots \\
\cdots\end{array}$ & $\begin{array}{l}\text { 3. 3MD (GM) } \\
\text { 3. } 0 \mathrm{ML}(\mathrm{PS}) \\
3.2 \mathrm{MD}(\mathrm{GM}) \\
\text { 3.2MD (GM) } \\
\text { 3.1ML(PS) }\end{array}$ & $\begin{array}{l}\ldots \\
\cdots \\
\cdots \\
\ldots\end{array}$ & $\begin{array}{l}\text { SEPT. } \\
\text { SEPT. } \\
\text { SEPT. } \\
\text { SEPT. } \\
\text { SEPT. }\end{array}$ & $\begin{array}{l}26 \\
26 \\
28 \\
29 \\
30\end{array}$ & $\begin{array}{l}12: 46 \\
14: 06 \\
07: 11 \\
09: 14 \\
01: 23\end{array}$ & $\begin{array}{l}T \\
T \\
T \\
T\end{array}$ \\
\hline
\end{tabular}


Table 1. Summary of U.S. earthquakes for 1984-Continued

\begin{tabular}{|c|c|c|c|c|c|c|c|c|c|c|c|c|c|}
\hline \multirow{2}{*}{ Date } & \multicolumn{2}{|c|}{$\begin{array}{l}\text { Origin time } \\
\text { (UTC) }\end{array}$} & \multirow{2}{*}{$\begin{array}{c}\text { Latitude } \\
\left({ }^{\circ}\right)\end{array}$} & \multirow{2}{*}{$\begin{array}{c}\text { Longitude } \\
\left({ }^{\circ}\right)\end{array}$} & \multirow{2}{*}{$\begin{array}{l}\text { Depth } \\
(\mathbf{k m})\end{array}$} & \multirow{2}{*}{$\begin{array}{l}\text { Hypo- } \\
\text { center } \\
\text { source }\end{array}$} & \multicolumn{3}{|c|}{ Magnitude } & \multirow{2}{*}{$\begin{array}{l}\text { Maximum } \\
\text { intensity }\end{array}$} & \multicolumn{3}{|c|}{ Local time } \\
\hline & hr $\min$ & ex & & & & & $\mathbf{m b}$ & MS & $\begin{array}{l}\text { ML, Mn } \\
M D, M w\end{array}$ & & Date & Hour & $\begin{array}{l}\text { Time } \\
\text { Tone }\end{array}$ \\
\hline
\end{tabular}

\section{CALIFORNIA-Continued}

OCT. $1114 \quad 3757.4 \quad 35.038 \mathrm{~N} .119 .073 \mathrm{~W}$.

OCT. $\quad 2 \quad 0259 \quad 16.7$

OCT. $\quad 2 \quad 09 \quad 38 \quad 14.5$

$\begin{array}{lllll}\text { OCT. } & 2 & 13 & 24 & 15.2\end{array}$

$\begin{array}{lllll}\text { OCT. } & 3 & 12 & 49 & 49.5\end{array}$

OCT. $\quad 4 \quad 07 \quad 53 \quad 12.5$

$\begin{array}{lllll}\text { OCT. } & 5 & 07 & 37 & 14 .\end{array}$

OCT. $5 \quad 5 \quad 16 \quad 16 \quad 30.0$

OCT. $\quad \begin{array}{llll}6 & 00 & 38 & 30.9\end{array}$

OCT. $\quad \begin{array}{llll}6 & 08 & 2105.9\end{array}$

OCT. $\quad \begin{array}{lllll}7 & 04 & 21 & 17.4\end{array}$

$\begin{array}{lllll}\text { OCT. } & 7 & 15 & 44 & 17.9\end{array}$

OCT. $\quad 8 \quad 86 \quad 3920.4$

OCT. $\quad 8 \quad 20 \quad 30 \quad 54.5$

$\begin{array}{lllll}\text { OCT. } & 9 & 06 & 01 & 05.3\end{array}$

OCT. $\quad 10 \quad 21 \quad 22 \quad 58.9$

OCT. $\quad \begin{array}{lllll}14 & 09 & 44 & 14.4\end{array}$

OCT. $15 \quad 08 \quad 12 \quad 36.0$

OCT. $\quad \begin{array}{lllll}15 & 17 & 44 & 04.4\end{array}$

OCT. $16 \quad 17 \quad \begin{array}{llll}17 & 49 & 34.1\end{array}$

OCT. $16 \quad 22 \quad 18 \quad 50.0$

OCT. $\begin{array}{lllll}18 & 00 & 57 & 14.2\end{array}$

OCT. $\begin{array}{lllll}20 & 15 & 05 & 07.0\end{array}$

$\begin{array}{llllll}\text { OCT. } & 22 & 04 & 06 & 31.4\end{array}$

$\begin{array}{lllll}\text { OCT. } & 22 & 05 & 44 & 34.8\end{array}$

OCT. $22 \quad 20 \quad 28 \quad 53.8$

OCT. $23 \quad 1008 \quad 34.7$

$\begin{array}{lllll}\text { OCT. } & 24 & 06 & 36 & 31.2\end{array}$

$\begin{array}{lllll}\text { OCT. } & 25 & 07 & 59 & 34.5\end{array}$

$\begin{array}{lllll}\text { OCT. } \quad 25 & 10 & 36 & 02.4\end{array}$

OCT. $\begin{array}{lllll}25 & 20 & 29 & 33.5\end{array}$

$\begin{array}{lllll}\text { OCT. } & 26 & 17 & 20 & 43.5\end{array}$

$\begin{array}{lllll}\text { OCT. } & 27 & 20 & 22 & 06.6\end{array}$

OCT. $\quad \begin{array}{lllll}29 & 08 & 35 & 29.3\end{array}$

$\begin{array}{lllll}\text { OCT. } & 29 & 17 & 58 & 13.5\end{array}$

OCT. $\begin{array}{lllll}30 & 20 & 50 & 06.6\end{array}$

$\begin{array}{lllll}\text { OCT. } \quad 30 & 33 & 44 & 00.7\end{array}$

OCT. $31 \quad 03 \quad 05 \quad 12.0$

$\begin{array}{llllll}\text { OCT. } & 31 & 14 & 12 & 27.1\end{array}$

$\begin{array}{llllll}\text { Oct. } & 15 & 08 & 12 & 21.7\end{array}$

NOV. $\quad \begin{array}{lllll}1 & 10 & 14 & 38.1\end{array}$ NOV. $2 \quad 10 \quad 2447.0$ NOV. $3 \quad 09 \quad 11 \quad 11.3$ NOV. $3 \quad 093450.0$ NOV. $\begin{array}{lllll}3 & 15 & 02 & 15.3\end{array}$

NOV. $\quad \begin{array}{lllll}4 & 11 & 20 & 20.7\end{array}$ NOV. $\quad 4 \quad 203953.7$ NOV. $\quad \begin{array}{llll}5 & 02 & 59 & 27.5\end{array}$ NOV. $\quad \begin{array}{lllll}8 & 08 & 02 & 51.2\end{array}$ NOV. $\quad 8 \quad 09 \quad 43 \quad 32.6$
$34.309 \mathrm{~N} .119 .250 \mathrm{~W}$

$34.310 \mathrm{~N} .119 .241 \mathrm{~W}$.

$36.219 \mathrm{~N} .120 .389 \mathrm{~W}$.

$33.984 \mathrm{~N} .118 .665 \mathrm{~W}$

$38.835 \mathrm{~N} .122 .777 \mathrm{~W}$.

$33.666 \mathrm{~N} .116 .703 \mathrm{~W}$.

$36.573 \mathrm{~N} .121 .203 \mathrm{~W}$.

$40.900 \mathrm{~N}$. $122.433 W$.

$35.023 \mathrm{~N} .118 .473 \mathrm{~W}$.

$34.242 \mathrm{~N} .120 .707 W$.

$33.258 \mathrm{~N} .116 .053 \mathrm{~W}$.

$36.855 \mathrm{~N} .121 .400 \mathrm{~W}$.

$36.340 \mathrm{~N} .120 .316 \mathrm{~W}$.

$33.939 \mathrm{~N} .116 .995 \mathrm{~W}$.

$33.138 \mathrm{~N} .116 .501 \mathrm{~W}$.

$37.414 \mathrm{~N} .118 .497 \mathrm{~W}$.

$36.034 \mathrm{~N} .119 .913 \mathrm{~W}$.

$33.698 \mathrm{~N} .118 .160 \mathrm{~W}$.

$33.697 \mathrm{~N} .118 .161 \mathrm{~W}$.

$33.815 \mathrm{~N} .117 .031 \mathrm{~W}$.

$33.927 \mathrm{~N}, 118.020 \mathrm{~W}$.

$36.253 \mathrm{~N} .120 .868 \mathrm{~W}$.

$36.582 \mathrm{~N} .121 .235 \mathrm{~W}$.

$37.496 \mathrm{~N} .118 .849 \mathrm{~W}$.

$35.099 \mathrm{~N} .118 .522 \mathrm{~W}$.

$38.705 \mathrm{~N}, 123.500 \mathrm{~W}$

$33.137 \mathrm{~N} .116 .505 \mathrm{~W}$.

$34.032 \mathrm{~N} .116 .848 \mathrm{~W}$

$34.737 \mathrm{~N} .120 .148 \mathrm{~W}$.

$34.729 \mathrm{~N} .120 .158 \mathrm{~W}$.

$34.016 \mathrm{~N} .118 .988 \mathrm{~W}$.

$33.280 \mathrm{~N} .115 .702 \mathrm{~W}$.

$33.017 \mathrm{~N} .116 .392 \mathrm{~W}$.

$37.490 \mathrm{~N} .118 .846 \mathrm{~W}$.

$38.495 \mathrm{~N} .122 .710 \mathrm{~W}$.

$38.500 \mathrm{~N} .122 .705 \mathrm{~W}$.

$37.293 \mathrm{~N} .121 .685 \mathrm{~W}$.

$37.305 \mathrm{~N} .121 .648 \mathrm{~W}$.

35.978 N. $120.177 W$.

$37.507 \mathrm{~N} .122 .190 \mathrm{~W}$.

$37.268 \mathrm{~N} .121 .665 \mathrm{~W}$.

$37.267 \mathrm{~N} .121 .663 \mathrm{~W}$.

$37.268 \mathrm{~N} .121 .652 \mathrm{~W}$.

$34.706 \mathrm{~N} .120 .294 \mathrm{~W}$.

$36.553 \mathrm{~N} .121 .172 \mathrm{~W}$

$37.453 \mathrm{~N} .118 .817 \mathrm{~W}$

$34.140 \mathrm{~N} .117 .433 \mathrm{~W}$

$36.605 \mathrm{~N} .120 .671 \mathrm{~W}$.

$33.901 \mathrm{~N} .118 .263 \mathrm{~W}$.
22 PS

2 PS

1 PS

2 PS

13 PS

$\begin{array}{rllll}5 & \text { BK } & \ldots & \ldots & 2.8 M L(B K) \\ 19 & \text { PS } & \ldots & \ldots & 3.9 M L(P S) \\ 9 & \text { BK } & \ldots & \ldots & 4.0 M L(B K) \\ 15 & \text { GM } & \ldots & \ldots & 3.2 M D(G M) \\ 7 & \text { PS } & \ldots & \ldots & 3.0 M L(P S)\end{array}$

3. $2 M L(P S)$

$\begin{array}{lll}\ldots & \ldots & 3.2 M L(P S) \\ \ldots & \ldots & 3.2 M L(P S)\end{array}$

.. $\quad \ldots$ 3.3ML(PS)

$\ldots \quad \ldots 3.4 M L$ (PS)

$\ldots \quad \ldots \quad 3.3 M L(P S)$

. OML(PS)

6 PS

1 PS

8 BK

6
15

$\ldots \quad \ldots 3.5 \mathrm{ML}$ (PS)

$\cdots \quad \cdots \quad 3.0 M L(P S)$

$\cdots \quad \cdots \quad 3.0 M L(P S)$

$\ldots \quad \ldots \quad 3.0 M L(P S)$

PS

12
6

6 PS

2 PS

4.3

.

$\cdots$

4. 5ML (PS)

$\ldots \quad$ 3. OML (PS)

$\ldots \quad \ldots 3.1 \mathrm{ML}$ (PS)

$\ldots \quad \ldots 3.7 \mathrm{ML}(\mathrm{PS})$

3. $7 \mathrm{ML}$ (PS)
3. $1 \mathrm{ML}$ (PS)

PS

PS

PS

BK

BK

PS

PS

BK

PS

PS

PS

PS

PS

PS

PS

PS

$\begin{array}{lll}\ldots & \ldots & 3.2 \mathrm{ML}(\mathrm{PS}) \\ \ldots & \ldots & 3.1 \mathrm{ML}(\mathrm{PS})\end{array}$

$\ldots$ 3. OML (BK)

$\ldots \quad \ldots 3.6 \mathrm{ML}(\mathrm{BK})$

... $\quad \ldots 3.1 \mathrm{ML}$ (PS)

$\ldots \quad \ldots 3.5 \mathrm{ML}$ (PS)

$\ldots \quad \ldots 3.1 \mathrm{ML}(\mathrm{BK})$

3.1ML(PS)

$\ldots \quad \ldots 3.1 \mathrm{ML}(\mathrm{PS})$

$4.5 \quad \ldots .4404$ (PS)

. $5 M L(P S)$

$\cdots \quad \cdots \quad 3.1 \mathrm{ML}$ (PS)

$\begin{array}{lll}4.3 & \ldots & 4.6 \mathrm{ML} \text { (PS) }\end{array}$

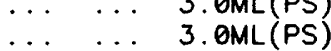

$\ldots$ 3. OML (PS)

BK

.. $\quad \ldots 2.7 M L(B K)$

BK

BK

BK

$\ldots \quad \ldots \quad 2.9 M L(B K)$

$\ldots \quad \ldots 3.2 \mathrm{ML}(\mathrm{BK})$

$\ldots \quad \ldots 3.6 \mathrm{ML}(\mathrm{BK})$

$\ldots$ 3.OMD (GM)

2. $4 M L(B K)$

$\ldots \quad \ldots \quad 2.4 M \mathrm{~L}(\mathrm{BK})$

$\ldots$ 3.3ML(BK)

$\ldots \quad \ldots 3.1 \mathrm{ML}(\mathrm{BK})$

$\ldots$ 3.2ML(PS)

$\begin{array}{lll}\ldots & \ldots & 3.1 \mathrm{ML}(\mathrm{BK}) \\ \ldots & \ldots & 3.0 \mathrm{MD}(\mathrm{GM})\end{array}$

$\cdots \quad \ldots .4 M$ 3. $\cdots$ (PS)

$\ldots 3.2 \mathrm{ML}$ (PS)

3. $2 M L(P S)$

.

$\begin{array}{rllll}\ldots & \text { OCT. } & 1 & 06: 37 & \text { PST } \\ \text { IV } & \text { OCT. } & 1 & 18: 59 & \text { PST } \\ \text { IV } & \text { OCT. } & 2 & 01: 38 & \text { PST } \\ \ldots & \text { OCT. } & 2 & 05: 24 & \text { PST } \\ \text { FELT } & \text { OCT. } & 3 & 04: 49 & \text { PST } \\ & & & & \\ \text { FELT } & \text { OCT. } & 3 & 23: 53 & \text { PST } \\ V & \text { OCT. } & 4 & 23: 37 & \text { PST } \\ \text { III } & \text { OCT. } & 5 & 08: 16 & \text { PST } \\ \ldots & \text { OCT. } & 5 & 16: 38 & \text { PST } \\ \ldots & \text { OCT. } & 6 & 00: 21 & \text { PST }\end{array}$

$\begin{array}{llll}\ldots & \text { OCT. } 6 & 20: 21 & \text { PST }\end{array}$

$\begin{array}{llll}\ldots & \text { OCT. } & 7 & 07: 44 \\ \text { PST }\end{array}$

$\begin{array}{lll}\ldots \text { OCT. } 7 & 72: 39 \text { PST }\end{array}$

$\begin{array}{lll}\ldots & \text { AUG. } 8 & 12: 30 \text { PST }\end{array}$

$\begin{array}{lll}\ldots \text { OCT. } 8 & 22: 01 \text { PST }\end{array}$

IV OCT. $10 \quad 13: 22$ PST

$\begin{array}{llll}\ldots & \text { OCT. } & 14 & 01: 44 \\ \text { PST }\end{array}$

OCT. 15 00:12 PST
OCT

IV OCT. 15 09:44 PST

$\begin{array}{llll}\ldots & \text { OCT. } 16 & 09: 49 & \text { PST }\end{array}$

II I OCT. $16 \quad 14: 18$ PST

$\begin{array}{llll}\ldots & \text { OCT. } 17 \text { 16:57 PST }\end{array}$

$\begin{array}{lll} & \text { OCT. } 20 & 07: 05 \\ \text { PST }\end{array}$

IV OCT. 21 20:06 PST

... OCT. $21 \quad 21: 44$ PST

$\begin{array}{llll}\ldots & \text { OCT. } 22 & 12: 28 & \text { PST }\end{array}$

$\begin{array}{llll} & \text { FELT OCT. } 23 & 02: 08 \text { PST }\end{array}$

$\begin{array}{lll}\ldots & \text { OCT. } 23 & 22: 36 \text { PST }\end{array}$

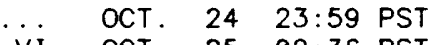

$\begin{array}{llll}V I & \text { OCT. } 25 & 02: 36 & \text { PST }\end{array}$

FELT OCT. $25 \quad 12: 29$ PST

$\checkmark$ OCT. 26 09:20 PST

$\begin{array}{lll}\ldots & \text { OCT. } 27 & 12: 22 \\ \ldots S T\end{array}$

$\begin{array}{llll}\ldots & \text { OCT. } 29 & 00: 35 \text { PST }\end{array}$

$\begin{array}{lll}\text { OCT. } 29 & 09: 58 \text { PST }\end{array}$

$\begin{array}{llll}\text { FELT OCT. } 30 & 12: 50 \text { PST }\end{array}$

FELT OCT. 30 15:44 PST

FELT OCT. 30 19:05 PST

II OCT. $31 \quad 06: 12$ PST

OCT 15 Ө0:12 PST

FELT NOV

NOV

NOV.

NOV.

$\begin{array}{lll}\ldots & \text { NOV. } 3 & 07: 02 \\ \text {... PST }\end{array}$

02:14 PST

$202: 24$ PST

.. NOV

NOV.

IV

NOV.

NOV.

NOV.
$03: 20$ PST

$12: 39$ PST

18:59 PST

00:02 PST

$01: 43$ PST 
Table 1. Summary of U.S. earthquakes for 1984-Continued

\begin{tabular}{|c|c|c|c|c|c|c|c|c|c|c|c|c|}
\hline \multirow{2}{*}{ Date } & $\begin{array}{c}\text { Origin time } \\
\text { (UTC) }\end{array}$ & \multirow{2}{*}{$\begin{array}{c}\text { Latitude } \\
\text { ( })\end{array}$} & \multirow{2}{*}{$\begin{array}{c}\text { Longitude } \\
\left({ }^{\circ}\right)\end{array}$} & \multirow{2}{*}{$\begin{array}{c}\text { Depth } \\
(\mathbf{k m})\end{array}$} & \multirow{2}{*}{$\begin{array}{l}\text { Hypo- } \\
\text { center } \\
\text { source }\end{array}$} & \multicolumn{3}{|c|}{ Magnitude } & \multirow{2}{*}{$\begin{array}{l}\text { Maximum } \\
\text { intensity }\end{array}$} & \multicolumn{3}{|c|}{ Local time } \\
\hline & hr $\min \sec$ & & & & & $\mathbf{m b}$ & MS & $\begin{array}{l}\text { ML, Mn } \\
\text { MD. Mw }\end{array}$ & & Date & Hour & $\begin{array}{l}\text { Time } \\
\text { zone }\end{array}$ \\
\hline
\end{tabular}

CALIFORNIA-Continued

\begin{tabular}{|c|c|c|c|c|c|c|c|c|c|c|c|c|c|c|c|c|}
\hline $\begin{array}{l}\text { NOV. } \\
\text { NOV. } \\
\text { NOV. } \\
\text { NOV. } \\
\text { NOV. }\end{array}$ & $\begin{array}{r}8 \\
10 \\
13 \\
14 \\
15\end{array}$ & $\begin{array}{l}16 \\
09 \\
00 \\
04 \\
02\end{array}$ & $\begin{array}{l}54 \\
54 \\
04 \\
02 \\
06\end{array}$ & $\begin{array}{l}43.1 \\
09.7 \\
13.9 \\
19.8 \\
32.4\end{array}$ & $\begin{array}{l}34.304 \mathrm{~N} . \\
37.235 \mathrm{~N} . \\
36.292 \mathrm{~N} . \\
36.213 \mathrm{~N} . \\
40.298 \mathrm{~N} .\end{array}$ & $\begin{array}{l}116.501 w . \\
121.647 w . \\
120.427 W . \\
120.400 W . \\
124.328 W .\end{array}$ & $\begin{array}{r}6 \\
5 \\
11 \\
1 \\
5\end{array}$ & $\begin{array}{l}\text { PS } \\
\text { BK } \\
\text { GM } \\
\text { PS } \\
\text { BK }\end{array}$ & $\begin{array}{l}\cdots \\
\cdots \\
\cdots \\
\cdots\end{array}$ & $\begin{array}{l}\cdots \\
\cdots \\
\cdots \\
\cdots\end{array}$ & $\begin{array}{l}\text { 3. } 0 \mathrm{ML}(\mathrm{PS}) \\
\text { 3. } 0 \mathrm{ML}(\mathrm{BK}) \\
\text { 3. } 1 \mathrm{MD}(\mathrm{GM}) \\
\text { 3. } 3 \mathrm{ML}(\mathrm{PS}) \\
\text { 3. } 6 \mathrm{ML}(\mathrm{BK})\end{array}$ & $\begin{array}{c}\text { FELT } \\
\ldots \\
\ldots \\
\cdots\end{array}$ & $\begin{array}{l}\text { NOV. } \\
\text { NOV. } \\
\text { NOV. } \\
\text { NOV. } \\
\text { NOV. }\end{array}$ & $\begin{array}{r}8 \\
10 \\
12 \\
13 \\
14\end{array}$ & $\begin{array}{l}08: 54 \\
01: 54 \\
16: 04 \\
20: 02 \\
18: 06\end{array}$ & $\begin{array}{l}\text { PST } \\
\text { PST } \\
\text { PST } \\
\text { PST } \\
\text { PST }\end{array}$ \\
\hline $\begin{array}{l}\text { NOV. } \\
\text { NOV. } \\
\text { NOV. } \\
\text { NOV. } \\
\text { NOV. }\end{array}$ & $\begin{array}{l}18 \\
19 \\
22 \\
23 \\
23\end{array}$ & $\begin{array}{l}05 \\
05 \\
23 \\
18 \\
18\end{array}$ & $\begin{array}{l}50 \\
02 \\
59 \\
08 \\
11\end{array}$ & $\begin{array}{l}44.8 \\
53.3 \\
08.0 \\
25.5 \\
21.9\end{array}$ & $\begin{array}{l}34.122 \mathrm{~N} . \\
35.858 \mathrm{~N} . \\
36.563 \mathrm{~N} . \\
37.458 \mathrm{~N} . \\
37.467 \mathrm{~N} .\end{array}$ & $\begin{array}{l}117.386 W . \\
117.724 W . \\
121.198 W . \\
118.605 W . \\
118.583 W .\end{array}$ & $\begin{array}{r}10 \\
5 \\
7 \\
12 \\
6\end{array}$ & $\begin{array}{l}\text { PS } \\
\text { PS } \\
\text { BK } \\
\text { BK } \\
\text { PS }\end{array}$ & $\begin{array}{l}\ldots \\
\ldots \\
5.6 \\
\ldots\end{array}$ & $\begin{array}{l}\ldots \\
\ldots \\
5.7 \\
\ldots\end{array}$ & $\begin{array}{l}\text { 3. } 0 \mathrm{ML}(\mathrm{PS}) \\
\text { 3. } 1 \mathrm{ML}(\mathrm{PS}) \\
\text { 3. } 2 \mathrm{ML}(\mathrm{BK}) \\
\text { 6. } 1 \mathrm{ML}(\mathrm{BK}) \\
\text { 3. } 4 \mathrm{ML}(\mathrm{PS})\end{array}$ & $\begin{array}{c}\text { FELT } \\
\cdots \\
\cdots \\
\cdots\end{array}$ & $\begin{array}{l}\text { NOV. } \\
\text { NOV. } \\
\text { NOV. } \\
\text { NOV. } \\
\text { NOV. }\end{array}$ & $\begin{array}{l}17 \\
18 \\
22 \\
23 \\
23\end{array}$ & $\begin{array}{l}21: 50 \\
21: 02 \\
15: 59 \\
10: 08 \\
10: 11\end{array}$ & $\begin{array}{l}\text { PST } \\
\text { PST } \\
\text { PST } \\
\text { PST } \\
\text { PST }\end{array}$ \\
\hline $\begin{array}{l}\text { NOV. } \\
\text { NOV. } \\
\text { NOV. } \\
\text { NOV. } \\
\text { NOV. }\end{array}$ & $\begin{array}{l}23 \\
23 \\
23 \\
23 \\
23\end{array}$ & $\begin{array}{l}18 \\
18 \\
18 \\
18 \\
18\end{array}$ & $\begin{array}{l}11 \\
15 \\
17 \\
17 \\
17\end{array}$ & $\begin{array}{l}47.9 \\
39.2 \\
21.8 \\
47.4 \\
47.9\end{array}$ & $\begin{array}{l}37.467 \mathrm{~N} . \\
37.467 \mathrm{~N} . \\
37.432 \mathrm{~N} . \\
37.467 \mathrm{~N} . \\
37.473 \mathrm{~N} .\end{array}$ & $\begin{array}{l}118.583 w . \\
118.583 w . \\
118.637 w . \\
118.583 w . \\
118.668 w .\end{array}$ & $\begin{array}{r}6 \\
6 \\
10 \\
6 \\
10\end{array}$ & $\begin{array}{l}P S \\
P S \\
\text { GM } \\
\text { PS } \\
\text { GM }\end{array}$ & $\begin{array}{l}\cdots \\
\cdots \\
\cdots \\
\cdots\end{array}$ & $\begin{array}{l}\ldots \\
\ldots \\
\ldots \\
\ldots \\
\ldots\end{array}$ & $\begin{array}{l}\text { 3. } 4 M L(P S) \\
\text { 3. } 1 \mathrm{ML}(\mathrm{PS}) \\
\text { 3. } 2 \mathrm{ML}(\mathrm{BK}) \\
\text { 3. } 1 \mathrm{ML}(\mathrm{PS}) \\
\text { 3. } 6 \mathrm{MD}(\mathrm{GM})\end{array}$ & $\begin{array}{l}\cdots \\
\cdots \\
\cdots \\
\cdots\end{array}$ & $\begin{array}{l}\text { NOV. } \\
\text { NOV. } \\
\text { NOV. } \\
\text { NOV. } \\
\text { APR. }\end{array}$ & $\begin{array}{l}23 \\
23 \\
23 \\
23 \\
23\end{array}$ & $\begin{array}{l}10: 11 \\
10: 15 \\
10: 17 \\
10: 17 \\
10: 17\end{array}$ & $\begin{array}{l}\text { PST } \\
\text { PST } \\
\text { PST } \\
\text { PST } \\
\text { PST }\end{array}$ \\
\hline $\begin{array}{l}\text { NOV. } \\
\text { NOV. } \\
\text { NOV. } \\
\text { NOV. } \\
\text { NOV. }\end{array}$ & $\begin{array}{l}23 \\
23 \\
23 \\
23 \\
23\end{array}$ & $\begin{array}{l}18 \\
18 \\
18 \\
18 \\
18\end{array}$ & $\begin{array}{l}21 \\
22 \\
23 \\
24 \\
27\end{array}$ & $\begin{array}{l}08.6 \\
52.3 \\
64.3 \\
57.5 \\
30.8\end{array}$ & $\begin{array}{l}37.425 \mathrm{~N} . \\
37.478 \mathrm{~N} . \\
37.467 \mathrm{~N} . \\
37.467 \mathrm{~N} . \\
37.425 \mathrm{~N} .\end{array}$ & $\begin{array}{l}118.610 W . \\
118.595 W . \\
118.583 w . \\
118.583 w . \\
118.645 W .\end{array}$ & $\begin{array}{r}10 \\
10 \\
6 \\
6 \\
4\end{array}$ & $\begin{array}{l}\text { BK } \\
\text { BK } \\
\text { PS } \\
\text { PS } \\
\text { GM }\end{array}$ & $\begin{array}{l}\ldots \\
\cdots \\
\cdots \\
\cdots\end{array}$ & $\begin{array}{l}\ldots \\
\ldots \\
\ldots \\
\ldots \\
\ldots\end{array}$ & $\begin{array}{l}\text { 3. } 6 \mathrm{ML}(\mathrm{BK}) \\
\text { 3. } 5 \mathrm{ML}(\mathrm{BK}) \\
\text { 3. } \mathrm{ML} \text { (PS) } \\
\text { 3. } 0 \mathrm{ML}(\mathrm{PS}) \\
\text { 3. } 1 \mathrm{MD}(\mathrm{GM})\end{array}$ & $\begin{array}{l}\cdots \\
\cdots \\
\cdots \\
\cdots\end{array}$ & $\begin{array}{l}\text { NOV. } \\
\text { NOV. } \\
\text { NOV. } \\
\text { NOV. } \\
\text { NOV. }\end{array}$ & $\begin{array}{l}23 \\
23 \\
23 \\
23 \\
23\end{array}$ & $\begin{array}{l}10: 21 \\
10: 22 \\
10: 23 \\
10: 24 \\
10: 27\end{array}$ & $\begin{array}{l}\text { PST } \\
\text { PST } \\
\text { PST } \\
\text { PST } \\
\text { PST }\end{array}$ \\
\hline $\begin{array}{l}\text { NOV. } \\
\text { NOV. } \\
\text { NOV. } \\
\text { NOV. } \\
\text { NOV. }\end{array}$ & $\begin{array}{l}23 \\
23 \\
23 \\
23 \\
23\end{array}$ & $\begin{array}{l}18 \\
18 \\
18 \\
18 \\
18\end{array}$ & $\begin{array}{l}34 \\
34 \\
38 \\
40 \\
40\end{array}$ & $\begin{array}{l}00.0 \\
35.9 \\
55.1 \\
00.5 \\
46.0\end{array}$ & $\begin{array}{l}37.470 \mathrm{~N} . \\
37.448 \mathrm{~N} . \\
37.427 \mathrm{~N} . \\
37.465 \mathrm{~N} . \\
37.467 \mathrm{~N} .\end{array}$ & $\begin{array}{l}118.610 W . \\
118.593 W \\
118.610 W \\
118.622 W \\
118.583 W .\end{array}$ & $\begin{array}{r}8 \\
6 \\
14 \\
5 \\
6\end{array}$ & $\begin{array}{l}\text { GM } \\
\text { GM } \\
\text { GM } \\
\text { GM } \\
\text { PS }\end{array}$ & $\begin{array}{l}\ldots \\
\ldots \\
\ldots \\
\ldots\end{array}$ & $\begin{array}{l}\ldots \\
\ldots \\
\ldots \\
\cdots \\
\cdots\end{array}$ & $\begin{array}{l}\text { 3. } 0 M D(G M) \\
\text { 3. } 3 M D(G M) \\
\text { 3. } 2 M L(P S) \\
\text { 3. } 4 M D(G M) \\
\text { 3. } 0 M L(P S)\end{array}$ & $\begin{array}{l}\cdots \\
\cdots \\
\cdots \\
\cdots \\
\cdots\end{array}$ & $\begin{array}{l}\text { NOV. } \\
\text { NOV. } \\
\text { NOV. } \\
\text { NOV. } \\
\text { NOV. }\end{array}$ & $\begin{array}{l}23 \\
23 \\
23 \\
23 \\
23\end{array}$ & $\begin{array}{l}10: 34 \\
10: 34 \\
10: 38 \\
10: 40 \\
10: 40\end{array}$ & $\begin{array}{l}\text { PST } \\
\text { PST } \\
\text { PST } \\
\text { PST } \\
\text { PST }\end{array}$ \\
\hline $\begin{array}{l}\text { NOV. } \\
\text { NOV. } \\
\text { NOV. } \\
\text { NOV. } \\
\text { NOV. }\end{array}$ & $\begin{array}{l}23 \\
23 \\
23 \\
23 \\
23\end{array}$ & $\begin{array}{l}18 \\
18 \\
18 \\
19 \\
19\end{array}$ & $\begin{array}{l}41 \\
43 \\
47 \\
08 \\
12\end{array}$ & $\begin{array}{l}10.7 \\
22.6 \\
34.8 \\
00.7 \\
34.6\end{array}$ & $\begin{array}{l}37.468 \mathrm{~N} . \\
37.440 \mathrm{~N} . \\
37.447 \mathrm{~N} . \\
37.448 \mathrm{~N} . \\
37.433 \mathrm{~N} .\end{array}$ & $\begin{array}{l}118.635 W . \\
118.648 W . \\
118.625 W . \\
118.613 W . \\
118.612 W .\end{array}$ & $\begin{array}{r}9 \\
12 \\
7 \\
10 \\
14\end{array}$ & $\begin{array}{l}\text { GM } \\
G M \\
G M \\
G M \\
G M\end{array}$ & $\begin{array}{l}\ldots \\
\ldots \\
\ldots \\
4.8\end{array}$ & $\begin{array}{l}\ldots \\
\ldots \\
\cdots \\
4.7\end{array}$ & $\begin{array}{l}\text { 3. } 0 M D(G M) \\
\text { 3. } 2 M D(G M) \\
\text { 3. } 4 M D(G M) \\
\text { 3. } 0 M D(G M) \\
5.5 M L(B K)\end{array}$ & $\begin{array}{l}\ldots \\
\ldots \\
\ldots \\
\text { IV }\end{array}$ & $\begin{array}{l}\text { NOV. } \\
\text { NOV. } \\
\text { NOV. } \\
\text { NOV. } \\
\text { NOV. }\end{array}$ & $\begin{array}{l}23 \\
23 \\
23 \\
23 \\
23\end{array}$ & $\begin{array}{l}10: 41 \\
10: 43 \\
10: 47 \\
11: 08 \\
11: 12\end{array}$ & $\begin{array}{l}\text { PST } \\
\text { PST } \\
\text { PST } \\
\text { PST } \\
\text { PST }\end{array}$ \\
\hline $\begin{array}{l}\text { NOV. } \\
\text { NOV. } \\
\text { NOV. } \\
\text { NOV. } \\
\text { NOV. }\end{array}$ & $\begin{array}{l}23 \\
23 \\
23 \\
23 \\
23\end{array}$ & $\begin{array}{l}19 \\
19 \\
19 \\
19 \\
19\end{array}$ & $\begin{array}{l}15 \\
17 \\
17 \\
21 \\
21\end{array}$ & $\begin{array}{l}29.7 \\
24.8 \\
48.7 \\
19.7 \\
29.4\end{array}$ & $\begin{array}{l}37.467 \mathrm{~N} . \\
37.467 \mathrm{~N} . \\
37.467 \mathrm{~N} . \\
37.425 \mathrm{~N} . \\
37.437 \mathrm{~N} .\end{array}$ & $\begin{array}{l}118.583 W . \\
118.583 W . \\
118.583 W \\
118.618 W . \\
118.650 W .\end{array}$ & $\begin{array}{r}6 \\
6 \\
6 \\
16 \\
8\end{array}$ & $\begin{array}{l}\text { PS } \\
\text { PS } \\
\text { PS } \\
\text { GM } \\
\text { BK }\end{array}$ & $\begin{array}{l}\ldots \\
\ldots \\
\cdots \\
\cdots \\
\cdots\end{array}$ & $\begin{array}{l}\ldots \\
\cdots \\
\cdots \\
\cdots \\
\cdots\end{array}$ & $\begin{array}{l}\text { 3. } 6 \mathrm{ML}(\mathrm{PS}) \\
\text { 3. } 0 \mathrm{ML} \text { (PS) } \\
\text { 3. } 0 \mathrm{ML}(\mathrm{PS}) \\
\text { 3. } 1 \mathrm{MD}(\mathrm{GM}) \\
\text { 3. } 6 \mathrm{ML} \text { (BK) }\end{array}$ & $\begin{array}{l}\ldots \\
\ldots \\
\ldots \\
\ldots\end{array}$ & $\begin{array}{l}\text { NOV. } \\
\text { NOV. } \\
\text { NOV. } \\
\text { NOV. } \\
\text { NOV. }\end{array}$ & $\begin{array}{l}23 \\
23 \\
23 \\
23 \\
23\end{array}$ & $\begin{array}{l}11: 15 \\
11: 17 \\
11: 17 \\
11: 21 \\
11: 21\end{array}$ & $\begin{array}{l}\text { PST } \\
\text { PST } \\
\text { PST } \\
\text { PST } \\
\text { PST }\end{array}$ \\
\hline $\begin{array}{l}\text { NOV. } \\
\text { NOV. } \\
\text { NOV. } \\
\text { NOV. } \\
\text { NOV. }\end{array}$ & $\begin{array}{l}23 \\
23 \\
23 \\
23 \\
23\end{array}$ & $\begin{array}{l}19 \\
19 \\
19 \\
19 \\
19\end{array}$ & $\begin{array}{l}21 \\
23 \\
25 \\
26 \\
27\end{array}$ & $\begin{array}{l}32.2 \\
21.8 \\
37.1 \\
40.3 \\
59.2\end{array}$ & $\begin{array}{l}37.368 \mathrm{~N} . \\
37.467 \mathrm{~N} . \\
37.442 \mathrm{~N} . \\
37.424 \mathrm{~N} . \\
37.434 \mathrm{~N} .\end{array}$ & $\begin{array}{l}118.309 W \\
118.583 W \\
118.638 W \\
118.662 W \\
118.631 W\end{array}$ & $\begin{array}{r}6 \\
6 \\
10 \\
6 \\
6\end{array}$ & $\begin{array}{l}\text { PS } \\
\text { PS } \\
\text { GM } \\
\text { PS } \\
\text { PS }\end{array}$ & $\begin{array}{l}\cdots \\
\cdots \\
\cdots \\
\ldots\end{array}$ & $\begin{array}{l}\ldots \\
\ldots \\
\cdots \\
\cdots \\
\cdots\end{array}$ & $\begin{array}{l}\text { 3. } 7 \mathrm{ML}(P S) \\
\text { 3. } 0 \mathrm{ML}(\mathrm{PS}) \\
\text { 3. } 0 \mathrm{MD}(\mathrm{GM}) \\
\text { 3. } 6 \mathrm{ML}(\mathrm{PS}) \\
\text { 3. } 4 \mathrm{ML} \text { (PS) }\end{array}$ & $\begin{array}{l}\cdots \\
\cdots \\
\cdots \\
\cdots\end{array}$ & $\begin{array}{l}\text { NOV. } \\
\text { NOV. } \\
\text { NOV. } \\
\text { NOV. } \\
\text { NOV. }\end{array}$ & $\begin{array}{l}23 \\
23 \\
23 \\
23 \\
23\end{array}$ & $\begin{array}{l}11: 21 \\
11: 23 \\
11: 25 \\
11: 26 \\
11: 27\end{array}$ & $\begin{array}{l}\text { PST } \\
\text { PST } \\
\text { PST } \\
\text { PST } \\
\text { PST }\end{array}$ \\
\hline $\begin{array}{l}\text { NOV. } \\
\text { NOV. } \\
\text { NOV. } \\
\text { NOV. } \\
\text { NOV. }\end{array}$ & $\begin{array}{l}23 \\
23 \\
23 \\
23 \\
23\end{array}$ & $\begin{array}{l}19 \\
19 \\
19 \\
20 \\
20\end{array}$ & $\begin{array}{l}39 \\
44 \\
48 \\
00 \\
01\end{array}$ & $\begin{array}{l}33.7 \\
43.2 \\
15.3 \\
42.8 \\
53.7\end{array}$ & $\begin{array}{l}37.440 \mathrm{~N} . \\
37.445 \mathrm{~N} . \\
37.437 \mathrm{~N} . \\
37.448 \mathrm{~N} . \\
37.465 \mathrm{~N}\end{array}$ & $\begin{array}{l}118.640 W . \\
118.635 W \\
118.645 W \\
118.615 W \\
118.588 W\end{array}$ & $\begin{array}{r}10 \\
6 \\
11 \\
9 \\
6\end{array}$ & $\begin{array}{l}G M \\
P S \\
G M \\
G M \\
G M\end{array}$ & $\begin{array}{l}\cdots \\
\cdots \\
\cdots \\
\cdots\end{array}$ & $\begin{array}{l}\ldots \\
\ldots \\
\cdots \\
\cdots \\
\cdots\end{array}$ & $\begin{array}{l}\text { 3. } 0 M D(G M) \\
\text { 3. } 2 M L(P S) \\
\text { 3.1MD(GM) } \\
\text { 3. } 2 M L(P S) \\
\text { 3. } 4 M D(G M)\end{array}$ & $\begin{array}{l}\cdots \\
\cdots \\
\cdots \\
\cdots \\
\cdots\end{array}$ & $\begin{array}{l}\text { NOV. } \\
\text { NOV. } \\
\text { NOV. } \\
\text { NOV. } \\
\text { NOV. }\end{array}$ & $\begin{array}{l}23 \\
23 \\
23 \\
23 \\
23\end{array}$ & $\begin{array}{l}11: 39 \\
11: 44 \\
11: 48 \\
12: 00 \\
12: 01\end{array}$ & $\begin{array}{l}\text { PST } \\
\text { PST } \\
\text { PST } \\
\text { PST } \\
\text { PST }\end{array}$ \\
\hline $\begin{array}{l}\text { NOV. } \\
\text { NOV. } \\
\text { NOV. } \\
\text { NOV. } \\
\text { NOV. }\end{array}$ & $\begin{array}{l}23 \\
23 \\
23 \\
23 \\
23\end{array}$ & $\begin{array}{l}20 \\
20 \\
20 \\
20 \\
20\end{array}$ & $\begin{array}{l}08 \\
09 \\
11 \\
20 \\
35\end{array}$ & $\begin{array}{l}00.1 \\
35.4 \\
06.6 \\
38.4 \\
51.7\end{array}$ & $\begin{array}{l}37.452 \mathrm{~N} . \\
37.422 \mathrm{~N} . \\
37.453 \mathrm{~N} . \\
37.424 \mathrm{~N} . \\
37.424 \mathrm{~N} .\end{array}$ & $\begin{array}{l}118.588 w . \\
118.617 w . \\
118.595 w . \\
118.653 w . \\
118.634 W .\end{array}$ & $\begin{array}{r}14 \\
13 \\
9 \\
6 \\
6\end{array}$ & $\begin{array}{l}\text { GM } \\
G M \\
G M \\
P S \\
P S\end{array}$ & $\begin{array}{l}\cdots \\
\cdots \\
\cdots \\
\cdots\end{array}$ & $\begin{array}{l}\ldots \\
\ldots \\
\ldots \\
\ldots\end{array}$ & $\begin{array}{l}3.5 \mathrm{ML}(\mathrm{BK}) \\
3.2 \mathrm{MD}(\mathrm{GM}) \\
3.5 \mathrm{ML}(\mathrm{PS}) \\
3.7 \mathrm{ML}(\mathrm{PS}) \\
3.2 \mathrm{ML}(\mathrm{PS})\end{array}$ & $\cdots$ & $\begin{array}{l}\text { NOV. } \\
\text { NOV. } \\
\text { NOV. } \\
\text { NOV. } \\
\text { NOV. }\end{array}$ & $\begin{array}{l}23 \\
23 \\
23 \\
23 \\
23\end{array}$ & $\begin{array}{l}12: 08 \\
12: 09 \\
12: 11 \\
12: 20 \\
12: 35\end{array}$ & $\begin{array}{l}\text { PST } \\
\text { PST } \\
\text { PST } \\
\text { PST } \\
\text { PST }\end{array}$ \\
\hline
\end{tabular}


Table 1. Summary of U.S. earthquakes for 1984--Continued

\begin{tabular}{|c|c|c|c|c|c|c|c|c|c|c|c|c|c|}
\hline Date & hr $\min$ & $\sec$ & $\begin{array}{c}\text { Latitude } \\
\left({ }^{\circ}\right)\end{array}$ & $\begin{array}{c}\text { Longitude } \\
\left({ }^{\circ}\right)\end{array}$ & $\begin{array}{c}\text { Depth } \\
(\mathbf{k m})\end{array}$ & $\begin{array}{l}\text { Hypo- } \\
\text { center } \\
\text { source }\end{array}$ & $\mathbf{m b}$ & MS & $\begin{array}{l}\text { ML. Mn } \\
\text { MD. Mw }\end{array}$ & $\begin{array}{l}\text { Maximum } \\
\text { intensity }\end{array}$ & Date & Hour & $\begin{array}{l}\text { Time } \\
\text { Tone }\end{array}$ \\
\hline
\end{tabular}

\section{CALIFORNIA-Continued}

\begin{tabular}{|c|c|c|c|c|c|c|c|c|c|c|c|c|c|c|c|}
\hline $\begin{array}{l}\text { NOV. } \\
\text { NOV. } \\
\text { NOV. } \\
\text { NOV. } \\
\text { NOV. }\end{array}$ & $\begin{array}{l}23 \\
23 \\
23 \\
23 \\
23\end{array}$ & $\begin{array}{l}20 \\
20 \\
20 \\
20 \\
20\end{array}$ & $\begin{array}{l}37 \\
40 \\
41 \\
42 \\
43\end{array}$ & $\begin{array}{l}36.6 \\
50.5 \\
13.0 \\
22.0 \\
54.4\end{array}$ & $\begin{array}{l}37.594 \mathrm{~N} \\
37.423 \mathrm{~N} \\
37.458 \mathrm{~N} \\
37.340 \mathrm{~N} \\
37.455 \mathrm{~N}\end{array}$ & $\begin{array}{l}119.244 W . \\
118.570 W . \\
118.593 W . \\
118.694 W . \\
118.613 W .\end{array}$ & $\begin{array}{r}6 \\
15 \\
15 \\
6 \\
10\end{array}$ & $\begin{array}{l}P S \\
G M \\
G M \\
P S \\
G M\end{array}$ & $\begin{array}{l}\cdots \\
\cdots \\
\cdots \\
\cdots\end{array}$ & $\begin{array}{l}\cdots \\
\cdots \\
\cdots \\
\cdots\end{array}$ & $\begin{array}{l}\text { 3.1ML(PS) } \\
\text { 3.5MD(GM) } \\
\text { 3. } 3 \mathrm{ML}(\mathrm{PS}) \\
\text { 3. } 3 \mathrm{ML}(\mathrm{PS}) \\
3.8 \mathrm{MD}(\mathrm{GM})\end{array}$ & $\begin{array}{l}\cdots \\
\cdots \\
\cdots \\
\cdots\end{array}$ & $\begin{array}{l}\text { NOV. } \\
\text { NOV. } \\
\text { NOV. } \\
\text { NOV. } \\
\text { NOV. }\end{array}$ & $\begin{array}{l}23 \\
23 \\
23 \\
23 \\
23\end{array}$ & $\begin{array}{l}12: 37 \\
12: 40 \\
12: 41 \\
12: 42 \\
12: 43\end{array}$ \\
\hline $\begin{array}{l}\text { NOV. } \\
\text { NOV. } \\
\text { NOV. } \\
\text { NOV. } \\
\text { NOV. }\end{array}$ & $\begin{array}{l}23 \\
23 \\
23 \\
23 \\
23\end{array}$ & $\begin{array}{l}20 \\
20 \\
21 \\
21 \\
21\end{array}$ & $\begin{array}{l}44 \\
48 \\
11 \\
16 \\
34\end{array}$ & $\begin{array}{l}17.7 \\
59.8 \\
45.0 \\
39.3 \\
42.0\end{array}$ & $\begin{array}{l}37.450 \mathrm{~N} \\
40.498 \mathrm{~N} \\
37.453 \mathrm{~N} \\
37.467 \mathrm{~N} \\
37.457 \mathrm{~N}\end{array}$ & $\begin{array}{l}118.570 W . \\
123.178 W . \\
118.652 W \\
118.634 W \\
118.615 W .\end{array}$ & $\begin{array}{r}7 \\
5 \\
6 \\
6 \\
15\end{array}$ & $\begin{array}{l}\text { GM } \\
\text { BK } \\
\text { PS } \\
\text { PS } \\
\text { GM }\end{array}$ & $\begin{array}{l}\cdots \\
4.3 \\
\cdots \\
\ldots\end{array}$ & $\begin{array}{l}\cdots \\
\cdots \\
\cdots \\
\cdots \\
\cdots\end{array}$ & $\begin{array}{l}\text { 3. 3ML (PS) } \\
\text { 3.9ML(BK) } \\
\text { 3.0ML(PS) } \\
\text { 3.0ML(PS) } \\
\text { 3. 3MD (GM) }\end{array}$ & $\begin{array}{l}\cdots \\
\cdots \\
\cdots \\
\cdots \\
\cdots\end{array}$ & $\begin{array}{l}\text { NOV. } \\
\text { NOV. } \\
\text { NOV. } \\
\text { NOV. } \\
\text { NOV. }\end{array}$ & $\begin{array}{l}23 \\
23 \\
23 \\
23 \\
23\end{array}$ & $\begin{array}{l}12: 44 \\
12: 48 \\
13: 11 \\
13: 16 \\
13: 34\end{array}$ \\
\hline $\begin{array}{l}\text { NOV. } \\
\text { NOV. } \\
\text { NOV. } \\
\text { NOV. } \\
\text { NOV. }\end{array}$ & $\begin{array}{l}23 \\
23 \\
23 \\
23 \\
23\end{array}$ & $\begin{array}{l}21 \\
21 \\
21 \\
21 \\
21\end{array}$ & $\begin{array}{l}34 \\
44 \\
51 \\
56 \\
57\end{array}$ & $\begin{array}{l}55.4 \\
11.8 \\
36.9 \\
23.6 \\
34.7\end{array}$ & $\begin{array}{l}37.437 \mathrm{~N} \\
37.446 \mathrm{~N} \\
37.452 \mathrm{~N} \\
37.452 \mathrm{~N} \\
37.457 \mathrm{~N}\end{array}$ & $\begin{array}{l}118.608 W . \\
118.653 w . \\
118.595 w \\
118.643 w . \\
118.612 W .\end{array}$ & $\begin{array}{r}10 \\
6 \\
5 \\
9 \\
9\end{array}$ & $\begin{array}{l}B K \\
P S \\
G M \\
G M \\
G M\end{array}$ & $\begin{array}{l}\cdots \\
\cdots \\
\cdots \\
\cdots\end{array}$ & $\begin{array}{l}\cdots \\
\ldots \\
\ldots \\
\cdots\end{array}$ & $\begin{array}{l}\text { 3.6ML(BK) } \\
\text { 3. } 1 \mathrm{ML}(\mathrm{PS}) \\
\text { 3.1MD(GM) } \\
\text { 3. } 3 \mathrm{MD}(\mathrm{GM}) \\
\text { 3. } \mathrm{MDD}(\mathrm{GM})\end{array}$ & $\begin{array}{l}\cdots \\
\cdots \\
\cdots \\
\cdots \\
\cdots\end{array}$ & $\begin{array}{l}\text { NOV. } \\
\text { NOV. } \\
\text { NOV. } \\
\text { NOV. } \\
\text { NOV. }\end{array}$ & $\begin{array}{l}23 \\
23 \\
23 \\
23 \\
23\end{array}$ & $\begin{array}{l}13: 34 \\
13: 44 \\
13: 51 \\
13: 56 \\
13: 57\end{array}$ \\
\hline $\begin{array}{l}\text { NOV. } \\
\text { NOV. } \\
\text { NOV. } \\
\text { NOV. } \\
\text { NOV. }\end{array}$ & $\begin{array}{l}23 \\
23 \\
23 \\
23 \\
23\end{array}$ & $\begin{array}{l}22 \\
22 \\
22 \\
22 \\
23\end{array}$ & $\begin{array}{l}00 \\
10 \\
21 \\
22 \\
43\end{array}$ & $\begin{array}{l}49.5 \\
16.9 \\
47.2 \\
42.2 \\
10.3\end{array}$ & $\begin{array}{l}37.445 \mathrm{~N} \\
37.463 \mathrm{~N} \\
37.477 \mathrm{~N} \\
37.463 \mathrm{~N} . \\
37.440 \mathrm{~N} .\end{array}$ & $\begin{array}{l}118.597 w . \\
118.603 W . \\
118.627 W . \\
118.587 W . \\
118.625 W .\end{array}$ & $\begin{array}{r}13 \\
7 \\
6 \\
7 \\
7\end{array}$ & $\begin{array}{l}\text { GM } \\
\text { BK } \\
P S \\
\text { GM } \\
\text { GM }\end{array}$ & $\begin{array}{l}\cdots \\
\cdots \\
\cdots \\
\cdots\end{array}$ & $\begin{array}{l}\cdots \\
\cdots \\
\cdots \\
\cdots\end{array}$ & $\begin{array}{l}\text { 3. } 2 M L(P S) \\
\text { 3.5ML(BK) } \\
\text { 3. OML (PS) } \\
\text { 3. OMD (GM) } \\
\text { 3.8ML(BK) }\end{array}$ & $\begin{array}{l}\cdots \\
\cdots \\
\cdots \\
\cdots \\
\cdots\end{array}$ & $\begin{array}{l}\text { NOV. } \\
\text { NOV. } \\
\text { NOV. } \\
\text { NOV. } \\
\text { NOV. }\end{array}$ & $\begin{array}{l}23 \\
23 \\
23 \\
23 \\
23\end{array}$ & $\begin{array}{l}14: 00 \\
14: 10 \\
14: 21 \\
14: 22 \\
15: 43\end{array}$ \\
\hline $\begin{array}{l}\text { NOV. } \\
\text { NOV. } \\
\text { NOV. } \\
\text { NOV. } \\
\text { NOV. }\end{array}$ & $\begin{array}{l}23 \\
23 \\
24 \\
24 \\
24\end{array}$ & $\begin{array}{l}23 \\
23 \\
00 \\
00 \\
01\end{array}$ & $\begin{array}{l}47 \\
54 \\
30 \\
31 \\
36\end{array}$ & $\begin{array}{l}33.1 \\
02.5 \\
06.9 \\
20.8 \\
07.1\end{array}$ & $\begin{array}{l}37.473 \mathrm{~N} . \\
37.430 \mathrm{~N} . \\
37.433 \mathrm{~N} . \\
37.433 \mathrm{~N} . \\
37.432 \mathrm{~N} .\end{array}$ & $\begin{array}{l}118.651 w . \\
118.637 w . \\
118.660 w . \\
118.665 w . \\
118.637 w .\end{array}$ & $\begin{array}{l}6 \\
5 \\
6 \\
6 \\
5\end{array}$ & $\begin{array}{l}P S \\
G M \\
P S \\
P S \\
G M\end{array}$ & $\begin{array}{l}\cdots \\
\cdots \\
\cdots \\
\cdots \\
\cdots\end{array}$ & $\begin{array}{l}\cdots \\
\cdots \\
\cdots \\
\cdots\end{array}$ & $\begin{array}{l}\text { 3. } 0 M L(P S) \\
3.2 M D(G M) \\
3.8 M L(P S) \\
3.6 M L(P S) \\
3.3 M D(G M)\end{array}$ & $\begin{array}{l}\cdots \\
\cdots \\
\cdots \\
\cdots \\
\cdots\end{array}$ & $\begin{array}{l}\text { NOV. } \\
\text { NOV. } \\
\text { NOV. } \\
\text { NOV. } \\
\text { NOV. }\end{array}$ & $\begin{array}{l}23 \\
23 \\
23 \\
23 \\
23\end{array}$ & $\begin{array}{l}15: 47 \\
15: 54 \\
16: 30 \\
16: 31 \\
17: 36\end{array}$ \\
\hline $\begin{array}{l}\text { NOV. } \\
\text { NOV. } \\
\text { NOV. } \\
\text { NOV. } \\
\text { NOV. }\end{array}$ & $\begin{array}{l}24 \\
24 \\
24 \\
24 \\
24\end{array}$ & $\begin{array}{l}01 \\
01 \\
02 \\
02 \\
02\end{array}$ & $\begin{array}{l}37 \\
38 \\
08 \\
10 \\
12\end{array}$ & $\begin{array}{l}34.7 \\
12.4 \\
14.4 \\
38.8 \\
02.1\end{array}$ & $\begin{array}{l}37.442 \mathrm{~N} \\
37.432 \mathrm{~N} \\
37.440 \mathrm{~N} \\
37.437 \mathrm{~N} \\
37.435 \mathrm{~N}\end{array}$ & $\begin{array}{l}118.622 W . \\
118.637 W . \\
118.632 W . \\
118.669 W . \\
118.678 W .\end{array}$ & $\begin{array}{l}6 \\
5 \\
7 \\
6 \\
6\end{array}$ & $\begin{array}{l}\text { GM } \\
\text { GM } \\
\text { GM } \\
\text { PS } \\
\text { PS }\end{array}$ & $\begin{array}{l}\cdots \\
\cdots \\
\cdots \\
\cdots\end{array}$ & $\begin{array}{l}\ldots \\
\cdots \\
\cdots \\
\cdots\end{array}$ & $\begin{array}{l}\text { 3. } 1 M D(G M) \\
\text { 3. } 1 M D(G M) \\
\text { 3. } 5 M L(B K) \\
\text { 3. } 7 M L(P S) \\
\text { 3. } 7 M L(P S)\end{array}$ & $\begin{array}{l}\cdots \\
\cdots \\
\cdots \\
\cdots \\
\cdots\end{array}$ & $\begin{array}{l}\text { NOV. } \\
\text { NOV. } \\
\text { NOV. } \\
\text { NOV. } \\
\text { NOV. }\end{array}$ & $\begin{array}{l}23 \\
23 \\
23 \\
23 \\
23\end{array}$ & $\begin{array}{l}17: 37 \\
17: 38 \\
18: 08 \\
18: 10 \\
18: 12\end{array}$ \\
\hline $\begin{array}{l}\text { NOV. } \\
\text { NOV. } \\
\text { NOV. } \\
\text { NOV. } \\
\text { NOV. }\end{array}$ & $\begin{array}{l}24 \\
24 \\
24 \\
24 \\
24\end{array}$ & $\begin{array}{l}02 \\
02 \\
02 \\
02 \\
02\end{array}$ & $\begin{array}{l}13 \\
14 \\
16 \\
22 \\
35\end{array}$ & $\begin{array}{l}34.0 \\
53.7 \\
20.4 \\
48.4 \\
20.3\end{array}$ & $\begin{array}{l}37.435 \mathrm{~N} \\
37.438 \mathrm{~N} \\
37.432 \mathrm{~N} \\
37.450 \mathrm{~N} \\
37.425 \mathrm{~N} .\end{array}$ & $\begin{array}{l}118.640 W . \\
118.640 W . \\
118.633 W . \\
118.657 W . \\
118.615 W .\end{array}$ & $\begin{array}{l}5 \\
5 \\
5 \\
5 \\
7\end{array}$ & $\begin{array}{l}G M \\
G M \\
G M \\
G M \\
G M\end{array}$ & $\begin{array}{l}\cdots \\
\cdots \\
\cdots \\
\cdots\end{array}$ & $\begin{array}{l}\ldots \\
\ldots \\
\cdots \\
\cdots\end{array}$ & $\begin{array}{l}\text { 3. } 6 \mathrm{MD}(\mathrm{GM}) \\
\text { 3. } \mathrm{MD}(\mathrm{GM}) \\
3.2 \mathrm{MD}(\mathrm{GM}) \\
3.1 \mathrm{MD}(\mathrm{GM}) \\
\text { 3.6ML(PS) }\end{array}$ & $\begin{array}{l}\cdots \\
\cdots \\
\cdots \\
\cdots \\
\cdots\end{array}$ & $\begin{array}{l}\text { NOV. } \\
\text { NOV. } \\
\text { NOV. } \\
\text { NOV. } \\
\text { NOV. }\end{array}$ & $\begin{array}{l}23 \\
23 \\
23 \\
23 \\
23\end{array}$ & $\begin{array}{l}18: 13 \\
18: 14 \\
18: 16 \\
18: 22 \\
18: 35\end{array}$ \\
\hline $\begin{array}{l}\text { NOV. } \\
\text { NOV. } \\
\text { NOV. } \\
\text { NOV. } \\
\text { NOV. }\end{array}$ & $\begin{array}{l}24 \\
24 \\
24 \\
24 \\
24\end{array}$ & $\begin{array}{l}02 \\
02 \\
02 \\
02 \\
02\end{array}$ & $\begin{array}{l}35 \\
35 \\
43 \\
46 \\
47\end{array}$ & $\begin{array}{l}21.1 \\
27.2 \\
29.3 \\
33.4 \\
20.2\end{array}$ & $\begin{array}{l}37.427 \mathrm{~N} \\
37.497 \mathrm{~N} \\
37.442 \mathrm{~N} \\
37.445 \mathrm{~N} \\
37.475 \mathrm{~N}\end{array}$ & $\begin{array}{l}118.623 W . \\
118.575 W . \\
118.645 W . \\
118.648 W \\
118.652 W .\end{array}$ & $\begin{array}{l}6 \\
6 \\
4 \\
4 \\
4\end{array}$ & $\begin{array}{l}P S \\
P S \\
G M \\
G M \\
G M\end{array}$ & $\begin{array}{l}\ldots \\
\ldots \\
\cdots \\
\cdots \\
\cdots\end{array}$ & $\begin{array}{l}\cdots \\
\cdots \\
\cdots \\
\ldots\end{array}$ & $\begin{array}{l}\text { 3.5ML(PS) } \\
\text { 3. } 3 \mathrm{ML}(\mathrm{PS}) \\
3.0 \mathrm{MD}(\mathrm{GM}) \\
\text { 3.8MD(GM) } \\
\text { 3.2MD(GM) }\end{array}$ & $\begin{array}{l}\ldots \\
\ldots \\
\cdots \\
\ldots \\
\ldots\end{array}$ & $\begin{array}{l}\text { NOV. } \\
\text { NOV. } \\
\text { NOV. } \\
\text { NOV. } \\
\text { NOV. }\end{array}$ & $\begin{array}{l}23 \\
23 \\
23 \\
23 \\
23\end{array}$ & $\begin{array}{l}18: 35 \\
18: 35 \\
18: 43 \\
18: 46 \\
18: 47\end{array}$ \\
\hline $\begin{array}{l}\text { NOV. } \\
\text { NOV. } \\
\text { NOV. } \\
\text { NOV. } \\
\text { NOV. }\end{array}$ & $\begin{array}{l}24 \\
24 \\
24 \\
24 \\
24\end{array}$ & $\begin{array}{l}03 \\
04 \\
04 \\
05 \\
07\end{array}$ & $\begin{array}{l}16 \\
02 \\
08 \\
10 \\
40\end{array}$ & $\begin{array}{l}29.9 \\
19.5 \\
17.6 \\
21.4 \\
36.8\end{array}$ & $\begin{array}{l}37.435 \mathrm{~N} \\
34.382 \mathrm{~N} . \\
37.664 \mathrm{~N} . \\
37.462 \mathrm{~N} . \\
37.452 \mathrm{~N} .\end{array}$ & $\begin{array}{l}118.672 W . \\
116.611 W . \\
118.693 W . \\
118.652 W . \\
118.620 W .\end{array}$ & $\begin{array}{l}6 \\
3 \\
6 \\
6 \\
7\end{array}$ & $\begin{array}{l}\text { PS } \\
\text { PS } \\
\text { PS } \\
\text { PS } \\
\text { GM }\end{array}$ & $\begin{array}{l}\ldots \\
\cdots \\
\cdots \\
\cdots\end{array}$ & $\begin{array}{l}\ldots \\
\cdots \\
\cdots \\
\cdots \\
\cdots\end{array}$ & $\begin{array}{l}\text { 3.0ML(PS) } \\
3.4 M L(P S) \\
3.0 M L(P S) \\
3.3 \mathrm{ML}(P S) \\
3.7 M D(G M)\end{array}$ & $\begin{array}{l}\cdots \\
\cdots \\
\cdots \\
\cdots \\
\cdots\end{array}$ & $\begin{array}{l}\text { NOV. } \\
\text { NOV. } \\
\text { NOV. } \\
\text { NOV. } \\
\text { NOV. }\end{array}$ & $\begin{array}{l}23 \\
23 \\
23 \\
23 \\
23\end{array}$ & $\begin{array}{l}19: 16 \\
20: 02 \\
20: 08 \\
21: 10 \\
23: 40\end{array}$ \\
\hline $\begin{array}{l}\text { NOV. } \\
\text { NOV. } \\
\text { NOV. } \\
\text { NOV. } \\
\text { NOV. }\end{array}$ & $\begin{array}{l}24 \\
24 \\
24 \\
24 \\
24\end{array}$ & $\begin{array}{l}07 \\
07 \\
08 \\
08 \\
08\end{array}$ & $\begin{array}{l}40 \\
49 \\
01 \\
44 \\
53\end{array}$ & $\begin{array}{l}52.8 \\
04.6 \\
22.8 \\
51.7 \\
00.2\end{array}$ & $\begin{array}{l}37.399 \mathrm{~N} . \\
37.435 \mathrm{~N} . \\
37.455 \mathrm{~N} . \\
37.462 \mathrm{~N} . \\
37.457 \mathrm{~N} .\end{array}$ & $\begin{array}{l}118.673 w . \\
118.655 W . \\
118.622 W . \\
118.590 w . \\
118.612 W .\end{array}$ & $\begin{array}{r}6 \\
6 \\
8 \\
3 \\
10\end{array}$ & $\begin{array}{l}\text { PS } \\
\text { PS } \\
\text { GM } \\
\text { GM } \\
\text { GM }\end{array}$ & $\begin{array}{l}\cdots \\
\cdots \\
\cdots\end{array}$ & $\begin{array}{l}\cdots \\
\cdots \\
\cdots \\
\cdots\end{array}$ & $\begin{array}{l}\text { 3. } 6 \mathrm{ML} \text { (PS) } \\
\text { 3.5ML(PS) } \\
3.0 \mathrm{MD}(\mathrm{GM}) \\
3.0 \mathrm{ML}(\mathrm{PS}) \\
\text { 3.2ML(BK) }\end{array}$ & $\begin{array}{l}\ldots \\
\ldots \\
\ldots \\
\ldots\end{array}$ & $\begin{array}{l}\text { NOV. } \\
\text { NOV. } \\
\text { NOV. } \\
\text { NOV. } \\
\text { NOV. }\end{array}$ & $\begin{array}{l}23 \\
23 \\
24 \\
24 \\
24\end{array}$ & $\begin{array}{l}23: 40 \\
23: 49 \\
\theta 0: \theta 1 \\
\theta 0: 44 \\
\theta 0: 53\end{array}$ \\
\hline
\end{tabular}


Table 1. Summary of U.S. earthquakes for 1984-Continued

\begin{tabular}{|c|c|c|c|c|c|c|c|c|c|c|c|c|c|}
\hline Date & hr $\min$ & $\sec$ & $\begin{array}{c}\text { Latihude } \\
\left({ }^{\circ}\right)\end{array}$ & $\begin{array}{l}\text { Longitude } \\
\left({ }^{\circ}\right)\end{array}$ & $\begin{array}{c}\text { Depth } \\
(\mathbf{k m})\end{array}$ & $\begin{array}{l}\text { Hypo- } \\
\text { center } \\
\text { source }\end{array}$ & $\mathbf{m b}$ & MS & $\begin{array}{l}\text { ML, Mn } \\
\text { MD. Mw }\end{array}$ & $\begin{array}{l}\text { Maximum } \\
\text { intensity }\end{array}$ & Date & Hour & $\begin{array}{l}\text { Time } \\
\text { 7ome }\end{array}$ \\
\hline
\end{tabular}

\section{CALIFORNIA-Continued}

\begin{tabular}{|c|c|c|c|c|c|c|c|c|c|c|c|c|c|c|c|c|}
\hline $\begin{array}{l}\text { NOV. } \\
\text { NOV. } \\
\text { NOV. } \\
\text { NOV. } \\
\text { NOV. }\end{array}$ & $\begin{array}{l}24 \\
24 \\
24 \\
24 \\
24\end{array}$ & $\begin{array}{l}09 \\
10 \\
11 \\
12 \\
12\end{array}$ & $\begin{array}{l}21 \\
13 \\
22 \\
15 \\
32\end{array}$ & $\begin{array}{l}17.1 \\
12.4 \\
49.6 \\
31.8 \\
26.1\end{array}$ & $\begin{array}{l}37.473 \mathrm{~N} . \\
37.447 \mathrm{~N} . \\
37.475 \mathrm{~N} . \\
37.455 \mathrm{~N} . \\
37.450 \mathrm{~N} .\end{array}$ & $\begin{array}{l}118.577 W . \\
118.617 W . \\
118.567 W . \\
118.612 W . \\
118.592 W .\end{array}$ & $\begin{array}{r}12 \\
9 \\
11 \\
10 \\
6\end{array}$ & $\begin{array}{l}G M \\
G M \\
G M \\
G M \\
G M\end{array}$ & $\begin{array}{l}\cdots \\
\cdots \\
\cdots \\
\cdots \\
\cdots\end{array}$ & $\begin{array}{l}\cdots \\
\cdots \\
\cdots \\
\cdots\end{array}$ & $\begin{array}{l}\text { 4. } 2 M L(B K) \\
\text { 3.6ML(BK) } \\
\text { 3. } 0 M D(G M) \\
\text { 3. } 6 M L(B K) \\
\text { 3.1ML(PS) }\end{array}$ & $\begin{array}{c}\text { FELT } \\
\ldots \\
\ldots \\
\cdots \\
\cdots\end{array}$ & $\begin{array}{l}\text { NOV. } \\
\text { NOV. } \\
\text { NOV. } \\
\text { NOV. } \\
\text { NOV. }\end{array}$ & $\begin{array}{l}24 \\
24 \\
24 \\
24 \\
24\end{array}$ & $\begin{array}{l}01: 21 \\
02: 13 \\
03: 22 \\
04: 15 \\
04: 32\end{array}$ & $\begin{array}{l}\text { PST } \\
\text { PST } \\
\text { PST } \\
\text { PST } \\
\text { PST }\end{array}$ \\
\hline $\begin{array}{l}\text { NOV. } \\
\text { NOV. } \\
\text { NOV. } \\
\text { NOV. } \\
\text { NOV. }\end{array}$ & $\begin{array}{l}24 \\
24 \\
24 \\
24 \\
24\end{array}$ & $\begin{array}{l}13 \\
13 \\
14 \\
15 \\
18\end{array}$ & $\begin{array}{l}01 \\
49 \\
00 \\
42 \\
01\end{array}$ & $\begin{array}{l}45.4 \\
21.0 \\
49.2 \\
57.4 \\
39.0\end{array}$ & $\begin{array}{l}37.463 \mathrm{~N} . \\
37.448 \mathrm{~N} . \\
37.525 \mathrm{~N} \\
36.132 \mathrm{~N} . \\
37.470 \mathrm{~N} .\end{array}$ & $\begin{array}{l}118.615 W . \\
118.588 W . \\
118.858 W . \\
120.021 W . \\
118.592 W .\end{array}$ & $\begin{array}{r}11 \\
6 \\
9 \\
6 \\
4\end{array}$ & $\begin{array}{l}\text { GM } \\
\text { GM } \\
\text { GM } \\
\text { PS } \\
\text { GM }\end{array}$ & $\begin{array}{l}\cdots \\
\cdots \\
\cdots \\
\cdots \\
\cdots\end{array}$ & $\begin{array}{l}\ldots \\
\cdots \\
\ldots \\
\cdots \\
\cdots\end{array}$ & $\begin{array}{l}\text { 3. } 4 M L(P S) \\
\text { 3. } 0 M D(G M) \\
\text { 3. } 3 M L(P S) \\
\text { 3. } 0 M L(P S) \\
\text { 3. } 2 M D(G M)\end{array}$ & $\begin{array}{l}\cdots \\
\cdots \\
\cdots \\
\cdots \\
\cdots\end{array}$ & $\begin{array}{l}\text { NOV. } \\
\text { NOV. } \\
\text { NOV. } \\
\text { NOV. } \\
\text { NOV. }\end{array}$ & $\begin{array}{l}24 \\
24 \\
24 \\
24 \\
24\end{array}$ & $\begin{array}{l}05: 01 \\
05: 49 \\
06: 00 \\
07: 42 \\
10: 01\end{array}$ & $\begin{array}{l}\text { PST } \\
\text { PST } \\
\text { PST } \\
\text { PST } \\
\text { PST }\end{array}$ \\
\hline $\begin{array}{l}\text { NOV. } \\
\text { NOV. } \\
\text { NOV. } \\
\text { NOV. } \\
\text { NOV. }\end{array}$ & $\begin{array}{l}24 \\
24 \\
24 \\
24 \\
24\end{array}$ & $\begin{array}{l}18 \\
19 \\
19 \\
20 \\
20\end{array}$ & $\begin{array}{l}30 \\
22 \\
39 \\
25 \\
29\end{array}$ & $\begin{array}{l}56.9 \\
09.8 \\
20.4 \\
32.9 \\
46.5\end{array}$ & $\begin{array}{l}37.457 \mathrm{~N} . \\
36.203 \mathrm{~N} . \\
37.412 \mathrm{~N} . \\
37.438 \mathrm{~N} . \\
37.428 \mathrm{~N} .\end{array}$ & $\begin{array}{l}118.613 W . \\
120.005 W . \\
118.618 W . \\
118.648 W . \\
118.640 W .\end{array}$ & $\begin{array}{r}8 \\
6 \\
15 \\
9 \\
12\end{array}$ & $\begin{array}{l}\text { GM } \\
P S \\
\text { GM } \\
\text { GM } \\
\text { GM }\end{array}$ & $\begin{array}{l}\ldots \\
\cdots \\
4.0 \\
\ldots\end{array}$ & $\begin{array}{l}\cdots \\
\cdots \\
\cdots \\
\cdots \\
\cdots\end{array}$ & $\begin{array}{l}\text { 3. } 2 M D(G M) \\
\text { 3. } 0 M L(P S) \\
\text { 3. } 3 M L(P S) \\
\text { 4.0ML(BK) } \\
\text { 3. } 1 M D(G M)\end{array}$ & $\begin{array}{c}\cdots \\
\cdots \\
\text { FELT } \\
\cdots\end{array}$ & $\begin{array}{l}\text { NOV. } \\
\text { NOV. } \\
\text { NOV. } \\
\text { NOV. } \\
\text { NOV. }\end{array}$ & $\begin{array}{l}24 \\
24 \\
24 \\
24 \\
24\end{array}$ & $\begin{array}{l}10: 30 \\
11: 22 \\
11: 39 \\
12: 25 \\
12: 29\end{array}$ & $\begin{array}{l}\text { PST } \\
\text { PST } \\
\text { PST } \\
\text { PST } \\
\text { PST }\end{array}$ \\
\hline $\begin{array}{l}\text { NOV. } \\
\text { NOV. } \\
\text { NOV. } \\
\text { NOV. } \\
\text { NOV. }\end{array}$ & $\begin{array}{l}24 \\
24 \\
24 \\
25 \\
25\end{array}$ & $\begin{array}{l}20 \\
20 \\
21 \\
00 \\
00\end{array}$ & $\begin{array}{l}33 \\
33 \\
10 \\
14 \\
59\end{array}$ & $\begin{array}{l}18.3 \\
59.8 \\
33.2 \\
58.9 \\
00.5\end{array}$ & $\begin{array}{l}37.435 \mathrm{~N} . \\
37.361 \mathrm{~N} . \\
37.452 \mathrm{~N} . \\
37.435 \mathrm{~N} . \\
37.417 \mathrm{~N} .\end{array}$ & $\begin{array}{l}118.643 W . \\
118.668 W . \\
118.593 w . \\
118.618 W . \\
118.645 W .\end{array}$ & $\begin{array}{r}4 \\
6 \\
5 \\
10 \\
10\end{array}$ & $\begin{array}{l}G M \\
P S \\
G M \\
G M \\
G M\end{array}$ & $\begin{array}{l}\ldots \\
\cdots \\
\cdots \\
\cdots \\
\cdots\end{array}$ & $\begin{array}{l}\cdots \\
\cdots \\
\cdots \\
\cdots\end{array}$ & $\begin{array}{l}\text { 3. } 1 \mathrm{ML}(P S) \\
\text { 3. } \mathrm{MLL}(\mathrm{PS}) \\
\text { 3. } 5 \mathrm{ML}(\mathrm{BK}) \\
\text { 3. } 0 \mathrm{MD}(\mathrm{GM}) \\
\text { 3. } \mathrm{ML} \text { (PS) }\end{array}$ & $\begin{array}{l}\cdots \\
\cdots \\
\cdots \\
\cdots \\
\cdots\end{array}$ & $\begin{array}{l}\text { NOV. } \\
\text { NOV. } \\
\text { NOV. } \\
\text { NOV. } \\
\text { NOV. }\end{array}$ & $\begin{array}{l}24 \\
24 \\
24 \\
24 \\
24\end{array}$ & $\begin{array}{l}12: 33 \\
12: 33 \\
13: 10 \\
16: 14 \\
16: 59\end{array}$ & $\begin{array}{l}\text { PST } \\
\text { PST } \\
\text { PST } \\
\text { PST } \\
\text { PST }\end{array}$ \\
\hline $\begin{array}{l}\text { NOV. } \\
\text { NOV. } \\
\text { NOV. } \\
\text { NOV. } \\
\text { NOV. }\end{array}$ & $\begin{array}{l}25 \\
25 \\
25 \\
25 \\
25\end{array}$ & $\begin{array}{l}02 \\
02 \\
02 \\
05 \\
05\end{array}$ & $\begin{array}{l}30 \\
30 \\
47 \\
08 \\
50\end{array}$ & $\begin{array}{l}16.3 \\
31.1 \\
00.8 \\
50.1 \\
38.4\end{array}$ & $\begin{array}{l}37.438 \mathrm{~N} \\
37.483 \mathrm{~N} \\
36.102 \mathrm{~N} \\
33.332 \mathrm{~N} \\
37.533 \mathrm{~N}\end{array}$ & $\begin{array}{l}118.643 w . \\
118.653 w . \\
120.123 w . \\
118.667 w . \\
118.865 w .\end{array}$ & $\begin{array}{r}10 \\
2 \\
2 \\
14 \\
10\end{array}$ & $\begin{array}{l}G M \\
G M \\
G M \\
P S \\
G M\end{array}$ & $\begin{array}{l}\cdots \\
\cdots \\
\cdots \\
\cdots\end{array}$ & $\begin{array}{l}\cdots \\
\cdots \\
\cdots \\
\cdots \\
\cdots\end{array}$ & $\begin{array}{l}\text { 3. } 5 \mathrm{MD}(\mathrm{GM}) \\
3.1 \mathrm{MD}(\mathrm{GM}) \\
\text { 3.1MD (GM) } \\
\text { 3.8ML(PS) } \\
\text { 3.5ML(PS) }\end{array}$ & $\begin{array}{c}\ldots \\
\ldots \\
\text { FELT } \\
\ldots\end{array}$ & $\begin{array}{l}\text { NOV. } \\
\text { NOV. } \\
\text { NOV. } \\
\text { NOV. } \\
\text { NOV. }\end{array}$ & $\begin{array}{l}24 \\
24 \\
24 \\
24 \\
24\end{array}$ & $\begin{array}{l}18: 30 \\
18: 30 \\
18: 47 \\
21: 08 \\
21: 50\end{array}$ & $\begin{array}{l}\text { PST } \\
\text { PST } \\
\text { PST } \\
\text { PST } \\
\text { PST }\end{array}$ \\
\hline $\begin{array}{l}\text { NOV. } \\
\text { NOV. } \\
\text { NOV. } \\
\text { NOV. } \\
\text { NOV. }\end{array}$ & $\begin{array}{l}25 \\
25 \\
25 \\
25 \\
25\end{array}$ & $\begin{array}{l}06 \\
06 \\
08 \\
08 \\
10\end{array}$ & $\begin{array}{l}25 \\
34 \\
27 \\
44 \\
37\end{array}$ & $\begin{array}{l}19.2 \\
12.6 \\
18.7 \\
54.5 \\
10.2\end{array}$ & $\begin{array}{l}37.408 \mathrm{~N} . \\
37.435 \mathrm{~N} . \\
37.450 \mathrm{~N} . \\
37.452 \mathrm{~N} . \\
37.400 \mathrm{~N} .\end{array}$ & $\begin{array}{l}118.686 w . \\
118.683 w . \\
118.677 w . \\
118.645 W . \\
118.655 w .\end{array}$ & $\begin{array}{l}6 \\
4 \\
4 \\
7 \\
6\end{array}$ & $\begin{array}{l}P S \\
G M \\
G M \\
G M \\
P S\end{array}$ & $\begin{array}{l}\cdots \\
\cdots \\
\cdots \\
\cdots\end{array}$ & $\begin{array}{l}\cdots \\
\cdots \\
\cdots \\
\cdots \\
\cdots\end{array}$ & $\begin{array}{l}\text { 3. } 1 \mathrm{ML}(\mathrm{PS}) \\
\text { 3. } 2 \mathrm{MD}(\mathrm{GM}) \\
\text { 3. } 3 \mathrm{MD}(\mathrm{GM}) \\
\text { 3. } 3 \mathrm{ML}(\mathrm{PS}) \\
\text { 3. } 2 \mathrm{ML} \text { (PS) }\end{array}$ & $\begin{array}{l}\ldots \\
\cdots \\
\cdots \\
\cdots \\
\cdots\end{array}$ & $\begin{array}{l}\text { NOV. } \\
\text { NOV. } \\
\text { NOV. } \\
\text { NOV. } \\
\text { NOV. }\end{array}$ & $\begin{array}{l}24 \\
24 \\
25 \\
25 \\
25\end{array}$ & $\begin{array}{l}22: 25 \\
22: 34 \\
00: 27 \\
00: 44 \\
02: 37\end{array}$ & $\begin{array}{l}\text { PST } \\
\text { PST } \\
\text { PST } \\
\text { PST } \\
\text { PST }\end{array}$ \\
\hline $\begin{array}{l}\text { NOV. } \\
\text { NOV. } \\
\text { NOV. } \\
\text { NOV. } \\
\text { NOV. }\end{array}$ & $\begin{array}{l}25 \\
25 \\
25 \\
25 \\
25\end{array}$ & $\begin{array}{l}13 \\
15 \\
16 \\
16 \\
16\end{array}$ & $\begin{array}{l}30 \\
56 \\
15 \\
15 \\
17\end{array}$ & $\begin{array}{l}42.2 \\
07.9 \\
03.2 \\
20.3 \\
19.1\end{array}$ & $\begin{array}{l}37.437 \mathrm{~N} \\
37.448 \mathrm{~N} \\
37.393 \mathrm{~N} \\
37.455 \mathrm{~N} \\
37.400 \mathrm{~N}\end{array}$ & $\begin{array}{l}118.733 w . \\
118.662 W . \\
118.627 w . \\
118.687 w . \\
118.640 W .\end{array}$ & $\begin{array}{l}0 \\
6 \\
2 \\
6 \\
2\end{array}$ & $\begin{array}{l}\text { GM } \\
\text { PS } \\
\text { GM } \\
\text { PS } \\
\text { GM }\end{array}$ & $\begin{array}{l}\ldots \\
\cdots \\
\cdots \\
\cdots \\
\cdots\end{array}$ & $\begin{array}{l}\cdots \\
\cdots \\
\cdots \\
\cdots \\
\cdots\end{array}$ & $\begin{array}{l}\text { 3. } 0 M D(G M) \\
\text { 3. } 2 M L(P S) \\
\text { 3. } 8 M D(G M) \\
\text { 4. } 1 \mathrm{ML}(\mathrm{PS}) \\
\text { 3. } 0 \mathrm{MD}(\mathrm{GM})\end{array}$ & $\begin{array}{l}\ldots \\
\ldots \\
\ldots\end{array}$ & $\begin{array}{l}\text { NOV. } \\
\text { NOV. } \\
\text { NOV. } \\
\text { NOV. } \\
\text { NOV. }\end{array}$ & $\begin{array}{l}25 \\
25 \\
25 \\
25 \\
25\end{array}$ & $\begin{array}{l}05: 30 \\
07: 56 \\
08: 15 \\
08: 15 \\
08: 17\end{array}$ & $\begin{array}{l}\text { PST } \\
\text { PST } \\
\text { PST } \\
\text { PST } \\
\text { PST }\end{array}$ \\
\hline $\begin{array}{l}\text { NOV. } \\
\text { NOV. } \\
\text { NOV. } \\
\text { NOV. } \\
\text { NOV. }\end{array}$ & $\begin{array}{l}25 \\
25 \\
25 \\
25 \\
25\end{array}$ & $\begin{array}{l}16 \\
16 \\
19 \\
21 \\
22\end{array}$ & $\begin{array}{l}22 \\
25 \\
18 \\
49 \\
54\end{array}$ & $\begin{array}{l}30.4 \\
23.6 \\
48.5 \\
10.9 \\
58.4\end{array}$ & $\begin{array}{l}37.448 \mathrm{~N} \\
37.383 \mathrm{~N} \\
37.456 \mathrm{~N} \\
37.438 \mathrm{~N} . \\
37.467 \mathrm{~N} .\end{array}$ & $\begin{array}{l}118.638 w . \\
118.613 w . \\
118.643 w . \\
118.650 w . \\
118.659 w .\end{array}$ & $\begin{array}{l}8 \\
2 \\
6 \\
5 \\
6\end{array}$ & $\begin{array}{l}\text { GM } \\
\text { GM } \\
\text { PS } \\
\text { GM } \\
\text { PS }\end{array}$ & $\begin{array}{l}\cdots \\
\cdots \\
\cdots \\
\cdots\end{array}$ & $\begin{array}{l}\cdots \\
\cdots \\
\cdots \\
\cdots \\
\cdots\end{array}$ & $\begin{array}{l}\text { 3. } 7 \mathrm{ML}(\mathrm{BK}) \\
\text { 3. } 8 \mathrm{MD}(\mathrm{GM}) \\
\text { 3. } 5 \mathrm{ML}(\mathrm{PS}) \\
\text { 3. } 4 \mathrm{ML}(\mathrm{BK}) \\
\text { 3. } \mathrm{ML}(\mathrm{PS})\end{array}$ & $\begin{array}{l}\cdots \\
\cdots \\
\cdots \\
\cdots \\
\cdots\end{array}$ & $\begin{array}{l}\text { NOV. } \\
\text { NOV. } \\
\text { NOV. } \\
\text { NOV. } \\
\text { NOV. }\end{array}$ & $\begin{array}{l}25 \\
25 \\
25 \\
25 \\
25\end{array}$ & $\begin{array}{l}08: 22 \\
08: 25 \\
11: 18 \\
13: 49 \\
14: 54\end{array}$ & $\begin{array}{l}\text { PST } \\
\text { PST } \\
\text { PST } \\
\text { PST } \\
\text { PST }\end{array}$ \\
\hline $\begin{array}{l}\text { NOV. } \\
\text { NOV. } \\
\text { NOV. } \\
\text { NOV. } \\
\text { NOV. }\end{array}$ & $\begin{array}{l}25 \\
26 \\
26 \\
26 \\
26\end{array}$ & $\begin{array}{l}23 \\
00 \\
02 \\
04 \\
08\end{array}$ & $\begin{array}{l}10 \\
58 \\
14 \\
22 \\
18\end{array}$ & $\begin{array}{l}09.6 \\
50.2 \\
23.6 \\
09.3 \\
48.9\end{array}$ & $\begin{array}{l}37.451 \mathrm{~N} \\
37.422 \mathrm{~N} . \\
37.455 \mathrm{~N} \\
37.433 \mathrm{~N} \\
34.238 \mathrm{~N}\end{array}$ & $\begin{array}{l}118.620 W . \\
118.637 w . \\
118.668 W . \\
118.692 W . \\
119.449 W .\end{array}$ & $\begin{array}{r}6 \\
6 \\
7 \\
8 \\
14\end{array}$ & $\begin{array}{l}\text { PS } \\
\text { PS } \\
\text { GM } \\
\text { GM } \\
\text { PS }\end{array}$ & $\begin{array}{l}4.3 \\
\ldots \\
\cdots \\
\cdots \\
\ldots\end{array}$ & $\begin{array}{l}3.1 \\
\ldots \\
\ldots \\
\ldots \\
\ldots\end{array}$ & $\begin{array}{l}\text { 4. } 7 M L(P S) \\
\text { 3.1ML (PS) } \\
\text { 3. } 3 \mathrm{MD}(\mathrm{GM}) \\
\text { 3. } 1 \mathrm{MD}(\mathrm{GM}) \\
\text { 3. } 1 \mathrm{ML}(\mathrm{PS})\end{array}$ & $\begin{array}{c}\text { FELT } \\
\ldots \\
\ldots \\
\ldots \\
\ldots\end{array}$ & $\begin{array}{l}\text { NOV. } \\
\text { NOV. } \\
\text { NOV. } \\
\text { NOV. } \\
\text { NOV. }\end{array}$ & $\begin{array}{l}25 \\
25 \\
25 \\
25 \\
26\end{array}$ & $\begin{array}{l}15: 10 \\
16: 58 \\
18: 14 \\
20: 22 \\
00: 18\end{array}$ & $\begin{array}{l}\text { PST } \\
\text { PST } \\
\text { PST } \\
\text { PST } \\
\text { PST }\end{array}$ \\
\hline $\begin{array}{l}\text { NOV. } \\
\text { NOV. } \\
\text { NOV. } \\
\text { NOV. } \\
\text { NOV. }\end{array}$ & $\begin{array}{l}26 \\
26 \\
26 \\
26 \\
26\end{array}$ & $\begin{array}{l}10 \\
16 \\
16 \\
16 \\
16\end{array}$ & $\begin{array}{l}31 \\
21 \\
21 \\
28 \\
31\end{array}$ & $\begin{array}{l}25.6 \\
41.5 \\
47.2 \\
29.2 \\
21.4\end{array}$ & $\begin{array}{l}37.422 \mathrm{~N} \\
37.448 \mathrm{~N} \\
37.448 \mathrm{~N} \\
37.460 \mathrm{~N} \\
37.422 \mathrm{~N}\end{array}$ & $\begin{array}{l}118.755 W . \\
118.652 W . \\
118.653 W . \\
118.647 W . \\
118.635 W .\end{array}$ & $\begin{array}{r}0 \\
9 \\
9 \\
8 \\
10\end{array}$ & $\begin{array}{l}\text { GM } \\
\text { BK } \\
\text { BK } \\
\text { GM } \\
\text { BK }\end{array}$ & $\begin{array}{l}\ldots \\
\ldots \\
\ldots \\
\ldots\end{array}$ & $\begin{array}{l}\cdots \\
\cdots \\
4.7 \\
\cdots \\
\cdots\end{array}$ & $\begin{array}{l}\text { 3. } 1 \mathrm{MD}(\mathrm{GM}) \\
\text { 3. } 9 \mathrm{ML}(\mathrm{BK}) \\
\text { 5. } 6 \mathrm{ML}(\mathrm{BK}) \\
\text { 3. } \mathrm{MLL}(\mathrm{BK}) \\
\text { 4. } \mathrm{ML}(\mathrm{BK})\end{array}$ & $\begin{array}{l}\cdots \\
\cdots \\
\cdots \\
\cdots\end{array}$ & $\begin{array}{l}\text { NOV. } \\
\text { NOV. } \\
\text { NOV. } \\
\text { NOV. } \\
\text { NOV. }\end{array}$ & $\begin{array}{l}26 \\
26 \\
26 \\
26 \\
26\end{array}$ & $\begin{array}{l}02: 31 \\
08: 21 \\
08: 21 \\
08: 28 \\
08: 31\end{array}$ & $\begin{array}{l}\text { PST } \\
\text { PST } \\
\text { PST } \\
\text { PST } \\
\text { PST }\end{array}$ \\
\hline
\end{tabular}


Table 1. Summary of U.S. earthquakes for 1984-Continued

\begin{tabular}{|c|c|c|c|c|c|c|c|c|c|c|c|c|c|}
\hline \multirow{2}{*}{ Date } & \multicolumn{2}{|c|}{$\begin{array}{c}\text { Origin time } \\
\text { (UTC) }\end{array}$} & \multirow{2}{*}{$\begin{array}{c}\text { Latitude } \\
\text { ( })\end{array}$} & \multirow{2}{*}{$\begin{array}{c}\text { Longitude } \\
\left({ }^{\circ}\right)\end{array}$} & \multirow{2}{*}{$\begin{array}{c}\text { Depth } \\
(\mathbf{k m})\end{array}$} & \multirow{2}{*}{$\begin{array}{l}\text { Ilypo- } \\
\text { center } \\
\text { source }\end{array}$} & \multicolumn{3}{|c|}{ Magnitude } & \multirow{2}{*}{$\begin{array}{l}\text { Maximum } \\
\text { intensity }\end{array}$} & \multicolumn{3}{|c|}{ lexal time } \\
\hline & hr $\min$ & $\sec$ & & & & & $\mathbf{m b}$ & MS & $\begin{array}{l}\text { ML, Mn } \\
M D, M w\end{array}$ & & Date & Hour & $\begin{array}{l}\text { Time } \\
\text { zone }\end{array}$ \\
\hline
\end{tabular}

CALIFORNIA-Continued

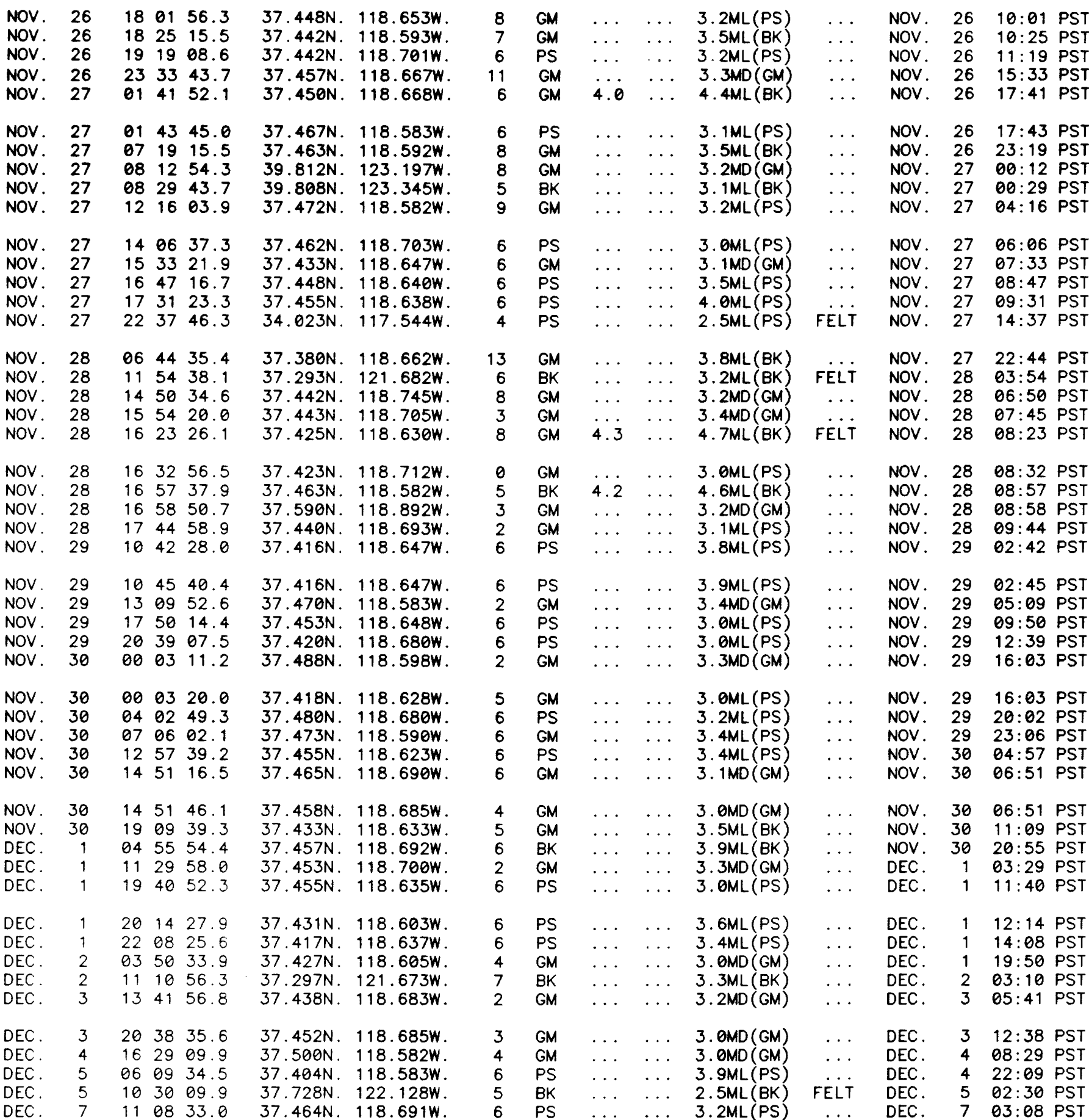


Table 1. Summary of U.S. earthquakes for 1984-Continued

\begin{tabular}{|c|c|c|c|c|c|c|c|c|c|c|c|c|}
\hline \multirow{2}{*}{ Date } & $\begin{array}{c}\begin{array}{c}\text { Origin time } \\
\text { (UTC) }\end{array} \\
\end{array}$ & \multirow{2}{*}{$\begin{array}{c}\text { Latitude } \\
\left({ }^{\circ}\right)\end{array}$} & \multirow{2}{*}{$\begin{array}{c}\text { Lomgitude } \\
\text { (9) }\end{array}$} & \multirow{2}{*}{$\begin{array}{l}\text { Depth } \\
(\mathbf{k m})\end{array}$} & \multirow{2}{*}{$\begin{array}{l}\text { Hypo- } \\
\text { center } \\
\text { source }\end{array}$} & \multicolumn{3}{|c|}{ Magnitude } & \multirow{2}{*}{$\begin{array}{l}\text { Maximum } \\
\text { intensity }\end{array}$} & \multicolumn{3}{|c|}{ Local time } \\
\hline & hr $\min \mathrm{sec}$ & & & & & $\mathbf{m b}$ & MS & $\begin{array}{l}\text { ML, Mn } \\
M D, M w\end{array}$ & & Date & Hour & $\begin{array}{l}\text { Time } \\
\text { Tone }\end{array}$ \\
\hline
\end{tabular}

CALIFORNIA-Continued

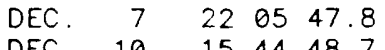

$\begin{array}{lllll}\text { DEC. } & 10 & 15 & 44 & 48.7\end{array}$

DEC. $12 \quad 07 \quad 36 \quad 37.9$

DEC. $13 \quad 1103 \quad 36.5$

DEC. $14 \quad 0923 \quad 57.0$

DEC. $\quad 14 \quad 19 \quad 33 \quad 23.5$

$\begin{array}{lllll}\text { DEC. } & 14 & 20 & 13 & 35.9\end{array}$

DEC. $\quad \begin{array}{llll}14 & 21 & 28 & 04.8\end{array}$

DEC. $15 \quad 06 \quad 19 \quad 41.1$

DEC. $17 \quad 12 \quad 27 \quad 24.1$

DEC. $\quad 17 \quad 21 \quad 17 \quad 23.6$

DEC. $19 \quad 0240 \quad 04.1$

DEC. $\quad 20 \quad 08 \quad 03 \quad 06.2$

DEC. $\quad 20 \quad 16 \quad 1959.7$

DEC. $20 \quad 2018 \quad 19 \quad 48.1$

DEC. $\quad 21 \quad 123904.8$

$\begin{array}{lllll}\text { DEC. } & 21 & 22 & 09 & 17.8\end{array}$

DEC. $22 \quad 02 \quad 09 \quad 23.5$

DEC. $23 \quad 04 \quad 45 \quad 20.9$

DEC. $23 \quad 165508.0$

DEC. $\quad 23 \quad 17 \quad 38 \quad 18.9$

DEC. $24 \quad 1922 \quad 29.4$

DEC. $\quad 25 \quad 0345 \quad 11.9$

DEC. $\quad 28 \quad 07 \quad 19 \quad 42.4$

DEC. $28 \quad 0908 \quad 08.2$

DEC. $\quad 29 \quad 16 \quad 21 \quad 13.1$
\end{abstract}

DEC. $\quad 29 \quad 21 \quad 42 \quad 25.9$
$37.428 \mathrm{~N}, 118.628 \mathrm{~W}$. $34.082 \mathrm{~N} .118 .105 \mathrm{~W}$. $37.445 \mathrm{~N} .118 .688 \mathrm{~W}$. $37.434 \mathrm{~N} .118 .661 \mathrm{~W}$. $40.357 \mathrm{~N} .124 .370 \mathrm{~W}$.

$37.467 \mathrm{~N} .118 .600 \mathrm{~W}$. $37.448 \mathrm{~N} .118 .593 \mathrm{~W}$. $33.890 \mathrm{~N} .117 .821 \mathrm{~W}$. $37.409 \mathrm{~N} .118 .616 \mathrm{~W}$. $37.457 \mathrm{~N} .118 .716 \mathrm{~W}$.

$37.425 \mathrm{~N} .118 .615 \mathrm{~W}$. $38.755 \mathrm{~N} .122 .587 \mathrm{~W}$. $37.428 \mathrm{~N} .118 .590 \mathrm{~W}$ $36.979 \mathrm{~N}, 116.006 \mathrm{~W}$ $37.462 \mathrm{~N} .118 .597 \mathrm{~W}$.

$36.520 \mathrm{~N} .121 .123 \mathrm{~W}$. $35.450 \mathrm{~N} .119 .807 \mathrm{~W}$. $40.467 \mathrm{~N} .124 .218 \mathrm{~W}$ $36.595 \mathrm{~N} .121 .112 \mathrm{~W}$. $37.431 \mathrm{~N} .118 .615 \mathrm{~W}$.

$37.454 \mathrm{~N} .118 .674 W$. $37.622 \mathrm{~N} .118 .863 \mathrm{~W}$. $37.453 \mathrm{~N} .118 .670 \mathrm{~W}$. $37.327 \mathrm{~N} .121 .718 \mathrm{~W}$ $40.658 \mathrm{~N}, 123.760 \mathrm{~W}$.

$38.837 \mathrm{~N} .122 .797 \mathrm{~W}$. $40.972 \mathrm{~N} .123 .550 \mathrm{~W}$.

$\begin{array}{rl}9 & \text { G } \\ 5 & P S \\ 6 & \text { PS } \\ 6 & \text { PS } \\ 5 & \text { BK } \\ & \\ 6 & P S \\ 15 & \text { GM } \\ 3 & \text { PS } \\ 6 & \text { PS } \\ 6 & \text { PS }\end{array}$

$\begin{array}{llll}\text { GM } & \ldots & \ldots & 3.1 \mathrm{MD}(\mathrm{GM}) \\ \text { PS } & \ldots & \ldots & 2.5 \mathrm{ML}(\mathrm{PS})\end{array}$

$\ldots$ 3.3ML(PS)

$\ldots$ 3.2ML(PS)

.. $\quad \ldots \quad 3.1 \mathrm{ML}(\mathrm{BK})$

.. $\quad \ldots \quad 3.6 \mathrm{ML}(\mathrm{PS})$

$\ldots \quad \ldots \quad$ 3.OMD (GM)

$\ldots \quad \ldots \quad 3.1 \mathrm{ML}(\mathrm{PS})$

$\begin{array}{lll}\cdots & \ldots & 3.1 M L(P S) \\ \cdots & \ldots & 3.1 M L(P S)\end{array}$

$\ldots$ 3.2ML(PS)

$\begin{array}{lll}\ldots & \ldots & 4.0 M L(P S) \\ \ldots & \ldots & 3.0 M L(B K)\end{array}$

$\begin{array}{lll}\ldots & \ldots & 3.0 \mathrm{ML}(\mathrm{BK}) \\ \ldots & \ldots & 3.5 \mathrm{ML}(\mathrm{BK})\end{array}$

$4.2 \quad \ldots .4 \% 4.2 M L(B K)$

$\ldots \quad \ldots .2 M D(G M)$

$\begin{array}{lll}\ldots & \ldots & 3.0 \mathrm{OL}(\mathrm{BK}) \\ \ldots & \ldots & 3.1 \mathrm{ML}(\mathrm{PS})\end{array}$

$\ldots$ 3.5ML(BK)

$\ldots \ldots$ 3.OML(BK)

3. $6 \mathrm{ML}$ (PS)

$\begin{array}{rllll}6 & \mathrm{PS} & \ldots & \ldots & 3.0 \mathrm{ML}(\mathrm{PS}) \\ 10 & \mathrm{GM} & \ldots & \ldots & 3.8 \mathrm{ML}(\mathrm{BK})\end{array}$

$\begin{array}{rllll}10 & \mathrm{GM} & \ldots & \ldots & 3.8 \mathrm{ML}(\mathrm{BK}) \\ 6 & \mathrm{GM} & \ldots & \ldots & 3.1 \mathrm{MD}(\mathrm{GM})\end{array}$

6 BK $\quad \ldots \quad \ldots l$ 3.8ML(BK)

5 GS

$\ldots$ 3.

3. OML (BK)

3. $1 \mathrm{ML}(\mathrm{BK})$
FEL $\dot{T}$

...

..

$\cdots$

$\ldots$

FELTT

...

$\cdots$

.

DEC. 18 18:40 PST

$\begin{array}{lll}\cdots & \text { DEC. } 20 \text { 00:03 PST }\end{array}$

... DEC. 20 08:19 PST

... DEC. $2010: 19$ PST

IV DEC. $21 \quad 04: 39$ PST

$\begin{array}{lll}\ldots & \text { DEC. } 21 & 14: 09 \\ & \text { PST }\end{array}$

$\begin{array}{lll}\ldots & \text { DEC. } 21 & 18: 09 \\ \text { PST }\end{array}$

‥ DEC. 22 20:45 PST

$\begin{array}{lll}\ldots & \text { DEC. } 23 \text { 08:55 PST }\end{array}$

$\begin{array}{lll}\ldots \text { DEC. } 23 & 09: 38 \text { PST }\end{array}$

II I DEC. $24 \quad 11: 22$ PST

$\begin{array}{lll}\ldots \text { DEC. } 24 & 19: 45 & \text { PST }\end{array}$

III DEC. $27 \quad 23: 19$ PST

$\begin{array}{lll}\ldots & \text { DEC. } 28 & 01: 08 \text { PST }\end{array}$

DEC. $29 \quad 08: 21$ PST DEC. 29 13:42 PST

\section{CALIFORNIA OFF THE COAST}

\begin{tabular}{|c|c|c|c|c|c|c|c|c|c|c|c|c|c|c|c|c|}
\hline $\begin{array}{l}\text { JAN. } \\
\text { JAN. } \\
\text { JAN. } \\
\text { JAN. } \\
\text { FEB. }\end{array}$ & $\begin{array}{r}17 \\
20 \\
30 \\
31 \\
7\end{array}$ & $\begin{array}{l}05 \\
07 \\
20 \\
16 \\
07\end{array}$ & $\begin{array}{l}34 \\
53 \\
15 \\
45 \\
52\end{array}$ & $\begin{array}{l}09.4 \\
17.2 \\
12.8 \\
40.7 \\
36.2\end{array}$ & $\begin{array}{l}40.375 \mathrm{~N} . \\
40.742 \mathrm{~N} . \\
40.820 \mathrm{~N} . \\
41.233 \mathrm{~N} . \\
33.865 \mathrm{~N} .\end{array}$ & $\begin{array}{l}126.877 w . \\
126.738 w . \\
124.718 w . \\
125.995 w . \\
120.307 w .\end{array}$ & $\begin{array}{r}5 \\
20 \\
5 \\
5 \\
6\end{array}$ & $\begin{array}{l}\text { BK } \\
\text { GM } \\
\text { BK } \\
\text { BK } \\
\text { PS }\end{array}$ & $\begin{array}{l}\cdots \\
\cdots \\
\cdots \\
\cdots \\
\cdots\end{array}$ & $\begin{array}{l}\cdots \\
\cdots \\
\cdots \\
\cdots \\
\cdots\end{array}$ & $\begin{array}{l}\text { 3. } 5 \mathrm{ML}(\mathrm{BK}) \\
\text { 3. } 2 \mathrm{MD}(\mathrm{GM}) \\
\text { 3. } 2 \mathrm{ML}(\mathrm{BK}) \\
\text { 3. } 5 \mathrm{ML}(\mathrm{BK}) \\
\text { 3. } 0 \mathrm{ML}(\mathrm{PS})\end{array}$ & $\begin{array}{l}\cdots \\
\cdots \\
\cdots \\
\cdots \\
\cdots\end{array}$ & $\begin{array}{l}\text { JAN. } \\
\text { JAN. } \\
\text { JAN. } \\
\text { JAN. } \\
\text { FEB. }\end{array}$ & $\begin{array}{r}16 \\
19 \\
30 \\
31 \\
6\end{array}$ & $\begin{array}{l}21: 34 \\
23: 53 \\
12: 15 \\
08: 45 \\
23: 52\end{array}$ & $\begin{array}{l}\text { PST } \\
\text { PST } \\
\text { PST } \\
\text { PST } \\
\text { PST }\end{array}$ \\
\hline $\begin{array}{l}\text { FEB. } \\
\text { FEB. } \\
\text { FEB. } \\
\text { FEB. } \\
\text { MAR. }\end{array}$ & $\begin{array}{l}10 \\
20 \\
27 \\
28 \\
15\end{array}$ & $\begin{array}{l}12 \\
01 \\
10 \\
15 \\
13\end{array}$ & $\begin{array}{l}33 \\
24 \\
18 \\
16 \\
35\end{array}$ & $\begin{array}{l}05.0 \\
34.3 \\
15.0 \\
06.7 \\
22.6\end{array}$ & $\begin{array}{l}37.637 \mathrm{~N} . \\
40.592 \mathrm{~N} . \\
33.471 \mathrm{~N} . \\
40.357 \mathrm{~N} . \\
41.087 \mathrm{~N} .\end{array}$ & $\begin{array}{l}125.560 w . \\
126.447 w . \\
118.061 w . \\
125.897 w . \\
124.673 w .\end{array}$ & $\begin{array}{l}8 \\
5 \\
6 \\
5 \\
5\end{array}$ & $\begin{array}{l}\text { GM } \\
\text { BK } \\
\text { PS } \\
\text { BK } \\
\text { BK }\end{array}$ & $\begin{array}{l}\cdots \\
\cdots \\
4.9 \\
\ldots\end{array}$ & $\begin{array}{l}\cdots \\
\cdots \\
\cdots \\
4.4 \\
\cdots\end{array}$ & $\begin{array}{l}\text { 3. } 8 \mathrm{MD}(\mathrm{GM}) \\
\text { 3. } 6 \mathrm{ML}(\mathrm{BK}) \\
\text { 4. } 0 \mathrm{ML}(\mathrm{PS}) \\
\text { 5. } 2 \mathrm{ML}(\mathrm{BK}) \\
\text { 3. } 6 \mathrm{ML}(\mathrm{BK})\end{array}$ & $\begin{array}{l}\cdots \\
\cdots \\
\text { IV } \\
\cdots\end{array}$ & $\begin{array}{l}\text { FEB. } \\
\text { FEB. } \\
\text { FEB. } \\
\text { FEB. } \\
\text { MAR. }\end{array}$ & $\begin{array}{l}10 \\
19 \\
27 \\
28 \\
15\end{array}$ & $\begin{array}{l}04: 33 \\
17: 24 \\
02: 18 \\
07: 16 \\
05: 35\end{array}$ & $\begin{array}{l}\text { PST } \\
\text { PST } \\
\text { PST } \\
\text { PST } \\
\text { PST }\end{array}$ \\
\hline $\begin{array}{l}\text { MAR. } \\
\text { APR. } \\
\text { APR. } \\
\text { APR. } \\
\text { APR. }\end{array}$ & $\begin{array}{r}23 \\
3 \\
18 \\
18 \\
25\end{array}$ & $\begin{array}{l}17 \\
13 \\
03 \\
23 \\
18\end{array}$ & $\begin{array}{l}58 \\
08 \\
30 \\
26 \\
03\end{array}$ & $\begin{array}{l}26.2 \\
23.9 \\
32.5 \\
02.7 \\
23.1\end{array}$ & $\begin{array}{l}41.448 \mathrm{~N} \\
40.502 \mathrm{~N} \\
40.435 \mathrm{~N} \\
40.413 \mathrm{~N} \\
41.727 \mathrm{~N}\end{array}$ & $\begin{array}{l}125.865 W . \\
126.010 W . \\
126.080 W . \\
125.212 W . \\
126.008 W .\end{array}$ & $\begin{array}{r}5 \\
20 \\
5 \\
5 \\
20\end{array}$ & $\begin{array}{l}\text { BK } \\
\text { GM } \\
\text { BK } \\
\text { BK } \\
\text { GM }\end{array}$ & $\begin{array}{l}\cdots \\
\cdots \\
\cdots \\
\ldots\end{array}$ & $\begin{array}{l}\cdots \\
\cdots \\
\cdots \\
\cdots \\
\cdots\end{array}$ & $\begin{array}{l}\text { 3. } 7 M L(B K) \\
\text { 3. } 9 M D(G M) \\
\text { 3. } 7 M L(B K) \\
\text { 3. } 9 M L(B K) \\
\text { 3. } 3 M D(G M)\end{array}$ & $\begin{array}{l}\cdots \\
\cdots \\
\cdots \\
\cdots \\
\cdots\end{array}$ & $\begin{array}{l}\text { MAR. } \\
\text { APR. } \\
\text { APR. } \\
\text { APR. } \\
\text { APR. }\end{array}$ & $\begin{array}{r}23 \\
3 \\
17 \\
18 \\
25\end{array}$ & $\begin{array}{l}09: 58 \\
05: 08 \\
19: 30 \\
15: 26 \\
10: 03\end{array}$ & $\begin{array}{l}\text { PST } \\
\text { PST } \\
\text { PST } \\
\text { PST } \\
\text { PST }\end{array}$ \\
\hline $\begin{array}{l}\text { MAY } \\
\text { MAY } \\
\text { JUNE } \\
\text { JUNE } \\
\text { JUNE }\end{array}$ & $\begin{array}{r}12 \\
13 \\
2 \\
11 \\
24\end{array}$ & $\begin{array}{l}20 \\
23 \\
10 \\
18 \\
02\end{array}$ & $\begin{array}{l}26 \\
51 \\
20 \\
01 \\
13\end{array}$ & $\begin{array}{l}38.6 \\
52.5 \\
47.8 \\
35.8 \\
27.6\end{array}$ & $\begin{array}{l}41.720 \mathrm{~N} . \\
40.487 \mathrm{~N} . \\
40.300 \mathrm{~N} \\
40.428 \mathrm{~N} \\
40.438 \mathrm{~N}\end{array}$ & $\begin{array}{l}125.885 W . \\
125.713 W . \\
124.738 W . \\
125.712 W . \\
125.217 W .\end{array}$ & $\begin{array}{r}20 \\
5 \\
11 \\
2 \\
17\end{array}$ & $\begin{array}{l}\text { GM } \\
\text { BK } \\
\text { GM } \\
\text { BK } \\
\text { GM }\end{array}$ & $\begin{array}{l}3.8 \\
3.9 \\
\ldots \\
\ldots \\
\ldots\end{array}$ & $\begin{array}{l}\cdots \\
\cdots \\
\cdots \\
\cdots\end{array}$ & $\begin{array}{l}\text { 3. } 1 M D(G M) \\
\text { 4. } 3 M L(B K) \\
\text { 3. } 9 M D(G M) \\
\text { 3. } 5 M L(B K) \\
\text { 3. } 4 M L(B K)\end{array}$ & $\begin{array}{l}\cdots \\
\cdots \\
\cdots \\
\cdots \\
\cdots\end{array}$ & $\begin{array}{l}\text { MAY } \\
\text { MAY } \\
\text { JUNE } \\
\text { JUNE } \\
\text { JUNE }\end{array}$ & $\begin{array}{r}12 \\
13 \\
2 \\
11 \\
23\end{array}$ & $\begin{array}{l}12: 26 \\
15: 51 \\
02: 20 \\
10: 01 \\
18: 13\end{array}$ & $\begin{array}{l}\text { PST } \\
\text { PST } \\
\text { PST } \\
\text { PST } \\
\text { PST }\end{array}$ \\
\hline
\end{tabular}


Table 1. Summary of U.S. earthquakes for 1984-Continued

\begin{tabular}{|c|c|c|c|c|c|c|c|c|c|c|c|c|c|}
\hline \multirow{2}{*}{ Date } & \multicolumn{2}{|c|}{$\begin{array}{c}\text { Origin time } \\
\text { (UTC) }\end{array}$} & \multirow{2}{*}{$\begin{array}{c}\text { Latitude } \\
\left(^{\circ}\right)\end{array}$} & \multirow{2}{*}{$\begin{array}{l}\text { Longitude } \\
\left.\text { ( }{ }^{\circ}\right)\end{array}$} & \multirow{2}{*}{$\begin{array}{l}\text { Depth } \\
(\mathbf{k m})\end{array}$} & \multirow{2}{*}{$\begin{array}{l}\text { Hypo- } \\
\text { center } \\
\text { source }\end{array}$} & \multicolumn{3}{|c|}{ Magnitude } & \multirow{2}{*}{$\begin{array}{l}\text { Maximum } \\
\text { intensity }\end{array}$} & \multicolumn{3}{|c|}{ Lexal time } \\
\hline & hr $\min$ & sec & & & & & $\mathbf{m b}$ & MS & $\begin{array}{l}\text { ML. Mn } \\
\text { MD. Mw }\end{array}$ & & Date & llour & $\begin{array}{l}\text { Time } \\
\text { 7one }\end{array}$ \\
\hline
\end{tabular}

CALIFORNIA OFF THE COAST-Continued

\begin{tabular}{|c|c|c|c|c|c|c|c|c|c|c|c|c|c|c|c|c|}
\hline $\begin{array}{l}\text { JUNE } \\
\text { JUNE } \\
\text { JUNE } \\
\text { JULY } \\
\text { JULY }\end{array}$ & $\begin{array}{r}25 \\
29 \\
29 \\
2 \\
28\end{array}$ & $\begin{array}{l}01 \\
06 \\
16 \\
19 \\
06\end{array}$ & $\begin{array}{l}24 \\
46 \\
20 \\
16 \\
30\end{array}$ & $\begin{array}{l}47.8 \\
40.1 \\
15.0 \\
55.2 \\
06.4\end{array}$ & $\begin{array}{l}41.123 \mathrm{~N} . \\
40.275 \mathrm{~N} . \\
40.470 \mathrm{~N} . \\
32.459 \mathrm{~N} . \\
40.558 \mathrm{~N} .\end{array}$ & $\begin{array}{l}124.975 w . \\
125.512 W . \\
124.860 W . \\
118.055 W . \\
124.748 w .\end{array}$ & $\begin{array}{r}3 \\
20 \\
5 \\
6 \\
11\end{array}$ & $\begin{array}{l}\text { BK } \\
\text { GM } \\
\text { BK } \\
\text { PS } \\
\text { BK }\end{array}$ & $\begin{array}{l}\cdots \\
\cdots \\
\cdots \\
\cdots \\
\cdots\end{array}$ & $\begin{array}{l}\cdots \\
\cdots \\
\cdots \\
\cdots\end{array}$ & $\begin{array}{l}\text { 3. } 3 M L(B K) \\
\text { 3. } 7 M D(G M) \\
\text { 3. } 1 \mathrm{ML}(\mathrm{BK}) \\
\text { 3. } 5 \mathrm{ML}(\mathrm{PS}) \\
\text { 3. } 3 \mathrm{ML}(\mathrm{BK})\end{array}$ & $\begin{array}{l}\cdots \\
\cdots \\
\cdots \\
\cdots \\
\cdots\end{array}$ & $\begin{array}{l}\text { JUNE } \\
\text { JUNE } \\
\text { JUNE } \\
\text { JULY } \\
\text { JULY }\end{array}$ & $\begin{array}{r}24 \\
28 \\
29 \\
2 \\
27\end{array}$ & $\begin{array}{l}17: 24 \\
22: 46 \\
08: 20 \\
11: 16 \\
22: 29\end{array}$ & $\begin{array}{l}\text { PST } \\
\text { PST } \\
\text { PST } \\
\text { PST } \\
\text { PST }\end{array}$ \\
\hline $\begin{array}{l}\text { AUG. } \\
\text { AUG. } \\
\text { AUG. } \\
\text { AUG. } \\
\text { AUG. }\end{array}$ & $\begin{array}{l}4 \\
4 \\
4 \\
4 \\
4\end{array}$ & $\begin{array}{l}01 \\
01 \\
03 \\
05 \\
21\end{array}$ & $\begin{array}{l}38 \\
38 \\
00 \\
29 \\
45\end{array}$ & $\begin{array}{l}49.0 \\
49.0 \\
53.9 \\
45.0 \\
53.2\end{array}$ & $\begin{array}{l}40.392 \mathrm{~N} \\
40.580 \mathrm{~N} \\
40.428 \mathrm{~N} \\
40.418 \mathrm{~N} . \\
40.255 \mathrm{~N} .\end{array}$ & $\begin{array}{l}125.415 w . \\
125.370 w . \\
125.335 w . \\
126.987 w . \\
124.578 w .\end{array}$ & $\begin{array}{r}15 \\
5 \\
5 \\
20 \\
5\end{array}$ & $\begin{array}{l}\text { GM } \\
\text { BK } \\
\text { BK } \\
\text { GM } \\
\text { BK }\end{array}$ & $\begin{array}{l}\cdots \\
\cdots \\
\cdots\end{array}$ & $\begin{array}{l}\ldots \\
\ldots \\
\cdots \\
\cdots \\
4.6\end{array}$ & $\begin{array}{l}\text { 3. } 2 \mathrm{MD}(\mathrm{GM}) \\
\text { 3. } 5 \mathrm{ML}(\mathrm{BK}) \\
\text { 4. } O \mathrm{ML}(\mathrm{BK}) \\
\text { 3. } 3 \mathrm{MD}(\mathrm{GM}) \\
\text { 4. } 7 \mathrm{ML}(\mathrm{BK})\end{array}$ & $\begin{array}{l}\cdots \\
\cdots \\
\cdots \\
\cdots\end{array}$ & $\begin{array}{l}A \cup G . \\
A \cup G . \\
A \cup G . \\
A \cup G . \\
A \cup G .\end{array}$ & $\begin{array}{l}3 \\
3 \\
3 \\
3 \\
4\end{array}$ & $\begin{array}{l}17: 38 \\
17: 38 \\
19: 00 \\
21: 29 \\
13: 45\end{array}$ & $\begin{array}{l}\text { PST } \\
\text { PST } \\
\text { PST } \\
\text { PST } \\
\text { PST }\end{array}$ \\
\hline $\begin{array}{l}\text { AUG. } \\
\text { AUG. } \\
\text { AUG. } \\
\text { SEPT. } \\
\text { SEPT. }\end{array}$ & $\begin{array}{r}25 \\
26 \\
27 \\
7 \\
7\end{array}$ & $\begin{array}{l}19 \\
03 \\
07 \\
06 \\
11\end{array}$ & $\begin{array}{l}12 \\
24 \\
53 \\
50 \\
03\end{array}$ & $\begin{array}{l}38.0 \\
18.4 \\
14.7 \\
37.5 \\
13.4\end{array}$ & $\begin{array}{l}32.758 \mathrm{~N} \\
40.398 \mathrm{~N} \\
32.697 \mathrm{~N} \\
32.798 \mathrm{~N} \\
32.945 \mathrm{~N}\end{array}$ & $\begin{array}{l}118.163 w . \\
125.590 W . \\
118.184 W . \\
118.138 w . \\
117.806 w .\end{array}$ & $\begin{array}{r}6 \\
20 \\
6 \\
6 \\
6\end{array}$ & $\begin{array}{l}\text { PS } \\
\text { GM } \\
\text { PS } \\
\text { PS } \\
\text { PS }\end{array}$ & $\begin{array}{l}3.9 \\
\ldots \\
4.8\end{array}$ & $\begin{array}{l}\cdots \\
\cdots \\
\cdots \\
\cdots\end{array}$ & $\begin{array}{l}\text { 3. } 3 M L(P S) \\
\text { 3. } 7 M L(B K) \\
\text { 3. } 3 M L(P S) \\
\text { 3. } 6 \mathrm{ML}(P S) \\
\text { 4. } 3 \mathrm{ML}(P S)\end{array}$ & $\begin{array}{l}\ldots \\
\ldots \\
\cdots \\
\text { IV }\end{array}$ & $\begin{array}{l}\text { AUG. } \\
\text { AUG. } \\
\text { AUG. } \\
\text { SEPT. } \\
\text { SEPT. }\end{array}$ & $\begin{array}{r}25 \\
25 \\
26 \\
6 \\
7\end{array}$ & $\begin{array}{l}11: 12 \\
19: 24 \\
23: 53 \\
22: 50 \\
03: 03\end{array}$ & $\begin{array}{l}\text { PST } \\
\text { PST } \\
\text { PST } \\
\text { PST } \\
\text { PST }\end{array}$ \\
\hline $\begin{array}{l}\text { SEPT. } \\
\text { SEPT. } \\
\text { SEPT. } \\
\text { SEPT. } \\
\text { SEPT. }\end{array}$ & $\begin{array}{l}10 \\
10 \\
10 \\
10 \\
10\end{array}$ & $\begin{array}{l}01 \\
03 \\
03 \\
03 \\
06\end{array}$ & $\begin{array}{l}53 \\
14 \\
29 \\
43 \\
43\end{array}$ & $\begin{array}{l}53.4 \\
10.1 \\
43.2 \\
45.8 \\
27.6\end{array}$ & $\begin{array}{l}40.693 \mathrm{~N} \\
40.503 \mathrm{~N} . \\
41.109 \mathrm{~N} . \\
40.787 \mathrm{~N} \\
40.296 \mathrm{~N} .\end{array}$ & $\begin{array}{l}124.657 w . \\
126.831 w . \\
129.390 w . \\
127.201 w . \\
126.903 w .\end{array}$ & $\begin{array}{r}5 \\
10 \\
10 \\
10 \\
10\end{array}$ & $\begin{array}{l}\text { BK } \\
\text { GS } \\
\text { GS } \\
\text { GS } \\
\text { GS }\end{array}$ & $\begin{array}{l}6.1 \\
\ldots \\
\ldots \\
4.5\end{array}$ & $\begin{array}{l}6.7 \\
\ldots \\
\ldots \\
\ldots\end{array}$ & $\begin{array}{l}\text { 3. } 2 M L(B K) \\
\text { 6. } 6 \mathrm{ML}(\mathrm{BK}) \\
\text { 3. } 5 \mathrm{ML}(\mathrm{BK}) \\
\text { 3. } 3 \mathrm{ML}(\mathrm{BK}) \\
\text { 4. } 1 \mathrm{ML}(\mathrm{BK})\end{array}$ & $\begin{array}{l}\cdots \\
\cdots \\
\cdots \\
\cdots\end{array}$ & $\begin{array}{l}\text { SEPT. } \\
\text { SEPT. } \\
\text { SEPT. } \\
\text { SEPT. } \\
\text { SEPT. }\end{array}$ & $\begin{array}{l}9 \\
9 \\
9 \\
9 \\
9\end{array}$ & $\begin{array}{l}17: 53 \\
19: 14 \\
19: 29 \\
19: 43 \\
22: 43\end{array}$ & $\begin{array}{l}\text { PST } \\
\text { PST } \\
\text { PST } \\
\text { PST } \\
\text { PST }\end{array}$ \\
\hline $\begin{array}{l}\text { SEPT. } \\
\text { SEPT. } \\
\text { SEPT. } \\
\text { SEPT. } \\
\text { SEPT. }\end{array}$ & $\begin{array}{l}10 \\
10 \\
10 \\
10 \\
10\end{array}$ & $\begin{array}{l}06 \\
07 \\
07 \\
09 \\
23\end{array}$ & $\begin{array}{l}58 \\
19 \\
47 \\
31 \\
52\end{array}$ & $\begin{array}{l}42.4 \\
34.1 \\
29.7 \\
49.6 \\
30.9\end{array}$ & $\begin{array}{l}40.324 \mathrm{~N} \\
40.315 \mathrm{~N} . \\
40.311 \mathrm{~N} . \\
40.171 \mathrm{~N} . \\
40.395 \mathrm{~N} .\end{array}$ & $\begin{array}{l}127.064 w . \\
127.089 w . \\
127.123 w . \\
126.857 w . \\
126.895 w .\end{array}$ & $\begin{array}{l}10 \\
10 \\
10 \\
10 \\
10\end{array}$ & $\begin{array}{l}\text { GS } \\
\text { GS } \\
\text { GS } \\
\text { GS } \\
\text { GS }\end{array}$ & $\begin{array}{l}4.2 \\
4.6 \\
4.7 \\
3.3 \\
4.9\end{array}$ & $\begin{array}{l}\ldots \\
\ldots \\
\ldots \\
3.8\end{array}$ & $\begin{array}{l}\text { 4. } 0 \mathrm{ML}(\mathrm{BK}) \\
\text { 3. } 7 \mathrm{ML}(\mathrm{BK}) \\
\text { 4. } 5 \mathrm{ML}(\mathrm{BK}) \\
\text { 3. } 3 \mathrm{ML}(\mathrm{BK}) \\
\text { 4. } 7 \mathrm{ML}(\mathrm{BK})\end{array}$ & $\begin{array}{l}\cdots \\
\cdots \\
\cdots \\
\cdots \\
\cdots\end{array}$ & $\begin{array}{l}\text { SEPT. } \\
\text { SEPT. } \\
\text { SEPT. } \\
\text { SEPT. } \\
\text { SEPT. }\end{array}$ & $\begin{array}{r}9 \\
9 \\
9 \\
10 \\
10\end{array}$ & $\begin{array}{l}22: 58 \\
23: 19 \\
23: 47 \\
01: 31 \\
15: 52\end{array}$ & $\begin{array}{l}\text { PST } \\
\text { PST } \\
\text { PST } \\
\text { PST } \\
\text { PST }\end{array}$ \\
\hline $\begin{array}{l}\text { SEPT. } \\
\text { SEPT. } \\
\text { SEPT. } \\
\text { SEPT. } \\
\text { SEPT. }\end{array}$ & $\begin{array}{l}11 \\
11 \\
11 \\
14 \\
14\end{array}$ & $\begin{array}{l}01 \\
11 \\
18 \\
09 \\
17\end{array}$ & $\begin{array}{l}31 \\
23 \\
43 \\
09 \\
43\end{array}$ & $\begin{array}{l}58.8 \\
08.3 \\
54.4 \\
24.3 \\
41.3\end{array}$ & $\begin{array}{l}40.499 \mathrm{~N} \\
40.353 \mathrm{~N} \\
40.364 \mathrm{~N} \\
40.322 \mathrm{~N} \\
40.455 \mathrm{~N}\end{array}$ & $\begin{array}{l}126.172 W . \\
126.993 w . \\
126.982 W . \\
127.216 W . \\
127.859 W .\end{array}$ & $\begin{array}{l}10 \\
10 \\
10 \\
10 \\
10\end{array}$ & $\begin{array}{l}\text { GS } \\
\text { GS } \\
\text { GS } \\
\text { GS } \\
\text { GS }\end{array}$ & $\begin{array}{l}4.2 \\
5.0 \\
4.5 \\
4.2 \\
\ldots .\end{array}$ & $\begin{array}{l}4.3 \\
\ldots \\
\ldots \\
\ldots\end{array}$ & $\begin{array}{l}\text { 4. } 2 M L(B K) \\
\text { 4. } 6 \mathrm{ML}(\mathrm{BK}) \\
\text { 4. } 3 \mathrm{ML}(\mathrm{BK}) \\
\text { 4. } 1 \mathrm{ML}(\mathrm{BK}) \\
\text { 3. } 5 \mathrm{ML}(\mathrm{BK})\end{array}$ & $\begin{array}{l}\ldots \\
\cdots \\
\cdots \\
\cdots \\
\cdots\end{array}$ & $\begin{array}{l}\text { SEPT. } \\
\text { SEPT. } \\
\text { SEPT. } \\
\text { SEPT. } \\
\text { SEPT. }\end{array}$ & $\begin{array}{l}10 \\
11 \\
11 \\
14 \\
14\end{array}$ & $\begin{array}{l}17: 31 \\
03: 23 \\
10: 43 \\
01: 09 \\
09: 43\end{array}$ & $\begin{array}{l}\text { PST } \\
\text { PST } \\
\text { PST } \\
\text { PST } \\
\text { PST }\end{array}$ \\
\hline $\begin{array}{l}\text { SEPT. } \\
\text { SEPT. } \\
\text { SEPT. } \\
\text { SEPT. } \\
\text { SEPT. }\end{array}$ & $\begin{array}{l}16 \\
20 \\
20 \\
22 \\
23\end{array}$ & $\begin{array}{l}21 \\
18 \\
19 \\
15 \\
23\end{array}$ & $\begin{array}{l}36 \\
30 \\
04 \\
10 \\
36\end{array}$ & $\begin{array}{l}24.7 \\
42.5 \\
35.5 \\
28.8 \\
46.3\end{array}$ & $\begin{array}{l}40.343 \mathrm{~N} . \\
40.382 \mathrm{~N} . \\
40.276 \mathrm{~N} . \\
40.405 \mathrm{~N} . \\
32.766 \mathrm{~N} .\end{array}$ & $\begin{array}{l}127.215 W . \\
125.617 W . \\
127.205 W . \\
125.063 W . \\
118.162 W .\end{array}$ & $\begin{array}{r}10 \\
5 \\
10 \\
5 \\
6\end{array}$ & $\begin{array}{l}\text { GS } \\
\text { BK } \\
\text { GS } \\
\text { BK } \\
\text { PS }\end{array}$ & $\begin{array}{l}4.2 \\
4.7 \\
4.5 \\
\ldots \\
\ldots\end{array}$ & $\begin{array}{l}\cdots \\
\cdots \\
\cdots \\
\cdots\end{array}$ & $\begin{array}{l}4.8 M L(B K) \\
4.8 M L(B K) \\
4.8 M L(B K) \\
\text { 3. } 3 \mathrm{ML}(B K) \\
\text { 3. } 1 \mathrm{ML}(\mathrm{PS})\end{array}$ & $\begin{array}{l}\text { IV } \\
\text { I I I } \\
\ldots \\
\ldots\end{array}$ & $\begin{array}{l}\text { SEPT. } \\
\text { SEPT. } \\
\text { SEPT. } \\
\text { SEPT. } \\
\text { SEPT. }\end{array}$ & $\begin{array}{l}16 \\
20 \\
20 \\
22 \\
23\end{array}$ & $\begin{array}{l}13: 36 \\
10: 30 \\
11: 04 \\
07: 10 \\
15: 36\end{array}$ & $\begin{array}{l}\text { PST } \\
\text { PST } \\
\text { PST } \\
\text { PST } \\
\text { PST }\end{array}$ \\
\hline $\begin{array}{l}\text { SEPT. } \\
\text { NOV. } \\
\text { NOV. } \\
\text { OEC. } \\
\text { DEC. }\end{array}$ & $\begin{array}{r}26 \\
3 \\
30 \\
4 \\
11\end{array}$ & $\begin{array}{l}10 \\
08 \\
01 \\
21 \\
05\end{array}$ & $\begin{array}{l}12 \\
51 \\
53 \\
53 \\
54\end{array}$ & $\begin{array}{l}59.6 \\
46.8 \\
38.9 \\
36.5 \\
36.2\end{array}$ & $\begin{array}{l}40.287 \mathrm{~N} \\
40.415 \mathrm{~N} \\
40.201 \mathrm{~N} \\
41.852 \mathrm{~N} \\
40.522 \mathrm{~N}\end{array}$ & $\begin{array}{l}126.886 w . \\
125.187 W . \\
127.084 W . \\
126.815 W . \\
127.645 W .\end{array}$ & $\begin{array}{r}10 \\
5 \\
10 \\
10 \\
10\end{array}$ & $\begin{array}{l}\text { GS } \\
\text { BK } \\
\text { GS } \\
\text { GS } \\
\text { GS }\end{array}$ & $\begin{array}{l}\cdots \\
\cdots .2 \\
4.2 \\
4.1\end{array}$ & $\begin{array}{l}\ldots \\
\ldots \\
\ldots \\
3.2\end{array}$ & $\begin{array}{c}\text { 4. } \theta \mathrm{ML}(\mathrm{BK}) \\
\text { 3. } \mathrm{ML}(\mathrm{BK}) \\
\text { 4. } 2 \mathrm{ML}(\mathrm{BK}) \\
\ldots \ldots \\
\ldots\end{array}$ & $\begin{array}{l}\ldots \\
\cdots \\
\cdots \\
\cdots \\
\cdots\end{array}$ & $\begin{array}{l}\text { SEPT. } \\
\text { NOV. } \\
\text { NOV. } \\
\text { DEC. } \\
\text { DEC. }\end{array}$ & $\begin{array}{r}26 \\
3 \\
29 \\
4 \\
10\end{array}$ & $\begin{array}{l}02: 12 \\
00: 51 \\
17: 53 \\
13: 53 \\
21: 54\end{array}$ & $\begin{array}{l}\text { PST } \\
\text { PST } \\
\text { PST } \\
\text { PST } \\
\text { PST }\end{array}$ \\
\hline $\begin{array}{l}\text { DEC. } \\
\text { DEC. }\end{array}$ & $\begin{array}{l}11 \\
31\end{array}$ & $\begin{array}{l}06 \\
10\end{array}$ & $\begin{array}{l}26 \\
35\end{array}$ & $\begin{array}{l}45.4 \\
42.7\end{array}$ & $\begin{array}{l}40.465 \mathrm{~N} . \\
40.388 \mathrm{~N} .\end{array}$ & $\begin{array}{l}126.744 W . \\
127.762 W .\end{array}$ & $\begin{array}{l}10 \\
10\end{array}$ & $\begin{array}{l}\text { GS } \\
\text { GS }\end{array}$ & $\begin{array}{l}3.9 \\
\ldots\end{array}$ & $\cdots$ & 3. $5 \mathrm{ML}(\mathrm{BK})$ & $\cdots$ & $\begin{array}{l}\text { DEC. } \\
\text { DEC. }\end{array}$ & $\begin{array}{l}10 \\
31\end{array}$ & $\begin{array}{l}22: 26 \\
02: 35\end{array}$ & $\begin{array}{l}\text { PST } \\
\text { PST }\end{array}$ \\
\hline
\end{tabular}

\section{COLORADO}

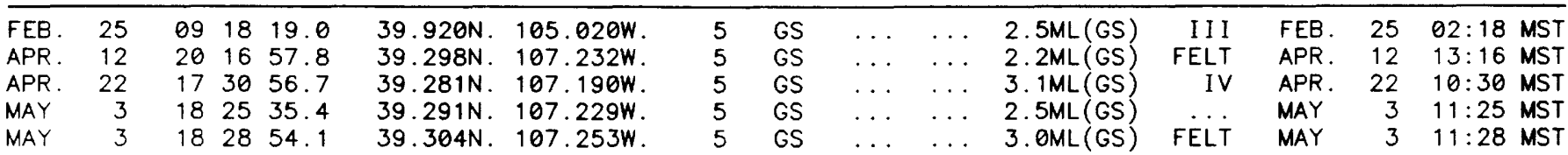


Table 1. Summary of U.S. earthquakes for 1984-Continued

\begin{tabular}{|c|c|c|c|c|c|c|c|c|c|c|c|c|c|}
\hline \multirow{2}{*}{ Date } & \multicolumn{2}{|c|}{$\begin{array}{l}\text { Origin time } \\
\text { (UTC) }\end{array}$} & \multirow{2}{*}{$\begin{array}{c}\text { Latitude } \\
\left({ }^{\circ}\right)\end{array}$} & \multirow{2}{*}{$\begin{array}{l}\text { Lompitude } \\
\text { (i) }\end{array}$} & \multirow{2}{*}{$\begin{array}{c}\text { Depth } \\
\text { (km) }\end{array}$} & \multirow{2}{*}{$\begin{array}{l}\text { Hypo- } \\
\text { center } \\
\text { source }\end{array}$} & \multicolumn{3}{|c|}{ Magnitude } & \multirow{2}{*}{$\begin{array}{l}\text { Maximum } \\
\text { intensity }\end{array}$} & \multicolumn{3}{|c|}{ Local time } \\
\hline & hr $\min$ & $\sec$ & & & & & mb & MS & $\begin{array}{l}\text { ML. Mn } \\
M D, M w\end{array}$ & & Date & Hour & $\begin{array}{l}\text { Time } \\
\text { rome }\end{array}$ \\
\hline
\end{tabular}

$\begin{array}{lllll}\text { MAY } & 3 & 19 & 18 & 23.0 \\ \text { MAY } & 4 & 01 & 17 & 10.4 \\ \text { MAY } & 4 & 02 & 13 & 33.1 \\ \text { MAY } & 4 & 18 & 44 & 37.4 \\ \text { MAY } & 6 & 02 & 00 & 56.6 \\ \text { MAY } & 6 & 02 & 12 & 49.7 \\ \text { MAY } & 6 & 02 & 13 & 34.4 \\ \text { MAY } & 6 & 02 & 51 & 36.9 \\ \text { MAY } & 6 & 04 & 17 & 35.5 \\ \text { MAY } & 6 & 04 & 21 & 37.7 \\ \text { MAY } & 10 & 01 & 20 & 14.9 \\ \text { MAY } & 10 & 01 & 53 & 51.8 \\ \text { MAY } & 11 & 13 & 34 & 57.9 \\ \text { MAY } & 14 & 10 & 14 & 17.2 \\ \text { MAY } & 17 & 09 & 11 & 20.2 \\ \text { MAY } & 27 & 23 & 30 & 19.3\end{array}$

$\begin{array}{lllll}\text { MAYNE } & 12 & 04 & 48 & 54.1\end{array}$
39.331 N. 107.253W. $39.334 \mathrm{~N} .107 .274 \mathrm{~W}$. $39.340 \mathrm{~N} .107 .245 \mathrm{~W}$. $39.283 \mathrm{~N} .107 .203 \mathrm{~W}$. $39.335 \mathrm{~N} .107 .249 \mathrm{~W}$.

39.339N. 107.248W. $39.349 \mathrm{~N} .107 .262 \mathrm{~W}$. $39.331 \mathrm{~N} .107 .232 \mathrm{~W}$. $39.354 \mathrm{~N} .107 .258 \mathrm{~W}$. $39.329 \mathrm{~N} .107 .239 \mathrm{~W}$.

$39.346 \mathrm{~N} .107 .249 \mathrm{~W}$. $39.360 \mathrm{~N} .107 .303 \mathrm{~W}$. $39.336 \mathrm{~N} .107 .259 \mathrm{~W}$. $39.322 \mathrm{~N} .107 .228 \mathrm{~W}$. 39.337 N. $107.245 W$.

$39.220 \mathrm{~N} .102 .155 \mathrm{~W}$. 39.143 N. $107.394 W$.
5 GS

5 GS

5 GS

5 GS

5 GS

5 GS

$\begin{array}{ll}5 & G S \\ 5 & G S\end{array}$

$\begin{array}{ll}5 & \text { GS } \\ 5 & \text { GS }\end{array}$

5 GS

5 GS

$\begin{array}{ll}5 & G S \\ 5 & G S\end{array}$

5 GS

5 GS

5 GS

1 GS $\ldots \quad \ldots 2.3 M L(G S)$

$\begin{array}{lll}\ldots & \ldots & 2.3 M L(G S) \\ \ldots & \ldots & 2.4 M L(G S) \\ \ldots & \ldots & 2.2 M L(G S)\end{array}$

$\ldots \quad \ldots 2.2 \mathrm{ML}(\mathrm{GS})$

$\ldots \quad \ldots 2.1 \mathrm{ML}(\mathrm{GS})$

.. $\quad \ldots \quad 2.3 \mathrm{ML}(\mathrm{GS})$

$\ldots \quad \ldots 2.6 \mathrm{ML}$ (GS)

$\ldots \quad \ldots \quad 2.2 \mathrm{ML}(\mathrm{GS})$

$\ldots \ldots 2.5 \mathrm{ML}$ (GS)

$\ldots \quad \ldots 2.7 \mathrm{ML}(\mathrm{GS})$

$\ldots \quad \ldots \quad 1.9 \mathrm{ML}$ (GS)

$\ldots \quad \ldots \quad 2.4 M L(G S)$

$\ldots \quad \ldots 2.3 M L(G S)$

$\ldots$ 3. $\ldots$ ML (GS)

.. $\ldots 2.4 \mathrm{ML}$ (GS)

$\ldots \quad \ldots \quad 3.6 \mathrm{ML}$ (GS)

$\ldots$ 3. OML (GS) $\begin{array}{llll}\text {... MAY } 3 & 12: 18 \text { MST }\end{array}$

FELT MAY 3 18:17 MST

FELT MAY 3 19:13 MST

‥ MAY 4 11:44 MST

FELT MAY 5 19:00 MST

FELT MAY 5 19:12 MST

FELT MAY 5 19:13 MST

FELT MAY 5 19:51 MST

... MAY 5 21:17 MST

... MAY 5 21:21 MST

.. MAY 9 18:20 MST

... MAY 9 18:53 MST

... MAY $11 \quad 06: 34$ MST

IV MAY 14 03:14 MST

FELT MAY 17 02:11 MST

$\begin{array}{lll}\ldots & \text { MAY } 27 \quad 17: 30 \text { CST }\end{array}$ $\begin{array}{lll}\ldots & \text { JUNE } 11 \text { 21:48 MST }\end{array}$

DELAWARE

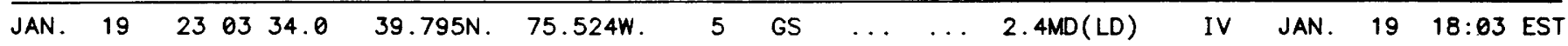

GEORGIA

\begin{tabular}{lllllllllllllllll}
\hline APR. & 18 & 05 & 41 & 00.6 & $33.861 \mathrm{~N}$. & $82.527 \mathrm{~W}$ & 0 & $\mathrm{GT}$ & $\ldots$ & $\ldots$ & $2.5 \ldots(\mathrm{GT})$ & $\ldots$ & APR. & 18 & B0:41 & EST
\end{tabular}

$\begin{array}{llllllllllllllll}\text { OCT. } & 9 & 11 & 54 & 26.2 & 34.775 \mathrm{~N} & 85.193 \mathrm{~W} & 15 & \mathrm{GT} & \ldots & \ldots & 4.0 \mathrm{Mn}(\mathrm{GS}) & \mathrm{VI} & \text { OCT. } & 9 & 05: 54 \text { CST }\end{array}$

\section{HAWAII}

\begin{tabular}{|c|c|c|c|c|c|c|c|c|c|c|c|c|c|c|c|c|}
\hline $\begin{array}{l}\text { JAN. } \\
\text { JAN. } \\
\text { JAN. } \\
\text { JAN. } \\
\text { JAN. }\end{array}$ & $\begin{array}{r}2 \\
3 \\
12 \\
13 \\
13\end{array}$ & $\begin{array}{l}06 \\
00 \\
10 \\
03 \\
22\end{array}$ & $\begin{array}{l}55 \\
10 \\
32 \\
48 \\
22\end{array}$ & $\begin{array}{l}57.9 \\
20.1 \\
27.8 \\
15.7 \\
36.2\end{array}$ & $\begin{array}{l}19.423 \mathrm{~N} . \\
20.588 \mathrm{~N} . \\
19.309 \mathrm{~N} . \\
19.306 \mathrm{~N} . \\
19.463 \mathrm{~N} .\end{array}$ & $\begin{array}{l}155.336 W . \\
154.916 W \\
155.237 W \\
155.235 W \\
155.398 W\end{array}$ & $\begin{array}{r}8 \\
0 \\
10 \\
10 \\
12\end{array}$ & $\begin{array}{l}\text { HV } \\
H V \\
H V \\
H V \\
H V\end{array}$ & $\begin{array}{l}\ldots \\
\cdots \\
\cdots \\
\cdots \\
\cdots\end{array}$ & $\begin{array}{l}\ldots \\
\cdots \\
\cdots \\
\cdots \\
\cdots\end{array}$ & $\begin{array}{l}\text { 3. OML (HV) } \\
\text { 3. } 7 \mathrm{ML}(\mathrm{HV}) \\
\text { 3. } 2 \mathrm{ML}(\mathrm{HV}) \\
\text { 3. } 4 \mathrm{ML}(\mathrm{HV}) \\
\text { 3. } 3 \mathrm{ML}(\mathrm{HV})\end{array}$ & $\begin{array}{l}\text { IV } \\
\cdots \\
\cdots \\
\cdots\end{array}$ & $\begin{array}{l}\text { JAN. } \\
\text { JAN. } \\
\text { JAN. } \\
\text { JAN. } \\
\text { JAN. }\end{array}$ & $\begin{array}{r}1 \\
2 \\
12 \\
12 \\
13\end{array}$ & $\begin{array}{l}20: 55 \\
14: 10 \\
00: 32 \\
17: 48 \\
12: 22\end{array}$ & $\begin{array}{l}\text { HST } \\
\text { HST } \\
\text { HST } \\
\text { HST } \\
\text { HST }\end{array}$ \\
\hline $\begin{array}{l}\text { AN. } \\
\text { AN. } \\
\text { AN. } \\
\text { AN. } \\
\text { AN. }\end{array}$ & $\begin{array}{l}17 \\
20 \\
26 \\
28 \\
28\end{array}$ & $\begin{array}{l}89 \\
07 \\
17 \\
12 \\
18\end{array}$ & $\begin{array}{l}29 \\
04 \\
16 \\
22 \\
23\end{array}$ & $\begin{array}{l}30.9 \\
48.6 \\
26.7 \\
07.8 \\
07.6\end{array}$ & $\begin{array}{l}18.855 \mathrm{~N} . \\
19.367 \mathrm{~N} . \\
19.433 \mathrm{~N} . \\
19.620 \mathrm{~N} . \\
19.644 \mathrm{~N} .\end{array}$ & $\begin{array}{l}155.477 W . \\
155.120 W . \\
155.356 W . \\
156.180 W . \\
156.043 W .\end{array}$ & $\begin{array}{r}36 \\
9 \\
10 \\
29 \\
43\end{array}$ & $\begin{array}{l}H V \\
H V \\
H V\end{array}$ & $\begin{array}{l}\ldots \\
\ldots \\
\ldots\end{array}$ & $\begin{array}{l}\cdots \\
\cdots \\
\cdots \\
\cdots \\
\cdots\end{array}$ & $\begin{array}{l}\text { 3. OML (HV) } \\
\text { 3. } 1 \mathrm{ML}(\mathrm{HV}) \\
\text { 3. } 0 \mathrm{ML}(\mathrm{HV}) \\
\text { 3. } 7 \mathrm{ML}(\mathrm{HV}) \\
\text { 3. } 0 \mathrm{ML}(\mathrm{HV})\end{array}$ & $\begin{array}{l}\ldots \\
\ldots\end{array}$ & $\begin{array}{l}\text { JAN. } \\
\text { JAN. } \\
\text { JAN. } \\
\text { JAN. } \\
\text { JAN. }\end{array}$ & $\begin{array}{l}16 \\
19 \\
26 \\
28 \\
28\end{array}$ & $\begin{array}{l}23: 29 \\
21: 04 \\
07: 16 \\
02: 22 \\
08: 23\end{array}$ & $\begin{array}{l}\text { HST } \\
\text { HST } \\
\text { HST } \\
\text { HST } \\
\text { HST }\end{array}$ \\
\hline $\begin{array}{l}=E B . \\
=E B . \\
=E B . \\
=E B . \\
=E B .\end{array}$ & $\begin{array}{r}31 \\
2 \\
3 \\
3 \\
4\end{array}$ & $\begin{array}{l}06 \\
21 \\
01 \\
16 \\
05\end{array}$ & $\begin{array}{l}26 \\
12 \\
51 \\
22 \\
45\end{array}$ & $\begin{array}{l}56.5 \\
20.9 \\
22.5 \\
25.1 \\
35.9\end{array}$ & $\begin{array}{l}19.566 \mathrm{~N} . \\
19.319 \mathrm{~N} . \\
19.395 \mathrm{~N} . \\
19.304 \mathrm{~N} . \\
19.192 \mathrm{~N} .\end{array}$ & $\begin{array}{l}156.028 W . \\
155.221 W . \\
155.611 W . \\
155.230 W . \\
155.610 W .\end{array}$ & $\begin{array}{r}16 \\
11 \\
6 \\
10 \\
9\end{array}$ & $\begin{array}{l}\text { HV } \\
H V \\
H V \\
H V\end{array}$ & $\begin{array}{l}\cdots \\
\cdots \\
\cdots \\
\cdots \\
\cdots\end{array}$ & $\begin{array}{l}\cdots \\
\cdots \\
\cdots \\
\cdots\end{array}$ & $\begin{array}{l}\text { 3. } 4 M L(H V) \\
\text { 3. } 5 M L(H V) \\
\text { 3. } 5 M L(H V) \\
\text { 3. } 5 M L(H V) \\
\text { 3. } 3 M L(H V)\end{array}$ & $\begin{array}{l}\text { I I I } \\
\text { I I I }\end{array}$ & $\begin{array}{l}\text { FEB. } \\
\text { FEB. } \\
\text { FEB. } \\
\text { FEB. }\end{array}$ & $\begin{array}{r}30 \\
2 \\
2 \\
3 \\
3\end{array}$ & $\begin{array}{l}20: 26 \\
11: 12 \\
15: 51 \\
86: 22 \\
19: 45\end{array}$ & $\begin{array}{l}\text { HST } \\
\text { HST } \\
\text { HST } \\
\text { HST } \\
\text { HST }\end{array}$ \\
\hline $\begin{array}{l}\text { FEB. } \\
\text { FEB. } \\
\text { FEB. } \\
\text { FEB. } \\
\text { FEB. }\end{array}$ & $\begin{array}{l}13 \\
13 \\
15 \\
16 \\
17\end{array}$ & $\begin{array}{l}02 \\
20 \\
17 \\
16 \\
12\end{array}$ & $\begin{array}{l}18 \\
27 \\
22 \\
52 \\
45\end{array}$ & $\begin{array}{l}11.2 \\
21.8 \\
41.1 \\
33.1 \\
06.3\end{array}$ & $\begin{array}{l}19.269 \mathrm{~N} . \\
19.400 \mathrm{~N} . \\
19.399 \mathrm{~N} . \\
19.481 \mathrm{~N} . \\
19.382 \mathrm{~N} .\end{array}$ & $\begin{array}{l}155.528 W \\
155.436 W \\
155.455 W \\
155.445 W \\
155.074 W .\end{array}$ & $\begin{array}{r}8 \\
9 \\
12 \\
12 \\
9\end{array}$ & $\begin{array}{l}H V \\
H V \\
H V \\
H V \\
H V\end{array}$ & $\begin{array}{l}\cdots \\
\cdots \\
\cdots\end{array}$ & $\begin{array}{l}\cdots \\
\cdots \\
\cdots \\
\cdots \\
\cdots\end{array}$ & $\begin{array}{l}\text { 3. } 2 M L(H V) \\
\text { 3. } 1 M L(H V) \\
\text { 3. } 5 M L(H V) \\
\text { 3. } 2 M L(H V) \\
\text { 3. } 3 M L(H V)\end{array}$ & $\begin{array}{l}\text { IV } \\
\text { II }\end{array}$ & $\begin{array}{l}\text { FEB. } \\
\text { FEB. } \\
\text { FEB. } \\
\text { FEB. } \\
\text { FEB. }\end{array}$ & $\begin{array}{l}12 \\
13 \\
15 \\
16 \\
17\end{array}$ & $\begin{array}{l}16: 18 \\
10: 27 \\
07: 22 \\
06: 52 \\
02: 45\end{array}$ & $\begin{array}{l}\text { HST } \\
\text { HST } \\
\text { HST } \\
\text { HST } \\
\text { HST }\end{array}$ \\
\hline
\end{tabular}


Table 1. Summary of U.S. earthquakes for 1984-Continued

\begin{tabular}{|c|c|c|c|c|c|c|c|c|c|c|c|c|c|}
\hline \multirow{2}{*}{ Date } & \multicolumn{2}{|c|}{$\begin{array}{c}\text { Origin time } \\
\text { (UTC) }\end{array}$} & \multirow{2}{*}{$\begin{array}{l}\text { Latihude } \\
\text { (9) }\end{array}$} & \multirow{2}{*}{$\begin{array}{l}\text { Lomgitude } \\
\text { (०) }\end{array}$} & \multirow{2}{*}{$\begin{array}{l}\text { Depth } \\
\text { (km) }\end{array}$} & \multirow{2}{*}{$\begin{array}{l}\text { Hypo- } \\
\text { center } \\
\text { source }\end{array}$} & \multicolumn{3}{|c|}{ Magnitude } & \multirow{2}{*}{$\begin{array}{l}\text { Maximum } \\
\text { intensity }\end{array}$} & \multicolumn{3}{|c|}{ Lacal time } \\
\hline & hr $\min$ & $\sec$ & & & & & $\mathbf{m b}$ & MS & $\begin{array}{l}\text { ML. Mn } \\
\text { MD, Mw }\end{array}$ & & Date & Hour & $\begin{array}{l}\text { Time } \\
\text { zome }\end{array}$ \\
\hline
\end{tabular}

HAWAII-Continued

\begin{tabular}{|c|c|c|c|c|c|c|c|c|c|c|c|c|c|c|c|c|}
\hline $\begin{array}{l}\text { FEB. } \\
\text { FEB. } \\
\text { MAR. } \\
\text { MAR. } \\
\text { MAR. }\end{array}$ & $\begin{array}{r}24 \\
29 \\
4 \\
8 \\
11\end{array}$ & $\begin{array}{l}06 \\
01 \\
09 \\
20 \\
16\end{array}$ & $\begin{array}{l}59 \\
20 \\
14 \\
58 \\
46\end{array}$ & $\begin{array}{l}09.6 \\
08.3 \\
37.5 \\
49.9 \\
46.0\end{array}$ & $\begin{array}{l}19.417 \mathrm{~N} \\
19.349 \mathrm{~N} \\
19.212 \mathrm{~N} \\
19.322 \mathrm{~N} \\
19.821 \mathrm{~N}\end{array}$ & $\begin{array}{l}155.503 w . \\
155.129 W . \\
155.586 w . \\
155.194 W \\
155.616 W\end{array}$ & $\begin{array}{r}12 \\
9 \\
2 \\
9 \\
19\end{array}$ & $\begin{array}{l}\text { HV } \\
H V \\
H V \\
H V \\
H V\end{array}$ & $\begin{array}{l}\cdots \\
\cdots \\
\cdots \\
\cdots\end{array}$ & $\begin{array}{l}\cdots \\
\cdots \\
\cdots \\
\cdots\end{array}$ & $\begin{array}{l}\text { 3. } 2 M L(H V) \\
\text { 3. } 1 M L(H V) \\
\text { 3. } 3 M L(H V) \\
\text { 3. } 5 M L(H V) \\
\text { 3. } 1 M L(H V)\end{array}$ & $\begin{array}{l}\text { I I } \\
\cdots \\
\text { I I I } \\
\text { I I }\end{array}$ & $\begin{array}{l}\text { FEB. } \\
\text { FEB. } \\
\text { MAR. } \\
\text { MAR. } \\
\text { MAR. }\end{array}$ & $\begin{array}{r}23 \\
28 \\
3 \\
8 \\
11\end{array}$ & $\begin{array}{l}20: 59 \\
15: 20 \\
23: 14 \\
10: 58 \\
06: 46\end{array}$ & $\begin{array}{l}\text { HST } \\
\text { HST } \\
\text { HST } \\
\text { HST } \\
\text { HST }\end{array}$ \\
\hline $\begin{array}{l}\text { MAR. } \\
\text { MAR. } \\
\text { MAR. } \\
\text { MAR. } \\
\text { MAR. }\end{array}$ & $\begin{array}{l}15 \\
18 \\
22 \\
25 \\
25\end{array}$ & $\begin{array}{l}23 \\
05 \\
08 \\
10 \\
10\end{array}$ & $\begin{array}{l}03 \\
27 \\
28 \\
50 \\
55\end{array}$ & $\begin{array}{l}00.8 \\
35.1 \\
24.7 \\
52.8 \\
13.6\end{array}$ & $\begin{array}{l}19.364 \mathrm{~N} \\
19.334 \mathrm{~N} \\
19.427 \mathrm{~N} \\
19.473 \mathrm{~N} \\
19.463 \mathrm{~N}\end{array}$ & $\begin{array}{l}155.116 w . \\
155.200 W . \\
155.627 W . \\
155.586 w . \\
155.598 W\end{array}$ & $\begin{array}{l}9 \\
9 \\
2 \\
0 \\
1\end{array}$ & $\begin{array}{l}\text { HV } \\
H V \\
H V \\
H V \\
H V\end{array}$ & $\begin{array}{l}\cdots \\
\cdots \\
\cdots \\
\cdots\end{array}$ & $\begin{array}{l}\cdots \\
\cdots \\
\cdots \\
\cdots \\
\cdots\end{array}$ & $\begin{array}{l}\text { 3. } 4 M L(H V) \\
\text { 3. } 1 \mathrm{ML}(\mathrm{HV}) \\
\text { 3. } 2 \mathrm{ML}(\mathrm{HV}) \\
\text { 3. } 3 \mathrm{ML}(\mathrm{HV}) \\
\text { 3. } 9 \mathrm{ML}(\mathrm{HV})\end{array}$ & $\begin{array}{l}\text { I I I } \\
\text { I I } \\
\ldots \\
\cdots \\
\ldots\end{array}$ & $\begin{array}{l}\text { MAR. } \\
\text { MAR. } \\
\text { MAR. } \\
\text { MAR. } \\
\text { MAR. }\end{array}$ & $\begin{array}{l}15 \\
17 \\
21 \\
25 \\
25\end{array}$ & $\begin{array}{l}13: 03 \\
19: 27 \\
22: 28 \\
00: 50 \\
00: 55\end{array}$ & \\
\hline $\begin{array}{l}\text { MAR. } \\
\text { MAR. } \\
\text { MAR. } \\
\text { MAR. } \\
\text { MAR. }\end{array}$ & $\begin{array}{l}25 \\
25 \\
25 \\
25 \\
25\end{array}$ & $\begin{array}{l}11 \\
11 \\
11 \\
11 \\
12\end{array}$ & $\begin{array}{l}00 \\
01 \\
04 \\
08 \\
10\end{array}$ & $\begin{array}{l}22.2 \\
20.3 \\
32.2 \\
08.5 \\
24.7\end{array}$ & $\begin{array}{l}19.467 \mathrm{~N} \\
19.456 \mathrm{~N} \\
19.437 \mathrm{~N} \\
19.452 \mathrm{~N} \\
19.478 \mathrm{~N}\end{array}$ & $\begin{array}{l}155.953 w . \\
155.580 w . \\
155.591 w . \\
155.619 w . \\
155.580 w .\end{array}$ & $\begin{array}{l}2 \\
2 \\
7 \\
6 \\
8\end{array}$ & $\begin{array}{l}H V \\
H V \\
H V \\
H V \\
H V\end{array}$ & $\begin{array}{l}\cdots \\
\cdots \\
\cdots \\
\cdots \\
\cdots\end{array}$ & $\begin{array}{l}\ldots \\
\cdots \\
\cdots \\
\cdots\end{array}$ & $\begin{array}{l}\text { 3. } 2 \mathrm{ML}(\mathrm{HV}) \\
\text { 3. } 8 \mathrm{ML}(\mathrm{HV}) \\
3.4 \mathrm{ML}(\mathrm{HV}) \\
\text { 3. } 1 \mathrm{ML}(\mathrm{HV}) \\
\text { 3. } 3 \mathrm{ML}(\mathrm{HV})\end{array}$ & $\begin{array}{l}\cdots \\
\cdots \\
\cdots \\
\cdots \\
\cdots\end{array}$ & $\begin{array}{l}\text { MAR. } \\
\text { MAR. } \\
\text { MAR. } \\
\text { MAR. } \\
\text { MAR. }\end{array}$ & $\begin{array}{l}25 \\
25 \\
25 \\
25 \\
25\end{array}$ & $\begin{array}{l}01: 00 \\
01: 01 \\
01: 04 \\
01: 08 \\
02: 10\end{array}$ & \\
\hline $\begin{array}{l}\text { MAR. } \\
\text { MAR. } \\
\text { MAR. } \\
\text { APR. } \\
\text { APR. }\end{array}$ & $\begin{array}{r}26 \\
27 \\
30 \\
1 \\
7\end{array}$ & $\begin{array}{l}07 \\
19 \\
20 \\
12 \\
01\end{array}$ & $\begin{array}{l}05 \\
14 \\
21 \\
22 \\
26\end{array}$ & $\begin{array}{l}50.3 \\
42.8 \\
15.1 \\
25.0 \\
12.3\end{array}$ & $\begin{array}{l}19.463 \mathrm{~N} \\
19.353 \mathrm{~N} \\
19.970 \mathrm{~N} \\
19.455 \mathrm{~N} \\
19.503 \mathrm{~N}\end{array}$ & $\begin{array}{l}155.599 w . \\
155.494 W . \\
155.091 W . \\
155.492 W . \\
156.266 w .\end{array}$ & $\begin{array}{r}6 \\
7 \\
36 \\
10 \\
36\end{array}$ & $\begin{array}{l}H V \\
H V \\
H V \\
H V \\
H V\end{array}$ & $\begin{array}{l}\ldots \\
\cdots \\
\cdots \\
\cdots \\
\cdots\end{array}$ & $\begin{array}{l}\cdots \\
\cdots \\
\ldots \\
\cdots\end{array}$ & $\begin{array}{l}\text { 3. } 3 \mathrm{ML}(\mathrm{HV}) \\
\text { 3. } 1 \mathrm{ML}(\mathrm{HV}) \\
\text { 3. } 8 \mathrm{ML}(\mathrm{HV}) \\
\text { 3. } 2 \mathrm{ML}(\mathrm{HV}) \\
\text { 3. } 0 \mathrm{ML}(\mathrm{HV})\end{array}$ & $\begin{array}{l}\cdots \\
\cdots \\
\cdots \\
\cdots\end{array}$ & $\begin{array}{l}\text { MAR. } \\
\text { MAR. } \\
\text { MAR. } \\
\text { APR. } \\
\text { APR. }\end{array}$ & $\begin{array}{r}25 \\
27 \\
30 \\
1 \\
6\end{array}$ & $\begin{array}{l}21: 05 \\
09: 14 \\
10: 21 \\
02: 22 \\
15: 26\end{array}$ & \\
\hline $\begin{array}{l}\text { APR. } \\
\text { APR. } \\
\text { APR. } \\
\text { APR. } \\
\text { APR. }\end{array}$ & $\begin{array}{r}7 \\
9 \\
9 \\
10 \\
18\end{array}$ & $\begin{array}{l}04 \\
17 \\
23 \\
21 \\
16\end{array}$ & $\begin{array}{l}43 \\
05 \\
13 \\
37 \\
40\end{array}$ & $\begin{array}{l}29.0 \\
39.2 \\
39.9 \\
02.7 \\
13.0\end{array}$ & $\begin{array}{l}19.312 \mathrm{~N} \\
19.468 \mathrm{~N} \\
19.318 \mathrm{~N} \\
19.177 \mathrm{~N} \\
19.298 \mathrm{~N}\end{array}$ & $\begin{array}{l}155.229 W . \\
155.606 W . \\
155.226 W . \\
156.795 W . \\
155.216 W .\end{array}$ & $\begin{array}{r}10 \\
2 \\
11 \\
28 \\
7\end{array}$ & $\begin{array}{l}\text { HV } \\
H V \\
H V \\
H V \\
H V\end{array}$ & $\begin{array}{l}\cdots \\
\cdots \\
\cdots \\
\cdots\end{array}$ & $\begin{array}{l}\ldots \\
\ldots \\
\cdots \\
\cdots\end{array}$ & $\begin{array}{l}\text { 3. } 4 M L(H V) \\
3.7 M L(H V) \\
3.9 M L(H V) \\
3.4 M L(H V) \\
3.0 M L(H V)\end{array}$ & $\begin{array}{l}\text { II I } \\
\text { II } \\
\text { IV } \\
\ldots \\
\ldots\end{array}$ & $\begin{array}{l}\text { APR. } \\
\text { APR. } \\
\text { APR. } \\
\text { APR. } \\
\text { APR. }\end{array}$ & $\begin{array}{r}6 \\
9 \\
9 \\
10 \\
18\end{array}$ & $\begin{array}{l}18: 43 \\
07: 05 \\
13: 13 \\
11: 37 \\
06: 40\end{array}$ & $H$ \\
\hline $\begin{array}{l}\text { APR. } \\
\text { APR. } \\
\text { APR. } \\
\text { APR. } \\
\text { APR. }\end{array}$ & $\begin{array}{l}22 \\
22 \\
28 \\
29 \\
30\end{array}$ & $\begin{array}{l}12 \\
12 \\
09 \\
06 \\
23\end{array}$ & $\begin{array}{l}23 \\
27 \\
48 \\
45 \\
49\end{array}$ & $\begin{array}{l}29.0 \\
03.6 \\
49.5 \\
34.4 \\
34.3\end{array}$ & $\begin{array}{l}19.305 \mathrm{~N} \\
19.307 \mathrm{~N} \\
19.320 \mathrm{~N} \\
19.173 \mathrm{~N} \\
19.724 \mathrm{~N}\end{array}$ & $\begin{array}{l}155.224 W . \\
155.223 W . \\
155.473 w . \\
155.677 w \\
154.880 W\end{array}$ & $\begin{array}{r}11 \\
8 \\
10 \\
9 \\
40\end{array}$ & $\begin{array}{l}\text { HV } \\
\text { HV } \\
H V \\
H V \\
H V\end{array}$ & $\begin{array}{l}\ldots \\
\ldots \\
\cdots \\
\cdots\end{array}$ & $\begin{array}{l}\ldots \\
\ldots \\
\ldots \\
\cdots\end{array}$ & $\begin{array}{l}3.7 M L(H V) \\
3.8 M L(H V) \\
3.2 M L(H V) \\
3.5 M L(H V) \\
3.5 M L(H V)\end{array}$ & $\begin{array}{l}\text { I I I } \\
\text { I I I } \\
\text { I I } \\
\text { I I } \\
\ldots\end{array}$ & $\begin{array}{l}\text { APR. } \\
\text { APR. } \\
\text { APR. } \\
\text { APR. } \\
\text { APR. }\end{array}$ & $\begin{array}{l}22 \\
22 \\
27 \\
28 \\
30\end{array}$ & $\begin{array}{l}02: 23 \\
02: 27 \\
23: 48 \\
20: 45 \\
13: 49\end{array}$ & $\begin{array}{l}H S \\
H S \\
H S \\
H S \\
H S\end{array}$ \\
\hline $\begin{array}{l}\text { MAY } \\
\text { MAY } \\
\text { MAY } \\
\text { MAY } \\
\text { MAY }\end{array}$ & $\begin{array}{r}1 \\
9 \\
10 \\
10 \\
17\end{array}$ & $\begin{array}{l}13 \\
05 \\
01 \\
19 \\
17\end{array}$ & $\begin{array}{l}55 \\
17 \\
45 \\
26 \\
45\end{array}$ & $\begin{array}{l}23.1 \\
34.3 \\
10.9 \\
58.8 \\
30.9\end{array}$ & $\begin{array}{l}19.211 \mathrm{~N} . \\
19.823 \mathrm{~N} . \\
19.371 \mathrm{~N} . \\
19.987 \mathrm{~N} . \\
19.506 \mathrm{~N}\end{array}$ & $\begin{array}{l}155.569 w \\
155.918 w . \\
155.486 w \\
155.898 w \\
155.565 w\end{array}$ & $\begin{array}{l}13 \\
40 \\
10 \\
22 \\
14\end{array}$ & $\begin{array}{l}\text { HV } \\
H V \\
H V \\
H V \\
H V\end{array}$ & $\begin{array}{l}\ldots \\
\cdots \\
\cdots \\
\cdots\end{array}$ & $\begin{array}{l}\ldots \\
\ldots \\
\ldots \\
\ldots\end{array}$ & $\begin{array}{l}\text { 4. OML (HV) } \\
\text { 3. } 1 \mathrm{ML}(\mathrm{HV}) \\
\text { 3. OML (HV) } \\
\text { 3. OML (HV) } \\
\text { 3.1ML(HV) }\end{array}$ & $\begin{array}{l}1 \mathrm{II} \\
\ldots \\
\ldots \\
\ldots\end{array}$ & $\begin{array}{l}\text { MAY } \\
\text { MAY } \\
\text { MAY } \\
\text { MAY } \\
\text { MAY }\end{array}$ & $\begin{array}{r}1 \\
8 \\
9 \\
10 \\
17\end{array}$ & $\begin{array}{l}03: 55 \\
19: 17 \\
15: 45 \\
09: 26 \\
07: 45\end{array}$ & $\begin{array}{l}\text { HST } \\
\text { HST } \\
\text { HST } \\
\text { HST } \\
\text { HST }\end{array}$ \\
\hline $\begin{array}{l}\text { MAY } \\
\text { MAY } \\
\text { MAY } \\
\text { JUNE } \\
\text { JUNE }\end{array}$ & $\begin{array}{r}20 \\
20 \\
31 \\
1 \\
4\end{array}$ & $\begin{array}{l}00 \\
04 \\
04 \\
09 \\
07\end{array}$ & $\begin{array}{l}18 \\
41 \\
07 \\
18 \\
12\end{array}$ & $\begin{array}{l}01.7 \\
02.7 \\
13.6 \\
37.6 \\
07.5\end{array}$ & $\begin{array}{l}19.322 \mathrm{~N} \\
19.343 \mathrm{~N} \\
19.483 \mathrm{~N} \\
19.204 \mathrm{~N} \\
18.904 \mathrm{~N}\end{array}$ & $\begin{array}{l}155.190 w . \\
155.217 w . \\
155.595 w . \\
155.461 w . \\
155.145 w .\end{array}$ & $\begin{array}{r}9 \\
10 \\
2 \\
8 \\
50\end{array}$ & $\begin{array}{l}H V \\
H V \\
H V \\
H V \\
H V\end{array}$ & $\begin{array}{l}\ldots \\
\ldots \\
\cdots \\
\cdots \\
\cdots\end{array}$ & $\begin{array}{l}\ldots \\
\ldots \\
\ldots \\
\ldots\end{array}$ & $\begin{array}{l}\text { 3. OML (HV) } \\
\text { 3. 3ML }(H V) \\
\text { 3. OML }(H V) \\
\text { 3. } 0 \mathrm{HV}(\mathrm{HV}) \\
\text { 3. } 4 \mathrm{ML}(\mathrm{HV})\end{array}$ & $\begin{array}{l}\text { II } \\
\ldots \\
\ldots \\
\ldots\end{array}$ & $\begin{array}{l}\text { MAY } \\
\text { MAY } \\
\text { MAY } \\
\text { MAY } \\
\text { JUNE }\end{array}$ & $\begin{array}{r}19 \\
19 \\
30 \\
30 \\
3\end{array}$ & $\begin{array}{l}14: 18 \\
18: 41 \\
18: 07 \\
23: 18 \\
21: 12\end{array}$ & $\begin{array}{l}\text { HST } \\
\text { HST } \\
\text { HST } \\
\text { HST } \\
\text { HST }\end{array}$ \\
\hline $\begin{array}{l}\text { JUNE } \\
\text { JUNE } \\
\text { JUNE } \\
\text { JUNE } \\
\text { JUNE }\end{array}$ & $\begin{array}{r}8 \\
8 \\
9 \\
12 \\
13\end{array}$ & $\begin{array}{l}19 \\
19 \\
03 \\
23 \\
03\end{array}$ & $\begin{array}{l}27 \\
33 \\
34 \\
39 \\
03\end{array}$ & $\begin{array}{l}50.7 \\
12.3 \\
10.6 \\
00.4 \\
20.1\end{array}$ & $\begin{array}{l}19.201 \mathrm{~N} \\
19.202 \mathrm{~N} . \\
20.055 \mathrm{~N} \\
19.624 \mathrm{~N} \\
19.352 \mathrm{~N} .\end{array}$ & $\begin{array}{l}155.520 W \\
155.520 W \\
157.875 w \\
155.930 w . \\
155.502 W .\end{array}$ & $\begin{array}{l}38 \\
37 \\
30 \\
20 \\
10\end{array}$ & $\begin{array}{l}H V \\
H V \\
H V \\
H V \\
H V\end{array}$ & $\begin{array}{l}\ldots \\
\ldots \\
\ldots \\
\cdots \\
\cdots\end{array}$ & $\begin{array}{l}\cdots \\
\cdots \\
\cdots \\
\cdots \\
\cdots\end{array}$ & $\begin{array}{l}\text { 3.7ML }(\mathrm{HV}) \\
3.1 \mathrm{ML}(\mathrm{HV}) \\
5.0 \mathrm{ML}(\mathrm{HV}) \\
3.5 \mathrm{ML}(\mathrm{HV}) \\
3.6 \mathrm{ML}(\mathrm{HV})\end{array}$ & $\begin{array}{l}\text { IV } \\
\cdots \\
\cdots \\
\text { II } \\
\text { I I }\end{array}$ & $\begin{array}{l}\text { JUNE } \\
\text { JUNE } \\
\text { JUNE } \\
\text { JUNE } \\
\text { JUNE }\end{array}$ & $\begin{array}{r}8 \\
8 \\
8 \\
12 \\
12\end{array}$ & $\begin{array}{l}09: 27 \\
09: 33 \\
17: 34 \\
13: 39 \\
17: 03\end{array}$ & $\begin{array}{l}\text { HS } \\
H S \\
H S\end{array}$ \\
\hline $\begin{array}{l}\text { JUNE } \\
\text { JUNE } \\
\text { JUNE } \\
\text { JUNE } \\
\text { JUNE }\end{array}$ & $\begin{array}{l}13 \\
16 \\
17 \\
25 \\
25\end{array}$ & $\begin{array}{l}11 \\
18 \\
21 \\
07 \\
23\end{array}$ & $\begin{array}{l}52 \\
22 \\
05 \\
23 \\
29\end{array}$ & $\begin{array}{l}31.2 \\
10.0 \\
23.5 \\
20.0 \\
43.7\end{array}$ & $\begin{array}{l}19.341 \mathrm{~N} . \\
20.742 \mathrm{~N} . \\
19.357 \mathrm{~N} . \\
19.337 \mathrm{~N} . \\
19.247 \mathrm{~N} .\end{array}$ & $\begin{array}{l}155.213 W . \\
157.505 W . \\
155.502 W . \\
155.181 W . \\
155.068 W .\end{array}$ & $\begin{array}{r}9 \\
34 \\
10 \\
10 \\
48\end{array}$ & $\begin{array}{l}H V \\
H V \\
H V \\
H V \\
H V\end{array}$ & $\begin{array}{l}\cdots \\
\cdots \\
\cdots \\
\cdots\end{array}$ & $\begin{array}{l}\cdots \\
\cdots \\
\cdots \\
\cdots\end{array}$ & $\begin{array}{l}\text { 3.5ML(HV) } \\
4.0 \mathrm{HL}(\mathrm{HV}) \\
3.1 \mathrm{ML}(\mathrm{HV}) \\
3.5 \mathrm{ML}(\mathrm{HV}) \\
3.1 \mathrm{ML}(\mathrm{HV})\end{array}$ & $\begin{array}{l}\text { I I I } \\
\text { IV } \\
\text { I I } \\
\text { I I I } \\
\ldots\end{array}$ & $\begin{array}{l}\text { JUNE } \\
\text { JUNE } \\
\text { JUNE } \\
\text { JUNE } \\
\text { JUNE }\end{array}$ & $\begin{array}{l}13 \\
16 \\
17 \\
24 \\
25\end{array}$ & $\begin{array}{l}01: 52 \\
08: 22 \\
11: 05 \\
21: 23 \\
13: 29\end{array}$ & $\begin{array}{l}-15 \\
-1\end{array}$ \\
\hline
\end{tabular}


Table 1. Summary of U.S. earthquakes for 1984-Continued

\begin{tabular}{|c|c|c|c|c|c|c|c|c|c|c|c|c|c|}
\hline \multirow{2}{*}{ Date } & \multicolumn{2}{|c|}{$\begin{array}{l}\text { Origin time } \\
\text { (UTC) }\end{array}$} & \multirow{2}{*}{$\begin{array}{l}\text { Latitude } \\
\left({ }^{\circ}\right)\end{array}$} & \multirow{2}{*}{$\begin{array}{c}\text { Longitude } \\
\left({ }^{\circ}\right)\end{array}$} & \multirow{2}{*}{$\begin{array}{c}\text { Depth } \\
\text { (km) }\end{array}$} & \multirow{2}{*}{$\begin{array}{l}\text { Hypo- } \\
\text { center } \\
\text { source }\end{array}$} & \multicolumn{3}{|c|}{ Mannitude } & \multirow{2}{*}{$\begin{array}{l}\text { Maximum } \\
\text { intensity }\end{array}$} & \multicolumn{3}{|c|}{ Local time } \\
\hline & hr $\min$ & $\sec$ & & & & & mh & MS & $\begin{array}{l}M L, M n \\
M D, M w\end{array}$ & & Date & Hour & $\begin{array}{l}\text { Time } \\
\text { zome }\end{array}$ \\
\hline
\end{tabular}

HAWAII-Continued

\begin{tabular}{|c|c|c|c|c|c|c|c|c|c|c|c|c|c|c|c|c|}
\hline $\begin{array}{l}\text { JUNE } \\
\text { JULY } \\
\text { JULY } \\
\text { JULY } \\
\text { JULY }\end{array}$ & $\begin{array}{r}27 \\
5 \\
9 \\
15 \\
20\end{array}$ & $\begin{array}{l}05 \\
00 \\
03 \\
08 \\
18\end{array}$ & $\begin{array}{l}14 \\
30 \\
26 \\
29 \\
47\end{array}$ & $\begin{array}{l}31.3 \\
13.2 \\
33.9 \\
59.7 \\
47.9\end{array}$ & $\begin{array}{l}20.018 \mathrm{~N} \\
19.080 \mathrm{~N} \\
21.552 \mathrm{~N} . \\
19.146 \mathrm{~N} . \\
20.050 \mathrm{~N} .\end{array}$ & $\begin{array}{l}155.358 w . \\
156.903 W . \\
154.938 w . \\
156.157 w . \\
155.728 W .\end{array}$ & $\begin{array}{r}8 \\
29 \\
27 \\
37 \\
7\end{array}$ & $\begin{array}{l}H V \\
H V \\
H V \\
H V \\
H V\end{array}$ & $\begin{array}{l}\cdots \\
\cdots \\
\cdots\end{array}$ & $\begin{array}{l}\ldots \\
\ldots \\
\ldots\end{array}$ & $\begin{array}{l}\text { 3. 3ML (HV) } \\
3.4 M L(\mathrm{HV}) \\
3.5 \mathrm{ML}(\mathrm{HV}) \\
3.0 \mathrm{HL}(\mathrm{HV}) \\
3.6 \mathrm{ML}(\mathrm{HV})\end{array}$ & $\begin{array}{l}\text { I I } \\
\cdots \\
\cdots \\
\text { I I I }\end{array}$ & $\begin{array}{l}\text { JUNE } \\
\text { JULY } \\
\text { JULY } \\
\text { JULY } \\
\text { JULY }\end{array}$ & $\begin{array}{r}26 \\
4 \\
8 \\
14 \\
20\end{array}$ & $\begin{array}{l}19: 14 \\
14: 30 \\
17: 26 \\
22: 29 \\
08: 47\end{array}$ & $\begin{array}{l}\text { HST } \\
\text { HST } \\
\text { HST } \\
\text { HST } \\
\text { HST }\end{array}$ \\
\hline $\begin{array}{l}\text { JULY } \\
\text { JULY } \\
\text { AUG. } \\
\text { AUG. } \\
\text { AUG. }\end{array}$ & $\begin{array}{r}25 \\
26 \\
3 \\
12 \\
14\end{array}$ & $\begin{array}{l}18 \\
15 \\
15 \\
09 \\
20\end{array}$ & $\begin{array}{l}32 \\
45 \\
08 \\
29 \\
10\end{array}$ & $\begin{array}{l}38.1 \\
11.1 \\
39.3 \\
30.1 \\
02.1\end{array}$ & $\begin{array}{l}19.369 \mathrm{~N} \\
19.352 \mathrm{~N} \\
19.326 \mathrm{~N} \\
19.458 \mathrm{~N} \\
19.786 \mathrm{~N}\end{array}$ & $\begin{array}{l}155.311 w . \\
155.123 w . \\
155.258 w . \\
155.489 w . \\
154.913 w .\end{array}$ & $\begin{array}{r}31 \\
8 \\
9 \\
10 \\
48\end{array}$ & $\begin{array}{l}H V \\
H V \\
H V \\
H V \\
H V\end{array}$ & $\begin{array}{l}\cdots \\
\cdots \\
\cdots\end{array}$ & $\begin{array}{l}\ldots \\
\cdots \\
\cdots \\
\cdots\end{array}$ & $\begin{array}{l}\text { 3. } 5 \mathrm{ML}(\mathrm{HV}) \\
3.3 \mathrm{ML}(\mathrm{HV}) \\
3.1 \mathrm{ML}(\mathrm{HV}) \\
3.0 \mathrm{ML}(\mathrm{HV}) \\
3.7 \mathrm{ML}(\mathrm{HV})\end{array}$ & $\begin{array}{c}\text { II I } \\
\ldots \\
\text { IV } \\
\text { FELT }\end{array}$ & $\begin{array}{l}\text { JULY } \\
\text { JULY } \\
\text { AUG. } \\
\text { AUG. } \\
\text { AUG. }\end{array}$ & $\begin{array}{r}25 \\
26 \\
3 \\
11 \\
14\end{array}$ & $\begin{array}{l}08: 32 \\
05: 45 \\
05: 08 \\
23: 29 \\
10: 10\end{array}$ & $\begin{array}{l}\text { HST } \\
\text { HST } \\
\text { HST } \\
\text { HST } \\
\text { HST }\end{array}$ \\
\hline $\begin{array}{l}\text { AUG. } \\
\text { AUG. } \\
\text { SEPT. } \\
\text { SEPT. } \\
\text { SEPT. }\end{array}$ & $\begin{array}{r}28 \\
31 \\
3 \\
7 \\
7\end{array}$ & $\begin{array}{l}17 \\
23 \\
02 \\
04 \\
23\end{array}$ & $\begin{array}{l}28 \\
33 \\
43 \\
56 \\
14\end{array}$ & $\begin{array}{l}48.9 \\
31.1 \\
14.4 \\
58.5 \\
56.8\end{array}$ & $\begin{array}{l}19.206 \mathrm{~N} \\
20.141 \mathrm{~N} . \\
19.355 \mathrm{~N} \\
19.335 \mathrm{~N} . \\
19.507 \mathrm{~N}\end{array}$ & $\begin{array}{l}155.608 \mathrm{~W} . \\
155.841 \mathrm{~W} \\
155.026 \mathrm{~W} . \\
154.871 \mathrm{w} . \\
155.941 \mathrm{w} .\end{array}$ & $\begin{array}{r}10 \\
39 \\
7 \\
41 \\
12\end{array}$ & $\begin{array}{l}H V \\
H V \\
H V \\
H V \\
H V\end{array}$ & $\ldots$ & $\begin{array}{l}\ldots \\
\ldots \\
\ldots \\
\ldots\end{array}$ & $\begin{array}{l}\text { 3. } 2 \mathrm{ML}(\mathrm{HV}) \\
3.3 \mathrm{ML}(\mathrm{HV}) \\
3.2 \mathrm{ML}(\mathrm{HV}) \\
3.0 \mathrm{HL}(\mathrm{HV}) \\
3.0 \mathrm{ML}(\mathrm{HV})\end{array}$ & $\begin{array}{l}\ldots \\
\text { III } \\
\ldots \\
\ldots\end{array}$ & $\begin{array}{l}\text { AUG. } \\
\text { AUG. } \\
\text { SEPT. } \\
\text { SEPT. } \\
\text { SEPT. }\end{array}$ & $\begin{array}{r}28 \\
31 \\
2 \\
6 \\
7\end{array}$ & $\begin{array}{l}07: 28 \\
13: 33 \\
16: 43 \\
18: 56 \\
13: 14\end{array}$ & $\begin{array}{l}\text { HST } \\
\text { HST } \\
\text { HST } \\
\text { HST } \\
\text { HST }\end{array}$ \\
\hline $\begin{array}{l}\text { SEPT } \\
\text { SEPT. } \\
\text { SEPT. } \\
\text { SEPT. } \\
\text { SEPT. }\end{array}$ & $\begin{array}{l}11 \\
11 \\
14 \\
25 \\
27\end{array}$ & $\begin{array}{l}05 \\
09 \\
06 \\
07 \\
17\end{array}$ & $\begin{array}{l}16 \\
51 \\
27 \\
27 \\
03\end{array}$ & $\begin{array}{l}06.5 \\
36.3 \\
59.5 \\
48.2 \\
12.7\end{array}$ & $\begin{array}{l}18.928 \mathrm{~N} \\
19.343 \mathrm{~N} \\
19.464 \mathrm{~N} \\
19.316 \mathrm{~N} \\
19.323 \mathrm{~N}\end{array}$ & $\begin{array}{l}155.277 w . \\
155.222 W . \\
154.886 W . \\
155.903 W . \\
155.183 W .\end{array}$ & $\begin{array}{r}15 \\
9 \\
9 \\
12 \\
10\end{array}$ & $\begin{array}{l}H V \\
H V \\
H V\end{array}$ & $\begin{array}{l}\cdots \\
\cdots \\
\cdots \\
\cdots\end{array}$ & $\begin{array}{l}\ldots \\
\cdots \\
\cdots \\
\cdots\end{array}$ & $\begin{array}{l}\text { 3.1ML(HV) } \\
3.4 \mathrm{ML} \text { (HV) } \\
3.0 \mathrm{HV} \text { (HV) } \\
\text { 4.3ML(HV) } \\
3.2 \mathrm{HL}(\mathrm{HV})\end{array}$ & $\begin{array}{l}\text { I I I } \\
\text { I IV } \\
\text { I I I }\end{array}$ & $\begin{array}{l}\text { SEPT. } \\
\text { SEPT. } \\
\text { SEPT. } \\
\text { SEPT. } \\
\text { SEPT. }\end{array}$ & $\begin{array}{l}10 \\
10 \\
13 \\
24 \\
27\end{array}$ & $\begin{array}{l}19: 16 \\
23: 51 \\
20: 27 \\
21: 27 \\
07: 03\end{array}$ & $\begin{array}{l}\text { HST } \\
\text { HST } \\
\text { HST } \\
\text { HST } \\
\text { HST }\end{array}$ \\
\hline $\begin{array}{l}\text { OCT. } \\
\text { OCT. } \\
\text { OCT. } \\
\text { OCT. } \\
\text { OCT. }\end{array}$ & $\begin{array}{r}3 \\
7 \\
8 \\
9 \\
10\end{array}$ & $\begin{array}{l}19 \\
03 \\
10 \\
02 \\
12\end{array}$ & $\begin{array}{l}34 \\
54 \\
00 \\
18 \\
33\end{array}$ & $\begin{array}{l}11.9 \\
56.8 \\
25.1 \\
10.7 \\
49.7\end{array}$ & & $\begin{array}{l}155.453 w . \\
155.181 w . \\
156.861 w . \\
155.490 w . \\
155.420 w .\end{array}$ & $\begin{array}{l}14 \\
32 \\
30 \\
18 \\
10\end{array}$ & $\begin{array}{l}\text { HV } \\
\text { HV }\end{array}$ & $\begin{array}{l}\ldots \\
\cdots \\
\cdots \\
\ldots\end{array}$ & $\begin{array}{l}\ldots \\
\ldots \\
\ldots \\
\ldots\end{array}$ & $\begin{array}{l}3.1 \mathrm{ML} \text { (HV) } \\
3.0 \mathrm{HL} \text { (HV) } \\
3.9 \mathrm{HL} \text { (HV) } \\
3.7 \mathrm{HL} \text { (HV) } \\
3.0 \mathrm{ML}(\mathrm{HV})\end{array}$ & $\begin{array}{l}\text { I I I } \\
\text { IV } \\
\text { I I I }\end{array}$ & $\begin{array}{l}\text { OCT. } \\
\text { OCT. } \\
\text { OCT. } \\
\text { OCT. } \\
\text { OCT. }\end{array}$ & $\begin{array}{r}3 \\
6 \\
8 \\
8 \\
10\end{array}$ & $\begin{array}{l}09: 34 \\
17: 54 \\
00: 00 \\
16: 18 \\
02: 33\end{array}$ & $\begin{array}{l}\text { HST } \\
\text { HST } \\
\text { HST } \\
\text { HST } \\
\text { HST }\end{array}$ \\
\hline $\begin{array}{l}\text { OCT. } \\
\text { OCT. } \\
\text { OCT. } \\
\text { OCT. } \\
\text { OCT. }\end{array}$ & $\begin{array}{l}13 \\
16 \\
17 \\
23 \\
29\end{array}$ & $\begin{array}{l}16 \\
15 \\
00 \\
13 \\
14\end{array}$ & $\begin{array}{l}45 \\
31 \\
20\end{array}$ & $\begin{array}{l}26.9 \\
14.7 \\
55.8 \\
48.6 \\
38.2\end{array}$ & $\begin{array}{l}19.344 \mathrm{~N} \\
18.910 \mathrm{~N} . \\
19.408 \mathrm{~N} . \\
19.341 \mathrm{~N} . \\
19.243 \mathrm{~N}\end{array}$ & $\begin{array}{l}155.130 w . \\
155.255 w . \\
155.489 w . \\
155.200 w . \\
154.889 w .\end{array}$ & $\begin{array}{r}9 \\
13 \\
10 \\
9 \\
41\end{array}$ & $\begin{array}{l}H V \\
H V\end{array}$ & $\begin{array}{l}\ldots \\
\cdots \\
\cdots \\
\ldots\end{array}$ & $\begin{array}{l}\ldots \\
\cdots \\
\cdots \\
\ldots\end{array}$ & $\begin{array}{l}\text { 3. } 0 M L \text { (HV) } \\
3.0 M L(H V) \\
3.5 M L(H V) \\
3.0 M L(H V) \\
3.2 M L(H V)\end{array}$ & $\begin{array}{l}\text { II I } \\
\ldots \\
\cdots\end{array}$ & $\begin{array}{l}\text { OCT. } \\
\text { OCT. } \\
\text { OCT. } \\
\text { OCT. } \\
\text { OCT. }\end{array}$ & $\begin{array}{l}13 \\
16 \\
16 \\
23 \\
29\end{array}$ & $\begin{array}{l}06: 16 \\
05: 34 \\
14: 45 \\
03: 31 \\
04: 20\end{array}$ & $\begin{array}{l}\text { HST } \\
\text { HST } \\
\text { HST } \\
\text { HST } \\
\text { HST }\end{array}$ \\
\hline $\begin{array}{l}\text { NOV. } \\
\text { NoV. } \\
\text { NOV. } \\
\text { NOV. } \\
\text { NOV. }\end{array}$ & $\begin{array}{r}1 \\
11 \\
12 \\
14 \\
16\end{array}$ & $\begin{array}{l}05 \\
15 \\
05 \\
11 \\
11\end{array}$ & $\begin{array}{l}22 \\
34 \\
03 \\
05 \\
01\end{array}$ & $\begin{array}{l}22.5 \\
06.2 \\
19.5 \\
56.2 \\
58.0\end{array}$ & $\begin{array}{l}19.208 \mathrm{~N} \\
18.931 \mathrm{~N} \\
19.343 \mathrm{~N} \\
19.863 \mathrm{~N} \\
19.371 \mathrm{~N}\end{array}$ & $\begin{array}{l}155.447 w . \\
155.222 w . \\
155.188 w . \\
155.148 w . \\
155.481 w .\end{array}$ & $\begin{array}{r}9 \\
9 \\
9 \\
16 \\
10\end{array}$ & HV & $\begin{array}{l}\cdots \\
\cdots \\
\cdots \\
\cdots\end{array}$ & $\begin{array}{l}\ldots \\
\ldots \\
\ldots \\
\ldots\end{array}$ & $\begin{array}{l}\text { 3. } 0 \mathrm{ML} \text { (HV) } \\
4.0 \mathrm{HL}(\mathrm{HV}) \\
3.4 \mathrm{ML}(\mathrm{VH}) \\
3.8 \mathrm{ML}(\mathrm{HV}) \\
3.0 \mathrm{ML}(\mathrm{HV})\end{array}$ & $\begin{array}{l}\text { I I } \\
\text { I I } \\
\text { IV } \\
\ldots\end{array}$ & $\begin{array}{l}\text { OCT. } \\
\text { NOV. } \\
\text { NOV. } \\
\text { NOV. } \\
\text { NOV. }\end{array}$ & $\begin{array}{l}31 \\
11 \\
11 \\
14 \\
16\end{array}$ & $\begin{array}{l}19: 22 \\
05: 34 \\
19: 03 \\
01: 05 \\
01: 01\end{array}$ & $\begin{array}{l}\text { HST } \\
\text { HST } \\
\text { HST } \\
\text { HST } \\
\text { HST }\end{array}$ \\
\hline $\begin{array}{l}\text { NOV. } \\
\text { NOV. } \\
\text { OV. } \\
\text { DEC. } \\
\text { DEC. }\end{array}$ & $\begin{array}{r}19 \\
28 \\
17 \\
5 \\
7\end{array}$ & $\begin{array}{l}00 \\
12 \\
06 \\
07 \\
11\end{array}$ & $\begin{array}{l}35 \\
15 \\
51 \\
34 \\
16\end{array}$ & $\begin{array}{l}10.0 \\
48.5 \\
59.4 \\
57.6 \\
19.3\end{array}$ & $\begin{array}{l}20.163 \mathrm{~N} \\
19.797 \mathrm{~N} . \\
19.695 \mathrm{~N} \\
19.896 \mathrm{~N} \\
19.087 \mathrm{~N}\end{array}$ & $\begin{array}{l}155.636 \mathrm{~W} . \\
155.394 \mathrm{~W} . \\
155.084 \mathrm{~W} . \\
155.377 \mathrm{~W} . \\
155.411 \mathrm{w} .\end{array}$ & $\begin{array}{r}32 \\
25 \\
6 \\
10 \\
34\end{array}$ & $\begin{array}{l}H V \\
H V \\
H V\end{array}$ & $\begin{array}{l}\cdots \\
\cdots \\
\cdots \\
\cdots\end{array}$ & $\begin{array}{l}\ldots \\
\ldots \\
\ldots \\
\ldots\end{array}$ & $\begin{array}{l}\text { 4. } 0 \mathrm{ML}(\mathrm{HV}) \\
\text { 3.1ML(HV) } \\
\text { 3. } 0 \mathrm{ML}(\mathrm{HV}) \\
\text { 3. } 3 \mathrm{ML}(\mathrm{HV}) \\
\text { 3. } 1 \mathrm{ML}(\mathrm{HV})\end{array}$ & $\begin{array}{l}\text { I I I } \\
\text { I I I } \\
\text { I I I }\end{array}$ & $\begin{array}{l}\text { NOV. } \\
\text { NOV. } \\
\text { NOV. } \\
\text { DEC. } \\
\text { DEC. }\end{array}$ & $\begin{array}{r}18 \\
28 \\
16 \\
4 \\
7\end{array}$ & $\begin{array}{l}14: 35 \\
02: 15 \\
20: 51 \\
21: 34 \\
01: 16\end{array}$ & $\begin{array}{l}\text { HST } \\
\text { HST } \\
\text { HST } \\
\text { HST } \\
\text { HST }\end{array}$ \\
\hline $\begin{array}{l}\text { DEC. } \\
\text { DEC. } \\
\text { DEC. }\end{array}$ & $\begin{array}{l}23 \\
23 \\
26\end{array}$ & $\begin{array}{l}16 \\
17 \\
03\end{array}$ & $\begin{array}{l}46 \\
22\end{array}$ & $\begin{array}{l}19.2 \\
18.5 \\
11.5\end{array}$ & $\begin{array}{l}19.269 \mathrm{~N} \\
19.269 \mathrm{~N} \\
19.980 \mathrm{~N}\end{array}$ & $\begin{array}{l}155.381 W . \\
155.385 w . \\
155.099 w .\end{array}$ & $\begin{array}{r}8 \\
8 \\
35\end{array}$ & & $\begin{array}{l}\cdots \\
\cdots \\
\cdots\end{array}$ & $\begin{array}{l}\ldots \\
\ldots \\
\ldots\end{array}$ & $\begin{array}{l}\text { 3. } 2 \mathrm{ML}(\mathrm{HV}) \\
\text { 3.2ML(HV) } \\
\text { 4.3ML(HV) }\end{array}$ & $\begin{array}{l}\text { I I I } \\
\text { I I I } \\
\text { I I I }\end{array}$ & $\begin{array}{l}\text { DEC. } \\
\text { DEC. } \\
\text { DEC. }\end{array}$ & $\begin{array}{l}23 \\
23 \\
25\end{array}$ & $\begin{array}{l}06: 14 \\
07: 46 \\
17: 22\end{array}$ & $\begin{array}{l}\text { HST } \\
\text { HST } \\
\text { HST }\end{array}$ \\
\hline
\end{tabular}

IDAHO

\begin{tabular}{|c|c|c|c|c|c|c|c|c|c|c|c|c|c|c|c|c|}
\hline $\begin{array}{l}\text { JAN. } \\
\text { JAN. } \\
\text { JAN. } \\
\text { JAN. } \\
\text { JAN. }\end{array}$ & $\begin{array}{r}1 \\
3 \\
3 \\
5 \\
11\end{array}$ & $\begin{array}{l}12 \\
09 \\
09 \\
02 \\
07\end{array}$ & $\begin{array}{l}27 \\
30 \\
40 \\
02 \\
35\end{array}$ & $\begin{array}{l}14.4 \\
08.4 \\
32.9 \\
51.3 \\
44.1\end{array}$ & $\begin{array}{l}44.261 \mathrm{~N} . \\
44.305 \mathrm{~N} . \\
44.281 \mathrm{~N} . \\
44.395 \mathrm{~N} . \\
44.287 \mathrm{~N} .\end{array}$ & $\begin{array}{l}113.985 W . \\
114.113 W . \\
114.107 W . \\
114.160 W . \\
114.093 W .\end{array}$ & $\begin{array}{l}10 \\
10 \\
10 \\
10 \\
10\end{array}$ & $\begin{array}{l}\text { GS } \\
\text { GS } \\
\text { GS } \\
\text { GS } \\
\text { GS }\end{array}$ & $\begin{array}{l}\cdots \\
\cdots \\
\cdots \\
\cdots \\
\cdots\end{array}$ & $\begin{array}{l}\cdots \\
\cdots \\
\cdots \\
\cdots \\
\ldots\end{array}$ & $\begin{array}{l}\text { 3. } 4 M L(G S) \\
\text { 3. } 0 M L(G S) \\
3.3 M L(G S) \\
3.2 M L(G S) \\
4.0 M L(G S)\end{array}$ & $\begin{array}{l}\cdots \\
\cdots \\
\cdots \\
\cdots \\
\cdots\end{array}$ & $\begin{array}{l}\text { JAN. } \\
\text { JAN. } \\
\text { JAN. } \\
\text { JAN. } \\
\text { JAN. }\end{array}$ & $\begin{array}{r}1 \\
3 \\
3 \\
4 \\
11\end{array}$ & $\begin{array}{l}05: 27 \\
01: 30 \\
02: 40 \\
19: 02 \\
00: 35\end{array}$ & $\begin{array}{l}\text { MST } \\
\text { PST } \\
\text { MST } \\
\text { MST } \\
\text { MST }\end{array}$ \\
\hline
\end{tabular}


Table 1. Summary of U.S. earthquakes for 1984-Continued

\begin{tabular}{|c|c|c|c|c|c|c|c|c|c|c|c|c|c|}
\hline \multirow{2}{*}{ Date } & \multicolumn{2}{|c|}{$\begin{array}{l}\text { Origin time } \\
\text { (UTC) }\end{array}$} & \multirow{2}{*}{$\begin{array}{c}\text { Latitude } \\
\left({ }^{\circ}\right)\end{array}$} & \multirow{2}{*}{$\begin{array}{l}\text { Longitude } \\
\text { (क) }\end{array}$} & \multirow{2}{*}{$\begin{array}{c}\text { Depth } \\
\text { (km) }\end{array}$} & \multirow{2}{*}{$\begin{array}{l}\text { Hypo- } \\
\text { center } \\
\text { source }\end{array}$} & \multicolumn{3}{|c|}{ Magnitude } & \multirow{2}{*}{$\begin{array}{l}\text { Maximum } \\
\text { intensity }\end{array}$} & \multicolumn{3}{|c|}{ Local time } \\
\hline & hr $\mathrm{min}$ & $\sec$ & & & & & $\mathbf{m b}$ & MS & $\begin{array}{l}\text { ML, Mn } \\
\text { MD. Mw }\end{array}$ & & Date & Hour & $\begin{array}{l}\text { Time } \\
\text { 7ome }\end{array}$ \\
\hline
\end{tabular}

IDAHO-Continued

\begin{tabular}{|c|c|c|c|c|c|c|c|c|c|c|c|c|c|c|c|c|}
\hline $\begin{array}{l}\text { JAN. } \\
\text { JAN. } \\
\text { JAN. } \\
\text { JAN. } \\
\text { JAN. }\end{array}$ & $\begin{array}{l}15 \\
24 \\
25 \\
29 \\
29\end{array}$ & $\begin{array}{l}00 \\
21 \\
19 \\
03 \\
07\end{array}$ & $\begin{array}{l}05 \\
07 \\
36 \\
22 \\
03\end{array}$ & $\begin{array}{l}34.1 \\
57.5 \\
12.0 \\
25.4 \\
46.1\end{array}$ & $\begin{array}{l}44.264 \mathrm{~N} \\
44.047 \mathrm{~N} \\
47.500 \mathrm{~N} \\
44.721 \mathrm{~N} \\
44.573 \mathrm{~N}\end{array}$ & $\begin{array}{l}114.157 W \\
114.442 W \\
116.000 W . \\
114.442 W . \\
114.264 W .\end{array}$ & $\begin{array}{r}10 \\
10 \\
1 \\
10 \\
10\end{array}$ & $\begin{array}{l}\text { GS } \\
\text { GS } \\
\text { GS } \\
\text { GS } \\
\text { GS }\end{array}$ & $\begin{array}{l}4.5 \\
\ldots \\
\ldots \\
\ldots\end{array}$ & $\begin{array}{l}\cdots \\
\cdots \\
\cdots \\
\cdots\end{array}$ & $\begin{array}{l}\text { 3. } 2 M L(G S) \\
\text { 4. } 6 M L(B U) \\
\text { 2. } 3 M L(G S) \\
\text { 3. } 6 M L(G S) \\
\text { 3. } 1 M L(G S)\end{array}$ & $\begin{array}{c}\text { IV } \\
\text { FELT } \\
\ldots \\
\ldots\end{array}$ & $\begin{array}{l}\text { JAN. } \\
\text { JAN. } \\
\text { JAN. } \\
\text { JAN. } \\
\text { JAN. }\end{array}$ & $\begin{array}{l}14 \\
24 \\
25 \\
28 \\
29\end{array}$ & $\begin{array}{l}17: 05 \\
14: 07 \\
12: 36 \\
20: 22 \\
00: 03\end{array}$ & $\begin{array}{l}\text { MST } \\
\text { MST } \\
\text { MST } \\
\text { MST } \\
\text { MST }\end{array}$ \\
\hline $\begin{array}{l}\text { JAN. } \\
\text { JAN. } \\
\text { JAN. } \\
\text { JAN. } \\
\text { JAN. }\end{array}$ & $\begin{array}{l}29 \\
31 \\
31 \\
31 \\
31\end{array}$ & $\begin{array}{l}12 \\
03 \\
08 \\
09 \\
11\end{array}$ & $\begin{array}{l}05 \\
24 \\
25 \\
57 \\
45\end{array}$ & $\begin{array}{l}06.9 \\
46.5 \\
35.7 \\
44.0 \\
25.6\end{array}$ & $\begin{array}{l}44.098 \mathrm{~N} \\
44.768 \mathrm{~N} \\
44.205 \mathrm{~N} \\
47.414 \mathrm{~N} \\
44.775 \mathrm{~N}\end{array}$ & $\begin{array}{l}113.905 W \\
114.488 W . \\
114.032 W \\
115.944 W . \\
114.460 W .\end{array}$ & $\begin{array}{r}10 \\
10 \\
10 \\
0 \\
10\end{array}$ & $\begin{array}{l}\text { GS } \\
\text { GS } \\
\text { GS } \\
\text { BU } \\
\text { GS }\end{array}$ & $\begin{array}{l}\ldots \\
\ldots \\
\cdots \\
3.7\end{array}$ & $\begin{array}{l}\cdots \\
\cdots \\
\cdots \\
\cdots \\
\cdots\end{array}$ & $\begin{array}{l}\text { 3. } 4 M L(G S) \\
\text { 3. } 2 M L(G S) \\
\text { 3. } 2 M L(G S) \\
\text { 3. } 1 M D(B U) \\
\text { 3. } 2 M L(G S)\end{array}$ & $\begin{array}{l}\cdots \\
\cdots \\
\cdots \\
\cdots \\
\cdots\end{array}$ & $\begin{array}{l}\text { JAN. } \\
\text { JAN. } \\
\text { JAN. } \\
\text { JAN. } \\
\text { JAN. }\end{array}$ & $\begin{array}{l}29 \\
30 \\
31 \\
31 \\
31\end{array}$ & $\begin{array}{l}05: 05 \\
20: 24 \\
01: 25 \\
02: 57 \\
04: 45\end{array}$ & $\begin{array}{l}\text { MST } \\
\text { MST } \\
\text { MST } \\
\text { MST } \\
\text { MST }\end{array}$ \\
\hline $\begin{array}{l}\text { JAN. } \\
\text { FEB. } \\
\text { FEB. } \\
\text { FEB. } \\
\text { FEB. }\end{array}$ & $\begin{array}{r}31 \\
1 \\
1 \\
1 \\
2\end{array}$ & $\begin{array}{l}16 \\
13 \\
16 \\
22 \\
16\end{array}$ & $\begin{array}{l}50 \\
18 \\
12 \\
49 \\
36\end{array}$ & $\begin{array}{l}07.5 \\
08.9 \\
35.4 \\
16.6 \\
33.2\end{array}$ & $\begin{array}{l}44.735 \mathrm{~N} \\
44.703 \mathrm{~N} \\
44.746 \mathrm{~N} \\
44.548 \mathrm{~N} \\
44.672 \mathrm{~N}\end{array}$ & $\begin{array}{l}114.470 W . \\
114.472 W . \\
114.370 W . \\
114.214 W . \\
114.506 W .\end{array}$ & $\begin{array}{l}10 \\
10 \\
10 \\
10 \\
10\end{array}$ & $\begin{array}{l}\text { GS } \\
\text { GS } \\
\text { GS } \\
\text { GS } \\
\text { GS }\end{array}$ & $\begin{array}{l}\cdots \\
\cdots \\
\cdots \\
\cdots \\
\cdots\end{array}$ & $\begin{array}{l}\cdots \\
\cdots \\
\cdots \\
\cdots \\
\cdots\end{array}$ & $\begin{array}{l}\text { 3. } 0 M L(G S) \\
\text { 3. } 4 M L(G S) \\
\text { 3. } 1 M L(G S) \\
\text { 3. } 2 M L(G S) \\
\text { 3. } 4 M L(G S)\end{array}$ & $\begin{array}{l}\cdots \\
\cdots \\
\cdots \\
\cdots\end{array}$ & $\begin{array}{l}\text { JAN. } \\
\text { FEB. } \\
\text { FEB. } \\
\text { FEB. } \\
\text { FEB. }\end{array}$ & $\begin{array}{r}31 \\
1 \\
1 \\
1 \\
2\end{array}$ & $\begin{array}{l}09: 50 \\
06: 18 \\
09: 12 \\
15: 49 \\
09: 36\end{array}$ & $\begin{array}{l}\text { MST } \\
\text { MST } \\
\text { MST } \\
\text { MST } \\
\text { MST }\end{array}$ \\
\hline $\begin{array}{l}\text { FEB. } \\
\text { FEB. } \\
\text { FEB. } \\
\text { FEB. } \\
\text { FEB. }\end{array}$ & $\begin{array}{l}3 \\
5 \\
5 \\
5 \\
6\end{array}$ & $\begin{array}{l}05 \\
03 \\
03 \\
14 \\
23\end{array}$ & $\begin{array}{l}05 \\
42 \\
44 \\
02 \\
04\end{array}$ & $\begin{array}{l}25.1 \\
29.6 \\
15.8 \\
54.7 \\
32.1\end{array}$ & $\begin{array}{l}44.726 \mathrm{~N} \\
44.276 \mathrm{~N} \\
44.275 \mathrm{~N} \\
44.093 \mathrm{~N} \\
44.540 \mathrm{~N}\end{array}$ & $\begin{array}{l}114.432 W . \\
114.124 W . \\
114.127 W . \\
114.196 \mathrm{~W} . \\
114.266 \mathrm{~W} .\end{array}$ & $\begin{array}{l}10 \\
10 \\
10 \\
10 \\
10\end{array}$ & $\begin{array}{l}\text { GS } \\
\text { GS } \\
\text { GS } \\
\text { GS } \\
\text { GS }\end{array}$ & $\begin{array}{l}\cdots \\
\cdots \\
\cdots \\
\cdots\end{array}$ & $\begin{array}{l}\cdots \\
\cdots \\
\cdots \\
\cdots\end{array}$ & $\begin{array}{l}\text { 3. } 2 M L(G S) \\
\text { 3. } 1 M L(G S) \\
\text { 3. } 9 M L(G S) \\
\text { 3. } 2 M L(G S) \\
\text { 3. } 1 M L(G S)\end{array}$ & $\begin{array}{c}\ldots \\
\ldots \\
F \\
\ldots\end{array}$ & $\begin{array}{l}\text { FEB. } \\
\text { FEB. } \\
\text { FEB. } \\
\text { FEB. } \\
\text { FEB. }\end{array}$ & $\begin{array}{l}2 \\
4 \\
4 \\
5 \\
6\end{array}$ & $\begin{array}{l}22: 05 \\
20: 42 \\
20: 44 \\
07: 02 \\
16: 04\end{array}$ & $\begin{array}{l}\text { MST } \\
\text { MST } \\
\text { MST } \\
\text { MST } \\
\text { MST }\end{array}$ \\
\hline $\begin{array}{l}\text { FEB. } \\
\text { FEB. } \\
\text { FEB. } \\
\text { FEB. } \\
\text { FEB. }\end{array}$ & $\begin{array}{r}7 \\
8 \\
8 \\
10 \\
15\end{array}$ & $\begin{array}{l}19 \\
15 \\
18 \\
16 \\
18\end{array}$ & $\begin{array}{l}00 \\
02 \\
17 \\
06 \\
34\end{array}$ & $\begin{array}{l}40.1 \\
32.4 \\
43.9 \\
15.7 \\
36.6\end{array}$ & $\begin{array}{l}44.438 \mathrm{~N} \\
44.702 \mathrm{~N} \\
44.431 \mathrm{~N} \\
44.333 \mathrm{~N} \\
44.294 \mathrm{~N}\end{array}$ & $\begin{array}{l}114.137 W . \\
114.437 W . \\
114.168 W . \\
114.107 W . \\
114.000 W .\end{array}$ & $\begin{array}{l}10 \\
10 \\
10 \\
10 \\
10\end{array}$ & $\begin{array}{l}\text { GS } \\
\text { GS } \\
\text { GS } \\
\text { GS } \\
\text { GS }\end{array}$ & $\begin{array}{l}\cdots \\
\cdots \\
\cdots \\
\cdots\end{array}$ & $\begin{array}{l}\cdots \\
\cdots \\
\cdots \\
\cdots \\
\cdots\end{array}$ & $\begin{array}{l}\text { 3. } 2 M L(G S) \\
\text { 3. } 0 M L(G S) \\
\text { 3. } 4 M L(G S) \\
\text { 3. } 1 M L(G S) \\
\text { 3. } 2 M L(G S)\end{array}$ & $\begin{array}{l}\cdots \\
\cdots \\
\cdots \\
\cdots \\
\cdots\end{array}$ & $\begin{array}{l}\text { FEB. } \\
\text { FEB. } \\
\text { FEB. } \\
\text { FEB. } \\
\text { FEB. }\end{array}$ & $\begin{array}{r}7 \\
8 \\
8 \\
10 \\
15\end{array}$ & $\begin{array}{l}12: 00 \\
08: 02 \\
11: 17 \\
09: 06 \\
11: 34\end{array}$ & $\begin{array}{l}\text { MST } \\
\text { MST } \\
\text { MST } \\
\text { MST } \\
\text { MST }\end{array}$ \\
\hline $\begin{array}{l}\text { FEB. } \\
\text { FEB. } \\
\text { FEB. } \\
\text { FEB. } \\
\text { FEB. }\end{array}$ & $\begin{array}{l}19 \\
20 \\
20 \\
25 \\
27\end{array}$ & $\begin{array}{l}21 \\
02 \\
16 \\
11 \\
11\end{array}$ & $\begin{array}{l}48 \\
58 \\
17 \\
11 \\
52\end{array}$ & $\begin{array}{l}50.5 \\
32.9 \\
25.5 \\
21.0 \\
11.2\end{array}$ & $\begin{array}{l}44.333 \mathrm{~N} \\
44.437 \mathrm{~N} \\
44.432 \mathrm{~N} \\
44.197 \mathrm{~N} \\
44.222 \mathrm{~N}\end{array}$ & $\begin{array}{l}114.105 W . \\
114.089 W . \\
114.194 W . \\
114.093 W . \\
114.003 W .\end{array}$ & $\begin{array}{l}10 \\
10 \\
10 \\
10 \\
10\end{array}$ & $\begin{array}{l}\text { GS } \\
\text { GS } \\
\text { GS } \\
\text { GS } \\
\text { GS }\end{array}$ & $\begin{array}{l}\ldots \\
\ldots \\
\ldots \\
\ldots \\
\ldots\end{array}$ & $\begin{array}{l}\cdots \\
\cdots \\
\cdots \\
\cdots \\
\cdots\end{array}$ & $\begin{array}{l}\text { 3. } 0 M L(G S) \\
3.7 M L(G S) \\
3.6 M L(G S) \\
3.4 M L(G S) \\
3.0 M L(G S)\end{array}$ & $\begin{array}{l}\cdots \\
\cdots \\
\cdots \\
\cdots\end{array}$ & $\begin{array}{l}\text { FEB. } \\
\text { FEB. } \\
\text { FEB. } \\
\text { FEB. } \\
\text { FEB. }\end{array}$ & $\begin{array}{l}19 \\
19 \\
20 \\
25 \\
27\end{array}$ & $\begin{array}{l}14: 48 \\
19: 58 \\
09: 17 \\
04: 11 \\
04: 52\end{array}$ & $\begin{array}{l}\text { MST } \\
\text { MST } \\
\text { MST } \\
\text { MST } \\
\text { MST }\end{array}$ \\
\hline $\begin{array}{l}\text { MAR. } \\
\text { MAR. } \\
\text { MAR. } \\
\text { MAR. } \\
\text { MAR. }\end{array}$ & $\begin{array}{l}2 \\
2 \\
6 \\
6 \\
7\end{array}$ & $\begin{array}{l}00 \\
00 \\
15 \\
19 \\
18\end{array}$ & $\begin{array}{l}29 \\
52 \\
22 \\
51 \\
32\end{array}$ & $\begin{array}{l}45.1 \\
25.3 \\
30.5 \\
35.3 \\
02.9\end{array}$ & $\begin{array}{l}44.350 \mathrm{~N} . \\
44.320 \mathrm{~N} . \\
44.197 \mathrm{~N} . \\
44.349 \mathrm{~N} . \\
44.335 \mathrm{~N} .\end{array}$ & $\begin{array}{l}114.186 W . \\
114.173 W . \\
114.457 W . \\
114.586 W . \\
114.387 W .\end{array}$ & $\begin{array}{l}10 \\
10 \\
10 \\
10 \\
10\end{array}$ & $\begin{array}{l}\text { GS } \\
\text { GS } \\
\text { GS } \\
\text { GS } \\
\text { GS }\end{array}$ & $\begin{array}{l}4.5 \\
\ldots \\
\ldots \\
\ldots \\
\ldots\end{array}$ & $\begin{array}{l}\cdots \\
\cdots \\
\cdots \\
\cdots \\
\cdots\end{array}$ & $\begin{array}{l}\text { 4. 3ML (GS) } \\
\text { 3. } 0 \mathrm{ML}(\mathrm{GS}) \\
3.3 \mathrm{ML}(\mathrm{GS}) \\
3.0 \mathrm{ML}(\mathrm{GS}) \\
3.0 \mathrm{ML}(\mathrm{GS})\end{array}$ & $\begin{array}{l}\text { IV } \\
\cdots \\
\cdots \\
\cdots \\
\cdots\end{array}$ & $\begin{array}{l}\text { MAR. } \\
\text { MAR. } \\
\text { MAR. } \\
\text { MAR. } \\
\text { MAR. }\end{array}$ & $\begin{array}{l}1 \\
1 \\
6 \\
6 \\
7\end{array}$ & $\begin{array}{l}17: 29 \\
17: 52 \\
08: 22 \\
12: 51 \\
11: 32\end{array}$ & $\begin{array}{l}\text { MST } \\
\text { MST } \\
\text { MST } \\
\text { MST } \\
\text { MST }\end{array}$ \\
\hline $\begin{array}{l}\text { MAR. } \\
\text { MAR. } \\
\text { MAR. } \\
\text { MAR. } \\
\text { MAR. }\end{array}$ & $\begin{array}{l}8 \\
9 \\
9 \\
9 \\
9\end{array}$ & $\begin{array}{l}11 \\
01 \\
01 \\
10 \\
23\end{array}$ & $\begin{array}{l}19 \\
16 \\
55 \\
49 \\
49\end{array}$ & $\begin{array}{l}43.6 \\
14.2 \\
34.0 \\
37.2 \\
12.4\end{array}$ & $\begin{array}{l}44.467 \mathrm{~N} \\
44.330 \mathrm{~N} \\
44.302 \mathrm{~N} \\
43.971 \mathrm{~N} \\
44.151 \mathrm{~N}\end{array}$ & $\begin{array}{l}112.643 w . \\
114.009 w . \\
114.102 W . \\
113.730 w . \\
113.977 w .\end{array}$ & $\begin{array}{r}5 \\
5 \\
10 \\
10 \\
10\end{array}$ & $\begin{array}{l}\text { GS } \\
\text { GS } \\
\text { GS } \\
\text { GS } \\
\text { GS }\end{array}$ & $\begin{array}{l}\ldots \\
\cdots \\
\cdots \\
\cdots\end{array}$ & $\begin{array}{l}\cdots \\
\cdots \\
\cdots \\
\cdots\end{array}$ & $\begin{array}{l}\text { 3. } 1 M L(B U) \\
\text { 3. } 3 M L(G S) \\
\text { 3. } 3 M L(G S) \\
\text { 3. } 3 M L(G S) \\
3.4 M L(G S)\end{array}$ & $\begin{array}{l}\cdots \\
\cdots \\
\cdots \\
\cdots\end{array}$ & $\begin{array}{l}\text { MAR. } \\
\text { MAR. } \\
\text { MAR. } \\
\text { MAR. } \\
\text { MAR. }\end{array}$ & $\begin{array}{l}8 \\
8 \\
8 \\
9 \\
9\end{array}$ & $\begin{array}{l}04: 19 \\
18: 16 \\
18: 55 \\
03: 49 \\
16: 49\end{array}$ & $\begin{array}{l}\text { MST } \\
\text { MST } \\
\text { MST } \\
\text { MST } \\
\text { MST }\end{array}$ \\
\hline $\begin{array}{l}\text { MAR. } \\
\text { MAR. } \\
\text { MAR. } \\
\text { MAR. } \\
\text { MAR. }\end{array}$ & $\begin{array}{l}10 \\
12 \\
16 \\
17 \\
21\end{array}$ & $\begin{array}{l}04 \\
83 \\
14 \\
82 \\
21\end{array}$ & $\begin{array}{l}53 \\
21 \\
35 \\
10 \\
43\end{array}$ & $\begin{array}{l}46.9 \\
56.7 \\
38.9 \\
20.4 \\
36.5\end{array}$ & $\begin{array}{l}44.437 \mathrm{~N} \\
44.347 \mathrm{~N} \\
44.309 \mathrm{~N} \\
44.266 \mathrm{~N} \\
44.730 \mathrm{~N}\end{array}$ & $\begin{array}{l}112.638 W \\
114.317 W \\
114.544 W \\
114.147 W \\
114.438 W\end{array}$ & $\begin{array}{r}5 \\
10 \\
10 \\
10 \\
10\end{array}$ & $\begin{array}{l}\text { GS } \\
\text { GS } \\
\text { GS } \\
\text { GS } \\
\text { GS }\end{array}$ & $\begin{array}{l}\cdots \\
\cdots \\
\cdots \\
\cdots\end{array}$ & $\begin{array}{l}\cdots \\
\cdots \\
\cdots \\
\cdots \\
\cdots\end{array}$ & $\begin{array}{l}\text { 3. } 3 \mathrm{ML}(\mathrm{GS}) \\
\text { 3. } 2 \mathrm{ML}(\mathrm{GS}) \\
3.2 \mathrm{ML}(\mathrm{BU}) \\
\text { 3. } 2 \mathrm{ML}(\mathrm{GS}) \\
\text { 3. } 8 \mathrm{ML}(\mathrm{GS})\end{array}$ & $\begin{array}{l}\ldots \\
\cdots \\
\cdots\end{array}$ & $\begin{array}{l}\text { MAR. } \\
\text { MAR. } \\
\text { MAR. } \\
\text { MAR. } \\
\text { MAR. }\end{array}$ & $\begin{array}{r}9 \\
11 \\
16 \\
16 \\
21\end{array}$ & $\begin{array}{l}21: 53 \\
20: 21 \\
07: 35 \\
19: 10 \\
14: 43\end{array}$ & $\begin{array}{l}\text { MST } \\
\text { MST } \\
\text { MST } \\
\text { MST } \\
\text { MST }\end{array}$ \\
\hline $\begin{array}{l}\text { MAR. } \\
\text { MAR. } \\
\text { MAR. } \\
\text { MAR. } \\
\text { MAR. }\end{array}$ & $\begin{array}{l}23 \\
24 \\
24 \\
24 \\
24\end{array}$ & $\begin{array}{l}01 \\
00 \\
02 \\
02 \\
03\end{array}$ & $\begin{array}{l}39 \\
07 \\
16 \\
31 \\
03\end{array}$ & $\begin{array}{l}37.3 \\
47.7 \\
52.8 \\
33.3 \\
15.5\end{array}$ & $\begin{array}{l}44.696 \mathrm{~N} \\
44.740 \mathrm{~N} \\
44.743 \mathrm{~N} \\
44.669 \mathrm{~N} \\
44.722 \mathrm{~N}\end{array}$ & $\begin{array}{l}114.531 W . \\
114.430 W . \\
114.373 w . \\
114.471 W . \\
114.461 W .\end{array}$ & $\begin{array}{l}10 \\
10 \\
10 \\
10 \\
10\end{array}$ & $\begin{array}{l}\text { GS } \\
\text { GS } \\
\text { GS } \\
\text { GS }\end{array}$ & $\begin{array}{l}4.2 \\
\ldots \\
\ldots \\
\ldots\end{array}$ & $\begin{array}{l}\ldots \\
\cdots \\
\cdots \\
\cdots \\
\cdots\end{array}$ & $\begin{array}{l}\text { 3. } 2 M L(G S) \\
\text { 4.3ML(BU) } \\
\text { 3. } 0 M L(G S) \\
\text { 3. } 2 M L(G S) \\
\text { 4.0ML(GS) }\end{array}$ & $\cdots$ & $\begin{array}{l}\text { MAR. } \\
\text { MAR. } \\
\text { MAR. } \\
\text { MAR. } \\
\text { MAR. }\end{array}$ & $\begin{array}{l}22 \\
23 \\
23 \\
23 \\
23\end{array}$ & $\begin{array}{l}18: 39 \\
17: 07 \\
19: 16 \\
19: 31 \\
20: 03\end{array}$ & $\begin{array}{l}\text { MST } \\
\text { MST } \\
\text { MST } \\
\text { MST } \\
\text { MST }\end{array}$ \\
\hline
\end{tabular}




\begin{tabular}{|c|c|c|c|c|c|c|c|c|c|c|c|c|c|}
\hline \multirow{2}{*}{ Date } & \multicolumn{2}{|c|}{$\begin{array}{c}\text { Origin time } \\
\text { (UTC) }\end{array}$} & \multirow{2}{*}{$\begin{array}{c}\text { Latitude } \\
\left.\text { ( }{ }^{\circ}\right)\end{array}$} & \multirow{2}{*}{$\begin{array}{l}\text { Longitude } \\
\text { ( })\end{array}$} & \multirow{2}{*}{$\begin{array}{c}\text { Depth } \\
(\mathbf{k m})\end{array}$} & \multirow{2}{*}{$\begin{array}{l}\text { Hypo- } \\
\text { center } \\
\text { source }\end{array}$} & \multicolumn{3}{|c|}{ Magnitude } & \multirow{2}{*}{$\begin{array}{l}\text { Maximum } \\
\text { intensity }\end{array}$} & \multicolumn{3}{|c|}{ Lucal time } \\
\hline & hr $\min$ & $\sec$ & & & & & $\mathbf{m b}$ & MS & $\begin{array}{l}\text { ML, Mn } \\
\text { MD, Mw }\end{array}$ & & Date & Hour & $\begin{array}{l}\text { Time } \\
\text { zome }\end{array}$ \\
\hline
\end{tabular}

IDAHO-Continued

\begin{tabular}{|c|c|c|c|c|c|c|c|c|c|c|c|c|c|c|c|c|}
\hline $\begin{array}{l}\text { MAR. } \\
\text { MAR. } \\
\text { MAR. } \\
\text { MAR. } \\
\text { MAR. }\end{array}$ & $\begin{array}{l}24 \\
24 \\
24 \\
24 \\
24\end{array}$ & $\begin{array}{l}08 \\
09 \\
16 \\
19 \\
20\end{array}$ & $\begin{array}{l}55 \\
53 \\
01 \\
20 \\
02\end{array}$ & $\begin{array}{l}14.5 \\
42.6 \\
32.6 \\
13.8 \\
06.0\end{array}$ & $\begin{array}{l}44.715 \mathrm{~N} \\
44.693 \mathrm{~N} \\
44.641 \mathrm{~N} \\
44.721 \mathrm{~N} \\
44.709 \mathrm{~N}\end{array}$ & $\begin{array}{l}114.423 W . \\
114.534 W . \\
114.670 W . \\
114.443 W . \\
114.452 W .\end{array}$ & $\begin{array}{l}10 \\
10 \\
10 \\
10 \\
10\end{array}$ & $\begin{array}{l}\text { GS } \\
\text { GS } \\
\text { GS } \\
\text { GS } \\
\text { GS }\end{array}$ & $\begin{array}{l}\cdots \\
\cdots \\
\cdots \\
\cdots\end{array}$ & $\begin{array}{l}\cdots \\
\cdots \\
\cdots \\
\cdots \\
\cdots\end{array}$ & $\begin{array}{l}\text { 3. } 3 M L(G S) \\
\text { 3. } 6 \mathrm{ML}(\mathrm{BU}) \\
\text { 3. } 1 \mathrm{ML}(\mathrm{GS}) \\
\text { 3. } 3 \mathrm{ML}(\mathrm{GS}) \\
\text { 3. } 5 \mathrm{ML}(\mathrm{GS})\end{array}$ & $\begin{array}{l}\cdots \\
\cdots \\
\cdots \\
\cdots \\
\cdots\end{array}$ & $\begin{array}{l}\text { MAR. } \\
\text { MAR. } \\
\text { MAR. } \\
\text { MAR. } \\
\text { MAR. }\end{array}$ & $\begin{array}{l}24 \\
24 \\
24 \\
24 \\
24\end{array}$ & $\begin{array}{l}01: 55 \\
02: 53 \\
09: 01 \\
12: 20 \\
13: 02\end{array}$ & $\begin{array}{l}\text { MST } \\
\text { MST } \\
\text { MST } \\
\text { MST } \\
\text { MST }\end{array}$ \\
\hline $\begin{array}{l}\text { MAR. } \\
\text { MAR. } \\
\text { MAR. } \\
\text { MAR. } \\
\text { MAR. }\end{array}$ & $\begin{array}{l}24 \\
24 \\
25 \\
25 \\
29\end{array}$ & $\begin{array}{l}21 \\
21 \\
00 \\
11 \\
11\end{array}$ & $\begin{array}{l}01 \\
03 \\
21 \\
24 \\
43\end{array}$ & $\begin{array}{l}12.3 \\
16.3 \\
14.5 \\
53.7 \\
55.0\end{array}$ & $\begin{array}{l}44.374 \mathrm{~N} \\
44.728 \mathrm{~N} \\
44.713 \mathrm{~N} \\
44.722 \mathrm{~N} \\
44.708 \mathrm{~N}\end{array}$ & $\begin{array}{l}114.086 W . \\
114.441 W . \\
114.507 W . \\
114.422 W . \\
114.505 W .\end{array}$ & $\begin{array}{l}10 \\
10 \\
10 \\
10 \\
10\end{array}$ & $\begin{array}{l}\text { GS } \\
\text { GS } \\
\text { GS } \\
\text { GS } \\
\text { GS }\end{array}$ & $\begin{array}{l}\ldots \\
\ldots \\
\ldots \\
\ldots\end{array}$ & $\begin{array}{l}\cdots \\
\cdots \\
\cdots \\
\cdots \\
\cdots\end{array}$ & $\begin{array}{l}\text { 3.5ML(BU) } \\
3.8 M L(G S) \\
3.2 M L(G S) \\
3.5 M L(G S) \\
3.2 M L(G S)\end{array}$ & $\begin{array}{l}\text { II I } \\
\ldots \\
\ldots \\
\ldots\end{array}$ & $\begin{array}{l}\text { MAR. } \\
\text { MAR. } \\
\text { MAR. } \\
\text { MAR. } \\
\text { MAR. }\end{array}$ & $\begin{array}{l}24 \\
24 \\
24 \\
25 \\
29\end{array}$ & $\begin{array}{l}14: 01 \\
14: 03 \\
17: 21 \\
04: 24 \\
04: 43\end{array}$ & $\begin{array}{l}\text { MST } \\
\text { MST } \\
\text { MST } \\
\text { MST } \\
\text { MST }\end{array}$ \\
\hline $\begin{array}{l}\text { MAR. } \\
\text { MAR. } \\
\text { MAR. } \\
\text { APR. } \\
\text { APR. }\end{array}$ & $\begin{array}{r}30 \\
31 \\
31 \\
1 \\
1\end{array}$ & $\begin{array}{l}16 \\
01 \\
23 \\
05 \\
85\end{array}$ & $\begin{array}{l}23 \\
12 \\
07 \\
15 \\
39\end{array}$ & $\begin{array}{l}59.2 \\
56.4 \\
10.1 \\
18.8 \\
27.8\end{array}$ & $\begin{array}{l}44.430 \mathrm{~N} \\
44.326 \mathrm{~N} \\
44.728 \mathrm{~N} \\
44.707 \mathrm{~N} \\
44.726 \mathrm{~N}\end{array}$ & $\begin{array}{l}114.173 w . \\
114.144 W . \\
114.356 w . \\
114.410 W . \\
114.375 W .\end{array}$ & $\begin{array}{l}10 \\
10 \\
10 \\
10 \\
10\end{array}$ & $\begin{array}{l}\text { GS } \\
\text { GS } \\
\text { GS } \\
\text { GS } \\
\text { GS }\end{array}$ & $\begin{array}{l}\ldots \\
\cdots \\
\cdots \\
\cdots\end{array}$ & $\begin{array}{l}\cdots \\
\cdots \\
\cdots \\
\cdots\end{array}$ & $\begin{array}{l}\text { 3.5ML(BU) } \\
\text { 3.3ML(GS) } \\
\text { 3.3ML(GS) } \\
\text { 3.2ML(GS) } \\
\text { 3.9ML(GS) }\end{array}$ & $\begin{array}{l}\cdots \\
\cdots \\
\cdots \\
\cdots \\
\cdots\end{array}$ & $\begin{array}{l}\text { MAR. } \\
\text { MAR. } \\
\text { MAR. } \\
\text { MAR. } \\
\text { MAR. }\end{array}$ & $\begin{array}{l}30 \\
30 \\
31 \\
31 \\
31\end{array}$ & $\begin{array}{l}09: 23 \\
18: 12 \\
16: 07 \\
22: 15 \\
22: 39\end{array}$ & $\begin{array}{l}\text { MST } \\
\text { MST } \\
\text { MST } \\
\text { MST } \\
\text { MST }\end{array}$ \\
\hline $\begin{array}{l}\text { APR. } \\
\text { APR. } \\
\text { APR. } \\
\text { APR. } \\
\text { APR. }\end{array}$ & $\begin{array}{r}1 \\
3 \\
3 \\
10 \\
10\end{array}$ & $\begin{array}{l}89 \\
00 \\
06 \\
02 \\
14\end{array}$ & $\begin{array}{l}13 \\
07 \\
58 \\
10 \\
30\end{array}$ & $\begin{array}{l}38.4 \\
26.7 \\
08.1 \\
18.8 \\
54.9\end{array}$ & $\begin{array}{l}44.739 \mathrm{~N} . \\
44.297 \mathrm{~N} . \\
44.200 \mathrm{~N} \\
44.654 \mathrm{~N} \\
44.270 \mathrm{~N}\end{array}$ & $\begin{array}{l}114.376 \mathrm{~W} . \\
114.507 \mathrm{~W} . \\
114.161 \mathrm{~W} . \\
114.310 \mathrm{~W} . \\
114.365 \mathrm{~W} .\end{array}$ & $\begin{array}{l}10 \\
10 \\
10 \\
10 \\
10\end{array}$ & $\begin{array}{l}\text { GS } \\
\text { GS } \\
\text { GS } \\
\text { GS } \\
\text { GS }\end{array}$ & $\begin{array}{l}\cdots \\
\cdots \\
\cdots \\
\cdots \\
\cdots\end{array}$ & $\begin{array}{l}\cdots \\
\cdots \\
\cdots \\
\cdots\end{array}$ & $\begin{array}{l}\text { 3. } 4 M L(G S) \\
\text { 3.1ML(BU) } \\
\text { 3. } 0 M L(B U) \\
\text { 3.1ML(GS) } \\
\text { 3. } 2 M L(B U)\end{array}$ & $\begin{array}{c}\cdots \\
\cdots \\
\text { FELT } \\
\text { FELT }\end{array}$ & $\begin{array}{l}\text { APR. } \\
\text { APR. } \\
\text { APR. } \\
\text { APR. } \\
\text { APR. }\end{array}$ & $\begin{array}{r}1 \\
2 \\
2 \\
9 \\
10\end{array}$ & $\begin{array}{l}02: 13 \\
17: 07 \\
23: 58 \\
19: 10 \\
07: 30\end{array}$ & $\begin{array}{l}\text { MST } \\
\text { MST } \\
\text { MST } \\
\text { MST } \\
\text { MST }\end{array}$ \\
\hline $\begin{array}{l}\text { APR. } \\
\text { APR } \\
\text { APR. } \\
\text { APR. } \\
\text { APR. }\end{array}$ & $\begin{array}{l}11 \\
11 \\
12 \\
13 \\
13\end{array}$ & $\begin{array}{l}08 \\
15 \\
09 \\
01 \\
07\end{array}$ & $\begin{array}{l}23 \\
51 \\
08 \\
14 \\
16\end{array}$ & $\begin{array}{l}20.9 \\
55.9 \\
02.0 \\
13.5 \\
39.5\end{array}$ & $\begin{array}{l}44.297 \mathrm{~N} \\
44.774 \mathrm{~N} \\
44.297 \mathrm{~N} \\
44.281 \mathrm{~N} \\
44.727 \mathrm{~N}\end{array}$ & $\begin{array}{l}114.295 W . \\
114.469 W \\
114.280 W . \\
114.193 W . \\
114.433 W .\end{array}$ & $\begin{array}{l}10 \\
10 \\
10 \\
10 \\
10\end{array}$ & $\begin{array}{l}\text { GS } \\
\text { GS } \\
\text { GS } \\
\text { GS } \\
\text { GS }\end{array}$ & $\begin{array}{l}3.7 \\
\ldots \\
\ldots \\
\ldots\end{array}$ & $\begin{array}{l}\cdots \\
\cdots \\
\cdots \\
\cdots \\
\cdots\end{array}$ & $\begin{array}{l}\text { 3. } 4 M L(B U) \\
\text { 4. } 1 \mathrm{ML}(\mathrm{GS}) \\
\text { 3. } 0 \mathrm{ML}(\mathrm{BU}) \\
\text { 3. } 4 \mathrm{ML}(\mathrm{BU}) \\
3.0 \mathrm{ML}(\mathrm{GS})\end{array}$ & $\begin{array}{c}\text { IV } \\
\text { FELT } \\
\ldots \\
\cdots\end{array}$ & $\begin{array}{l}\text { APR. } \\
\text { APR. } \\
\text { APR. } \\
\text { APR. } \\
\text { APR. }\end{array}$ & $\begin{array}{l}11 \\
11 \\
12 \\
12 \\
13\end{array}$ & $\begin{array}{l}01: 23 \\
08: 51 \\
02: 08 \\
18: 14 \\
00: 16\end{array}$ & $\begin{array}{l}\text { MST } \\
\text { MST } \\
\text { MST } \\
\text { MST } \\
\text { MST }\end{array}$ \\
\hline $\begin{array}{l}\text { APR } \\
\text { APR } \\
\text { APR. } \\
\text { APR. } \\
\text { APR. }\end{array}$ & $\begin{array}{l}13 \\
18 \\
19 \\
20 \\
23\end{array}$ & $\begin{array}{l}12 \\
01 \\
01 \\
17 \\
05\end{array}$ & $\begin{array}{l}16 \\
15 \\
58 \\
25 \\
54\end{array}$ & $\begin{array}{l}05.4 \\
09.4 \\
49.5 \\
31.2 \\
14.6\end{array}$ & $\begin{array}{l}44.742 \mathrm{~N} . \\
44.342 \mathrm{~N} . \\
44.363 \mathrm{~N} \\
44.608 \mathrm{~N} \\
44.133 \mathrm{~N}\end{array}$ & $\begin{array}{l}114.424 W . \\
114.099 W \\
114.137 W \\
114.408 W \\
113.867 W\end{array}$ & $\begin{array}{l}10 \\
10 \\
10 \\
10 \\
10\end{array}$ & $\begin{array}{l}\text { GS } \\
\text { GS } \\
\text { GS } \\
\text { GS } \\
\text { GS }\end{array}$ & $\begin{array}{l}\cdots \\
\cdots \\
\cdots \\
\cdots \\
\cdots\end{array}$ & $\begin{array}{l}\cdots \\
\cdots \\
\cdots \\
\cdots\end{array}$ & $\begin{array}{l}\text { 3. } 1 \mathrm{ML}(\mathrm{GS}) \\
\text { 4. } \mathrm{OML}(\mathrm{GS}) \\
\text { 3. } 3 \mathrm{ML}(\mathrm{GS}) \\
\text { 3. } 0 \mathrm{ML}(\mathrm{BU}) \\
\text { 3. } 3 \mathrm{ML}(\mathrm{BU})\end{array}$ & $\begin{array}{l}\ldots \\
\cdots \\
\cdots \\
\cdots\end{array}$ & $\begin{array}{l}\text { APR. } \\
\text { APR. } \\
\text { APR. } \\
\text { APR. } \\
\text { APR. }\end{array}$ & $\begin{array}{l}13 \\
17 \\
18 \\
20 \\
22\end{array}$ & $\begin{array}{l}05: 16 \\
18: 15 \\
18: 58 \\
10: 25 \\
22: 54\end{array}$ & $\begin{array}{l}\text { MST } \\
\text { MST } \\
\text { MST } \\
\text { MST } \\
\text { MST }\end{array}$ \\
\hline $\begin{array}{l}\text { APR. } \\
\text { MAY } \\
\text { MAY } \\
\text { JUNE } \\
\text { JUNE }\end{array}$ & $\begin{array}{l}29 \\
16 \\
30 \\
15 \\
26\end{array}$ & $\begin{array}{l}04 \\
18 \\
04 \\
23 \\
19\end{array}$ & $\begin{array}{l}42 \\
02 \\
11 \\
04 \\
15\end{array}$ & $\begin{array}{l}55.8 \\
20.0 \\
01.7 \\
32.7 \\
35.9\end{array}$ & $\begin{array}{l}44.244 \mathrm{~N} \\
44.302 \mathrm{~N} \\
44.264 \mathrm{~N} \\
46.641 \mathrm{~N} \\
44.221 \mathrm{~N}\end{array}$ & $\begin{array}{l}114.135 W . \\
114.126 W . \\
114.136 W . \\
116.245 W . \\
114.046 W .\end{array}$ & $\begin{array}{r}10 \\
10 \\
10 \\
1 \\
10\end{array}$ & $\begin{array}{l}\text { GS } \\
\text { GS } \\
\text { GS } \\
\text { BU } \\
\text { GS }\end{array}$ & $\begin{array}{l}\cdots \\
\cdots \\
\cdots \\
\cdots \\
\cdots\end{array}$ & $\begin{array}{l}\cdots \\
\cdots \\
\cdots \\
\cdots\end{array}$ & $\begin{array}{l}\text { 3. } 4 M L(G S) \\
3.2 M L(G S) \\
3.5 M L(G S) \\
2.5 M L(B U) \\
3.5 M L(G S)\end{array}$ & $\begin{array}{l}\cdots \\
\cdots \\
\cdots \\
\cdots\end{array}$ & $\begin{array}{l}\text { APR. } \\
\text { MAY } \\
\text { MAY } \\
\text { JUNE } \\
\text { JUNE }\end{array}$ & $\begin{array}{l}28 \\
16 \\
29 \\
15 \\
26\end{array}$ & $\begin{array}{l}21: 42 \\
11: 02 \\
21: 11 \\
16: 04 \\
12: 15\end{array}$ & $\begin{array}{l}\text { MST } \\
\text { MST } \\
\text { MST } \\
\text { MST } \\
\text { MST }\end{array}$ \\
\hline $\begin{array}{l}\text { JUNE } \\
\text { JULY } \\
\text { JULY } \\
\text { JULY } \\
\text { JULY }\end{array}$ & $\begin{array}{r}30 \\
5 \\
14 \\
16 \\
29\end{array}$ & $\begin{array}{l}23 \\
19 \\
17 \\
01 \\
11\end{array}$ & $\begin{array}{l}39 \\
32 \\
46 \\
34 \\
54\end{array}$ & $\begin{array}{l}07.5 \\
59.0 \\
02.1 \\
52.8 \\
14.3\end{array}$ & $\begin{array}{l}44.651 \mathrm{~N} \\
44.574 \mathrm{~N} \\
44.628 \mathrm{~N} \\
44.302 \mathrm{~N} \\
44.620 \mathrm{~N}\end{array}$ & $\begin{array}{l}114.396 W . \\
114.286 W . \\
114.293 W . \\
114.333 W . \\
114.490 W .\end{array}$ & $\begin{array}{l}10 \\
10 \\
10 \\
10 \\
10\end{array}$ & $\begin{array}{l}\text { GS } \\
\text { GS } \\
\text { GS } \\
\text { GS } \\
\text { GS }\end{array}$ & $\begin{array}{l}\cdots \\
\cdots \\
\cdots \\
\cdots\end{array}$ & $\begin{array}{l}\ldots \\
\ldots \\
\cdots \\
\ldots \\
\cdots\end{array}$ & $\begin{array}{l}\text { 3. } 8 M L(G S) \\
\text { 3. } 4 M L(G S) \\
3.6 M L(G S) \\
3.3 M L(G S) \\
3.4 M L(G S)\end{array}$ & $\begin{array}{l}\cdots \\
\cdots \\
\cdots \\
\cdots \\
\cdots\end{array}$ & $\begin{array}{l}\text { JUNE } \\
\text { JULY } \\
\text { JULY } \\
\text { JULY } \\
\text { JULY }\end{array}$ & $\begin{array}{r}30 \\
5 \\
14 \\
15 \\
29\end{array}$ & $\begin{array}{l}16: 39 \\
12: 32 \\
10: 46 \\
18: 34 \\
04: 54\end{array}$ & $\begin{array}{l}\text { MST } \\
\text { MST } \\
\text { MST } \\
\text { MST } \\
\text { MST }\end{array}$ \\
\hline $\begin{array}{l}\text { JULY } \\
\text { AUG. } \\
\text { AUG. } \\
\text { AUG. } \\
\text { AUG. }\end{array}$ & $\begin{array}{l}30 \\
15 \\
21 \\
22 \\
22\end{array}$ & $\begin{array}{l}23 \\
22 \\
23 \\
09 \\
10\end{array}$ & $\begin{array}{l}06 \\
07 \\
42 \\
46 \\
10\end{array}$ & $\begin{array}{l}48.2 \\
52.4 \\
13.7 \\
30.2 \\
05.7\end{array}$ & $\begin{array}{l}48.872 \mathrm{~N} \\
44.126 \mathrm{~N} \\
44.087 \mathrm{~N} \\
44.467 \mathrm{~N} \\
44.082 \mathrm{~N}\end{array}$ & $\begin{array}{l}116.204 w . \\
114.717 w . \\
114.759 w . \\
114.008 w . \\
114.687 w .\end{array}$ & $\begin{array}{l}11 \\
10 \\
10 \\
10 \\
10\end{array}$ & $\begin{array}{l}\text { BU } \\
\text { GS } \\
\text { GS } \\
\text { GS } \\
\text { GS }\end{array}$ & $\begin{array}{l}\cdots \\
\cdots \\
\cdots \\
\ldots \\
\cdots\end{array}$ & $\begin{array}{l}\ldots \\
\ldots \\
\ldots \\
5.1 \\
\ldots\end{array}$ & $\begin{array}{l}\text { 3. } 4 M L(B U) \\
\text { 3. } 4 M L(G S) \\
3.2 M L(G S) \\
5.8 M L(U U) \\
3.8 M L(G S)\end{array}$ & $\begin{array}{l}\cdots \\
\cdots \\
\cdots \\
\cdots\end{array}$ & $\begin{array}{l}\text { JULY } \\
\text { AUG. } \\
\text { AUG. } \\
\text { AUG. } \\
\text { AUG. }\end{array}$ & $\begin{array}{l}30 \\
15 \\
21 \\
22 \\
22\end{array}$ & $\begin{array}{l}15: 06 \\
15: 07 \\
16: 42 \\
02: 46 \\
03: 10\end{array}$ & $\begin{array}{l}\text { PST } \\
\text { MST } \\
\text { MST } \\
\text { MST } \\
\text { MST }\end{array}$ \\
\hline $\begin{array}{l}\text { AUG. } \\
A \cup G . \\
A \cup G . \\
A \cup G . \\
A \cup G .\end{array}$ & $\begin{array}{l}22 \\
22 \\
22 \\
22 \\
22\end{array}$ & $\begin{array}{l}10 \\
10 \\
10 \\
11 \\
11\end{array}$ & $\begin{array}{l}48 \\
50 \\
52 \\
09 \\
44\end{array}$ & $\begin{array}{l}24.2 \\
25.0 \\
01.1 \\
19.7 \\
24.9\end{array}$ & $\begin{array}{l}44.454 \mathrm{~N} . \\
44.452 \mathrm{~N} . \\
44.481 \mathrm{~N} . \\
44.468 \mathrm{~N} . \\
44.419 \mathrm{~N} .\end{array}$ & $\begin{array}{l}114.153 w . \\
114.113 w . \\
114.204 W . \\
114.084 W . \\
114.118 W .\end{array}$ & $\begin{array}{l}10 \\
10 \\
10 \\
10 \\
10\end{array}$ & $\begin{array}{l}\text { GS } \\
\text { GS } \\
G S \\
\text { GS } \\
\text { GS }\end{array}$ & $\begin{array}{l}\cdots \\
\cdots \\
\cdots \\
\cdots \\
\cdots\end{array}$ & $\begin{array}{l}\ldots \\
\ldots \\
\ldots \\
\ldots \\
\ldots\end{array}$ & $\begin{array}{l}\text { 3. } 3 M L(G S) \\
\text { 3. 3ML (GS) } \\
\text { 4. } 9 M L(G S) \\
\text { 3. } 3 M L(G S) \\
\text { 3. } 2 M L(G S)\end{array}$ & $\begin{array}{l}\cdots \\
\cdots \\
\cdots \\
\cdots\end{array}$ & $\begin{array}{l}\text { AUG. } \\
\text { AUG. } \\
\text { AUG. } \\
\text { AUG. } \\
\text { AUG. }\end{array}$ & $\begin{array}{l}22 \\
22 \\
22 \\
22 \\
22\end{array}$ & $\begin{array}{l}03: 48 \\
03: 50 \\
03: 52 \\
04: 09 \\
04: 44\end{array}$ & $\begin{array}{l}\text { ST } \\
\text { IST } \\
\text { IST } \\
\text { ST }\end{array}$ \\
\hline
\end{tabular}


Table 1. Summary of U.S. earthquakes for 1984-Continued

\begin{tabular}{|c|c|c|c|c|c|c|c|c|c|c|c|c|c|}
\hline Date & hr $\min$ & $\sec$ & $\begin{array}{l}\text { Latitude } \\
\left({ }^{\circ}\right)\end{array}$ & $\begin{array}{c}\text { Longitude } \\
\text { (1) }\end{array}$ & $\begin{array}{c}\text { Depth } \\
(\mathbf{k m})\end{array}$ & $\begin{array}{l}\text { Hypo- } \\
\text { center } \\
\text { source }\end{array}$ & $\mathbf{m b}$ & MS & $\begin{array}{l}\text { ML, } \mathbf{M n} \\
M D, M w\end{array}$ & $\begin{array}{l}\text { Maximum } \\
\text { intensity }\end{array}$ & Date & Hour & $\begin{array}{l}\text { Time } \\
\text { zone }\end{array}$ \\
\hline
\end{tabular}

IDAHO-Continued

\begin{tabular}{|c|c|c|c|c|c|c|c|c|c|c|c|c|c|c|c|c|}
\hline $\begin{array}{l}\text { AUG. } \\
\text { AUG. } \\
\text { AUG. } \\
\text { AUG. } \\
\text { AUG. }\end{array}$ & $\begin{array}{l}22 \\
22 \\
22 \\
22 \\
23\end{array}$ & $\begin{array}{l}11 \\
12 \\
13 \\
15 \\
80\end{array}$ & $\begin{array}{l}52 \\
06 \\
34 \\
35 \\
01\end{array}$ & $\begin{array}{l}30.8 \\
31.6 \\
21.6 \\
41.5 \\
58.1\end{array}$ & $\begin{array}{l}44.186 \mathrm{~N} \\
44.377 \mathrm{~N} \\
44.471 \mathrm{~N} \\
44.419 \mathrm{~N} \\
44.369 \mathrm{~N}\end{array}$ & $\begin{array}{l}114.658 W . \\
114.181 w . \\
114.152 W . \\
114.094 W . \\
114.203 w .\end{array}$ & $\begin{array}{l}10 \\
10 \\
10 \\
10 \\
10\end{array}$ & $\begin{array}{l}\text { GS } \\
\text { GS } \\
\text { GS } \\
\text { GS } \\
\text { GS }\end{array}$ & $\begin{array}{l}\ldots \\
\ldots \\
\ldots \\
\ldots\end{array}$ & $\begin{array}{l}\cdots \\
\cdots \\
\cdots \\
\cdots\end{array}$ & $\begin{array}{l}\text { 3. } 2 M L(G S) \\
\text { 3. } 1 M L(G S) \\
\text { 4.1ML(GS) } \\
\text { 3. } 2 M L(G S) \\
\text { 3. } 2 M L(G S)\end{array}$ & $\begin{array}{l}\cdots \\
\cdots \\
\cdots \\
\cdots\end{array}$ & $\begin{array}{l}\text { AUG. } \\
A \cup G . \\
A \cup G . \\
\text { AUG. } \\
\text { AUG. }\end{array}$ & $\begin{array}{l}22 \\
22 \\
22 \\
22 \\
22\end{array}$ & $\begin{array}{l}04: 52 \\
05: 06 \\
06: 34 \\
08: 35 \\
17: 01\end{array}$ & $\begin{array}{l}\text { MST } \\
\text { MST } \\
\text { MST } \\
\text { MST } \\
\text { MST }\end{array}$ \\
\hline $\begin{array}{l}A \cup G . \\
A \cup G . \\
A \cup G . \\
A \cup G . \\
A \cup G .\end{array}$ & $\begin{array}{l}23 \\
23 \\
24 \\
25 \\
25\end{array}$ & $\begin{array}{l}06 \\
13 \\
13 \\
07 \\
17\end{array}$ & $\begin{array}{l}13 \\
21 \\
53 \\
43 \\
43\end{array}$ & $\begin{array}{l}33.2 \\
53.0 \\
48.2 \\
34.0 \\
53.0\end{array}$ & $\begin{array}{l}44.445 \mathrm{~N} \\
44.462 \mathrm{~N} \\
44.437 \mathrm{~N} \\
44.459 \mathrm{~N} \\
44.416 \mathrm{~N}\end{array}$ & $\begin{array}{l}114.089 W . \\
114.137 W . \\
114.063 W . \\
114.141 W . \\
114.100 W .\end{array}$ & $\begin{array}{l}10 \\
10 \\
10 \\
10 \\
10\end{array}$ & $\begin{array}{l}\text { GS } \\
\text { GS } \\
\text { GS } \\
\text { GS } \\
\text { GS }\end{array}$ & $\begin{array}{l}\cdots \\
\cdots \\
\cdots \\
\cdots\end{array}$ & $\begin{array}{l}\ldots \\
\cdots \\
\cdots \\
\cdots\end{array}$ & $\begin{array}{l}\text { 3. } 4 M L(G S) \\
3.8 M L(G S) \\
3.4 M L(G S) \\
3.1 M L(G S) \\
3.0 M L(G S)\end{array}$ & $\begin{array}{l}\cdots \\
\cdots \\
\cdots \\
\cdots\end{array}$ & $\begin{array}{l}\text { AUG. } \\
\text { AUG. } \\
\text { AUG. } \\
\text { AUG. } \\
\text { AUG. }\end{array}$ & $\begin{array}{l}22 \\
23 \\
24 \\
25 \\
25\end{array}$ & $\begin{array}{l}23: 13 \\
06: 21 \\
06: 53 \\
00: 43 \\
10: 43\end{array}$ & $\begin{array}{l}\text { MST } \\
\text { MST } \\
\text { MST } \\
\text { MST } \\
\text { MST }\end{array}$ \\
\hline $\begin{array}{l}\text { AUG. } \\
\text { AUG. } \\
\text { AUG. } \\
\text { AUG. } \\
\text { AUG. }\end{array}$ & $\begin{array}{l}27 \\
28 \\
28 \\
29 \\
30\end{array}$ & $\begin{array}{l}02 \\
11 \\
20 \\
09 \\
18\end{array}$ & $\begin{array}{l}12 \\
19 \\
40 \\
47 \\
42\end{array}$ & $\begin{array}{l}08.8 \\
44.2 \\
30.0 \\
51.5 \\
27.6\end{array}$ & $\begin{array}{l}44.420 \mathrm{~N} \\
44.456 \mathrm{~N} \\
44.444 \mathrm{~N} \\
44.386 \mathrm{~N} \\
44.452 \mathrm{~N}\end{array}$ & $\begin{array}{l}114.174 W . \\
114.138 W . \\
114.172 W . \\
114.060 W . \\
114.126 W .\end{array}$ & $\begin{array}{l}10 \\
10 \\
10 \\
10 \\
10\end{array}$ & $\begin{array}{l}\text { GS } \\
\text { GS } \\
\text { GS } \\
\text { GS } \\
\text { GS }\end{array}$ & $\begin{array}{l}\ldots \\
\ldots \\
\cdots \\
\cdots \\
\cdots\end{array}$ & $\begin{array}{l}\ldots \\
\cdots \\
\cdots \\
\cdots\end{array}$ & $\begin{array}{l}\text { 3. } 1 M L(G S) \\
\text { 3.1ML(GS) } \\
\text { 3. } 4 M L(G S) \\
\text { 3. } 3 M L(G S) \\
3.5 M L(G S)\end{array}$ & $\begin{array}{c}\cdots \\
\cdots \\
\cdots \\
\text { FËLT }\end{array}$ & $\begin{array}{l}\text { AUG. } \\
\text { AUG. } \\
\text { AUG. } \\
\text { AUG. } \\
\text { AUG. }\end{array}$ & $\begin{array}{l}26 \\
28 \\
28 \\
29 \\
30\end{array}$ & $\begin{array}{l}19: 12 \\
04: 19 \\
13: 40 \\
02: 47 \\
11: 42\end{array}$ & $\begin{array}{l}\text { MST } \\
\text { MST } \\
\text { MST } \\
\text { MST } \\
\text { MST }\end{array}$ \\
\hline $\begin{array}{l}\text { AUG. } \\
\text { SEPT. } \\
\text { SEPT. } \\
\text { SEPT. } \\
\text { SEPT. }\end{array}$ & $\begin{array}{r}30 \\
1 \\
3 \\
4 \\
4\end{array}$ & $\begin{array}{l}21 \\
14 \\
20 \\
05 \\
05\end{array}$ & $\begin{array}{l}06 \\
11 \\
28 \\
50 \\
57\end{array}$ & $\begin{array}{l}20.7 \\
18.3 \\
00.1 \\
41.3 \\
21.8\end{array}$ & $\begin{array}{l}44.455 \mathrm{~N} \\
44.439 \mathrm{~N} \\
44.359 \mathrm{~N} \\
44.449 \mathrm{~N} \\
44.466 \mathrm{~N}\end{array}$ & $\begin{array}{l}114.154 W . \\
114.117 W . \\
114.126 W . \\
114.107 W . \\
114.115 W .\end{array}$ & $\begin{array}{l}10 \\
10 \\
10 \\
10 \\
10\end{array}$ & $\begin{array}{l}\text { GS } \\
\text { GS } \\
\text { GS } \\
\text { GS } \\
\text { GS }\end{array}$ & $\begin{array}{l}\ldots \\
\ldots \\
\cdots \\
\cdots \\
\cdots\end{array}$ & $\begin{array}{l}\ldots \\
\ldots \\
\ldots \\
\ldots\end{array}$ & $\begin{array}{l}\text { 3.9ML(GS) } \\
\text { 3.1ML(GS) } \\
\text { 3. } 2 M L(G S) \\
\text { 3.7ML (GS) } \\
\text { 3.1ML(GS) }\end{array}$ & $\begin{array}{c}\text { FELT } \\
\ldots \\
\cdots \\
\text { IV } \\
\ldots\end{array}$ & $\begin{array}{l}\text { AUG. } \\
\text { SEPT. } \\
\text { SEPT. } \\
\text { SEPT. } \\
\text { SEPT. }\end{array}$ & $\begin{array}{r}30 \\
1 \\
3 \\
3 \\
3\end{array}$ & $\begin{array}{l}14: 06 \\
07: 11 \\
13: 28 \\
22: 50 \\
22: 57\end{array}$ & $\begin{array}{l}\text { MST } \\
\text { MST } \\
\text { MST } \\
\text { MST } \\
\text { MST }\end{array}$ \\
\hline $\begin{array}{l}\text { SEPT. } \\
\text { SEPT. } \\
\text { SEPT. } \\
\text { SEPT. } \\
\text { SEPT. }\end{array}$ & $\begin{array}{l}4 \\
5 \\
5 \\
5 \\
6\end{array}$ & $\begin{array}{l}14 \\
09 \\
17 \\
20 \\
19\end{array}$ & $\begin{array}{l}10 \\
39 \\
38 \\
28 \\
50\end{array}$ & $\begin{array}{l}03.6 \\
54.8 \\
58.4 \\
01.2 \\
22.3\end{array}$ & $\begin{array}{l}44.491 \mathrm{~N} \\
44.432 \mathrm{~N} \\
44.421 \mathrm{~N} \\
44.218 \mathrm{~N} \\
44.450 \mathrm{~N}\end{array}$ & $\begin{array}{l}114.127 W . \\
114.154 W . \\
114.183 W . \\
114.134 W . \\
114.077 W .\end{array}$ & $\begin{array}{l}10 \\
10 \\
10 \\
10 \\
10\end{array}$ & $\begin{array}{l}\text { GS } \\
\text { GS } \\
\text { GS } \\
\text { GS } \\
\text { GS }\end{array}$ & $\begin{array}{l}\ldots \\
\ldots \\
\ldots \\
\ldots\end{array}$ & $\begin{array}{l}\ldots \\
\ldots \\
\cdots \\
\cdots\end{array}$ & $\begin{array}{l}\text { 2.9ML(GS) } \\
3.4 \mathrm{ML}(\mathrm{GS}) \\
3.7 \mathrm{ML}(\mathrm{GS}) \\
3.1 \mathrm{ML}(\mathrm{GS}) \\
3.4 \mathrm{ML}(\mathrm{BU})\end{array}$ & $\begin{array}{c}\ldots \\
\text { FELT } \\
\ldots \\
\ldots\end{array}$ & $\begin{array}{l}\text { SEPT. } \\
\text { SEPT. } \\
\text { SEPT. } \\
\text { SEPT. } \\
\text { SEPT. }\end{array}$ & $\begin{array}{l}4 \\
5 \\
5 \\
5 \\
6\end{array}$ & $\begin{array}{l}07: 10 \\
02: 39 \\
10: 38 \\
13: 28 \\
12: 50\end{array}$ & $\begin{array}{l}\text { MST } \\
\text { MST } \\
\text { MST } \\
\text { MST } \\
\text { MST }\end{array}$ \\
\hline $\begin{array}{l}\text { SEPT. } \\
\text { SEPT. } \\
\text { SEPT. } \\
\text { SEPT. } \\
\text { SEPT. }\end{array}$ & $\begin{array}{l}8 \\
8 \\
8 \\
8 \\
8\end{array}$ & $\begin{array}{l}06 \\
06 \\
08 \\
08 \\
08\end{array}$ & $\begin{array}{l}16 \\
26 \\
01 \\
03 \\
08\end{array}$ & $\begin{array}{l}40.1 \\
46.0 \\
02.3 \\
16.8 \\
32.9\end{array}$ & $\begin{array}{l}44.439 \mathrm{~N} \\
44.418 \mathrm{~N} \\
44.284 \mathrm{~N} \\
44.415 \mathrm{~N} \\
44.409 \mathrm{~N}\end{array}$ & $\begin{array}{l}114.154 \mathrm{~W} . \\
114.136 \mathrm{~W} . \\
114.256 \mathrm{~W} . \\
114.062 \mathrm{~W} . \\
114.149 \mathrm{~W} .\end{array}$ & $\begin{array}{l}10 \\
10 \\
10 \\
10 \\
10\end{array}$ & $\begin{array}{l}\text { GS } \\
\text { GS } \\
\text { GS } \\
\text { GS } \\
\text { GS }\end{array}$ & $\begin{array}{l}5.0 \\
\ldots \\
\ldots \\
\ldots \\
\ldots\end{array}$ & $\begin{array}{l}4.4 \\
\ldots \\
\ldots \\
\cdots \\
\cdots\end{array}$ & $\begin{array}{l}\text { 4. } 3 \mathrm{MD}(\mathrm{UU}) \\
3.5 \mathrm{ML}(\mathrm{GS}) \\
3.0 \mathrm{ML}(\mathrm{GS}) \\
3.5 \mathrm{ML}(\mathrm{GS}) \\
3.3 \mathrm{ML}(\mathrm{GS})\end{array}$ & $\begin{array}{l}\text { IV } \\
\cdots \\
\cdots \\
\cdots \\
\cdots\end{array}$ & $\begin{array}{l}\text { SEPT. } \\
\text { SEPT. } \\
\text { SEPT. } \\
\text { SEPT. } \\
\text { SEPT. }\end{array}$ & $\begin{array}{l}7 \\
7 \\
8 \\
8 \\
8\end{array}$ & $\begin{array}{l}23: 16 \\
23: 26 \\
01: 01 \\
01: 03 \\
01: 08\end{array}$ & $\begin{array}{l}\text { MST } \\
\text { MST } \\
\text { MST } \\
\text { MST } \\
\text { MST }\end{array}$ \\
\hline $\begin{array}{l}\text { SEPT. } \\
\text { SEPT. } \\
\text { SEPT. } \\
\text { SEPT. } \\
\text { SEPT. }\end{array}$ & $\begin{array}{l}8 \\
8 \\
8 \\
8 \\
8\end{array}$ & $\begin{array}{l}08 \\
08 \\
08 \\
09 \\
09\end{array}$ & $\begin{array}{l}16 \\
35 \\
55 \\
22 \\
27\end{array}$ & $\begin{array}{l}28.1 \\
02.9 \\
50.0 \\
26.4 \\
28.3\end{array}$ & $\begin{array}{l}44.441 \mathrm{~N} \\
44.441 \mathrm{~N} \\
44.444 \mathrm{~N} \\
44.441 \mathrm{~N} \\
44.438 \mathrm{~N}\end{array}$ & $\begin{array}{l}114.081 W . \\
114.141 W \\
114.152 W \\
114.115 W \\
114.091 W .\end{array}$ & $\begin{array}{l}10 \\
10 \\
10 \\
10 \\
10\end{array}$ & $\begin{array}{l}\text { GS } \\
\text { GS } \\
\text { GS } \\
\text { GS } \\
\text { GS }\end{array}$ & $\begin{array}{l}\cdots \\
\cdots \\
\cdots \\
\cdots\end{array}$ & $\begin{array}{l}\ldots \\
\cdots \\
\cdots \\
\cdots\end{array}$ & $\begin{array}{l}\text { 3. } 3 \mathrm{ML}(G S) \\
3.1 \mathrm{ML}(G S) \\
3.9 \mathrm{ML}(G S) \\
\text { 3. } 9 \mathrm{ML}(G S) \\
\text { 3.0ML(GS) }\end{array}$ & I I I & $\begin{array}{l}\text { SEPT. } \\
\text { SEPT. } \\
\text { SEPT. } \\
\text { SEPT. } \\
\text { SEPT. }\end{array}$ & $\begin{array}{l}8 \\
8 \\
8 \\
8 \\
8\end{array}$ & $\begin{array}{l}01: 16 \\
01: 35 \\
01: 55 \\
02: 22 \\
02: 27\end{array}$ & $\begin{array}{l}\text { MST } \\
\text { MST } \\
\text { MST } \\
\text { MST } \\
\text { MST }\end{array}$ \\
\hline $\begin{array}{l}\text { SEPT. } \\
\text { SEPT. } \\
\text { SEPT. } \\
\text { SEPT. } \\
\text { SEPT. }\end{array}$ & $\begin{array}{r}8 \\
8 \\
13 \\
14 \\
15\end{array}$ & $\begin{array}{l}11 \\
13 \\
11 \\
10 \\
02\end{array}$ & $\begin{array}{l}36 \\
56 \\
12 \\
55 \\
55\end{array}$ & $\begin{array}{l}13.7 \\
37.7 \\
48.9 \\
82.4 \\
02.6\end{array}$ & $\begin{array}{l}44.420 \mathrm{~N} \\
44.416 \mathrm{~N} \\
44.434 \mathrm{~N} \\
45.250 \mathrm{~N} \\
47.460 \mathrm{~N}\end{array}$ & $\begin{array}{l}114.150 W . \\
114.147 W . \\
114.150 w . \\
114.621 W . \\
115.987 w .\end{array}$ & $\begin{array}{r}10 \\
10 \\
10 \\
10 \\
1\end{array}$ & $\begin{array}{l}\text { GS } \\
\text { GS } \\
\text { GS } \\
\text { GS } \\
\text { GS }\end{array}$ & $\begin{array}{l}\ldots \\
4.4 \\
\ldots \\
\ldots \\
\ldots\end{array}$ & $\begin{array}{l}\cdots \\
\cdots \\
\cdots \\
\cdots \\
\cdots\end{array}$ & $\begin{array}{l}\text { 4. } 0 \mathrm{ML}(\mathrm{GS}) \\
\text { 4.6MD(BU) } \\
\text { 3.9ML(GS) } \\
\text { 3.6ML(GS) } \\
2.8 \mathrm{ML}(\mathrm{GS})\end{array}$ & $\begin{array}{r}\text { FELT } \\
\text { III } \\
\text { IV } \\
\text { FELiT }\end{array}$ & $\begin{array}{l}\text { SEPT. } \\
\text { SEPT. } \\
\text { SEPT. } \\
\text { SEPT. } \\
\text { SEPT. }\end{array}$ & $\begin{array}{r}8 \\
8 \\
13 \\
14 \\
14\end{array}$ & $\begin{array}{l}04: 36 \\
06: 56 \\
04: 12 \\
03: 55 \\
18: 55\end{array}$ & $\begin{array}{l}\text { MST } \\
\text { MST } \\
\text { MST } \\
\text { MST } \\
\text { PST }\end{array}$ \\
\hline $\begin{array}{l}\text { SEPT. } \\
\text { SEPT. } \\
\text { SEPT. } \\
\text { SEPT. } \\
\text { SEPT. }\end{array}$ & $\begin{array}{l}18 \\
18 \\
19 \\
19 \\
21\end{array}$ & $\begin{array}{l}15 \\
16 \\
01 \\
15 \\
08\end{array}$ & $\begin{array}{l}09 \\
27 \\
32 \\
33 \\
40\end{array}$ & $\begin{array}{l}58.0 \\
30.6 \\
06.9 \\
22.6 \\
40.2\end{array}$ & $\begin{array}{l}44.408 \mathrm{~N} \\
44.420 \mathrm{~N} \\
45.079 \mathrm{~N} \\
44.334 \mathrm{~N} \\
44.817 \mathrm{~N}\end{array}$ & $\begin{array}{l}114.124 W . \\
114.107 w . \\
116.766 w . \\
114.205 W . \\
112.899 W .\end{array}$ & $\begin{array}{r}10 \\
10 \\
5 \\
10 \\
10\end{array}$ & $\begin{array}{l}\text { GS } \\
\text { GS } \\
\text { GS } \\
\text { GS } \\
\text { GS }\end{array}$ & $\begin{array}{l}\cdots \\
\cdots \\
\cdots \\
\cdots\end{array}$ & $\begin{array}{l}\ldots \\
\cdots \\
\cdots \\
\cdots\end{array}$ & $\begin{array}{l}\text { 4. } 0 M L(G S) \\
\text { 3. } 2 M L(G S) \\
\text { 3. } 4 M L(G S) \\
\text { 3.5ML(GS) } \\
\text { 3. } 6 M L(G S)\end{array}$ & $\begin{array}{c}\text { FELT } \\
\ldots \text { III } \\
\ldots \\
\ldots\end{array}$ & $\begin{array}{l}\text { SEPT. } \\
\text { SEPT. } \\
\text { SEPT. } \\
\text { SEPT. } \\
\text { SEPT. }\end{array}$ & $\begin{array}{l}18 \\
18 \\
18 \\
19 \\
21\end{array}$ & $\begin{array}{l}08: 09 \\
09: 27 \\
18: 32 \\
08: 33 \\
01: 40\end{array}$ & $\begin{array}{l}\text { MST } \\
\text { MST } \\
\text { MST } \\
\text { MST } \\
\text { MST }\end{array}$ \\
\hline $\begin{array}{l}\text { SEPT } . \\
\text { SEPT. } \\
\text { SEPT. } \\
\text { SEPT. } \\
\text { OCT. }\end{array}$ & $\begin{array}{r}21 \\
23 \\
26 \\
29 \\
2\end{array}$ & $\begin{array}{l}18 \\
08 \\
10 \\
14 \\
09\end{array}$ & $\begin{array}{l}21 \\
19 \\
19 \\
34 \\
51\end{array}$ & $\begin{array}{l}55.5 \\
44.1 \\
20.6 \\
40.8 \\
31.8\end{array}$ & $\begin{array}{l}44.428 \mathrm{~N} \\
44.420 \mathrm{~N} \\
44.514 \mathrm{~N} \\
44.614 \mathrm{~N} \\
44.392 \mathrm{~N}\end{array}$ & $\begin{array}{l}114.242 W . \\
114.150 W . \\
114.228 W . \\
115.057 W . \\
114.184 W .\end{array}$ & $\begin{array}{c}10 \\
10 \\
10 \\
10 \\
10\end{array}$ & $\begin{array}{l}\text { GS } \\
\text { GS } \\
\text { GS } \\
\text { GS } \\
\text { GS }\end{array}$ & $\begin{array}{l}\cdots \\
\cdots \\
\cdots \\
\cdots\end{array}$ & $\begin{array}{l}\cdots \\
\ldots \\
\cdots \\
\cdots\end{array}$ & $\begin{array}{l}\text { 3. } 4 M L(G S) \\
\text { 3. } 4 M L(G S) \\
\text { 3. } 0 M L(G S) \\
\text { 3. } 3 M L(G S) \\
\text { 3.6ML(GS) }\end{array}$ & $\begin{array}{l}\ldots \\
\ldots \\
\cdots \\
\ldots\end{array}$ & $\begin{array}{l}\text { SEPT. } \\
\text { SEPT. } \\
\text { SEPT. } \\
\text { SEPT. } \\
\text { OCT. }\end{array}$ & $\begin{array}{c}21 \\
2 \overline{3} \\
26 \\
29 \\
2\end{array}$ & $\begin{array}{l}11: 21 \\
01: 19 \\
03: 19 \\
07: 34 \\
02: 51\end{array}$ & $\begin{array}{l}\text { MST } \\
\text { MST } \\
\text { MST } \\
\text { MST } \\
\text { MST }\end{array}$ \\
\hline
\end{tabular}


Table 1. Summary of U.S. earthquakes for 1984-Continued

\begin{tabular}{|c|c|c|c|c|c|c|c|c|c|c|c|c|c|}
\hline \multirow{2}{*}{ Date } & \multicolumn{2}{|c|}{$\begin{array}{l}\text { Origin time } \\
\text { (UTC) }\end{array}$} & \multirow{2}{*}{$\begin{array}{c}\text { Latitude } \\
\left({ }^{\circ}\right)\end{array}$} & \multirow{2}{*}{$\begin{array}{c}\text { Longitude } \\
\left({ }^{\circ}\right)\end{array}$} & \multirow{2}{*}{$\begin{array}{l}\text { Depth } \\
(\mathbf{k m})\end{array}$} & \multirow{2}{*}{$\begin{array}{l}\text { Hypo- } \\
\text { center } \\
\text { source }\end{array}$} & \multicolumn{3}{|c|}{ Magnitude } & \multirow{2}{*}{$\begin{array}{l}\text { Maximum } \\
\text { intensity }\end{array}$} & \multicolumn{3}{|c|}{ local time } \\
\hline & hr $\min$ & $\sec$ & & & & & $m b$ & MS & $\begin{array}{l}\text { ML, Mn } \\
\text { MD. Mw }\end{array}$ & & Date & Hour & $\begin{array}{l}\text { Time } \\
\text { zome }\end{array}$ \\
\hline
\end{tabular}

IDAHO-Continued

\begin{tabular}{|c|c|c|c|c|c|c|c|c|c|c|c|c|c|c|}
\hline $\begin{array}{lr}\text { OCT. } & 3 \\
\text { OCT. } & 4 \\
\text { OCT. } & 12 \\
\text { OCT. } & 19 \\
\text { OCT. } & 25\end{array}$ & $\begin{array}{l}05 \\
17 \\
04 \\
07 \\
12\end{array}$ & $\begin{array}{l}32 \\
02 \\
49 \\
35 \\
10\end{array}$ & $\begin{array}{l}01.2 \\
45.2 \\
24.3 \\
09.1 \\
36.5\end{array}$ & $\begin{array}{l}44.333 \mathrm{~N} . \\
44.282 \mathrm{~N} . \\
44.315 \mathrm{~N} . \\
44.424 \mathrm{~N} . \\
44.340 \mathrm{~N} .\end{array}$ & $\begin{array}{l}114.033 W . \\
114.090 W . \\
114.083 W . \\
114.021 W . \\
114.157 W .\end{array}$ & $\begin{array}{l}10 \\
10 \\
10 \\
10 \\
10\end{array}$ & $\begin{array}{l}\text { GS } \\
\text { GS } \\
\text { GS } \\
\text { GS } \\
\text { GS }\end{array}$ & $\begin{array}{l}\cdots \\
\cdots \\
\cdots \\
\cdots \\
\ldots\end{array}$ & $\begin{array}{l}\cdots \\
\cdots \\
\cdots \\
\ldots\end{array}$ & $\begin{array}{l}\text { 3.2ML(GS) } \\
3.8 M L(G S) \\
3.5 M L(G S) \\
3.7 M L(G S) \\
3.6 M L(G S)\end{array}$ & $\begin{array}{l}\cdots \\
\cdots \\
\cdots \\
\ldots \\
\ldots\end{array}$ & $\begin{array}{l}\text { OCT. } \\
\text { OCT. } \\
\text { OCT. } \\
\text { OCT. } \\
\text { OCT. }\end{array}$ & $\begin{array}{r}2 \\
4 \\
11 \\
19 \\
25\end{array}$ & $\begin{array}{l}22: 32 \\
10: 02 \\
21: 49 \\
00: 35 \\
05: 10\end{array}$ \\
\hline $\begin{array}{lr}\text { OCT. } & 30 \\
\text { NOV. } & 13 \\
\text { NOV. } & 27 \\
\text { DEC. } & 8 \\
\text { DEC. } & 19\end{array}$ & $\begin{array}{l}23 \\
03 \\
12 \\
09 \\
11\end{array}$ & $\begin{array}{l}05 \\
11 \\
07 \\
41 \\
32\end{array}$ & $\begin{array}{l}30.6 \\
14.5 \\
50.6 \\
02.0 \\
29.3\end{array}$ & $\begin{array}{l}44.430 \mathrm{~N} . \\
44.346 \mathrm{~N} . \\
48.871 \mathrm{~N} . \\
42.379 \mathrm{~N} . \\
42.601 \mathrm{~N} .\end{array}$ & $\begin{array}{l}114.112 W . \\
114.210 W . \\
116.381 W . \\
111.542 W . \\
111.282 W .\end{array}$ & $\begin{array}{r}10 \\
10 \\
5 \\
5 \\
1\end{array}$ & $\begin{array}{l}\text { GS } \\
\text { GS } \\
\text { GS } \\
\text { GS } \\
\text { UU }\end{array}$ & $\begin{array}{l}\cdots \\
\cdots \\
\cdots \\
\ldots\end{array}$ & $\begin{array}{l}\ldots \\
\ldots \\
\cdots \\
\cdots \\
\cdots\end{array}$ & $\begin{array}{l}\text { 3.9ML(GS) } \\
3.6 \mathrm{ML}(\mathrm{GS}) \\
2.8 \mathrm{ML}(\mathrm{GS}) \\
2.9 \mathrm{ML}(\mathrm{GS}) \\
2.7 \mathrm{MD}(\mathrm{UU})\end{array}$ & $\begin{array}{l}\text { IV } \\
\ldots \\
\ldots \\
\ldots \\
\ldots\end{array}$ & $\begin{array}{l}\text { OCT. } \\
\text { NOV. } \\
\text { NOV. } \\
\text { DEC. } \\
\text { DEC. }\end{array}$ & $\begin{array}{r}30 \\
12 \\
27 \\
8 \\
19\end{array}$ & $\begin{array}{l}16: 05 \\
20: 11 \\
04: 07 \\
02: 41 \\
04: 32\end{array}$ \\
\hline DEC. & 13 & 20 & 27.9 & $44.241 \mathrm{~N}$. & $114.059 W$. & 10 & GS & $\cdots$ & $\ldots$ & 3.2ML (GS) & $\ldots$ & DEC. & 22 & $06: 20$ \\
\hline
\end{tabular}

\section{ILLINOIS}

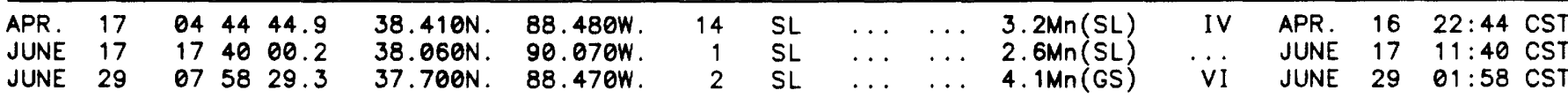

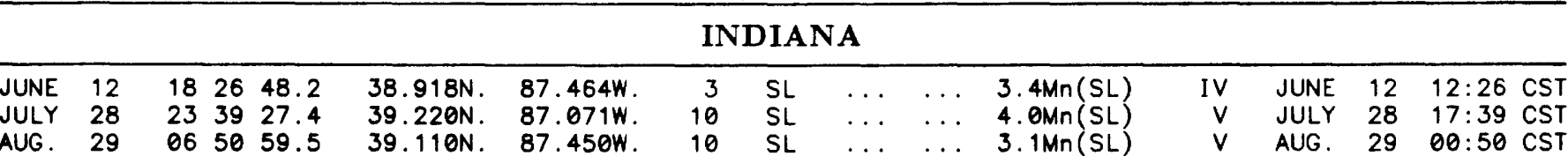

\begin{tabular}{|c|c|c|c|c|c|c|c|c|c|c|c|c|c|c|}
\hline $\begin{array}{ll}\text { FEB. } & 13 \\
\text { FEB. } & 14 \\
\text { FEB. } & 14 \\
\text { FEB. } & 16 \\
\text { FEB. } & 25\end{array}$ & $\begin{array}{l}22 \\
03 \\
22 \\
11 \\
21\end{array}$ & $\begin{array}{l}42 \\
53 \\
56 \\
30 \\
01\end{array}$ & $\begin{array}{l}45.3 \\
11.7 \\
10.4 \\
32.8 \\
57.2\end{array}$ & $\begin{array}{l}37.210 \mathrm{~N} . \\
37.210 \mathrm{~N} . \\
37.210 \mathrm{~N} . \\
37.210 \mathrm{~N} \\
37.220 \mathrm{~N}\end{array}$ & $\begin{array}{l}89.020 W . \\
89.010 W . \\
89.000 W . \\
89.020 W . \\
89.010 W .\end{array}$ & $\begin{array}{l}5 \\
2 \\
2 \\
2\end{array}$ & $\begin{array}{l}S L \\
S L \\
S L \\
S L \\
S L\end{array}$ & $\begin{array}{l}\cdots \\
\ldots \\
\ldots \\
\cdots \\
\cdots\end{array}$ & $\begin{array}{l}\ldots \\
\cdots \\
\cdots \\
\cdots \\
\cdots\end{array}$ & $\begin{array}{l}\text { 3. } 2 \mathrm{Mn}(\mathrm{SL}) \\
\text { 2. } 6 \mathrm{Mn}(\mathrm{SL}) \\
\text { 3. } 6 \mathrm{Mn}(\mathrm{SL}) \\
\text { 2. } 5 \mathrm{Mn}(\mathrm{SL}) \\
\text { 3. } \mathrm{Mnn}(\mathrm{SL})\end{array}$ & $\begin{array}{l}\text { IV } \\
\ldots \dot{\text { IV }} \\
\ldots \\
\ldots\end{array}$ & $\begin{array}{l}\text { FEB. } \\
\text { FEB. } \\
\text { FEB. } \\
\text { FEB. } \\
\text { FEB. }\end{array}$ & $\begin{array}{l}13 \\
13 \\
14 \\
16 \\
25\end{array}$ & $\begin{array}{lll}16: 42 & \text { CST } \\
21: 53 & \text { CST } \\
16: 56 & \text { CST } \\
05: 30 & \text { CST } \\
15: 01 & \text { CST }\end{array}$ \\
\hline $\begin{array}{lr}\text { MAR. } & 1 \\
\text { MAR. } & 11 \\
\text { SEPT. } & 19 \\
\text { SEPT. } & 20 \\
\text { SEPT. } & 27\end{array}$ & $\begin{array}{l}16 \\
12 \\
16 \\
20 \\
08\end{array}$ & $\begin{array}{l}18 \\
50 \\
25 \\
42 \\
08\end{array}$ & $\begin{array}{l}06.0 \\
57.5 \\
49.7 \\
31.0 \\
37.2\end{array}$ & $\begin{array}{l}37.210 \mathrm{~N} . \\
37.020 \mathrm{~N} \\
37.210 \mathrm{~N} \\
37.170 \mathrm{~N} . \\
37.200 \mathrm{~N}\end{array}$ & $\begin{array}{l}89.010 W . \\
88.930 W . \\
89.010 W . \\
87.490 W . \\
89.000 W .\end{array}$ & $\begin{array}{l}2 \\
7 \\
1 \\
5 \\
5\end{array}$ & $\begin{array}{l}S L \\
S L \\
S L \\
S L \\
S L\end{array}$ & $\begin{array}{l}\ldots \\
\ldots \\
\ldots \\
\ldots \\
\ldots\end{array}$ & $\begin{array}{l}\ldots \\
\ldots \\
\ldots \\
\ldots\end{array}$ & $\begin{array}{l}\text { 2. } 9 \mathrm{Mn}(S L) \\
2.6 \mathrm{Mn}(S L) \\
2.5 \mathrm{Mn}(\mathrm{SL}) \\
2.5 \mathrm{Mn}(\mathrm{SL}) \\
2.8 \mathrm{Mn}(\mathrm{SL})\end{array}$ & $\begin{array}{l}\cdots \\
\cdots \\
\cdots \\
\cdots\end{array}$ & $\begin{array}{l}\text { MAR. } \\
\text { MAR. } \\
\text { SEPT } \\
\text { SEPT } \\
\text { SEPT. }\end{array}$ & $\begin{array}{l}1 \\
11 \\
19 \\
20 \\
27\end{array}$ & 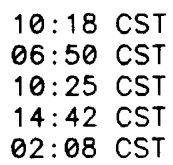 \\
\hline
\end{tabular}

\begin{tabular}{|c|c|c|c|c|c|c|c|c|c|c|c|c|c|c|c|}
\hline \multicolumn{16}{|c|}{ MAINE } \\
\hline $\begin{array}{l}\text { JAN. } \\
\text { JAN. } \\
\text { JAN. } \\
\text { JAN. } \\
\text { JUNE }\end{array}$ & $\begin{array}{r}14 \\
14 \\
14 \\
19 \\
8\end{array}$ & $\begin{array}{l}05 \\
09 \\
09 \\
05 \\
06\end{array}$ & $\begin{array}{l}34 \\
08 \\
09 \\
26 \\
47\end{array}$ & $\begin{array}{l}34.2 \\
34.6 \\
33.1 \\
08.8 \\
34.9\end{array}$ & $\begin{array}{l}45.025 \mathrm{~N} \\
45.051 \mathrm{~N} \\
45.050 \mathrm{~N} \\
44.893 \mathrm{~N} \\
43.232 \mathrm{~N}\end{array}$ & $\begin{array}{l}67.279 w . \\
67.181 w . \\
67.180 w . \\
67.301 w . \\
70.206 w .\end{array}$ & $\begin{array}{r}12 \\
8 \\
8 \\
12 \\
11\end{array}$ & $\begin{array}{l}\text { WO } \\
\text { WO } \\
\text { WO } \\
\text { WO } \\
\text { wo }\end{array}$ & $\begin{array}{l}\ldots \\
\cdots \\
\cdots \\
\cdots\end{array}$ & $\begin{array}{l}\ldots \\
\ldots \\
\cdots \\
\cdots \\
\cdots\end{array}$ & $\begin{array}{l}2.6 \mathrm{Mn} \text { (EP) } \\
3.4 \mathrm{Mn} \text { (WO) } \\
3.6 \mathrm{Mn} \text { (WO) } \\
3.8 \mathrm{Mn} \text { (WO) } \\
2.5 \mathrm{Mn} \text { (WO) }\end{array}$ & $\begin{array}{r}\text { FELT } \\
V \\
\text { FELT } \\
V \\
\ldots\end{array}$ & $\begin{array}{l}\text { JAN. } \\
\text { JAN. } \\
\text { JAN. } \\
\text { JAN. } \\
\text { JUNE }\end{array}$ & $\begin{array}{r}14 \\
14 \\
14 \\
19 \\
8\end{array}$ & $\begin{array}{ll}00: 34 & \text { EST } \\
04: 08 & \text { EST } \\
04: 09 & \text { EST } \\
00: 26 & \text { EST } \\
01: 47 & \text { EST }\end{array}$ \\
\hline $\begin{array}{l}\text { SEPT. } \\
\text { OCT. } \\
\text { DEC. }\end{array}$ & $\begin{array}{l}20 \\
18 \\
22\end{array}$ & $\begin{array}{l}03 \\
07 \\
16\end{array}$ & $\begin{array}{l}43 \\
16 \\
05\end{array}$ & $\begin{array}{l}35.8 \\
15.9 \\
14.3\end{array}$ & $\begin{array}{l}44.486 \mathrm{~N} \\
45.525 \mathrm{~N} \\
44.900 \mathrm{~N}\end{array}$ & $\begin{array}{l}70.381 W . \\
68.780 W . \\
68.037 W .\end{array}$ & $\begin{array}{l}8 \\
7 \\
8\end{array}$ & $\begin{array}{l}\text { wo } \\
\text { wo } \\
\text { wo }\end{array}$ & $\begin{array}{l}\cdots \\
\cdots \\
\cdots\end{array}$ & $\begin{array}{l}\ldots \\
\ldots \\
\ldots\end{array}$ & $\begin{array}{l}2.5 \mathrm{Mn} \text { (WO) } \\
2.5 \mathrm{Mn} \text { (WO) } \\
2.5 \mathrm{Mn} \text { (WO) }\end{array}$ & $\begin{array}{l}\cdots \\
\cdots \\
\cdots\end{array}$ & $\begin{array}{l}\text { SEPT. } \\
\text { OCT. } \\
\text { DEC. }\end{array}$ & $\begin{array}{l}19 \\
18 \\
22\end{array}$ & $\begin{array}{ll}22: 43 & \text { EST } \\
02: 16 & \text { EST } \\
11: 05 & \text { EST }\end{array}$ \\
\hline
\end{tabular}

\section{MASSACHUSETTS}

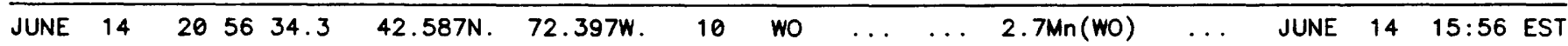


Table 1. Summary of U.S. earthquakes for 1984--Continued

\begin{tabular}{|c|c|c|c|c|c|c|c|c|c|c|c|c|c|c|c|c|}
\hline \multirow{2}{*}{ Date } & & \multicolumn{3}{|c|}{$\begin{array}{l}\text { Origin time } \\
\text { (UTC) }\end{array}$} & \multirow{2}{*}{$\begin{array}{c}\text { Latitude } \\
\left({ }^{\circ}\right)\end{array}$} & \multirow{2}{*}{$\begin{array}{c}\text { Lonngitude } \\
\left({ }^{\circ}\right)\end{array}$} & \multirow{2}{*}{$\begin{array}{c}\text { Depth } \\
(\mathbf{k m})\end{array}$} & \multirow{2}{*}{$\begin{array}{l}\text { Ilypo- } \\
\text { center } \\
\text { source }\end{array}$} & \multicolumn{3}{|c|}{ Magnitude } & \multirow{2}{*}{$\begin{array}{l}\text { Maximum } \\
\text { intensity }\end{array}$} & \multicolumn{4}{|c|}{ local time } \\
\hline & & hr & $\min$ & $\sec$ & & & & & $\mathbf{m b}$ & MS & $\begin{array}{l}\text { ML, Mn } \\
\text { MD, Mw }\end{array}$ & & Date & & Hour & $\begin{array}{l}\text { Time } \\
\text { rone }\end{array}$ \\
\hline \multicolumn{17}{|c|}{ MISSISSIPPI } \\
\hline SEPT. & 25 & 01 & 53 & 26.8 & $34.064 \mathrm{~N}$ & $89.818 W$. & 6 & TC & $\ldots$ & $\ldots$ & $2.5 \mathrm{MD}(\mathrm{TC})$ & $\ldots$ & SEPT. & 24 & $19: 53$ & CST \\
\hline \multicolumn{17}{|c|}{ MISSOURI } \\
\hline $\begin{array}{l}\text { JAN. } \\
\text { JAN. } \\
\text { JAN. } \\
\text { FEB. } \\
\text { MAY }\end{array}$ & $\begin{array}{l}12 \\
27 \\
28 \\
22 \\
25\end{array}$ & $\begin{array}{l}02 \\
09 \\
21 \\
17 \\
09\end{array}$ & $\begin{array}{l}48 \\
25 \\
29 \\
06 \\
45\end{array}$ & $\begin{array}{l}15.7 \\
02.2 \\
22.1 \\
40.7 \\
44.5\end{array}$ & $\begin{array}{l}37.590 \mathrm{~N} . \\
36.110 \mathrm{~N} . \\
36.610 \mathrm{~N} . \\
37.970 \mathrm{~N} . \\
36.490 \mathrm{~N} .\end{array}$ & $\begin{array}{l}89.750 w . \\
89.750 w . \\
89.920 w . \\
90.530 w . \\
89.550 w .\end{array}$ & $\begin{array}{r}2 \\
7 \\
1 \\
18 \\
8\end{array}$ & $\begin{array}{l}S L \\
S L \\
S L \\
S L \\
S L\end{array}$ & $\begin{array}{l}\ldots \\
\ldots \\
\cdots \\
\cdots \\
\cdots\end{array}$ & $\begin{array}{l}\cdots \\
\ldots \\
\cdots \\
\cdots \\
\cdots\end{array}$ & $\begin{array}{l}\text { 3. } 0 \mathrm{Mn}(\mathrm{SL}) \\
\text { 2. } 7 \mathrm{Mn}(\mathrm{SL}) \\
\text { 3. } 2 \mathrm{Mn}(\mathrm{SL}) \\
\text { 2. } 6 \mathrm{Mn}(\mathrm{SL}) \\
\text { 2. } 6 \mathrm{Mn}(\mathrm{SL})\end{array}$ & $\begin{array}{l}\text { I I I } \\
\cdots \\
\text { IV } \\
\ldots \\
\ldots\end{array}$ & $\begin{array}{l}\text { JAN. } \\
\text { JAN. } \\
\text { JAN. } \\
\text { FEB. } \\
\text { MAY }\end{array}$ & $\begin{array}{l}11 \\
27 \\
28 \\
22 \\
25\end{array}$ & $\begin{array}{l}20: 48 \\
03: 25 \\
15: 29 \\
11: 06 \\
03: 45\end{array}$ & $\begin{array}{l}\text { CST } \\
\text { CST } \\
\text { CST } \\
\text { CST } \\
\text { CST }\end{array}$ \\
\hline $\begin{array}{l}\text { JULY } \\
\text { JULY } \\
\text { OCT. } \\
\text { NOV. } \\
\text { DEC. }\end{array}$ & $\begin{array}{r}16 \\
30 \\
4 \\
10 \\
3\end{array}$ & $\begin{array}{l}03 \\
07 \\
13 \\
11 \\
11\end{array}$ & $\begin{array}{l}50 \\
33 \\
12 \\
25 \\
55\end{array}$ & $\begin{array}{l}53.5 \\
46.5 \\
23.4 \\
33.4 \\
44.5\end{array}$ & $\begin{array}{l}36.500 \mathrm{~N} . \\
37.828 \mathrm{~N} . \\
36.850 \mathrm{~N} . \\
37.390 \mathrm{~N} . \\
36.150 \mathrm{~N} .\end{array}$ & $\begin{array}{l}89.530 W . \\
90.921 W . \\
91.910 W . \\
90.050 W . \\
89.700 W .\end{array}$ & $\begin{array}{r}7 \\
7 \\
5 \\
5 \\
12\end{array}$ & $\begin{array}{l}S L \\
S L \\
S L \\
S L \\
S L\end{array}$ & $\begin{array}{l}\ldots \\
\ldots \\
\ldots \\
\cdots \\
\ldots\end{array}$ & $\begin{array}{l}\cdots \\
\cdots \\
\cdots \\
\cdots \\
\cdots\end{array}$ & $\begin{array}{l}\text { 3. } 0 \mathrm{Mn}(\mathrm{SL}) \\
\text { 3. } 0 \mathrm{Mn}(\mathrm{SL}) \\
\text { 2. } 8 \mathrm{Mn}(\mathrm{SL}) \\
\text { 2. } 5 \mathrm{Mn}(\mathrm{SL}) \\
\text { 3. } 0 \mathrm{Mn}(\mathrm{SL})\end{array}$ & $\begin{array}{c}\text { FELT } \\
\ldots \\
\text { IV }\end{array}$ & $\begin{array}{l}\text { JULY } \\
\text { JULY } \\
\text { OCT. } \\
\text { NOV. } \\
\text { DEC. }\end{array}$ & $\begin{array}{r}15 \\
30 \\
4 \\
10 \\
3\end{array}$ & $\begin{array}{l}21: 50 \\
01: 33 \\
07: 12 \\
05: 25 \\
05: 55\end{array}$ & $\begin{array}{l}\text { CST } \\
\text { CST } \\
\text { CST } \\
\text { CST } \\
\text { CST }\end{array}$ \\
\hline
\end{tabular}

\section{MONTANA}

\begin{tabular}{|c|c|c|c|c|c|c|c|c|c|c|c|c|c|c|c|c|}
\hline $\begin{array}{l}\text { JAN. } \\
\text { JAN. } \\
\text { JAN. } \\
\text { JAN. } \\
\text { JAN. }\end{array}$ & $\begin{array}{r}1 \\
14 \\
14 \\
14 \\
14\end{array}$ & $\begin{array}{l}02 \\
18 \\
21 \\
21 \\
22\end{array}$ & $\begin{array}{l}29 \\
30 \\
38 \\
57 \\
24\end{array}$ & $\begin{array}{l}24.8 \\
54.9 \\
09.2 \\
54.3 \\
01.9\end{array}$ & $\begin{array}{l}46.101 \mathrm{~N} \\
44.877 \mathrm{~N} \\
44.996 \mathrm{~N} \\
45.827 \mathrm{~N} \\
44.826 \mathrm{~N}\end{array}$ & $\begin{array}{l}111.470 \mathrm{~W} . \\
111.290 \mathrm{~W} . \\
111.816 \mathrm{~W} . \\
111.818 \mathrm{~W} . \\
111.056 \mathrm{~W}\end{array}$ & $\begin{array}{l}5 \\
0 \\
5 \\
2 \\
5\end{array}$ & $\begin{array}{l}\text { GS } \\
\text { BU } \\
\text { GS } \\
\text { BU } \\
\text { GS }\end{array}$ & $\begin{array}{l}\cdots \\
\cdots \\
\cdots \\
\cdots\end{array}$ & $\begin{array}{l}\cdots \\
\cdots \\
\cdots \\
\cdots \\
\cdots\end{array}$ & $\begin{array}{l}\text { 4. } 0 \mathrm{ML}(\mathrm{GS}) \\
2.8 \mathrm{ML}(\mathrm{BU}) \\
3.3 \mathrm{ML}(\mathrm{GS}) \\
3.1 \mathrm{ML}(\mathrm{BU}) \\
3.9 \mathrm{ML}(\mathrm{GS})\end{array}$ & $\begin{array}{l}\ldots \\
\cdots \\
\cdots \\
\cdots \\
\cdots\end{array}$ & $\begin{array}{l}\text { DEC. } \\
\text { JAN. } \\
\text { JAN. } \\
\text { JAN. } \\
\text { JAN. }\end{array}$ & $\begin{array}{l}31 \\
14 \\
14 \\
14 \\
14\end{array}$ & $\begin{array}{l}19: 29 \\
11: 30 \\
14: 38 \\
14: 57 \\
15: 24\end{array}$ & $\begin{array}{l}\text { MST } \\
\text { MST } \\
\text { MST } \\
\text { MST } \\
\text { MST }\end{array}$ \\
\hline $\begin{array}{l}\text { JAN. } \\
\text { JAN. } \\
\text { JAN. } \\
\text { JAN. } \\
\text { JAN. }\end{array}$ & $\begin{array}{l}14 \\
15 \\
15 \\
15 \\
16\end{array}$ & $\begin{array}{l}23 \\
03 \\
08 \\
17 \\
19\end{array}$ & $\begin{array}{l}45 \\
56 \\
18 \\
11 \\
50\end{array}$ & $\begin{array}{l}10.5 \\
22.9 \\
38.5 \\
34.2 \\
25.2\end{array}$ & $\begin{array}{l}44.823 \mathrm{~N} \\
44.828 \mathrm{~N} \\
44.591 \mathrm{~N} \\
44.847 \mathrm{~N} \\
47.149 \mathrm{~N}\end{array}$ & $\begin{array}{l}111.085 W \\
111.036 w \\
111.987 w \\
111.225 W \\
110.845 W\end{array}$ & $\begin{array}{r}5 \\
5 \\
13 \\
1 \\
5\end{array}$ & $\begin{array}{l}\text { GS } \\
\text { GS } \\
\text { BU } \\
\text { BU } \\
\text { GS }\end{array}$ & $\begin{array}{l}\cdots \\
\cdots \\
\cdots \\
\cdots\end{array}$ & $\begin{array}{l}\ldots \\
\ldots \\
\ldots \\
\cdots \\
\cdots\end{array}$ & $\begin{array}{l}\text { 3. } 0 \mathrm{ML}(\mathrm{GS}) \\
3.0 \mathrm{ML}(\mathrm{BU}) \\
2.8 \mathrm{ML}(\mathrm{BU}) \\
2.7 \mathrm{ML}(\mathrm{BU}) \\
\text { 3. } 3 \mathrm{ML}(\mathrm{BU})\end{array}$ & $\begin{array}{l}\cdots \\
\cdots \\
\cdots \\
\text { IV }\end{array}$ & $\begin{array}{l}\text { JAN. } \\
\text { JAN. } \\
\text { JAN. } \\
\text { JAN. } \\
\text { JAN. }\end{array}$ & $\begin{array}{l}14 \\
14 \\
15 \\
15 \\
16\end{array}$ & $\begin{array}{l}16: 45 \\
20: 56 \\
01: 18 \\
10: 11 \\
12: 50\end{array}$ & $\begin{array}{l}\text { MST } \\
\text { MST } \\
\text { MST } \\
\text { MST } \\
\text { MST }\end{array}$ \\
\hline $\begin{array}{l}\text { JAN. } \\
\text { JAN. } \\
\text { JAN. } \\
\text { JAN. } \\
\text { FEB. }\end{array}$ & $\begin{array}{r}17 \\
17 \\
19 \\
24 \\
2\end{array}$ & $\begin{array}{l}16 \\
16 \\
07 \\
19 \\
16\end{array}$ & $\begin{array}{l}41 \\
50 \\
25 \\
18 \\
32\end{array}$ & $\begin{array}{l}07.1 \\
45.8 \\
42.9 \\
23.2 \\
53.8\end{array}$ & $\begin{array}{l}44.707 \mathrm{~N} \\
44.724 \mathrm{~N} \\
47.435 \mathrm{~N} \\
44.868 \mathrm{~N} \\
44.782 \mathrm{~N}\end{array}$ & $\begin{array}{l}111.304 w . \\
111.228 w . \\
115.503 w . \\
111.943 w . \\
112.753 w .\end{array}$ & $\begin{array}{r}6 \\
3 \\
1 \\
0 \\
19\end{array}$ & $\begin{array}{l}\text { BU } \\
\text { BU } \\
\text { GS } \\
\text { BU } \\
\text { BU }\end{array}$ & $\begin{array}{l}\cdots \\
\cdots \\
\cdots \\
\cdots\end{array}$ & $\begin{array}{l}\cdots \\
\cdots \\
\cdots \\
\cdots \\
\cdots\end{array}$ & $\begin{array}{l}2.5 \mathrm{ML}(\mathrm{BU}) \\
2.9 \mathrm{ML}(\mathrm{BU}) \\
2.5 \mathrm{ML}(\mathrm{GS}) \\
2.6 \mathrm{ML}(\mathrm{BU}) \\
3.1 \mathrm{ML}(\mathrm{BU})\end{array}$ & $\begin{array}{c}\cdots \\
\ldots \dot{E} \dot{L} T \\
\cdots \\
\cdots\end{array}$ & & $\begin{array}{r}17 \\
17 \\
18 \\
24 \\
2\end{array}$ & $\begin{array}{l}08: 41 \\
08: 50 \\
23: 25 \\
12: 18 \\
09: 32\end{array}$ & $\begin{array}{l}\text { MST } \\
\text { MST } \\
\text { PST } \\
\text { MST } \\
\text { MST }\end{array}$ \\
\hline $\begin{array}{l}\text { FEB. } \\
\text { FEB. } \\
\text { FEB. } \\
\text { FEB. } \\
\text { FEB. }\end{array}$ & $\begin{array}{r}3 \\
3 \\
18 \\
14 \\
27\end{array}$ & $\begin{array}{l}08 \\
22 \\
12 \\
11 \\
16\end{array}$ & $\begin{array}{l}45 \\
52 \\
57 \\
31 \\
26\end{array}$ & $\begin{array}{l}16.6 \\
49.9 \\
54.6 \\
34.9 \\
21.9\end{array}$ & $\begin{array}{l}44.839 \mathrm{~N} \\
47.750 \mathrm{~N} \\
44.849 \mathrm{~N} \\
44.722 \mathrm{~N} \\
44.744 \mathrm{~N}\end{array}$ & $\begin{array}{l}112.789 W \\
113.849 W \\
112.892 W \\
111.753 W \\
111.405 W\end{array}$ & $\begin{array}{r}1 \\
16 \\
9 \\
5 \\
13\end{array}$ & $\begin{array}{l}\text { BU } \\
\text { GS } \\
\text { BU }\end{array}$ & $\begin{array}{l}\cdots \\
\cdots \\
\cdots \\
\cdots\end{array}$ & $\begin{array}{l}\ldots \\
\cdots \\
\cdots \\
\cdots \\
\cdots\end{array}$ & $\begin{array}{l}\text { 3. } 6 \mathrm{ML}(\mathrm{BU}) \\
2.9 \mathrm{ML}(\mathrm{BU}) \\
2.6 \mathrm{ML}(\mathrm{BU}) \\
3.3 \mathrm{ML}(\mathrm{GS}) \\
3.0 \mathrm{ML}(\mathrm{BU})\end{array}$ & $\begin{array}{l}\cdots \\
\cdots \\
\cdots \\
\cdots\end{array}$ & & $\begin{array}{r}3 \\
3 \\
10 \\
14 \\
27\end{array}$ & $\begin{array}{l}01: 45 \\
15: 52 \\
05: 57 \\
04: 31 \\
09: 26\end{array}$ & $\begin{array}{l}\text { MST } \\
\text { MST } \\
\text { MST } \\
\text { MST } \\
\text { MST }\end{array}$ \\
\hline $\begin{array}{l}\text { FEB. } \\
\text { FEB. } \\
\text { MAR. } \\
\text { MAR. } \\
\text { MAR. }\end{array}$ & $\begin{array}{r}28 \\
28 \\
6 \\
12 \\
17\end{array}$ & $\begin{array}{l}00 \\
04 \\
22 \\
17 \\
04\end{array}$ & $\begin{array}{l}31 \\
25 \\
00 \\
56 \\
56\end{array}$ & $\begin{array}{l}33.5 \\
11.4 \\
31.5 \\
15.6 \\
20.2\end{array}$ & $\begin{array}{l}46.184 \mathrm{~N} \\
46.179 \mathrm{~N} \\
44.790 \mathrm{~N} \\
45.744 \mathrm{~N} \\
44.778 \mathrm{~N}\end{array}$ & $\begin{array}{l}111.497 \mathrm{~W} . \\
111.460 \mathrm{~W} . \\
111.155 \mathrm{~W} . \\
113.820 \mathrm{~W} . \\
111.666 \mathrm{~W} .\end{array}$ & $\begin{array}{r}5 \\
5 \\
14 \\
26 \\
5\end{array}$ & $\begin{array}{l}\text { GS } \\
\text { GS } \\
\text { BU } \\
\text { BU } \\
\text { GS }\end{array}$ & $\begin{array}{l}\cdots \\
\cdots \\
\cdots \\
\cdots\end{array}$ & $\begin{array}{l}\cdots \\
\cdots \\
\cdots \\
\cdots\end{array}$ & $\begin{array}{l}\text { 3. } 2 \mathrm{ML}(\mathrm{BU}) \\
3.8 \mathrm{ML}(\mathrm{BU}) \\
2.7 \mathrm{ML}(\mathrm{BU}) \\
2.5 \mathrm{ML}(\mathrm{BU}) \\
3.3 \mathrm{ML}(\mathrm{BU})\end{array}$ & $\begin{array}{c}\text { FELT } \\
\quad \vee \\
\ldots \\
\cdots \\
\cdots\end{array}$ & $\begin{array}{l}\text { FEB. } \\
\text { FEB. } \\
\text { MAR. } \\
\text { MAR. } \\
\text { MAR. }\end{array}$ & $\begin{array}{r}27 \\
27 \\
6 \\
12 \\
16\end{array}$ & $\begin{array}{l}17: 31 \\
21: 25 \\
15: 00 \\
10: 56 \\
21: 56\end{array}$ & $\begin{array}{l}\text { MST } \\
\text { MST } \\
\text { MST } \\
\text { MST } \\
\text { MST }\end{array}$ \\
\hline $\begin{array}{l}\text { MAR. } \\
\text { MAR. } \\
\text { MAR. } \\
\text { MAR. } \\
\text { APR. }\end{array}$ & $\begin{array}{r}19 \\
19 \\
24 \\
26 \\
1\end{array}$ & $\begin{array}{l}02 \\
09 \\
21 \\
07 \\
20\end{array}$ & $\begin{array}{l}52 \\
44 \\
06 \\
08 \\
43\end{array}$ & $\begin{array}{l}42.2 \\
04.4 \\
31.8 \\
32.0 \\
09.5\end{array}$ & $\begin{array}{l}44.611 \mathrm{~N} \\
44.935 \mathrm{~N} \\
46.547 \mathrm{~N} \\
44.963 \mathrm{~N} \\
44.799 \mathrm{~N}\end{array}$ & $\begin{array}{l}112.079 W \\
111.921 W \\
112.095 W \\
111.808 W \\
111.607 W\end{array}$ & $\begin{array}{r}10 \\
18 \\
11 \\
5 \\
17\end{array}$ & $\begin{array}{l}\text { BU } \\
\text { BU } \\
\text { BU } \\
\text { GS } \\
\text { BU }\end{array}$ & $\begin{array}{l}\cdots \\
\cdots \\
\cdots \\
\cdots\end{array}$ & $\begin{array}{l}\cdots \\
\cdots \\
\cdots \\
\cdots \\
\cdots\end{array}$ & $\begin{array}{l}3.0 \mathrm{ML}(\mathrm{BU}) \\
2.7 \mathrm{ML}(\mathrm{BU}) \\
3.1 \mathrm{ML}(\mathrm{BU}) \\
3.5 \mathrm{ML}(\mathrm{GS}) \\
2.7 \mathrm{ML}(\mathrm{BU})\end{array}$ & $\begin{array}{l}\ldots \\
\text { III } \\
\ldots \\
\ldots\end{array}$ & $\begin{array}{l}\text { MAR. } \\
\text { MAR. } \\
\text { MAR. } \\
\text { MAR. } \\
\text { APR. }\end{array}$ & $\begin{array}{r}18 \\
19 \\
24 \\
26 \\
1\end{array}$ & $\begin{array}{l}19: 52 \\
02: 44 \\
14: 06 \\
00: 08 \\
13: 43\end{array}$ & $\begin{array}{l}\text { MST } \\
\text { MST } \\
\text { MST } \\
\text { MST } \\
\text { MST }\end{array}$ \\
\hline $\begin{array}{l}\text { APR. } \\
\text { APR. } \\
\text { APR. } \\
\text { APR. } \\
\text { APR. }\end{array}$ & $\begin{array}{r}2 \\
6 \\
6 \\
7 \\
13\end{array}$ & $\begin{array}{l}20 \\
08 \\
08 \\
13 \\
02\end{array}$ & $\begin{array}{l}20 \\
25 \\
57 \\
16 \\
34\end{array}$ & $\begin{array}{l}21.3 \\
42.9 \\
07.6 \\
21.3 \\
32.1\end{array}$ & $\begin{array}{l}45.263 \mathrm{~N} \\
44.830 \mathrm{~N} \\
44.889 \mathrm{~N} \\
45.582 \mathrm{~N} \\
44.833 \mathrm{~N}\end{array}$ & $\begin{array}{l}112.915 W \\
111.552 W \\
111.484 W \\
111.815 W \\
111.421 W\end{array}$ & $\begin{array}{r}2 \\
14 \\
10 \\
5 \\
10\end{array}$ & $\begin{array}{l}\text { BU } \\
\text { BU } \\
\text { BU } \\
\text { GS } \\
\text { BU }\end{array}$ & $\cdots$ & $\begin{array}{l}\ldots \\
\ldots \\
\ldots \\
\ldots \\
\ldots\end{array}$ & $\begin{array}{l}2.5 \mathrm{ML}(\mathrm{BU}) \\
2.8 \mathrm{ML}(\mathrm{BU}) \\
2.5 \mathrm{ML}(\mathrm{BU}) \\
3.0 \mathrm{ML}(\mathrm{BU}) \\
2.7 \mathrm{ML}(\mathrm{BU})\end{array}$ & $\begin{array}{l}\ldots \\
\ldots \\
\cdots \\
\ldots\end{array}$ & $\begin{array}{l}\text { APR. } \\
\text { APR. } \\
\text { APR. } \\
\text { APR. } \\
\text { APR. }\end{array}$ & $\begin{array}{r}2 \\
6 \\
6 \\
7 \\
12\end{array}$ & $\begin{array}{l}13: 20 \\
01: 25 \\
01: 57 \\
06: 16 \\
19: 34\end{array}$ & $\begin{array}{l}\text { MST } \\
\text { MST } \\
\text { MST } \\
\text { MST } \\
\text { MST }\end{array}$ \\
\hline
\end{tabular}


Table 1. Summary of U.S. earthquakes for 1984-Continued

\begin{tabular}{|c|c|c|c|c|c|c|c|c|c|c|c|c|c|}
\hline \multirow{2}{*}{ Date } & \multicolumn{2}{|c|}{$\begin{array}{c}\text { Origin time } \\
\text { (UTC) }\end{array}$} & \multirow{2}{*}{$\begin{array}{c}\text { Latitude } \\
\left({ }^{\circ}\right)\end{array}$} & \multirow{2}{*}{$\begin{array}{c}\text { Longitude } \\
\left({ }^{\circ}\right)\end{array}$} & \multirow{2}{*}{$\begin{array}{c}\text { Depth } \\
\text { (km) }\end{array}$} & \multirow{2}{*}{$\begin{array}{l}\text { Hypo- } \\
\text { center } \\
\text { surce }\end{array}$} & \multicolumn{3}{|c|}{ Magnitude } & \multirow{2}{*}{$\begin{array}{l}\text { Maximum } \\
\text { intensity }\end{array}$} & \multicolumn{3}{|c|}{ Local time } \\
\hline & hr $\min$ & $\sec$ & & & & & $\mathbf{m b}$ & MS & $\begin{array}{l}\text { ML, Mn } \\
M D, M w\end{array}$ & & Date & Hour & $\begin{array}{l}\text { Time } \\
\text { zone }\end{array}$ \\
\hline
\end{tabular}

MONTANA-Continued

\begin{tabular}{|c|c|c|c|c|c|c|c|c|c|c|c|c|c|c|c|c|}
\hline $\begin{array}{l}\text { APR. } \\
\text { APR. } \\
\text { APR. } \\
\text { APR. } \\
\text { APR. }\end{array}$ & $\begin{array}{l}17 \\
23 \\
28 \\
28 \\
28\end{array}$ & $\begin{array}{l}01 \\
18 \\
00 \\
00 \\
03\end{array}$ & $\begin{array}{l}19 \\
37 \\
13 \\
54 \\
11\end{array}$ & $\begin{array}{l}36.8 \\
41.0 \\
26.7 \\
41.2 \\
51.2\end{array}$ & $\begin{array}{l}44.748 \mathrm{~N} \\
46.639 \mathrm{~N} . \\
46.027 \mathrm{~N} . \\
48.010 \mathrm{~N} . \\
44.706 \mathrm{~N} .\end{array}$ & $\begin{array}{l}111.114 W . \\
112.359 w . \\
111.546 w . \\
113.275 w . \\
111.745 W .\end{array}$ & $\begin{array}{l}15 \\
13 \\
11 \\
41 \\
16\end{array}$ & $\begin{array}{l}\text { BU } \\
B U \\
B U \\
U \\
U\end{array}$ & $\begin{array}{l}\cdots \\
\cdots \\
\cdots \\
\cdots\end{array}$ & $\begin{array}{l}\cdots \\
\cdots \\
\cdots \\
\cdots\end{array}$ & $\begin{array}{l}2.6 \mathrm{ML}(\mathrm{BU}) \\
2.7 \mathrm{ML}(\mathrm{BU}) \\
2.6 \mathrm{ML}(\mathrm{BU}) \\
2.6 \mathrm{ML}(\mathrm{BU}) \\
2.8 \mathrm{ML}(\mathrm{BU})\end{array}$ & $\begin{array}{l}\cdots \\
\ldots \\
\cdots \\
\cdots\end{array}$ & $\begin{array}{l}\text { APR. } \\
\text { APR. } \\
\text { APR. } \\
\text { APR. } \\
\text { APR. }\end{array}$ & $\begin{array}{l}16 \\
23 \\
27 \\
27 \\
27\end{array}$ & $\begin{array}{l}18: 19 \\
11: 37 \\
17: 13 \\
17: 54 \\
20: 11\end{array}$ & $\begin{array}{l}\text { MST } \\
\text { MST } \\
\text { MST } \\
\text { MST } \\
\text { MST }\end{array}$ \\
\hline $\begin{array}{l}\text { APR. } \\
\text { APR. } \\
\text { APR. } \\
\text { MAY } \\
\text { MAY }\end{array}$ & $\begin{array}{r}29 \\
30 \\
30 \\
3 \\
7\end{array}$ & $\begin{array}{l}01 \\
08 \\
19 \\
11 \\
20\end{array}$ & $\begin{array}{l}11 \\
22 \\
16 \\
07 \\
57\end{array}$ & $\begin{array}{l}36.7 \\
54.1 \\
54.6 \\
54.4 \\
16.9\end{array}$ & $\begin{array}{l}44.792 \mathrm{~N} \\
44.789 \mathrm{~N} \\
44.586 \mathrm{~N} \\
47.885 \mathrm{~N} \\
44.662 \mathrm{~N}\end{array}$ & $\begin{array}{l}111.597 W . \\
111.419 W . \\
111.182 W . \\
113.684 W . \\
111.851 W .\end{array}$ & $\begin{array}{r}15 \\
18 \\
14 \\
5 \\
5\end{array}$ & $\begin{array}{l}\text { U } \\
\text { BU } \\
\text { BU } \\
\text { GS } \\
\text { GS }\end{array}$ & $\begin{array}{l}\cdots \\
\cdots \\
\cdots \\
\cdots\end{array}$ & $\begin{array}{l}\cdots \\
\cdots \\
\cdots \\
\cdots\end{array}$ & $\begin{array}{l}2.5 \mathrm{ML}(\mathrm{BU}) \\
2.8 \mathrm{ML}(\mathrm{BU}) \\
2.5 \mathrm{ML}(\mathrm{BU}) \\
3.4 \mathrm{ML}(\mathrm{BU}) \\
\text { 4. } \mathrm{MML}(\mathrm{GS})\end{array}$ & $\begin{array}{l}\cdots \\
\cdots \\
\cdots \\
\text { III }\end{array}$ & $\begin{array}{l}\text { APR. } \\
\text { APR. } \\
\text { APR. } \\
\text { MAY } \\
\text { MAY }\end{array}$ & $\begin{array}{r}28 \\
30 \\
30 \\
3 \\
7\end{array}$ & $\begin{array}{l}18: 11 \\
01: 22 \\
12: 16 \\
04: 07 \\
13: 57\end{array}$ & $\begin{array}{l}\text { MST } \\
\text { MST } \\
\text { MST } \\
\text { MST } \\
\text { MST }\end{array}$ \\
\hline $\begin{array}{l}\text { MAY } \\
\text { MAY } \\
\text { MAY } \\
\text { JUNE } \\
\text { JUNE }\end{array}$ & $\begin{array}{r}14 \\
19 \\
30 \\
5 \\
9\end{array}$ & $\begin{array}{l}23 \\
05 \\
07 \\
11 \\
10\end{array}$ & $\begin{array}{l}55 \\
32 \\
22 \\
39 \\
36\end{array}$ & $\begin{array}{l}42.0 \\
15.7 \\
27.7 \\
39.0 \\
19.0\end{array}$ & $\begin{array}{l}45.384 \mathrm{~N} \\
45.767 \mathrm{~N} \\
46.032 \mathrm{~N} \\
44.769 \mathrm{~N} \\
45.025 \mathrm{~N}\end{array}$ & $\begin{array}{l}111.885 W . \\
111.954 W . \\
111.568 W . \\
111.527 W . \\
113.123 W .\end{array}$ & $\begin{array}{r}7 \\
8 \\
13 \\
12 \\
10\end{array}$ & $\begin{array}{l}\text { BU } \\
\text { BU } \\
\text { BU } \\
\text { BU } \\
\text { BU }\end{array}$ & $\begin{array}{l}\cdots \\
\cdots \\
\cdots \\
\cdots\end{array}$ & $\begin{array}{l}\cdots \\
\cdots \\
\cdots \\
\cdots \\
\cdots\end{array}$ & $\begin{array}{l}2.6 \mathrm{ML}(\mathrm{BU}) \\
2.5 \mathrm{ML}(\mathrm{BU}) \\
2.5 \mathrm{ML}(\mathrm{BU}) \\
3.1 \mathrm{ML}(\mathrm{BU}) \\
2.5 \mathrm{ML}(\mathrm{BU})\end{array}$ & $\begin{array}{l}\cdots \\
\cdots \\
\cdots \\
\cdots\end{array}$ & $\begin{array}{l}\text { MAY } \\
\text { MAY } \\
\text { MAY } \\
\text { JUNE } \\
\text { JUNE }\end{array}$ & $\begin{array}{r}14 \\
18 \\
30 \\
5 \\
9\end{array}$ & $\begin{array}{l}16: 55 \\
22: 32 \\
00: 22 \\
04: 39 \\
03: 36\end{array}$ & $\begin{array}{l}\text { MST } \\
\text { MST } \\
\text { MST } \\
\text { MST } \\
\text { MST }\end{array}$ \\
\hline $\begin{array}{l}\text { JUNE } \\
\text { JUNE } \\
\text { JUNE } \\
\text { JULY } \\
\text { JULY }\end{array}$ & $\begin{array}{r}17 \\
22 \\
22 \\
2 \\
7\end{array}$ & $\begin{array}{l}14 \\
05 \\
08 \\
22 \\
11\end{array}$ & $\begin{array}{l}02 \\
10 \\
09 \\
42 \\
43\end{array}$ & $\begin{array}{l}01.0 \\
29.0 \\
12.7 \\
49.4 \\
32.4\end{array}$ & $\begin{array}{l}44.797 \mathrm{~N} \\
44.759 \mathrm{~N} . \\
44.767 \mathrm{~N} . \\
44.956 \mathrm{~N} \\
47.401 \mathrm{~N} .\end{array}$ & $\begin{array}{l}112.186 W \\
112.275 W \\
112.233 W \\
112.938 W \\
113.177 w .\end{array}$ & $\begin{array}{r}10 \\
10 \\
10 \\
10 \\
9\end{array}$ & $\begin{array}{l}\text { BU } \\
\text { BU } \\
\text { BU } \\
\text { BU } \\
\text { BU }\end{array}$ & $\begin{array}{l}\cdots \\
\cdots \\
\cdots \\
\cdots\end{array}$ & $\begin{array}{l}\cdots \\
\ldots \\
\cdots \\
\cdots\end{array}$ & $\begin{array}{l}2.8 \mathrm{ML}(\mathrm{BU}) \\
2.6 \mathrm{ML}(\mathrm{BU}) \\
2.7 \mathrm{ML}(\mathrm{BU}) \\
2.5 \mathrm{ML}(\mathrm{BU}) \\
2.8 \mathrm{ML}(\mathrm{BU})\end{array}$ & $\begin{array}{l}\cdots \\
\cdots \\
\cdots \\
\ldots\end{array}$ & $\begin{array}{l}\text { JUNE } \\
\text { JUNE } \\
\text { JUNE } \\
\text { JULY } \\
\text { JULY }\end{array}$ & $\begin{array}{r}17 \\
21 \\
22 \\
2 \\
7\end{array}$ & $\begin{array}{l}07: 02 \\
22: 10 \\
01: 09 \\
15: 42 \\
04: 43\end{array}$ & $\begin{array}{l}\text { MST } \\
\text { MST } \\
\text { MST } \\
\text { MST } \\
\text { MST }\end{array}$ \\
\hline $\begin{array}{l}\text { JULY } \\
\text { JULY } \\
\text { JULY } \\
\text { JULY } \\
\text { JULY }\end{array}$ & $\begin{array}{r}9 \\
9 \\
13 \\
14 \\
14\end{array}$ & $\begin{array}{l}21 \\
21 \\
19 \\
05 \\
07\end{array}$ & $\begin{array}{l}32 \\
42 \\
13 \\
42 \\
17\end{array}$ & $\begin{array}{l}04.9 \\
05.6 \\
18.0 \\
47.7 \\
45.2\end{array}$ & $\begin{array}{l}45.058 \mathrm{~N} \\
45.029 \mathrm{~N} \\
44.811 \mathrm{~N} \\
44.717 \mathrm{~N} \\
44.771 \mathrm{~N}\end{array}$ & $\begin{array}{l}110.882 W . \\
110.870 W . \\
112.754 W . \\
112.855 W . \\
112.660 W .\end{array}$ & $\begin{array}{r}10 \\
10 \\
10 \\
9 \\
10\end{array}$ & $\begin{array}{l}\text { BU } \\
B U \\
B U \\
B U \\
B U\end{array}$ & $\begin{array}{l}\cdots \\
\ldots \\
\cdots \\
\cdots \\
\cdots\end{array}$ & $\begin{array}{l}\ldots \\
\cdots \\
\cdots \\
\cdots \\
\cdots\end{array}$ & $\begin{array}{l}\text { 3. } 0 \mathrm{ML}(\mathrm{BU}) \\
\text { 3. } 0 \mathrm{ML}(\mathrm{BU}) \\
2.8 \mathrm{ML}(\mathrm{BU}) \\
2.7 \mathrm{ML}(\mathrm{BU}) \\
\text { 3. } \mathrm{ML}(\mathrm{BU})\end{array}$ & $\begin{array}{l}\ldots \\
\ldots \\
\ldots \\
\ldots\end{array}$ & $\begin{array}{l}\text { JULY } \\
\text { JULY } \\
\text { JULY } \\
\text { JULY } \\
\text { JULY }\end{array}$ & $\begin{array}{r}9 \\
9 \\
13 \\
13 \\
14\end{array}$ & $\begin{array}{l}14: 32 \\
14: 42 \\
12: 13 \\
22: 42 \\
00: 17\end{array}$ & $\begin{array}{l}\text { MST } \\
\text { MST } \\
\text { MST } \\
\text { MST } \\
\text { MST }\end{array}$ \\
\hline $\begin{array}{l}\text { JULY } \\
\text { JULY } \\
\text { JULY } \\
\text { JULY } \\
\text { JULY }\end{array}$ & $\begin{array}{l}14 \\
14 \\
16 \\
21 \\
21\end{array}$ & $\begin{array}{l}07 \\
13 \\
23 \\
09 \\
17\end{array}$ & $\begin{array}{l}29 \\
52 \\
39 \\
18 \\
42\end{array}$ & $\begin{array}{l}55.5 \\
45.7 \\
13.7 \\
52.6 \\
53.6\end{array}$ & $\begin{array}{l}47.929 \mathrm{~N} \\
44.806 \mathrm{~N} \\
44.686 \mathrm{~N} \\
44.974 \mathrm{~N} . \\
44.762 \mathrm{~N} .\end{array}$ & $\begin{array}{l}114.955 W . \\
112.721 W . \\
112.818 W . \\
112.905 w . \\
112.758 w .\end{array}$ & $\begin{array}{r}14 \\
10 \\
10 \\
13 \\
7\end{array}$ & $\begin{array}{l}\text { BU } \\
B U \\
B U \\
B U \\
B U\end{array}$ & $\begin{array}{l}\cdots \\
\cdots \\
\cdots \\
\cdots\end{array}$ & $\begin{array}{l}\ldots \\
\ldots \\
\cdots \\
\cdots \\
\cdots\end{array}$ & $\begin{array}{l}\text { 3. } 0 \mathrm{MD}(\mathrm{BU}) \\
\text { 2. } 7 \mathrm{ML}(\mathrm{BU}) \\
\text { 3. } 3 \mathrm{ML}(\mathrm{BU}) \\
\text { 3. } O M L(\mathrm{BU}) \\
\text { 3. } 2 \mathrm{ML}(\mathrm{BU})\end{array}$ & $\begin{array}{l}\ldots \\
\cdots \\
\ldots \\
\ldots\end{array}$ & $\begin{array}{l}\text { JULY } \\
\text { JULY } \\
\text { JULY } \\
\text { JULY } \\
\text { JULY }\end{array}$ & $\begin{array}{l}14 \\
14 \\
16 \\
21 \\
21\end{array}$ & $\begin{array}{l}08: 29 \\
06: 52 \\
16: 39 \\
02: 18 \\
10: 42\end{array}$ & $\begin{array}{l}\text { UST } \\
\text { UST } \\
\text { UST } \\
\text { UST } \\
\text { UST }\end{array}$ \\
\hline $\begin{array}{l}\text { JULY } \\
\text { JULY } \\
\text { JULY } \\
\text { AUG } \\
\text { AUG. }\end{array}$ & $\begin{array}{r}25 \\
28 \\
31 \\
19 \\
4\end{array}$ & $\begin{array}{l}22 \\
19 \\
02 \\
20 \\
07\end{array}$ & $\begin{array}{l}37 \\
12 \\
30 \\
59 \\
47\end{array}$ & $\begin{array}{l}08.4 \\
10.9 \\
22.6 \\
50.5 \\
17.3\end{array}$ & $\begin{array}{l}44.926 \mathrm{~N} \\
44.905 \mathrm{~N} \\
45.024 \mathrm{~N} \\
44.957 \mathrm{~N} \\
44.853 \mathrm{~N}\end{array}$ & $\begin{array}{l}112.731 W . \\
112.612 W . \\
112.745 W . \\
112.925 W . \\
112.934 W .\end{array}$ & $\begin{array}{r}13 \\
1 \\
0 \\
7 \\
10\end{array}$ & $\begin{array}{l}\text { BU } \\
B U \\
B U \\
B U \\
B U\end{array}$ & $\begin{array}{l}\cdots \\
\cdots \\
\cdots \\
\cdots \\
\cdots\end{array}$ & $\begin{array}{l}\cdots \\
\cdots \\
\cdots \\
\cdots \\
\cdots\end{array}$ & $\begin{array}{l}2.5 \mathrm{ML}(\mathrm{BU}) \\
2.6 \mathrm{ML}(\mathrm{BU}) \\
2.5 \mathrm{ML}(\mathrm{BU}) \\
2.8 \mathrm{ML}(\mathrm{BU}) \\
2.9 \mathrm{ML}(\mathrm{BU})\end{array}$ & $\begin{array}{l}\ldots \\
\cdots \\
\ldots \\
\ldots\end{array}$ & $\begin{array}{l}\text { JULY } \\
\text { JULY } \\
\text { JULY } \\
\text { AUG. } \\
\text { AUG. }\end{array}$ & $\begin{array}{r}25 \\
28 \\
30 \\
19 \\
4\end{array}$ & $\begin{array}{l}15: 37 \\
12: 12 \\
19: 30 \\
13: 59 \\
00: 47\end{array}$ & $\begin{array}{l}\text { MST } \\
\text { MST } \\
\text { MST } \\
\text { MST } \\
\text { MST }\end{array}$ \\
\hline $\begin{array}{l}\text { AUG. } \\
\text { AUG. } \\
\text { AUG. } \\
\text { AUG. } \\
\text { AUG. }\end{array}$ & $\begin{array}{l}5 \\
5 \\
5 \\
5 \\
5\end{array}$ & $\begin{array}{l}09 \\
15 \\
18 \\
19 \\
23\end{array}$ & $\begin{array}{l}35 \\
58 \\
25 \\
42 \\
13\end{array}$ & $\begin{array}{l}08.2 \\
19.9 \\
45.4 \\
58.6 \\
20.9\end{array}$ & $\begin{array}{l}45.022 \mathrm{~N} \\
45.044 \mathrm{~N} \\
45.029 \mathrm{~N} \\
45.018 \mathrm{~N} \\
45.037 \mathrm{~N}\end{array}$ & $\begin{array}{l}112.961 w . \\
112.757 w . \\
112.759 w . \\
113.009 w . \\
112.856 w .\end{array}$ & $\begin{array}{r}6 \\
5 \\
1 \\
8 \\
18\end{array}$ & $\begin{array}{l}\text { BU } \\
\text { BU } \\
\text { BU } \\
\text { BU } \\
\text { BU }\end{array}$ & $\begin{array}{l}\cdots \\
\cdots \\
\cdots \\
\cdots \\
\cdots\end{array}$ & $\begin{array}{l}\cdots \\
\cdots \\
\cdots \\
\cdots \\
\cdots\end{array}$ & $\begin{array}{l}2.5 \mathrm{ML}(\mathrm{BU}) \\
2.5 \mathrm{ML}(\mathrm{BU}) \\
2.8 \mathrm{ML}(\mathrm{BU}) \\
2.5 \mathrm{ML}(\mathrm{BU}) \\
2.5 \mathrm{ML}(\mathrm{BU})\end{array}$ & $\begin{array}{l}\ldots \\
\ldots \\
\ldots \\
\ldots \\
\ldots\end{array}$ & $\begin{array}{l}\text { AUG. } \\
\text { AUG. } \\
\text { AUG. } \\
\text { AUG. } \\
\text { AUG. }\end{array}$ & $\begin{array}{l}5 \\
5 \\
5 \\
5 \\
5\end{array}$ & $\begin{array}{l}02: 35 \\
08: 58 \\
11: 25 \\
12: 42 \\
16: 13\end{array}$ & $\begin{array}{l}\text { MST } \\
\text { MST } \\
\text { MST } \\
\text { MST } \\
\text { MST }\end{array}$ \\
\hline $\begin{array}{l}\text { AUG. } \\
\text { AUG. } \\
\text { AUG. } \\
\text { AUG. } \\
\text { AUG. }\end{array}$ & $\begin{array}{r}6 \\
7 \\
9 \\
10 \\
12\end{array}$ & $\begin{array}{l}18 \\
02 \\
18 \\
12 \\
20\end{array}$ & $\begin{array}{l}52 \\
45 \\
59 \\
58 \\
30\end{array}$ & $\begin{array}{l}23.3 \\
09.7 \\
57.3 \\
29.5 \\
06.7\end{array}$ & $\begin{array}{l}44.896 \mathrm{~N} \\
45.021 \mathrm{~N} \\
44.767 \mathrm{~N} \\
45.061 \mathrm{~N} \\
44.903 \mathrm{~N}\end{array}$ & $\begin{array}{l}112.820 w . \\
112.780 w . \\
112.831 w . \\
112.905 w . \\
112.801 w .\end{array}$ & $\begin{array}{r}6 \\
11 \\
0 \\
4 \\
12\end{array}$ & $\begin{array}{l}\text { BU } \\
\text { BU } \\
\text { BU } \\
\text { BU } \\
\text { BU }\end{array}$ & $\begin{array}{l}\cdots \\
\cdots \\
\cdots \\
\cdots\end{array}$ & $\begin{array}{l}\cdots \\
\cdots \\
\cdots \\
\cdots\end{array}$ & $\begin{array}{l}2.7 \mathrm{ML}(\mathrm{BU}) \\
2.7 \mathrm{ML}(\mathrm{BU}) \\
2.6 \mathrm{ML}(\mathrm{BU}) \\
2.5 \mathrm{ML}(\mathrm{BU}) \\
3.6 \mathrm{ML}(\mathrm{BU})\end{array}$ & $\begin{array}{l}\ldots \\
\ldots \\
\cdots \\
\ldots\end{array}$ & $\begin{array}{l}\text { AUG. } \\
A \cup G . \\
A \cup G . \\
A \cup G . \\
A \cup G .\end{array}$ & $\begin{array}{r}6 \\
6 \\
9 \\
10 \\
12\end{array}$ & $\begin{array}{l}11: 52 \\
19: 45 \\
11: 59 \\
05: 58 \\
13: 30\end{array}$ & $\begin{array}{l}\text { MST } \\
\text { MST } \\
\text { MST } \\
\text { MST } \\
\text { MST }\end{array}$ \\
\hline $\begin{array}{l}\text { AUG. } \\
\text { AUG. } \\
\text { AUG. } \\
\text { AUG. } \\
\text { AUG. }\end{array}$ & $\begin{array}{l}13 \\
15 \\
23 \\
23 \\
24\end{array}$ & $\begin{array}{l}06 \\
00 \\
04 \\
18 \\
10\end{array}$ & $\begin{array}{l}47 \\
02 \\
17 \\
26 \\
10\end{array}$ & $\begin{array}{l}32.2 \\
21.5 \\
44.2 \\
24.4 \\
48.0\end{array}$ & $\begin{array}{l}44.861 \mathrm{~N} . \\
44.950 \mathrm{~N} . \\
46.842 \mathrm{~N} \\
44.999 \mathrm{~N} . \\
44.941 \mathrm{~N} .\end{array}$ & $\begin{array}{l}111.530 \mathrm{~W} . \\
113.141 \mathrm{~W} . \\
112.907 \mathrm{~W} \\
112.995 \mathrm{~W} . \\
112.616 \mathrm{~W} .\end{array}$ & $\begin{array}{r}15 \\
16 \\
7 \\
9 \\
1\end{array}$ & $\begin{array}{l}\text { BU } \\
\text { BU } \\
\text { BU } \\
\text { BU } \\
\text { BU }\end{array}$ & $\begin{array}{l}\cdots \\
\cdots \\
\cdots \\
\cdots\end{array}$ & $\begin{array}{l}\cdots \\
\cdots \\
\cdots \\
\cdots \\
\cdots\end{array}$ & $\begin{array}{l}2.9 \mathrm{ML}(\mathrm{BU}) \\
2.5 \mathrm{ML}(\mathrm{BU}) \\
2.9 \mathrm{ML}(\mathrm{BU}) \\
2.5 \mathrm{ML}(\mathrm{BU}) \\
2.6 \mathrm{ML}(\mathrm{BU})\end{array}$ & $\begin{array}{l}\cdots \\
\ldots \\
\cdots \\
\cdots\end{array}$ & $\begin{array}{l}\text { AUG. } \\
\text { AUG. } \\
\text { AUG. } \\
\text { AUG. } \\
\text { AUG. }\end{array}$ & $\begin{array}{l}12 \\
14 \\
22 \\
23 \\
24\end{array}$ & $\begin{array}{l}23: 47 \\
17: 02 \\
21: 17 \\
11: 26 \\
03: 10\end{array}$ & $\begin{array}{l}\text { UST } \\
\text { UST } \\
\text { UST } \\
\text { UST } \\
\text { UST }\end{array}$ \\
\hline
\end{tabular}


Table 1. Summary of U.S. earthquakes for 1984-Continued

\begin{tabular}{|c|c|c|c|c|c|c|c|c|c|c|c|c|c|}
\hline \multirow{2}{*}{ Date } & \multicolumn{2}{|c|}{$\begin{array}{l}\text { Origin time } \\
\text { (UTC) }\end{array}$} & \multirow{2}{*}{$\begin{array}{c}\text { Latitude } \\
\left(^{\circ}\right)\end{array}$} & \multirow{2}{*}{$\begin{array}{c}\text { Lomgitude } \\
\left({ }^{\circ}\right)\end{array}$} & \multirow{2}{*}{$\begin{array}{c}\text { Depth } \\
(\mathbf{k m})\end{array}$} & \multirow{2}{*}{$\begin{array}{l}\text { Hypo- } \\
\text { center } \\
\text { siurce }\end{array}$} & \multicolumn{3}{|c|}{ Magnitude } & \multirow{2}{*}{$\begin{array}{l}\text { Maximum } \\
\text { intensity }\end{array}$} & \multicolumn{3}{|c|}{ Lexal time } \\
\hline & hr $\min$ & $\sec$ & & & & & $\mathbf{m b}$ & MS & $\begin{array}{l}\text { ML. Mn } \\
\text { MD. Mw }\end{array}$ & & Date & Hour & $\begin{array}{l}\text { Time } \\
\text { Tome }\end{array}$ \\
\hline \multicolumn{14}{|c|}{ MONTANA-Continued } \\
\hline
\end{tabular}

AUG. $\quad \begin{array}{lllll}27 & 16 & 04 & 00.7\end{array}$

$\begin{array}{lllll}\text { SEPT. } & 3 & 12 & 28 & 50.8\end{array}$

SEPT. $3 \quad 203531.7$

SEPT. $6 \quad \begin{array}{llll}6 & 180 & 29.0\end{array}$

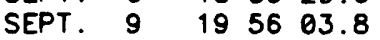

SEPT. $13 \quad 1754 \quad 22.3$

SEPT. $16 \quad 0128 \quad 54.4$

SEPT. $20 \quad 02 \quad 3208.6$

$\begin{array}{llll}\text { SEPT. } 20 & 17 & 18 & 39.1\end{array}$

SEPT. $20 \quad 17 \quad 1903.6$

SEPT. $20 \quad 20 \quad 28 \quad 45.9$

SEPT. $21 \quad 040102.6$

SEPT. $21 \quad 19 \quad 11 \quad 17.0$

SEPT. $21 \quad 192904.9$

SEPT. $23 \quad 02 \quad 44 \quad 14.6$

SEPT. $24 \quad 17 \quad 09 \quad 02.0$

SEPT. $26 \quad 21 \quad 1035.2$

OCT. $3 \quad 05 \quad 45 \quad 16.6$

OCT. $3 \quad 2038 \quad 59.7$

OCT. $5 \quad 175750.9$

OCT. $\quad 8 \quad 02 \quad 40 \quad 07.1$

OCT. $11 \quad 14 \quad 2530.0$

OCT. $18 \quad 011207.8$

OCT. $20 \quad 20 \quad 13 \quad 41.3$

OCT. $25 \quad 225748.6$

OCT. $26 \quad 2251 \quad 30.3$

OCT. $\quad 30 \quad 2137 \quad 34.2$

OCT. $31 \quad 313 \quad 36 \quad 20.1$

NOV. $\quad 1 \quad 050755.6$

NOV. $\quad \begin{array}{llll}6 & 21 & 03 & 38.6\end{array}$

NOV. $\quad \begin{array}{llll}6 & 22 & 25 & 45.4\end{array}$

NOV. $7 \quad 170904.8$

NOV. $\begin{array}{lllll}8 & 09 & 02 & 02.7\end{array}$

NOV. $11 \quad 16 \quad 3251.6$

NOV. $17 \quad 145941.3$

NOV. $20 \quad 04 \quad 4945.4$

NOV. $20 \quad 095401.8$

NOV. $\quad 20 \quad 18 \quad 46 \quad 53.1$

DEC. $\quad 4 \quad 023910.0$

DEC. $\quad 7 \quad 215857.3$

DEC. $\quad \begin{array}{llll}8 & 09 & 39 & 00.6\end{array}$

DEC. $10 \quad 03 \quad 3839.2$

DEC. $20 \quad 00 \quad 43 \quad 44.4$

$\begin{array}{lllll}\text { DEC. } & 22 & 07 & 17 & 49.8\end{array}$
$45.095 \mathrm{~N} .113 .080 \mathrm{~W}$. $45.019 \mathrm{~N} .112 .963 \mathrm{~W}$. $47.778 \mathrm{~N} .113 .293 \mathrm{~W}$. $46.660 \mathrm{~N}$. $112.960 \mathrm{~W}$. $44.638 \mathrm{~N}$. $111.912 \mathrm{~W}$.

$44.830 N .112 .842 W$. $44.653 \mathrm{~N} .111 .851 \mathrm{~W}$. $45.043 \mathrm{~N} .113 .025 \mathrm{~W}$. $44.930 \mathrm{~N}, 112.838 \mathrm{~W}$. $44.913 N$. $112.912 W$.

$44.931 \mathrm{~N} .112 .912 \mathrm{~W}$ $44.835 N$. $112.827 W$. $44.899 \mathrm{~N} .113 .006 \mathrm{~W}$. $44.833 \mathrm{~N} .113 .052 \mathrm{~W}$. $44.803 \mathrm{~N} .112 .919 \mathrm{~W}$.

44.876 N. $113.123 W$. $44.879 \mathrm{~N} .112 .847 \mathrm{~W}$. $44.912 \mathrm{~N}, 112.784 \mathrm{~W}$. $45.000 \mathrm{~N} .112 .800 \mathrm{~W}$. $44.957 \mathrm{~N}$. 112.723W.

$44.645 \mathrm{~N} .112 .903 W$. $44.821 \mathrm{~N} .112 .735 \mathrm{~W}$ $48.174 \mathrm{~N} .114 .184 \mathrm{~W}$. $47.791 \mathrm{~N} .115 .131 \mathrm{~W}$. $44.811 \mathrm{~N} .112 .708 \mathrm{~W}$.

$44.806 \mathrm{~N} .112 .694 \mathrm{~W}$. $48.166 \mathrm{~N}, 114.175 \mathrm{~W}$. $44.912 \mathrm{~N} .112 .758 \mathrm{~W}$. $44.669 \mathrm{~N} .111 .968 \mathrm{~W}$ $45.985 \mathrm{~N} .111 .360 \mathrm{~W}$

$44.767 \mathrm{~N} .111 .479 \mathrm{~W}$. $45.046 \mathrm{~N} .112 .900 \mathrm{~W}$ $45.283 \mathrm{~N} .112 .645 \mathrm{~W}$. $44.790 \mathrm{~N} .111 .593 \mathrm{~W}$. $44.907 \mathrm{~N} .112 .543 \mathrm{~W}$.

$44.715 \mathrm{~N} .112 .051 \mathrm{~W}$. $44.658 \mathrm{~N} .111 .858 \mathrm{~W}$. $44.807 \mathrm{~N} .112 .904 \mathrm{~W}$. $44.769 \mathrm{~N} .112 .880 \mathrm{~W}$. $44.952 \mathrm{~N} .112 .767 \mathrm{~W}$.

$44.940 \mathrm{~N} .112 .762 \mathrm{~W}$. $44.780 \mathrm{~N} .111 .620 \mathrm{~W}$. $45.800 \mathrm{~N} .111 .796 \mathrm{~W}$. $44.826 \mathrm{~N}, 112.748 \mathrm{~W}$.

$\begin{array}{rr}1 & \text { B } \\ 5 & \text { CS } \\ 19 & \text { BU } \\ 7 & \text { BU } \\ 8 & \text { BU } \\ 2 & \\ 2 & \\ 10 & \\ 8 & \\ 3 & \\ 11 & \end{array}$

BU $\quad \ldots \quad \ldots 2.5 \mathrm{ML}(\mathrm{BU})$

$\ldots 3.2 \mathrm{ML}(\mathrm{BU})$

$\ldots \quad \ldots 2.7 M L(B U)$

$\ldots \ldots 2.5 M L(B U)$

$\ldots \quad \ldots 2.5 M L(B U)$

$\ldots \quad \ldots 2.5 \mathrm{ML}(\mathrm{BU})$

$\ldots \ldots 2.7 M L(B U)$

$\ldots \quad \ldots .9 M L(B U)$

$\ldots \ldots 2.8 M L(B U)$

$\ldots 3.7 \mathrm{ML}(\mathrm{BU})$

$\begin{array}{lll}\ldots & \ldots & 3.2 \mathrm{ML}(\mathrm{BU}) \\ \ldots & \ldots & 2.9 \mathrm{ML}(\mathrm{BU})\end{array}$

$\begin{array}{lll}\ldots & \ldots & 2.9 \mathrm{ML}(\mathrm{BU}) \\ \ldots & \ldots & 3.1 \mathrm{ML}(\mathrm{BU})\end{array}$

$\begin{array}{lll}\ldots & \ldots & 3.1 \mathrm{ML}(\mathrm{BU}) \\ \ldots & \ldots & 3.1 \mathrm{ML}(\mathrm{BU})\end{array}$

$\ldots \quad \ldots 3$ 3.OML(BU)

$\ldots \quad \ldots 2.6 \mathrm{ML}(\mathrm{BU})$

$\ldots \quad \ldots .72 .7 M L(B U)$

$\ldots \ldots$ 2.9ML(BU)

$\ldots 1.3 .3 M L(B U)$

$\ldots \ldots$ 2.7ML(BU)

$\ldots \quad \ldots 2.7 M L(B U)$

$\ldots \quad \ldots 3.2 \mathrm{ML}(\mathrm{BU})$

$\ldots \quad \ldots 3.1 \mathrm{ML}(\mathrm{BU})$

$\ldots \ldots 2.9 M D(B U)$

$\ldots \quad \ldots 3.1 \mathrm{ML}(\mathrm{BU})$

.. $\quad \ldots \quad 3.0 M L(B U)$

$\ldots \ldots 3.9 M L(B U)$

$\ldots$ 3.3ML(BU)

$\ldots$... $\ldots 3$ 3.2ML(BU)

$\ldots \ldots$ 2.8ML(BU)

$\begin{array}{lll}\ldots & \ldots & 2.7 \mathrm{ML}(\mathrm{BU}) \\ \ldots & \ldots & 2.8 \mathrm{ML}(\mathrm{BU})\end{array}$

$\ldots \quad \ldots 2$ 2.6ML(BU)

$\ldots \ldots 3.1 \mathrm{ML}(\mathrm{BU})$

... $\ldots 2.8 \mathrm{ML}(\mathrm{BU})$

$\begin{array}{lll}\ldots & \ldots & 3.0 \mathrm{OL}(\mathrm{BU}) \\ \ldots & \ldots & 2.9 \mathrm{ML}(\mathrm{BU})\end{array}$

2. $6 \mathrm{ML}(\mathrm{BU})$

3. $1 \mathrm{ML}(\mathrm{BU})$

3. $O M L(B U)$

3. $0 \mathrm{ML}(\mathrm{BU})$

2. $8 \mathrm{ML}(\mathrm{BU})$

3. $5 \mathrm{ML}$ (GS)

3. $3 \mathrm{ML}(\mathrm{BU})$

$\begin{array}{rl}14 & B \\ 16 & B \\ 5 & \text { G } \\ 8 & B\end{array}$

$\begin{array}{lll}\ldots & \text { AUG. } 27 & 09: 04 \\ \text { MST }\end{array}$ ... SEPT. 3 05:28 MST ... SEPT. 3 13:35 MST ... SEPT. 6 11:50 MST ... SEPT. 9 12:56 MST

... SEPT. 13 10:54 MST $\ldots$ SEPT. 15 18:28 MST

... SEPT. 19 19:32 MST

... SEPT. 20 10:18 MST

$\ldots$ SEPT. 20 10:19 MST

... SEPT. $20 \quad 13: 28$ MST ... SEPT. 20 21:01 MST $\ldots$ SEPT. 21 12:11 MST ... SEPT. 21 12:29 MST ... SEPT. 22 19:44 MST

... SEPT. 24 10:09 MST

... SEPT. 26 12:10 MST

... OCT. 2 22:45 MST

... OCT. 3 13:38 MST

... OCT. 5 10:57 MST

$\begin{array}{llll}\ldots & \text { OCT. } 7 & 19: 40 \text { MST }\end{array}$

$\begin{array}{llll}\ldots & \text { OCT. } & 11 & 07: 25 \text { MST }\end{array}$

... OCT. 17 18:12 MST

... OCT. 20 13:13 MST

$\begin{array}{lll}\ldots & \text { OCT. } 25 \text { 15:57 MST }\end{array}$

$\begin{array}{llll}\text {.. OCT. } 26 & 15: 51 & \text { MST }\end{array}$

$\begin{array}{llll}\ldots & \text { OCT. } 30 & 14: 37 \text { MST }\end{array}$

... OCT. 31 06:36 MST

$\begin{array}{llll}\ldots & \text { OCT. } 31 & 22: 07 & \text { MST }\end{array}$

... NOV. 6 14:03 MST

$\begin{array}{lll}\text { NOV. } 6 & 15: 25 \text { MST }\end{array}$

... NOV. 7 10:09 MST

... NOV. 8 02:02 MST

$\begin{array}{lll}\ldots \text { NOV. } 11 & 09: 32 & \text { MST }\end{array}$

$\begin{array}{lll}\ldots & \text { NOV. } 17 \text { 07:59 MST }\end{array}$

.. NOV. $19 \quad 21: 49$ MST

... NOV. 20 02:54 MST

... NOV. $20 \quad 11: 46$ MST

.. DEC. 3 19:39 MST

$\begin{array}{lll}\ldots & \text { DEC. } 7 & 14: 58 \text { MST }\end{array}$

DEC. $8 \quad 02: 39$ MST

DEC. 9 20:38 MST

DEC. 19 17:43 MST

DEC. 22 00:17 MST

\section{NEVADA}

\begin{tabular}{|c|c|c|c|c|c|c|c|c|c|c|c|c|c|c|c|c|}
\hline $\begin{array}{l}\text { JAN. } \\
\text { JAN. } \\
\text { JAN. }\end{array}$ & $\begin{array}{r}2 \\
3 \\
26\end{array}$ & $\begin{array}{l}89 \\
09 \\
22\end{array}$ & $\begin{array}{l}29 \\
01 \\
27\end{array}$ & $\begin{array}{l}41.4 \\
01.1 \\
56.8\end{array}$ & $\begin{array}{l}37.993 \mathrm{~N} \\
38.809 \mathrm{~N} . \\
38.217 \mathrm{~N} .\end{array}$ & $\begin{array}{l}118.131 W . \\
118.843 W . \\
117.879 w\end{array}$ & $\begin{array}{l}1 \\
8 \\
4\end{array}$ & $\begin{array}{l}\text { RN } \\
\text { RN } \\
\text { RN }\end{array}$ & $\begin{array}{l}\cdots \\
\cdots \\
\cdots\end{array}$ & $\begin{array}{l}\cdots \\
\cdots \\
\cdots\end{array}$ & $\begin{array}{l}\text { 3. } 6 \mathrm{ML}(\mathrm{RN}) \\
\text { 2. } 6 \mathrm{ML}(\mathrm{RN}) \\
\text { 3. } \mathrm{ML}(\mathrm{RN})\end{array}$ & $\begin{array}{l}\cdots \\
\cdots\end{array}$ & $\begin{array}{l}\text { JAN. } \\
\text { JAN. } \\
\text { JAN. }\end{array}$ & $\begin{array}{r}2 \\
3 \\
26\end{array}$ & $\begin{array}{l}01: 29 \\
01: 01 \\
14: 27\end{array}$ & $\begin{array}{l}\text { PST } \\
\text { PST } \\
\text { PST }\end{array}$ \\
\hline
\end{tabular}


Table 1. Summary of U.S. earthquakes for 1984-Continued

\begin{tabular}{|c|c|c|c|c|c|c|c|c|c|c|c|c|c|}
\hline \multirow{2}{*}{ Date } & \multicolumn{2}{|c|}{$\begin{array}{l}\text { Origin time } \\
\text { (UTC) }\end{array}$} & \multirow{2}{*}{$\begin{array}{c}\text { Latitude } \\
\text { ( })\end{array}$} & \multirow{2}{*}{$\begin{array}{c}\text { Longitude } \\
\text { () }\end{array}$} & \multirow{2}{*}{$\begin{array}{l}\text { Depth } \\
(\mathbf{k m})\end{array}$} & \multirow{2}{*}{$\begin{array}{l}\text { Hypo- } \\
\text { center } \\
\text { source }\end{array}$} & \multicolumn{3}{|c|}{ Magnitude } & \multirow{2}{*}{$\begin{array}{l}\text { Maximum } \\
\text { intensity }\end{array}$} & \multicolumn{3}{|c|}{ Lecal time } \\
\hline & hr $\min$ & sec & & & & & $\mathbf{m b}$ & MS & $\begin{array}{l}\text { ML, Mn } \\
\text { MD. Mw }\end{array}$ & & Date & Hour & $\begin{array}{l}\text { Time } \\
\text { rome }\end{array}$ \\
\hline
\end{tabular}

NEVADA-Continued

JAN. $31 \quad 15 \quad 3000.0 \quad 37.113 \mathrm{~N}, 116.122 \mathrm{~W}$

FEB. $15 \quad 170000.1 \quad 37.221 \mathrm{~N} .116 .181 \mathrm{~W}$.

$\begin{array}{lllll}\text { FEB. } & 16 & 11 & 14 & 57.5\end{array}$

FEB. $\quad \begin{array}{lllll}16 & 11 & 27 & 41.5\end{array}$

FEB. $\quad \begin{array}{lllll}17 & 12 & 03 & 56.2\end{array}$

FEB. $17 \quad 12 \quad 16 \quad 46.6$

FEB. $19 \quad 10 \quad 22 \quad 41.2$

FEB. $\quad 25 \quad 07 \quad 04 \quad 44.3$

MAR. 1174500.0

MAR. $\quad 7 \quad 2204 \quad 43.1$

MAR. $\quad 9 \quad 17 \quad 18 \quad 30.0$

MAR. $10 \quad 12 \quad 46 \quad 52.4$

MAR. $\quad 18 \quad 09 \quad 4459.8$

MAR. $31 \quad 143000.0$

APR. $\quad 17 \quad 11 \quad 18 \quad 52.0$

$\begin{array}{lllll}\text { APR. } \quad 27 \quad 00 & 31 & 34.2\end{array}$

APR. $\quad 28 \quad 17 \quad 36 \quad 06.0$

APR. $\quad \begin{array}{lllll}30 & 13 & 44 & 05.2\end{array}$

MAY 1190500.0

$\begin{array}{lllll}\text { MAY } & 1 & 20 & 06 & 48.0\end{array}$

$\begin{array}{lllll}\text { MAY } & 2 & 13 & 49 & 59.6\end{array}$

$\begin{array}{lllll}\text { MAY } & 10 & 16 & 52 & 45.7\end{array}$

MAY $\quad 16 \quad 15 \quad 59 \quad 59.3$

MAY $17 \quad 20 \quad 23 \quad 25.0$

$\begin{array}{lllll}\text { MAY } & 17 & 20 & 31 & 33.7\end{array}$

$\begin{array}{lllll}\text { MAY } & 31 & 13 & 04 & 00.1\end{array}$

JUNE $17 \quad 20 \quad 23 \quad 24.0$

JUNE $20 \quad 15 \quad 1500.0$

$\begin{array}{lllll}\text { JUNE } & 20 & 16 & 10 & 00.4\end{array}$

JULY $10 \quad 17 \quad 40 \quad 21.6$

JULY $10 \quad 17 \quad 40 \quad 22.9$

JULY $11 \quad 22 \quad 32 \quad 43.1$

$\begin{array}{lllll}\text { JULY } & 11 & 22 & 32 & 47.7\end{array}$

$\begin{array}{lllll}\text { JULY } & 11 & 23 & 19 & 21.4\end{array}$

JULY $12 \quad 13 \quad 5959.9$

JULY $25 \quad 1530 \quad 00.0$

AUG. $\quad 2 \quad 1101 \quad 34.9$

AUG. $2 \quad 1500 \quad 00.0$

AUG. $12 \quad 05 \quad 44 \quad 07.6$

AUG. $13 \quad 11 \quad 30 \quad 55.5$

AUG. $\quad \begin{array}{llll}16 & 09 & 32 & 34.4\end{array}$

AUG. $\quad \begin{array}{lllll}18 & 11 & 05 & 36.4\end{array}$

AUG. $\quad \begin{array}{llll}19 & 02 & 22 & 34.2\end{array}$

AUG. $\quad \begin{array}{lllll}19 & 12 & 25 & 36.8\end{array}$

AUG. $\quad \begin{array}{llll}20 & 00 & 49 & 32.7\end{array}$

AUG. $28 \quad 02 \quad 33 \quad 43.6$

AUG. $28 \quad 03 \quad 11 \quad 59.5$

AUG. $28 \quad 2006 \quad 05.0$

AUG. $30 \quad 14 \quad 4500.1$
$39.933 \mathrm{~N}, 117.759 \mathrm{~W}$.

$39.835 \mathrm{~N} .117 .548 \mathrm{~W}$.

$38.902 \mathrm{~N} .119 .608 \mathrm{~W}$.

$39.976 \mathrm{~N} .117 .825 \mathrm{~W}$.

$41.753 \mathrm{~N} .114 .882 \mathrm{~W}$.

$37.066 \mathrm{~N} .116 .046 \mathrm{~W}$.

$39.934 \mathrm{~N} .117 .760 \mathrm{~W}$.

$37.278 \mathrm{~N} .116 .330 \mathrm{~W}$.

$38.819 \mathrm{~N} .118 .007 \mathrm{~W}$.

$39.480 \mathrm{~N} .115 .220 \mathrm{~W}$.

$37.146 \mathrm{~N} .116 .084 \mathrm{~W}$.

$38.037 \mathrm{~N} .118 .712 \mathrm{~W}$.

$38.047 \mathrm{~N} .118 .713 W$.

$38.304 \mathrm{~N} .118 .702 \mathrm{~W}$.

$38.303 \mathrm{~N} .118 .696 \mathrm{~W}$.

$37.106 \mathrm{~N} .116 .022 \mathrm{~W}$.

$37.085 \mathrm{~N}, 115.966 \mathrm{~W}$.

$37.189 \mathrm{~N} .116 .016 \mathrm{~W}$.

$37.857 \mathrm{~N} .118 .144 \mathrm{~W}$.

$37.091 \mathrm{~N} .115 .994 \mathrm{~W}$.

$38.224 \mathrm{~N} .117 .876 \mathrm{~W}$.

$38.225 \mathrm{~N} .117 .875 \mathrm{~W}$.

$37.103 \mathrm{~N} .116 .048 \mathrm{~W}$.

$38.247 \mathrm{~N} .117 .851 \mathrm{~W}$.

$37.000 \mathrm{~N} .116 .043 \mathrm{~W}$.

$37.002 \mathrm{~N} .116 .031 \mathrm{~W}$.

$40.445 \mathrm{~N} .119 .882 \mathrm{~W}$.

$40.363 \mathrm{~N} .119 .895 \mathrm{~W}$.

$38.220 \mathrm{~N}, 117.868 \mathrm{~W}$.

$38.000 \mathrm{~N} .118 .108 \mathrm{~W}$.

$38.225 \mathrm{~N} .117 .836 \mathrm{~W}$.

$37.186 \mathrm{~N} .116 .012 \mathrm{~W}$.

$37.268 \mathrm{~N} .116 .411 \mathrm{~W}$.

$37.295 \mathrm{~N}$. 114.936W.

$37.017 \mathrm{~N} .116 .008 \mathrm{~W}$.

$39.535 \mathrm{~N} .119 .163 \mathrm{~W}$.

$38.220 \mathrm{~N}$. $117.868 \mathrm{~W}$.

$39.473 \mathrm{~N} .119 .395 \mathrm{~W}$.

$37.947 \mathrm{~N} .118 .220 \mathrm{~W}$.

$38.356 \mathrm{~N}, 118.062 \mathrm{~W}$.

$38.354 \mathrm{~N} .118 .124 \mathrm{~W}$.

$38.185 \mathrm{~N} .116 .767 \mathrm{~W}$.

$37.884 \mathrm{~N} .118 .074 \mathrm{~W}$.

$38.386 \mathrm{~N} .118 .739 \mathrm{~W}$.

$37.883 \mathrm{~N}, 118.076 \mathrm{~W}$.

$37.090 \mathrm{~N} .115 .998 \mathrm{~W}$.
$38.880 \mathrm{~N} .119 .637 \mathrm{~W}$

\begin{tabular}{|c|c|c|c|c|c|c|c|c|c|}
\hline $\begin{array}{l}0 \\
0\end{array}$ & $\begin{array}{l}\text { EN } \\
\text { EN }\end{array}$ & $\begin{array}{l}4.1 \\
5.0\end{array}$ & $\cdots$ & $\begin{array}{l}\text { 4. } 4 \mathrm{ML}(\mathrm{BK}) \\
\text { 4.8ML(BK) }\end{array}$ & $\cdots$ & $\begin{array}{l}\text { JAN. } \\
\text { FEB. }\end{array}$ & $\begin{array}{l}31 \\
15\end{array}$ & $\begin{array}{l}07: 30 \\
09: 00\end{array}$ & $\begin{array}{l}\text { PST } \\
\text { PST }\end{array}$ \\
\hline $\begin{array}{r}8 \\
0 \\
25 \\
25 \\
10\end{array}$ & $\begin{array}{l}\text { GS } \\
\text { RN } \\
\text { BK } \\
\text { BK } \\
\text { GS }\end{array}$ & $\begin{array}{l}4.8 \\
\ldots \\
4.1 \\
\ldots \\
\ldots\end{array}$ & $\begin{array}{l}\ldots \\
\ldots \\
\ldots \\
\ldots \\
\ldots\end{array}$ & $\begin{array}{l}\text { 5. } 2 \mathrm{ML}(\mathrm{BK}) \\
\text { 2. } 7 \mathrm{ML}(\mathrm{RN}) \\
\text { 4. } 3 \mathrm{ML}(\mathrm{BK}) \\
\text { 3. } 6 \mathrm{ML}(\mathrm{BK}) \\
\text { 4. } \mathrm{OML}(\mathrm{GS})\end{array}$ & $\begin{array}{r}\text { IV } \\
\cdots \\
\text { FELT } \\
\text { IV }\end{array}$ & $\begin{array}{l}\text { FEB. } \\
\text { FEB. } \\
\text { FEB. } \\
\text { FEB. } \\
\text { FEB. }\end{array}$ & $\begin{array}{l}16 \\
16 \\
17 \\
17 \\
19\end{array}$ & $\begin{array}{l}03: 14 \\
03: 27 \\
04: 03 \\
04: 16 \\
02: 22\end{array}$ & $\begin{array}{l}\text { PST } \\
\text { PST } \\
\text { PST } \\
\text { PST } \\
\text { PST }\end{array}$ \\
\hline $\begin{array}{r}5 \\
0 \\
7 \\
7 \\
13\end{array}$ & $\begin{array}{l}\text { GS } \\
\text { EN } \\
\text { RN } \\
\text { GS } \\
\text { RN }\end{array}$ & $\begin{array}{l}5.9 \\
\ldots \\
\ldots \\
\ldots\end{array}$ & $\begin{array}{l}4.4 \\
\ldots \\
\ldots \\
\ldots\end{array}$ & $\begin{array}{l}\text { 4. } 9 \mathrm{ML}(\mathrm{GS}) \\
5.5 \mathrm{ML}(\mathrm{BK}) \\
2.7 \mathrm{ML}(\mathrm{RN}) \\
3.2 \mathrm{ML}(\mathrm{GS}) \\
2.6 \mathrm{ML}(\mathrm{RN})\end{array}$ & $\begin{array}{l}\cdots \\
\cdots \\
\cdots \\
\dot{F E L T}\end{array}$ & $\begin{array}{l}\text { FEB. } \\
\text { MAR. } \\
\text { MAR. } \\
\text { MAR. } \\
\text { MAR. }\end{array}$ & $\begin{array}{r}24 \\
1 \\
7 \\
9 \\
10\end{array}$ & $\begin{array}{l}23: 04 \\
09: 45 \\
14: 04 \\
09: 18 \\
04: 46\end{array}$ & $\begin{array}{l}\text { PST } \\
\text { PST } \\
\text { PST } \\
\text { PST } \\
\text { PST }\end{array}$ \\
\hline $\begin{array}{l}2 \\
0 \\
0 \\
0 \\
6\end{array}$ & $\begin{array}{l}\text { UU } \\
\text { EN } \\
\text { RN } \\
\text { RN } \\
\text { RN }\end{array}$ & $\begin{array}{l}\ddot{4} \dot{1} \\
\ldots \\
\cdots \\
\cdots\end{array}$ & $\begin{array}{l}\cdots \\
\ldots \\
\ldots \\
\ldots \\
\cdots\end{array}$ & $\begin{array}{l}\text { 3. } 2 M D(U U) \\
4.2 M L(B K) \\
2.5 M L(R N) \\
2.7 M L(R N) \\
2.7 M L(R N)\end{array}$ & $\begin{array}{l}\ldots \\
\cdots \\
\cdots \\
\ldots\end{array}$ & $\begin{array}{l}\text { MAR. } \\
\text { MAR. } \\
\text { APR. } \\
\text { APR. } \\
\text { APR. }\end{array}$ & $\begin{array}{l}18 \\
31 \\
17 \\
26 \\
28\end{array}$ & $\begin{array}{l}01: 44 \\
06: 30 \\
03: 18 \\
16: 31 \\
09: 36\end{array}$ & $\begin{array}{l}\text { MST } \\
\text { PST } \\
\text { PST } \\
\text { PST } \\
\text { PST }\end{array}$ \\
\hline $\begin{array}{l}1 \\
0 \\
0 \\
5 \\
9\end{array}$ & $\begin{array}{l}\text { RN } \\
\text { EN } \\
\text { GS } \\
\text { GS } \\
\text { RN }\end{array}$ & $\begin{array}{l}5.3 \\
4.2 \\
\ldots \\
\ldots\end{array}$ & $\begin{array}{l}\ddot{4} \\
\ldots \\
\ldots \\
\cdots\end{array}$ & $\begin{array}{c}2.9 M L(R N) \\
5.3 M L(B K) \\
\ldots \ldots \\
3.3 M L(B K) \\
2.7 M L(R N)\end{array}$ & $\begin{array}{l}\ldots \\
\ldots \\
\cdots \\
\cdots\end{array}$ & $\begin{array}{l}\text { APR. } \\
\text { MAY } \\
\text { MAY } \\
\text { MAY } \\
\text { MAY }\end{array}$ & $\begin{array}{r}30 \\
1 \\
1 \\
2 \\
10\end{array}$ & $\begin{array}{l}05: 44 \\
11: 05 \\
12: 06 \\
05: 49 \\
08: 52\end{array}$ & $\begin{array}{l}\text { PST } \\
\text { PST } \\
\text { PST } \\
\text { PST } \\
\text { PST }\end{array}$ \\
\hline $\begin{array}{l}5 \\
6 \\
1 \\
0 \\
6\end{array}$ & $\begin{array}{l}\text { GS } \\
\text { RN } \\
\text { RN } \\
\text { EN } \\
\text { GS }\end{array}$ & $\begin{array}{l}\cdots \\
\cdots \\
5.8 \\
\ldots\end{array}$ & $\begin{array}{l}\ldots \\
\ldots \\
\cdots \\
4.1 \\
\ldots\end{array}$ & $\begin{array}{l}\text { 3. } 5 \mathrm{ML}(\mathrm{BK}) \\
\text { 4.1ML(RN) } \\
2.9 \mathrm{ML}(\mathrm{RN}) \\
5.6 \mathrm{ML}(\mathrm{BK}) \\
\text { 3. } \mathrm{ML}(\mathrm{BK})\end{array}$ & $\begin{array}{l}\ldots \\
\ldots \\
\cdots \\
\cdots\end{array}$ & $\begin{array}{l}\text { MAY } \\
\text { MAY } \\
\text { MAY } \\
\text { MAY } \\
\text { JUNE }\end{array}$ & $\begin{array}{l}16 \\
17 \\
17 \\
31 \\
17\end{array}$ & $\begin{array}{l}07: 59 \\
12: 23 \\
12: 31 \\
05: 04 \\
12: 23\end{array}$ & $\begin{array}{l}\text { PST } \\
\text { PST } \\
\text { PST } \\
\text { PST } \\
\text { PST }\end{array}$ \\
\hline $\begin{array}{l}0 \\
0 \\
5 \\
0 \\
6\end{array}$ & $\begin{array}{l}\text { EN } \\
\text { GS } \\
\text { GS } \\
\text { RN } \\
\text { RN }\end{array}$ & $\begin{array}{l}4.6 \\
4.2 \\
\ldots \\
\ldots \\
\ldots\end{array}$ & $\begin{array}{l}\ldots \\
\ldots \\
\ldots \\
\ldots \\
\ldots\end{array}$ & $\begin{array}{l}\text { 4. } 5 \mathrm{ML}(\mathrm{BK}) \\
\text { 4.1ML(BK) } \\
\text { 3. } 8 \mathrm{ML}(\mathrm{BK}) \\
2.5 \mathrm{ML}(\mathrm{RN}) \\
\text { 3. } \mathrm{ML}(\mathrm{RN})\end{array}$ & $\begin{array}{l}\ldots \\
\ldots \\
\ldots \\
\cdots\end{array}$ & $\begin{array}{l}\text { JUNE } \\
\text { JUNE } \\
\text { JULY } \\
\text { JULY } \\
\text { JULY }\end{array}$ & $\begin{array}{l}20 \\
20 \\
10 \\
10 \\
11\end{array}$ & $\begin{array}{l}07: 15 \\
08: 10 \\
09: 40 \\
09: 40 \\
14: 32\end{array}$ & $\begin{array}{l}\text { PST } \\
\text { PST } \\
\text { PST } \\
\text { PST } \\
\text { PST }\end{array}$ \\
\hline $\begin{array}{l}6 \\
0 \\
5 \\
0 \\
5\end{array}$ & $\begin{array}{l}\text { GM } \\
\text { RN } \\
\text { GS } \\
\text { EN } \\
\text { GS }\end{array}$ & $\begin{array}{l}\ldots \\
3.6 \\
5.3 \\
\ldots\end{array}$ & $\begin{array}{l}\cdots \\
\cdots \\
\cdots \\
\cdots \\
\cdots\end{array}$ & $\begin{array}{l}\text { 3. } 0 \mathrm{MD}(\mathrm{GM}) \\
\text { 2.8ML(RN) } \\
\text { 3.8ML(BK) } \\
5.2 \mathrm{ML}(\mathrm{BK}) \\
\text { 3.7ML(GS) }\end{array}$ & $\begin{array}{l}\cdots \\
\cdots \\
\ldots \\
\ldots\end{array}$ & $\begin{array}{l}\text { JULY } \\
\text { JULY } \\
\text { JULY } \\
\text { JULY } \\
\text { AUG. }\end{array}$ & $\begin{array}{r}11 \\
11 \\
12 \\
25 \\
2\end{array}$ & $\begin{array}{l}14: 32 \\
15: 19 \\
05: 59 \\
07: 30 \\
03: 01\end{array}$ & $\begin{array}{l}\text { PST } \\
\text { PST } \\
\text { PST } \\
\text { PST } \\
\text { PST }\end{array}$ \\
\hline $\begin{array}{r}0 \\
18 \\
2 \\
16 \\
3\end{array}$ & $\begin{array}{l}\text { EN } \\
\text { RN } \\
\text { RN } \\
\text { RN } \\
\text { RN }\end{array}$ & $\begin{array}{l}4.7 \\
\ldots \\
\cdots \\
\ldots \\
\ldots\end{array}$ & $\dot{.}$. & $\begin{array}{l}\text { 4. } 4 \mathrm{ML}(\mathrm{BK}) \\
2.8 \mathrm{ML}(\mathrm{RN}) \\
2.6 \mathrm{ML}(\mathrm{RN}) \\
3.5 \mathrm{ML}(\mathrm{RN}) \\
2.6 \mathrm{ML}(\mathrm{RN})\end{array}$ & $\begin{array}{l}\cdots \\
\cdots \\
\cdots \\
\cdots\end{array}$ & $\begin{array}{l}\text { AUG. } \\
\text { AUG. } \\
\text { AUG. } \\
\text { AUG. } \\
\text { AUG. }\end{array}$ & $\begin{array}{r}2 \\
11 \\
13 \\
16 \\
18\end{array}$ & $\begin{array}{l}07: 00 \\
21: 44 \\
03: 30 \\
01: 32 \\
03: 05\end{array}$ & $\begin{array}{l}\text { PST } \\
\text { PST } \\
\text { PST } \\
\text { PST } \\
\text { PST }\end{array}$ \\
\hline $\begin{array}{l}5 \\
8 \\
3 \\
4 \\
0\end{array}$ & $\begin{array}{l}\text { GS } \\
\text { RN } \\
\text { RN } \\
\text { RN } \\
\text { RN }\end{array}$ & $\begin{array}{l}3.8 \\
\ldots \\
\ldots \\
\ldots \\
\ldots\end{array}$ & . & $\begin{array}{l}\text { 4. } 1 \mathrm{ML}(\mathrm{BK}) \\
2.6 \mathrm{ML}(\mathrm{RN}) \\
2.9 \mathrm{ML}(\mathrm{RN}) \\
2.9 \mathrm{ML}(\mathrm{RN}) \\
2.5 \mathrm{ML}(\mathrm{RN})\end{array}$ & $\begin{array}{l}\quad v \\
\cdots \\
\cdots \\
\cdots \\
\cdots\end{array}$ & $\begin{array}{l}A \cup G . \\
A \cup G . \\
A \cup G . \\
A \cup G . \\
A \cup G .\end{array}$ & $\begin{array}{l}18 \\
19 \\
19 \\
27 \\
27\end{array}$ & $\begin{array}{l}18: 22 \\
04: 25 \\
16: 49 \\
18: 33 \\
19: 11\end{array}$ & $\begin{array}{l}\text { PST } \\
\text { PST } \\
\text { PST } \\
\text { PST } \\
\text { PST }\end{array}$ \\
\hline $\begin{array}{l}4 \\
0\end{array}$ & $\begin{array}{l}\text { RN } \\
\text { EN }\end{array}$ & $\ddot{4} .5$ & & $\begin{array}{l}\text { 2. } 8 \mathrm{ML}(\mathrm{RN}) \\
\text { 4. } 3 \mathrm{ML}(\mathrm{BK})\end{array}$ & $\cdots$ & $\begin{array}{l}\text { AUG. } \\
A \cup G \text {. }\end{array}$ & $\begin{array}{l}28 \\
30\end{array}$ & $\begin{array}{l}12: 06 \\
06: 45\end{array}$ & $\begin{array}{l}\text { PST } \\
\text { PST }\end{array}$ \\
\hline
\end{tabular}


Table 1. Summary of U.S. earthquakes for 1984 Continued

\begin{tabular}{|c|c|c|c|c|c|c|c|c|c|c|c|}
\hline \multirow{2}{*}{ Date } & $\begin{array}{c}\begin{array}{c}\text { Origin time } \\
\text { (UTC) }\end{array} \\
\end{array}$ & \multirow{2}{*}{$\begin{array}{c}\text { Latitude } \\
\left({ }^{\circ}\right)\end{array}$} & \multirow{2}{*}{$\begin{array}{c}\text { Longitude } \\
\left({ }^{\circ}\right)\end{array}$} & \multirow{2}{*}{$\begin{array}{c}\text { Depth } \\
(\mathbf{k m})\end{array}$} & \multirow{2}{*}{$\begin{array}{l}\text { Hypo- } \\
\text { center } \\
\text { source }\end{array}$} & \multicolumn{3}{|c|}{ Magnitude } & \multirow{2}{*}{$\begin{array}{l}\text { Maximum } \\
\text { intensity }\end{array}$} & \multicolumn{2}{|c|}{ Lecal time } \\
\hline & hr $\min \sec$ & & & & & $\mathbf{m b}$ & MS & $\begin{array}{l}\text { ML. Mn } \\
M D, M w\end{array}$ & & Date & Hour \\
\hline
\end{tabular}

NEVADA-Continued

SEPT. $\quad \begin{array}{lllll}1 & 11 & 45 & 03.9\end{array}$ $\begin{array}{lllll}\text { SEPT. } & 1 & 16 & 45 & 34.4\end{array}$ $\begin{array}{lllll}\text { SEPT. } 3 & 00 & 23 & 29.1\end{array}$

SEPT. $\quad 3 \quad 00 \quad 31 \quad 12.7$ $\begin{array}{lllll}\text { SEPT. } & 4 & 04 & 08 & 57.2\end{array}$ SEPT. $5 \quad 03 \quad 3032.5$ $\begin{array}{llllll} & \text { SEPT. } & 6 & 13 & 49 & 56.5\end{array}$ SEPT. $12 \quad 12 \quad 53 \quad 43.1$

$\begin{array}{lllll}\text { SEPT. } 13 & 10 & 37 & 29.8\end{array}$ SEPT. 13 SEPT. 13 SEPT. 16 SEPT . 17

SEPT. 26 SEPT. 26 SEPT. 26 SEPT. 27 SEPT. 27

SEPT. 29 SEPT. 29 SEPT. 30

OCT. 2

OCT. 3

$\begin{array}{lll}14 & 00 & 00.0\end{array}$ $\begin{array}{lll}21 & 01 & 36.7\end{array}$ $06 \quad 49 \quad 35.0$ $\begin{array}{lll}19 & 11 & 07.8\end{array}$

202024.4 $\begin{array}{llll}22 & 05 & 19.4\end{array}$ $\begin{array}{lll}22 & 06 & 12.1\end{array}$ $\begin{array}{lll}01 & 45 & 04.3\end{array}$ $\begin{array}{lll}13 & 17 & 20.8\end{array}$

$\begin{array}{lll}17 & 12 & 59.9\end{array}$ $\begin{array}{lll}17 & 14 & 48.4\end{array}$ $\begin{array}{llll}19 & 03 & 10.4\end{array}$ $\begin{array}{lll}18 & 13 & 59.3\end{array}$ $\begin{array}{lll}09 & 02 & 28.9\end{array}$

OCT. 19

OCT. 21

$\begin{array}{lll}22 & 12 & 24.6\end{array}$

OCT. $21 \quad 145145.6$

$\begin{array}{lllll}\text { OCT. } & 21 & 14 & 55 & 11.8\end{array}$

OCT. 22

011023.4

OCT. 22

OCT. 23

OCT. 27

NOV. 5

$\begin{array}{lll}11 & 27 & 40.7\end{array}$

$\begin{array}{lll}18 & 44 & 47.3\end{array}$

$\begin{array}{lll}18 & 22 & 07.2\end{array}$

$\begin{array}{lll}11 & 21 & 15.1\end{array}$

NOV. $\begin{array}{rrrr}10 & 16 \quad 40 & 00.0\end{array}$

NOV. $\begin{array}{lllll}11 & 06 & 03 & 28.2\end{array}$

NOV. $18 \quad 08 \quad 3505.3$

$\begin{array}{lllll}\text { DEC } & 10 & 00 & 29 & 56.9\end{array}$

DEC. $\quad 4 \quad 2053 \quad 06.3$

DEC. $\quad 5 \quad 10 \quad 14 \quad 44.2$

DEC. $\quad 5 \quad 1456 \quad 56.3$

DEC. $9 \quad 0234 \quad 35.5$

DEC. $\quad \begin{array}{lllll}9 & 09 & 28 & 29.6\end{array}$

DEC. $\quad 9 \quad 18 \quad 48 \quad 59.0$

DEC. $9 \quad 1940 \quad 00.0$

DEC. $\quad \begin{array}{rrrrr}9 & 21 & 48 & 58.6\end{array}$

DEC. $10 \quad 00 \quad 2954.7$

DEC. 15144500.0

DEC. $\quad 21 \quad 21 \quad 23 \quad 41.1$

DEC. $23 \quad 000029.0$

DEC. 23
$39.629 \mathrm{~N} .118 .193 \mathrm{~W}$. $37.836 \mathrm{~N} .118 .176 \mathrm{~W}$

$37.885 \mathrm{~N} .118 .075 \mathrm{~W}$.

$37.886 \mathrm{~N}, 118.072 \mathrm{~W}$. $39.396 \mathrm{~N}, 117.665 \mathrm{~W}$

$39.071 \mathrm{~N} .115 .646 \mathrm{~W}$.

$37.883 \mathrm{~N} .118 .879 \mathrm{~W}$

$38.038 \mathrm{~N} .118 .715 \mathrm{~W}$.

38.479N. 118.353W. $37.087 \mathrm{~N} .116 .071 \mathrm{~W}$ $37.073 \mathrm{~N} .116 .097 \mathrm{~W}$. $39.098 \mathrm{~N} .115 .602 \mathrm{~W}$. $39.077 \mathrm{~N} .115 .616 \mathrm{~W}$

$38.028 \mathrm{~N} .118 .671 W$. $38.028 \mathrm{~N} .118 .671 W$. $38.028 \mathrm{~N} .118 .667 \mathrm{~W}$. $38.043 \mathrm{~N} .118 .575 W$. $38.218 \mathrm{~N} .117 .875 \mathrm{~W}$.

$37.836 \mathrm{~N} .118 .146 \mathrm{~W}$. $37.835 \mathrm{~N} .118 .146 \mathrm{~W}$ $37.910 \mathrm{~N} .118 .479 \mathrm{~W}$. $37.076 \mathrm{~N} .115 .989 \mathrm{~W}$. $38.222 \mathrm{~N} .117 .869 \mathrm{~W}$.

$38.864 \mathrm{~N}, 117.875 \mathrm{~W}$. $38.810 \mathrm{~N} .119 .297 \mathrm{~W}$. $38.454 \mathrm{~N} .118 .280 \mathrm{~W}$. $38.830 \mathrm{~N}, 119.317 \mathrm{~W}$. $40.005 \mathrm{~N} .119 .326 \mathrm{~W}$.

$39.967 \mathrm{~N} .119 .479 \mathrm{~W}$. $40.373 \mathrm{~N} .119 .040 \mathrm{~W}$. $40.302 \mathrm{~N} .119 .040 \mathrm{~W}$. $38.251 \mathrm{~N} .117 .852 \mathrm{~W}$ $37.000 \mathrm{~N} .116 .017 \mathrm{~W}$.

$38.553 \mathrm{~N}, 116.463 \mathrm{~W}$ $37.840 \mathrm{~N} .118 .153 \mathrm{~W}$ $38.087 \mathrm{~N} .118 .510 \mathrm{~W}$. $36.091 \mathrm{~N} .115 .069 \mathrm{~W}$ $38.641 \mathrm{~N} .119 .483 \mathrm{~W}$.

$38.643 \mathrm{~N} .119 .483 \mathrm{~W}$ $37.353 \mathrm{~N} .117 .254 \mathrm{~W}$ $38.641 \mathrm{~N} .119 .485 \mathrm{~W}$ $38.490 \mathrm{~N} .116 .490 \mathrm{~W}$. $37.270 \mathrm{~N}$. 116.498W.

$38.223 \mathrm{~N} .118 .423 \mathrm{~W}$. $38.224 \mathrm{~N} .118 .425 \mathrm{~W}$. $37.281 \mathrm{~N} .116 .305 \mathrm{~W}$ $38.920 \mathrm{~N} .114 .567 \mathrm{~W}$. $38.881 \mathrm{~N} .114 .599 \mathrm{~W}$.
$2 \mathrm{RN}$

$2 \quad \mathrm{RN} \quad \ldots \quad \ldots 2.7 \mathrm{ML}(\mathrm{RN})$

$\begin{array}{lllll}7 & \mathrm{RN} & \ldots & \ldots & 3.0 \mathrm{ML}(\mathrm{RN}) \\ 2 & \mathrm{RN} & \ldots & \ldots & 3.3 \mathrm{ML}(\mathrm{RN})\end{array}$

5 RN

4 RN

5 GS

0 GS

5 GS

$2 \mathrm{RN}$

1 RN

$\begin{array}{rr}2 & \mathrm{RN} \\ 10 & \mathrm{RN}\end{array}$

$2 \mathrm{RN}$

5 RN

$\begin{array}{ll}4 & \mathrm{RN} \\ 7 & \mathrm{RN}\end{array}$

2 GS

8 RN

12 RN

16 BK

$19 \mathrm{RN}$

5 GS

11 RN

5 GS

- EN

5 GS

o RN

0 RN

- EN

$9 \mathrm{RN}$

$38.864 N .114 .613 W$. $\begin{array}{ll}1 & \mathrm{RN} \\ 2 & \mathrm{RN}\end{array}$

$\begin{array}{ll}2 & \mathrm{RN} \\ 0 & \mathrm{EN}\end{array}$

5 GS

3 R

7 RN

$\begin{array}{ll}6 & \text { GM } \\ 5 & \text { GS }\end{array}$

0 RN

o RN

9 RN

1 UU
5

$\begin{array}{lll}\ldots & \ldots & 2.5 \mathrm{ML}(\mathrm{RN}) \\ \ldots & \ldots & 2.8 \mathrm{ML}(\mathrm{RN}) \\ \ldots & \ldots & 3.8 \mathrm{ML}(\mathrm{GS}) \\ \ldots & \ldots & 2.8 \mathrm{ML}(\mathrm{RN}) \\ \ldots & \ldots & 3.1 \mathrm{ML}(\mathrm{RN})\end{array}$

$\ldots \quad \ldots 2.9 M L(R N)$

$\begin{array}{lll}5.0 & \ldots & 5.0 \mathrm{ML}(\mathrm{BK})\end{array}$

4.3

3. OML (GS)

$\ldots 3.3 .7 M L(G S)$

$\begin{array}{lll}\ldots & \ldots & 2.7 M L(R N)\end{array}$

$\ldots \quad \ldots \quad 2.5 \mathrm{ML}(\mathrm{RN})$

$\ldots \quad \ldots 3.8 \mathrm{ML}(\mathrm{RN})$

$\ldots \quad \ldots 2.7 M L(R N)$

$\ldots \quad \ldots 2.7 M L(R N)$

$\ldots \quad \ldots 2.5 \mathrm{ML}(\mathrm{RN})$

$\ldots \quad \ldots \quad 3.9 \mathrm{ML}$ (RN)

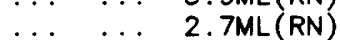

$4.2 \ldots .34$ 4.3ML(BK)

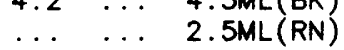

$\ldots \quad \ldots \quad 2.7 \mathrm{ML}(\mathrm{RN})$

$\ldots \quad \ldots \quad 3.9 M L(B K)$

$\ldots \quad \ldots 2.7 \mathrm{ML}(\mathrm{RN})$

$3.5 \mathrm{ML}(\mathrm{BK})$

$\ldots \quad \ldots 2.7 \mathrm{ML}(\mathrm{RN})$

.. $\quad \ldots \quad 3.9 \mathrm{ML}(\mathrm{BK})$

$\ldots \quad \ldots \quad 2.6 \mathrm{ML}(\mathrm{RN})$

$\ldots \quad \ldots 2.6 \mathrm{ML}(\mathrm{RN})$

$\ldots 3.7 M L(B K)$

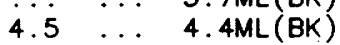

$\begin{array}{lll}\ldots & \ldots & 2.8 M L(G S)\end{array}$

$\ldots \quad \ldots 2.5 \mathrm{ML}(\mathrm{RN})$

$\ldots \quad \ldots 3.2 \mathrm{MD}(\mathrm{GM})$

$\ldots \quad \ldots 2.9 M L(G S)$

$\ldots \quad \ldots 2.5 \mathrm{ML}(\mathrm{RN})$

$\ldots \quad \ldots 2.7 \mathrm{ML}(\mathrm{RN})$

$\begin{array}{lll}\ldots & \ldots & 2.7 \mathrm{ML}(\mathrm{RN}) \\ \ldots & \ldots & 2.5 \mathrm{ML}(\mathrm{RN})\end{array}$

$\ldots \quad \ldots 2.5 \mathrm{ML}(\mathrm{RN})$

$\begin{array}{lll}5.5 & 4.2 & 5.4 \mathrm{ML}(\mathrm{BK})\end{array}$

$\ldots \quad \ldots 2.6 \mathrm{ML}(\mathrm{RN})$

$\ldots \quad \ldots \quad 3.3 \mathrm{ML}(\mathrm{RN})$

$\begin{array}{lll}3.4 & \ldots & 5.4 \mathrm{ML}(\mathrm{BK})\end{array}$

$\ldots \ldots 2.5 M D(U U)$

$\ldots \ldots 2.9 M L(G S)$

5 GS $\ldots \quad \ldots 2.8 \mathrm{ML}(\mathrm{RN})$
SEPT. $1 \quad 03: 45$ PST SEPT. 1 08:45 PST SEPT. 2 16:23 PST

SEPT. 2 16:31 PST SEPT. 3 20:08 PST SEPT. 4 19:30 PST SEPT. 6 05:49 PST SEPT. $12 \quad 04: 53$ PST

SEPT. $13 \quad 02: 37$ PST SEPT. 13 06:00 PST SEPT. 13 13:01 PST SEPT. 15 22:49 PST SEPT. 17 11:11 PST

SEPT. $26 \quad 12: 20$ PST SEPT. $26 \quad 14: 05$ PST SEPT. 26 14:06 PST SEPT. $26 \quad 17: 45$ PST SEPT. 27 05:17 PST

SEPT. $2909: 12$ PST SEPT. 29 09:14 PST SEPT. 30 11:03 PST OCT. 2 10:13 PST OCT. 3 01:01 PST

$\begin{array}{llll}\ldots & \text { OCT. } 19 & 14: 12 & \text { PST }\end{array}$ IV OCT. $21 \quad 06: 51$ PST $\begin{array}{lllll} & \text { OCT. } & 21 & 06: 51 & \text { PST }\end{array}$ FELT OCT. 21 06:55 PST OCT. 21 17:10 PST

$\begin{array}{llll}\text { IV OCT. } 22 & 03: 27 & \text { PST }\end{array}$ $\begin{array}{lll}\text {... OCT. } 23 & 10: 44 \text { PST }\end{array}$ ... OCT. 27 10:22 PST $\begin{array}{lll}\ldots & \text { NOV } 5 & 03: 21 \\ & \text { PST }\end{array}$ ... NOV. 10 08:40 PST

$\begin{array}{lll}\ldots & \text { NOV. } 10 & 22: 03 \\ \text { PST }\end{array}$ $\begin{array}{lll}\ldots & \text { NOV. } 18 & 00: 35 \\ \ldots & \text { PST }\end{array}$

‥ DEC. 9 16:29 PST

III DEC. 4 12:53 PST

... DEC. 5 02:14 PST

DEC. $5 \quad 06: 56$ PST

DEC. 8 18:34 PST

DEC. $9 \quad 01: 28$ PST

DEC. $910: 48$ PST

DEC. 9 11:40 PST

DEC. $913: 48$ PST

DEC. $916: 29$ PST

DEC. 15 06:45 PST

DEC. 21 13:23 PST

DEC. 22 16:00 PST

DEC. $22 \quad 17: 47$ PST 
Table 1. Summary of U.S. earthquakes for 1984-Continued

\begin{tabular}{|c|c|c|c|c|c|c|c|c|c|c|c|c|c|}
\hline \multirow{2}{*}{ Date } & \multicolumn{2}{|c|}{$\begin{array}{l}\text { Origin time } \\
\text { (UTC) }\end{array}$} & \multirow{2}{*}{$\begin{array}{c}\text { Latitude } \\
\left({ }^{\circ}\right)\end{array}$} & \multirow{2}{*}{$\begin{array}{c}\text { Longitude } \\
\text { (क) }\end{array}$} & \multirow{2}{*}{$\begin{array}{c}\text { Depth } \\
(\mathbf{k m})\end{array}$} & \multirow{2}{*}{$\begin{array}{l}\text { Hypo- } \\
\text { center } \\
\text { source }\end{array}$} & \multicolumn{3}{|c|}{ Magnitude } & \multirow{2}{*}{$\begin{array}{l}\text { Maximum } \\
\text { intensity }\end{array}$} & \multicolumn{3}{|c|}{ Local time } \\
\hline & hr $\min$ & $\sec$ & & & & & $\mathbf{m b}$ & MS & $\begin{array}{l}\text { ML, Mn } \\
\text { MD, Mw }\end{array}$ & & Date & Hour & $\begin{array}{l}\text { Time } \\
\text { rome }\end{array}$ \\
\hline
\end{tabular}

NEW HAMPSHIRE

\begin{tabular}{llllllllllllllllllll}
\hline FEB. & 26 & 12 & 37 & 19.2 & $44.486 \mathrm{~N}$ & $71.303 W$ & 12 & WO & $\ldots$ & $\ldots$ & $2.7 \mathrm{Mn}($ WO $)$ & $\ldots$ & FEB. & 26 & $07: 37$ & EST
\end{tabular}

NEW MEXICO

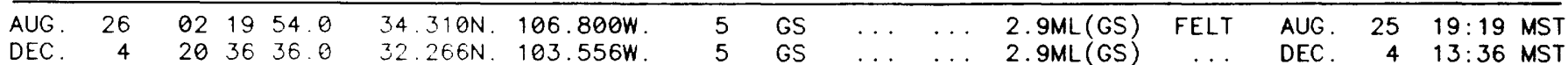

\section{NEW YORK}

\begin{tabular}{|c|c|c|c|c|c|c|c|c|c|c|c|c|c|c|c|c|}
\hline JAN. & 14 & 07 & 34 & 39.3 & $43.480 \mathrm{~N}$ & $74.621 W$. & 5 & LD & & & $2.5 \mathrm{MD}(\mathrm{LD})$ & & JAN. & 14 & $02: 34$ & EST \\
\hline MAR. & 17 & 21 & 34 & 16.2 & $43.943 \mathrm{~N}$ & $74.253 w$. & 9 & LD & & & $2.6 \mathrm{MD}(\mathrm{LD})$ & & MAR. & 17 & $16: 34$ & EST \\
\hline MAR. & 25 & 11 & 12 & 49.2 & $40.350 \mathrm{~N}$. & $72.166 \mathrm{~W}$. & 13 & WO & $\ldots$ & $\ldots$ & $2.5 \mathrm{MD}(\mathrm{WO})$ & & MAR. & 25 & $06: 12$ & EST \\
\hline MAR. & 26 & 06 & 28 & 37.8 & $43.944 \mathrm{~N}$ & $74.248 W$. & 8 & LD & $\ldots$ & $\ldots$ & $2.6 \mathrm{MD}(\mathrm{LD})$ & & MAR. & 26 & $01: 28$ & EST \\
\hline JUN & 1 & 21 & 28 & 10.0 & $43.191 \mathrm{~N}$. & $75.170 \mathrm{w}$. & 4 & LD & $\ldots$ & $\ldots$ & 2. $5 \mathrm{MD}$ & $\ldots$ & JUNE & 1 & $16: 28$ & EST \\
\hline JULY & 28 & 10 & 07 & 03.2 & 44.7 & 74.5 & 7 & LD & & & 2. $6 \mathrm{MD}(\mathrm{LD})$ & & JULY & 28 & 07 & EST \\
\hline AUG & 20 & 10 & 58 & 17.0 & $44.875 \mathrm{~N}$ & $73.480 \mathrm{~W}$. & 11 & LD & & & 3. $2 \mathrm{MD}(\mathrm{LD})$ & v & AUG & 20 & $05: 58$ & EST \\
\hline SEPT . & 28 & 14 & 39 & 32.0 & $44.356 \mathrm{~N}$. & $74.112 W$. & 14 & LD & & & 2.7MD (LD) & & SEPT. & 28 & $09: 39$ & EST \\
\hline SEPT. & 29 & 13 & 05 & 19.4 & $42.974 \mathrm{~N}$ & 77.0 & 2 & LD & $\ldots$ & & $2.7 \mathrm{MD}(\mathrm{LI}$ & & SEPT. & 29 & $08: 05$ & EST \\
\hline OCT. & 23 & 06 & 26 & 21.5 & $43.592 \mathrm{~N}$ & $73.937 w$. & $\overline{1}$ & LD & $\ldots$ & $\ldots$ & 3. $2 \mathrm{Mn}(\mathrm{GS})$ & IV & OCT. & 23 & $01: 26$ & EST \\
\hline DEC. & 8 & 18 & 40 & 14.8 & $43.951 \mathrm{~N}$. & $74.259 w$. & 7 & LD & & $\cdots$ & $2.5 \mathrm{MD}(\mathrm{LD})$ & & DEC. & 8 & $13: 40$ & EST \\
\hline
\end{tabular}

NORTH CAROLINA

\begin{tabular}{llllllllllllllll}
\hline OCT. & 22 & 18 & 58 & 41.8 & $36.364 \mathrm{~N}$ & $81.677 \mathrm{~W}$. & 11 & $\mathrm{TC}$ & $\ldots$ & $\ldots$ & $3.1 \mathrm{Mn}(\mathrm{VP})$ & $\mathrm{V}$ & OCT. & 22 & $13: 58$ EST
\end{tabular}

\section{OHIO}

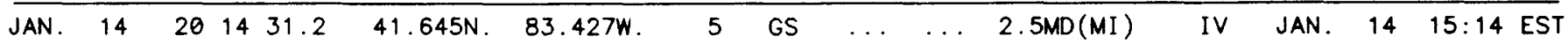

\section{OKLAHOMA}

\begin{tabular}{|c|c|c|c|c|c|c|c|c|c|c|c|c|c|c|c|c|}
\hline $\begin{array}{l}\text { JAN. } \\
\text { JAN. } \\
\text { FEB. } \\
\text { FEB. } \\
\text { MAR. }\end{array}$ & $\begin{array}{r}6 \\
24 \\
3 \\
10 \\
3\end{array}$ & $\begin{array}{l}17 \\
15 \\
04 \\
18 \\
11\end{array}$ & $\begin{array}{l}14 \\
34 \\
38 \\
39 \\
42\end{array}$ & $\begin{array}{l}49.8 \\
09.6 \\
28.0 \\
13.6 \\
02.4\end{array}$ & $\begin{array}{l}36.161 \mathrm{~N} . \\
35.033 \mathrm{~N} \\
34.665 \mathrm{~N} . \\
34.049 \mathrm{~N} . \\
35.514 \mathrm{~N} .\end{array}$ & $\begin{array}{l}95.582 W . \\
96.366 w . \\
97.356 w . \\
97.415 W . \\
96.301 W .\end{array}$ & $\begin{array}{l}5 \\
5 \\
5 \\
5 \\
5\end{array}$ & $\begin{array}{l}\text { TU } \\
\text { TU } \\
\text { TU } \\
\text { TU } \\
\text { TU }\end{array}$ & $\begin{array}{l}\cdots \\
\cdots \\
\cdots \\
\cdots \\
\cdots\end{array}$ & $\begin{array}{l}\cdots \\
\cdots \\
\cdots\end{array}$ & $\begin{array}{l}2.5 \mathrm{MD}(T U) \\
2.8 \mathrm{Mn}(T U) \\
3.2 \mathrm{Mn}(\mathrm{TU}) \\
2.2 \mathrm{MD}(\mathrm{TU}) \\
2.6 \mathrm{Mn}(T U)\end{array}$ & $\begin{array}{l}\text { IV } \\
V \\
\text { V } \\
\text { IV } \\
\text { V }\end{array}$ & $\begin{array}{l}\text { JAN. } \\
\text { JAN. } \\
\text { FEB. } \\
\text { FEB. } \\
\text { MAR. }\end{array}$ & $\begin{array}{r}6 \\
24 \\
2 \\
10 \\
3\end{array}$ & $\begin{array}{l}11: 14 \\
09: 34 \\
22: 38 \\
12: 39 \\
05: 42\end{array}$ & $\begin{array}{l}\text { CST } \\
\text { CST } \\
\text { CST } \\
\text { CST } \\
\text { CST }\end{array}$ \\
\hline $\begin{array}{l}\text { AUG. } \\
\text { OCT. } \\
\text { NOV. }\end{array}$ & $\begin{array}{r}17 \\
4 \\
20\end{array}$ & $\begin{array}{l}18 \\
12 \\
10\end{array}$ & $\begin{array}{l}04 \\
25 \\
57\end{array}$ & $\begin{array}{l}01.9 \\
09.3 \\
32.0\end{array}$ & $\begin{array}{l}34.741 \mathrm{~N} \\
34.707 \mathrm{~N}\end{array}$ & $\begin{array}{l}97.326 \mathrm{~W} . \\
97.502 \mathrm{~W} . \\
97.410 \mathrm{~W} .\end{array}$ & $\begin{array}{l}5 \\
5 \\
5\end{array}$ & $\begin{array}{l}\text { TU } \\
\text { TU } \\
\text { TU }\end{array}$ & & & $\begin{array}{l}\text { 2. } 5 \mathrm{Mn}(T U) \\
\text { 2. } 6 \mathrm{Mn}(\mathrm{TU}) \\
\text { 3. } 1 \mathrm{Mn}(\mathrm{TU})\end{array}$ & IV & $\begin{array}{l}\text { AUG. } \\
\text { OCT. } \\
\text { NOV. }\end{array}$ & $\begin{array}{r}17 \\
4 \\
20\end{array}$ & $\begin{array}{l}12: 04 \\
06: 25 \\
04: 57\end{array}$ & CST \\
\hline
\end{tabular}

\section{OREGON}

\begin{tabular}{|c|c|c|c|c|c|c|c|c|c|c|c|c|c|c|c|c|}
\hline $\begin{array}{l}\text { JAN. } \\
\text { MAR. } \\
\text { MAY } \\
\text { JUNE } \\
\text { JUNE }\end{array}$ & $\begin{array}{r}31 \\
4 \\
7 \\
6 \\
18\end{array}$ & $\begin{array}{l}05 \\
11 \\
07 \\
19 \\
19\end{array}$ & $\begin{array}{l}29 \\
01 \\
12 \\
49 \\
34\end{array}$ & $\begin{array}{l}15.6 \\
10.8 \\
02.6 \\
52.5 \\
08.9\end{array}$ & $\begin{array}{l}45.579 \mathrm{~N} \\
45.048 \mathrm{~N} \\
45.306 \mathrm{~N} \\
45.974 \mathrm{~N} \\
45.486 \mathrm{~N}\end{array}$ & $\begin{array}{l}116.571 W . \\
122.603 W . \\
121.594 W . \\
118.437 W . \\
118.840 W .\end{array}$ & $\begin{array}{r}5 \\
15 \\
4 \\
6 \\
6\end{array}$ & $\begin{array}{l}\text { GS } \\
\text { WA } \\
\text { WA } \\
\text { WA } \\
\text { WA }\end{array}$ & $\begin{array}{l}. \\
\cdots \\
.\end{array}$ & $\begin{array}{l}\cdots \\
\cdots \\
\cdots \\
\cdots\end{array}$ & $\begin{array}{l}\text { 3. 5ML (GS) } \\
2.8 \mathrm{MD} \text { (WA) } \\
2.9 \mathrm{MD} \text { (WA) } \\
2.7 \mathrm{MD} \text { (WA) } \\
3.1 \mathrm{MD} \text { (WA) }\end{array}$ & $\begin{array}{l}\text { IV } \\
\ldots \\
\cdots \\
\cdots \\
\cdots\end{array}$ & $\begin{array}{l}\text { JAN. } \\
\text { MAR. } \\
\text { MAY } \\
\text { JUNE } \\
\text { JUNE }\end{array}$ & $\begin{array}{r}30 \\
4 \\
6 \\
6 \\
18\end{array}$ & $\begin{array}{l}22: 29 \\
03: 01 \\
23: 12 \\
11: 49 \\
11: 34\end{array}$ & $\begin{array}{l}\text { MST } \\
\text { PST } \\
\text { PST } \\
\text { PST } \\
\text { PST }\end{array}$ \\
\hline $\begin{array}{l}\text { JULY } \\
\text { AUG. } \\
\text { DEC. }\end{array}$ & $\begin{array}{l}30 \\
10 \\
11\end{array}$ & $\begin{array}{l}21 \\
07 \\
06\end{array}$ & $\begin{array}{l}39 \\
26 \\
34\end{array}$ & $\begin{array}{l}04.5 \\
38.3 \\
49.9\end{array}$ & $\begin{array}{l}42.150 \mathrm{~N} \\
44.986 \mathrm{~N} \\
45.469 \mathrm{~N} .\end{array}$ & $\begin{array}{l}121.877 W . \\
116.952 W . \\
122.829 W\end{array}$ & $\begin{array}{r}8 \\
5 \\
22\end{array}$ & $\begin{array}{l}\text { GS } \\
\text { WA }\end{array}$ & $\cdots$ & $\begin{array}{l}\cdots \\
\cdots \\
\cdots\end{array}$ & $\begin{array}{l}\text { 3. } 5 \mathrm{MD}(\mathrm{GM}) \\
3.5 \mathrm{ML}(\mathrm{GS}) \\
2.5 \mathrm{MD}(W A)\end{array}$ & $\begin{array}{l}\text { IV } \\
\text { IV } \\
\text { FELT }\end{array}$ & $\begin{array}{l}\text { JULY } \\
\text { AUG. } \\
\text { DEC. }\end{array}$ & $\begin{array}{l}30 \\
10 \\
10\end{array}$ & $\begin{array}{l}13: 39 \\
00: 26 \\
22: 34\end{array}$ & $\begin{array}{l}\text { PST } \\
\text { MST } \\
\text { PST }\end{array}$ \\
\hline
\end{tabular}


Table 1. Summary of U.S. earthquakes for 1984-Continued

\begin{tabular}{|c|c|c|c|c|c|c|c|c|c|c|c|c|c|c|c|c|}
\hline \multirow{2}{*}{\multicolumn{2}{|c|}{ Date }} & \multicolumn{3}{|c|}{$\begin{array}{c}\text { Origin time } \\
\text { (UTC) }\end{array}$} & \multirow{2}{*}{$\begin{array}{c}\text { Lotitude } \\
\left({ }^{\circ}\right)\end{array}$} & \multirow{2}{*}{$\begin{array}{c}\text { Longitude } \\
\left({ }^{\circ}\right)\end{array}$} & \multirow{2}{*}{$\begin{array}{c}\text { Depth } \\
(\mathbf{k m})\end{array}$} & \multirow{2}{*}{$\begin{array}{l}\text { Hypo- } \\
\text { center } \\
\text { source }\end{array}$} & \multicolumn{3}{|c|}{ Magnitude } & \multirow{2}{*}{$\begin{array}{l}\text { Maximum } \\
\text { intensity }\end{array}$} & \multicolumn{4}{|c|}{ local time } \\
\hline & & hr & $\min$ & Isec & & & & & $\mathbf{m b}$ & MS & $\begin{array}{l}\text { ML, Mn } \\
\text { MD, Mw }\end{array}$ & & Date & & Hour & $\begin{array}{l}\text { Time } \\
\text { Jome }\end{array}$ \\
\hline \multicolumn{17}{|c|}{ OREGON OFF THE COAST } \\
\hline $\begin{array}{l}\text { JAN. } \\
\text { FEB. } \\
\text { FEB. } \\
\text { FEB. } \\
\text { MAR. }\end{array}$ & $\begin{array}{r}5 \\
10 \\
12 \\
13 \\
30\end{array}$ & $\begin{array}{l}08 \\
01 \\
11 \\
10 \\
04\end{array}$ & $\begin{array}{l}42 \\
53 \\
51 \\
21 \\
38\end{array}$ & $\begin{array}{l}19.4 \\
03.9 \\
42.0 \\
55.0 \\
18.4\end{array}$ & $\begin{array}{l}44.483 \mathrm{~N} \\
44.340 \mathrm{~N} \\
42.416 \mathrm{~N} \\
43.188 \mathrm{~N} \\
43.652 \mathrm{~N}\end{array}$ & $\begin{array}{l}129.136 w . \\
129.072 w . \\
126.371 w . \\
126.228 w . \\
127.205 w .\end{array}$ & $\begin{array}{l}10 \\
10 \\
10 \\
10 \\
10\end{array}$ & $\begin{array}{l}\text { GS } \\
\text { GS } \\
\text { GS } \\
\text { GS } \\
\text { GS }\end{array}$ & $\begin{array}{l}3.8 \\
4.2 \\
4.6 \\
4.2 \\
4.4\end{array}$ & $\begin{array}{l}\cdots \\
4.0 \\
4.3 \\
3.6\end{array}$ & $\begin{array}{l}\cdots \\
\cdots \\
\cdots \\
\cdots \\
\cdots\end{array}$ & $\begin{array}{l}\cdots \\
\cdots \\
\cdots \\
\cdots \\
\cdots\end{array}$ & $\begin{array}{l}\text { JAN. } \\
\text { FEB. } \\
\text { FEB. } \\
\text { FEB. } \\
\text { MAR. }\end{array}$ & $\begin{array}{r}5 \\
9 \\
12 \\
13 \\
29\end{array}$ & $\begin{array}{l}00: 42 \\
17: 53 \\
03: 51 \\
02: 21 \\
20: 38\end{array}$ & $\begin{array}{l}\text { PST } \\
\text { PST } \\
\text { PST } \\
\text { PST } \\
\text { PST }\end{array}$ \\
\hline $\begin{array}{l}\text { APR. } \\
\text { APR. } \\
\text { APR. } \\
\text { APR. } \\
\text { JULY }\end{array}$ & $\begin{array}{l}7 \\
14 \\
18 \\
26 \\
10\end{array}$ & $\begin{array}{l}04 \\
14 \\
23 \\
13 \\
04\end{array}$ & $\begin{array}{l}25 \\
58 \\
18 \\
47 \\
28\end{array}$ & $\begin{array}{l}52.1 \\
17.8 \\
47.2 \\
07.2 \\
03.8\end{array}$ & $\begin{array}{l}43.169 \mathrm{~N} \\
44.181 \mathrm{~N} \\
43.139 \mathrm{~N} \\
44.202 \mathrm{~N} \\
43.489 \mathrm{~N}\end{array}$ & $\begin{array}{l}126.318 w \\
129.348 w \\
126.331 W \\
128.327 w \\
126.658 w\end{array}$ & $\begin{array}{l}10 \\
10 \\
10 \\
10 \\
10\end{array}$ & $\begin{array}{l}\text { GS } \\
\text { GS } \\
\text { GS } \\
\text { GS } \\
\text { GS }\end{array}$ & $\begin{array}{l}4.7 \\
4.3 \\
4.8 \\
4.0 \\
3.9\end{array}$ & $\begin{array}{l}\ldots \\
3.8 \\
\ldots \\
\ldots\end{array}$ & $\begin{array}{c}4.1 \mathrm{ML}(\mathrm{BK}) \\
\ldots \\
\ldots \\
\ldots \\
\ldots\end{array}$ & $\begin{array}{l}\cdots \\
\cdots \\
\cdots \\
\cdots \\
\cdots\end{array}$ & $\begin{array}{l}\text { APR. } \\
\text { APR. } \\
\text { APR. } \\
\text { APR. } \\
\text { JULY }\end{array}$ & $\begin{array}{r}6 \\
14 \\
18 \\
26 \\
9\end{array}$ & $\begin{array}{l}20: 25 \\
06: 58 \\
15: 18 \\
05: 47 \\
20: 28\end{array}$ & $\begin{array}{l}\text { PST } \\
\text { PST } \\
\text { PST } \\
\text { PST } \\
\text { PST }\end{array}$ \\
\hline $\begin{array}{l}\text { JULY } \\
\text { AUG. } \\
\text { AUG. } \\
\text { AUG. } \\
\text { SEPT. }\end{array}$ & $\begin{array}{l}19 \\
20 \\
25 \\
25 \\
13\end{array}$ & $\begin{array}{l}07 \\
07 \\
12 \\
12 \\
08\end{array}$ & $\begin{array}{l}54 \\
02 \\
55 \\
58 \\
38\end{array}$ & $\begin{array}{l}12.3 \\
43.2 \\
46.7 \\
47.5 \\
32.0\end{array}$ & $\begin{array}{l}43.606 \mathrm{~N} \\
43.480 \mathrm{~N} \\
44.664 \mathrm{~N} \\
44.711 \mathrm{~N} \\
42.960 \mathrm{~N}\end{array}$ & $\begin{array}{l}127.011 w . \\
126.821 w . \\
129.723 w . \\
129.794 w . \\
126.459 w .\end{array}$ & $\begin{array}{l}10 \\
10 \\
10 \\
10 \\
10\end{array}$ & $\begin{array}{l}\text { GS } \\
\text { GS } \\
\text { GS } \\
\text { GS } \\
\text { GS }\end{array}$ & $\begin{array}{l}3.8 \\
4.5 \\
4.2 \\
4.5 \\
4.3\end{array}$ & $\begin{array}{l}\cdots \\
\cdots \\
\cdots \\
\cdots \\
\cdots\end{array}$ & $\begin{array}{l}\cdots \\
\cdots \\
\cdots \\
\cdots \\
\cdots\end{array}$ & $\begin{array}{l}\ldots \\
\ldots \\
\cdots \\
\cdots \\
\cdots\end{array}$ & $\begin{array}{l}\text { JULY } \\
\text { AUG. } \\
\text { AUG. } \\
\text { AUG. } \\
\text { SEPT. }\end{array}$ & $\begin{array}{l}18 \\
19 \\
25 \\
25 \\
13\end{array}$ & $\begin{array}{l}23: 54 \\
23: 02 \\
04: 55 \\
04: 58 \\
00: 38\end{array}$ & $\begin{array}{l}\text { PST } \\
\text { PST } \\
\text { PST } \\
\text { PST } \\
\text { PST }\end{array}$ \\
\hline $\begin{array}{l}\text { SEPT. } \\
\text { OCT. } \\
\text { OCT. } \\
\text { OCT. } \\
\text { OCT. }\end{array}$ & $\begin{array}{l}13 \\
17 \\
24 \\
27 \\
27\end{array}$ & $\begin{array}{l}08 \\
00 \\
10 \\
03 \\
04\end{array}$ & $\begin{array}{l}44 \\
44 \\
36 \\
20 \\
48\end{array}$ & $\begin{array}{l}18.3 \\
49.6 \\
52.4 \\
35.5 \\
25.2\end{array}$ & $\begin{array}{l}43.028 \mathrm{~N} \\
43.964 \mathrm{~N} \\
44.296 \mathrm{~N} \\
43.983 \mathrm{~N} \\
43.947 \mathrm{~N}\end{array}$ & $\begin{array}{l}126.760 w . \\
128.393 w . \\
129.554 w . \\
127.836 w . \\
127.996 w\end{array}$ & $\begin{array}{l}10 \\
10 \\
10 \\
10 \\
10\end{array}$ & $\begin{array}{l}\text { GS } \\
\text { GS } \\
\text { GS } \\
\text { GS } \\
\text { GS }\end{array}$ & $\begin{array}{l}4.5 \\
4.7 \\
3.9 \\
4.1 \\
4.7\end{array}$ & $\begin{array}{l}\ddot{4} .6 \\
\ddot{4} . \dot{2} \\
4.3\end{array}$ & $\begin{array}{l}\cdots \\
\cdots \\
\cdots \\
\cdots \\
\cdots\end{array}$ & $\begin{array}{l}\cdots \\
\cdots \\
\cdots \\
\cdots \\
\cdots\end{array}$ & $\begin{array}{l}\text { SEPT. } \\
\text { OCT. } \\
\text { OCT. } \\
\text { OCT. } \\
\text { OCT. }\end{array}$ & $\begin{array}{l}13 \\
16 \\
24 \\
26 \\
26\end{array}$ & $\begin{array}{l}00: 44 \\
16: 44 \\
02: 36 \\
19: 20 \\
20: 48\end{array}$ & $\begin{array}{l}\text { PST } \\
\text { PST } \\
\text { PST } \\
\text { PST } \\
\text { PST }\end{array}$ \\
\hline $\begin{array}{l}\text { OCT. } \\
\text { NOV. } \\
\text { NOV. } \\
\text { NOV. } \\
\text { NOV. }\end{array}$ & $\begin{array}{r}29 \\
8 \\
8 \\
9 \\
23\end{array}$ & $\begin{array}{l}83 \\
12 \\
17 \\
16 \\
03\end{array}$ & $\begin{array}{l}04 \\
01 \\
36 \\
58 \\
45\end{array}$ & $\begin{array}{l}38.6 \\
25.3 \\
48.5 \\
04.4 \\
45.8\end{array}$ & $\begin{array}{l}43.569 \mathrm{~N} \\
43.406 \mathrm{~N} \\
43.388 \mathrm{~N} \\
43.140 \mathrm{~N} \\
43.516 \mathrm{~N}\end{array}$ & $\begin{array}{l}126.973 w . \\
126.794 w . \\
126.819 w . \\
126.455 w . \\
126.701 w .\end{array}$ & $\begin{array}{l}10 \\
10 \\
10 \\
10 \\
10\end{array}$ & $\begin{array}{l}\text { GS } \\
\text { GS } \\
\text { GS } \\
\text { GS } \\
\text { GS }\end{array}$ & $\begin{array}{l}4.3 \\
4.2 \\
4.1 \\
4.2 \\
4.4\end{array}$ & $\begin{array}{l}3.5 \\
3.9 \\
3.4 \\
4.5 \\
\ldots\end{array}$ & $\begin{array}{l}\cdots \\
\cdots \\
\cdots \\
\cdots \\
\cdots\end{array}$ & $\begin{array}{l}\cdots \\
\cdots \\
\cdots \\
\cdots \\
\cdots\end{array}$ & $\begin{array}{l}\text { OCT. } \\
\text { NOV. } \\
\text { NOV. } \\
\text { NOV. } \\
\text { NOV. }\end{array}$ & $\begin{array}{r}28 \\
8 \\
8 \\
9 \\
22\end{array}$ & $\begin{array}{l}19: 04 \\
04: 01 \\
09: 36 \\
08: 58 \\
19: 45\end{array}$ & $\begin{array}{l}\text { PST } \\
\text { PST } \\
\text { PST } \\
\text { PST } \\
\text { PST }\end{array}$ \\
\hline $\begin{array}{l}\text { DEC. } \\
\text { DEC. }\end{array}$ & $\begin{array}{l}24 \\
30\end{array}$ & $\begin{array}{l}06 \\
15\end{array}$ & $\begin{array}{l}39 \\
49\end{array}$ & $\begin{array}{l}30.4 \\
26.3\end{array}$ & $\begin{array}{l}43.727 \mathrm{~N} \\
44.313 \mathrm{~N}\end{array}$ & $\begin{array}{l}127.638 w \\
128.701 w .\end{array}$ & $\begin{array}{l}10 \\
10\end{array}$ & $\begin{array}{l}\text { GS } \\
\text { GS }\end{array}$ & $\begin{array}{l}4.2 \\
4.3\end{array}$ & $\begin{array}{l}\cdots \\
\cdots\end{array}$ & $\cdots$ & $\begin{array}{l}\cdots \\
\cdots\end{array}$ & $\begin{array}{l}\text { DEC. } \\
\text { DEC. }\end{array}$ & $\begin{array}{l}23 \\
30\end{array}$ & $\begin{array}{l}22: 39 \\
07: 49\end{array}$ & $\begin{array}{l}\text { PST } \\
\text { PST }\end{array}$ \\
\hline
\end{tabular}

\section{PENNSYLVANIA}

\begin{tabular}{llllllllllllllll}
\hline APR. & 19 & 04 & 54 & 58.3 & $39.923 \mathrm{~N}$ & $76.316 \mathrm{~W}$ & 5 & GS & $\ldots$ & $\ldots$ & $3.0 \mathrm{Mn}(\mathrm{VP})$ & $\mathrm{V}$ & APR. & 18 & $23: 54$ \\
APR. & 23 & 01 & 36 & 00.1 & $39.921 \mathrm{~N}$. & $76.355 \mathrm{~W}$ & 5 & GS & 4.2 & $\ldots$ & $4.1 \mathrm{Mn}(\mathrm{GS})$ & VI & APR. & 22 & $20: 36$ EST
\end{tabular}

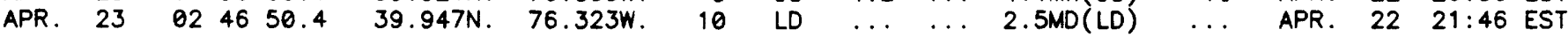

\section{TENNESSEE}

\begin{tabular}{|c|c|c|c|c|c|c|c|c|c|c|c|c|c|c|c|}
\hline $\begin{array}{l}\text { FEB. } \\
\text { MAR. } \\
\text { APR. } \\
\text { JUNE } \\
\text { JULY }\end{array}$ & $\begin{array}{l}14 \\
17 \\
21 \\
26 \\
21\end{array}$ & $\begin{array}{l}20 \\
23 \\
02 \\
15 \\
18\end{array}$ & $\begin{array}{l}54 \\
26 \\
28 \\
15 \\
09\end{array}$ & $\begin{array}{l}30.9 \\
11.4 \\
08.2 \\
19.9 \\
33.5\end{array}$ & $\begin{array}{l}36.125 \mathrm{~N} . \\
35.832 \mathrm{~N} . \\
36.280 \mathrm{~N} . \\
36.102 \mathrm{~N} . \\
36.170 \mathrm{~N} .\end{array}$ & $\begin{array}{l}83.737 w . \\
84.050 w . \\
89.450 w . \\
89.391 w . \\
89.440 w .\end{array}$ & $\begin{array}{r}10 \\
7 \\
6 \\
12 \\
11\end{array}$ & $\begin{array}{l}\text { TC } \\
\text { TC }\end{array}$ & $\begin{array}{l}\cdots \\
\cdots \\
\cdots \\
\ldots \\
\ldots\end{array}$ & $\begin{array}{l}\cdots \\
\cdots \\
\cdots \\
\cdots\end{array}$ & $\begin{array}{l}\text { 3. } 5 \mathrm{MD}(\mathrm{TC}) \\
\text { 3. } \mathrm{OML}(\mathrm{GS}) \\
2.5 \mathrm{Mn}(\mathrm{SL}) \\
\text { 3. } \mathrm{Mn}(\mathrm{SL}) \\
2.5 \mathrm{Mn}(\mathrm{SL})\end{array}$ & $\begin{array}{l}\text { VI } \\
\text { IV } \\
\dddot{I} \ddot{I} \\
\cdots\end{array}$ & & $\begin{array}{l}14 \\
17 \\
20 \\
26 \\
21\end{array}$ & $\begin{array}{l}15: 54 \\
18: 26 \\
20: 28 \\
09: 15 \\
12: 09\end{array}$ \\
\hline $\begin{array}{l}\text { JG. } \\
\text { JG. } \\
\text { EPT. } \\
\text { EPT. } \\
\text { OPV. }\end{array}$ & $\begin{array}{r}30 \\
30 \\
6 \\
21 \\
20\end{array}$ & $\begin{array}{l}16 \\
16 \\
16 \\
08 \\
10\end{array}$ & $\begin{array}{l}26 \\
41 \\
06 \\
46 \\
52\end{array}$ & $\begin{array}{l}28.4 \\
52.3 \\
08.1 \\
12.6 \\
54.9\end{array}$ & $\begin{array}{l}35.565 \mathrm{~N} . \\
35.564 \mathrm{~N} . \\
36.100 \mathrm{~N} . \\
36.320 \mathrm{~N} . \\
35.590 \mathrm{~N} .\end{array}$ & $\begin{array}{l}84.338 w . \\
84.343 w . \\
89.350 w . \\
89.530 w . \\
89.760 w .\end{array}$ & $\begin{array}{r}14 \\
14 \\
9 \\
6 \\
3\end{array}$ & $\begin{array}{l}10 \\
\text { SL } \\
\text { SL } \\
\text { SL }\end{array}$ & $\begin{array}{l}\cdots \\
\cdots \\
\cdots \\
\cdots\end{array}$ & & $\begin{array}{l}\text { 3. } 2 \mathrm{Mn}(\mathrm{GS}) \\
2.4 \mathrm{MD}(\mathrm{TC}) \\
2.9 \mathrm{Mn}(\mathrm{SL}) \\
2.9 \mathrm{Mn}(\mathrm{SL}) \\
2.6 \mathrm{Mn}(\mathrm{SL})\end{array}$ & $\begin{array}{c}\ldots \\
\text { FË } \\
\ldots \\
\ldots\end{array}$ & $\begin{array}{l}\text { i. } \\
\text { T. } \\
\text { T. }\end{array}$ & $\begin{array}{r}30 \\
30 \\
6 \\
21 \\
20\end{array}$ & $\begin{array}{l}11: 26 \\
11: 41 \\
10: 06 \\
02: 46 \\
04: 52\end{array}$ \\
\hline EC. & 17 & 18 & 48 & 28.2 & $35.415 \mathrm{~N}$ & $84.309 w$. & 14 & TC & $\cdots$ & $\cdots$ & $2.8 \mathrm{MD}(\mathrm{TC})$ & & DEC. & 17 & $13: 48$ \\
\hline
\end{tabular}

\section{TEXAS}

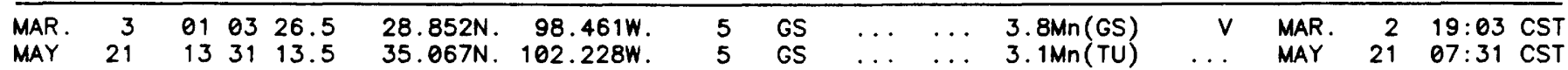

130 U.S. Earthquakes, 1984 
Table 1. Summary of U.S. earthquakes for 1984-Continued

\begin{tabular}{|c|c|c|c|c|c|c|c|c|c|c|c|c|c|}
\hline Date & hr $\min$ & $=c$ & $\begin{array}{l}\text { Latitude } \\
\text { (") }\end{array}$ & $\begin{array}{c}\text { Lompitude } \\
\text { (1) }\end{array}$ & $\begin{array}{c}\text { Depth } \\
(\mathbf{k m})\end{array}$ & $\begin{array}{l}\text { Hypo- } \\
\text { center } \\
\text { source }\end{array}$ & $\mathbf{m b}$ & MS & $\begin{array}{l}\text { ML, Mn } \\
\text { MD, Mw }\end{array}$ & $\begin{array}{l}\text { Maximum } \\
\text { intensity }\end{array}$ & Date & Hour & $\begin{array}{l}\text { Time } \\
\text { none }\end{array}$ \\
\hline
\end{tabular}

TEXAS-Continued

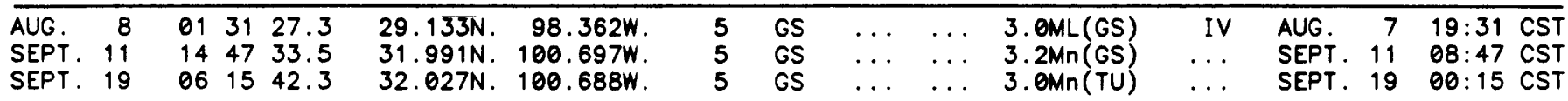

\section{UTAH}

\begin{tabular}{|c|c|c|c|c|c|c|c|c|c|c|c|c|c|c|c|c|}
\hline $\begin{array}{l}\text { JAN. } \\
\text { MAR. } \\
\text { MAY } \\
\text { MAY } \\
\text { JUN. }\end{array}$ & $\begin{array}{r}8 \\
21 \\
5 \\
12 \\
2\end{array}$ & $\begin{array}{l}81 \\
11 \\
04 \\
15 \\
13\end{array}$ & $\begin{array}{l}59 \\
19 \\
03 \\
20 \\
52\end{array}$ & $\begin{array}{l}07.2 \\
30.6 \\
00.9 \\
04.4 \\
34.4\end{array}$ & $\begin{array}{l}39.040 \mathrm{~N} . \\
39.344 \mathrm{~N} . \\
40.323 \mathrm{~N} . \\
42.003 \mathrm{~N} . \\
39.996 \mathrm{~N} .\end{array}$ & $\begin{array}{l}111.509 W . \\
111.109 W . \\
111.314 W . \\
112.552 W . \\
111.844 W .\end{array}$ & $\begin{array}{l}0 \\
0 \\
7 \\
4 \\
2\end{array}$ & $\begin{array}{l}\text { UU } \\
\text { UU } \\
\text { UU } \\
\text { UU }\end{array}$ & $\begin{array}{l}\ldots \\
\ldots \\
\cdots \\
\cdots \\
\cdots\end{array}$ & $\begin{array}{l}\ldots \\
\ldots \\
\ldots \\
\ldots \\
\ldots\end{array}$ & $\begin{array}{l}2.7 \mathrm{ML}(U U) \\
3.5 \mathrm{ML}(\mathrm{GS}) \\
2.6 \mathrm{ML} \text { (UU) } \\
3.0 \mathrm{ML} \text { (UU) } \\
2.6 \mathrm{ML} \text { (UU) }\end{array}$ & $\begin{array}{c}\text { FELT } \\
\ldots \\
\ldots \\
\ldots\end{array}$ & $\begin{array}{l}\text { JAN. } \\
\text { MAR. } \\
\text { MAY } \\
\text { MAY } \\
\text { JUN. }\end{array}$ & $\begin{array}{r}7 \\
21 \\
4 \\
12 \\
2\end{array}$ & $\begin{array}{l}18: 59 \\
04: 19 \\
21: 03 \\
08: 20 \\
06: 52\end{array}$ & $\begin{array}{l}\text { MST } \\
\text { MST } \\
\text { MST } \\
\text { MST } \\
\text { MST }\end{array}$ \\
\hline $\begin{array}{l}\text { JUN. } \\
\text { JUN. } \\
\text { AUG. } \\
\text { AUG. } \\
\text { AUG. }\end{array}$ & $\begin{array}{r}8 \\
18 \\
6 \\
9 \\
16\end{array}$ & $\begin{array}{l}21 \\
14 \\
22 \\
19 \\
14\end{array}$ & $\begin{array}{l}52 \\
10 \\
30 \\
08 \\
19\end{array}$ & $\begin{array}{l}21.6 \\
30.9 \\
38.7 \\
07.0 \\
21.7\end{array}$ & $\begin{array}{l}39.733 \mathrm{~N} . \\
40.753 \mathrm{~N} . \\
41.876 \mathrm{~N} . \\
37.650 \mathrm{~N} . \\
39.392 \mathrm{~N} .\end{array}$ & $\begin{array}{l}110.940 W . \\
112.068 W . \\
112.373 W . \\
112.471 W . \\
111.936 W .\end{array}$ & $\begin{array}{l}1 \\
1 \\
2 \\
1 \\
6\end{array}$ & $\begin{array}{l}U U \\
U U \\
U U \\
U U \\
U U\end{array}$ & $\begin{array}{l}\ldots \\
\ldots \\
\ldots \\
\ldots \\
\ldots\end{array}$ & $\begin{array}{l}\cdots \\
\cdots \\
\cdots \\
\cdots \\
\cdots\end{array}$ & $\begin{array}{l}2.6 \mathrm{MD}(\mathrm{UU}) \\
2.7 \mathrm{MD} \text { (UU) } \\
3.0 \mathrm{ML}(\mathrm{UU}) \\
2.5 \mathrm{MD} \text { (UU) } \\
3.7 \mathrm{ML} \text { (UU) }\end{array}$ & $\begin{array}{l}\cdots \\
\cdots \\
\cdots \\
\text { IV }\end{array}$ & $\begin{array}{l}\text { JUN. } \\
\text { JUN. } \\
\text { AUG. } \\
\text { AUG. } \\
\text { AUG. }\end{array}$ & $\begin{array}{r}8 \\
10 \\
6 \\
9 \\
16\end{array}$ & $\begin{array}{l}14: 52 \\
07: 10 \\
15: 30 \\
12: 08 \\
07: 19\end{array}$ & $\begin{array}{l}\text { MST } \\
\text { MST } \\
\text { MST } \\
\text { MST } \\
\text { MST }\end{array}$ \\
\hline $\begin{array}{l}\text { AUG. } \\
\text { SEPT. } \\
\text { SEPT. } \\
\text { OCT. }\end{array}$ & $\begin{array}{r}29 \\
7 \\
30 \\
15\end{array}$ & $\begin{array}{l}09 \\
03 \\
20 \\
23\end{array}$ & $\begin{array}{l}89 \\
12 \\
46 \\
23\end{array}$ & $\begin{array}{l}30.6 \\
26.8 \\
31.7 \\
56.5\end{array}$ & $\begin{array}{l}39.320 \mathrm{~N} . \\
38.536 \mathrm{~N} . \\
41.455 \mathrm{~N} . \\
41.805 \mathrm{~N} .\end{array}$ & $\begin{array}{l}111.162 W . \\
112.287 W . \\
112.410 W . \\
112.402 W .\end{array}$ & $\begin{array}{l}0 \\
1 \\
0 \\
4\end{array}$ & $\begin{array}{l}\text { UU } \\
\text { UU } \\
\text { UU }\end{array}$ & $\begin{array}{l}\cdots \\
\cdots \\
\cdots \\
\cdots\end{array}$ & $\begin{array}{l}\cdots \\
\cdots \\
\cdots \\
\cdots\end{array}$ & $\begin{array}{l}2.7 \mathrm{MD}(\mathrm{UU}) \\
2.5 \mathrm{MD} \text { (UU) } \\
2.8 \mathrm{ML}(\mathrm{UU}) \\
3.4 \mathrm{ML}(\mathrm{UU})\end{array}$ & $\begin{array}{l}\cdots \\
\cdots \\
\text { IV }\end{array}$ & $\begin{array}{l}\text { AUG. } \\
\text { SEPT. } \\
\text { SEPT. } \\
\text { OCT. }\end{array}$ & $\begin{array}{r}29 \\
6 \\
30 \\
15\end{array}$ & $\begin{array}{l}02: 09 \\
20: 12 \\
13: 46 \\
16: 23\end{array}$ & $\begin{array}{l}\text { MST } \\
\text { MST } \\
\text { MST } \\
\text { MST }\end{array}$ \\
\hline
\end{tabular}

\section{VERMONT}

\begin{tabular}{lllllllllllllllll}
\hline AUG. & 25 & 10 & 27 & 04.7 & $44.052 \mathrm{~N}$. & $73.394 W$. & 5 & WO & $\ldots$ & $\ldots$ & $2.5 \mathrm{Mn}($ WO $)$ & $\ldots$ & AUG. & 25 & $05: 27$ & EST
\end{tabular}

VIRGINIA

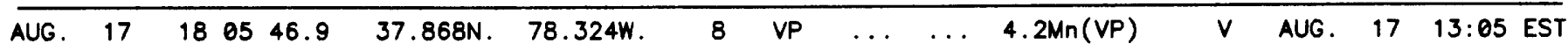

WASHINGTON

\begin{tabular}{|c|c|c|c|c|c|c|c|c|c|c|c|c|c|c|c|}
\hline $\begin{array}{l}\text { JAN. } \\
\text { JAN. } \\
\text { FEB. } \\
\text { FEB. } \\
\text { MAR. }\end{array}$ & $\begin{array}{r}4 \\
13 \\
5 \\
7 \\
14\end{array}$ & $\begin{array}{l}01 \\
21 \\
15 \\
04 \\
08\end{array}$ & $\begin{array}{l}47 \\
07 \\
34 \\
25 \\
50\end{array}$ & $\begin{array}{l}06.1 \\
52.1 \\
30.2 \\
22.2 \\
05.3\end{array}$ & $\begin{array}{l}47.655 \mathrm{~N} \\
46.268 \mathrm{~N} \text {. } \\
46.206 \mathrm{~N} \text {. } \\
46.205 \mathrm{~N} \text {. } \\
47.779 \mathrm{~N} .\end{array}$ & $\begin{array}{l}122.514 W . \\
118.141 W . \\
122.190 W . \\
122.188 W . \\
122.324 W .\end{array}$ & $\begin{array}{r}19 \\
0 \\
1 \\
1 \\
23\end{array}$ & $\begin{array}{l}\text { WA } \\
\text { WA } \\
\text { WA } \\
\text { WA } \\
\text { WA }\end{array}$ & $\begin{array}{l}\ldots \\
\cdots \\
\cdots \\
\cdots \\
\cdots\end{array}$ & $\begin{array}{l}\ldots \\
\cdots \\
\cdots \\
\cdots \\
\cdots\end{array}$ & $\begin{array}{l}2.8 \mathrm{MD} \text { (WA) } \\
2.7 \mathrm{MD} \text { (WA) } \\
2.8 \mathrm{MD} \text { (WA) } \\
2.8 \mathrm{MD} \text { (WA) } \\
2.7 \mathrm{MD} \text { (WA) }\end{array}$ & $\begin{array}{l}\cdots \\
\cdots \\
\cdots \\
\cdots \\
\cdots\end{array}$ & $\begin{array}{l}\text { JAN. } \\
\text { JAN. } \\
\text { FEB. } \\
\text { FEB. } \\
\text { MAR. }\end{array}$ & $\begin{array}{r}3 \\
13 \\
5 \\
6 \\
14\end{array}$ & $\begin{array}{l}17: 47 \\
13: 07 \\
07: 34 \\
20: 25 \\
00: 50\end{array}$ \\
\hline $\begin{array}{l}\text { IAR. } \\
\text { ARR. } \\
\text { AR. } \\
\text { AR. } \\
\text { AR. }\end{array}$ & $\begin{array}{l}16 \\
16 \\
23 \\
28 \\
28\end{array}$ & $\begin{array}{l}02 \\
17 \\
17 \\
11 \\
14\end{array}$ & $\begin{array}{l}35 \\
16 \\
50 \\
50 \\
58\end{array}$ & $\begin{array}{l}30.8 \\
37.2 \\
43.6 \\
03.3 \\
26.4\end{array}$ & $\begin{array}{l}48.482 \mathrm{~N} \\
48.454 \mathrm{~N} \\
47.709 \mathrm{~N} . \\
46.206 \mathrm{~N} \\
46.208 \mathrm{~N} .\end{array}$ & $\begin{array}{l}121.685 w . \\
121.705 w . \\
122.642 W . \\
122.184 W . \\
122.189 w .\end{array}$ & $\begin{array}{r}5 \\
0 \\
19 \\
1 \\
1\end{array}$ & $\begin{array}{l}\text { WA } \\
\text { WA } \\
\text { WA } \\
\text { WA } \\
\text { WA }\end{array}$ & $\begin{array}{l}\ldots \\
\ldots \\
\ldots \\
\cdots \\
\cdots\end{array}$ & $\begin{array}{l}\ldots \\
\ldots \\
\cdots \\
\cdots \\
\cdots\end{array}$ & $\begin{array}{l}2.6 \mathrm{MD} \text { (WA) } \\
3.4 \mathrm{MD} \text { (WA) } \\
2.9 \mathrm{MD} \text { (WA) } \\
2.7 \mathrm{MD} \text { (WA) } \\
3.0 \mathrm{MD} \text { (WA) }\end{array}$ & $\begin{array}{c}\text { FELT } \\
\text { FELT } \\
\ldots \\
\ldots \\
\ldots\end{array}$ & $\begin{array}{l}\text { MAR. } \\
\text { MAR. } \\
\text { MAR. } \\
\text { MAR. } \\
\text { MAR. }\end{array}$ & $\begin{array}{l}15 \\
16 \\
23 \\
28 \\
28\end{array}$ & $\begin{array}{l}18: 35 \\
09: 16 \\
09: 50 \\
03: 50 \\
06: 58\end{array}$ \\
\hline $\begin{array}{l}\text { MAR. } \\
\text { MAR. } \\
\text { APR. } \\
\text { APR. } \\
\text { APR. }\end{array}$ & $\begin{array}{r}29 \\
29 \\
8 \\
11 \\
27\end{array}$ & $\begin{array}{l}02 \\
02 \\
12 \\
03 \\
23\end{array}$ & $\begin{array}{l}25 \\
56 \\
56 \\
07 \\
06\end{array}$ & $\begin{array}{l}19.1 \\
26.1 \\
59.8 \\
42.1 \\
58.1\end{array}$ & $\begin{array}{l}46.205 \mathrm{~N} \\
46.208 \mathrm{~N} \\
46.800 \mathrm{~N} \\
47.535 \mathrm{~N} \\
47.609 \mathrm{~N} .\end{array}$ & $\begin{array}{l}122.188 w . \\
122.189 w . \\
122.470 w . \\
120.186 w . \\
121.964 W .\end{array}$ & $\begin{array}{r}1 \\
0 \\
67 \\
8 \\
10\end{array}$ & $\begin{array}{l}\text { WA } \\
\text { WA } \\
\text { WA } \\
\text { WA } \\
\text { WA }\end{array}$ & $\begin{array}{l}\cdots \\
\cdots \\
\cdots \\
\cdots \\
\cdots\end{array}$ & $\begin{array}{l}\ldots \\
\cdots \\
\cdots \\
\cdots \\
\cdots\end{array}$ & $\begin{array}{l}\text { 2. 7MD (WA) } \\
2.9 M D \text { (WA) } \\
3.3 \mathrm{MD} \text { (WA) } \\
3.6 \mathrm{ML} \text { (GS) } \\
2.9 \mathrm{MD} \text { (WA) }\end{array}$ & $\begin{array}{l}\cdots \\
\cdots \\
\cdots \\
\text { IV }\end{array}$ & $\begin{array}{l}\text { MAR. } \\
\text { MAR. } \\
\text { APR. } \\
\text { APR. } \\
\text { APR. }\end{array}$ & $\begin{array}{r}28 \\
28 \\
8 \\
10 \\
27\end{array}$ & $\begin{array}{l}18: 25 \\
18: 56 \\
04: 56 \\
19: 07 \\
15: 06\end{array}$ \\
\hline $\begin{array}{l}\text { APR. } \\
\text { APR. } \\
\text { JUNE } \\
\text { JUNE } \\
\text { JUNE }\end{array}$ & $\begin{array}{r}29 \\
30 \\
2 \\
2 \\
4\end{array}$ & $\begin{array}{l}22 \\
11 \\
12 \\
13 \\
04\end{array}$ & $\begin{array}{l}11 \\
02 \\
57 \\
02 \\
46\end{array}$ & $\begin{array}{l}34.5 \\
32.8 \\
21.0 \\
40.3 \\
01.7\end{array}$ & $\begin{array}{l}46.672 \mathrm{~N} \\
46.041 \mathrm{~N} . \\
47.415 \mathrm{~N} . \\
47.416 \mathrm{~N} . \\
46.214 \mathrm{~N} .\end{array}$ & $\begin{array}{l}118.975 w . \\
119.878 w . \\
122.702 W . \\
122.703 w . \\
123.062 W .\end{array}$ & $\begin{array}{r}2 \\
0 \\
21 \\
23 \\
53\end{array}$ & $\begin{array}{l}\text { WA } \\
\text { WA } \\
\text { WA } \\
\text { WA } \\
\text { WA }\end{array}$ & $\begin{array}{l}\ldots \\
\ldots \\
\ldots \\
\ldots \\
\ldots\end{array}$ & $\begin{array}{l}\cdots \\
\cdots \\
\cdots \\
\ldots\end{array}$ & $\begin{array}{l}2.8 \mathrm{MD} \text { (WA) } \\
2.8 \mathrm{MD} \text { (WA) } \\
3.5 \mathrm{ML} \text { (GS) } \\
2.8 \mathrm{MD} \text { (WA) } \\
3.7 \mathrm{MD} \text { (WA) }\end{array}$ & $\begin{array}{l}\cdots \\
\text { IV } \\
\text { IV }\end{array}$ & $\begin{array}{l}\text { APR. } \\
\text { APR. } \\
\text { JUNE } \\
\text { JUNE } \\
\text { JUNE }\end{array}$ & $\begin{array}{r}29 \\
30 \\
2 \\
2 \\
3\end{array}$ & $\begin{array}{l}14: 11 \\
03: 02 \\
04: 57 \\
05: 02 \\
20: 46\end{array}$ \\
\hline
\end{tabular}


Table 1. Summary of U.S. earthquakes for 1984-Continued

\begin{tabular}{|c|c|c|c|c|c|c|c|c|c|c|c|c|c|}
\hline \multirow{2}{*}{ Date } & \multicolumn{2}{|c|}{$\begin{array}{c}\text { Origin time } \\
\text { (UTC) }\end{array}$} & \multirow{2}{*}{$\begin{array}{c}\text { Latitude } \\
\left({ }^{\circ}\right)\end{array}$} & \multirow{2}{*}{$\begin{array}{c}\text { Longitude } \\
\text { (') }\end{array}$} & \multirow{2}{*}{$\begin{array}{c}\text { Depth } \\
(\mathbf{k m})\end{array}$} & \multirow{2}{*}{$\begin{array}{l}\text { Hypo- } \\
\text { center } \\
\text { source }\end{array}$} & \multicolumn{3}{|c|}{ Magnitude } & \multirow{2}{*}{$\begin{array}{l}\text { Maximum } \\
\text { intensity }\end{array}$} & \multicolumn{3}{|c|}{ local time } \\
\hline & hr $\min$ & $\sec$ & & & & & $\mathbf{m b}$ & MS & $\begin{array}{l}\text { ML, Mn } \\
M D, M w\end{array}$ & & Date & Hour & $\begin{array}{l}\text { Time } \\
\text { zone }\end{array}$ \\
\hline
\end{tabular}

\section{WASHINGTON-Continued}

\begin{tabular}{|c|c|c|c|c|c|c|c|c|c|c|c|c|c|c|c|}
\hline $\begin{array}{l}\text { JUNE } \\
\text { JULY } \\
\text { JULY } \\
\text { JULY } \\
\text { AUG. }\end{array}$ & $\begin{array}{r}19 \\
10 \\
16 \\
24 \\
5\end{array}$ & $\begin{array}{l}03 \\
12 \\
07 \\
22 \\
19\end{array}$ & $\begin{array}{l}23 \\
42 \\
38 \\
12 \\
11\end{array}$ & $\begin{array}{l}57.5 \\
33.4 \\
53.9 \\
56.3 \\
50.7\end{array}$ & $\begin{array}{l}47.703 \mathrm{~N} . \\
48.612 \mathrm{~N} \\
46.429 \mathrm{~N} . \\
47.740 \mathrm{~N} . \\
46.447 \mathrm{~N} .\end{array}$ & $\begin{array}{l}122.957 w . \\
122.057 w . \\
122.272 w . \\
122.423 w . \\
122.261 w .\end{array}$ & $\begin{array}{r}9 \\
3 \\
15 \\
21 \\
11\end{array}$ & $\begin{array}{l}\text { WA } \\
\text { WA } \\
\text { WA } \\
\text { WA } \\
\text { WA }\end{array}$ & $\begin{array}{l}\cdots \\
\cdots \\
\cdots \\
\cdots \\
\cdots\end{array}$ & $\begin{array}{l}\cdots \\
\cdots \\
\cdots \\
\cdots \\
\cdots\end{array}$ & $\begin{array}{l}\text { 3. } 0 \mathrm{MD} \text { (WA) } \\
2.4 \mathrm{ML} \text { (GS) } \\
2.8 \mathrm{MD} \text { (WA) } \\
2.7 \mathrm{MD} \text { (WA) } \\
3.1 \mathrm{MD} \text { (WA) }\end{array}$ & $\begin{array}{l}\text { I I I } \\
\cdots \\
\cdots \\
\cdots\end{array}$ & $\begin{array}{l}\text { JUNE } \\
\text { JULY } \\
\text { JULY } \\
\text { JULY } \\
\text { AUG. }\end{array}$ & $\begin{array}{r}18 \\
10 \\
15 \\
24 \\
5\end{array}$ & $\begin{array}{l}19: 23 \\
04: 42 \\
23: 38 \\
14: 12 \\
11: 11\end{array}$ \\
\hline $\begin{array}{l}\text { AUG. } \\
\text { SEPT. } \\
\text { SEPT. } \\
\text { OCT. } \\
\text { NOV. }\end{array}$ & $\begin{array}{l}24 \\
18 \\
20 \\
10 \\
16\end{array}$ & $\begin{array}{l}04 \\
16 \\
02 \\
03 \\
20\end{array}$ & $\begin{array}{l}43 \\
54 \\
16 \\
24 \\
59\end{array}$ & $\begin{array}{l}07.2 \\
13.3 \\
03.5 \\
18.2 \\
54.1\end{array}$ & $\begin{array}{l}47.650 \mathrm{~N} . \\
48.142 \mathrm{~N} . \\
47.494 \mathrm{~N} . \\
47.904 \mathrm{~N} . \\
48.275 \mathrm{~N} .\end{array}$ & $\begin{array}{l}120.955 w . \\
122.596 w . \\
122.306 w . \\
119.079 w . \\
122.190 w .\end{array}$ & $\begin{array}{r}1 \\
2 \\
27 \\
15 \\
14\end{array}$ & $\begin{array}{l}\text { WA } \\
\text { WA } \\
\text { WA } \\
\text { WA } \\
\text { WA }\end{array}$ & $\begin{array}{l}\cdots \\
\cdots \\
\cdots \\
\cdots \\
\cdots\end{array}$ & $\begin{array}{l}\cdots \\
\cdots \\
\cdots \\
\cdots \\
\cdots\end{array}$ & $\begin{array}{l}2.8 \mathrm{ML} \text { (GS) } \\
3.0 \mathrm{MD} \text { (WA) } \\
2.7 \mathrm{MD} \text { (WA) } \\
2.5 \mathrm{ML} \text { (GS) } \\
2.7 \mathrm{MD} \text { (WA) }\end{array}$ & $\begin{array}{c}\text { FELT } \\
\cdots \\
\cdots \\
\quad \cdots \\
\cdots\end{array}$ & $\begin{array}{l}\text { AUG. } \\
\text { SEPT } \\
\text { SEPT } \\
\text { OCT. } \\
\text { NOV. }\end{array}$ & $\begin{array}{r}23 \\
18 \\
19 \\
9 \\
16\end{array}$ & $\begin{array}{l}20: 43 \\
08: 54 \\
18: 16 \\
19: 24 \\
12: 59\end{array}$ \\
\hline $\begin{array}{l}\text { NOV. } \\
\text { NOV. } \\
\text { NOV. } \\
\text { DEC. } \\
\text { DEC. }\end{array}$ & $\begin{array}{r}17 \\
21 \\
30 \\
2 \\
3\end{array}$ & $\begin{array}{l}00 \\
09 \\
11 \\
16 \\
19\end{array}$ & $\begin{array}{l}28 \\
50 \\
07 \\
17 \\
03\end{array}$ & $\begin{array}{l}04.9 \\
47.5 \\
00.0 \\
24.6 \\
21.9\end{array}$ & $\begin{array}{l}46.905 \mathrm{~N} . \\
46.899 \mathrm{~N} . \\
47.682 \mathrm{~N} \\
48.592 \mathrm{~N} . \\
48.588 \mathrm{~N} .\end{array}$ & $\begin{array}{l}121.783 \mathrm{~W} . \\
123.684 \mathrm{~W} . \\
122.179 \mathrm{~W} . \\
121.806 \mathrm{~W} . \\
121.808 \mathrm{~W}\end{array}$ & $\begin{array}{r}1 \\
36 \\
24 \\
0 \\
0\end{array}$ & $\begin{array}{l}\text { WA } \\
\text { WA } \\
\text { WA } \\
\text { WA } \\
\text { WA }\end{array}$ & $\begin{array}{l}\cdots \\
\cdots \\
\cdots\end{array}$ & $\begin{array}{l}\cdots \\
\cdots \\
\cdots\end{array}$ & $\begin{array}{l}\text { 3. } 1 \mathrm{MD} \text { (WA) } \\
\text { 2. } 8 \mathrm{MD}(\mathrm{TU}) \\
\text { 2. } 7 \mathrm{MD} \text { (WA) } \\
\text { 3. 3ML (GS) } \\
\text { 2.9ML (GS) }\end{array}$ & $\begin{array}{l}\text { I I I } \\
\text { I I I }\end{array}$ & $\begin{array}{l}\text { NOV. } \\
\text { NOV. } \\
\text { NOV. } \\
\text { DEC. } \\
\text { DEC. }\end{array}$ & $\begin{array}{r}16 \\
21 \\
30 \\
2 \\
3\end{array}$ & $\begin{array}{l}16: 28 \\
01: 50 \\
03: 07 \\
08: 17 \\
11: 03\end{array}$ \\
\hline $\begin{array}{l}\text { DEC. } \\
\text { DEC. } \\
\text { DEC. } \\
\text { DEC. }\end{array}$ & $\begin{array}{l}17 \\
18 \\
23 \\
27\end{array}$ & $\begin{array}{l}08 \\
18 \\
19 \\
20\end{array}$ & $\begin{array}{l}30 \\
24 \\
07 \\
50\end{array}$ & $\begin{array}{l}12.6 \\
20.4 \\
56.2 \\
39.2\end{array}$ & $\begin{array}{l}47.274 \mathrm{~N} . \\
47.280 \mathrm{~N} . \\
48.591 \mathrm{~N} . \\
46.841 \mathrm{~N} .\end{array}$ & $\begin{array}{l}122.832 W . \\
117.136 w . \\
121.810 W . \\
121.953 W .\end{array}$ & $\begin{array}{r}46 \\
25 \\
3 \\
12\end{array}$ & $\begin{array}{l}\text { WA } \\
\text { WA } \\
\text { WA } \\
\text { WA }\end{array}$ & $\begin{array}{l}\cdots \\
\cdots \\
\cdots \\
\cdots\end{array}$ & $\begin{array}{l}\cdots \\
\cdots \\
\cdots \\
\cdots\end{array}$ & $\begin{array}{l}\text { 3. } 2 \mathrm{MD} \text { (WA) } \\
2.8 \mathrm{MD} \text { (WA) } \\
2.9 \mathrm{MD} \text { (WA) } \\
2.7 \mathrm{MD} \text { (WA) }\end{array}$ & $\cdots$ & $\begin{array}{l}\text { DEC. } \\
\text { DEC. } \\
\text { DEC. } \\
\text { DEC. }\end{array}$ & $\begin{array}{l}17 \\
18 \\
23 \\
27\end{array}$ & $\begin{array}{l}00: 30 \\
10: 24 \\
11: 07 \\
12: 50\end{array}$ \\
\hline
\end{tabular}

WYOMING

\begin{tabular}{|c|c|c|c|c|c|c|c|c|c|c|c|c|c|c|c|c|}
\hline $\begin{array}{l}\text { AN. } \\
\text { AN. } \\
\text { AN. } \\
\text { AN. } \\
\text { AR. }\end{array}$ & $\begin{array}{r}5 \\
14 \\
17 \\
24 \\
1\end{array}$ & $\begin{array}{l}20 \\
19 \\
20 \\
07 \\
18\end{array}$ & $\begin{array}{l}10 \\
54 \\
54 \\
12 \\
13\end{array}$ & $\begin{array}{l}24.3 \\
25.1 \\
40.2 \\
47.1 \\
00.9\end{array}$ & $\begin{array}{l}43.316 \mathrm{~N} . \\
44.801 \mathrm{~N} . \\
44.130 \mathrm{~N} . \\
44.453 \mathrm{~N} . \\
41.539 \mathrm{~N} .\end{array}$ & $\begin{array}{l}110.757 w . \\
111.033 w . \\
110.441 w . \\
110.519 w . \\
108.638 w .\end{array}$ & $\begin{array}{l}5 \\
5 \\
1 \\
5 \\
2\end{array}$ & $\begin{array}{l}\text { UU } \\
\text { GS } \\
\text { UU }\end{array}$ & $\begin{array}{l}\cdots \\
\cdots \\
\cdots \\
\cdots\end{array}$ & $\begin{array}{l}\cdots \\
\cdots \\
\cdots \\
\cdots\end{array}$ & $\begin{array}{l}\text { 3. } 0 \mathrm{ML} \text { (GS) } \\
\text { 3. } 4 \mathrm{ML}(\mathrm{GS}) \\
\text { 2.6MD(UU) } \\
\text { 3. } 3 \mathrm{ML}(\mathrm{BU}) \\
\text { 3. } \mathrm{MD} \text { (UU) }\end{array}$ & $\begin{array}{c}\text { FELT } \\
\ldots \\
\ldots \\
\text { IV } \\
\ldots\end{array}$ & $\begin{array}{l}\text { JAN. } \\
\text { JAN. } \\
\text { JAN. } \\
\text { JAN. } \\
\text { MAR. }\end{array}$ & $\begin{array}{r}5 \\
14 \\
17 \\
24 \\
1\end{array}$ & $\begin{array}{l}13: 10 \\
12: 54 \\
13: 54 \\
00: 12 \\
11: 13\end{array}$ & $\begin{array}{l}\text { MST } \\
\text { MST } \\
\text { MST } \\
\text { MST } \\
\text { MST }\end{array}$ \\
\hline $\begin{array}{l}\text { AR. } \\
\text { AY } \\
\text { AY } \\
\text { AY } \\
\text { JNE }\end{array}$ & $\begin{array}{r}24 \\
4 \\
29 \\
29 \\
13\end{array}$ & $\begin{array}{l}05 \\
04 \\
07 \\
20 \\
06\end{array}$ & $\begin{array}{l}21 \\
02 \\
45 \\
18 \\
17\end{array}$ & $\begin{array}{l}51.8 \\
30.0 \\
33.7 \\
32.6 \\
08.9\end{array}$ & $\begin{array}{l}43.329 \mathrm{~N} . \\
44.637 \mathrm{~N} \\
44.425 \mathrm{~N} \\
44.232 \mathrm{~N} \\
44.857 \mathrm{~N} .\end{array}$ & $\begin{array}{l}110.782 W . \\
110.063 W . \\
111.007 w . \\
105.965 W . \\
110.920 W .\end{array}$ & $\begin{array}{r}5 \\
5 \\
7 \\
18 \\
4\end{array}$ & $\begin{array}{l}\text { GS } \\
\text { BU }\end{array}$ & $\begin{array}{l}\ldots \\
\ldots \\
5.0 \\
\ldots\end{array}$ & $\begin{array}{l}\ldots \\
\ldots \\
\cdots \\
\cdots\end{array}$ & $\begin{array}{c}2.8 \mathrm{ML}(\mathrm{GS}) \\
3.1 \mathrm{ML}(\mathrm{BU}) \\
2.8 \mathrm{ML}(\mathrm{BU}) \\
\ldots \ldots \\
2.5 \mathrm{ML}(\mathrm{BU})\end{array}$ & $\begin{array}{l}\text { I I } \\
\cdots \\
\cdots \\
\cdots\end{array}$ & $\begin{array}{l}\text { MAR. } \\
\text { MAY } \\
\text { MAY } \\
\text { MAY } \\
\text { JUNE }\end{array}$ & $\begin{array}{r}23 \\
3 \\
29 \\
29 \\
12\end{array}$ & $\begin{array}{l}22: 21 \\
21: 02 \\
00: 45 \\
13: 18 \\
23: 17\end{array}$ & $\begin{array}{l}\text { MST } \\
\text { MST } \\
\text { MST } \\
\text { MST } \\
\text { MST }\end{array}$ \\
\hline $\begin{array}{l}\text { EP. } \\
\text { EPT. } \\
\text { CT. } \\
\text { CT. } \\
\text { CT. }\end{array}$ & $\begin{array}{r}14 \\
8 \\
1 \\
1 \\
6\end{array}$ & $\begin{array}{l}19 \\
00 \\
15 \\
17 \\
20\end{array}$ & $\begin{array}{l}04 \\
59 \\
01 \\
48 \\
00\end{array}$ & $\begin{array}{l}26.3 \\
31.1 \\
55.2 \\
12.1 \\
03.5\end{array}$ & $\begin{array}{l}41.610 \mathrm{~N} . \\
44.240 \mathrm{~N} . \\
44.754 \mathrm{~N} . \\
44.743 \mathrm{~N} . \\
44.917 \mathrm{~N} .\end{array}$ & $\begin{array}{l}108.582 W . \\
106.019 W . \\
110.504 W . \\
110.491 W . \\
110.484 W .\end{array}$ & $\begin{array}{r}2 \\
20 \\
2 \\
2 \\
5\end{array}$ & & $\begin{array}{l}5.1 \\
\cdots \\
\ldots \\
\ldots\end{array}$ & $\begin{array}{l}\ldots \\
\cdots \\
\cdots \\
\cdots \\
\cdots\end{array}$ & $\begin{array}{l}\text { 3. } 2 \mathrm{MD}(\mathrm{UU}) \\
5.6 \mathrm{ML}(\mathrm{BU}) \\
2.9 \mathrm{ML}(\mathrm{BU}) \\
2.9 \mathrm{ML}(\mathrm{BU}) \\
2.5 \mathrm{ML}(\mathrm{BU})\end{array}$ & $\begin{array}{l}\cdots \\
\dot{v} \\
\cdots \\
\cdots\end{array}$ & $\begin{array}{l}\text { SEP. } \\
\text { SEPT. } \\
\text { OCT. } \\
\text { OCT. } \\
\text { OCT. }\end{array}$ & $\begin{array}{r}14 \\
7 \\
1 \\
1 \\
6\end{array}$ & $\begin{array}{l}12: 04 \\
17: 59 \\
08: 01 \\
10: 48 \\
13: 00\end{array}$ & $\begin{array}{l}\text { MST } \\
\text { MST } \\
\text { MST } \\
\text { MST } \\
\text { MST }\end{array}$ \\
\hline $\begin{array}{l}\text { CT. } \\
\text { CT. } \\
\text { CT. } \\
\text { CT. }\end{array}$ & $\begin{array}{l}6 \\
6 \\
6 \\
6 \\
7\end{array}$ & $\begin{array}{l}20 \\
20 \\
20 \\
21 \\
04\end{array}$ & $\begin{array}{l}04 \\
30 \\
34 \\
29 \\
37\end{array}$ & $\begin{array}{l}02.7 \\
13.5 \\
28.2 \\
31.8 \\
21.9\end{array}$ & $\begin{array}{l}44.680 \mathrm{~N} . \\
44.743 \mathrm{~N} . \\
44.819 \mathrm{~N} . \\
44.782 \mathrm{~N} . \\
44.769 \mathrm{~N} .\end{array}$ & $\begin{array}{l}110.625 W . \\
110.528 W . \\
110.667 W . \\
110.602 W . \\
110.590 W .\end{array}$ & $\begin{array}{r}3 \\
5 \\
5 \\
14 \\
1\end{array}$ & & $\begin{array}{l}\ldots \\
\ldots \\
\ldots \\
\ldots\end{array}$ & $\begin{array}{l}\ldots \\
\ldots \\
\ldots \\
\cdots \\
\cdots\end{array}$ & $\begin{array}{l}2.5 \mathrm{ML}(\mathrm{BU}) \\
3.2 \mathrm{ML}(\mathrm{GS}) \\
2.6 \mathrm{ML}(\mathrm{BU}) \\
2.8 \mathrm{ML}(\mathrm{BU}) \\
2.7 \mathrm{ML}(\mathrm{BU})\end{array}$ & $\begin{array}{l}\ldots \\
\text { IV } \\
\ldots \\
\cdots\end{array}$ & $\begin{array}{l}\text { OCT. } \\
\text { OCT. } \\
\text { OCT. } \\
\text { OCT. } \\
\text { OCT. }\end{array}$ & $\begin{array}{l}6 \\
6 \\
6 \\
6 \\
6\end{array}$ & $\begin{array}{l}13: 04 \\
13: 30 \\
13: 34 \\
14: 29 \\
21: 37\end{array}$ & $\begin{array}{l}\text { MST } \\
\text { MST } \\
\text { MST } \\
\text { MST } \\
\text { MST }\end{array}$ \\
\hline OCT . & $\begin{array}{l}12 \\
18 \\
18 \\
18 \\
19\end{array}$ & $\begin{array}{l}05 \\
15 \\
15 \\
17 \\
16\end{array}$ & $\begin{array}{l}52 \\
30 \\
57 \\
38 \\
29\end{array}$ & $\begin{array}{l}32.7 \\
23.0 \\
37.3 \\
27.4 \\
04.4\end{array}$ & $\begin{array}{l}44.784 \mathrm{~N} . \\
42.375 \mathrm{~N} \\
42.365 \mathrm{~N} \\
42.412 \mathrm{~N} \\
42.408 \mathrm{~N} .\end{array}$ & $\begin{array}{l}110.634 W . \\
105.720 W . \\
105.805 W . \\
105.767 W . \\
105.772 W .\end{array}$ & $\begin{array}{c}4 \\
33 N \\
33 N \\
33 N \\
33 N\end{array}$ & $\begin{array}{l}\text { BU } \\
\text { GS } \\
\text { GS } \\
\text { GS } \\
\text { GS }\end{array}$ & $\begin{array}{l}5.4 \\
4.5 \\
\ldots \\
\ldots\end{array}$ & $\begin{array}{l}5.1 \\
\ldots \\
\cdots \\
\cdots\end{array}$ & $\begin{array}{l}\text { 2.5ML(BU) } \\
5.5 \mathrm{ML}(\mathrm{GS}) \\
\text { 4.2ML(GS) } \\
\text { 3. } 8 \mathrm{ML}(\mathrm{GS}) \\
\text { 3. } 3 \mathrm{ML}(\mathrm{GS})\end{array}$ & $\begin{array}{l}\text { VI } \\
\text { IV }\end{array}$ & & $\begin{array}{l}11 \\
18 \\
18 \\
18 \\
19\end{array}$ & $\begin{array}{l}22: 52 \\
08: 30 \\
08: 57 \\
10: 38 \\
09: 29\end{array}$ & $\begin{array}{l}\text { MST } \\
\text { MST } \\
\text { MST } \\
\text { MST } \\
\text { MST }\end{array}$ \\
\hline $\begin{array}{l}\text { OCT. } \\
\text { OCT. } \\
\text { OCT. } \\
\text { NOV. }\end{array}$ & $\begin{array}{r}20 \\
22 \\
24 \\
29 \\
3\end{array}$ & $\begin{array}{l}11 \\
11 \\
09 \\
19 \\
04\end{array}$ & $\begin{array}{l}51 \\
17 \\
03 \\
08 \\
23\end{array}$ & $\begin{array}{l}08.6 \\
56.3 \\
54.7 \\
00.1 \\
06.0\end{array}$ & $\begin{array}{l}42.401 \mathrm{~N} . \\
42.396 \mathrm{~N} . \\
42.322 \mathrm{~N} . \\
44.351 \mathrm{~N} . \\
43.551 \mathrm{~N} .\end{array}$ & $\begin{array}{l}105.874 W . \\
105.877 w . \\
105.717 w . \\
105.995 W . \\
110.813 w .\end{array}$ & $\begin{array}{l}33 N \\
33 N \\
22 \\
20 \\
5\end{array}$ & $\begin{array}{l}\text { GS } \\
\text { GS } \\
\text { GS } \\
\text { GS }\end{array}$ & $\begin{array}{l}\cdots \\
\cdots \\
\ldots\end{array}$ & $\begin{array}{l}\ldots \\
\ldots \\
\ldots \\
\ldots \\
\ldots\end{array}$ & $\begin{array}{l}\text { 3.5ML (GS) } \\
\text { 3.1ML (GS) } \\
\text { 3.2ML (GS) } \\
2.5 M L(G S) \\
2.6 M L(G S)\end{array}$ & $\begin{array}{r}\text { FELT } \\
\text { I I I }\end{array}$ & $\begin{array}{l}\text { OCT. } \\
\text { OCT. } \\
\text { OCT. } \\
\text { NOV. }\end{array}$ & $\begin{array}{r}20 \\
22 \\
24 \\
29 \\
2\end{array}$ & $\begin{array}{l}04: 51 \\
04: 17 \\
02: 03 \\
12: 08 \\
21: 23\end{array}$ & $\begin{array}{l}\text { MST } \\
\text { MST } \\
\text { MST } \\
\text { MST } \\
\text { MST }\end{array}$ \\
\hline
\end{tabular}


Table 1. Summary of U.S. earthquakes for 1984-Continued

\begin{tabular}{|c|c|c|c|c|c|c|c|c|c|c|c|c|c|c|c|c|}
\hline \multirow{2}{*}{ Dat } & & \multicolumn{3}{|c|}{$\begin{array}{l}\text { Origin time } \\
\text { (UTC) }\end{array}$} & \multirow{2}{*}{$\begin{array}{l}\text { Latitude } \\
\left({ }^{\circ}\right)\end{array}$} & \multirow{2}{*}{$\begin{array}{l}\text { Longitude } \\
\text { ( })\end{array}$} & \multirow{2}{*}{$\begin{array}{c}\text { Depth } \\
(\mathbf{k m})\end{array}$} & \multirow{2}{*}{$\begin{array}{l}\text { Hypo- } \\
\text { center } \\
\text { source }\end{array}$} & \multicolumn{3}{|c|}{ Magnitude } & \multirow{2}{*}{$\begin{array}{l}\text { Maximum } \\
\text { intensity }\end{array}$} & \multicolumn{4}{|c|}{ Local time } \\
\hline & & hr & $\min$ & $\sec$ & & & & & $\mathbf{m b}$ & MS & $\begin{array}{l}\text { ML. Mn } \\
\text { MD. Mw }\end{array}$ & & Date & & Hour & $\begin{array}{l}\text { Time } \\
\text { zome }\end{array}$ \\
\hline \multicolumn{17}{|c|}{ W YOMING-Continued } \\
\hline $\begin{array}{l}\text { NOV. } \\
\text { NOV. } \\
\text { NOV. } \\
\text { DEC. } \\
\text { DEC. }\end{array}$ & $\begin{array}{r}3 \\
6 \\
27 \\
6 \\
17\end{array}$ & $\begin{array}{l}09 \\
11 \\
20 \\
04 \\
09\end{array}$ & $\begin{array}{l}30 \\
38 \\
00 \\
04 \\
31\end{array}$ & $\begin{array}{l}08.5 \\
52.5 \\
07.2 \\
52.3 \\
32.2\end{array}$ & $\begin{array}{l}42.494 \mathrm{~N} \\
42.306 \mathrm{~N} \\
41.595 \mathrm{~N} \\
42.442 \mathrm{~N} \\
42.362 \mathrm{~N}\end{array}$ & $\begin{array}{l}108.854 W . \\
105.707 W . \\
108.692 W . \\
105.821 W . \\
105.733 W .\end{array}$ & $\begin{array}{c}5 \\
33 N \\
0 \\
20 \\
33 N\end{array}$ & $\begin{array}{l}\text { GS } \\
\text { GS } \\
\text { GS } \\
\text { GS } \\
\text { GS }\end{array}$ & $\begin{array}{l}5.0 \\
\ldots \\
\ldots \\
\ldots \\
\ldots\end{array}$ & $\begin{array}{l}4.1 \\
\ldots \\
\ldots \\
\ldots \\
\ldots\end{array}$ & $\begin{array}{l}\text { 5. } 1 M L \text { (UU) } \\
\text { 3. } 3 M L(G S) \\
\text { 3. } 2 M L(G S) \\
\text { 2. } 9 M L(G S) \\
\text { 3. } 3 M L(G S)\end{array}$ & $\begin{array}{l}V I \\
\cdots \\
\cdots \\
\cdots \\
\cdots\end{array}$ & $\begin{array}{l}\text { NOV. } \\
\text { NOV. } \\
\text { NOV. } \\
\text { DEC. } \\
\text { DEC. }\end{array}$ & $\begin{array}{r}3 \\
6 \\
27 \\
5 \\
17\end{array}$ & $\begin{array}{l}02: 30 \\
04: 38 \\
13: 00 \\
21: 04 \\
02: 31\end{array}$ & $\begin{array}{l}\text { MST } \\
\text { MST } \\
\text { MST } \\
\text { MST } \\
\text { MST }\end{array}$ \\
\hline
\end{tabular}





\section{NETWORK OPERATIONS}

\section{Eastern Aleutian Seismicity}

\author{
By J. J. Taber, M. A. Luckman and S. Rosen \\ Lamont-Doherty Geological Observatory of \\ Columbia University \\ Palisades, NY 10964
}

There were 655 events located by the Shumagin seismic network in 1984, with 73 events greater than or equal to magnitude 3.0 . This seismicity is shown in map view in figure 29 and in cross section in figure 30 . The coverage within the network is uniform at about the magnitude 2.0 level but events as small as 0.4 can be located in some areas. The overall pattern over this time period is similar to previous years, though the double-planed Benioff zone between 50 and 150 kilometers depth is only weakly determined. The highest concentration of events occurs along the base of the shallow thrust zone on a line roughly from station NGI to station SNK (fig. 31). The thrust zone itself, which is assumed to be the contact between the Pacific and North American plates, is poorly defined. West of the network the seismicity is more diffuse and extends further offshore, i.e., closer to the trench.

The only unusual activity consisted of a shallow (less than 10 kilometers) earthquake swarm that began in August under Mt. Dutton, an extinct volcano of uncertain age near $55.25^{\prime \prime} \mathrm{N}$., $162.25^{\prime \prime} \mathrm{W}$. This was the largest swarm we have recorded on the peninsula since the network was installed in 1973. There were 35 events located between 8-20-84 and 12-30-84 with at least 20 additional smaller events near the beginning of the swarm. Occasional events continued into March 1985. The biggest event in the swarm was $\mathbf{M}_{L}=2.9$ with most magnitudes measuring below 1.5 .

The Shumagin seismic network consists of shortperiod, high-gain seismic stations, a few low-gain stations, and strong motion accelerographs. The data from the different sets of instrumentation are being applied to ground motion, seismic source, earthquake prediction, tectonic and volcanological studies. The network includes 13 remote stations plus four stations in the Pavlof Volcano subarray and the local station at Sand Point (SAN) (fig. 31). There are 12 stations with a single vertical seismometer and 6 threecomponent stations. There is one digital strong-motion recorder and 11 analog strong-motion accelerographs (SMA-1) with the network. Nine of these SMA's are co-located with high-gain stations and are connected to the telemetry system, allowing us to know the exact time at which the SMA began recording a given earthquake.

A bulletin listing solutions for all located events and individual pickfiles for all events greater than magnitude 3.0 (including some regional events not shown on the map) can be obtained from the authors.

\section{Northern and Central California Earthquakes, 1984}

By Robert A. Uhrhammer

Seismographic Stations

475 Earth Sciences Building

University of California

Berkeley, CA 94720

The seismographic stations operated a network of 19 stations (shown as triangles in fig. 32) during 1984. The most significant change in the network occurred at Jamestown (JAS), where the station was moved to a temporary site (JAS1; $\left.37^{\prime \prime} 55.6^{\prime} \mathrm{N} ., 120^{\prime \prime} 25.2^{\prime} \mathrm{W}.\right), 3$ kilometers to the south. A search is currently underway for a new permanent site.

During 1984, the occurrences of approximately 5,280 seismic events were cataloged on summary sheets, and 905 teleseisms and 567 local earthquakes were analyzed. The Bulletin of the Seismographic Stations, v. 54, nos. 1 and 2 (Lomax and Uhrhammer, 1985), contains locations and magnitude information for 335 earthquakes (3.0 $\leq \mathbf{M}_{L} \leq$ to 6.6 ) located in northern and central California and adjoining regions. The epicentral locations are plotted in figure 32 . 


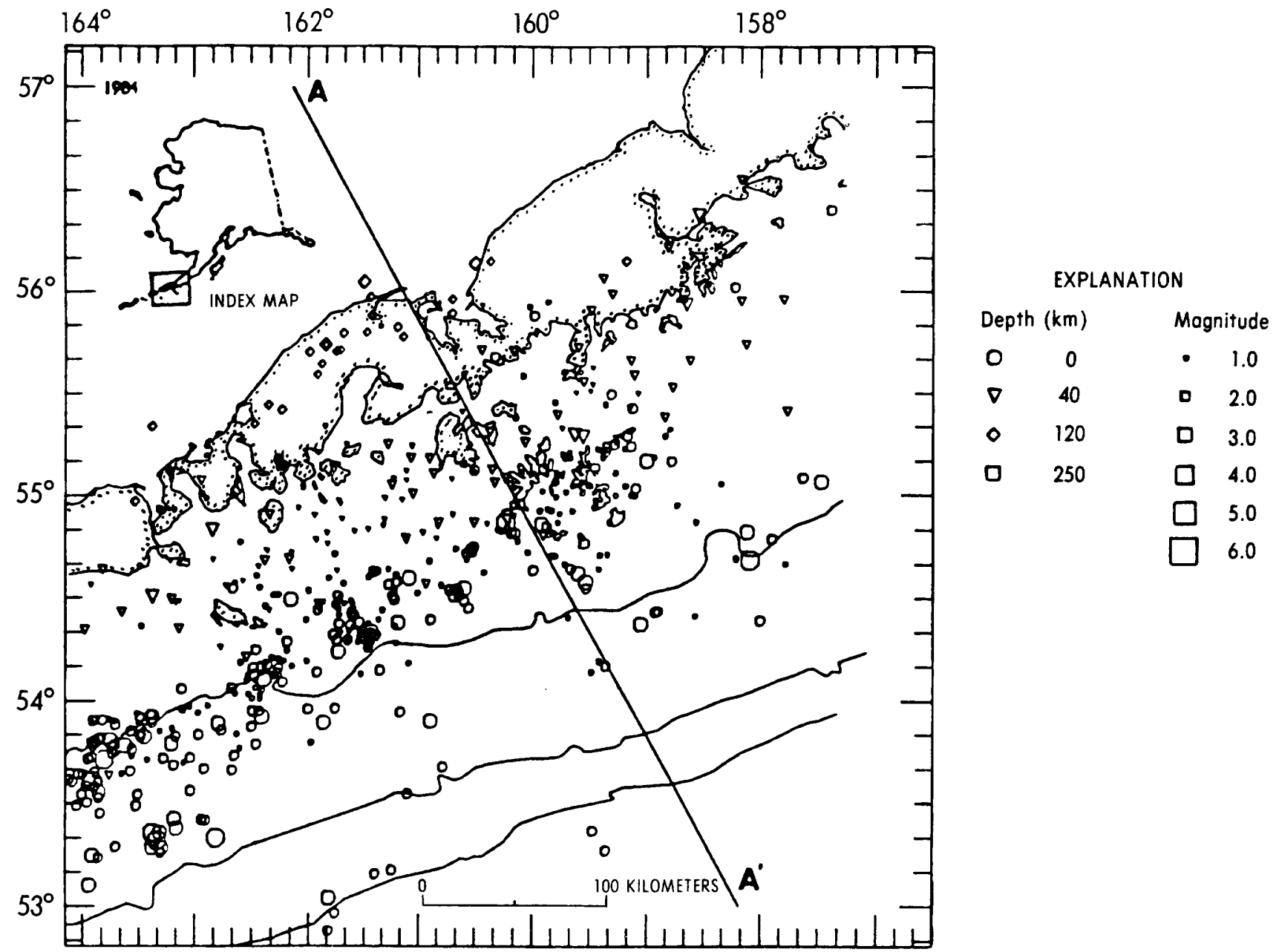

Figure 29. Seismicity recorded by the Shumagin Island seismic network from January 1 to December 30,1984. Depth is shown by symbol type and magnitude by symbol size.

The 1984 Bulletin of the Seismographic Stations lists, for the first time, the seismic moment ( $\left.\mathbf{M}_{\text {., }}\right)$ in dyne-cm for regional earthquakes as calculated from standard Wood-Anderson torsion seismographs recorded within 300 kilometers of the epicenter with peak-to-peak amplitudes of at least 3 millimeters. Seismic moment in dyne-cm is defined by Bolt and Herraiz (1983) as:

$$
\log \left(\mathbf{M}_{\vartheta}\right)=16.74+1.22 \log (\mathbf{C D E})
$$

where $\mathbf{C}$ is the maximum peak-to-peak amplitude in $\mathrm{mm}, \mathbf{D}$ is the duration in seconds from the S-wave onset to the last point where the peak-to-peak amplitude exceeds $\mathrm{C} / 3$, and $\mathbf{E}$ is the epicentral distance in kilometers.
As part of our ongoing seismicity monitoring program, a comparison is made between the observed 1984 seismicity $\left(\mathbf{M}_{L} \geq 3\right)$ in northern and central California and the 1949-1983 seismicity. The Bulletin of the Seismographic Stations lists 3,638 earthquakes $\left(3.0 \leq \mathbf{M}_{L}\right.$ $\leq 6.9$ ), which occurred in the 280,000 -kilometers ${ }^{2}$ area of northern and central California from 1949 through 1983. The 3,638 earthquakes were declustered into 2,312 earthquake sequences (of one or more earthquakes). The cumulative number of earthquake sequences $(N)$ was fit to the Gutenburg-Richter relation by the maximum likelihood method and the result is:

$$
\log \mathbf{N}=\mathbf{4 . 2 3}-0.815 \mathbf{M}_{L}
$$

with a corresponding variance of: 


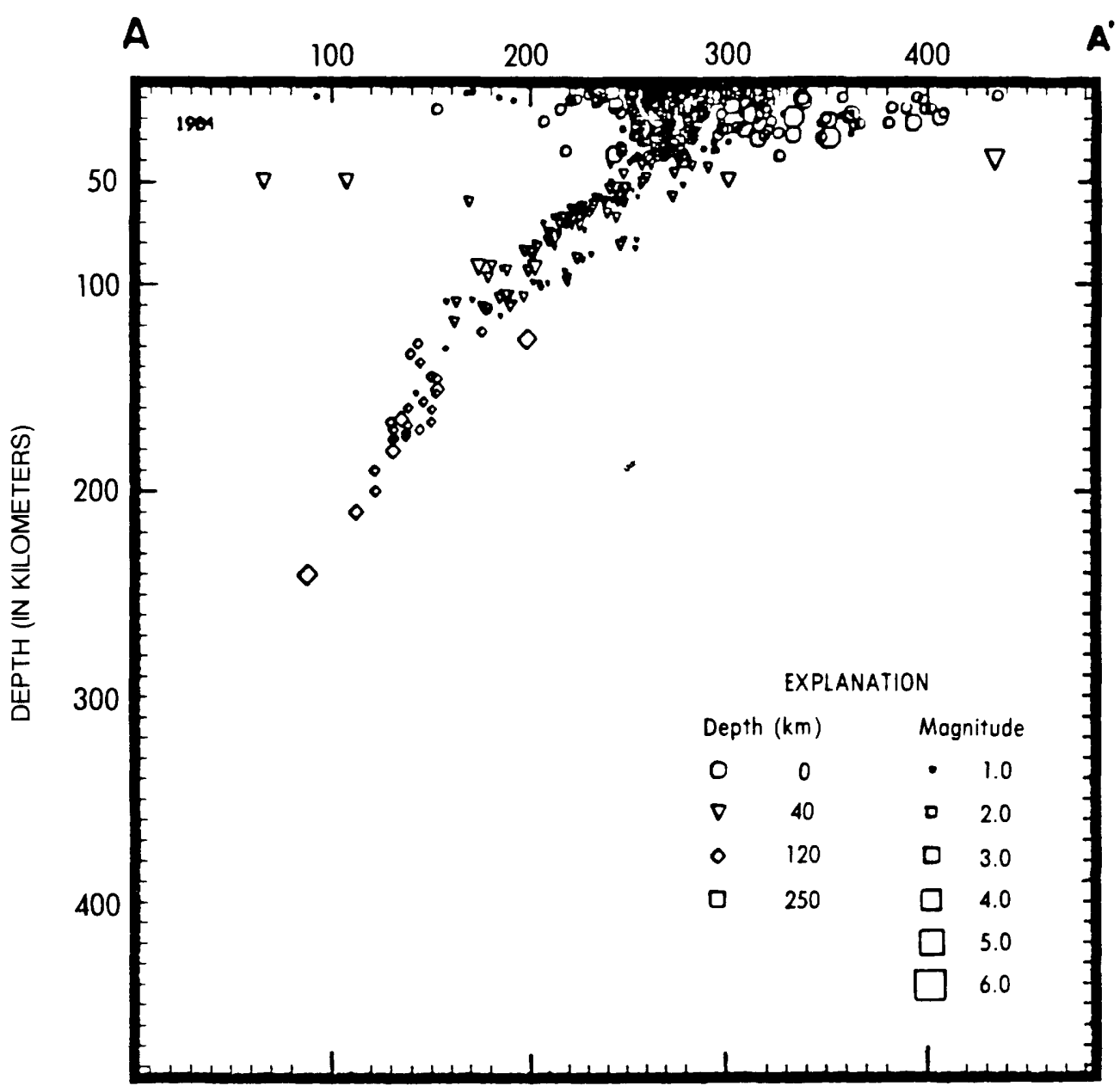

Figure 30. Cross section of seismicity projected along the line A-A' in figure 29.

$$
\sigma_{l, a, N}^{2}=0.000781-0.000792 \mathbf{M}_{L}+0.000525 \mathbf{M}_{L}^{2}
$$

(normalized to earthquake sequences per year per 280,000 kilometers $\left.^{2}\right)$. The annual rate of seismicity $\left(\mathrm{r}=10^{l, g, I N}\right)$ in earthquake sequences per year and the percent probability of one or more earthquakes of magnitude $\mathbf{M}_{L}$ or larger occurring in one day, week, month, year, or decade is given in table 2. During 1984, 78 earthquake sequences $\left(\mathbf{M}_{L} \geq 3.0\right)$ occurred in northern and central California. Thus the observed rate of seismicity in 1984 is significantly higher than the aver- age annual rate of seismicity during the preceding 35 years.

The Bulletin of the Seismographic Stations lists seven earthquakes of $\mathbf{M}_{L} \geq 5$ (table 3 ) which occurred in northern and central California and adjoining areas during 1984. The most significant of these earthquakes is the Halls Valley (Morgan Hill) earthquake $\left(6.2 \mathbf{M}_{L}\right)$ which occurred along the Calaveras fault zone 15 kilometers east of San Jose. The maximum observed intensity was MM VII in the Morgan Hill area. This earthquake was felt from Bakersfield to Sacramento, and from San Francisco to Reno, Nevada. 

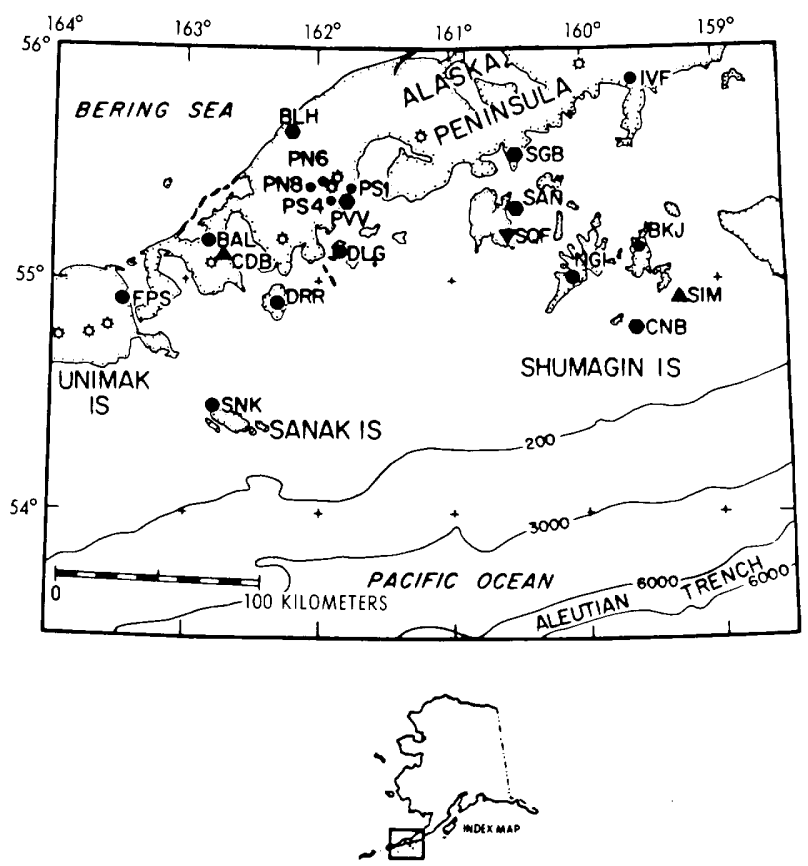

Figure 31. The Shumagin seismic network, Alaska, during 1984. Solid circles are short-period vertical seismic stations. Hexagons are short-period, threecomponent seismic stations. The inverted triangle (SQF) indicates a low-gain site with a three-component force balance accelerometer. Strong-motion accelerographs (SMA-1) are located at the seismic stations SNK, DRR, DLG, SGB, SAN, NGI, BKJ, IVF, and $\mathrm{CNB}$, and at upward pointing triangles. Instrumentation at $\mathrm{SAN}$ also includes a digitally recording PDR-1 strong-motion recorder with FBA sensors.

Table 2. Northern and central California probability of earthquake occurrence

\begin{tabular}{ccccccc}
\hline$M_{Z} \geq$ & $\begin{array}{c}r \\
\text { eq/yr) }\end{array}$ & day & week & month & year & decade \\
3 & 60 & 15 & 69 & 99 & 100 & 100 \\
3.5 & 24 & 6.3 & 36 & 86 & 100 & 100 \\
4 & 9.2 & 2.5 & 16 & 54 & 100 & 100 \\
4.5 & 3.6 & 0.99 & 6.7 & 26 & 97 & 100 \\
5 & 1.4 & 0.39 & 2.7 & 11 & 76 & 100 \\
5.5 & 0.55 & 0.15 & 1.1 & 4.5 & 43 & 100 \\
6 & 0.22 & 0.059 & 0.42 & 1.8 & 19 & 89 \\
6.5 & 0.085 & 0.023 & 0.16 & 0.71 & 8.1 & 57 \\
7 & 0.033 & 0.009 & 0.064 & 0.28 & 3.3 & 28 \\
\hline
\end{tabular}

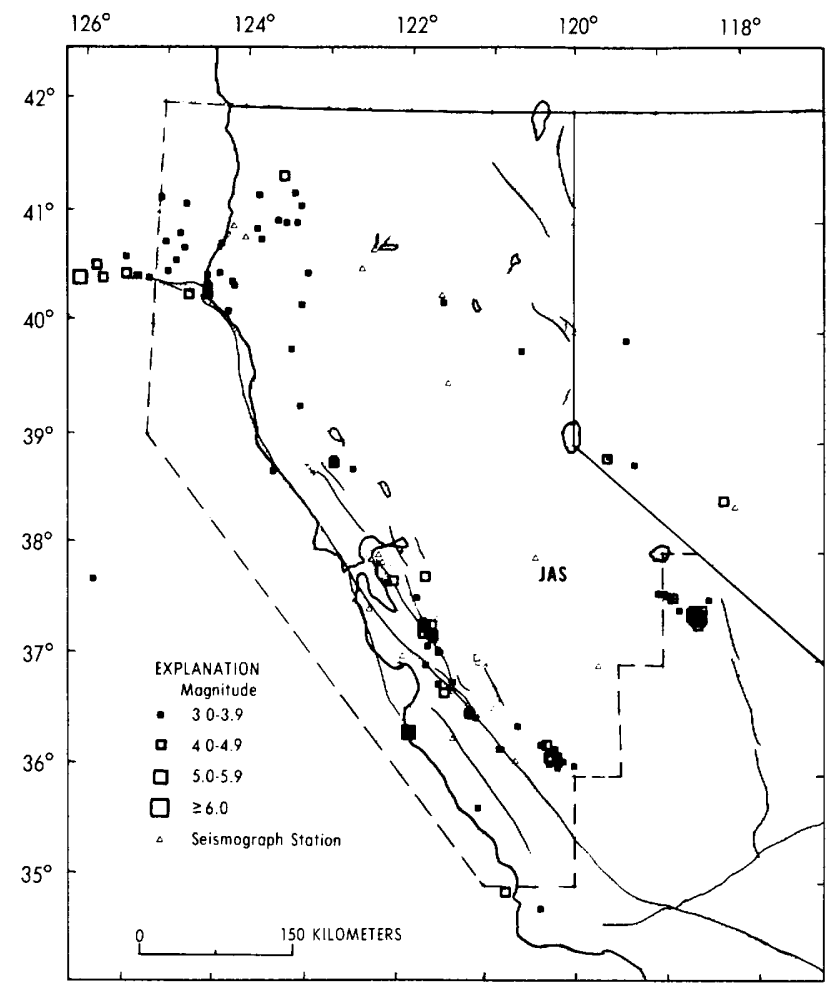

Figure 32. Northern and central California seismicity during 1984 .

Table 3. Earthquakes of $M_{L} \geq 5.0$ which occurred in northern and central California and adjoining areas during 1984

\begin{tabular}{lllll}
\hline DATE & $\begin{array}{l}\text { TIME } \\
\text { (UTC) }\end{array}$ & $\begin{array}{l}M \\
\text { (dyne-cm) }\end{array}$ & $\begin{array}{l}M_{0} \\
\text { LOCATION }\end{array}$ \\
$23 \mathrm{Jan}$ & 0540 & 5.1 & $3.5 E 23$ & $25 \mathrm{~km}$ south of Monterey \\
$28 \mathrm{Feb}$ & 1516 & 5.2 & $1.5 E 23$ & $160 \mathrm{~km}$ wSW of Eureka \\
$24 \mathrm{APr}$ & 2115 & 6.2 & $1.1 E 25$ & Halls Velley, $15 \mathrm{~km}$ east of San Jose \\
$10 \mathrm{Sep}$ & 0314 & 6.6 & & $260 \mathrm{~km}$ west of Eureka \\
$23 \mathrm{Nov}$ & 1808 & 6.1 & $2.6 \mathrm{E} 25$ & $25 \mathrm{~km}$ northwest of Bishop \\
$23 \mathrm{Nov}$ & 1912 & 5.5 & $5.2 E 23$ & $25 \mathrm{~km}$ northwest of B1shop \\
$26 \mathrm{Nov}$ & 1621 & 5.6 & $2.4 E 23$ & $25 \mathrm{~km}$ northwest of B1shop \\
\hline
\end{tabular}

\section{Seismicity and Volcanic Activity in Hawaii, 1984}

\section{By Robert $Y$. Koyanagi \\ U.S. Geological Survey \\ Hawaiian Volcano Observatory \\ Hawaii National Park, HI 96718}

The U.S. Geological Survey's Hawaiian Volcano Observatory (HVO) continued to operate an islandwide network (fig. 33) of short-period seismograph stations consisting of 13 three-component and 27 singlecomponent-vertical FM systems, radio-telemetered to 


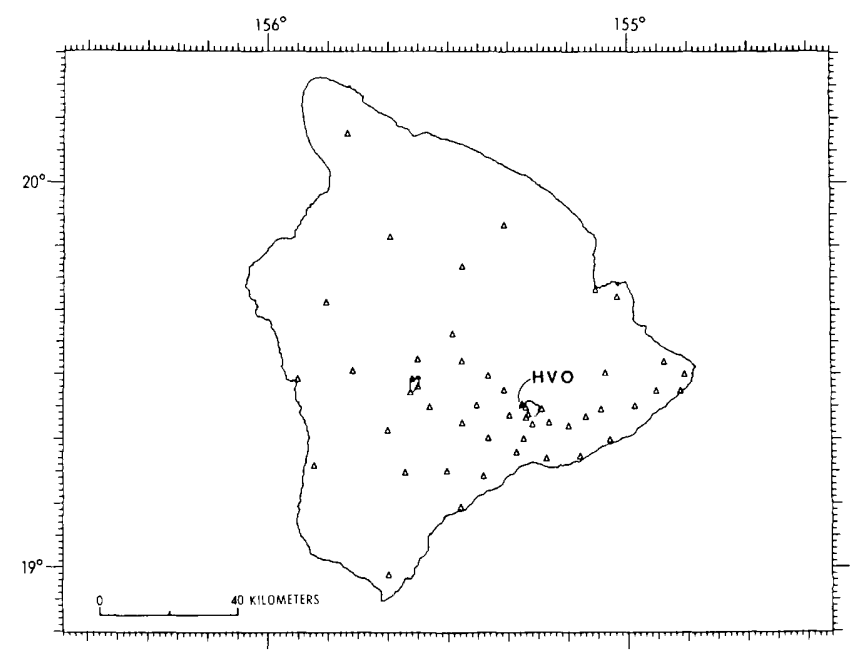

Figure 33. Seismograph stations (triangles) for the Island of Hawaii during 1984.

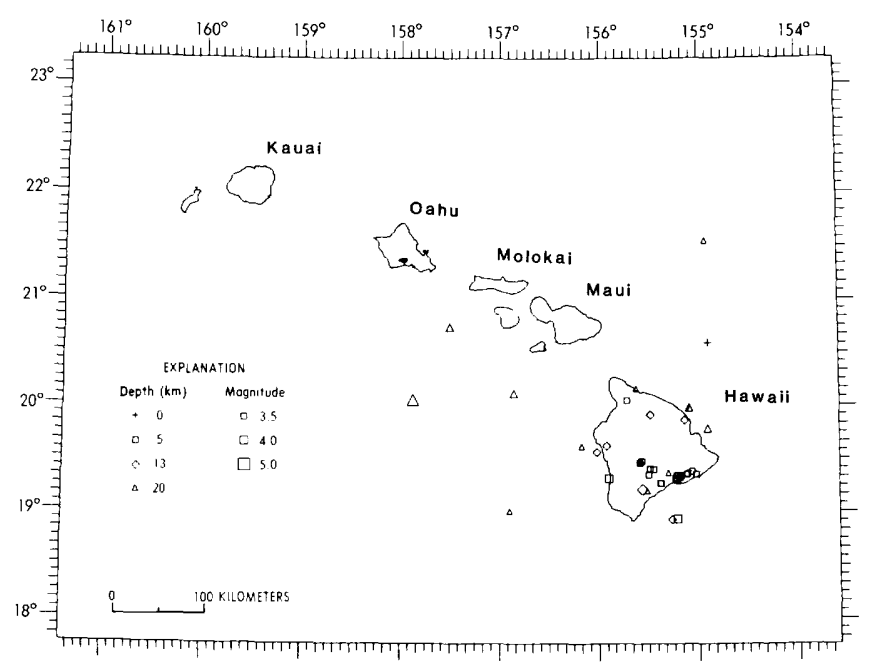

Figure 34. Earthquakes in Hawaii during 1984 (0-60 kilometer depths, $M \geq 3.5$ ).

HVO, and monitored continuously on eight drum recorders, two Develocorders, and a magnetic tape recorder. Optical-drum seismographs were also operated independently on the islands of Hawaii, Maui, and Oahu. These consisted of one two-component shortperiod and one three-component long-period systems at HVO, a short-period system with one high-gain vertical-component and two Wood-Anderson horizontal components at Hilo-Hawaii, a short-period system with one high-gain vertical-component and two WoodAnderson horizontal components at Haleakala-Maui, and one short-period vertical-component at KipapaOahu.

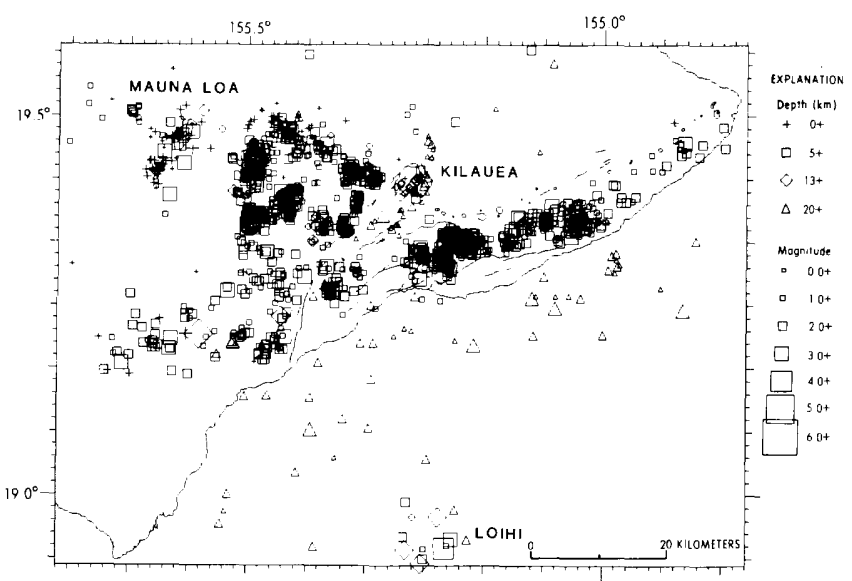

Figure 35. Southeastern Hawaii Island earthquakes $(\mathrm{M} \geq 1.5)$ during 1984.

Earthquakes instrumentally documented over the past 3 decades in Hawaii follow a pattern of distribution that closely relates to volcanism. Shallow earthquakes less than 5 kilometers in depth that occur episodically in swarms during times of accelerated magma influx accompany inflation at the summit and intrusions along the rift zones, and set the stage for eruptions. Earthquakes in outlying crustal regions that result form longterm stresses induced by magma pressure from the tectonic framework of the growing volcanoes. The persistent sources of seismicity emphasized at depths of 5 13 kilometers produce high-magnitude earthquakes of damaging intensities and major aftershock sequences. Deeper earthquakes that extend into the upper mantle to a depth of about 60 kilometers outline steeply dipping plumbing systems beneath the active volcanoes and the bordering regions of stress induced by lithostatic loading.

In 1984, there were 65 earthquakes of magnitude 3.5-5.0 located in the Hawaiian Islands with a high concentration beneath the southeastern region of active volcanism (fig. 34). Twenty-four hundred earthquakes with a magnitude threshold of about 1.5 were recorded and located beneath the active volcanoes $\mathrm{Ki}$ lauea, Mauna Loa, and newly developing Loihi (fig. $35)$. Numerous earthquakes too small to locate were detected beneath southeastern Hawaii at rates that varied from a few to many hundreds per day depending on the state of the volcanoes.

The year 1984 was an active one for volcanoes in Hawaii. The eruption on the east rift of Kilauea that started in January 1983 continued episodically for the second year. Episodes of high lava production and fountains lasting from 0.2-2.5 days issued from its active vent $\mathrm{Puu} \mathrm{Oo}$; intervals of repose between eruptive episodes ranged from 8-50 days. Sixteen eruptive 
episodes occurred in 1984 and produced an estimated 149 million meters ${ }^{3}$ of lava on the southeast flank of the volcano. Increased numbers of shallow earthquakes accompanied inflation of Kilauea summit preceding the outbreaks, strong harmonic tremor in the east rift zone occurred during vigorous eruption, and harmonic tremor and long-period events increased at the summit following the onset of sustained deflation associated with eruption. Between eruptive episodes, amplitude fluctuations of weak tremor in the east rift zone documented movement of magma beneath the eruption site. Crustal earthquakes persisted in the south flank of Kilauea in response to stresses gradually induced by repeated intrusions of magma prior to eruptions at Puu Oo.

Uninterrupted by the continuing activity at $\mathrm{Ki}$ lauea, Mauna Loa erupted on March 25, following a period of continuous inflation since its small summit eruption in 1975. The outbreak of lava migrated quickly from the summit to the northeast rift zone, where the eruption continued until April 15. The eruption generated a lava flow eastward to within 6 kilometers of Hilo, the principal cultural center of the island. Two hundred twenty million meters ${ }^{3}$ of lava were extruded. The sustained high count of shallow (less than 5 kilometers) earthquakes at the summit, which followed a pattern of gradual increase since 1980, developed into a swarm about 2.5 hours prior to the estimated start of eruption at 01:25 HST, March 25. The swarm of earthquakes and harmonic tremor continued as the eruption migrated into the northeast rift zone. The seismicity continued for the duration of the eruption as it responded to the pattern of lava output on the northeast. rift zone and deflation at the summit. Tremor amplitude varied generally in proportion to the intensity of lava fountaining and rate of lava output at the vent. Shallow earthquakes and tremor amplitude increased at the summit with sustained deflation as magma withdrew from the summit reservoir to supply the eruption on the northeast rift. The decreased level of post eruption seismic activity from the continuing adjustments to shallow gravitational and thermal stresses decayed gradually over the subsequent months.

\section{Kansas-Nebraska Seismicity, 1984}

By Greg M. Hildebrand and Don W. Steeples

Kansas Geological Survey

Lawrence, KS 66046

The Kansas Geological Survey operates a seismic network of 15 single-component, short-period stations throughout eastern Kansas and eastern Nebraska. The network is operated under a contract with the U.S. Nuclear Regulatory Commission and is designed to monitor seismic activity in eastern Kansas and eastern $\mathrm{Ne}$ -

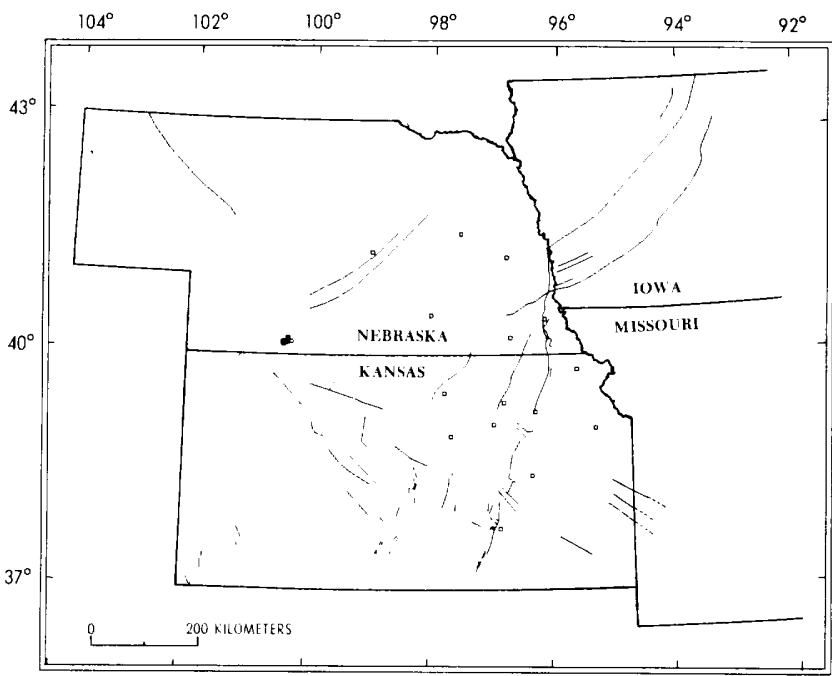

Figure 36. Seismograph stations in Kansas and Nebraska in January 1984. Sleepy Hollow stations are shown by the cluster of squares in southwestern Nebraska.

braska. A dense network of eight stations was also operated in the Sleepy Hollow oil field near $40.2^{\prime \prime} \mathrm{N}$., $100.4^{\prime \prime} \mathrm{W}$. through 1984. The Sleepy Hollow network was funded by the USGS and a NSF grant. The addition of digital acquisition capability to the Sleepy Hollow network made it possible to achieve better resolution on several of the 62 events (table 5) located in 1984. Figure 36 shows the station locations on January 1, 1984. Operation of the Sleepy Hollow network ceased in late December 1984.

Figure 37 shows cumulative microearthquake epicenters recorded between August 1977 and December 1984 (table 4 for 1984 epicenters). Four major microearthquake trends can be identified from this figure. One trend is associated with the Humbolt fault zone and runs south-southwesterly from near $40^{\prime \prime} \mathrm{N}$., $96^{\prime \prime} \mathrm{W}$. to near $37^{\prime \prime} \mathrm{N}$., $97^{\prime \prime} \mathrm{W}$. A second trend is nearly parallel to the first and is associated with the northwest flank of the Midcontinent geophysical anomaly and runs southsouthwesterly from near $40^{\prime \prime} \mathrm{N}$., $97.3^{\prime \prime} \mathrm{W}$. to near $37^{\prime \prime} \mathrm{N}$., $98.5^{\prime \prime} \mathrm{W}$. A third trend is generally associated with the Central Kansas uplift and runs from near $38^{\prime \prime} \mathrm{N}$., $98^{\prime \prime} \mathrm{W}$. northwesterly to the Sleepy Hollow area near $40.2^{\prime \prime} \mathrm{N}$., $100.4^{\prime \prime} \mathrm{W}$. The final trend runs northeastward across $\mathrm{Ne}-$ braska from Sleepy Hollow to near $42^{\prime \prime} \mathrm{N}$., $97^{\prime \prime} \mathrm{W}$.

Table 4. Kansas and Nebraska earthquakes, 1984

\begin{tabular}{|c|c|c|c|c|c|c|c|c|c|c|}
\hline DATE & 0 (6161 & TAT & LONG & DEPTH & $\overline{n A G}$ & 50 & GAP DMIN & $\operatorname{ans}$ & ERE & Eत20 \\
\hline $\begin{array}{l}840107 \\
840113 \\
840127 \\
840203 \\
840812 \\
840828 \\
841023 \\
841110 \\
841204 \\
841218\end{array}$ & 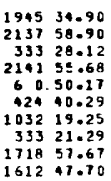 & $\begin{array}{l}40-3.99 \\
39-22.49 \\
1-906 \\
39-31.70 \\
38-42.02 \\
38-16.42 \\
38-8.76 \\
10-30.60 \\
35-52.06 \\
41-33.57\end{array}$ & $\begin{array}{l}97-49.64 \\
96-14.20 \\
98-28.52 \\
96-18.80 \\
96-46.89 \\
93-50.92 \\
95-55.84 \\
95-36.13 \\
99-58.55 \\
97-11.01\end{array}$ & $\begin{array}{r}5.00 \\
.83 \\
9.53 \\
1.50 \\
19.83 \\
5.00 \\
5.00 \\
5.00 \\
5.00 \\
5.00\end{array}$ & $\begin{array}{l}1.8 \\
1.7 \\
1.7 \\
1.9 \\
1.9 \\
2.1 \\
1.7 \\
1.7 \\
2.3 \\
1.8\end{array}$ & $\begin{array}{r}10 \\
5 \\
10 \\
5 \\
12 \\
8 \\
11 \\
15 \\
9\end{array}$ & 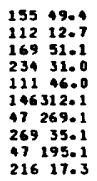 & $\begin{array}{l}.52 \\
.01 \\
.86 \\
.09 \\
.32 \\
.30 \\
.36 \\
.57 \\
.30 \\
.47\end{array}$ & $\begin{array}{l}2.4 \\
.2 \\
5.7 \\
1.7 \\
1.6 \\
5.0 \\
3.4 \\
3.8 \\
4.7 \\
4.3\end{array}$ & $\begin{array}{rl}9.4 & 0 \\
9-4 & c \\
93.1 & 0 \\
96.3 & 0 \\
1.8 & c \\
2.6 & 0 \\
7.2 & 0 \\
4.6 & 0 \\
6.7 & 0 \\
1.9 & 0\end{array}$ \\
\hline
\end{tabular}


Table 5. Earthquakes located by the Sleepy Hollow, Nebraska, seismic network, 1984

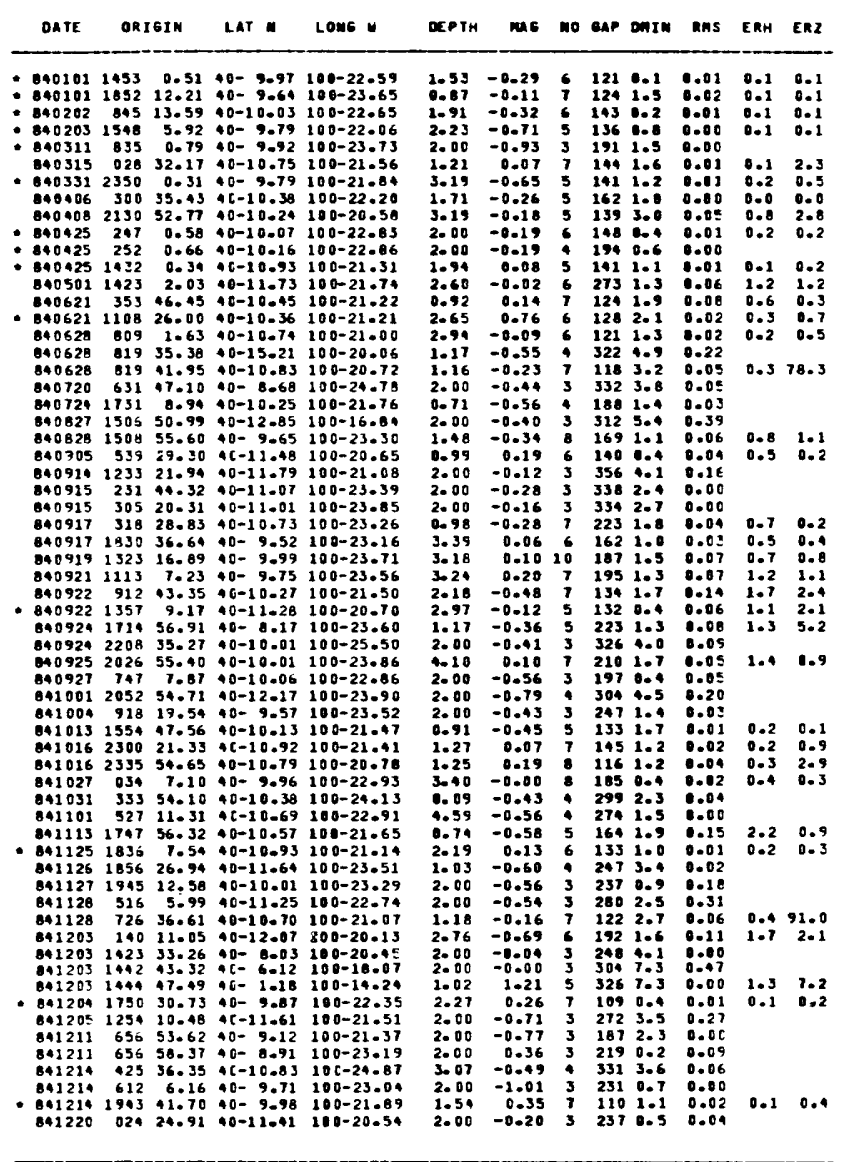

- Cocation using digital dota

Explanation of tables 4 and 5

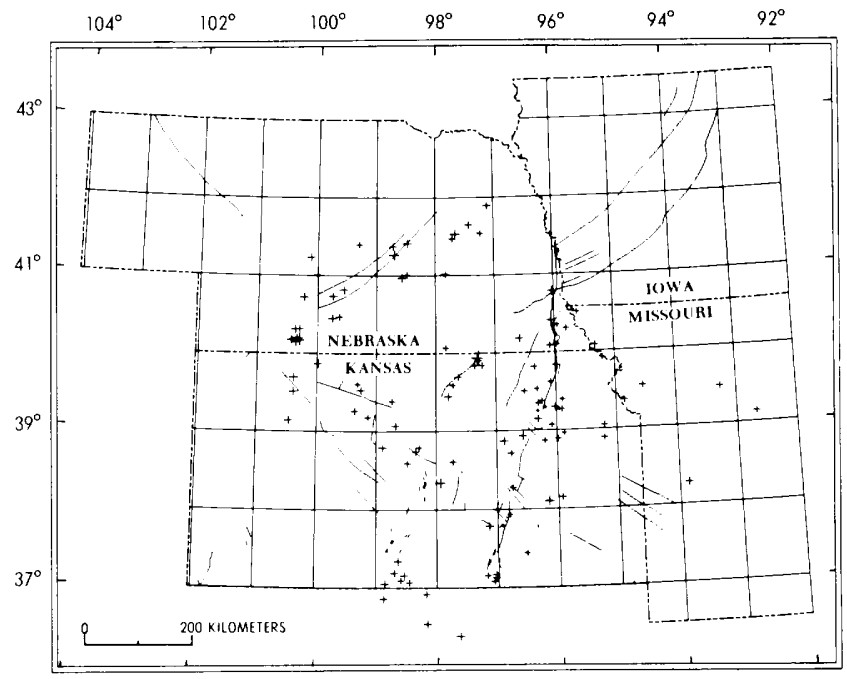

Figure 37. Microearthquakes located by the Kansas-Nebraska seismograph network between August 1977 and December 1984, shown by sizecoded plus signs. The largest event had a magnitude of 3.3. The smallest events had magnitudes of about 1.0 (local duration scale).

\section{Central Mississippi Valley Earthquakes, 1984}

By W. Stauder, R. Herrmann, J. Chulick, C. Finn, P. Leu, T. Shin, K. Rinderknecht, J. Mascarenas, $H$. Yepes, V. John, and C. Carr

Department of Earth and Atmospheric Sciences

Saint Louis University

P.O. Box 9099 Laclede Station

Saint Louis, MO 63156

In 1984, 357 earthquakes were located and 77 other nonlocatable earthquakes were detected by the 38 station regional telemetered microearthquake network operated by Saint Louis University for the U.S. Geological Survey and the Nuclear Regulatory Commission. Figure 38 shows 336 earthquakes located within a $4^{\prime \prime} \times 5^{\prime \prime}$ region centered on $36.5^{\prime \prime} \mathrm{N}$., $89.5^{\circ} \mathrm{W}$. Seismograph stations are denoted by triangles and are labeled by the station code. Figure 39 shows the locations and magnitudes of 121 earthquakes located within a $1.5^{\prime \prime} \times 1.5^{\prime \prime}$ region centered at $36.25^{\prime \prime} \mathrm{N} ., 89.75^{\prime \prime} \mathrm{W}$. Figures 40 and 41 are similar to figures 38 and 39 , but the epicenter symbols (squares) are scaled to focal depth.

The PDP 11/34 microcomputer recorded 165 teleseisms in 1984. Epicentral coordinates were determined by assuming a plane wave front propagating across the network and using the travel-time curves to determine back azimuth and slowness, and by assuming a focal depth of 15 kilometers using spherical geometry. Arrival-time information for teleseismic $P$ and 


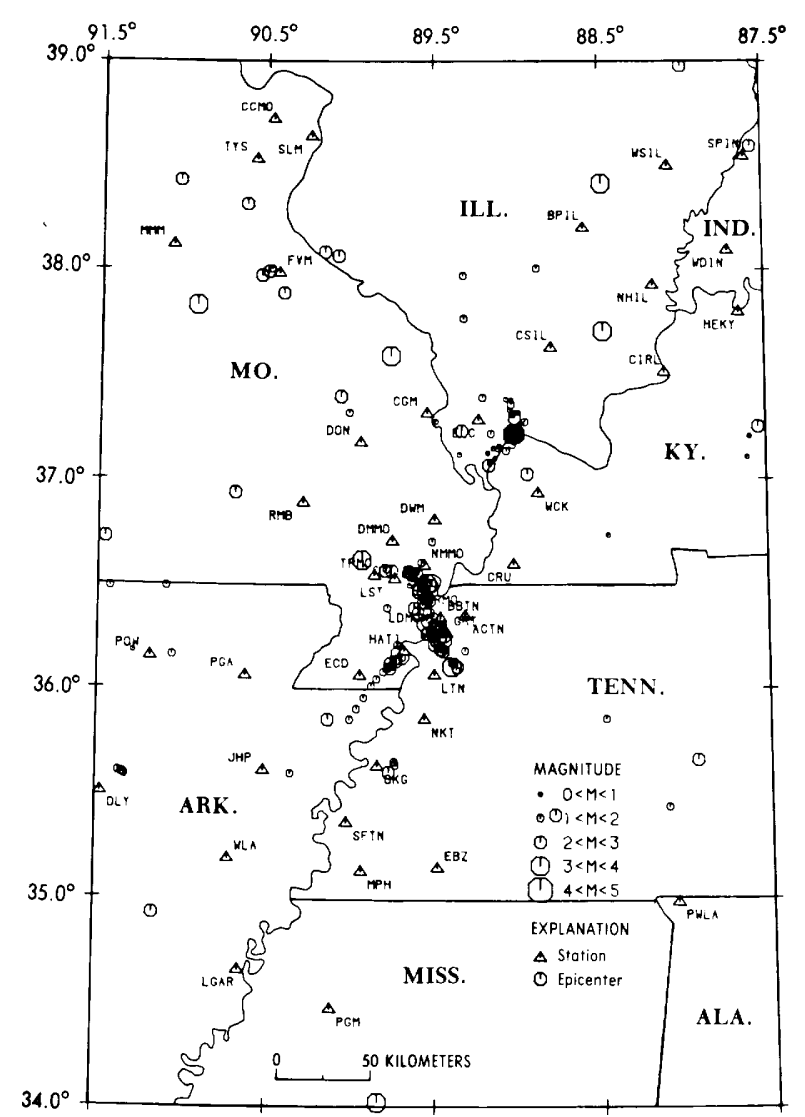

Figure 38. Central Mississippi Valley earthquakes during 1984 within a $4^{\prime \prime} \times 5^{\prime \prime}$ region centered at $36.5^{\prime \prime} \mathrm{N}$., $89.5^{\prime \prime} \mathrm{W}$.

PKP phases has been published in the quarterly earthquake bulletin (Saint Louis University, 1984).

The significant earthquakes occurring in 1984 are the following:

1- January 12, 1984, 0248 UTC, $97.59^{\prime \prime} N$., 89.75" W.; felt in an area about 10 miles south of Perryville, Missouri. $m_{L, g}(10 \mathrm{~Hz})=3.0($ SLM $)$.

2-January 28, 1984, 2129 UTC, 36.61"4N., 89.92"W.; felt (IV) in Malden and Gideon, Missouri. $m_{L_{g}}(10 \mathrm{HZ})$ = 3.2(SLM).

9- An earthquake swarm began in November 1989 in southern Illinois near Ohio River Lock and Dam No. 59 and continued through this year. Over 200 events were detected. Peak activity occurred in February 1984 with the event on February 19, 2242 UTC, 37.21"N., 89.02'W.; felt (IV) at Bandana, Kentucky. Felt (III) at Kevil and La Center, Kentucky. Also felt (III) at Belknap, Cairo, Grand Chain, Mounds, Tamms, and Ullin, Illinois. $m_{L !}(10 \mathrm{~Hz})=3.2(S L M)$, $m_{L ! 9}(3 H z)=3.0(F V M), m_{1, L,}=3.9(B L A)$.

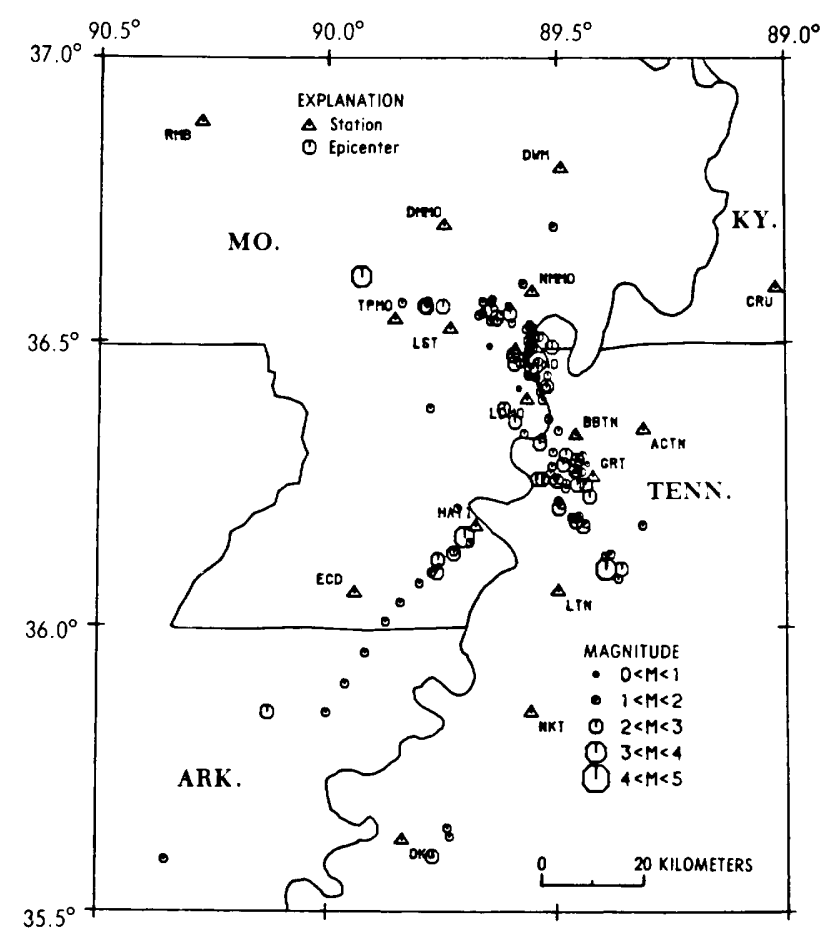

Figure 39. Central Mississippi Valley earthquakes during 1984 within a $1.5^{\prime \prime} \times 1.5^{\prime \prime}$ region centered at $36.25^{\prime \prime} \mathrm{N}$., $89.75^{\prime \prime} \mathrm{W}$.

A slightly larger event occurred on February 14, 1984, 22 56 UTC, 37.21'N., 89.00' W.; felt (IV) at Belknap, Cairo, Karnak, Olmstead, and Perks, Illinois. Also felt (IV) at Bandana and Kevil, Kentucky. Felt (III) in parts of western Kentucky, southeastern Missouri, and southern Illinois. $m_{L g}(10 H z)=9.6$ (SLM), $m_{b L g}=3.8(B L A)$.

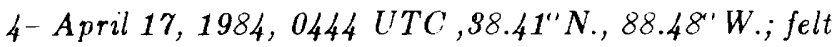
(IV) at Fairfield, Ellery, Dix, Griffin, Keenes, and Mill Shoals, Illinois. Felt (III) at Barnhill, Bluford, Geff, Macedonia, and Mount Erie, Illinois. $m_{L !}(10 \mathrm{~Hz})=3.2$ (SLM), $M_{L g}(3 \mathrm{~Hz})=2.7(F V M), m_{D}=2.8(T E I C)$.

5- June 26, 1984, 1515 UTC, $96.10^{\prime \prime} N$., 89.99'4 W.; felt in the Dyersburg, Tennessee, area. $m_{L !}(10 \mathrm{~Hz})=3.2$ (SLM), $m_{D}=3.0$ (TEIC).

6- June 29, 1984, 0758 UTC, 37.70'N., 89.47'W.; slight damage (VI) at Harrisburg, Illinois. Felt (V) at Raleigh and (IV) at Equality, Herod, Karbers Ridge, Muddy, and Shawneetown. Felt in Gallatin, Hamilton, Hardin, Johnson, Saline, and Williamson Counties. Also felt in several communities in northwestern Kentucky. $m_{L ! l}(10 \mathrm{~Hz})=3.8(\mathrm{SLM}), m_{l, L ! l}=4.1(G S)$, $M_{D}=3.9($ TEIC $)$.

7- July 28, 1984, 2999 UTC, 39.22'N., 87.07'W.; felt (V) at Clay City and Coal City, Indiana. Felt (IV) at Bloomfield, Bowling Green, Carlisle, Coal- 


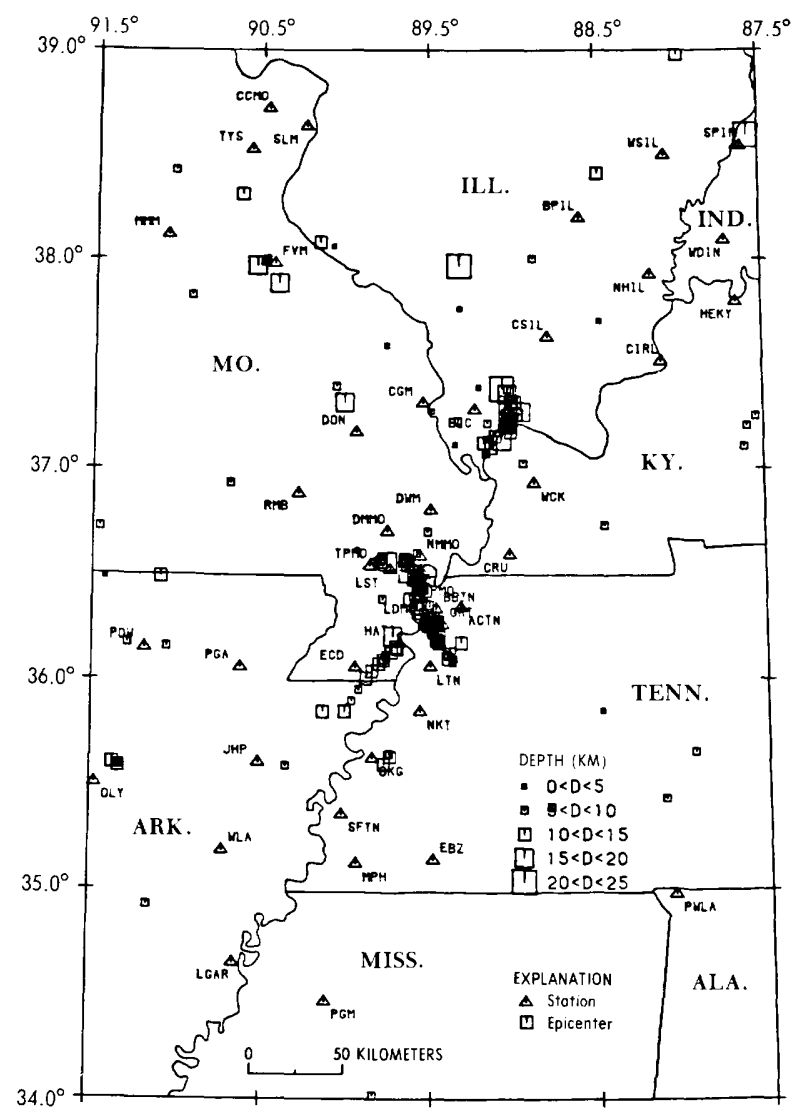

Figure 40. Central Mississippi Valley earthquakes during 1984 in the same region as figure 38 , scaled by focal depth.

mont, Cory, Freedom, Hymera, Jasonville, Lewis, Linton, Midland, Patricksburg, and Worthington, Indiana. $m_{L, 9}(10 \mathrm{~Hz})=4.0($ SLM $), m_{D}=9.8($ TEIC $)$.

8- July 90, 1984, 0799 UTC, 37.82'N., 90.92'W.; felt at Bonne Terre, Farmington, and Flat River, Missouri. Also felt at Carbondale, Illinois. $m_{L g}(10 \mathrm{~Hz})=9.0$ $(S L M), m_{D}=2.6(T E I C)$.

9- August 29, 1984, 0650 UTC, 39.11'N., 87.45"W.; felt (V) at Clay City, Indiana. Felt (III) at Bowling Green, (rane, Fairbanks, and Lewis, Indiana. $m_{L ! 1}(10 H z)=3.1(S L M), m_{D}=2.7(T E I C)$.

10-September 6, 1984, 1606 UTC, $96.10^{\prime} \mathrm{N} ., 89.95^{\prime \prime} \mathrm{W}$.; felt at Brazil, Tennessee. $m_{L !}(10 \mathrm{~Hz})=2.9(S L M), m_{D}$ $=2.4(T E I C)$.

11- September 27, 1984, 1909 UTC, 95.25" N., 92.21" W.; felt (IV) at Enola, Arkansas. $m_{L, 1}(10 \mathrm{~Hz})=9.4$ (SLM), $m_{D}=3.2(T E I C), m_{l, L !}=9.9(T U L)$.

$A$ second event occurred at 1916 UTC. $m_{L g}(10 \mathrm{~Hz})=$ 3.0(SLM), $m_{l, L_{9}}=2.4(T U L), m_{D}=2.7(T E I C)$.

12-October 9, 1984, 1154 UTC, 34.74"N., 85.16'W.; slight damage (VI) south of Ringgold, Georgia. Mi-

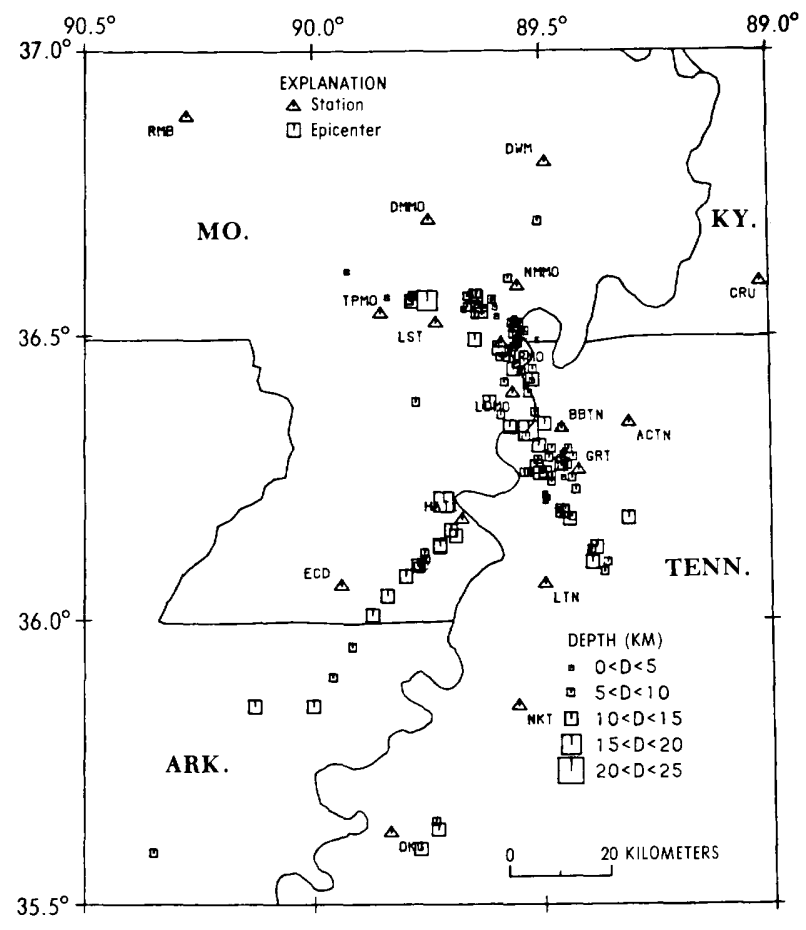

Figure 41. Central Mississippi Valley earthquakes during 1984 in the same region as figure 39 , scaled by focal depth.

nor damage reported in the Chattanooga, Tennessee area. Felt (V) at La Fayette, Chickamauga, and Trenton, Georgia. Felt in northwestern Georgia, northeastern Alabama, and parts of south-central Tennessee. $m_{L ! l}(10 \mathrm{~Hz})=4.2(S L M), m_{1, L !}=4.0(G S), m_{D}=9.8$ (TEIC). Consult Tennessee Earthquake Information Center (TEIC) (1984).

19-December \$, 1984, 1155 UTC, $96.15^{\circ} \mathrm{N}$., 89.70 W.; felt (IV) at Cooter, Missouri, and (III) at Gabler, Missouri and Miston, Tennessee. Also felt at Caruthersville, Missouri. $m_{L ! 9}(10 \mathrm{~Hz})=9.0(\mathrm{SLM}), m_{L^{\prime !}}(\Omega H z)=$ \$.2 (FVM), $m_{D}=9.0(T E I C)$.

\section{Nevada and Eastern California Earthquakes, 1984}

By Ute Vetter

Seismological Laboratory

University of Nevada

Reno, NV 89557-0018

In 1984, the University of Nevada-Reno Seismological Laboratory recorded data from a network of 79 seismographic stations, of which 59 were in operation the whole year. Six of the stations were three component, three of them were wide-band digital, and three of them were short-period analog. The remainder of the stations were short-period vertical instruments. 


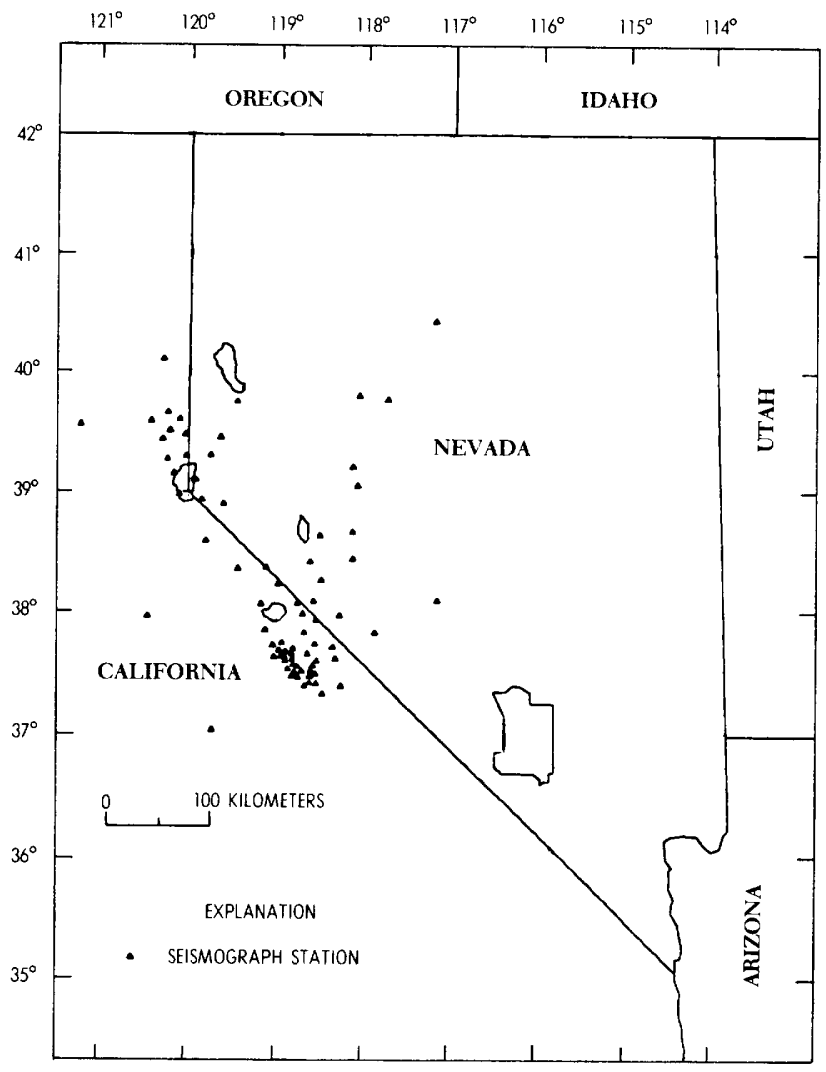

Figure 42. The Nevada-eastern California network during 1984 .

A map of all stations from which we received signals at any time in 1984 is shown in figure 42. The data were telemetered to the laboratory over radio and telephone links and recorded on analog tape; key stations were also monitored on Helicorders. In May 1984 the data recording was changed to an event-triggered online recording system.

Figure 43 shows a map of about 3,700 events which were located for the 1984 period using the HYPO71 algorithm of Lee and Lahr (1975). As in the previous 3 years, the most active region was the Mammoth Lakes area, where more than 3,000 events were located (fig. 44). The most significant seismic event of the 1984 was the November mainshock-aftershock sequence in the Round Valley region, a region which was only moderately active after the Wheeler Crest swarm in 1978. The seismicity in the Mammoth Lakes area before November 23 was concentrated around the southern boundary of Long Valley caldera and the Sierra block south of the caldera. Most of the seismicity near the Round Valley fault occurred on November 23 and afterwards; the sequence started November 23 with a small foreshock (1808 UTC), followed 5 seconds later by an event of magnitude 6.0 . The aftershocks of this

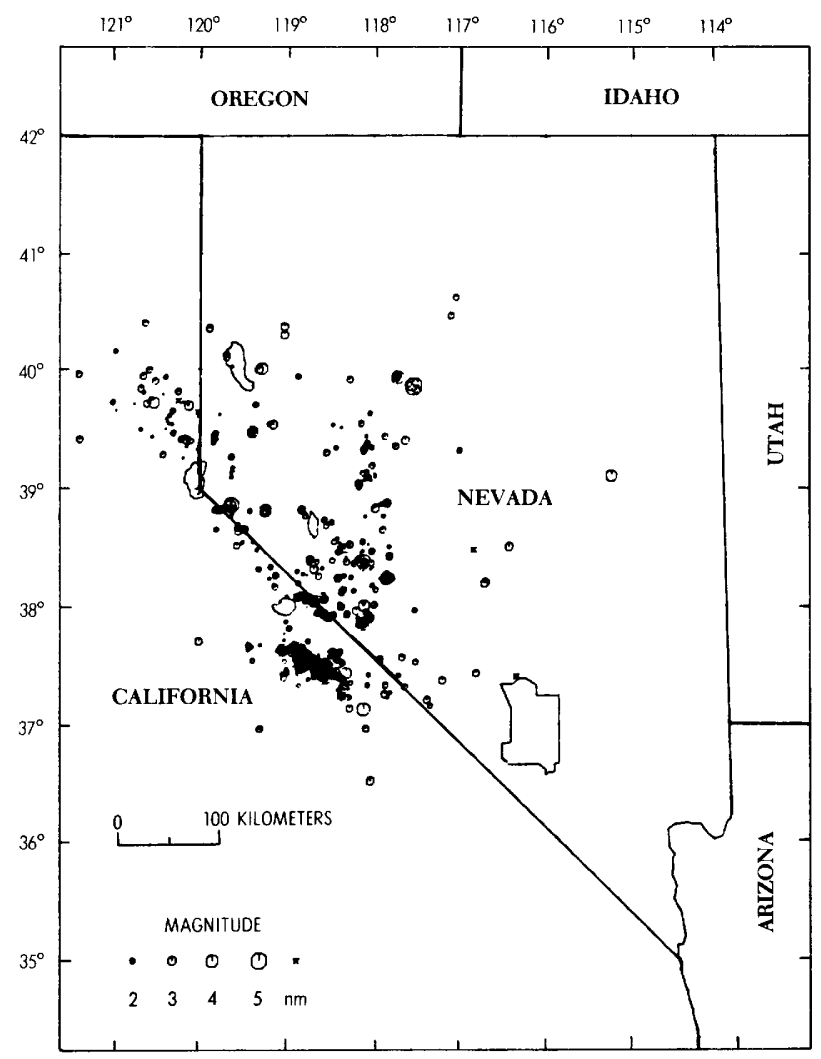

Figure 43. Seismicity of the Nevada-eastern California area during 1984.

earthquake (about 1,100 events between November 23 and December 9) occurred in a more or less triangular shaped area with about 12-kilometer sides, which is seen in figure 44. During this time the commonly most active region south of Long Valley caldera was unusually quiescent. This situation lasted for months and the "normal" pattern was not restored until about mid-1985.

Many of the swarm earthquakes were reported felt in the Bishop and Mammoth Lakes area, especially the strong events on November 23, 1808 UTC and on November 26, 1621 and 1631 UTC. The seismicity in 1984 was not particularly strong in terms of energy release; only 19 earthquakes with local magnitude greater than 4.0 occurred, and only two of them were of magnitude 5.2 and 6.0. Figure 45 shows all earthquakes with magnitudes greater than 3.5. Besides the events of the Round Valley series, the following earthquakes were reported felt:

February 16, 1115 UTC, 5.2 $\mathbf{M}_{L}, 48$ miles south of Battle Mountain, and the aftershock at 1209 UTC, 4.0 $\mathbf{M}_{L}$; felt reports from Lovelock, Fernley, and Fallon.

February 17, 1209 UTC, $4.6 \mathbf{M}_{L} 10$ miles southeast 


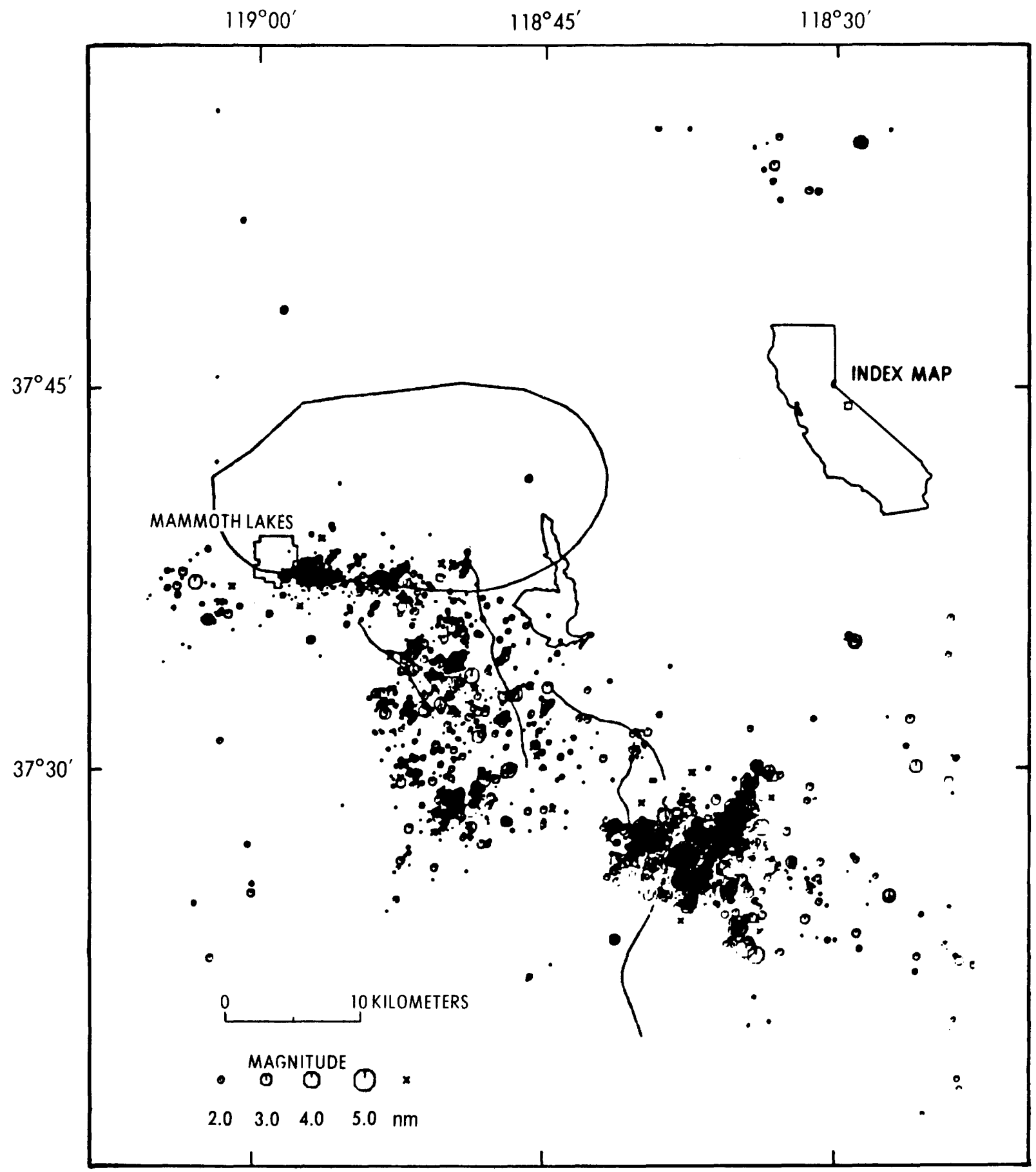

Figure 44. Mammoth Lakes, California area, earthquakes during 1984. 


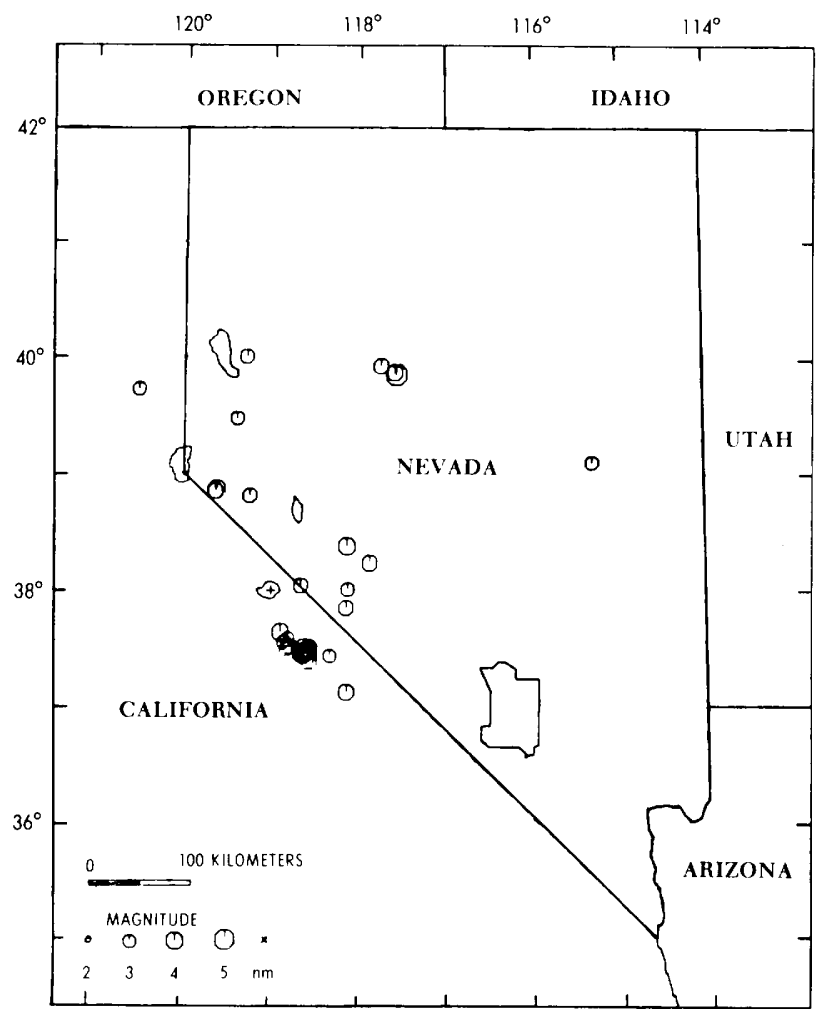

Figure 45. Earthquakes in the Nevada-eastern California area, with magnitude $\geq 3.5$.

of Gardnerville, and aftershock at 1216 UTC, $9.8 \mathbf{M}_{L}$; felt reports from Carson City, Gardnerville, Wellington, Yerington, and South Lake Tahoe.

February 19, 1022 UTC, 4.0 $\mathbf{M}_{L}$, at the western part of Dixie Valley; no felt reports.

March 10, 1246 UTC, 2.6 $\mathbf{M}_{L}$, near Gabbs, Nevada; felt in Gabbs.

October 21, 1451 UTC, $4.1 \mathbf{M}_{L}$, in the Mason Valley south of Yerington, and aftershock at 1455 UTC, 3.6 $\mathbf{M}_{L}$; felt in Mason Valley.

October 22, 1127 UTC, $9.9 \mathbf{M}_{L}$, south of Sutcliffe/Pyramid Lake; felt in Sutcliffe.

\section{New England Earthquakes, 1984}

By James P. Mc Caffrey, S.J.

Department of Geology and Geophysics

Weston Observatory, Boston College

Weston, MA 02199

Weston Observatory operated 30 stations in New England during 1984; all but one of these, WES, are remote, unattended stations. The data for the bulletin of the Northeastern U.S. Seismic Network prepared at Weston Observatory are provided by the members of this network. Additional data are provided for some of the events in Canada by the Earth Physics Branch Department of Energy, Mines, and Resources, Ottawa, Canada (fig. 46).

During 1984, 24 New England earthquakes were recorded by stations of the Northeastern U.S. Seismic Network (table 6 and fig. 46). Ten earthquakes occurred in Maine, six in New Hampshire, five in Massachusetts, and three in Vermont. The magnitudes of these events ranged from 0.8 to 3.6 on a coda magnitude scale $\left(\mathbf{M}_{\text {.. }}\right)$ that was developed for events in New England.

During the period August 1983 to January 1984, five felt earthquakes of magnitude greater than 3.0 were recorded from coastal Maine near the New Brunswick border. The occurrence of these earthquakes prompted a study of the historical seismicity of the region. The largest event reported from this region was that of March 21, 1904. Intensities of VIII (MM), at the epicenter near Calais, Maine, and VII (MM), at St. John, New Brunswick, 85 kilometers away, were reported. The two predominant tectonic features of the area are Passamaquoddy Bay, which is subsiding at a maximum rate of about $9 \mathrm{~mm} / \mathrm{yr}$, and the Oak Bay fault which trends northwest and is nearly perpendicular to the subsidence axis. All the recent events were located west of Passamaquoddy Bay and south of the Oak Bay fault. A fault plane solution, which is poorly constrained, indicates a thrust mechanism striking WNW for the largest and latest of these recent events $\left(\mathbf{M}_{y_{1}}=3.8\right)$.

Table 6. Earthquakes of magnitude $\geq 2.0$ which occurred in New England during 1984

\begin{tabular}{|c|c|c|c|c|c|c|c|}
\hline no & De & $\begin{array}{l}\text { Origin Tive } \\
\text { Ar Min Sec }\end{array}$ & Letr(u) & $\operatorname{Lom}_{0}(\mathrm{U})$ & $\begin{array}{l}\text { DEPTH } \\
(\mathbf{M H})\end{array}$ & $\operatorname{mac}_{\text {Mc }}$ & LOCATIOW \\
\hline Jan. & 14 & 053434.20 & $45 \quad 01.47$ & 6716.74 & 13.38 & 2.8 & Maine \\
\hline Jan. & 14 & $0908 \quad 34.57$ & 4503.04 & $67 \quad 10.85$ & 7.57 & 2.8 & Maine \\
\hline Jan. & 14 & 090933 & 4503 & 6711 & 7 & $3 . ?$ & Maine \\
\hline Jan. & 16 & 004321.12 & 4701.44 & $68 \quad 13.06$ & 4.14 & 2.0 & Maine \\
\hline Jan. & 19 & $05 \quad 26 \quad 08.84$ & 4453.60 & $67 \quad 18.06$ & 11.96 & 3.6 & Maine \\
\hline Jan. & 27 & $2105 \quad 36.30$ & 4326.16 & 71.36 .30 & 0.95 & 2.0 & New Hampshire \\
\hline Feb. & 10 & 074302.04 & 4228.37 & 7131.84 & 2.45 & 2.1 & Massachusetts \\
\hline Feb. & 26 & $12 \quad 37 \quad 19.22$ & 4429.17 & $71 \quad 18.17$ & 12.07 & 2.9 & New Hampshire \\
\hline Mar. & 13 & 185809.14 & 4359.68 & $70 \quad 07.98$ & 13.78 & 2.1 & Maine \\
\hline Apr. & 12 & $1154 \quad 05.83$ & 4711.81 & 7607.66 & 14.00 & 2.1 & Maine \\
\hline June & 8 & $06 \quad 47 \quad 34.87$ & 4313.90 & $70 \quad 12.35$ & 10.87 & 2.2 & Maine \\
\hline June & 14 & $2056 \quad 34.29$ & 4235.24 & 7223.84 & 10.47 & 2.4 & Massachusetts \\
\hline June & 16 & $18 \quad 20 \quad 59.90$ & 4301.42 & $72 \quad 23.12$ & 14.67 & 2.3 & New Hampshire \\
\hline July & 31 & 212146.22 & 4257.01 & 7224.88 & 5.00 & 2.1 & New Hampshire \\
\hline Aug. & 25 & $1027 \quad 04.72$ & 4403.14 & 7323.62 & 5.00 & 2.4 & Vermont \\
\hline Sep. & 9 & $12 \quad 18 \quad 35.24$ & 4408.86 & $\begin{array}{ll}68 & 09.72\end{array}$ & 0.00 & 2.1 & Maine \\
\hline Oct. & 18 & $\begin{array}{lll}07 & 16 & 15.89\end{array}$ & 4531.75 & $68 \quad 46.67$ & 6.25 & 2.3 & Maine \\
\hline Nov. & 17 & 022046.60 & 4412.03 & $72 \quad 19.13$ & 4.92 & 2.4 & Vermont \\
\hline Dec. & 22 & $16 \quad 05 \quad 14.32$ & 4454.00 & 6802.24 & 7.57 & 2.4 & Maine \\
\hline
\end{tabular}




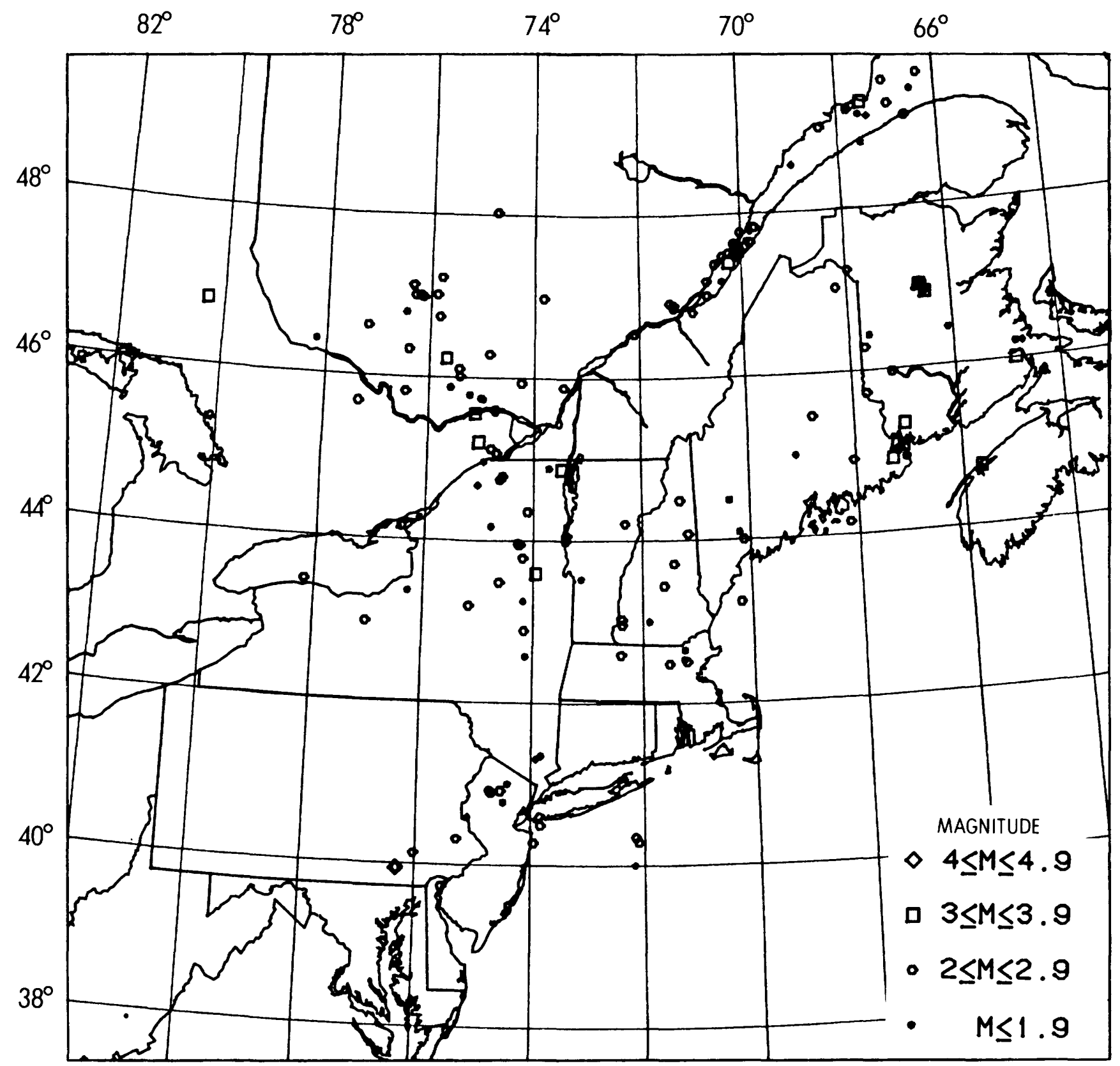

Figure 46. Northeastern United States earthquakes during 1984. 
In January 1984, during this period of moderate earthquake activity, two strong-motion instruments were installed at two places in eastern Maine. The sites chosen for their location were about 20 kilometers from the $\mathbf{M}_{n}=3.8$ earthquake which occurred on January 19 . At both places the strongest ground motions did not exceed the trigger-levels of the instruments, $0.01 \mathrm{~g}$ (Ebel, 1984).

By March 1984, the entire Weston Observatory seismic network was being recorded digitally as well as in the analog form on photographic film. The digital system had been developed over the period 1982-1984. During the course of the year, 16 events were recorded by the digital system, five of which were in New England (Ebel, 1984).

The WWSSN seismic data available at Weston Observatory (WES) was used to study the crustal structure directly under WES. This study involved the calculation of long-period synthetic seismograms and their comparison with data collected at WES. The crust beneath WES was clearly different from that described in the Chiburis and Ahner (1980) and the Taylor and Toksoz (1979) models. The best fitting model differs from other refraction results developed for New England and may reflect the location of WES just east of the Bloody Bluff fault (Ebel, 1984).

Investigation of source and path properties of short-period Rayleigh waves $\left(R_{y}\right)$ recorded by the network has been made. This study provides information about the shallow crustal structure and depths of seismic sources in New England (Ebel, 1984).

\section{Seismicity of New Mexico, 1984}

By Allan R. Sanford

Geoscience Department and Geophysical

Reasearch Center

New Mexico Institute of Mining and Technology

Socorro, NM 87801,

Lawrence Jaksha

U.S. Geological Survey

Socorro, NM 87801,

and Dan Cash

Geophysics Group, ESS-\$

Los Alamos National Laboratory

Los Alamos, NM 87545

The distribution of earthquakes in the state of New Mexico during 1984 is shown in Figure 47. Plotted on this map are epicenters for 37 shocks whose duration magnitudes were 1.5 or greater. Data are from networks of seismic stations operated by Los Alamos National Laboratory (LANL) and New Mexico Institute of Mining and Technology (NMIMT) in
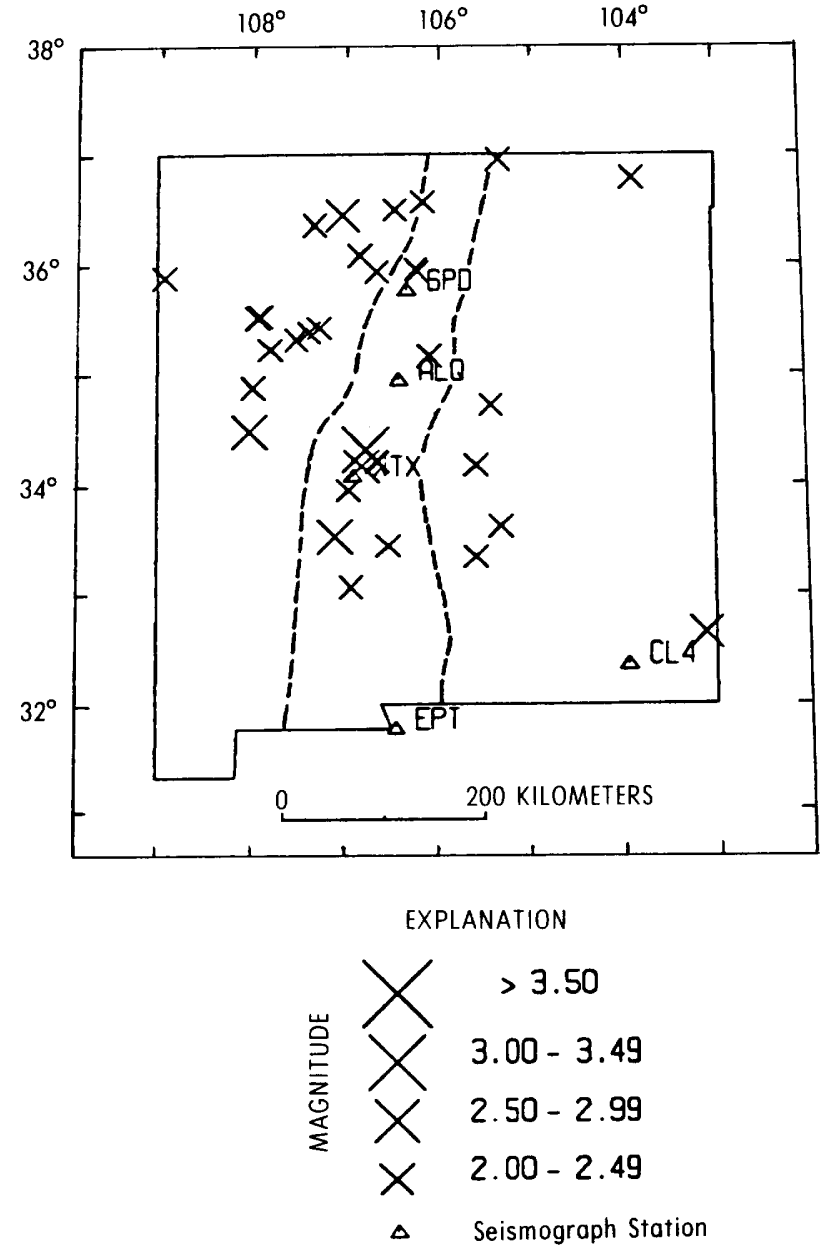

Figure 47. Eartliquakes in New Mexico during 1984.

collaboration with the United States Geological Survey (USGS). The LANL network is centered on the Rio Grande rift in north-central New Mexico; the principal NMIMT/USGS network is centered on the rift near the middle of the state. Stations SPD and WTX (fig. 47) are located near the centers of these two networks. An additional small network of stations in southeastern New Mexico, which includes station CL4 (fig. 47), is operated by the NMIMT/USGS group.

Although the recording stations are concentrated in the central regions of the state, the geographical distribution of earthquake activity in figure 47 is believed to be nearly free of station location bias. Earthquakes with magnitudes of 1.5 in the farthest regions of the state are well within the detection capabilities of all stations in the two principal networks. However, because the azimuthal distribution of stations narrows as distance from the networks increases, the accuracy of earthquake locations diminishes progressively toward the boundaries of the state. 


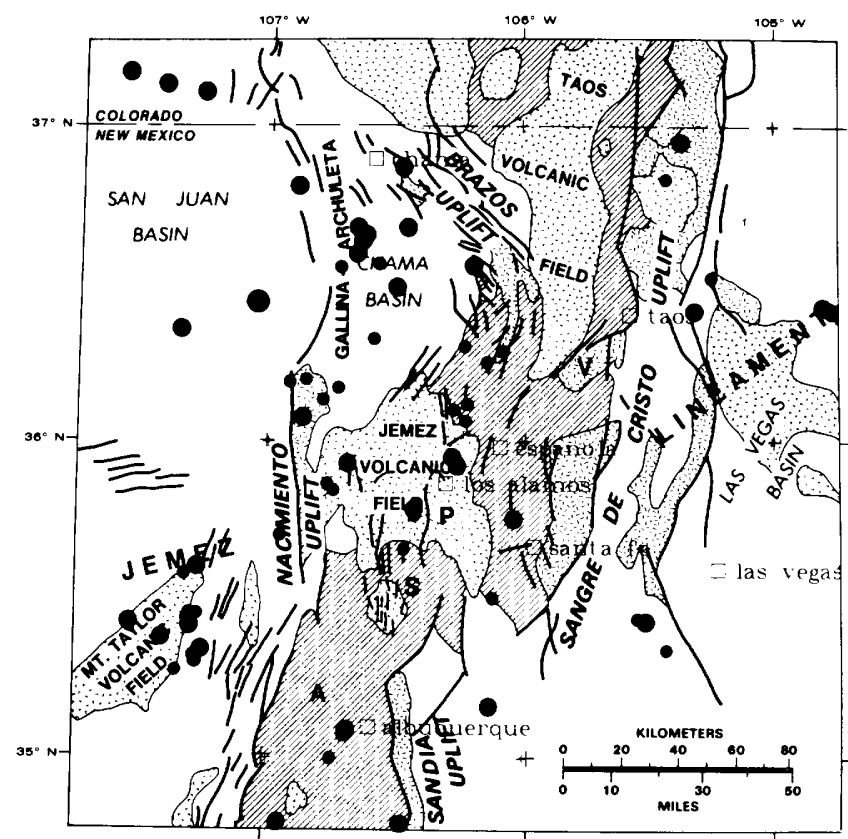

EXPLANATION

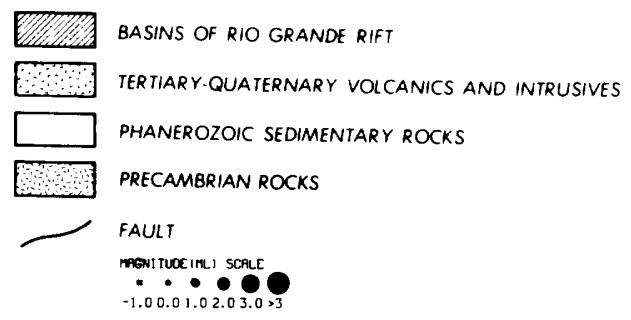

Figure 48. Seismicity of north-central New Mexico during 1984 superimposed on a generalized geologic and tectonic map.

For the most part, the distribution of earthquake activity in 1984 was similar to what has been observed since instrumental studies began in New Mexico (Sanford, 1965; Sanford and Cash, 1969; Toppozada and Sanford, 1972; Sanford, and others, 1981). Earthquakes were most numerous within or near the Rio Grande rift, a major extensional structure that runs north-south through the center of the state (approximate boundaries shown by dashed lines in fig. 47). Major differences between the 1984 seismicity and the long-term (since 1962) distribution of earthquake activity were (1) the near absence of epicenters in the Great Plains of eastern New Mexico and (2) the total absence of epicenters in the Datil-Mogollon volcanic field and southern Basin and Range in the southwestern quadrant of the state.

The strongest earthquake during the year occurred 30 kilometers $\mathrm{N} 26^{\prime \prime} \mathrm{E}$ of station WTX at 0219 UTC on August 26. The shock, which measured 2.5 on a duration magnitude scale, was felt in two small communities near the epicenter.
The seismograph networks in New Mexico are positioned to obtain detailed information on seismic activity where a prominent northeast-trending volcanic lineament crosses the Rio Grande rift (LANL network) and where a thin layer of magma exists at midcrustal level beneath the Rio Grande rift (NMIMT/USGS network). Discussions of seismic activity in these two areas during 1984 appear elsewhere in this publication.

\section{Seismic Activity in North-Central New Mexico, 1984}

\author{
By Joyce J. Wolff and Daniel J. Cash \\ Geophysics Group, ESS-9 \\ Los Alamos National Laboratory \\ Los Alamos, NM 87545
}

The Los Alamos Seismograph Network (LASN) has operated continuously since 1973 with as many as 24 stations operating at various times during that period. In 1983, the number of stations dropped to ten or fewer (Cash and Wolff, 1987), and in 1984, the number was reduced to six. We continued running locations, occasionally supplementing our data with times provided by the U.S. Geological Survey and the New Mexico Institute of Mining and Technology (both in Socorro). The reduced number of stations prevented us from locating numerous small events that occurred in our normal coverage area. Since we had fewer earthquakes to report in 1984, we published only one catalog for the entire year instead of issuing quarterly ones as we had done in the past. The Earthquake Catalog for Northern New Mexico, 1984 (Wolff and others, 1985), is the last one we will publish.

As the number of stations declined, it became impossible to monitor as broad an area as we had before. In 1984, we reduced our coverage from that shown in previous catalogs from:

$$
34.00^{\prime \prime}-37.500^{\prime \prime} \mathrm{N} \text {. by } 102.50-109.50^{\prime \prime} \mathrm{W} \text {. }
$$

to:

$$
34.75^{\prime \prime}-37.25^{\prime \prime} \mathrm{N} \text {. by } 104.75-107.75^{\prime \prime} \mathrm{W} \text {. }
$$

We chose these particular coordinates to include three active seismic areas: the Albuquerque volcanoes, the northern end of the Gallina-Archuleta arch as it trends into Colorado, and the Mt. Taylor volcanic field. This reduction eliminates two areas where we continually have problems. The first is the section of San Juan Basin that extends from the Four Corners area southward to Gallup. Here it is difficult to distinguish between earthquakes and coal mine explosions. The second is the Great Plains east of the Sangre de Cristo Mountains where our location attempts are frequently unsatisfactory. The location accuracy of events that 


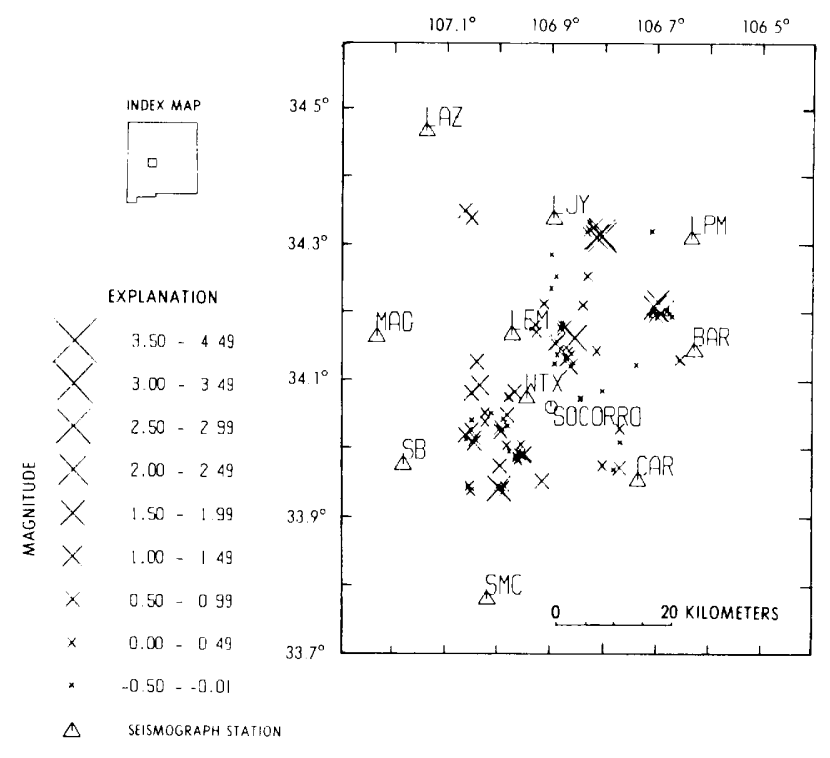

Figure 49. Seismicity of the Socorro, New Mexico, area during 1984. Plotted are 103 epicenters for which HYPO71 Revised (Lee and Lahr, 1975) solutions were quality $\mathrm{B}$ or better.

occur anywhere outside the network has always been suspect, even when we used many stations in earlier years.

Epicenters are determined using HYPOINVERSE (Klein, 1978) with a $6.15 \mathrm{~km} / \mathrm{sec}$ half-space velocity model applying no station corrections. Figure 48 shows the 1984 summary of epicenters (smaller coverage) superimposed on a generalized geologic and tectonic map.

Depths are only calculated if an event occurs within 10 kilometers of an operating station and if we can read a $P$ or an $S$ arrival time. In 1984, there were eight earthquakes that met this criteria and were run unconstrained. Depths ranged from 0.9 to 8.5 kilometers, not unusual in this region of extensional tectonics.

Magnitudes are calculated by the formula:

$$
\mathbf{M}_{L}=2.79 \log \mathbf{T}(\mathrm{sec})-3.63,
$$

where $\mathbf{T}$ is the event duration measured from the first $P$-wave arrival to the time when the signal disappears into the background (Newton and others, 1976). The formula was determined by fitting about ten large northern New Mexico earthquakes with the $\mathbf{M}_{L}$ value calculated from WWSSN station, ALQ. It works well for most events in central New Mexico in magnitude range 1-4 (Ake and others, 1983).

Every effort is made to identify and disregard manmade events. We communicate with individual mine

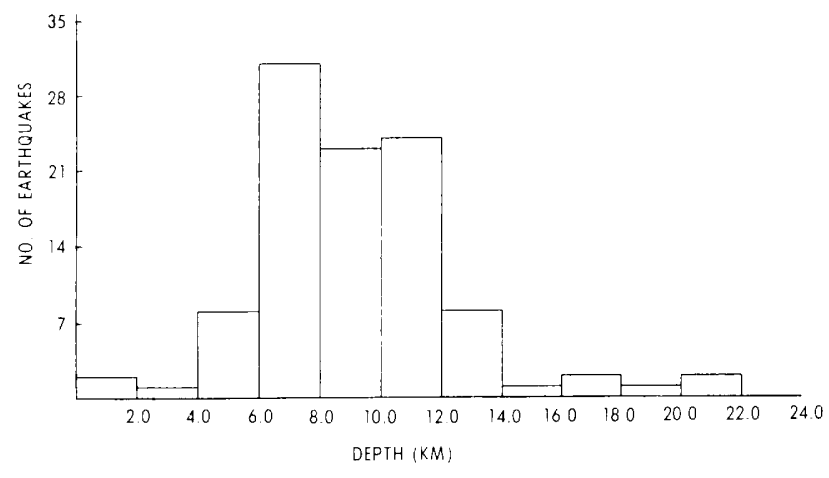

Figure 50. Distribution of focal depths for the 103 earthquakes plotted in figure 49 .

operators and frequently consult the state mine inspector and the highway department. Still, a few earthquakes may have been inadvertantly discarded because they occurred near known mining sites and had other explosion characteristics. The likelihood of confusing earthquakes and explosions has heightened because we have fewer stations to provide known signatures.

In 1984, 68 earthquakes (none felt) were cataloged in north-central New Mexico. They were all located in areas of known seismicity monitored by Los Alamos (Cash and Wolff, 1984) and provided no surprises.

\section{Socorro, New Mexico, Area Earthquakes, 1984}

By Allan Sanford, Shirley Wade, and Kevin King Geoscience Department and Geophysical

Research Center

New Mexico Institute of Mining and Technology

Socorro, NM 87801

and Lawrence Jaksha

U.S. Geological Survey

Socorro, NM 87801

The Socorro, New Mexico, network is a collaborative project of New Mexico Institute of Mining and Technology and the U.S. Geological Survey. For the first 9 months in 1984, the network consisted of nine stations; all of those shown in figure 49, except LJY and LEM, plus station MLM which is off the map 40 kilometers north of station LAZ. Station LJY was added to the network on October 2, 1984; station LEM was added June 22, 1985.

Earthquakes recorded by the Socorro network are located using the algorithm HYPO71 Revised (Lee and Lahr, 1975) with a half-space velocity of $5.85 \mathrm{~km} / \mathrm{sec}$ and a Poisson's ratio of 0.25 (Ward and others, 1981). Station corrections ranging from -0.33 to 0.28 second 
are used to account for differences in near-surface geology and elevation within the network (Ward, 1980; and Ake, 1984).

Magnitudes of the earthquakes are calculated from durations of recorded signals using an empirical equation based on northern New Mexico earthquakes (Newton and others, 1976). The same equation was found to be applicable to earthquakes in the Socorro area in the magnitude range 1.0-4.0 (Ake and others, 1983).

The 103 epicenters plotted in figure 49 are the total number of earthquakes in 1984 for which the HYPO71 solutions were of $\mathrm{B}$ or better quality. The total number of located events during 1984 was 312, but many of these had epicenters near or beyond the perimeter of the array where it is difficult to obtain high-quality solutions. For the interior of the array, the distribution of epicenters shown here for 1984 is quite similar to the pattern of seismicity observed in the same restricted area since installation of the network in June 1982 (Sanford and Wieder, 1985; Sanford and others, 1987).

The strongest earthquake within the Socorro network in 1984 was also the strongest shock in the entire state for the year. The 2.5 magnitude event, which took place at 0219 UTC on August 26, had epicentral coordinates of $34.314^{\prime \prime} \mathrm{N}$., $106.807^{\prime \prime} \mathrm{W}$; these are approximately 5 kilometers east of the center of a major swarm which occurred 18 months earlier (Jarpe, 1984). The earthquake was felt at La Joya and Contreras, two small villages approximately 5 and 8 kilometers east and northeast, respectively, of station LJY.

Figure 50 shows the distribution of focal depths for the 103 earthquakes in figure 49. The base of the seismogenic zone is believed to be around 13 kilometers even though about $10 \%$ of the HYPO71 solutions have focal depths which are greater.

\section{Oklahoma Earthquakes, 1984}

By James E. Lawson, Jr.

Oklahoma Geological Survey

Oklahoma Geophysical Observatory

Leonard, OK 74049,

and Kenneth $V$. Luza

Oklahoma Geological Survey

University of Oklahoma

Norman, OK 79019

A statewide network of 13 seismograph stations is recording seismological data in Oklahoma (fig. 51). The Oklahoma Geophysical Observatory (OGO) station, TUL, has been recording earthquake data since December 1961. The Observatory, located near
Leonard, Oklahoma, in southern Tulsa County, operates seven seismometers, three long period and four short period, which are installed in a vault detached from the main building. The seismic responses at TUL are recorded on 14 paper-drum recorders and one digital recorder. Accurate timing is assured by a microprocessor clock that is continually locked to the National Bureau of Standards cesium-beam clocks by low-frequency radio transmissions broadcast by WWVB (Lawson, 1980). Nine semipermanent volunteer-operated seismograph stations and three radio-telemetry seismograph stations complete the $\mathrm{Ok}$ lahoma Geological Survey's seismic network. The operation and maintenance of 12 of the remote stations is partially supported by the U.S. Nuclear Regulatory Commission.

Each of the nine volunteer-operated seismograph stations consists of a Geotech S-13, short-period, vertical seismometer; a Sprengnether MEQ-800 B unit, including amplifier, filters, ink-recording unit, and clock; and a Kinemetrics time-signal-radio receiver for highfrequency WWV time signals. Each radio-telemetry system consists of one Geotech S-13 seismometer and one radio-telemetry unit. The telemetry unit amplifies the seismometer output and uses this output to frequency-modulate an audiotone. A 500-milliwatt, crystal-controlled transmitter limits the line-of-sight transmission to 80 kilometers. Seismograms from the radio-telemetry stations are recorded at the Oklahoma Geophysical Observatory.

Station MFO was installed in Comanche County, southwestern Oklahoma, on April 20, 1984. Although this station was positioned near the Meers fault to monitor possible microseismic activity, it has proved to be an excellent detector of Oklahoma earthquakes as well.

Station OCO, which contains equipment similar to the volunteer-operated stations, is located at the Omniplex museum in Oklahoma City. Omniplex staff members change the seismic records daily as well as maintain the equipment. Oklahoma Geophysical Observatory staff help interpret the seismic data and archive the seismograms with all other Oklahoma network seismograms.

A triggered-digital recording station, SCE, which was installed in December 1980 as part of a five-station array in Canadian and Grady Counties was closed in November 1983. The site became too noisy because of an increase in oil-field-related activities.

All Oklahoma earthquakes recorded on seismograms from three or more stations are located. In 1984, 36 Oklahoma earthquakes were located (fig. 52 ; table 7). Six of these earthquakes were reported felt. The 


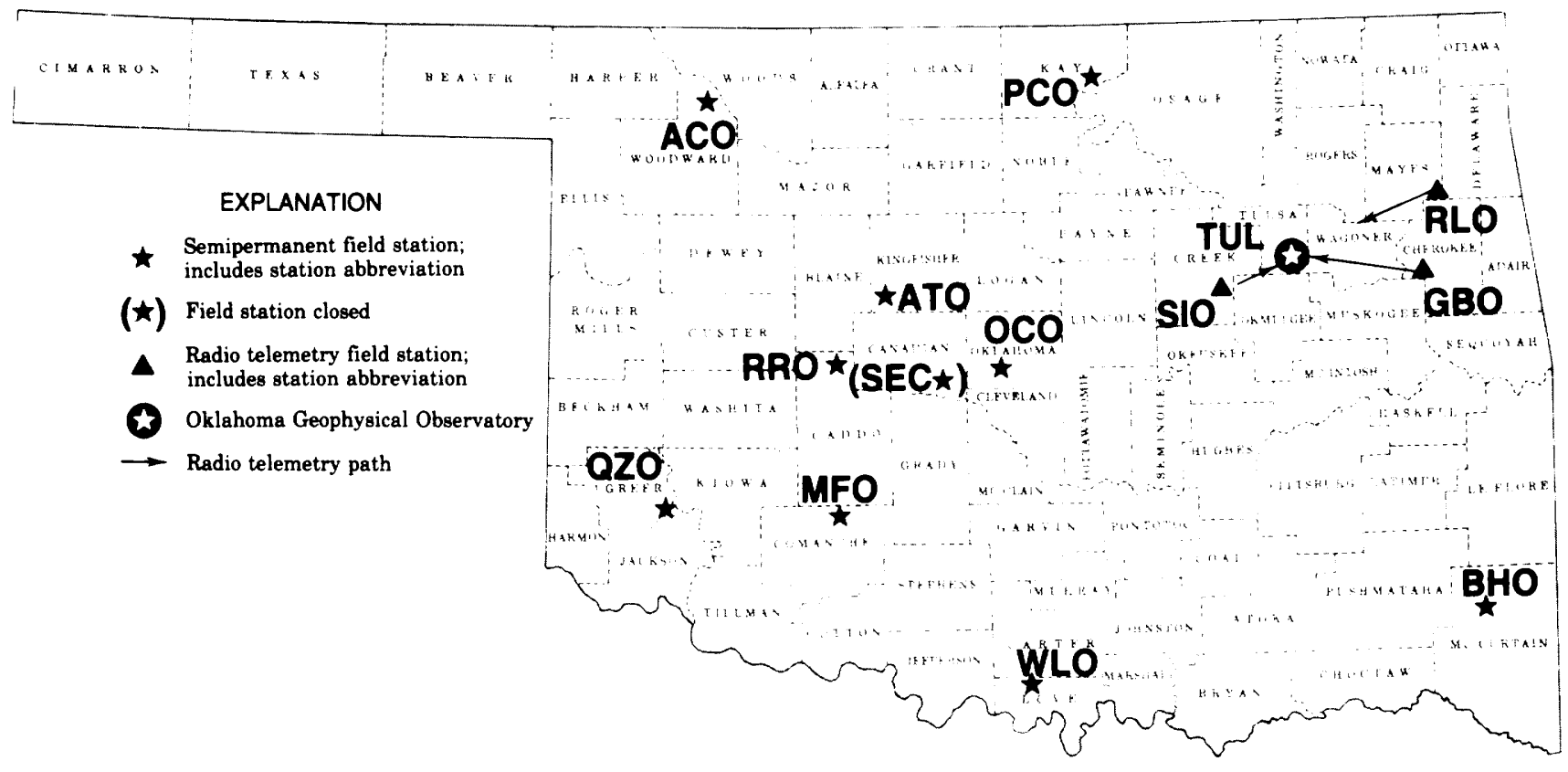

Figure 51. Active seismograph stations in Oklahoma for 1984.

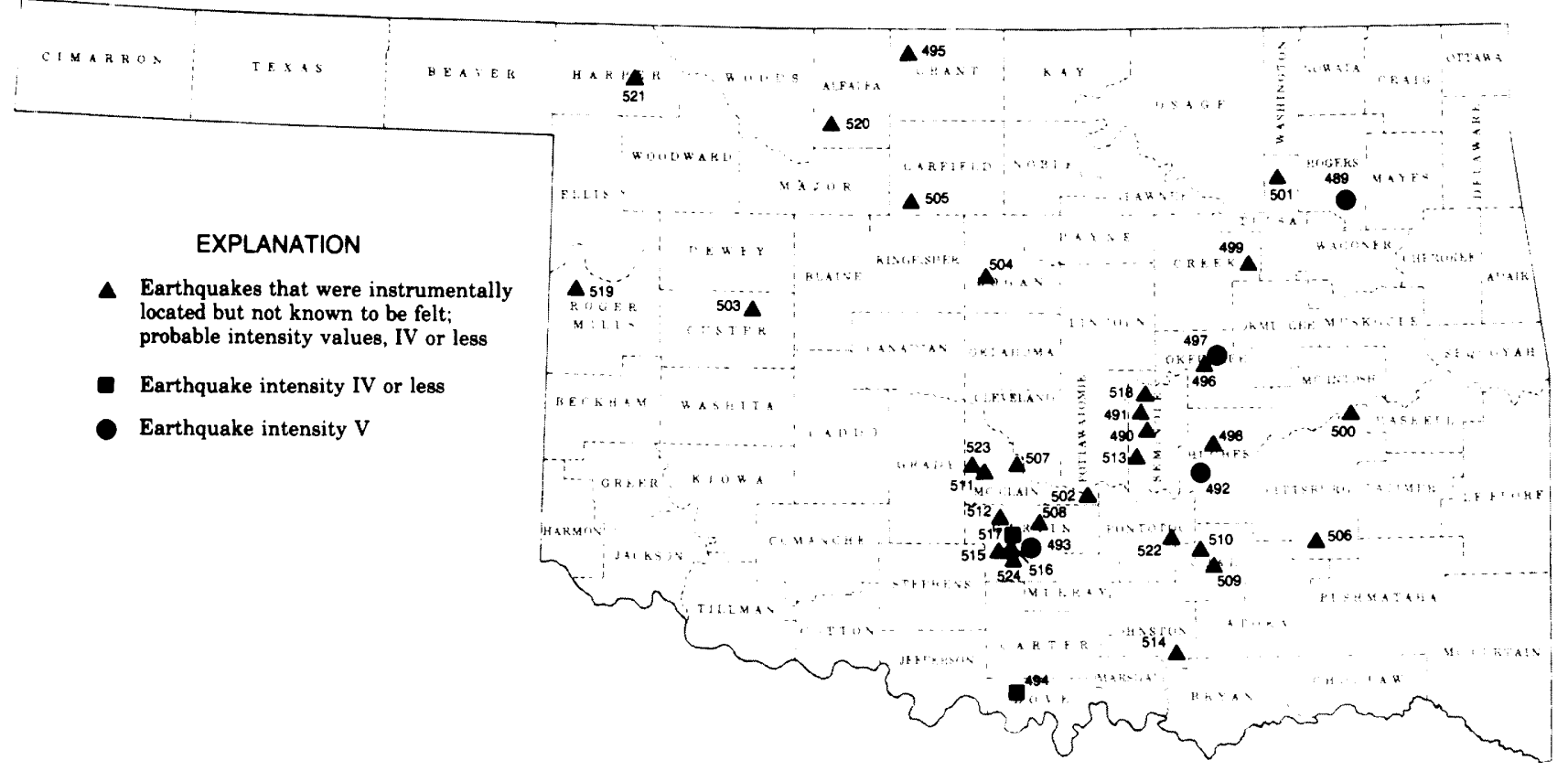

Figure 52. Distribution of Oklahoma earthquakes for 1984 . Numbers correspond to event numbers in table 7 . 
Table 7. Oklahoma earthquake catalog for 1984

\begin{tabular}{|c|c|c|c|c|c|c|c|c|c|c|}
\hline $\begin{array}{l}\text { Event } \\
\text { Number }\end{array}$ & $\begin{array}{l}\text { Dute } \\
\text { (UTC) }\end{array}$ & $\begin{array}{l}\text { Ongin and } \\
\text { Time }\end{array}$ & County & $\begin{array}{l}\text { Int } \\
\text { MM }\end{array}$ & $3 \mathrm{~Hz}$ & $\begin{array}{c}\text { Magnitude } \\
\text { bLg }\end{array}$ & DUR & $\begin{array}{c}\text { Lotitude } \\
\text { iN }\end{array}$ & $\begin{array}{c}\text { Longitude D } \\
\text { W }\end{array}$ & $\begin{array}{l}\text { Depth } \\
(\mathrm{km})^{\prime}\end{array}$ \\
\hline 489 & JANo & 17144981 & ROGERS & $v$ & 2.5 & & 25 & 36.161 & 95582 & $50 \mathrm{~K}$ \\
\hline 490 & IAN 23 & 10370430 & SEMINOLE & & 15 & & 13 & $35.20^{7}$ & 2064 & $50 R$ \\
\hline & JAN 23 & 11015621 & $\begin{array}{l}\text { SEMINLLE } \\
\text { HECHES }\end{array}$ & $\mathrm{v}$ & $\begin{array}{l}22 \\
31\end{array}$ & & 27 & $\begin{array}{l}35.234 \\
35.033\end{array}$ & $\begin{array}{l}06001 \\
0 \times 60\end{array}$ & $\begin{array}{l}50 R \\
50 R\end{array}$ \\
\hline 年4 & FEB 3 & 043828.04 & GARVIN & $v$ & & 32 & 31 & 34065 & 97356 & $50 \mathrm{R}$ \\
\hline 494 & FEB io & $\begin{array}{l}183997356 \\
1836\end{array}$ & LOLE & IV & 2.4 & & 22 & 34049 & 97415 & $5.0 R$ \\
\hline 195 & FEB 20 & 01472200 & GRANT & & 21 & & 20 & 36.870 & 98068 & UR \\
\hline 490 & $\mathrm{FEB} 20$ & $1148+951$ & OKFLSKEE & & 23 & & 21 & $\begin{array}{l}35+455 \\
35.514\end{array}$ & 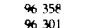 & $\begin{array}{l}5.0 R \\
5 \pi R R\end{array}$ \\
\hline 499 & $\begin{array}{l}\text { MAR } 3 \\
\text { MAR ID }\end{array}$ & $\begin{array}{l}11420236 \\
1713018\end{array}$ & $\begin{array}{l}\text { OOFLLSNEE } \\
\text { HLCGHES }\end{array}$ & $\mathrm{v}$ & 27 & 20 & $\begin{array}{l}27 \\
23\end{array}$ & $\begin{array}{l}35.514 \\
35117\end{array}$ & 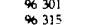 & $\begin{array}{l}5,0 R \\
5,0 R\end{array}$ \\
\hline $\begin{array}{l}498 \\
499\end{array}$ & $\begin{array}{l}\text { MAR 16 } \\
\text { APR } 18\end{array}$ & $\begin{array}{l}1713130.18 \\
08363 ; 22\end{array}$ & $\begin{array}{l}\text { HLGHES } \\
\text { CREEK }\end{array}$ & & $\begin{array}{l}2.5 \\
12\end{array}$ & & $\begin{array}{l}2.3 \\
1.5\end{array}$ & $\begin{array}{l}35117 \\
35.922\end{array}$ & $\begin{array}{r}\quad 315 \\
96074 \\
96074\end{array}$ & $\begin{array}{l}5.0 R \\
5.0 R\end{array}$ \\
\hline $\begin{array}{l}1990 \\
500\end{array}$ & APR 21 & $\begin{array}{l}053380212 \\
0536\end{array}$ & $\begin{array}{l}\text { PIITSSLL'RG } \\
\text { PITSK }\end{array}$ & & 1.9 & 15 & 1.8 & $\begin{array}{l}35.922 \\
35.233\end{array}$ & $\begin{array}{l}0.414 \\
9556 ?\end{array}$ & $50 R$ \\
\hline 501 & APR 28 & 2554071 & TLLSI & & 1.1 & & 1.2 & & 95921 & $50 \mathrm{R}$ \\
\hline 502 & MAY 27 & 12132731 & MC CLAN & & 2.1 & 17 & 2.0 & 34884 & $\$ 965$ & $50 \mathrm{R}$ \\
\hline 503 & MAY 31 & 023658312 & CLSTER & & 1.5 & & 20 & 35735 & 98013 & $5 \mathrm{OR}$ \\
\hline 504 & $14 \times 3$ & $081413 ! 1$ & LOGAII & & 2.0 & & 22 & & $\begin{array}{l}97593 \\
97979\end{array}$ & $\begin{array}{l}50 R \\
50 R\end{array}$ \\
\hline 505 & $\operatorname{lin} 11$ & $0508456-$ & GARFIELD, & & 15 & & 1.8 & $\begin{array}{l}361 \% 0 \\
34029\end{array}$ & $\begin{array}{l}97.978 \\
9.9584\end{array}$ & $5 \mathrm{BR}$ \\
\hline $\begin{array}{l}506 \\
507\end{array}$ & $\begin{array}{l}1614 \\
14213\end{array}$ & $\begin{array}{l}030000373 \\
165 \times 1235\end{array}$ & $\begin{array}{l}\text { PIITSBERG } \\
\text { MC CLAIK }\end{array}$ & & 19 & 22 & $\begin{array}{l}24 \\
22\end{array}$ & $\begin{array}{l}34029 \\
35020\end{array}$ & $\begin{array}{l}958.4 \\
97358\end{array}$ & $\begin{array}{l}5,8 R \\
50 R\end{array}$ \\
\hline 508 & ALC 1- & $180(40185$ & GARYIN & & 27 & 25 & 2.4 & 34.67 & 97326 & $50 \mathrm{R}$ \\
\hline 504 & ACC IS & $0.04+2163$ & & & 1.8 & 17 & 1.9 & & os 336 & $50 \mathrm{R}$ \\
\hline $\begin{array}{l}516 \\
511\end{array}$ & $\begin{array}{l}\text { SEP \& } \\
\text { SEP I- }\end{array}$ & 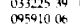 & $\begin{array}{l}\text { CAAI } \\
\text { MC CLAIX }\end{array}$ & & $\begin{array}{l}2.1 \\
1.0\end{array}$ & $\begin{array}{l}18 \\
20\end{array}$ & $\begin{array}{l}14 \\
22\end{array}$ & $\begin{array}{l}34.625 \\
35009\end{array}$ & 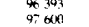 & $\begin{array}{ll}50 R \\
50 R\end{array}$ \\
\hline 512 & OCT 4 & $122 \times 1027$ & GARS & & 25 & 2.6 & 25 & 3471 & $9-502$ & 5 OR \\
\hline 513 & Not: & 07012288 & SE: & & 15 & & $1:$ & & $\% 745$ & 50 作 \\
\hline $\begin{array}{l}514 \\
515\end{array}$ & Nor & $\begin{array}{l}083.79976 \\
150451\end{array}$ & & & 2.2 & 2.10 & 20 & & Fis 518 & $\begin{array}{c}50 R \\
500\end{array}$ \\
\hline & $\begin{array}{l}\text { vot io } \\
\text { you is }\end{array}$ & $\begin{array}{l}11500451 \\
13140984\end{array}$ & $\begin{array}{l}\text { CARYKL } \\
\text { CARYII }\end{array}$ & & $\begin{array}{l}21 \\
24\end{array}$ & 2.1 & $\begin{array}{l}20 \\
21\end{array}$ & $\begin{array}{l}34+1+1 \\
340+4\end{array}$ & & $\begin{array}{l}50 R \\
50 R\end{array}$ \\
\hline 517 & Nor 20 & $105^{7}, 319$ & CARITI & N & 31 & 31 & $2=$ & 34707 & 9: 410 & $5 \pi R$ \\
\hline 518 & Nov 25 & $03.635 \mathrm{CH}$ & SEVI & & 19 & & 21 & 35340 & & $50 \mathrm{R}$ \\
\hline 519 & Nov 2 & $11202478 \%$ & ROGER MILLS & & 15 & & 19 & & & \\
\hline 520 & $\mathrm{NOH}_{30}$ & 132000792 & ALF & & 21 & & 22 & 36 & ant the & $50 \mathrm{R}$ \\
\hline 521 & DEC \& & $05 \times 2+1$ & $\begin{array}{l}\text { HAR } \\
\text { PON }\end{array}$ & & 20 & & 21 & & & \\
\hline $\begin{array}{l}522 \\
523\end{array}$ & $\begin{array}{l}\text { DEC } 11 \\
\text { DEC } 20\end{array}$ & & $\begin{array}{l}\text { PONTOTOX } \\
\text { MC CLAIY }\end{array}$ & & 18 & 17 & 19 & 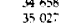 & 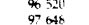 & $\begin{array}{l}50 k \\
50 R\end{array}$ \\
\hline 524 & DEC 29 & 04305923 & GARYIN & & 21 & 19 & 21 & 34609 & $9=437$ & $50 \mathrm{R}$ \\
\hline
\end{tabular}

felt areas for most of those six earthquakes are probably restricted to a few tens of kilometers ${ }^{2}$ away from the epicentral locations. No damage was reported from any of the felt earthquakes. On January 6 , a magnitude 2.5 $(\mathrm{m} 3 \mathrm{~Hz})$ earthquake, intensity MM V, occurred near Inola in northeastern Oklahoma. The last felt earthquake reported for this region occurred in March 1960. In late January, an intensity MM V earthquake, event number 492, was reported felt 7 kilometers east of Holdenville in Hughes County. On February 3, intensity MM V effects were reported 13 kilometers north of Elmore City. The same earthquake produced intensity MM II-IV effects in the Elmore City, Maysville, and Pauls Valley areas. A magnitude $2.4(\mathrm{~m} 3 \mathrm{~Hz})$ earthquake was located in Love County on February 10. Intensity MM IV effects were experienced 1 kilometer north of station WLO. Intensity MM V effects were reported in Okemah and Castle, Okfuskee County, on March 3. A magnitude $3.1(\mathrm{~m} 3 \mathrm{~Hz})$ earthquake occurred in Garvin County on November 20. This earthquake produced intensities of MM IV at Antioch and MM III at Elmore City, and was felt at Paoli.

The greatest concentration of earthquakes, 11, occurred in McClain and Garvin Counties. This region has been one of the most active since 1979. The Canadian County area, which has been the site of numerous earthquakes since 1908, contained no locatable earthquakes in 1984. Except for the February 10 earthquake, event number 494, the Wilson area was conspicuously quiet. The Arkoma Basin region experienced a modest number of earthquakes in 1984. The first known earthquake for Harper County occurred on December 8.

\section{Southeastern United States Earthquakes, 1984}

\author{
By G. A. Bollinger, Martin C. Chapman and \\ Matthew S. Sibol \\ Seismological Observatory \\ Virginia Polytechnic Institute and State University \\ Blacksburg, Virginia 24061
}

There were 83 earthquakes $(0.0 \leq \mathbf{M} \leq 4.2)$ detected instrumentally and located in the southeastern United States during 1984 (fig. 53). Six of these earthquakes were felt (table 8). The largest shock that occurred in the region during the year was a magnitude 4.2 event in central Virginia. The area experiencing the largest number of earthquakes (39/83 or $47 \%$ ) was southeastern Tennessee. For the report period, 16 earthquakes $(1.0 \leq \mathbf{M} \leq 2.0)$ in the vicinity of the Monticello Reservoir $\left(34.3^{\prime \prime} \mathrm{N}\right.$., $81.3^{\prime \prime} \mathrm{W}$.) in central South Carolina were reported by Pradeep Talwani of the University of South Carolina. A total of 136 seismograph stations were operational in the region at the end of 1984 (fig. 54).

Data listings for earthquakes during 1984 are contained in Southeastern United States Seismic Network (SEUSSN) Bulletins No. 14 and 15 available from the authors. Bulletin $14 \mathrm{~A}$ is a cumulative listing of hypocenters and magnitudes (July 1977-July 1983) and is also available from the authors. Bulletin 15 introduced a section on SEUSSN earthquake statistics (table 9 , fig. 55).

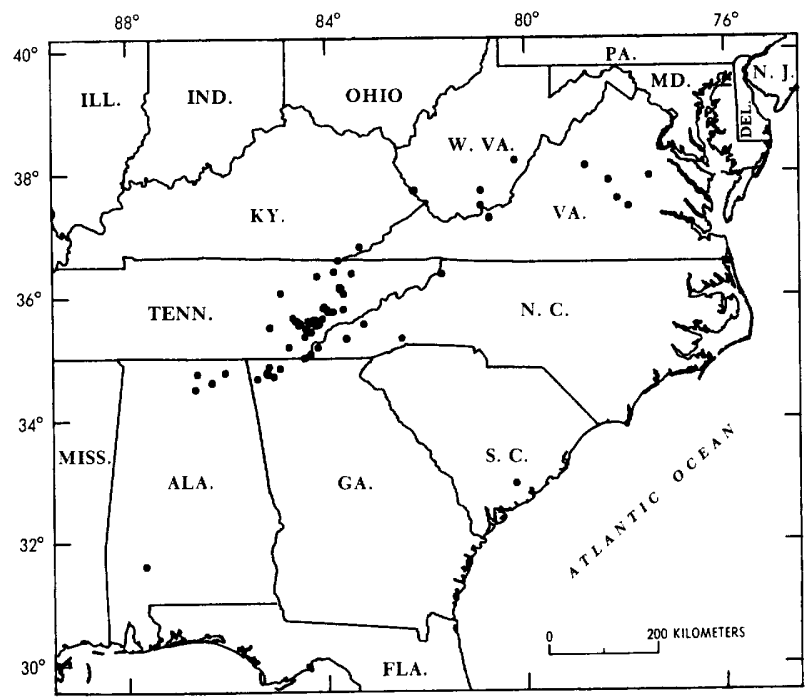

Figure 53. Southeastern United States earthquakes during 1984. 


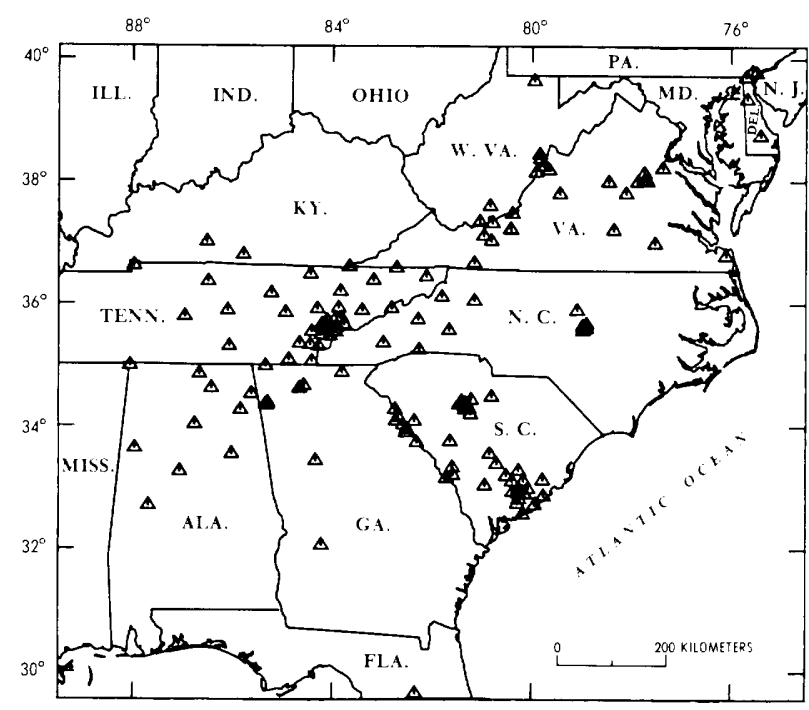

Figure 54. Southeastern United States seismic network stations during 1984 .
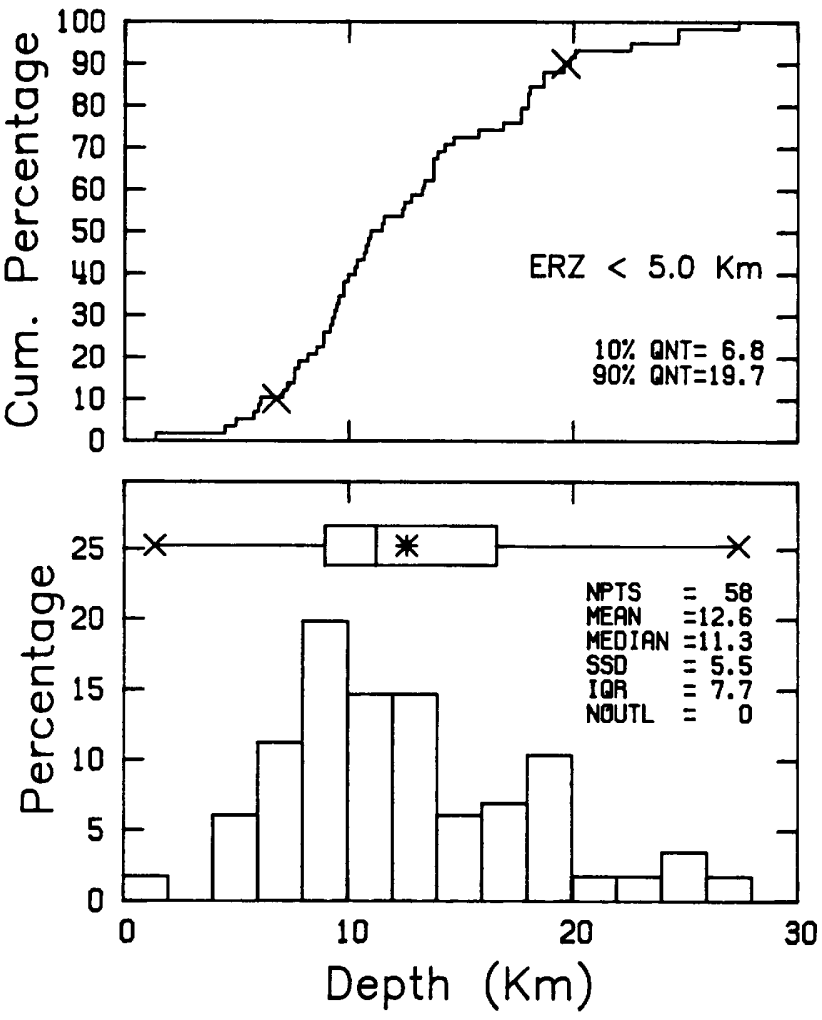

Figure 55. Earthquake statistics for the southeastern United States during 1984.
Table 8. Southeastern United States earthquakes, 1984

\begin{tabular}{|c|c|c|c|c|c|c|c|}
\hline $\begin{array}{l}\text { Date } \\
(1984)\end{array}$ & $\begin{array}{l}\text { Origin Time } \\
\text { (UTC) }\end{array}$ & $\begin{array}{l}\text { Lat. } \\
\text { (N.) }\end{array}$ & $\begin{array}{l}\text { Lons. } \\
\text { (ㅂ.) }\end{array}$ & $\begin{array}{l}\text { Depth } \\
\text { (III) }\end{array}$ & $\begin{array}{l}\text { Mag. } \\
\text { (mbLg/Md) }\end{array}$ & $\begin{array}{l}I_{0} \\
\text { (ry) })\end{array}$ & $\begin{array}{l}\text { State } \\
\text { Pelt }\end{array}$ \\
\hline 6 Jan. & $03: 04: 20.9$ & 31.607 & 87.808 & $5.0 F$ & 3.0 & $\nabla$ & $\perp$ \\
\hline 14 Feb. & $20: 54: 30.9$ & 36.125 & 83.737 & 9.8 & 3.5 & $v$ & TN \\
\hline $17 \mathrm{Mar}$. & $23: 26: 11.4$ & 35.832 & 84.050 & 7.3 & 3.0 & $\mathrm{IV}$ & $\mathbf{T N}$ \\
\hline 17 Aug. & $18: 05: 46.9$ & 37.868 & 78.324 & 8.2 & 4.2 & $\nabla$ & $\nabla A$ \\
\hline 9 Oct. & $11: 54: 26.3$ & 34.729 & 85.174 & $5.0 \mathrm{~F}$ & 4.0 & $\nabla I$ & GA \\
\hline 22 oct. & $18: 58: 41.8$ & 36.364 & 81.677 & 10.7 & 3.2 & $v$ & NC \\
\hline
\end{tabular}

Table 9. SEUSSN earthquake statistics, 1984

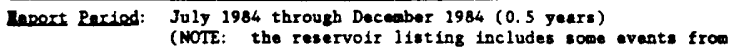
the first belf of 1984)

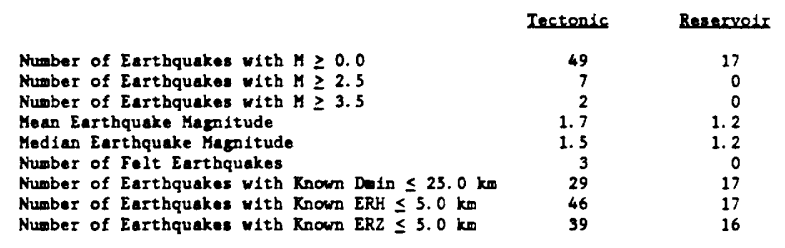

Lergest Earthquake Reported 17 August, 1984; 18:05 - Cunningham, vs $\operatorname{Mb}(28)=4.2$, MAII $=y$

Number of Blasts Reported

$\begin{array}{lr}\text { Number of Blasts Reported } & 1 \\ \text { Number of Events Outside the SEUSSN Reported } & 0 \\ \text { Number of Seismograph Stetions Operating } & 144\end{array}$

Repert Rertod: July 1977 through December 2984 ( 7.5 years)

Number of Earthquakes with $M \geq 0.0$

Number of Earthquakes with $M \geq 2$.

Number of Earthquakez with $M \geq 3.5$

Mean Earthquake Magnitude

Median Earthquake Magnitude

Number of Folt Earthquakes

Number of Earthquakes with Known Dmin $\leq 25.0 \mathrm{~km}$

Number of Earthquakes with Known ERH $\leq 5.0 \mathrm{~km}$

Number of Earthquakes with Known ERZ $\leq 5.0 \mathrm{~km}$

Tectonic Reserveit

Lergest Earthquake Roported

Number of Blests Reported

Number of Events Outside the sEUSSN Reported $1980 ; 18: 52-$ Sharpsburg, KY
$\mathrm{HB}(\mathrm{Lg})=5.2, \mathrm{MMI}=\mathrm{VII}$

415
100
11
1.9
2.0
61
184
316
259
$18: 52-$ Sharpsbu
5.2, MOII $=$ VII
152

\section{Utah Earthquakes, 1984}

\section{By Ethan D. Brown}

Seismograph Stations,

University of Utah

Salt Lake City, IT 84112-1183

The University of Utah Seismograph Stations records a regional seismic network that consisted of 81 short-period vertical stations, 27 of which are operated and maintained by other agencies (fig. 56). In addition to recording vertical components, four stations have horizontal short-period seismometers. Dugway, Utah (DUG), operates as a WWSSN station, and WoodAnderson type seismographs operate at Dugway and 


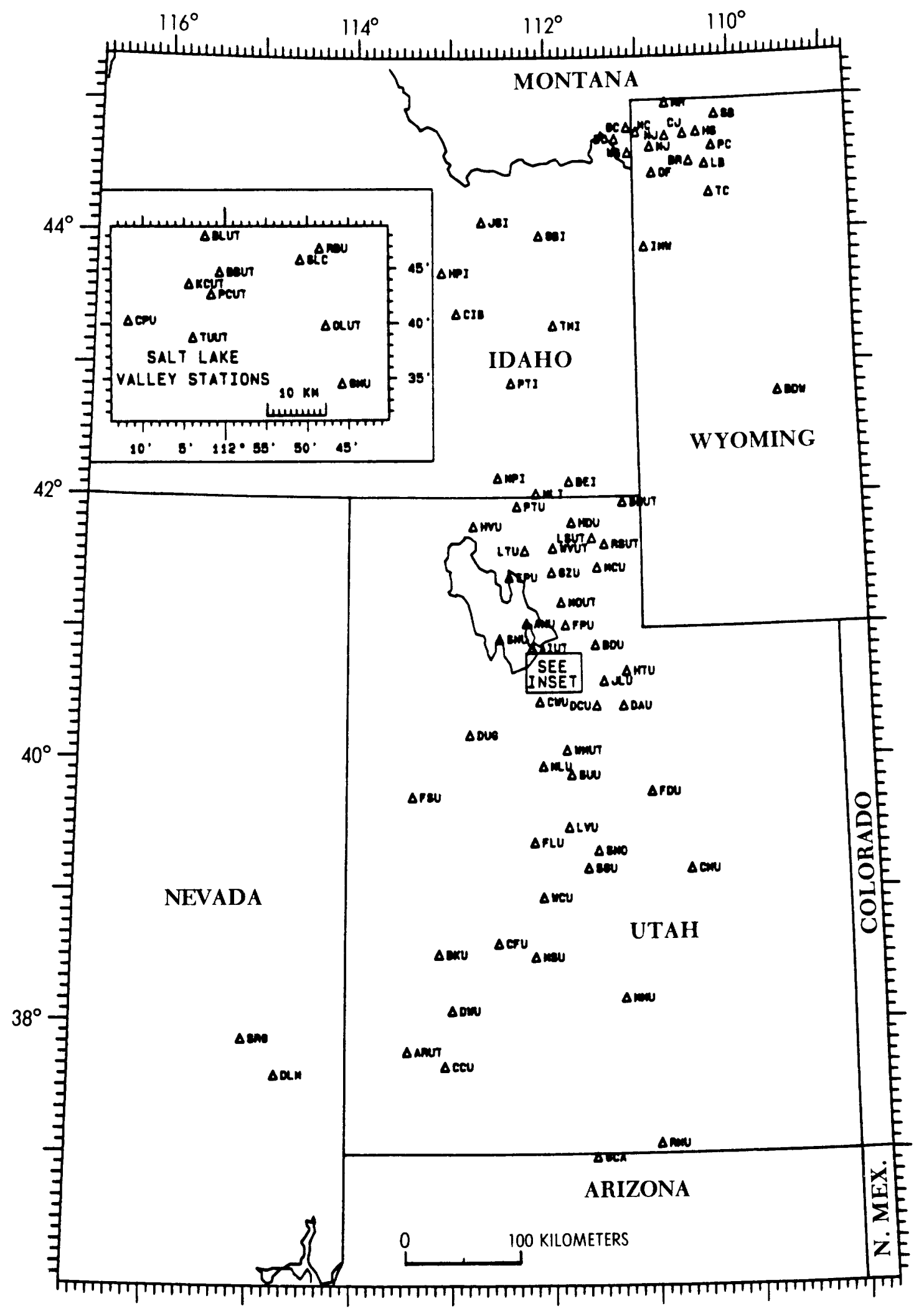

Figure 56. University of Utah seismograph station network during 1984. 


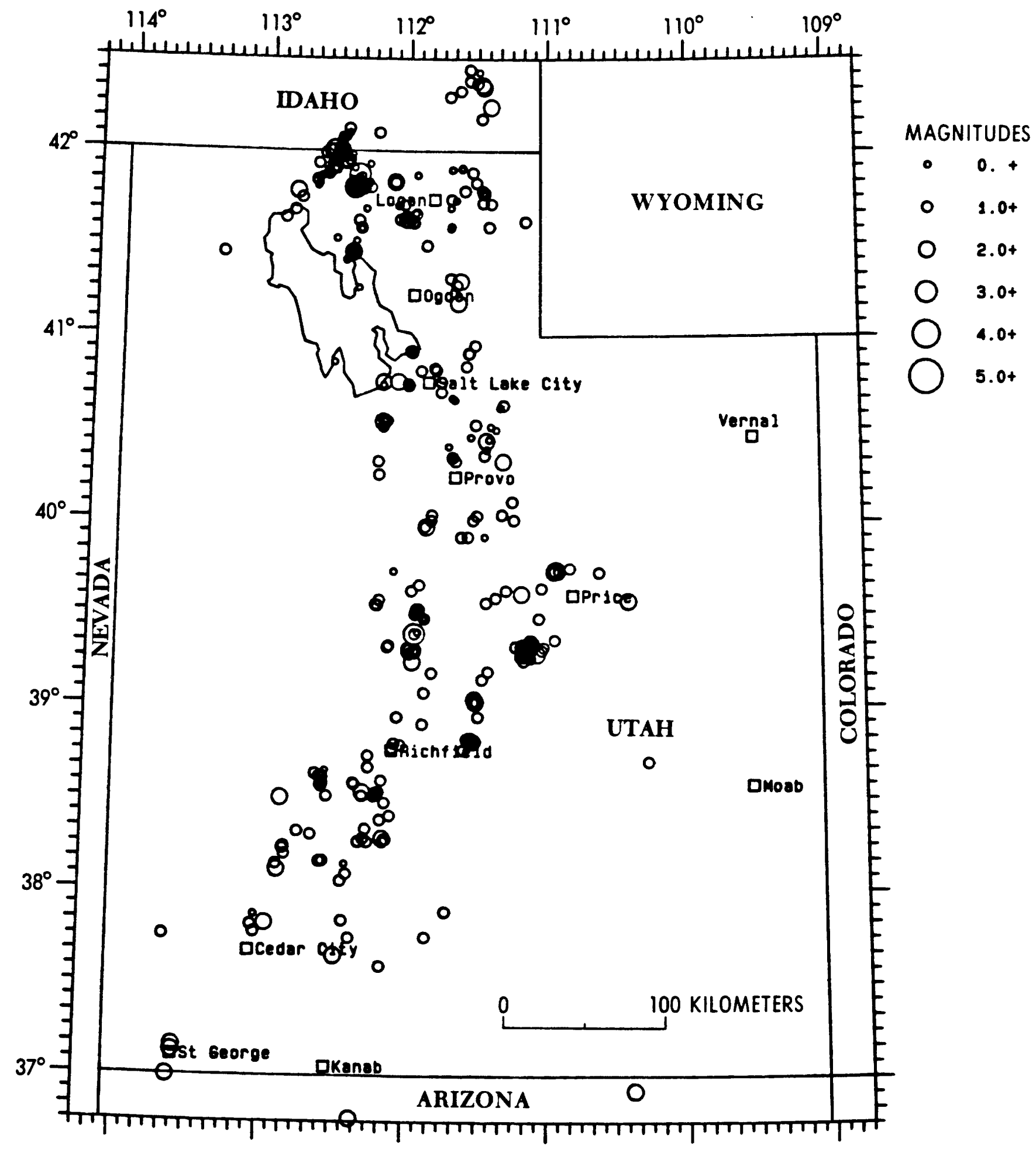

Figure 57. Utah and adjacent areas earthquakes during 1984. Squares indicate the location of cities. 
Salt Lake City. The network has average station spacings of 15-35 kilometers in north-central Utah, and 30100 kilometers in central and southwestern Utah and southeastern Idaho.

Seismic data from each seismometer are telemetered via telephone, microwave, and/or radio data transmission lines to a central recording facility located on the University of Utah campus in Salt Lake City. A discriminator bank decodes the incoming signals which are then sent through an $\mathrm{A} / \mathrm{D}$ converter to a DEC PDP$11 / 34$ computer. The $11 / 34$ monitors incoming signals using an event detection algorithm and digitally records the network data.

Epicenters for 426 earthquakes located in the Utah region during 1984 are shown in figure 57 and reflect typical earthquake activity scattered throughout Utah's main seismic region. The largest event during $1984\left(M_{L}=3.7\right)$ occurred on August 16, 1984, 75 kilometers north of Richfield near the southern end of the Wasatch fault. This earthquake was widely felt in nearby communities. Other significant aspects of earthquake activity shown in figure 57 are (from north to south):

1- Earthquake activity near Soda Springs, Idaho (approximately $42^{\prime \prime} 20^{\prime} \mathrm{N} ., 111^{\prime \prime} 90^{\prime} \mathrm{W}$.), in the same general area as a swarm sequence $\left(\mathbf{M}_{L} \leq 4.7\right)$ in 1982 .

2- Clusters of events along the Utah-Idaho border, north of the Great Salt Lake, which include late aftershocks of the magnitude 6.0 Pocatello Valley, Idaho, earthquake of March 1975 and related activity extending into northernmost Utah. A total of 98 events occurred there during 1984. This seismicity was characterized by two periods of swarm activity in February and October. A period of marked quiescence in March and April followed the first swarm.

9- Clusters of earthquakes within a 50-kilometer radius of Price, predominantly related to extensive underground coal mining. The rate of activity was nearly constant throughout 1984 with an average rate of 5-6 events/month.

4- Scattered activity within a broad north-south-trending zone between Salt Lake City and Richfield, Utah, in the general vicinity of the southern. Wasatch fault.

5- Scattered small earthquakes throughout southwestern Utah in a broad northeast-southwest-trending belt encompassing the Elsinore, Tushar, Sevier, and Hurricane fault zones between Richfield and Cedar City.

6- Three events near St. George including two small felt earthquakes (magnitudes 2.2 and 2.5) on November 25,1984 .
Details of Utah seismicity and information in bulletin format are available by contacting the University of Utah Seismograph Stations, 704 William C. Browing Building, Salt Lake City, Utah 48112.

\section{Washington Earthquakes, 1984}

By R. S. Ludwin, S. D. Malone, and R. S. Crosson University of Washington

Seattle, WA 98195

The University of Washington operated up to 111 telemetered seismic stations in Washington and northern Oregon during 1984. Our network configuration was described and depicted in a previous report (Noson and others, 1984). Minor changes in station coverage and equipment quality were made in 1984, including installation of three new stations: one on the Olympic Peninsula (OSD) in October 1984 and two in eastern Washington at Wenas and Moxie City in July and October, respectively. Station MOW was replaced in July by station BHW. On the Olympic Peninsula, OTR replaced OEM and OOW replaced OCT in June. Three stations (VBP, VGT, VLO) in north-central Oregon were permanently discontinued in October. Replacements for these stations may be reinstalled in 1986 . One station (BLS) was reinstalled in the Skagit Valley in December, replacing stations removed in 1982. Data acquisition and processing procedures are similar to those of the previous three years. A detailed description of procedures is available from the University of Washington (U.W.) Geophysics Program.

During 1984, 1,405 earthquakes were located within Washington and northern Oregon in the area shown in figure 58. Figure 58 shows epicenters of earthquakes which were reported as felt or had coda-length magnitudes $\left(\mathbf{M}_{\mathbf{r}}\right)$ greater than or equal to 2.7 (table 10 ). During 1984, 11 felt earthquakes were located in the area of figure 58 , and 33 additional events had magnitudes greater than or equal to 2.7. Earthquakes with felt reports are indicated by filled symbols. Of all earthquakes located by the U.W. network in $1984,54 \%$ were located in the vicinity of $\mathrm{Mt}$. Saint Helens, reflecting both greater station density and a higher level of activity at small magnitudes there. In 1984, $25 \%$ of the earthquakes were located west of the Cascade range outside of the Mt. Saint Helens area, $10 \%$ occurred within the Cascade Range, and the remaining $11 \%$ were east of the Cascade Range.

The largest event in Washington or northern Oregon during 1984 was a $\mathbf{M}_{1}:=4.3$ earthquake which occurred just north of Wenatchee on April 11, 1984 at 


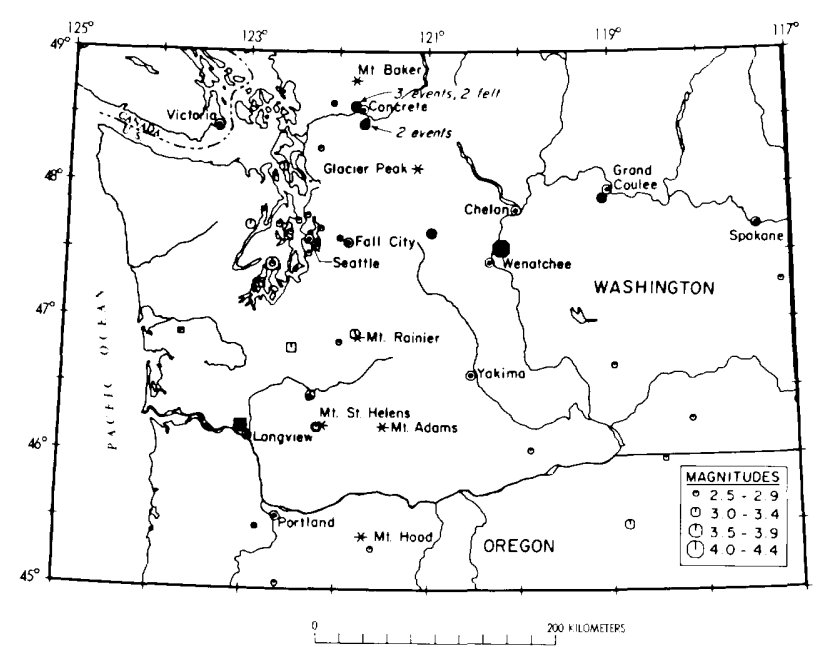

Figure 58. Earthquakes in Washington and northern Oregon during 1984 which had coda-length magnitudes $\geq 2.7$ and felt events of smaller magnitude. All felt events are filled. Round symbols represent events with depths shallower than 30 kilometers, while square symbols are events 30 kilometers or deeper.

0307 UTC. The event was felt in Yakima, Wenatchee, Leavenworth, Grand Coulee, and the southern Lake Chelan area. Secondhand reports of minor damage at Grand Coulee could not be confirmed. The maximum intensity would appear to be $M M=I V$ from Wenatchee. The location for this event (47'32.1'N., 120'11.13'W.) is about 20 kilometers south of a zone where seismic activity has persisted for more than 10 years. There was no other activity, including aftershocks, in the immediate vicinity of this event.

The largest and most significant earthquake in western Washington during 1984 was a $\mathbf{M}_{t}:=3.7$ earthquake located at a depth of 50 kilometers beneath Longview and widely felt in the Longview-Kelso area on June 4. During 1984 four well-located deep events (depth greater than or equal to 35 kilometers) of coda magnitude greater than or equal to 2.0 occurred south of latitude $47^{\prime \prime}$ north. An equal number of deep earthquakes were located in the same area in the 14 years preceding 1984. Instrumental coverage of the area improved in 1980 however, and older catalogs are probably not complete at magnitude 2.0 .

Two felt earthquakes of magnitudes 2.6 and $3.4 \mathrm{oc}-$ curred just south of Concrete, Washington, on March 16. On July 10 , a $\mathbf{M}_{1:=2.9}$ earthquake was felt 20 kilometers west of Concrete, near Mt. Vernon. Felt everts of magnitudes 3.1 and 3.2 were located just northwest of Concrete on December 2nd and 3rd. Five more earthquakes occurred in a tight cluster at the same spot during the remainder of December, including an unfelt $\mathbf{M}_{r}:=2.9$ event on December 23. All were shallower than 5 kilometers.

An event of $\mathbf{M}_{1}:=3.0$ located at a depth of 16 kilometers was felt at Grand Coulee Dam and in Elmer City on October 10. On April 27, an earthquake of $\mathbf{M}_{r:}=2.9$, felt at Fall City, was located at a depth of about 10 kilometers. On August 24, a camper felt a very shallow (about 1 kilometer depth) $\mathbf{M}_{i}:=3.0$ earthquake in the Cascades near the headwaters of the Yakima River. A $\mathbf{M}_{t}:=2.5$ event on December 11 occurred at a depth of about 25 kilometers and was felt in Portland, Oregon. A magnitude 3.6 earthquake on June 2, which was

Table 10. Washington area hypocenters for events with magnitudes $\geq 2.7$

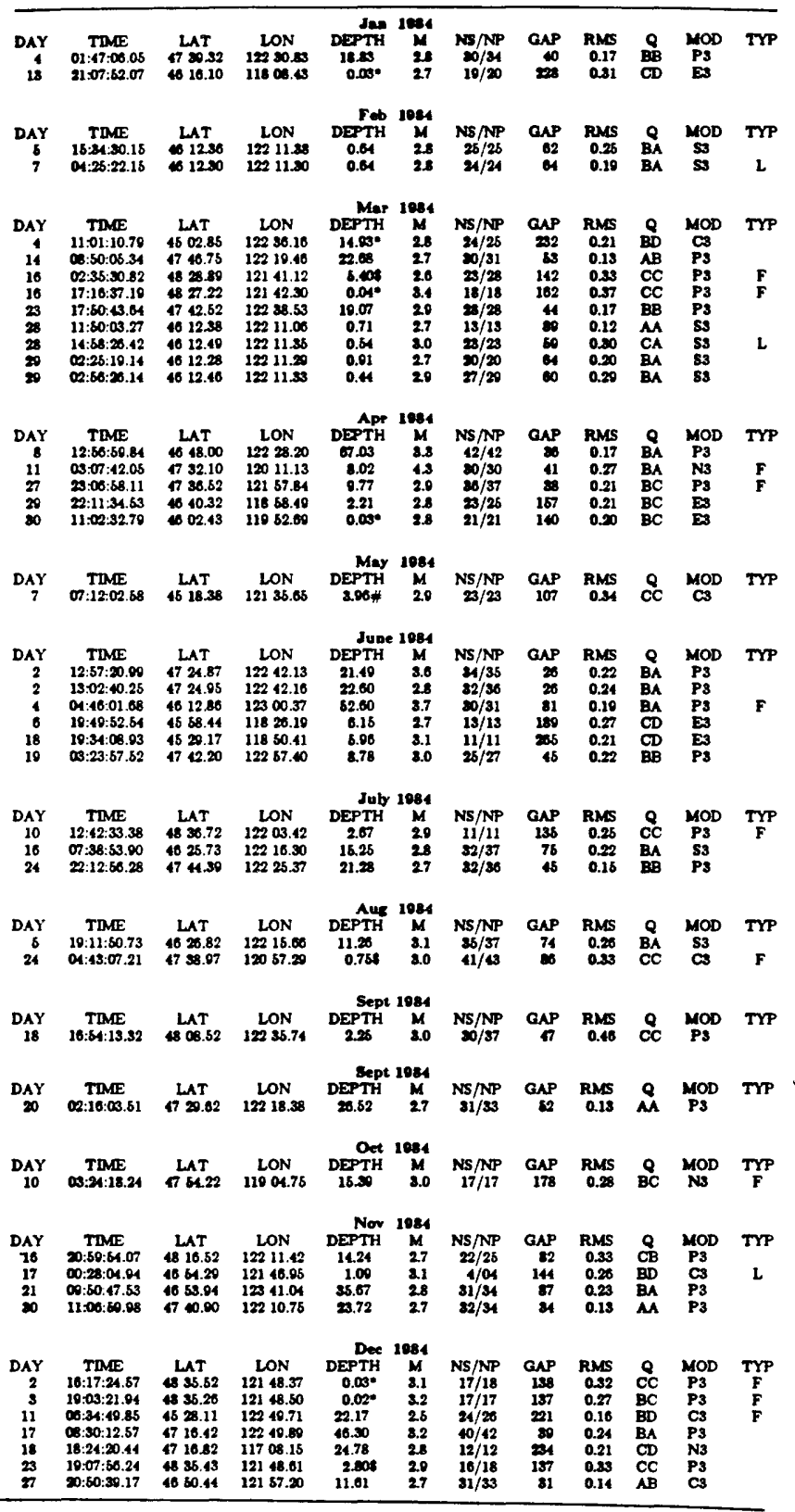


tem. On January 31 at 0529 UTC, a $\mathbf{M}_{L}=3.5$ (GS) earthquake was felt in Idaho and at Clarkston in southeastern Washington in an area of extremely sparse seismicity. The USGS gave a location of $45.59^{\prime \prime} \mathrm{N}$., $116.55^{\prime \prime} \mathrm{W}$., with a shallow depth. On February 11, at 1338 UTC an event of $m_{l}=4.5$ (GS), located by USGS in British Columbia at $49.19^{\prime \prime} \mathrm{N}$., $114.41^{\circ} \mathrm{W}$. at 18 kilometers depth, was reported felt in northeastern Washington. Two $\mathbf{M}_{L}=3.8$ earthquakes were felt at Richland and Halfway, Oregon, on August 10 at 0726 UTC, and September 19 at 0132 UTC, respectively. These events, and a number of aftershocks located using data from portable recorders, have been discussed by Zollweg and Jacobson (1986).

Mt. Saint Helens went through four dome-building eruptions in 1984. The first eruption was a continuation of a long-lasting eruption which began early in 1983 and ended in February 1984. The remaining eruptions, in late March, in June, and in September, were brief. The March eruption was preceded and accompanied by a swarm of shallow seismicity which peaked on March 28. The September eruption was accompanied by an intense earthquake swarm (with over 500 events large enough to trigger the data acquisition system) occurring from September 9 through 12. Most of the earthquakes were low-frequency type, although medium-high-frequency earthquakes also occurred. A large rock and snow avalanche occurred on May 23, plumes were reported on May 14, 16, and 27, and mud flows occurred on May 14 and 26. 


\section{MISCELLANEOUS ACTIVITIES}

Table 11. Principal earthquakes of the world during 1984

Table 11 lists principal world earthquakes for 1984. It includes earthquakes of magnitude 6.8 or greater, those of smaller magnitude that were locally destructive to life and property, and events of unusual interest. The principal source of table 11 was the Preliminary Determination of Epicenters, Monthly Listing.

\begin{tabular}{|c|c|c|c|c|c|c|c|c|c|c|}
\hline \multicolumn{2}{|c|}{$\begin{array}{l}\text { Date } \\
(\mathbf{1 9 8 4})\end{array}$} & $\begin{array}{l}\text { Origin time } \\
\text { (UTC) } \\
\text { hr min sec }\end{array}$ & \multicolumn{2}{|c|}{$\begin{array}{l}\text { Geographic } \\
\text { Coordinates }\end{array}$} & $\begin{array}{l}\text { Depth } \\
\text { (km) }\end{array}$ & $\begin{array}{l}\text { USC } \\
\text { Magn } \\
\text { mb }\end{array}$ & $\begin{array}{l}S \\
\text { tude } \\
M_{S}\end{array}$ & $\begin{array}{l}\text { Other } \\
\text { Magni tudes }\end{array}$ & Region & Remarks \\
\hline Jan. & 8 & 152413.5 & $02.823 \mathrm{~S}$. & $118.806 \mathrm{E}$. & 33 & 6.0 & 6.6 & $6.7 \mathrm{M}_{\mathrm{s}}(\mathrm{BK})$ & Sulawesi & $\begin{array}{l}\text { Two killed, } 23 \text { injured, and damage in the } \\
\text { Mamuju area. }\end{array}$ \\
\hline Feb. & 1 & 142207.9 & $34.616 \mathrm{~N}$. & $70.484 \mathrm{E}$. & 33 & 5.9 & 5.8 & & Afghanistan & $\begin{array}{l}\text { One killed, } 35 \text { injured, and damage in the } \\
\text { Jalalabad area. }\end{array}$ \\
\hline Feb. & 7 & 213321.4 & $10.012 \mathrm{~S}$. & $160.469 \mathrm{E}$. & 18 & 6.6 & 7.5 & $\begin{array}{l}7.5 \mathrm{M}_{\mathrm{s}}(\mathrm{BK}) \\
7.3 \mathrm{M}_{\mathrm{s}}(\mathrm{PS}) \\
7.7 \mathrm{M}_{\mathrm{s}}(\mathrm{LD})\end{array}$ & $\begin{array}{l}\text { Solomon } \\
\text { Islands }\end{array}$ & Damage on Guadalcanal Island. \\
\hline Feb. & 16 & 171841.6 & $36.431 \mathrm{~N}$. & $70.826 \mathrm{E}$. & 208 & 6.1 & & & Afghanistan & $\begin{array}{l}\text { Four killed, } 13 \text { injured, and considerable } \\
\text { damage in the Chitral-Landi Kotal, Pakistan } \\
\text { area. Minor damage in southern Tadzhik } \\
\text { SSR. Felt in Afghanistan, India, and USSR. }\end{array}$ \\
\hline Mar. & 19 & 202838.2 & $40.320 \mathrm{~N}$. & $63.350 \mathrm{E}$. & 15 & 6.5 & 7.0 & $7.1 \mathrm{M}_{\mathrm{s}}(\mathrm{BK})$ & Uzbek, USSR & $\begin{array}{l}\text { About } 100 \text { injured and extensive damage (IX) in } \\
\text { the Gazli area. Damage (VIII) at Dzhangeldy } \\
\text { and in the Budhara areas. }\end{array}$ \\
\hline Mar. & 24 & 094402.6 & $44.117 \mathrm{~N}$. & $148.192 \mathrm{E}$. & 44 & 6.1 & 7.0 & $6.7 \mathrm{M}_{\mathrm{s}}(\mathrm{BK})$ & Kuril Islands & $\begin{array}{l}\text { Felt on the southern Kuril Islands and on } \\
\text { Hokkaido and Honshu Islands of Japan. }\end{array}$ \\
\hline Mar. & 27 & 200633.2 & $04.647 \mathrm{~S}$. & $145.805 \mathrm{E}$. & 28 & 5.8 & 6.6 & $6.8 \mathrm{M}_{\mathrm{S}}(\mathrm{BK})$ & $\begin{array}{l}\text { Papua, New Guinea } \\
\text { area }\end{array}$ & $\begin{array}{l}\text { Eleven injured and many buildings destroyed } \\
\text { (VIII) on Karkar Island. }\end{array}$ \\
\hline Apr. & 24 & 211519.0 & $37.320 \mathrm{~N}$. & $121.698 \mathrm{~W}$. & 8 & 5.7 & 6.1 & $6.2 \mathrm{M}_{\mathrm{L}}(\mathrm{BK})$ & Central California & $\begin{array}{l}\text { Twenty-seven injured and } \$ 8 \text { million damage in } \\
\text { the Morgan Hill-San Jose area. }\end{array}$ \\
\hline Apr. & 29 & 050300.0 & $43.260 \mathrm{~N}$. & $12.558 \mathrm{E}$. & 12 & 5.2 & 5.3 & $5.6 \mathrm{M}_{\mathrm{L}}(\mathrm{TI})$ & Central Italy & $\begin{array}{l}\text { Thirty-six injured and extensive damage (VIII) in } \\
\text { the Assisi-Gubbio-Perugia area. About } 7500 \\
\text { people homeless. }\end{array}$ \\
\hline May & 7 & 174941.6 & $41.765 \mathrm{~N}$. & $13.898 \mathrm{E}$. & 10 & 5.5 & 5.8 & $\begin{array}{l}5.8 \mathrm{M}_{\mathrm{s}}(\mathrm{BK}) \\
6.0 \mathrm{M}_{\mathrm{L}}(\mathrm{TI})\end{array}$ & Southern Italy & $\begin{array}{l}\text { Three killed, about } 100 \text { injured, and extensive } \\
\text { damage (VIII) in the Abruzzo area. }\end{array}$ \\
\hline May & 11 & 104149.9 & $41.831 \mathrm{~N}$. & $13.961 \mathrm{E}$. & 14 & 5.2 & 5.2 & $5.4 \mathrm{M}_{\mathrm{L}}(\mathrm{TI})$ & Southern Italy & $\begin{array}{l}\text { Three deaths from heart attacks and about } 63 \\
\text { injured. Damage (VIII) in the Abruzzo area. }\end{array}$ \\
\hline
\end{tabular}




\begin{tabular}{|c|c|c|c|c|c|c|c|c|c|}
\hline May 13 & 124555.8 & $42.967 \mathrm{~N}$. & $17.734 \mathrm{E}$. & 30 & 5.1 & 5.1 & & Yugoslavia & $\begin{array}{l}\text { One killed. Damage (VII) at Dubrovnik. Felt at } \\
\text { Triste, Italy. }\end{array}$ \\
\hline June 24 & 181851.0 & $18.006 \mathrm{~N}$. & $69.197 \mathrm{~W}$. & 32 & 5.1 & 4.7 & & Dominican Republic & Five killed in the Bayaguana area. \\
\hline July 5 & 052148.9 & $06.056 \mathrm{~S}$. & $154.424 \mathrm{E}$. & 33 & 6.0 & 6.5 & $\begin{array}{l}6.8 \mathrm{M}_{\mathrm{s}}(\mathrm{BK}) \\
6.7 \mathrm{M}_{\mathrm{s}}(\mathrm{PS})\end{array}$ & Solomon Islands. & No report. \\
\hline Aug. 6 & 190638.3 & $32.386 \mathrm{~N}$. & $131.945 \mathrm{E}$. & 46 & 6.3 & 6.7 & $6.9 \mathrm{M}_{\mathrm{s}}(\mathrm{BK})$ & Kyushu, Japan & $\begin{array}{l}\text { Nine injured and slight damage. An 18- } \\
\text { centimeter tsunami at Nobeoka. }\end{array}$ \\
\hline Aug. 27 & 064126.2 & $01.761 \mathrm{~N}$. & $99.075 \mathrm{E}$. & 33 & 5.1 & 5.2 & & $\begin{array}{l}\text { Sumatra, } \\
\text { Indonesia }\end{array}$ & $\begin{array}{l}\text { One hundred twenty-three injured and } 350 \\
\text { homes and } 65 \text { government buildings damaged } \\
\text { in the Tarutung area. }\end{array}$ \\
\hline Sept. 13 & 234849.9 & $35.789 \mathrm{~N}$ & $137.488 \mathrm{E}$ & 10 & 6.0 & 6.1 & $6.3 \mathrm{M}_{\mathrm{s}}(\mathrm{BK})$ & Honshu, Japan & $\begin{array}{l}\text { Twenty-four killed, severe damage, and } \\
\text { landslides in the Otaki area. }\end{array}$ \\
\hline Sept. 18 & 132601.8 & $40.885 \mathrm{~N}$. & $42.219 \mathrm{E}$ & 10 & 5.3 & 6.4 & & Turkey & $\begin{array}{l}\text { Three killed, } 38 \text { injured, and } 75,000 \text { houses } \\
\text { destroyed or damaged in the Olur-Senkaya } \\
\text { area. }\end{array}$ \\
\hline Sept. 18 & 170244.3 & $34.006 \mathrm{~N}$. & $141.500 \mathrm{E}$ & 48 & 6.6 & 6.9 & $6.9 \mathrm{M}_{\mathrm{s}}(\mathrm{BK})$ & $\begin{array}{l}\text { Near Honshu, } \\
\text { Japan }\end{array}$ & $\begin{array}{l}\text { Local tsunami at Hachijo-jima }(11 \mathrm{~cm}) \text {, } \\
\text { Ishinomaki }(10 \mathrm{~cm}) \text {, Onahama }(5 \mathrm{~cm}) \text {, and } \\
\text { Tateyama }(3 \mathrm{~cm}) .\end{array}$ \\
\hline Sept. 28 & 000334.5 & $25.849 \mathrm{~S}$. & $175.911 \mathrm{~W}$. & 21 & 6.4 & 6.8 & $6.7 \mathrm{M}_{\mathrm{S}}(\mathrm{BK})$ & $\begin{array}{l}\text { South of the } \\
\text { Tonga Islands }\end{array}$ & Felt on Raoul Island. \\
\hline Oct. 18 & 094624.6 & $40.545 \mathrm{~N}$. & $42.403 \mathrm{E}$ & 60 & 5.3 & & & Turkey & $\begin{array}{l}\text { Three killed, } 35 \text { injured, and 75,000 homes } \\
\text { damaged in the Erzurum-Senkaya area. }\end{array}$ \\
\hline Nov. 1 & 044850.2 & $08.185 \mathrm{~N}$. & 38.794 W. & 10 & 6.5 & 7.1 & $\begin{array}{l}7.4 \mathrm{M}_{\mathrm{S}}(\mathrm{BK}) \\
6.9 \mathrm{M}_{\mathrm{S}}(\mathrm{PS})\end{array}$ & $\begin{array}{l}\text { Central Mid- } \\
\text { Atlan tic Ridge }\end{array}$ & Felt in nor theastern Brazil. \\
\hline Nov. 17 & 064930.0 & $00.197 \mathrm{~N}$. & $98.027 \mathrm{E}$. & 33 & 6.3 & 7.2 & $\begin{array}{l}7.4 \mathrm{M}_{\mathrm{s}}(\mathrm{BK}) \\
7.2 \mathrm{M}_{\mathrm{S}}(\mathrm{PS})\end{array}$ & $\begin{array}{l}\text { Sumatra, } \\
\text { Indonesia }\end{array}$ & $\begin{array}{l}\text { One injured and two buildings damaged on Nias } \\
\text { Island. }\end{array}$ \\
\hline Nov. 23 & 044606.3 & $14.312 \mathrm{~S}$. & $171.284 \mathrm{E}$. & 33 & 6.0 & & $\begin{array}{l}6.7 \mathrm{M}_{\mathrm{s}}(\mathrm{BK}) \\
6.8 \mathrm{M}_{\mathrm{s}}(\mathrm{PS})\end{array}$ & $\begin{array}{l}\text { Vanuatu, Islands } \\
\text { Region }\end{array}$ & No report. \\
\hline Dec. 28 & 103753.7 & $56.194 \mathrm{~N}$. & $163.460 \mathrm{E}$ & 33 & 6.2 & 7.0 & $\begin{array}{l}6.7 \mathrm{M}_{\mathrm{s}}(\mathrm{BK}) \\
6.4 \mathrm{M}_{\mathrm{s}}(\mathrm{PS})\end{array}$ & $\begin{array}{l}\text { Near east coast } \\
\text { of Kamchatka, } \\
\text { USSR }\end{array}$ & No report. \\
\hline Dec. 30 & 213656.4 & $36.663 \mathrm{~S}$. & $177.512 \mathrm{E}$. & 39 & 6.2 & 6.8 & $\begin{array}{l}6.8 \mathrm{M}_{\mathrm{s}}(\mathrm{BK}) \\
6.6 \mathrm{M}_{\mathrm{s}}(\mathrm{PS})\end{array}$ & $\begin{array}{l}\text { Near Northern } \\
\text { Island, New Zealand }\end{array}$ & Felt at Auckland and along the Bay of Plenty. \\
\hline Dec. 30 & 233337.7 & $24.641 \mathrm{~N}$. & 92.891 E. & 23 & 5.6 & & & $\begin{array}{l}\text { Assam, India } \\
\text { Region }\end{array}$ & $\begin{array}{l}\text { Twenty killed, about } 100 \text { injured, and extensive } \\
\text { damage in southern Assam. }\end{array}$ \\
\hline
\end{tabular}




\section{STRONG-MOTION SEISMOGRAPH DATA}

\author{
By Ronald L. Porcella and Josephine C. Switzer \\ U.S. Geological Survey \\ Menlo Park, California 94025
}

\section{Introduction}

The first engineering seismology program in the United States was administered by the Seismological Field Survey of the Coast and Geodetic Survey (C\&GS). This Program was begun in 1931 and effectively remained the responsibility of the Seismological Field Survey (SFS) for more than 40 years. During this period the SFS was shifted from one acronymic agency to another, including C\&GS, ESSA, NOS, NOAA, ERL, ESL, and finally, in 1973, USGS; soon afterwards the SFS became the Seismic Engineering Branch of the USGS. In spite of these numerous high-level administrative changes, the Program has retained its distinct identity and its basic objectives, and field-level operations have remained remarkably constant throughout the years.

The current program of strong-motion instrumentation is administered by the USGS in cooperation with both private industry and educational institutions, as well as numerous Federal, State, and local agencies and organizations. The objectives of the program are to record strong ground motions and the response of representative types of engineered structures during potentially damaging earthquakes and to disseminate processed data and information about the records, sites, and structures to external users in earthquake engineering research and design practice and engineering seismology. The dissemination of this information and data is achieved in various ways.

Preliminary earthquake reports and a summary of recent accerlerograph records are presented on a regular basis in Strong Motion Program Reports, a USGS Circular. These summaries include a brief description of the earthquake and strong-motion recording station, the results of routine scalings of those records that con- tain peak accelerations greater than $0.05 \mathrm{~g}$, and photographic reproductions of many of the more significant accelerograms. The program reports also contain abstracts of recent reports, notes on strong-motion information sources and the availability of digitized data, and other information pertinent to the USGS and other strong-motion programs.

Strong-motion event and strong-motion data reports are periodically published as USGS Open-file Reports and include the results of digitization and routine analyses of strong-motion accelerograms that contain peak accelerations greater than $0.10 \mathrm{~g}$ or are related to a specific event, particular strong-motion station, or geographic group of stations. The minimum acceleration level is based primarily on the current capability of USGS to process strong- motion records and may vary with both the degree of seismic activity and number of personnel available at any given time. Although maximum acceleration is not directly related to frequency content or duration of strong motion, the peak acceleration can be readily obtained from an accelerogram and thus the value is commonly used as a general indicator of the potential significance of the record. Detailed information on the availability of digitized data from various sources is published regularly in Strong-Motion Program Reports.

The Strong-Motion Accelerograph Station List is periodically published as a USGS Open-file Report and includes information on all of the accelerograph stations in the Western Hemisphere known to the USGS. Because of the ever-changing nature of this information, it is impossible to have a complete list of all of the stations in existence at any one time. Rather, the list is intended to provide that community of persons interested in strong-motion programs with a reasonably complete indication of the current status of the various strong-motion networks. Information presented in this list includes the station name and geographic coordinates, site characteristics, type and size of structure, location of instruments, and the primary sources of data. The current list contains information on approximately 1350 stations located in the United States, Canada, the Caribbean, and throughout Central and South America (Switzer and others, 1981). 


\section{Accelerograph Data}

During 1984 more than 160 strong-motion records were recovered from the U.S. Geological Survey's National Strong-Motion Network; approximately 40 of these were recorded during the magnitude $6.1\left(\mathrm{M}_{L}\right)$ Morgan Hill, California, earthquake of April 24. The main shock was felt throughout most of central California and produced a maximum recorded ground acceleration of $0.40 \mathrm{~g}$ at the Anderson Dam downstream station (see table 12). Additional records were recovered at California Division of Mines and Geology stations; altogether, about 75 stations in the two networks were triggered at epicentral distances ranging from about 4 kilometers at Halls Valley to more than 100 kilometers (Brady and others, 1987).

A swarm of earthquakes occurred in the Mammoth Lakes, California area on November 23, including a magnitude $6.1\left(\mathbf{M}_{L}\right)$ event at 1808 UTC and a magnitude $5.5\left(\mathbf{M}_{L}\right)$ event at 1912 UTC. Approximately 140 channels of acceleration data were recorded during the period November 23-29, including 117 channels from the McGee Creek downhole array.

Additional accelerograph data were recovered at USGS strong-motion stations located in North Dakota, Idaho, California, Alaska, and Hawaii during 1984 (table 12). 


\section{Table 12. Summary of U.S. accelerograph records recovered during 1984}

[Station owners: ACOE, U.S. Army Corps of Engineers; CDOT, California Department of Transportation; CDWR, Cal ifornia Department of Water Resources; CHY, City of Hayward; UCB, University of California at Berkeley; USBR, U.S. Bureau of Reclamation; USGS, U.S. Geological Survey; VA, U.S. Veterans Administration. Instrument trigger time in seconds after the minute or the following minute listed in event column. S-minus trigger denotes $S$-wave-arrival-minus-trigger-time ( $S$ - $t$ ) or $S$-wave-minus-P-wave-arrival time ( $\underline{S}-\underline{P}$, in parentheses) interval. Direction is of case acceleration For upward trace deflection on accélerogram; horizontal components are listed as azimuth, and vertical components as "up" or "down." Maximum amplitude is peak acceleration recorded at ground level on one vertical and two orthogonal horizontal components unless otherwise noted. Duration is interval between first and last peaks of acceleration greater than $0.10 \mathrm{~g}$.

\begin{tabular}{|c|c|c|c|c|c|c|c|}
\hline Earthquake & $\begin{array}{l}\text { Station name } \\
\text { (owner) }\end{array}$ & $\begin{array}{l}\text { Station } \\
\text { location }\end{array}$ & $\begin{array}{l}\text { Trigger } \\
\text { time }\end{array}$ & $\begin{array}{l}\text { S-minus } \\
\text { trigger } \\
\quad(s)\end{array}$ & $\begin{array}{l}\text { Direction } \\
\quad(a z)\end{array}$ & $\begin{array}{l}\text { Maximum } \\
\text { amplitude } \\
(\underline{g})\end{array}$ & $\begin{array}{l}\text { Duration } \\
\qquad(\mathrm{s})\end{array}$ \\
\hline $\begin{array}{l}3 \text { January } 1984 \\
\text { l051 G.m.t. } \\
\text { Central Calif. } \\
\text { Epicenter and } \\
\text { magnitude unknown }\end{array}$ & $\begin{array}{l}\text { Bear Valley Station } 10 \\
\text { Webb Residence } \\
\text { (USGS) }\end{array}$ & $\begin{array}{r}36.532^{\circ} \mathrm{N} \\
121.143^{\circ} \mathrm{W}\end{array}$ & 5.3 & (2) & & (1) & \\
\hline $\begin{array}{l}23 \text { January } 1984 \\
0540: 19.7 \text { G.m.t. } \\
\text { Central Calif. } \\
36.373 \mathrm{~N}, 121.907 \mathrm{~W}\end{array}$ & $\begin{array}{l}\text { Bear Valley Station } 6 \\
\text { James Ranch } \\
\text { (USGS) }\end{array}$ & $\begin{array}{r}36.504^{\circ} \mathrm{N} \\
121.101^{\circ} \mathrm{W}\end{array}$ & 43.6 & (2) & & (1) & \\
\hline Magnitude $5.4 \mathrm{ML}$ & $\begin{array}{l}\text { Bear Valley Station } 14 \\
\text { Upper Butts Ranch } \\
\text { (USGS) }\end{array}$ & $\begin{array}{r}36.569^{\circ} \mathrm{N} \\
121.043^{\circ} \mathrm{W}\end{array}$ & 45.9 & (2) & & (1) & \\
\hline $\begin{array}{l}27 \text { January } 1984 \\
0444: 35.8 \text { G.m.t. } \\
\text { Central Calif. } \\
36.277 \mathrm{~N}, 120.400 \mathrm{~W} \\
\text { Magnitude } 3.7 \mathrm{ML}\end{array}$ & $\begin{array}{l}\text { Coalinga } \\
\text { Oil City } \\
\text { (USGS) }\end{array}$ & $\begin{array}{r}36.229^{\circ} \mathrm{N} \\
120.360^{\circ} \mathrm{W}\end{array}$ & 37.1 & 1.6 & & (1) & \\
\hline $\begin{array}{l}10 \text { April 1983- } \\
14 \text { February } 1984 \\
\text { North Dakota } \\
\text { Epicenter and } \\
\text { magnitude unknown }\end{array}$ & $\begin{array}{l}\text { Garrison Dam } \\
\text { (ACOE) } \\
\text { Crest }\end{array}$ & $\begin{array}{r}47.50^{\circ} \mathrm{N} \\
101.43^{\circ} \mathrm{W}\end{array}$ & (2) & (2) & & (1) & \\
\hline $\begin{array}{l}19 \text { February } 1984 \\
\text { 0943:10.8 G.m.t. } \\
\text { Central Calif. } \\
36.285 \mathrm{~N}, 120.317 \mathrm{~W}\end{array}$ & $\begin{array}{l}\text { Coalinga } \\
\text { Burnett Company } \\
\text { (USGS) }\end{array}$ & $\begin{array}{r}36.138^{\circ} \mathrm{N} \\
120.357^{\circ} \mathrm{W}\end{array}$ & (2) & 3.1 & & (1) & \\
\hline Magnitude $4.1 \mathrm{ML}$ & $\begin{array}{l}\text { Coalinga } \\
\text { Oil City } \\
\text { (USGS) }\end{array}$ & $\begin{array}{r}36.229^{\circ} \mathrm{N} \\
120.360^{\circ} \mathrm{W}\end{array}$ & 12.4 & 2.0 & $\begin{array}{l}360^{\circ} \\
U p \\
270^{\circ}\end{array}$ & $\begin{array}{l}.18 \\
.05 \\
.20\end{array}$ & $\begin{array}{l}0.3 \\
--- \\
0.1\end{array}$ \\
\hline $\begin{array}{l}20 \text { February } 1984 \\
1617: 25.5 \text { G.m.t. } \\
\text { Western Idaho } \\
44.432 \mathrm{~N}, 114.194 \mathrm{~W} \\
\text { Magnitude } 3.6 \mathrm{ML}\end{array}$ & $\begin{array}{l}\text { Dickey, Idaho } \\
\text { Hatch Ranch } \\
\text { (USGS) }\end{array}$ & $\begin{array}{r}44.338^{\circ} \mathrm{N} \\
114.051^{\circ} \mathrm{W}\end{array}$ & 29 & 1.2 & & (1) & \\
\hline $\begin{array}{l}23 \text { February } 1984 \\
\text { 0959 G.m.t. } \\
\text { Central Calif. } \\
\text { Epicenter and } \\
\text { magnitude unknown }\end{array}$ & $\begin{array}{l}\text { Bear Valley Station } 10 \\
\text { Webb Residence } \\
\text { (USGS) }\end{array}$ & $\begin{array}{r}36.532^{\circ} \mathrm{N} \\
121.143^{\circ} \mathrm{W}\end{array}$ & 31.3 & .8 & & (1) & \\
\hline $\begin{array}{l}31 \text { July 1983- } \\
26 \text { February } 1984 \\
\text { Eastern Calif. } \\
\text { Epicenter and } \\
\text { magnitude unknown }\end{array}$ & $\begin{array}{l}\text { Long Valley Dam } \\
\text { Lake Crowley } \\
\text { (USGS) } \\
\quad \text { Left abutment }\end{array}$ & $\begin{array}{r}37.588^{\circ} \mathrm{N} \\
118.705^{\circ} \mathrm{W}\end{array}$ & (2) & (2) & & (1) & \\
\hline
\end{tabular}

See footnotes at end of table. 
Table 12. Summary of U.S. accelerograph records recovered during 1984-Continued

\begin{tabular}{|c|c|c|c|c|c|c|c|}
\hline Earthquake & $\begin{array}{l}\text { Station name } \\
\text { (owner) }\end{array}$ & $\begin{array}{l}\text { Station } \\
\text { location }\end{array}$ & $\begin{array}{l}\text { Trigger } \\
\text { time }\end{array}$ & $\begin{array}{c}\text { S-minus } \\
\text { trigger } \\
(\mathrm{s})\end{array}$ & $\begin{array}{l}\text { Direction } \\
\quad(\mathrm{az})\end{array}$ & $\begin{array}{l}\text { Maximum } \\
\text { amplitude } \\
(\mathrm{g})\end{array}$ & $\begin{array}{l}\text { Duration } \\
\qquad(\mathrm{s})\end{array}$ \\
\hline $\begin{array}{l}29 \text { February } 1984 \\
0207: 31.7 \text { G.m.t. } \\
\text { Southern Cal if. } \\
33.140 \mathrm{~N}, 116.070 \mathrm{~W} \\
\text { Magnitude } 4.3 \mathrm{ML}\end{array}$ & $\begin{array}{l}\text { Ocotillo wells } \\
\text { Burro Bend Cafe } \\
\text { (USGS) }\end{array}$ & $\begin{array}{l}33.140^{\circ} \mathrm{N} \\
116.130^{\circ} \mathrm{W}\end{array}$ & 33.7 & 1.4 & & (1) & \\
\hline $\begin{array}{l}2 \text { March } 1984 \\
0029: 45.1 \text { G.m.t. } \\
\text { Western Idaho } \\
44.350 \mathrm{~N}, 114.186 \mathrm{~W} \\
\text { Magnitude } 4.3 \mathrm{ML}\end{array}$ & $\begin{array}{l}\text { Dickey, Idaho } \\
\text { Hatch Ranch } \\
\text { (USGS) }\end{array}$ & $\begin{array}{r}44.338^{\circ} \mathrm{N} \\
114.051^{\circ} \mathrm{W}\end{array}$ & 48 & (2) & & (1) & \\
\hline $\begin{array}{l}6 \text { May 1983- } \\
7 \text { March } 1984 \\
\text { Central Calif. } \\
\text { Epicenter and } \\
\text { magnitude unknown }\end{array}$ & $\begin{array}{l}\text { Hollister } \\
\text { Damler Residence } \\
\text { (UCB) }\end{array}$ & $\begin{array}{r}36.81^{\circ} \mathrm{N} \\
121.41^{\circ} \mathrm{W}\end{array}$ & (2) & 2.6 & $\begin{array}{l}118^{\circ} \\
\text { Up } \\
028^{\circ}\end{array}$ & $\begin{array}{l}.05 \\
.02 \\
.05\end{array}$ & $\begin{array}{l}--- \\
---\end{array}$ \\
\hline \multirow{3}{*}{$\begin{array}{l}26 \text { March } 1984 \\
0758: 39.7 \text { G.m.t. } \\
\text { Centra7 Calif. } \\
36.740 \mathrm{~N}, 121.507 \mathrm{~W} \\
\text { Magnitude } 4.0 \mathrm{ML}\end{array}$} & $\begin{array}{l}\text { San Justo Damsite } \\
\text { (USBR) }\end{array}$ & $\begin{array}{r}36.827^{\circ} \mathrm{N} \\
121.445^{\circ} \mathrm{W}\end{array}$ & & & & & \\
\hline & Left abutment & & 44.9 & (2) & & (1) & \\
\hline & $\begin{array}{l}\text { Right abutment } \\
\text { (Dike) }\end{array}$ & & 45.2 & (2) & & (1) & \\
\hline \multirow{4}{*}{$\begin{array}{l}27 \text { March } 1984 \\
0336: 35.6 \text { G.m.t. } \\
\text { Central Calif. } \\
37.727 \mathrm{~N}, 122.130 \mathrm{~W} \\
\text { Magnitude } 4.4 \mathrm{ML}\end{array}$} & $\begin{array}{l}\text { Hayward City Hall } \\
(\mathrm{CHY})^{3}\end{array}$ & $\begin{array}{r}37.68^{\circ} \mathrm{N} \\
122.08^{\circ} \mathrm{W}\end{array}$ & (2) & (2) & & & \\
\hline & Basement & & & & & (1) & \\
\hline & Ground floor & & & & & (2) & \\
\hline & l1th floor & & & & & (1) & \\
\hline $\begin{array}{l}30 \text { March } 1984 \\
1021 \text { G.m.t. } \\
\text { Hawai } i \\
\text { Epicenter and } \\
\text { magnitude unknown }\end{array}$ & $\begin{array}{l}\text { Honokaa } \\
\text { Fire Station } \\
\text { (USGS) }\end{array}$ & $\begin{array}{r}20.080^{\circ} \mathrm{N} \\
155.465^{\circ} \mathrm{W}\end{array}$ & 30 & (2) & & (1) & \\
\hline $\begin{array}{l}\text { 31 March } 1984 \\
\text { 0112:56.4 G.m.t. } \\
\text { Western Idaho } \\
44.326 \mathrm{~N}, 114.144 \mathrm{~W} \\
\text { Magnitude } 3.3 \mathrm{ML}\end{array}$ & $\begin{array}{l}\text { Dickey, Idaho } \\
\text { Hatch Ranch } \\
\text { (USGS) }\end{array}$ & $\begin{array}{r}44.338^{\circ} \mathrm{N} \\
114.051^{\circ} \mathrm{W}\end{array}$ & 59 & (2) & & (1) & \\
\hline $\begin{array}{l}8 \text { April } 1984 \\
\text { Time unknown } \\
\text { Central Calif. } \\
\text { Epicenter and } \\
\text { magnitude unknown }\end{array}$ & $\begin{array}{l}\text { Coalinga } \\
\text { Oil City } \\
\text { (USGS) }\end{array}$ & $\begin{array}{r}36.229^{\circ} \mathrm{N} \\
120.360^{\circ} \mathrm{W}\end{array}$ & 14.5 & 1.8 & & (1) & \\
\hline $\begin{array}{l}\text { 10 April } 1984 \\
1812: 11.5 \text { G.m.t. } \\
\text { Central Calif. } \\
36.250 \mathrm{~N}, 120.222 \mathrm{~W} \\
\text { Magnitude } 3.4 \mathrm{ML}\end{array}$ & $\begin{array}{l}\text { Coalinga } \\
\text { Oil City } \\
\text { (USGS) }\end{array}$ & $\begin{array}{r}36.229^{\circ} \mathrm{N} \\
120.360^{\circ} \mathrm{W}\end{array}$ & 17 & (2) & & (1) & \\
\hline
\end{tabular}

See footnotes at end of table. 
Table 12. Summary of U.S. accelerograph records recovered during 1984-Continued

\begin{tabular}{|c|c|c|c|c|c|c|c|}
\hline Earthquake & $\begin{array}{l}\text { Station name } \\
\text { (owner) }\end{array}$ & $\begin{array}{l}\text { Station } \\
\text { location }\end{array}$ & $\begin{array}{l}\text { Trigger } \\
\text { time }\end{array}$ & $\begin{array}{l}\text { S-minus } \\
\text { trigger } \\
\quad(s)\end{array}$ & $\begin{array}{l}\text { Direction } \\
(a z)\end{array}$ & $\begin{array}{l}\text { Maximum } \\
\text { amplitude } \\
\text { (g) }\end{array}$ & $\begin{array}{l}\text { Duration } \\
\qquad(\mathrm{s})\end{array}$ \\
\hline $\begin{array}{l}18 \text { April } 1984 \\
0115: 09.4 \text { G.m.t. } \\
\text { Western Idaho } \\
44.342 \mathrm{~N}, 114.099 \mathrm{~W} \\
\text { Magnitude } 4.0 \mathrm{ML}\end{array}$ & $\begin{array}{l}\text { Dickey, Idaho } \\
\text { Hatch Ranch } \\
\text { (USGS) }\end{array}$ & $\begin{array}{r}44.338^{\circ} \mathrm{N} \\
114.051^{\circ} \mathrm{W}\end{array}$ & (2) & $(2)$ & & (1) & \\
\hline $\begin{array}{l}23 \text { April } 1984 \\
2142 \text { G.m.t. } \\
\text { Idaho } \\
\text { Epicenter and } \\
\text { magnitude unknown }\end{array}$ & $\begin{array}{l}\text { Dickey, Idaho } \\
\text { Smith Ranch } \\
\text { (USGS) }\end{array}$ & $\begin{array}{r}44.134^{\circ} \mathrm{N} \\
113.901^{\circ} \mathrm{W}\end{array}$ & 2 & 1.2 & & (1) & \\
\hline $\begin{array}{l}24 \text { April } 1984 \\
0452: 51.5 \text { G.m.t. } \\
\text { Central Calif. }\end{array}$ & $\begin{array}{l}\text { Coalinga } \\
\text { Burnett Company } \\
\text { (USGS) }\end{array}$ & $\begin{array}{r}36.138^{\circ} \mathrm{N} \\
120.357^{\circ} \mathrm{W}\end{array}$ & 54 & 2.3 & & $(1)$ & \\
\hline Magnitude $4.0 \mathrm{ML}$ & $\begin{array}{l}\text { Coalinga } \\
\text { Oil City } \\
\text { (USGS) }\end{array}$ & $\begin{array}{r}36.229^{\circ} \mathrm{N} \\
120.360^{\circ} \mathrm{W}\end{array}$ & 57 & $(2)$ & & (1) & \\
\hline \multirow{10}{*}{$\begin{array}{l}24 \text { April } 1984 \\
2115: 19.0 \text { G.m.t. } \\
\text { Central Calif. } \\
37.320 \mathrm{~N}, 121.698 \mathrm{~W} \\
\text { Magnitude } 6.2 \mathrm{ML}\end{array}$} & $\begin{array}{l}\text { Anderson Dam } \\
\text { (USGS) }\end{array}$ & & & & & & \\
\hline & Crest & $\begin{array}{r}37.166^{\circ} \mathrm{N} \\
121.626^{\circ} \mathrm{W}\end{array}$ & 23 & (2) & $\begin{array}{l}340^{\circ} \\
\text { Up } \\
250^{\circ}\end{array}$ & $\begin{array}{l}.39 \\
.20 \\
.63\end{array}$ & $\begin{array}{l}8.7 \\
3.3 \\
7.8\end{array}$ \\
\hline & Downstream & $\begin{array}{r}37.165^{\circ} \mathrm{N} \\
121.631^{\circ} \mathrm{W}\end{array}$ & (2) & (2) & $\begin{array}{l}340^{\circ} \\
\text { Up } \\
250^{\circ}\end{array}$ & $\begin{array}{l}.30 \\
.20 \\
.40\end{array}$ & $\begin{array}{l}4.0 \\
5.2 \\
4.4\end{array}$ \\
\hline & $\begin{array}{l}\text { Bear Valley Station } 1 \\
\text { Fire Station } \\
(\text { USGS })^{3}\end{array}$ & $\begin{array}{r}36.573^{\circ} \mathrm{N} \\
121.184^{\circ} \mathrm{W}\end{array}$ & (2) & (2) & & (1) & \\
\hline & $\begin{array}{l}\text { Bear Valley Station } 2 \\
\text { Stone Canyon West } \\
\text { (USGS) }\end{array}$ & $\begin{array}{r}36.636^{\circ} \mathrm{N} \\
121.234^{\circ} \mathrm{W}\end{array}$ & 36.1 & (2) & & (1) & \\
\hline & $\begin{array}{l}\text { Bear Valley Station } 5 \\
\text { Callens Ranch } \\
\text { (USGS) }\end{array}$ & $\begin{array}{r}36.673^{\circ} \mathrm{N} \\
121.195^{\circ} \mathrm{W}\end{array}$ & 35.3 & $(2)$ & & (1) & \\
\hline & $\begin{array}{l}\text { Bear Valley Station } 6 \\
\text { James Ranch } \\
\text { (USGS) }\end{array}$ & $\begin{array}{r}36.504^{\circ} \mathrm{N} \\
121.101^{\circ} \mathrm{W}\end{array}$ & 40.5 & (2) & & (1) & \\
\hline & $\begin{array}{l}\text { Bear Valley Station } 10 \\
\text { Webb Residence } \\
\text { (USGS) }\end{array}$ & $\begin{array}{r}36.532^{\circ} \mathrm{N} \\
121.143^{\circ} \mathrm{W}\end{array}$ & 41.2 & (2) & & (1) & \\
\hline & $\begin{array}{l}\text { Bear Valley Station } 12 \\
\text { Williams Ranch } \\
\text { (USGS) }\end{array}$ & $\begin{array}{r}36.658^{\circ} \mathrm{N} \\
121.249^{\circ} \mathrm{W}\end{array}$ & 34.7 & (2) & $\begin{array}{l}310^{\circ} \\
\text { Up } \\
220^{\circ}\end{array}$ & $\begin{array}{l}.06 \\
.14 \\
.04\end{array}$ & $\begin{array}{l}--- \\
0.6 \\
---\end{array}$ \\
\hline & $\begin{array}{l}\text { Bear Valley Station } 14 \\
\text { Upper Butts Kanch } \\
\text { (USGS) }\end{array}$ & $\begin{array}{r}36.569^{\circ} \mathrm{N} \\
120.043^{\circ} \mathrm{W}\end{array}$ & (2) & (2) & & (1) & \\
\hline
\end{tabular}

See footnotes at end of table. 
Table 12. Summary of $U$. S. accelerograph records recovered during 1984-Continued

\begin{tabular}{|c|c|c|c|c|c|c|c|}
\hline Earthquake & $\begin{array}{l}\text { Station name } \\
\text { (owner) }\end{array}$ & $\begin{array}{l}\text { Station } \\
\text { location }\end{array}$ & $\begin{array}{l}\text { Trigger } \\
\text { time }\end{array}$ & $\begin{array}{l}\text { S-minus } \\
\text { trigger } \\
\quad(s)\end{array}$ & $\begin{array}{l}\text { Direction } \\
(a z)\end{array}$ & $\begin{array}{l}\text { Maximum } \\
\text { amplitude } \\
\text { (g) }\end{array}$ & $\begin{array}{l}\text { Duration } \\
\qquad(\mathrm{s})\end{array}$ \\
\hline & $\begin{array}{l}\text { Dos Amigos } \\
\text { Pumping Plant } \\
\text { (CDWR })^{3}\end{array}$ & $\begin{array}{r}36.92^{\circ} \mathrm{N} \\
120.83^{\circ} \mathrm{W}\end{array}$ & (2) & 9.6 & & & \\
\hline & Level 1 & & & & & (1) & \\
\hline & $\begin{array}{l}\text { Hollister } \\
\text { City Hall Annex } \\
\text { (USGS) }\end{array}$ & $\begin{array}{r}36.85^{\circ} \mathrm{N} \\
121.40^{\circ} \mathrm{W}\end{array}$ & (2) & (2) & & & \\
\hline & Basement & & & & $\begin{array}{l}360^{\circ} \\
\text { Up } \\
270^{\circ}\end{array}$ & $\begin{array}{l}.08 \\
.42 \\
.08\end{array}$ & $\overline{---}$ \\
\hline & $\begin{array}{l}\text { Hollister } \\
\text { Differential Array } \\
\text { (USGS) }\end{array}$ & $\begin{array}{r}36.888^{\circ} \mathrm{N} \\
121.413^{\circ} \mathrm{W}\end{array}$ & & & & & \\
\hline & Freefield & & 29.2 & (2) & $\begin{array}{l}255^{\circ} \\
\text { Up } \\
165^{\circ}\end{array}$ & $\begin{array}{l}.09 \\
.22 \\
.09\end{array}$ & $\begin{array}{l}-- \\
2.0 \\
---\end{array}$ \\
\hline & Station 1 & & (2) & (2) & $\begin{array}{l}255^{\circ} \\
U p \\
345^{\circ}\end{array}$ & $\begin{array}{l}.09 \\
.21 \\
.09\end{array}$ & $\begin{array}{l}--- \\
2.1 \\
---\end{array}$ \\
\hline & Station 3 & & (2) & (2) & $\begin{array}{l}255^{\circ} \\
\text { Up } \\
345^{\circ}\end{array}$ & $\begin{array}{l}.08 \\
.24 \\
.08\end{array}$ & $\begin{array}{l}--- \\
2.2 \\
---\end{array}$ \\
\hline & Station 4 & & (2) & (2) & $\begin{array}{l}255^{\circ} \\
\text { Up } \\
345^{\circ}\end{array}$ & $\begin{array}{l}.10 \\
.28 \\
.10\end{array}$ & $\begin{array}{l}1 \text { peak } \\
2.1 \\
1 \text { peak }\end{array}$ \\
\hline & Station 5 & & $(2)$ & $(2)$ & $\begin{array}{l}255^{\circ} \\
\text { Up } \\
345^{\circ}\end{array}$ & $\begin{array}{l}.08 \\
.25 \\
.10\end{array}$ & $\overline{2.1}$ \\
\hline & $\begin{array}{l}\text { Hollister } \\
\text { Damler Residence } \\
(\text { UCB })^{3}\end{array}$ & $\begin{array}{r}36.81^{\circ} \mathrm{N} \\
121.41^{\circ} \mathrm{W}\end{array}$ & (2) & (2) & $\begin{array}{l}118^{\circ} \\
\text { Up } \\
028^{\circ}\end{array}$ & $\begin{array}{l}.08 \\
.08 \\
.06\end{array}$ & $\begin{array}{l}--- \\
--- \\
---\end{array}$ \\
\hline
\end{tabular}

Note: One additional record ${ }^{1}$ recovered at Damler Residence.

Livermore VA Hospital $37.62^{\circ} \mathrm{N}$ Building 62 $(V A)^{3}$

Basement

Roof

Palo Alto

VA Hospital

(VA)

Basement

Roof (7th level)

$\begin{array}{lr}\text { San Jose Interchange } & 37.340^{\circ} \mathrm{N} \\ \text { Freeways } 101 / 680 / 280 & 121.851^{\circ} \mathrm{W}\end{array}$

(USGS/CDOT)

$37.40^{\circ} \mathrm{N}$ $122.14^{\circ} \mathrm{W}$
(2) (2)

(2)

(2)

(1)

(1)

(2) (2)

$322^{\circ}$
Up $232^{\circ}$
.12

$.12 \quad 4-$ peak

.08 -..-

See footnotes at end of table. 
Table 12. Summary of U.S. accelerograph records recovered during 1984-Continued

\begin{tabular}{|c|c|c|c|c|c|c|c|}
\hline Earthquake & $\begin{array}{l}\text { Station name } \\
\text { (owner) }\end{array}$ & $\begin{array}{l}\text { Station } \\
\text { location }\end{array}$ & $\begin{array}{l}\text { Trigger } \\
\text { time }\end{array}$ & $\begin{array}{l}\text { S-minus } \\
\text { trigger } \\
\quad(s)\end{array}$ & $\begin{array}{l}\text { Direction } \\
\quad(a z)\end{array}$ & $\begin{array}{l}\text { Maximum } \\
\text { ampl itude } \\
\text { (g) }\end{array}$ & $\begin{array}{l}\text { Duration } \\
\qquad(\mathrm{s})\end{array}$ \\
\hline & $\begin{array}{l}\text { San Justo Damsite } \\
\text { (USBR) }\end{array}$ & & & & & & \\
\hline & $\begin{array}{l}\text { Right abutment } \\
\text { (dike) }\end{array}$ & $\begin{array}{r}36.827^{\circ} \mathrm{N} \\
121.445^{\circ} \mathrm{W}\end{array}$ & 31.0 & (2) & $\begin{array}{l}360^{\circ} \\
U p \\
270^{\circ}\end{array}$ & $\begin{array}{l}.06 \\
.06 \\
.08\end{array}$ & --- \\
\hline & Left abutment & $\begin{array}{r}36.815^{\circ} \mathrm{N} \\
121.447^{\circ} \mathrm{W}\end{array}$ & 31.5 & (2) & $\begin{array}{l}360^{\circ} \\
U p \\
270^{\circ}\end{array}$ & $\begin{array}{l}.07 \\
.03 \\
.08\end{array}$ & --- \\
\hline & $\begin{array}{l}\text { San Francisco } \\
\text { Transamerica Tower } \\
(\text { USGS })^{3}\end{array}$ & $\begin{array}{r}37.80^{\circ} \mathrm{N} \\
122.40^{\circ} \mathrm{W}\end{array}$ & $(2)$ & (2) & & & \\
\hline & Basement & & & & & $(1)$ & \\
\hline & 24 th floor & & & & & (1) & \\
\hline & 49 th floor & & & & & (1) & \\
\hline & 58th level & & & & $\begin{array}{l}261^{\circ} \\
\operatorname{Up} \\
171^{\circ}\end{array}$ & $\begin{array}{l}.10 \\
.04 \\
.08\end{array}$ & 1-peak \\
\hline & $\begin{array}{l}\text { San Francisco } \\
\text { Standard } 0 i 1, \text { SMA-1 } \\
(\text { USGS) }\end{array}$ & $\begin{array}{r}37.79^{\circ} \mathrm{N} \\
122.40^{\circ} \mathrm{W}\end{array}$ & (2) & (2) & & & \\
\hline & Basement & & & & & (1) & \\
\hline & $\begin{array}{l}\text { San Francisco } \\
\text { Standard } 0 i 1, \text { CRA-1 } \\
(\text { USGS) }\end{array}$ & $\begin{array}{r}37.79^{\circ} \mathrm{N} \\
122.40^{\circ} \mathrm{W}\end{array}$ & (2) & (2) & & & \\
\hline & $\begin{array}{l}41 \text { st floor (NW end) } \\
\text { channel (1) }\end{array}$ & & & & $045^{\circ}$ & (1) & \\
\hline & $\begin{array}{l}4 \text { lst floor (center) } \\
\text { channel (2) }\end{array}$ & & & & $225^{\circ}$ & (1) & \\
\hline & $\begin{array}{l}41 \text { st floor (center) } \\
\text { channel (3) }\end{array}$ & & & & $135^{\circ}$ & (1) & \\
\hline & $\begin{array}{l}33 r d \text { floor (NW end) } \\
\text { channel (4) }\end{array}$ & & & & $045^{\circ}$ & (1) & \\
\hline & $\begin{array}{l}33 r d \text { floor (center) } \\
\text { channel (5) }\end{array}$ & & & & $225^{\circ}$ & (1) & \\
\hline & $\begin{array}{l}33 r d \text { floor (center) } \\
\text { channel (6) }\end{array}$ & & & & $135^{\circ}$ & (1) & \\
\hline & $\begin{array}{l}24 \text { th floor (NW end) } \\
\text { channel ( } 7 \text { ) }\end{array}$ & & & & $045^{\circ}$ & (1) & \\
\hline & $\begin{array}{l}24 \text { th floor (center) } \\
\text { channe } 1(8)\end{array}$ & & & & $225^{\circ}$ & (1) & \\
\hline & $\begin{array}{l}24 \text { th floor (center) } \\
\text { channel (9) }\end{array}$ & & & & $135^{\circ}$ & (1) & \\
\hline
\end{tabular}

See footnotes at end of table. 
Table 12. Summary of $U$. S. accelerograph records recovered during 1984-Continued

\begin{tabular}{|c|c|c|c|c|c|c|c|}
\hline Earthquake & $\begin{array}{l}\text { Station name } \\
\text { (owner) }\end{array}$ & $\begin{array}{l}\text { Station } \\
\text { location }\end{array}$ & $\begin{array}{l}\text { Trigger } \\
\text { time }\end{array}$ & $\begin{array}{l}\text { S-minus } \\
\operatorname{trigger} \\
\quad(s)\end{array}$ & $\begin{array}{l}\text { Direction } \\
\quad(a z)\end{array}$ & $\begin{array}{l}\text { Maximum } \\
\text { amplitude } \\
(\underline{g})\end{array}$ & $\begin{array}{l}\text { Duration } \\
\qquad(s)\end{array}$ \\
\hline \multicolumn{5}{|c|}{$\begin{array}{l}\text { lst basement (center) } \\
\text { channel }(10)\end{array}$} & $045^{\circ}$ & (1) & \\
\hline \multicolumn{5}{|c|}{$\begin{array}{l}\text { lst basement (center) } \\
\text { channel (11) }\end{array}$} & $315^{\circ}$ & (1) & \\
\hline & $\begin{array}{l}\text { Stanford University } \\
\text { Quadrangle } \\
\text { (USGS) }^{3}\end{array}$ & $\begin{array}{r}37.429^{\circ} \mathrm{N} \\
122.169^{\circ} \mathrm{W}\end{array}$ & $(2)$ & (2) & & (1) & \\
\hline & $\begin{array}{l}\text { Stanford University } \\
\text { Survey Hill } \\
\text { (USGS) }\end{array}$ & $\begin{array}{r}37.417^{\circ} \mathrm{N} \\
122.198^{\circ} \mathrm{W}\end{array}$ & (2) & (2) & & (1) & \\
\hline & $\begin{array}{l}\text { Stanford University } \\
\text { Test Laboratory } \\
\text { (USGS) }\end{array}$ & $\begin{array}{r}37.419^{\circ} \mathrm{N} \\
122.205^{\circ} \mathrm{W}\end{array}$ & (2) & (2) & & (1) & \\
\hline \multirow{2}{*}{$\begin{array}{l}26 \text { April } 1984 \\
0042: 26.6 \text { G.m.t. } \\
\text { Central Calif. } \\
37.140 \mathrm{~N}, 121.580 \mathrm{~W} \\
\text { Magnitude } 3.9 \mathrm{ML}\end{array}$} & $\begin{array}{l}\text { Anderson Lake } \\
\text { Cochrane Bridge } \\
\text { (USGS) }\end{array}$ & $\begin{array}{r}37.152^{\circ} \mathrm{N} \\
121.583^{\circ} \mathrm{W}\end{array}$ & (2) & (2) & & & \\
\hline & Abutment & & & & $\begin{array}{l}360^{\circ} \\
U p \\
270^{\circ}\end{array}$ & $\begin{array}{l}.05 \\
.03 \\
.05\end{array}$ & --- \\
\hline \multirow{2}{*}{$\begin{array}{l}26 \text { April } 1984 \\
\text { 0629:51.9 G.m.t. } \\
\text { Central Calif. } \\
37.138 \mathrm{~N}, 121.570 \mathrm{~W} \\
\text { Magnitude } 4.0 \mathrm{ML}\end{array}$} & $\begin{array}{l}\text { Anderson Lake } \\
\text { Cochrane Bridge } \\
\text { (USGS) }\end{array}$ & $\begin{array}{r}37.152^{\circ} \mathrm{N} \\
121.583^{\circ} \mathrm{W}\end{array}$ & (2) & (2) & & & \\
\hline & Abutment & & & & $\begin{array}{l}360^{\circ} \\
\text { Up } \\
270^{\circ}\end{array}$ & $\begin{array}{l}.02 \\
.03 \\
.05\end{array}$ & $\begin{array}{l}--- \\
---\end{array}$ \\
\hline $\begin{array}{l}29 \text { April } 1984 \\
2358: 20.2 \text { G.m.t. } \\
\text { Central Calif. } \\
37.142 \mathrm{~N}, 121.572 \mathrm{~W} \\
\text { Magnitude } 3.2 \mathrm{ML}\end{array}$ & $\begin{array}{l}\text { Anderson Lake } \\
\text { Cochrane Bridge } \\
\text { (USGS) } \\
\quad \text { Abutment }\end{array}$ & $\begin{array}{r}37.152^{\circ} \mathrm{N} \\
127.583^{\circ} \mathrm{W}\end{array}$ & (2) & $(2)$ & & (1) & \\
\hline $\begin{array}{l}7 \text { March } 1984- \\
8 \text { May } 1984 \\
\text { Central Calif. } \\
\text { Epicenter and } \\
\text { magnitude unknown }\end{array}$ & $\begin{array}{l}\text { Hollister } \\
\text { Vault } \\
\text { (UCB) }\end{array}$ & $\begin{array}{r}37.76^{\circ} \mathrm{N} \\
121.45^{\circ} \mathrm{W}\end{array}$ & (2) & (2) & & (1) & \\
\hline \multirow{2}{*}{$\begin{array}{l}8 \text { May } 1984 \\
1921: 14.0 \text { G.m.t. } \\
\text { Central Calif. } \\
36.168 \mathrm{~N}, 120.297 \mathrm{~W} \\
\text { Magnitude } 4.1 \mathrm{ML}\end{array}$} & $\begin{array}{l}\text { Coalinga } \\
\text { Burnett Company } \\
\text { (USGS) }\end{array}$ & $\begin{array}{r}36.138^{\circ} \mathrm{N} \\
120.357^{\circ} \mathrm{W}\end{array}$ & 16 & 2.2 & $\begin{array}{l}360^{\circ} \\
U p \\
270^{\circ}\end{array}$ & $\begin{array}{l}.06 \\
.01 \\
.05\end{array}$ & $\begin{array}{l}--- \\
--- \\
---\end{array}$ \\
\hline & $\begin{array}{l}\text { Coalinga } \\
\text { Oil City } \\
\text { (USGS) }\end{array}$ & $\begin{array}{r}36.229^{\circ} \mathrm{N} \\
120.360^{\circ} \mathrm{W}\end{array}$ & 18.6 & .2 & $\begin{array}{l}360^{\circ} \\
\mathrm{Up} \\
270^{\circ}\end{array}$ & $\begin{array}{l}.12 \\
.03 \\
.11\end{array}$ & $\begin{array}{l}0.2 \\
--- \\
1-\text { peak }\end{array}$ \\
\hline $\begin{array}{l}17 \text { May } 1984 \\
0843: 03.6 \mathrm{G} . \mathrm{n} . \mathrm{t} . \\
\text { Central Calif. } \\
37.298 \mathrm{~N}, 121.688 \mathrm{~W} \\
\text { Magnitude } 3.6 \mathrm{ML}\end{array}$ & $\begin{array}{l}\text { Anderson Lake } \\
\text { Cochrane Bridge } \\
\text { (USGS) } \\
\text { Abutment }\end{array}$ & $\begin{array}{r}37.152^{\circ} \mathrm{N} \\
121.583^{\circ} \mathrm{W}\end{array}$ & (2) & (2) & & (1) & \\
\hline
\end{tabular}

See footnotes at end of table. 
Table 12. Summary of U.S. accelerograph records recovered during 1984-Continued

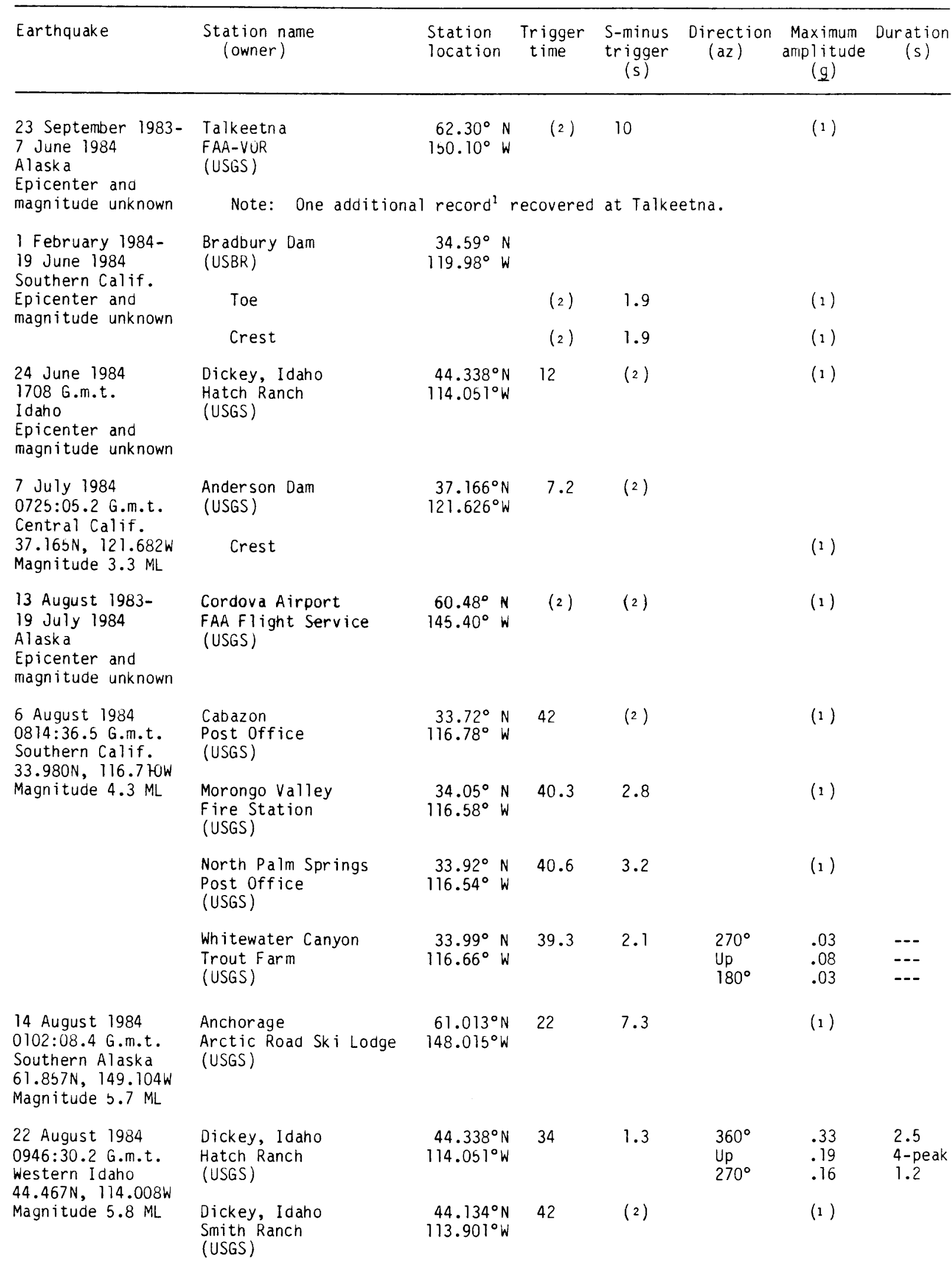

See footnotes at end of table. 
Table 12. Summary of U. S. accelerograph records recovered during 1984-Continued

\begin{tabular}{|c|c|c|c|c|c|c|c|}
\hline Earthquake & $\begin{array}{l}\text { Station name } \\
\text { (owner) }\end{array}$ & $\begin{array}{l}\text { Station } \\
\text { location }\end{array}$ & $\begin{array}{l}\text { Trigger } \\
\text { time }\end{array}$ & $\begin{array}{l}\text { S-minus } \\
\text { trigger } \\
(\mathrm{s})\end{array}$ & $\begin{array}{l}\text { Direction } \\
\quad(a z)\end{array}$ & $\begin{array}{l}\text { Maximum } \\
\text { amplitude } \\
\text { (g) }\end{array}$ & $\begin{array}{l}\text { Duration } \\
\qquad(\mathrm{s})\end{array}$ \\
\hline $\begin{array}{l}22 \text { August } 1984 \\
1052: 01.1 \mathrm{G} . \mathrm{m} . \mathrm{t} \text {. } \\
\text { Western Idaho } \\
44.48 \mathrm{~N}, 114.20 \mathrm{~W} \\
\text { Magnitude } 4.0 \mathrm{ML}\end{array}$ & $\begin{array}{l}\text { Dickey, Idaho } \\
\text { Hatch Ranch } \\
\text { (USGS) }\end{array}$ & $\begin{array}{r}44.338^{\circ} \mathrm{N} \\
114.051^{\circ} \mathrm{W}\end{array}$ & 5 & 1.3 & $\begin{array}{l}360^{\circ} \\
\text { Up } \\
270^{\circ}\end{array}$ & $\begin{array}{l}.07 \\
.02 \\
.06\end{array}$ & --- \\
\hline $\begin{array}{l}23 \text { August } 1984 \\
1321: 53.0 \mathrm{G} . \mathrm{m} . \mathrm{t} \text {. } \\
\text { Western Idaho } \\
44.462 \mathrm{~N}, 114.137 \mathrm{~W} \\
\text { Magnitude } 3.8 \mathrm{ML}\end{array}$ & $\begin{array}{l}\text { Dickey, Idaho } \\
\text { Hatch Ranch } \\
\text { (USGS) }\end{array}$ & $\begin{array}{r}44.338^{\circ} \mathrm{N} \\
114.051^{\circ} \mathrm{W}\end{array}$ & 57 & (2) & & (1) & \\
\hline $\begin{array}{l}30 \text { August } 1984 \\
2106: 20.7 \mathrm{G.m} . \mathrm{t} \text {. } \\
\text { Western Idaho } \\
44.455 \mathrm{~N}, 114.154 \mathrm{~W} \\
\text { Magnitude } 3.9 \mathrm{ML}\end{array}$ & $\begin{array}{l}\text { Dickey, Idaho } \\
\text { Hatch Ranch } \\
\text { (USGS) }\end{array}$ & $\begin{array}{r}44.338^{\circ} \mathrm{N} \\
114.051^{\circ} \mathrm{W}\end{array}$ & 25 & (2) & & (1) & \\
\hline $\begin{array}{l}8 \text { September } 1984 \\
0616: 40.1 \text { G.m.t. } \\
\text { Western Idaho } \\
44.439 \mathrm{~N}, 114.154 \mathrm{~W} \\
\text { Magnitude } 5.0 \mathrm{MB}\end{array}$ & $\begin{array}{l}\text { Dickey, Idaho } \\
\text { Hatch Ranch } \\
\text { (USGS) }\end{array}$ & $\begin{array}{r}44.338^{\circ} \mathrm{N} \\
114.051^{\circ} \mathrm{W}\end{array}$ & 43 & 1.8 & $\begin{array}{l}360^{\circ} \\
U p \\
270^{\circ}\end{array}$ & $\begin{array}{l}.09 \\
.03 \\
.06\end{array}$ & --- \\
\hline $\begin{array}{l}8 \text { September } 1984 \\
1356: 37.7 \text { G.m.t. }\end{array}$ & $\begin{array}{l}\text { Dickey, Idaho } \\
\text { Hatch Ranch }\end{array}$ & $\begin{array}{r}44.338^{\circ} \mathrm{N} \\
114.051^{\circ} \mathrm{W}\end{array}$ & 41 & 1.6 & & (1) & \\
\hline $\begin{array}{l}\text { Western Idaho } \\
44.416 \mathrm{~N}, 114.147 \mathrm{~W} \\
\text { Magnitude } 4.4 \mathrm{MB}\end{array}$ & (USGS) & & & & & & \\
\hline $\begin{array}{l}31 \text { May 1984- } \\
11 \text { September } 1984 \\
\text { Alaska } \\
\text { Epicenter and }\end{array}$ & $\begin{array}{l}\text { Anchorage } \\
\text { USGS Building } \\
\text { (USGS) }\end{array}$ & $\begin{array}{r}61.223^{\circ} \mathrm{N} \\
149.892^{\circ} \mathrm{W}\end{array}$ & (2) & (2) & & & \\
\hline magnitude unknown & Basement & & & & & (1) & \\
\hline $\begin{array}{l}18 \text { September } 1984 \\
1509: 58.0 \mathrm{G} . \mathrm{m} . \mathrm{t} \text {. } \\
\text { Western Idaho } \\
44.408 \mathrm{~N}, 114.124 \mathrm{~W} \\
\text { Magnitude } 4.0 \mathrm{ML}\end{array}$ & $\begin{array}{l}\text { Dickey, Idaho } \\
\text { Hatch Ranch } \\
\text { (USGS) }\end{array}$ & $\begin{array}{r}44.338^{\circ} \mathrm{N} \\
114.051^{\circ} \mathrm{W}\end{array}$ & 1 & 1.0 & & (1) & \\
\hline $\begin{array}{l}19 \text { September } 1984 \\
1533: 22.6 \mathrm{G} . \mathrm{m} . \mathrm{t} \text {. } \\
\text { Western Idaho } \\
44.334 \mathrm{~N}, 114.20 \mathrm{bW} \\
\text { Magnitude } 3.5 \mathrm{ML}\end{array}$ & $\begin{array}{l}\text { Dickey, Idaho } \\
\text { Hatch Ranch } \\
\text { (USGS) }\end{array}$ & $\begin{array}{r}44.338^{\circ} \mathrm{N} \\
114.051^{\circ} \mathrm{W}\end{array}$ & 26 & 1.2 & & (1) & \\
\hline \multirow{4}{*}{$\begin{array}{l}5 \text { October } 1984 \\
1616: 30.0 \text { G.m.t. } \\
\text { Central Calif. } \\
36.573 \mathrm{~N}, 121.203 \mathrm{~W} \\
\text { Magnitude } 4.0 \mathrm{ML}\end{array}$} & $\begin{array}{l}\text { Bear Valley Station } 1 \\
\text { Fire Station } \\
\text { (USGS) }\end{array}$ & $\begin{array}{r}36.573^{\circ} \mathrm{N} \\
121.184^{\circ} \mathrm{W}\end{array}$ & 33.3 & (2) & & (1) & \\
\hline & $\begin{array}{l}\text { Bear Valley Station } 6 \\
\text { James Ranch } \\
\text { (USGS) }\end{array}$ & $\begin{array}{r}36.504^{\circ} \mathrm{N} \\
121.101^{\circ} \mathrm{W}\end{array}$ & 32.9 & 2.1 & & (1) & \\
\hline & $\begin{array}{l}\text { Bear Valley Station } 10 \\
\text { Webb Residence } \\
\text { (USGS) }\end{array}$ & $\begin{array}{r}36.532^{\circ} \mathrm{N} \\
121.143^{\circ} \mathrm{W}\end{array}$ & 32.2 & 1.5 & $\begin{array}{l}310^{\circ} \\
\text { Up } \\
220^{\circ}\end{array}$ & $\begin{array}{l}.07 \\
.01 \\
.09\end{array}$ & $\overline{---}$ \\
\hline & $\begin{array}{l}\text { Bear Valley Station } 14 \\
\text { Upper Butts Ranch } \\
\text { (USGS) }\end{array}$ & $\begin{array}{r}36.569^{\circ} \mathrm{N} \\
121.043^{\circ} \mathrm{W}\end{array}$ & 33.6 & 2.5 & $\begin{array}{l}310^{\circ} \\
\text { Up } \\
220^{\circ}\end{array}$ & $\begin{array}{l}.04 \\
.04 \\
.06\end{array}$ & $\begin{array}{l}--- \\
--- \\
---\end{array}$ \\
\hline
\end{tabular}

See footnotes at end of table. 
Table 12. Summary of U.S. accelerograph records recovered during 1984-Continued

\begin{tabular}{|c|c|c|c|c|c|c|c|}
\hline Earthquake & $\begin{array}{l}\text { Station name } \\
\text { (owner) }\end{array}$ & $\begin{array}{l}\text { Station } \\
\text { location }\end{array}$ & $\begin{array}{l}\text { Trigger } \\
\text { time }\end{array}$ & $\begin{array}{l}\text { S-minus } \\
\text { trigger } \\
(\mathrm{s})\end{array}$ & $\begin{array}{l}\text { Direction } \\
\quad(a z)\end{array}$ & $\begin{array}{l}\text { Maximum } \\
\text { amplitude } \\
(\mathrm{g})\end{array}$ & $\begin{array}{c}\text { Duration } \\
(\mathrm{s})\end{array}$ \\
\hline \multirow{4}{*}{$\begin{array}{l}22 \text { October } 1984 \\
0406: 31.4 \mathrm{G} . \mathrm{m} . \mathrm{t} \text {. } \\
\text { Central Calif. } \\
36.582 \mathrm{~N}, 121.235 \mathrm{~W} \\
\text { Magnitude } 3.7 \mathrm{ML}\end{array}$} & $\begin{array}{l}\text { Bear Valley Station } 1 \\
\text { Fire Station } \\
\text { (USGS) }\end{array}$ & $\begin{array}{r}36.573^{\circ} \mathrm{N} \\
121.184^{\circ} \mathrm{W}\end{array}$ & 33.3 & 1.2 & $\begin{array}{l}310^{\circ} \\
U p \\
220^{\circ}\end{array}$ & $\begin{array}{l}.07 \\
.02 \\
.05\end{array}$ & $\begin{array}{l}--\cdot \\
--- \\
--\end{array}$ \\
\hline & $\begin{array}{l}\text { Bear Valley Station } 6 \\
\text { James Ranch } \\
\text { (USGS) }\end{array}$ & $\begin{array}{r}36.504^{\circ} \mathrm{N} \\
121.101^{\circ} \mathrm{W}\end{array}$ & 34.6 & 2.5 & & (1) & \\
\hline & $\begin{array}{l}\text { Bear Valley Station } 10 \\
\text { Webb Residence } \\
\text { (USGS) }\end{array}$ & $\begin{array}{r}36.532^{\circ} \mathrm{N} \\
121.143^{\circ} \mathrm{W}\end{array}$ & 33.9 & 1.8 & $\begin{array}{l}310^{\circ} \\
\text { Up } \\
220^{\circ}\end{array}$ & $\begin{array}{l}.07 \\
.04 \\
.09\end{array}$ & $\begin{array}{l}--- \\
--- \\
---\end{array}$ \\
\hline & $\begin{array}{l}\text { Bear Valley Station } 14 \\
\text { Upper Butts Ranch } \\
\text { (USGS) }\end{array}$ & $\begin{array}{r}36.569^{\circ} \mathrm{N} \\
121.043^{\circ} \mathrm{W}\end{array}$ & 35.3 & 3.1 & & (1) & \\
\hline \multirow{2}{*}{$\begin{array}{l}4 \text { November } 1984 \\
1120: 19.7 \mathrm{G.m} . \mathrm{t} \text {. } \\
\text { Central Cal if. } \\
36.553 \mathrm{~N}, 121.172 \mathrm{~W} \\
\text { Magnitude } 3.1 \mathrm{ML}\end{array}$} & $\begin{array}{l}\text { Bear Valley Station } 1 \\
\text { Fire Station } \\
\text { (USGS) }\end{array}$ & $\begin{array}{r}36.573^{\circ} \mathrm{N} \\
121.184^{\circ} \mathrm{W}\end{array}$ & 20.7 & .8 & $\begin{array}{l}310^{\circ} \\
\mathrm{Up} \\
220^{\circ}\end{array}$ & $\begin{array}{l}.04 \\
.02 \\
.05\end{array}$ & $\begin{array}{l}--- \\
--- \\
---\end{array}$ \\
\hline & $\begin{array}{l}\text { Bear Valley Station } 10 \\
\text { Webb Residence } \\
\text { (USGS) }\end{array}$ & $\begin{array}{r}36.532^{\circ} \mathrm{N} \\
121.143^{\circ} \mathrm{W}\end{array}$ & 20.9 & 1.1 & & (1) & \\
\hline $\begin{array}{l}11 \text { November } 1984 \\
0405 \text { G.m.t. } \\
\text { Central Calif. } \\
\text { Epicenter and } \\
\text { magnitude unknown }\end{array}$ & $\begin{array}{l}\text { Bear Valley Station } 10 \\
\text { Webb Residence } \\
\text { (USGS) }\end{array}$ & $\begin{array}{r}36.532^{\circ} \mathrm{N} \\
121.143^{\circ} \mathrm{W}\end{array}$ & 21.9 & .9 & $\begin{array}{l}310^{\circ} \\
U p \\
220^{\circ}\end{array}$ & $\begin{array}{l}.05 \\
.05 \\
.06\end{array}$ & $\begin{array}{l}--- \\
--- \\
---\end{array}$ \\
\hline $\begin{array}{l}22 \text { November } 1984 \\
2359: 08.4 \text { G.m.t. } \\
\text { Central Calif. } \\
36.563 \mathrm{~N}, 121.198 \mathrm{~W} \\
\text { Magnitude } 3.2 \mathrm{ML}\end{array}$ & $\begin{array}{l}\text { Bear Valley Station } 10 \\
\text { Webb Residence } \\
\text { (USGS) }\end{array}$ & $\begin{array}{r}36.532^{\circ} \mathrm{N} \\
121.143^{\circ} \mathrm{W}\end{array}$ & 10.1 & 1.3 & & (1) & \\
\hline \multirow{8}{*}{$\begin{array}{l}23 \text { November } 1984 \\
1808: 25.3 \text { G.m.t. } \\
\text { Eastern Calif. } \\
37.480 \mathrm{~N}, 118.655 \mathrm{~W} \\
\text { Magnitude } 6.1 \mathrm{ML}\end{array}$} & $\begin{array}{l}\text { Buchanan Dam } \\
\text { (ACOE) }\end{array}$ & $\begin{array}{r}37.22^{\circ} \mathrm{N} \\
119.98^{\circ} \mathrm{W}\end{array}$ & 47.6 & 13.7 & & & \\
\hline & Left crest & & & & & (1) & \\
\hline & $\begin{array}{l}\text { Hidden Dam } \\
\text { (ACOE) }\end{array}$ & $\begin{array}{r}37.112^{\circ} \mathrm{N} \\
119.883^{\circ} \mathrm{W}\end{array}$ & .3 & (2) & & & \\
\hline & Left crest & & & & & (1) & \\
\hline & $\begin{array}{l}\text { McGee Creek } \\
\text { (USGS) }\end{array}$ & $\begin{array}{r}37.550^{\circ} \mathrm{N} \\
118.811^{\circ} \mathrm{W}\end{array}$ & 30 & 2.9 & & & \\
\hline & 166-m downhole & & & & & (1) & \\
\hline & 35-m downhole & & & & & (1) & \\
\hline & Surface & & & & $\begin{array}{l}360^{\circ} \\
\text { Up } \\
270^{\circ}\end{array}$ & $\begin{array}{l}.11 \\
.09 \\
.10\end{array}$ & $\begin{array}{l}0.3 \\
--. \\
0.2\end{array}$ \\
\hline
\end{tabular}

See footnotes at end of table. 
Table 12. Summary of U. S. accelerograph records recovered during 1984-Continued

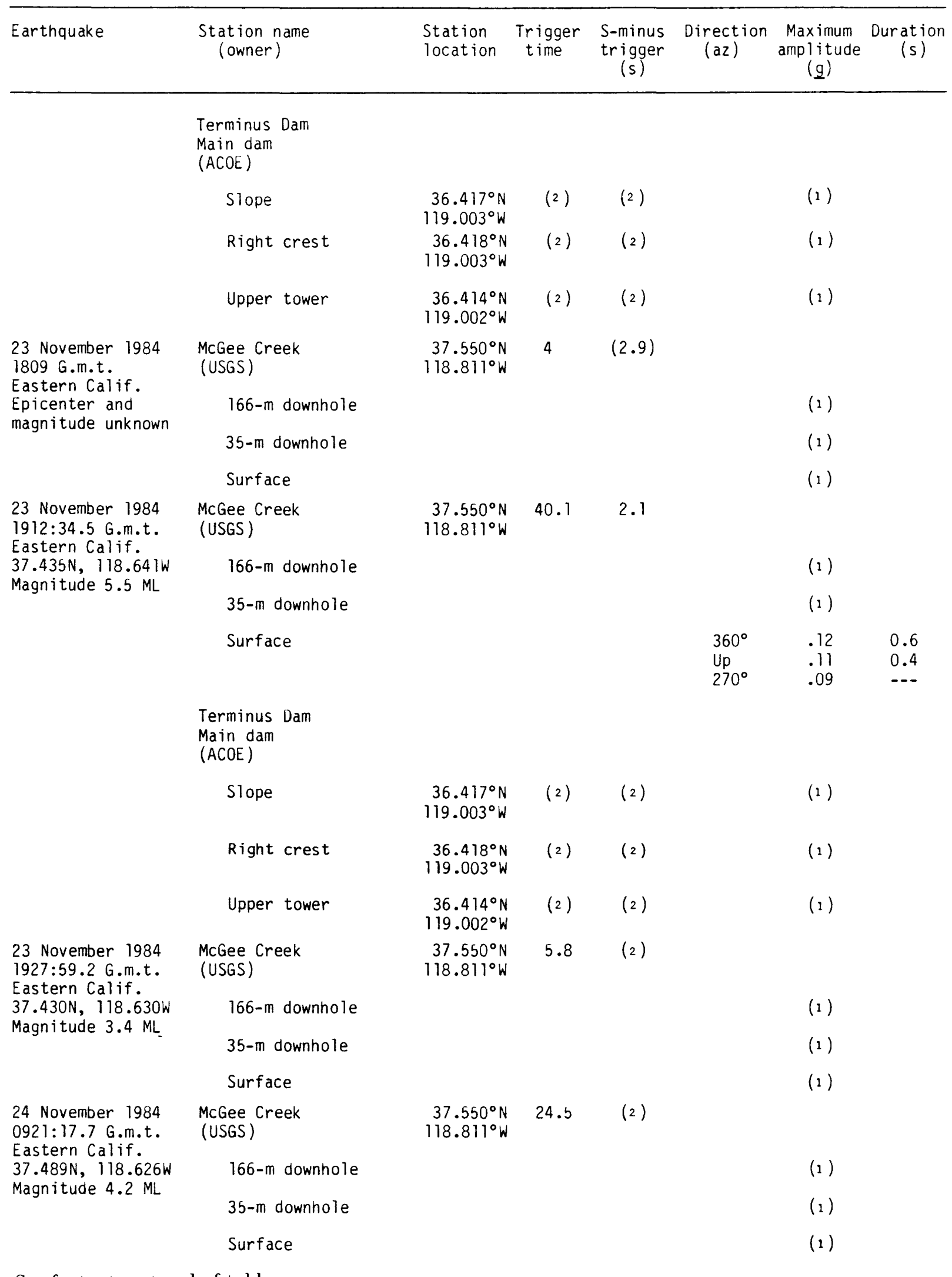

See footnotes at end of table. 
Table 12. Summary of U. S. accelerograph records recovered during 1984-Continued

\begin{tabular}{ccccc}
\hline Earthquake & $\begin{array}{c}\text { Station name } \\
\text { (owner) }\end{array}$ & $\begin{array}{l}\text { Station Trigger S-minus Direction Maximum Duration } \\
\text { location time trigger (az) }\end{array}$ & $\begin{array}{c}\text { Matitude } \\
\text { (s) }\end{array}$ & $\begin{array}{c}(\mathrm{s}) \\
\text { (g) }\end{array}$ \\
\hline
\end{tabular}

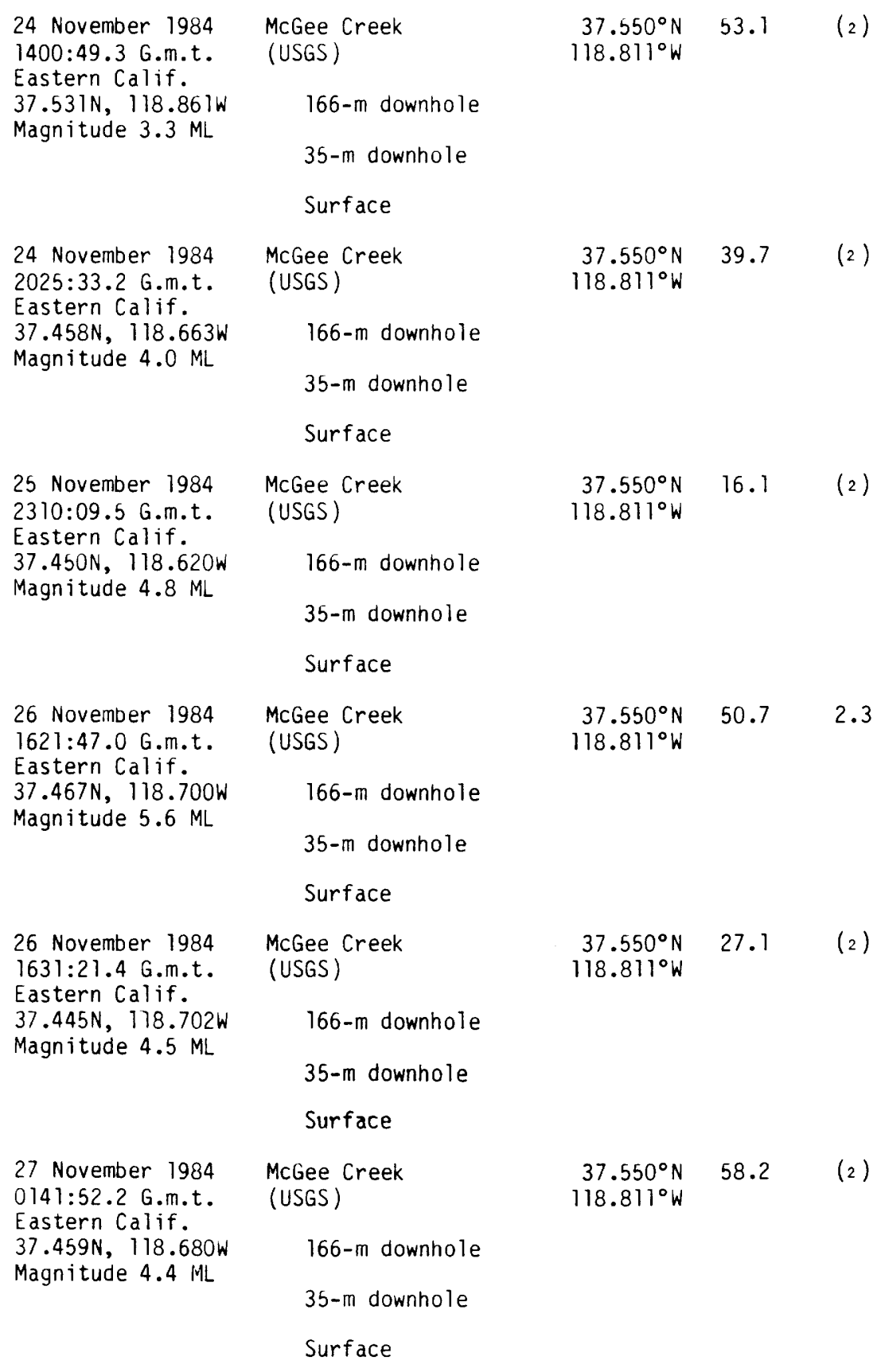

(1)

(1)

(1)

2)

(1)

(1)

(1)

(1)

(1)

(1)

(1)

(1)

(1)

(1)

(1)

(1)

(1)

(1)

(1)

See footnotes at end of table. 
Table 12. Summary of U.S. accelerograph records recovered during 1984-Continued

\begin{tabular}{|c|c|c|c|c|c|c|c|}
\hline Earthquake & $\begin{array}{l}\text { Station name } \\
\text { (owner) }\end{array}$ & $\begin{array}{l}\text { Station } \\
\text { location }\end{array}$ & $\begin{array}{l}\text { Trigger } \\
\text { time }\end{array}$ & $\begin{array}{l}\text { S-minus } \\
\text { trigger } \\
\quad(\mathrm{s})\end{array}$ & $\begin{array}{l}\text { Direction } \\
(\mathrm{az})\end{array}$ & $\begin{array}{l}\text { Maximum } \\
\text { amplitude } \\
(g)\end{array}$ & $\begin{array}{l}\text { Duration } \\
\qquad(\mathrm{s})\end{array}$ \\
\hline \multirow{5}{*}{$\begin{array}{l}26 \text { February } 1984- \\
28 \text { November } 1984 \\
\text { Eastern Calif. } \\
\text { Epicenters and } \\
\text { magnitudes unknown }\end{array}$} & $\begin{array}{l}\text { Long Valley Dam } \\
\text { Lake Crowley } \\
\text { (USGS) }\end{array}$ & \multirow[t]{4}{*}{$\begin{array}{r}37.588^{\circ} \mathrm{N} \\
118.705^{\circ} \mathrm{W}\end{array}$} & & & & & \\
\hline & \multirow[t]{3}{*}{ Left abutment } & & (2) & (2) & $\begin{array}{l}275^{\circ} \\
\operatorname{Up} \\
185^{\circ}\end{array}$ & $\begin{array}{l}.06 \\
.06 \\
.08\end{array}$ & --- \\
\hline & & & $(2)$ & 2.2 & $\begin{array}{l}275^{\circ} \\
\operatorname{Up} \\
185^{\circ}\end{array}$ & $\begin{array}{l}.03 \\
.03 \\
.06\end{array}$ & --- \\
\hline & & & (2) & (2) & $\begin{array}{l}275^{\circ} \\
\text { Up } \\
185^{\circ}\end{array}$ & $\begin{array}{l}.01 \\
.06 \\
.02\end{array}$ & --- \\
\hline & \multicolumn{2}{|c|}{$\begin{array}{l}\text { Note: Three additional records } \\
\text { left abutment. }\end{array}$} & \multicolumn{3}{|c|}{ recovered at Long Valley } & Dam, & \\
\hline \multirow{4}{*}{$\begin{array}{l}29 \text { November } 1984 \\
1042: 28.0 \mathrm{G} . \mathrm{m} . \mathrm{t} . \\
\text { Eastern Calif. } \\
37.420 \mathrm{~N}, 118.650 \mathrm{~W} \\
\text { Magnitude } 3.7 \mathrm{ML}\end{array}$} & $\begin{array}{l}\text { McGee Creek } \\
\text { (USGS) }\end{array}$ & $\begin{array}{r}37.550^{\circ} \mathrm{N} \\
118.811^{\circ} \mathrm{W}\end{array}$ & 35.3 & $(2)$ & & & \\
\hline & 166-m downhole & & & & & (1) & \\
\hline & $3 b-m$ downhole & & & & & (1) & \\
\hline & Surface & & & & & (1) & \\
\hline \multirow{4}{*}{$\begin{array}{l}29 \text { November } 1984 \\
1045: 40.4 \text { G.m.t. } \\
\text { Eastern Calif. } \\
37.420 \mathrm{~N}, 118.6 \mathrm{bOW} \\
\text { Magnitude } 3.5 \mathrm{ML}\end{array}$} & $\begin{array}{l}\text { McGee Creek } \\
\text { (USGS) }\end{array}$ & $\begin{array}{r}37.550^{\circ} \mathrm{N} \\
118.811^{\circ} \mathrm{W}\end{array}$ & 44.6 & (2) & & & \\
\hline & \multicolumn{2}{|l|}{ 166-m downhole } & & & & (1) & \\
\hline & \multicolumn{2}{|l|}{ 35-m downhole } & & & & (1) & \\
\hline & Surface & & & & & (1) & \\
\hline \multirow{2}{*}{$\begin{array}{l}21 \text { December } 1984 \\
\text { 1239:04.4 G.m.t. } \\
\text { Central Calif. } \\
36.508 \mathrm{~N}, 121.113 \mathrm{~W} \\
\text { Magnitude } 3.0 \mathrm{ML}\end{array}$} & $\begin{array}{l}\text { Bear Valley Station } 6 \\
\text { James Ranch } \\
\text { (USGS) }\end{array}$ & $\begin{array}{r}36.504^{\circ} \mathrm{N} \\
121.101^{\circ} \mathrm{W}\end{array}$ & 6.1 & 1.4 & & (1) & \\
\hline & $\begin{array}{l}\text { Bear Valley Station } 10 \\
\text { Webb Residence } \\
(\text { USGS) }\end{array}$ & $\begin{array}{r}36.532^{\circ} \mathrm{N} \\
121.143^{\circ} \mathrm{W}\end{array}$ & 6.1 & .7 & & (1) & \\
\hline
\end{tabular}

\footnotetext{
${ }^{1}$ Less than $0.05 \mathrm{~g}$ at ground-level or less than $0.10 \mathrm{~g}$ at non-ground-level stations.

${ }^{2}$ Questionable or undeterminable.

${ }^{3}$ WWVB time code illegible, or instrument not equipped with a radio receiver;

correlation of accelerogram with event may be questionable.
}

See footnotes at end of table. 


\section{REFERENCES CITED}

Abrahamson, N. A., and Darragh, R. B., 1985, The Morgan Hill Earthquake of April 24, 1984 - The $1.29 \mathrm{~g}$ acceleration at Coyote Lake Dam- Due to directivity, a double event, or both: EERI, Earthquake Spectra, v. 1 , no. 3 , p. 445-455.

Ake, J. P., 1984, An analysis of the May and July, 1983, Socorro Mountain microearthquake swarms: New Mexico Institute of Mining and Technology, Geophysics Open-File Report 49, 107 p.

Ake, J. P., Sanford, A. R., and Jarpe, S., 1983, A magnitude scale for central New Mexico based on signal duration: New Mexico Insitute of Mining and Technology, Geophysics Open-File Report 45, 26 p.

Armbruster, J. G., and Seeber, Leonardo, 1987, The 23 April 1984 Martic earthquake and the Lancaster seismic zone in eastern Pennsylvania: Seismological Society of America Bulletin, v. 77, no. 3, p. 877-890.

Bath, Markus, 1966, Earthquake energy and magnitude, in Physics and Chemistry of the Earth, v. 7:

New York, Pergamon Press, p. 115-165.

Bennett, J. H., and Sherburne, R. W., eds., 1984, The 1984 Morgan Hill, California earthquake: California Division of Mines and Geology Special Publication $68,271 \mathrm{p}$.

Bolt, B. A., and Herraiz, Miguel, 1983, Simplified estimation of seismic moment from seismograms: Seismological Society of America Bulletin, v. 73, no. 3, p. 735-748.

Brady, A. G., Porcella, R. L., Bycroft, G. N., Etheredge, E. C., Mork, P. N., Silverstein, B. L., and Shakal, A. F., 1987, Processing of strongmotion recordings from the main shock, in Hoose, S. N., The Morgan Hill, California, earthquake of April 24, 1984: U.S. Geological Survey Bulletin 1639, p. 53-60 and 139-256.

Cash, D. J., and Wolff, J. J., 1984, Seismicity of the Rio Grande Rift in Northern New Mexico, 1978-1983: New Mexico Geological Society Guidebook, 35th Field Conference, Rio Grande Rift, Northern New Mexico.

- 1987, Seismic activity in North-Central New Mexico, 1983, in Stover, C. W., ed., United States Earthquakes, 1983: U.S. Geological Survey Bulletin $1698,196 \mathrm{p}$.

Chiburis, E. F., and Ahner, R. O., 1980, Seismicity of the Northeastern United States, April 1, 1979 - June 30, 1979: Northeastern U.S. Seismic Network Bulletin no. 15, Weston Observatory, Weston Mass.
Davison, F. C., Chapman, M. C., Munsey, J. W., and Bollinger, G. A., 1984, A note on the Cunningham, Virginia earthquake of August 17, 1984, in the central Virginia seismic zone: Eastern Section, Seismological Society of America, Earthquake Notes, v. 55 , no. 4 , p. 26-33.

Ebel, J. E., 1984, A study of seismicity and tectonics in New England, prepared for U.S. Nuclear Regulatory Commission, Final Report, NUREG/CR4354.

Gutenberg, Beno, and Richter, C. F., 1956, Magnitude and energy of earthquakes: Annali di Geofisica, v. 9, no. 1, p. 1-15.

Hanks, T. C., and Kanamori, Hiroo, 1979, A moment magnitude scale: Journal of Geophysical Research, v. 84 , no. B5, p. 2348-2350.

Hindmarsh, J. V., 1984, State emergency services response to the 1984 Morgan Hill earthquake, in The 1984 Morgan Hill, California, earthquake: California Division of Mines and Geology Special Publication 68, p. 93-98.

Hoose, S. N., 1984, The Morgan Hill, California earthquake of April 24, 1984 (a preliminary report): U.S. Geological Survey Open-File Report 84-498A, 147 p.

Irby, W. L., Presgrave, B. W., and Schmieder, W. S., 1982, Preliminary Determination of Epicenters, Monthly Listing, January 1982: U.S. Geological Survey, $16 \mathrm{p}$.

Jarpe, Stephen, 1984, Characteristics and possible cause of an earthquake swarm in the central Rio Grande rift 28 kilometers north of Socorro, New Mexico, February and March 1983: New Mexico Institute of Mining and Technology Geophysics Open-File Report 50, 74 p.

Klein, F. W., 1978, Hypocenter location program, HYPOINVERSE: U.S. Geological Survey Open-File Report 78-694, 114 p.

Lawson, J. E., Jr., 1980, Geophysical observatory establishes continuous time synchronization: Oklahoma Geology Notes, v. 40, p. 214 p.

Lee, W. H. K., and Lahr, J. C., 1975, HYPO71 (Revised) - a computer program for determining hypocenter, magnitude, and first motion pattern of local earthquakes: U.S. Geological Survey OpenFile Report 75-311, $113 \mathrm{p}$.

Lomax, A. J., and Uhrhammer, R. A., 1985, Bulletin of the Seismographic Stations: University of California, Berkeley, v. 54, nos. 1 and 2, 88 p.

Newton, C. A., Cash, D. J., Olsen, K. H., and Homuth, E. F., 1976, LASL seismic programs in the vicinity of Los Alamos, New Mexico: Los Alamos Scientific Laboratory Informal Report LA-6406-MS, 42 p.

Noson, L. L., Crosson, R. S., Malone, S. D., and Zollweg, J. E., 1984, Washington earthquakes in 
Stover, C. W., ed., United States Earthquakes, 1981: U.S. Geological Survey Special Publication p. 115-117.

Nuttli, O. W., 1973, Seismic wave attenuation and magnitude relations for eastern North America: Journal of Geophysical Research, v. 78, no. 5, p. 876885 .

Richter, C. F., 1958, Elementary Seismology: San Francisco, W. H. Freeman and Company, 768 p.

Saint Louis University, 1984, Central Mississippi Valley Earthquake Bulletins nos. 39-42: Saint Louis University, Department of Earth and Atmospheric Sciences.

Sanford, A. R., 1965, An instrumental study of New Mexico earthquakes: New Mexico Bureau of Mines and Mineral Resources Circular 78, $12 \mathrm{p}$.

Sanford, A. R., and Cash, D. J., 1969, An instrumental study of New Mexico earthquakes, July 1, 1964 through December 31, 1967: New Mexico Bureau of Mines and Mineral Resources Circular 102, $7 \mathrm{p}$.

Sanford, A. R., Olsen, K. H., and Jaksha, L. H., 1981, Earthquakes in New Mexico, 1949-1977: New Mexico Bureau of Mines and Mineral Resources Circular 171, $20 \mathrm{p}$.

Sanford, Allan, and Wieder, Daniel, 1985, Socorro, New Mexico area earthquakes, 1982, in Stover, C. W., ed., United States Earthquakes, 1982: U.S. Geological Survey Bulletin 1655, p. 114-115.

Sanford, Allan, Ake, Jon, Jarpe, Stephen, and Jashka, Lawrence, 1987, Socorro, New Mexico area earthquakes, 1983, in Stover, C. W., ed., United States Earthquakes, 1983: U.S. Geological Survey Bulletin 1698, p. 142-143.

Scharnberger C. K., and Howell, B. F., Jr., 1985, Intensities and structural setting of the earthquakes of 19 April and 23 April, 1984, Lancaster County, Pennsylvania: Eastern Section, Seismological Society of America, Earthquake Notes, v. 56, no. 2, p. 43-46.

Stover, C. W., 1984, Intensity distribution and isoseismal map for the Morgan Hill, California, earthquake of April 24, 1984, in The 1984 Morgan
Hill, California, earthquake: California Division of Mines and Geology Special Publication 68, p. 1-5.

- 1985, Preliminary isoseismal map and intensity distribution for the Laramie Mountains, Wyoming, earthquake of October 18, 1984: U.S. Geological Survey Open-File Report 85-137, 8 p.

Switzer, J. C., Johnson, D. A., Maley, R. P., and Matthieson, R. B., 1981, Western hemisphere strong-motion accelerograph station list - 1980: U.S. Geological Survey Open-File Report 81-664, $162 \mathrm{p}$.

Taylor, S. R., and Toksoz, M. N., 1979, Three-dimensional crust and upper mantle structure of the Northeastern United States: Journal of Geophysical Research, v. 84, p. 7627-7644.

Thiel, C. C., Jr., ed., 1985, The Morgan Hill, California earthquake of April 24, 1984: EERI, Earthquake Spectra, v. 1, no. 3, 694 p.

Toppozada, T. R., and Sanford, A. R., 1972, Instrumental study of New Mexico earthquakes, January 1968 through June 1971: New Mexico Bureau of Mines and Mineral Resources Circular 126, 6 p.

Ward, R. M., 1980, Determination of three-dimensional anomalies within the upper crust in the vicinity of Socorro, New Mexico, using first $P$-arrival times from local earthquakes: Socorro, New Mexico Institute of Mining and Technology, Ph. D. Dissertation, $236 \mathrm{p}$.

Ward, R. M., Schlue, J. W., and Sanford, A. R., 1981, Three-dimensional velocity anomalies in the upper crust near Socorro, New Mexico: Geophysical Research Letters, v. 8, p. 553-556.

Wolff, J. J., Cash, D. J., Olsen, K. H., and Stewart, J. N., 1985, Earthquake catalog for Northern New Mexico, 1984: Los Alamos National Laboratory Progress Report LA-10598-PR, 16 p.

Wood, H. O., and Newmann, Frank, 1931, Modified Mercalli Intensity Scale of 1931: Seismological Society of America Bulletin, v. 21, no. 4, p. 277-283.

Zollweg, J. E., and Jacobson, R. S., 1986, A seismic zone on the Oregon-Idaho border- The Powder River earthquakes of 1984: Seismological Society of America Bulletin, v. 76, no. 4, p. 985-999. 


\section{ERRATA}

United States Earthquakes, 1983

U.S. Geological Survey Bulletin 1698

"Earthquake Descriptions"

1. The following event descriptions should be changed to the following:

Page 16

Page 38

Page 47

Page 50

Page 55

Page 55

Page 66

Page 66

Page 85

\section{January (TC) Central Arkansas}

This earthquake is one of a series that began on January 12,1982 .

22 July (BK) Central California Origin time: 032902.9 Epicenter: $36.223 \mathrm{~N} ., 120.450 \mathrm{~W}$.

9 May (HV) Island of Hawaii

Origin time: 161652.3

Epicenter: $20.653 \mathrm{~N} ., 155.921 \mathrm{~W}$.

16 November (HV) Island of Hawaii

Origin time: 161300.1

Epicenter: $19.429 \mathrm{~N} ., 155.452 \mathrm{~W}$.

19 November (HV) Island of Hawaii

Origin time: 113751.7

Epicenter: $19.290 \mathrm{~N} ., 155.261 \mathrm{~W}$.

27 November (HV) Island of Hawaii

Origin time: 192731.7

Epicenter: 19.358 N., $155.050 \mathrm{~W}$.

13 December (GS) Central Idaho

Origin time: 171338.6

Epicenter: $44.244 \mathrm{~N} ., 114.074 \mathrm{~W}$.

15 December (GS) Central Idaho

Origin time: 061334.8

Epicenter: $44.365 \mathrm{~N} ., 114.138 \mathrm{~W}$.

29 August (UU) Northern Utah

Origin time: 125311.5

Epicenter: $41.083 \mathrm{~N} ., 11.427 \mathrm{~W}$.

2. Captions for figure 75 and figure 76 on page 147 should be interchánged with each other. 
United States Earthquakes, 1984

"Earthquake Descriptions"

The following event description was omitted:

19 December (GS) Central Alaska

Origin time: 005152.2

Epicenter: 66.067N., 154.489 W.

Dep th: $27 \mathrm{~km}$

Magnitude: $3.8 \mathrm{M}_{\mathrm{L}}(\mathrm{PM})$

Intensity II: Hughes (PM).

\section{"Table 1"}

The following hypocenter on page 113 should be listed in CALIFORNIA OFF THE COAST:

Nov. 25050850.1 



\section{SELECTED SERIES OF U.S. GEOLOGICAL SURVEY PUBLICATIONS}

\section{Perlodicals}

Earthquakes \& Volcanoes (issued bimonthly).

Preliminary Determination of Epicenters (issued monthly).

\section{Technical Books and Reports}

Professional Papers are mainly comprehensive scientific reports of wide and lasting interest and importance to professional scientists and engineers. Included are reports on the results of resource studies and of topographic, hydrologic, and geologic investigations. They also include collections of related papers addressing different aspects of a single scientific topic.

Bulletins contain significant data and interpretations that are of lasting scientific interest but are generally more limited in scope or geographic coverage than Professional Papers. They include the results of resource studies and of geologic and topographic investigations; as well as collections of short papers related to a specific topic.

Water-Supply Papers are comprehensive reports that present significant interpretive results of hydrologic investigations of wide interest to professional geologists, hych ologists, and engineers. The series covers investigations in all phases of hydrology, including hydrogeology, availability of water, quality of water, and use of water.

Clrculars present administrative information or important scientific information of wide popular interest in a format designed for distribution at no cost to the public. Information is usually of short-term interest.

Water-Resources Investigations Reports are papers of an interpretive nature made available to the public outside the formal USGS publications series. Copies are reproduced on request unlike formal USGS publications, and they are also available for public inspection at depositories indicated in USGS catalogs.

Open-File Reports include unpublished manuscript reports, maps, and other material that are made available for public consultation at depositories. They are a nonpermanent form of publication that may be cited in other publications as sources of information.

\section{Maps}

Geologic Quadrangle Maps are multicolor geologic maps on topographic bases in 71/2-or 15 -minute quadrangle formats (scales mainly $1: 24,000$ or $1: 62,500$ ) showing bedrock, surficial, or engineering geology. Maps generally include brief texts; some maps include structure and columnar sections only.

Geophysical Investigations Maps are on topographic or planimetric bases at various scales; they show results of surveys using geophysical techniques, such as gravity, magnetic, seismic, or radioactivity, which reflect subsurface structures that are of economic or geologic significance. Many maps include correlations with the geology.

Miscellaneous Investigations Series Maps are on planimetric or topographic bases of regular and irregular areas at various scales; they present a wide variety of format and subject matter. The series also includes 7 1/2-minute quadrangle photogeologic maps on planimetric bases which show geology as interpreted from aerial photographs. Series also includes maps of Mars and the Moon.
Coal Investigations Maps are geologic maps on topographic o: planimetric bases at various scales showing bedrock or surficial geol ogy, stratigraphy, and structural relations in certain coal-resource areas

Oll and Gas Investigations Charts show stratigraphic informatior for certain oil and gas fields and other areas having petroleum potential

Miscellaneous Field Studies Maps are multicolor or black-and white maps on topographic or planimetric bases on quadrangle or ir regular areas at various scales. Pre-1971 maps show bedrock geolog) in relation to specific mining or mineral-deposit problems; post-1971 maps are primarily black-and-white maps on various subjects such a environmental studies or wilderness mineral investigations.

Hydrologic Investigations Atlases are multicolored or black-and. white maps on topographic or planimetric bases presenting a wide range of geohydrologic data of both regular and irregular areas; principal scale is $1: 24,000$ and regional studies are at $1: 250,000$ scale or smaller.

\section{Catalogs}

Permanent catalogs, as well as some others, giving comprehen sive listings of U.S. Geological Survey publications are available unde the conditions indicated below from the U.S. Geological Survey, Book. and Open-File Reports Section, Federal Center, Box 25425, Denver. CO 80225 . (See latest Price and Availability List.)

"Publications of the Geological Survey, 1879-1961" may be purchased by mail and over the counter in paperback book form and as a set of microfiche.

"Publications of the Geological Survey, 1962-1970" may be purchased by mail and over the counter in paperback book form and as a set of microfiche.

"Publications of the U.S. Geological Survey, 1971- 1981" may be purchased by mail and over the counter in paperback book form (two volumes, publications listing and index) and as a set of microfiche.

Supplements for 1982, 1983, 1984, 1985, 1986, and for subsequent years since the last permanent catalog may be purchased by mail and over the counter in paperback book form.

State catalogs, "List of U.S. Geological Survey Geologic and Water-Supply Reports and Maps For (State)," may be purchased by mail and over the counter in paperback booklet form only

"Price and Avallability List of U.S. Geological Survey Publications," issued annually, is available free of charge in paperback booklet form only.

Selected coples of a monthly catalog "New Publications of the U.S. Geological Survey" available free of charge by mail or may be obtained over the counter in paperback booklet form only. Those wishing a free subscription to the monthly catalog "New Publications of the U.S. Geological Survey" should write to the U.S. Geological Survey, 582 National Center, Reston, VA 22092.

Note.--Prices of Government publications listed in older catalogs, announcements, and publications may be incorrect. Therefore, the prices charged may differ from the prices in catalogs, announcements, and publications. 


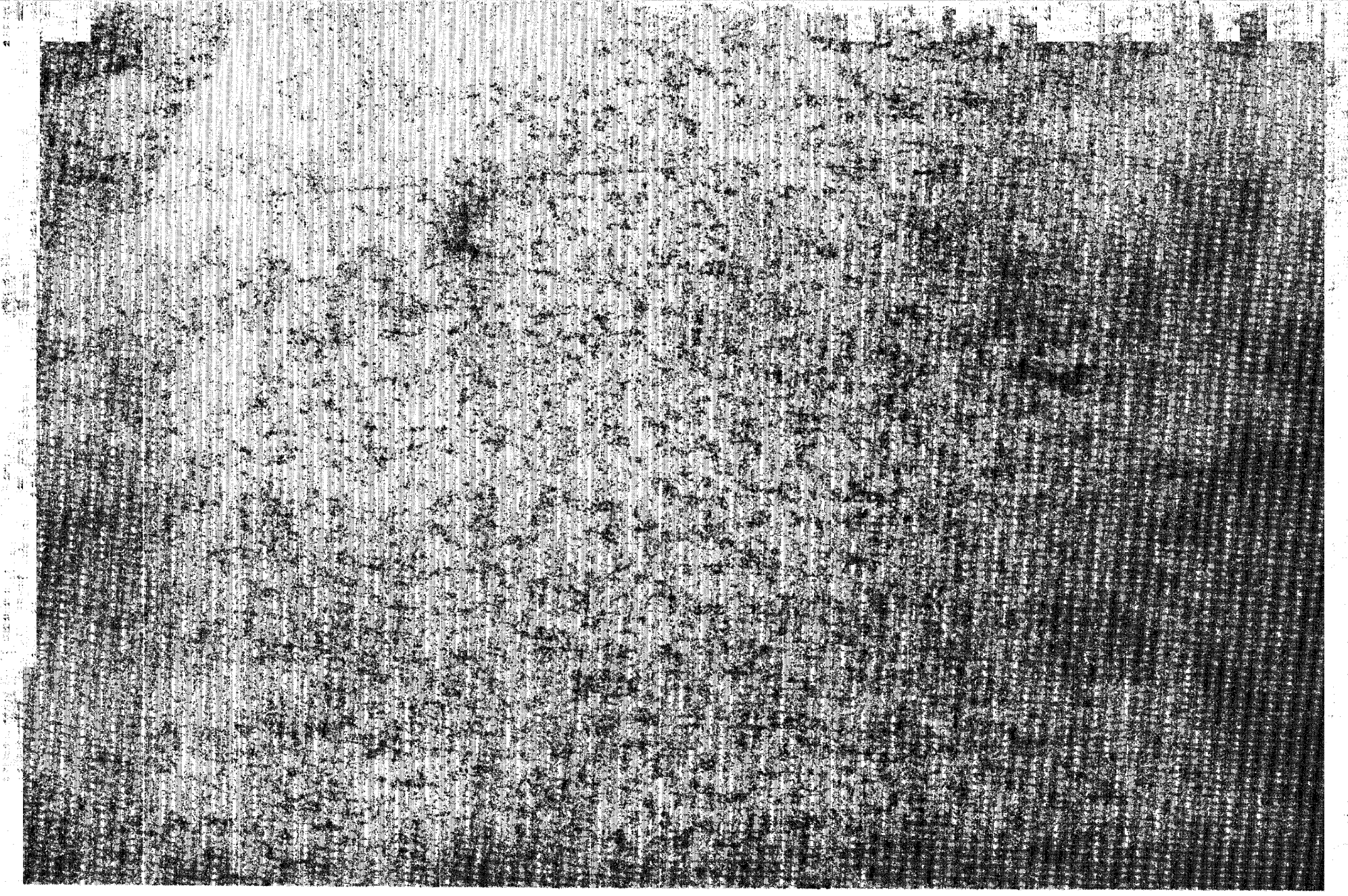

